
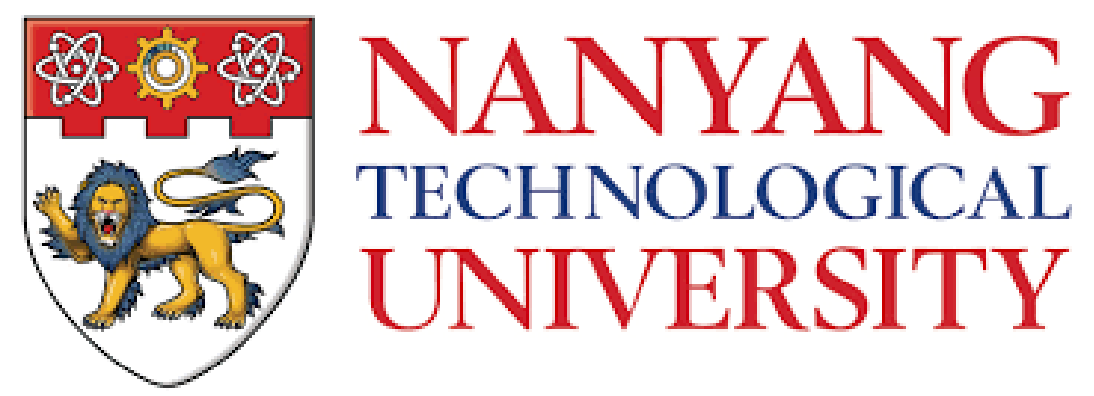

EXPERIMENTAL AND ANALYTICAL INVESTIGATIONS ON SEISMIC BEHAVIOR OF NON-RECTANGULAR REINFORCED CONCRETE SQUAT WALLS

\author{
MA JIAXING
}

SCHOOL OF CIVIL AND ENVIRONMENTAL ENGINEERING NANYANG TECHNOLOGICAL UNIVERSITY 


\section{EXPERIMENTAL AND ANALYTICAL INVESTIGATIONS ON SEISMIC BEHAVIOR OF NON-RECTANGULAR REINFORCED CONCRETE SQUAT WALLS}

MA JIAXING

School of Civil and Environmental Engineering

A thesis submitted to the Nanyang Technological University in fulfillment of the requirements for the degree of

Doctor of Philosophy 


\section{ACKNOWLEDGEMENTS}

The study presented in this thesis was conducted at the School of Civil and Environment Engineering (CEE) of Nanyang Technological University, Singapore.

The author would like to express his sincerest appreciation to his supervisor, Associate Professor $\mathrm{Li}$ Bing, for his patience and continued guidance, his constructive advice and his invaluable support throughout these years. His encouragement and help were essential in the fulfillment of the study. It was such a privilege to learn from a great mentor.

Great appreciation was given to the academic staffs of School of CEE, who offered their knowledge without reservation, and all technicians in the Protective Engineering Laboratory, including Mr. Chelladurai Subasanran, Mr. Jee Kim Tian, Mr. Tui Cheng Hoon, Mr. Chan Chiew Choon, Mr. Goh Choon Kiang, Mr. Phua Kok Soon and Mr. Ho Yaow Chan, who provided pertinent suggestions and continuous help. Special thanks were given to Mr. Yao Ming from Fongsoon Engineering for the advice and kindness.

Thanks were also extended to his colleagues in the same research team. They included Mr. Qian Kai, Mr. Zhang Zhongwen, Mr. Xiao Yao, Mr. Ning Chaolie, Mr. Pooya Alaee, Mr. Prakash Agarwal, Mr. Kang Shaobo and Mr. Vu Ngoc Son. They kindly assisted the author in his research work. Special appreciations were given to Mr. Pooya Alaee, for the inspiration and encouragement, and Mr. Ning Chaolie, for the guidance and meaningful discussions on topics covered in this thesis.

Last and the most important, the author would like to express his deepest gratitude to his beloved family, especially Mr. Ma Changwang and Ms. Chen Tingfang. This study could not be done without their endless and unconditional love, support, and encouragement. 


\begin{abstract}
Non-rectangular reinforced concrete (RC) squat walls are widely used in existing buildings and safety-related nuclear facilities. Compared with rectangular walls, these walls possess significant stiffness in both principal directions, which contributes to their resistance to biaxial bending actions during earthquakes. In spite of their popularity and practicability, the experimental data on these non-rectangular RC squat walls are rather sparse, especially for walls subjected to non-principal bending action. Moreover, current design procedures are developed for rectangular slender walls, which have been found by many researchers to be unsuitable for nonrectangular RC squat walls. To improve the understanding of the seismic behavior of such walls, both experimental and analytical investigations are conducted in this study.
\end{abstract}

Twelve non-rectangular RC squat walls with L-shaped, H-shaped, and T-shaped sections are tested under simulated gravity and reversed cyclic lateral loading at Nanyang Technological University. Variables of interest include axial loads and lateral loading directions. The seismic performance of the specimens is studied and discussed regarding cracking patterns, failure mechanisms, hysteretic responses, displacement components and strain profiles. After that, using nonlinear section analyses and methods available in the literature, further investigations and comparisons are carried out on several important subjects such as failure modes, shear lag effect, and effective stiffness.

Following that, the peak shear strength of non-rectangular RC squat walls is thoroughly studied. The equations provided by building codes and different researchers are initially assessed through comparisons between the predicted and experimental results. A modified strut-and-tie model, including effects of large flanges, is then developed to account for the force-resisting mechanism in nonrectangular RC squat walls and to generate the function form of the peak shear strength equation. Nonlinear regression is conducted to obtain the values of unknown coefficients in the function form. The finalized equation is evaluated statistically with methods available in the literature. Finally, the probabilistic 
approach is adopted to reconcile large scatters and to avoid the biased estimate found in the deterministic prediction of the peak shear strength of non-rectangular $\mathrm{RC}$ squat walls.

To improve the understanding of the seismic behavior of non-rectangular RC squat walls under different lateral loading directions, 3D finite element analyses are performed using the nonlinear finite element software Diana 9.4. Simulations of test specimens are first conducted to verify the capability of the introduced finite element approaches. A comprehensive parametric study is then carried out to investigate the influence of lateral loading direction on the peak shear strength of non-rectangular RC squat walls. The effects of section shapes, flange lengths and axial loads on the peak shear strength are also reported. 


\section{TABLE OF CONTENTS}

ACKNOWLEDGEMENTS

$\begin{array}{ll}\text { ABSTRACT } & \text { ii }\end{array}$

TABLE OF CONTENTS iv

$\begin{array}{ll}\text { LIST OF TABLES } & \text { ix }\end{array}$

LIST OF FIGURES - Xi

LIST OF SYMBOLS XXV

$\begin{array}{lll}\text { Chapter } 1 & \text { INTRODUCTION } & 1\end{array}$

$\begin{array}{lll}1.1 & \text { General Background } & 1\end{array}$

1.2 Objectives and Scope 4

1.3 Organization of the Thesis 5

$\begin{array}{lll}\text { Chapter } 2 \text { LITERATURE REVIEW } & 6\end{array}$

$\begin{array}{lll}2.1 & \text { Introduction } & 6\end{array}$

2.2 Estimation of the Peak Shear Strength of RC Squat Walls 6

2.2.1 Section 11.5 of ACI 318-14 6

$\begin{array}{lll}\text { 2.2.2 Section } 18.10 \text { of ACI 318-14 } & 7\end{array}$

$\begin{array}{lll}\text { 2.2.3 ASCE 43-05 } & 8\end{array}$

2.2.4 Barda et al. (1977) 9

2.2.5 Wood (1990) 9

$\begin{array}{ll}\text { 2.2.6 Hwang et al. (2005) } & 10\end{array}$

$\begin{array}{lll}\text { 2.2.7 } & \text { Gulec and Whittaker (2011) }\end{array}$

$\begin{array}{lll}2.2 .8 & \text { Kassem (2014) } & 11\end{array}$

$2.3 \quad$ Previously Research on Non-rectangular RC Squat Walls 12

2.3.1 Research conducted by Barda et al. (1972) 13 
$\begin{array}{lll}\text { 2.3.2 Research conducted by Synge (1980) } & 14\end{array}$

2.3.3 Research conducted by Mo and Chan (1996) 16

2.3.4 Research conducted by Kitada et al. (1997) 17

2.3.5 Research conducted by Palermo and Vecchio (2002) 19

2.3.6 Research conducted by Farvashany et al. (2008) 20

2.3.7 Database of non-rectangular RC squat walls 22

$\begin{array}{lll}2.4 & \text { Summary } & 38\end{array}$

Chapter 3 EXPERIMENTAL PROGRAM OF NON-RECTANGULAR

RC SQUAT WALLS 40

$\begin{array}{lll}3.1 & \text { Introduction } & 40\end{array}$

3.2 Design of Specimens 40

3.2.1 Specimen details $\quad 40$

3.2.2 Material properties 49

$\begin{array}{lll}\text { 3.2.3 Design considerations } & 50\end{array}$

3.3 Test Setup and Loading Sequence 51

$\begin{array}{lll}3.4 & \text { Instrumentation } & 53\end{array}$

3.4.1 Measurement of loads 53

3.4.2 Measurement of strains in reinforcing bars 54

3.4.3 Measurement of lateral displacements and deformations $\quad 61$

$\begin{array}{lll}3.5 & \text { Summary } & 62\end{array}$

Chapter 4 PRESENTATION OF THE EXPERIMENTAL RESULTS 63

$\begin{array}{lll}4.1 & \text { Introduction } & 63\end{array}$

4.2 Experimental Results of the L-shaped Specimens 63

$\begin{array}{lll}\text { 4.2.1 Cracking patterns } & 63\end{array}$

$\begin{array}{lll}\text { 4.2.2 Failure mechanisms } & 69\end{array}$

4.2.3 Hysteretic responses 72 
$\begin{array}{lll}\text { 4.2.4 Displacement components } & 78\end{array}$

$\begin{array}{lll}\text { 4.2.5 Strain profiles } & 81\end{array}$

4.3 Experimental Results of the H-shaped Specimens 94

$\begin{array}{lll}\text { 4.3.1 Cracking patterns } & 94\end{array}$

$\begin{array}{lll}\text { 4.3.2 } & \text { Failure mechanisms } & 100\end{array}$

4.3.3 Hysteretic responses 103

$\begin{array}{ll}\text { 4.3.4 Displacement components } & 110\end{array}$

$\begin{array}{lll}\text { 4.3.5 Strain profiles } & 113\end{array}$

4.4 Experimental Results of the T-shaped Specimens 124

$\begin{array}{lll}\text { 4.4.1 Cracking patterns } & 124\end{array}$

$\begin{array}{lll}\text { 4.4.2 Failure mechanisms } & 129\end{array}$

4.4.3 Hysteretic responses 132

$\begin{array}{lll}\text { 4.4.4 Displacement components } & 138\end{array}$

$\begin{array}{lll}\text { 4.4.5 Strain profiles } & 141\end{array}$

$\begin{array}{lll}4.5 & \text { Summary } & 155\end{array}$

Chapter 5 DISCUSSIONS AND COMPARISONS OF THE EXPERIMENTAL RESULTS 158

$\begin{array}{lll}5.1 & \text { Introduction } & 158\end{array}$

5.2 Cracking Patterns and Failure Mechanisms 158

5.3 Load-displacement Relationships 166

$\begin{array}{lll}\text { 5.3.1 Backbone curve } & 166\end{array}$

$\begin{array}{lll}\text { 5.3.2 } & \text { Effective stiffness } & 167\end{array}$

5.3.3 Yield displacement and deformation capacity 170

$\begin{array}{lll}5.4 & \text { Shear Deformation } & 172\end{array}$

5.5 Strain in Horizontal Reinforcement 173

$\begin{array}{lll}5.6 & \text { Shear Lag Effect } & 175\end{array}$ 
5.7.1 Effects of axial loads

$\begin{array}{lll}\text { 5.7.2 } & \text { Effects of loading directions } & 181\end{array}$

\section{Chapter 6}

6.1

6.2

6.3.1 Development of the function form

6.3.2 Nonlinear regression

6.3.3 Equations for the peak shear strength of non-rectangular RC squat walls

6.4 Probabilistic Approach for the Peak Shear Strength of Non-rectangular RC Squat Walls

6.4.1 Introduction

6.4.2 Probabilistic approach

6.4.3 Uncertainty estimation of unknown model parameters

6.4.4 Sensitivity analysis of the GLUE method

6.4.5 Evaluation of the proposed probabilistic equation

6.4.6 Simplification of the proposed probabilistic equation

6.4.7 Uncertainty bounds of the prediction of shear strength

6.4.8 Conclusion

\section{Chapter 7 INFLUENCE OF LATERAL LOADING DIRECTION ON THE PEAK SHEAR STRENGTH OF NON-RECTANGULAR RC SQUAT WALLS}


$\begin{array}{lll}7.1 & \text { Introduction } & 235\end{array}$

$\begin{array}{lll}7.2 & \text { Finite Element Model } & 236\end{array}$

$\begin{array}{lll}\text { 7.2.1 Element types } & 236\end{array}$

$\begin{array}{lll}\text { 7.2.2 Constitutive laws for concrete } & 238\end{array}$

7.2.3 Constitutive laws for reinforcement 243

$\begin{array}{lll}\text { 7.2.4 Iterative solution methods } & 244\end{array}$

7.3 Validation of the Finite Element Models 247

7.4 Parametric Study on the Peak Shear Strength of Non-rectangular RC Squat Walls 262

$\begin{array}{lll}\text { 7.4.1 Model layouts } & 262\end{array}$

7.4.2 Results of finite element analyses 265

$\begin{array}{lll}7.5 & \text { Summary } & 272\end{array}$

$\begin{array}{lll}\text { Chapter } 8 & \text { SUMMARY AND CONCLUSIONS }\end{array}$

$\begin{array}{lll}8.1 & \text { Summary and Conclusions } & 274\end{array}$

8.1.1 Seismic performance of specimens 275

8.1.2 Peak shear strength of non-rectangular RC squat walls 277

8.1.3 Influence of lateral loading direction on the peak shear strength of non-rectangular RC squat walls 278

8.2 Suggestions for Future Research 279

$\begin{array}{ll}\text { REFERENCES } & 280\end{array}$ 


\section{LIST OF TABLES}

Table

Page

\section{Chapter 2}

Table 2.1 Constants $A$ and $B$ for the calculation of $\rho s e$ [A3]

Table 2.2 Geometric properties of non-rectangular RC squat walls

Table 2.3 Material properties of non-rectangular RC squat walls

Table $2.4 \quad$ Performance of non-rectangular RC squat walls

\section{Chapter 3}

Table $3.1 \quad$ Summary of specimens

Table 3.2

Concrete properties

Table 3.3 Reinforcement properties

Table 3.4

Flexural and shear capacities of specimens $(\mathrm{kN})$

\section{Chapter 4}

Table 4.1 Test results of the L-shaped specimens

Table 4.2 Test results of the H-shaped specimens

Table 4.3 Test results of the T-shaped specimens

\section{Chapter 5}

Table 5.1 Comparisons between the predicted and experimental effective stiffness

Table 5.2 Summary of test results

Table 5.3 Ratios of experimental to predicted base moments at 


\section{Chapter 6}

Table 6.1 Predicted peak shear strength of test specimens $(\mathrm{kN})$

Table 6.2 Statistics for ratios of the predicted to measured shear strength using different sets of equations

Table 6.3 Calculated coefficients for the proposed equation and the refined ACI and ASCE equations

Table 6.4 Statistics for ratios of the calculated to measured peak shear strength using the eleven sets of equations

Table 6.5 Experimental database of non-rectangular RC squat walls

Table 6.6 Critical values and tested results of the

Kolmogorov-Smirnov test

Table 6.7 Distributed parameters of theoretical probability

distributions for $k_{1}, k_{2}, k_{3}$ and $k_{4}$

Table 6.8 Prior and posterior correlation coefficients of

$k_{1}, k_{2}, k_{3}$ and $k_{4}$

Table 6.9 Probabilities of the predicted shear strength

underestimating the measured shear strength

\section{Chapter 7}

Table 7.1 Material properties of test specimens

Table 7.2

Summary of parameters 


\section{LIST OF FIGURES}

Figure

Page

\section{Chapter 1}

Figure 1.1 Floor plan of the Chernobyl nuclear power plant

(courtesy of Carl Willis)

Figure 1.2 Typical story framing plan [B5]

Figure 1.3 Failure of a shear wall in 2010 Chile earthquake [M2]

\section{Chapter 2}

Figure 2.1 Details of specimens tested by Barda et al. [B1]

Figure 2.2 Details of Wall 3 tested by Synge [S10]

Figure 2.3 Details of specimens tested by Mo and Chan [M4]

Figure 2.4 Details of specimens tested by Kitada et al. [K2]

Figure 2.5 Details of specimens tested by Palermo and Vecchio [P2]

Figure 2.6 Details of specimens tested by Farvashany et al. [F1]

\section{Chapter 3}

Figure 3.1 Details of the H-shaped specimens in plan view

Figure 3.2 Details of the L-shaped specimens in plan view

Figure 3.3 Details of the T-shaped specimens in plan view

Figure 3.4 Details of specimens in front view

Figure 3.5 Photo of a H-shaped specimen

Figure 3.6 Photo of a L-shaped specimen 
$\begin{array}{lll}\text { Figure } 3.7 & \text { Photo of a T-shaped specimen } & 48\end{array}$

$\begin{array}{lll}\text { Figure 3.8 } & \text { Test setup in isometric view }\end{array}$

Figure 3.9 Photo of the test setup 52

$\begin{array}{lll}\text { Figure } 3.10 \quad \text { Loading history } & 53\end{array}$

Figure 3.11 Positions of strain gauges in Specimens HP0D0 and HP5D0 55

Figure 3.12 Positions of strain gauges in Specimens HP0D45 and 56 HP5D45

Figure 3.13 Positions of strain gauges in Specimens LP0D0 and LP5D0 57

Figure 3.14 Positions of strain gauges in Specimens LP0D45 and 58 LP5D45

Figure 3.15 Positions of strain gauges in Specimens TP0D0 and TP5D0 59

Figure 3.16 Positions of strain gauges in Specimens TP0D45 and 60 TP5D45

$\begin{array}{lll}\text { Figure } 3.17 \quad \text { Arrangements of LVDTs in specimens } & 61\end{array}$

\section{Chapter 4}

Figure 4.1 Cracking patterns of Specimen LP0D0 64

Figure 4.2 Cracking patterns of Specimen LP0D45 65

Figure 4.3 Cracking patterns of Specimen LP5D0 66

Figure 4.4 Cracking patterns of Specimen LP5D45 68

Figure 4.5 Failure mechanism of Specimen LP0D0 70

$\begin{array}{lll}\text { Figure 4.6 } & \text { Failure mechanism of Specimen LP0D45 }\end{array}$

$\begin{array}{lll}\text { Figure 4.7 Failure mechanism of Specimen LP5D0 } & 71\end{array}$

Figure 4.8 Failure mechanism of Specimen LP5D45 72 
Figure 4.9 Lateral load-displacement curve of Specimen LP0D0 73

$\begin{array}{lll}\text { Figure 4.10 } & \text { Lateral load-displacement curve of Specimen LP0D45 } & 74\end{array}$

Figure 4.11 Lateral load-displacement curve of Specimen LP5D0 74

Figure 4.12 Lateral load-displacement curve of Specimen LP5D45 75

$\begin{array}{lll}\text { Figure 4.13 Backbone curves of the L-shaped specimens } & 77\end{array}$

$\begin{array}{lll}\text { Figure 4.14 Secant stiffness of the L-shaped specimens } & 77\end{array}$

$\begin{array}{lll}\text { Figure 4.15 Energy dissipation capacities of the L-shaped specimens } & 78\end{array}$

Figure 4.16 Displacement components of Specimen LP0D0 80

Figure 4.17 Displacement components of Specimen LP0D45 80

$\begin{array}{lll}\text { Figure 4.18 Displacement components of Specimen LP5D0 } & 81\end{array}$

Figure 4.19 Displacement components of Specimen LP5D45 81

Figure 4.20 Strain profiles of longitudinal reinforcement along the 84 web of Specimen LPOD0 in the positive loading direction

Figure 4.21 Strain profiles of longitudinal reinforcement along the web of Specimen LP0D45 in the positive loading direction

Figure 4.22 Strain profiles of longitudinal reinforcement along the web of Specimen LP5D0 in the positive loading direction

Figure 4.23 Strain profiles of longitudinal reinforcement along the web of Specimen LP5D45 in the positive loading direction

Figure 4.24 Strain profiles of longitudinal reinforcement along the web of Specimen LP0D0 in the negative loading direction

Figure 4.25 Strain profiles of longitudinal reinforcement along the web of Specimen LP0D45 in the negative loading direction 
Figure 4.26 Strain profiles of longitudinal reinforcement along the web of Specimen LP5D0 in the negative loading direction

Figure 4.27 Strain profiles of longitudinal reinforcement along the web of Specimen LP5D45 in the negative loading direction

Figure 4.28 Strain profiles of longitudinal reinforcement along the

flange of Specimen LPOD0 in the positive loading direction

Figure 4.29 Strain profiles of longitudinal reinforcement along the

flange of Specimen LP5D0 in the positive loading direction

Figure 4.30 Strain profiles of longitudinal reinforcement along the

flange of Specimen LPOD0 in the negative loading direction

Figure 4.31 Strain profiles of longitudinal reinforcement along the

flange of Specimen LP5D0 in the negative loading direction

Figure 4.32 Strain profiles of No.1 horizontal rebar of Specimen LP0D0

Figure 4.33 Strain profiles of No.7 horizontal rebar of Specimen LP0D0

Figure 4.34 Strain profiles of No.1 horizontal rebar of Specimen LP0D45 91

Figure 4.35 Strain profiles of No.7 horizontal rebar of Specimen LP0D45 91

Figure 4.36 Strain profiles of No.1 horizontal rebar of Specimen LP5D0 92

Figure 4.37 Strain profiles of No.7 horizontal rebar of Specimen LP5D0 92

Figure 4.38 Strain profiles of No.1 horizontal rebar of Specimen LP5D45 93

Figure 4.39 Strain profiles of No.7 horizontal rebar of Specimen LP5D45 93

Figure 4.40 Cracking patterns of Specimen HPOD0 95

Figure 4.41 Cracking patterns of Specimen HP0D45 96

Figure 4.42 Cracking patterns of Specimen HP5D0 98

Figure 4.43 Cracking patterns of Specimen HP5D45 99 
Figure 4.44 Failure mechanism of Specimen HP0D0

Figure 4.45 Failure mechanism of Specimen HP0D45

Figure 4.46 Failure mechanism of Specimen HP5D0

Figure 4.47 Failure mechanism of Specimen HP5D45

Figure 4.48 Lateral load-displacement curve of Specimen HP0D0

Figure 4.49 Lateral load-displacement curve of Specimen HP0D45

Figure 4.50 Lateral load-displacement curve of Specimen HP5D0

Figure 4.51 Lateral load-displacement curve of Specimen HP5D45

Figure 4.52 Backbone curves of the H-shaped specimens

Figure 4.53 Secant stiffness of the H-shaped specimens

Figure 4.54 Energy dissipation capacities of the H-shaped specimens

Figure 4.55 Displacement components of Specimen HP0D0

Figure 4.56 Displacement components of Specimen HP0D45

Figure 4.57 Displacement components of Specimen HP5D0

Figure 4.58 Displacement components of Specimen HP5D45

Figure 4.59 Strain profiles of longitudinal reinforcement along the web of Specimen HP0D0 in the positive loading direction

Figure 4.60 Strain profiles of longitudinal reinforcement along the web of Specimen HP0D45 in the positive loading direction

Figure 4.61 Strain profiles of longitudinal reinforcement along the web of Specimen HP5D0 in the positive loading direction

Figure 4.62 Strain profiles of longitudinal reinforcement along the web of Specimen HPOD0 in the negative loading direction 
Figure 4.63 Strain profiles of longitudinal reinforcement along the web of Specimen HP0D45 in the negative loading direction

Figure 4.64 Strain profiles of longitudinal reinforcement along the web of Specimen HP5D0 in the negative loading direction

Figure 4.65 Strain profiles of longitudinal reinforcement along the

flange of Specimen HPOD0 in the positive loading direction

Figure 4.66 Strain profiles of longitudinal reinforcement along the

flange of Specimen HP0D45 in the positive loading direction

Figure 4.67 Strain profiles of longitudinal reinforcement along the

flange of Specimen HP5D0 in the positive loading direction

Figure 4.68 Strain profiles of longitudinal reinforcement along the

flange of Specimen HP5D45 in the positive loading direction

Figure 4.69 Strain profiles of longitudinal reinforcement along the

flange of Specimen HPOD0 in the negative loading direction

Figure 4.70 Strain profiles of longitudinal reinforcement along the

flange of Specimen HP0D45 in the negative loading direction

Figure 4.71 Strain profiles of longitudinal reinforcement along the

flange of Specimen HP5D0 in the negative loading direction

Figure 4.72 Strain profiles of longitudinal reinforcement along the

flange of Specimen HP5D45 in the negative loading direction

Figure 4.73 Strain profiles of No.1 horizontal rebar of Specimen HP0D45 122

Figure 4.74 Strain profiles of No.7 horizontal rebar of Specimen HP0D45 122

Figure 4.75 Strain profiles of No.1 horizontal rebar of Specimen HP5D0 123

Figure 4.76 Strain profiles of No.7 horizontal rebar of Specimen HP5D0 123

Figure 4.77 Cracking patterns of Specimen TP0D0 
Figure 4.78 Cracking patterns of Specimen TP0D45

Figure 4.79 Cracking patterns of Specimen TP5D0

Figure 4.80 Cracking patterns of Specimen TP5D45

Figure 4.81 Failure mechanism of Specimen TP0D0

Figure 4.82 Failure mechanism of Specimen TP0D45

Figure 4.83 Failure mechanism of Specimen TP5D0

Figure 4.84 Failure mechanism of Specimen TP5D45

Figure 4.85 Lateral load-displacement curve of Specimen TP0D0

Figure 4.86 Lateral load-displacement curve of Specimen TP0D45

Figure 4.87 Lateral load-displacement curve of Specimen TP5D0

Figure 4.88 Lateral load-displacement curve of Specimen TP5D45

Figure 4.89 Backbone curves of the T-shaped specimens

Figure 4.90 Secant stiffness of the T-shaped specimens

Figure 4.91 Energy dissipation capacities of the T-shaped specimens

Figure 4.92 Displacement components of Specimen TP0D0

Figure 4.93 Displacement components of Specimen TP0D45

Figure 4.94 Displacement components of Specimen TP5D0

Figure 4.95 Displacement components of Specimen TP5D45

Figure 4.96 Strain profiles of longitudinal reinforcement along the web of Specimen TPOD0 in the positive loading direction

Figure 4.97 Strain profiles of longitudinal reinforcement along the web of Specimen TP0D45 in the positive loading direction 
Figure 4.98 Strain profiles of longitudinal reinforcement along the web of Specimen TP5D0 in the positive loading direction

Figure 4.99 Strain profiles of longitudinal reinforcement along the web of Specimen TP5D45 in the positive loading direction

Figure 4.100 Strain profiles of longitudinal reinforcement along the web of Specimen TP0D0 in the negative loading direction

Figure 4.101 Strain profiles of longitudinal reinforcement along the web of Specimen TP0D45 in the negative loading direction

Figure 4.102 Strain profiles of longitudinal reinforcement along the web of Specimen TP5D0 in the negative loading direction

Figure 4.103 Strain profiles of longitudinal reinforcement along the web of Specimen TP5D45 in the negative loading direction

Figure 4.104 Strain profiles of longitudinal reinforcement along the flange of Specimen TPOD0 in the positive loading direction

Figure 4.105 Strain profiles of longitudinal reinforcement along the flange of Specimen TP0D45 in the positive loading direction

Figure 4.106 Strain profiles of longitudinal reinforcement along the

flange of Specimen TP5D0 in the positive loading direction

Figure 4.107 Strain profiles of longitudinal reinforcement along the flange of Specimen TP5D45 in the positive loading direction

Figure 4.108 Strain profiles of longitudinal reinforcement along the flange of Specimen TPOD0 in the negative loading direction

Figure 4.109 Strain profiles of longitudinal reinforcement along the flange of Specimen TP0D45 in the negative loading direction

Figure 4.110 Strain profiles of longitudinal reinforcement along the flange of Specimen TP5D0 in the negative loading direction 
Figure 4.111 Strain profiles of longitudinal reinforcement along the

flange of Specimen TP5D45 in the negative loading direction

Figure 4.112 Strain profiles of No.1 horizontal rebar of Specimen TP0D0 152

Figure 4.113 Strain profiles of No.7 horizontal rebar of Specimen TP0D0 152

Figure 4.114 Strain profiles of No.1 horizontal rebar of Specimen TP0D45 153

Figure 4.115 Strain profiles of No.7 horizontal rebar of Specimen TP0D45 153

Figure 4.116 Strain profiles of No.1 horizontal rebar of Specimen TP5D0 154

Figure 4.117 Strain profiles of No.7 horizontal rebar of Specimen TP5D0 154

Figure 4.118 Strain profiles of No.1 horizontal rebar of Specimen TP5D45 155

Figure 4.119 Strain profiles of No.7 horizontal rebar of Specimen TP5D45 155

\section{Chapter 5}

Figure 5.1 Comparison of the diagonal cracks on the web of

Specimens HP0D0 and HP5D0

Figure 5.2 Failure mechanisms of Specimens HP0D0 and DP2

Figure 5.3 Failure mechanisms of Specimens HP5D0 and DP1

Figure 5.4 Deformation incompatibility in Specimen HP5D0

Figure 5.5 Comparison of the backbone curves of the L-shaped and

$\mathrm{T}$-shaped specimens in the direction of the flange in tension

Figure 5.6 Equilibrium of a shear wall in the softened truss model [H1] 174

Figure 5.7 The diagonal mechanism of the softened strut-and-tie model [H4]

Figure 5.8 Effective flange width [S1]

Figure 5.9 Models of the H-shaped specimens in Xtract [X2] 
Figure 5.10 Models of the L-shaped specimens in Xtract [X2]

Figure 5.11 Models of the T-shaped specimens in Xtract [X2]

\section{Chapter 6}

Figure 6.1 Diagonal mechanism, horizontal and vertical mechanism

of the proposed strut-and-tie model

Figure 6.2 Force distribution in the proposed strut-and-tie model

Figure 6.3 Calculated peak shear strength using equations from

Section 11.5 of ACI 318-14 [A1] versus the measured peak shear strength

Figure 6.4 Calculated peak shear strength using the equation from

Section 18.10 of ACI 318-14 [A1] versus the measured peak shear strength

Figure 6.5 Calculated peak shear strength using the equation from ASCE 43-05 [A3] versus the measured peak shear strength

Figure 6.6 Calculated peak shear strength using the equation from

Barda et al. [B1] versus the measured peak shear strength

Figure 6.7 Calculated peak shear strength using the equation from

Wood [W7] versus the measured peak shear strength

Figure 6.8 Calculated peak shear strength using equations from

Hwang et al. [H4] versus the measured peak shear strength

Figure 6.9 Calculated peak shear strength using the equation from

Gulec and Whittaker [G3] versus the measured peak shear strength

Figure 6.10 Calculated peak shear strength using equations from

Kassem [K1] versus the measured peak shear strength 
Figure 6.11 Calculated peak shear strength using the proposed

equation versus the measured peak shear strength

Figure 6.12 Calculated peak shear strength using the refined

ACI equation versus the measured peak shear strength

Figure 6.13 Calculated peak shear strength using the refined

ASCE equation versus the measured peak shear strength

Figure 6.14 Variation of ratios of predicted and measured shear

strengths (normalized) with the shear span ratio

Figure 6.15 Estimated posterior cumulative distributions and

theoretical cumulative distributions of $k_{1}, k_{2}, k_{3}$ and $k_{4}$

Figure 6.16 Posterior cumulative distributions of $k_{1}, k_{2}, k_{3}$ and $k_{4}$

estimated from the uniform and the normal prior distributions

Figure 6.17 Posterior cumulative distributions of $k_{1}, k_{2}, k_{3}$ and $k_{4}$

estimated with threshold values of $0.6,0.7$ and 0.8

Figure 6.18 Posterior cumulative distributions of $k_{1}, k_{2}, k_{3}$ and $k_{4}$

estimated with different likelihood functions

Figure 6.19 Probability density function of the predicted shear strength

in comparison with the measured shear strength and deterministic predictions for Specimen W12-3 [S2]

Figure 6.20 Probability density function of the predicted shear strength

in comparison with the measured shear strength and deterministic predictions for Specimen LN4-3 [M4]

Figure 6.21 Mean prediction, Mean \pm STD and Mean $\pm 2 \times$ STD for the shear strength of non-rectangular RC squat walls

Figure 6.22 Average relative interval length versus the confidence interval and percentage of measurement coverage versus the confidence interval 
Figure 6.23 Evaluation of the deterministic shear strength equations in a probabilistic manner

\section{Chapter 7}

Figure 7.1 CHX60 element [D1]

Figure 7.2 Reinforcement in the beam element [D1]

Figure 7.3 Reinforcement in the curved shell element [D1]

Figure 7.4 Multi-directional fixed crack model [D1]

Figure 7.5 Predefined stress-strain curves for concrete

in compression [D1]

Figure 7.6 Predefined stress-strain curves for concrete in tension [D1]

Figure 7.7 Regular Newton-Raphson iteration method [D1]

Figure 7.8 Modified Newton-Raphson iteration method [D1]

Figure 7.9 Secant method [D1]

Figure 7.10 Linear stiffness iteration method [D1]

Figure 7.11 Loads and boundary constraints in a representative

finite element model

Figure 7.12 Mesh and reinforcement layout of the L-shaped models

Figure 7.13 Mesh and reinforcement layout of the H-shaped models

Figure 7.14 Mesh and reinforcement layout of the T-shaped models

Figure 7.15 Comparison between experimental and analytical lateral load-displacement curves of Specimen LP0D45

Figure 7.16 Comparison between experimental and analytical lateral load-displacement curves of Specimen LP5D45 
Figure 7.17 Comparison between experimental and analytical lateral

load-displacement curves of Specimen HPOD0

Figure 7.18 Comparison between experimental and analytical lateral load-displacement curves of Specimen HP5D0

Figure 7.19 Comparison between experimental and analytical lateral load-displacement curves of Specimen TP5D0

Figure 7.20 Comparison between experimental and analytical lateral load-displacement curves of Specimen TP5D45

Figure 7.21 Comparison between experimental and analytical strain profiles of longitudinal reinforcement along the web of Specimen LP0D45

Figure 7.22 Comparison between experimental and analytical strain profiles of longitudinal reinforcement along the web of Specimen LP5D45

Figure 7.23 Comparison between experimental and analytical strain profiles of longitudinal reinforcement along the web of Specimen HP0D0

Figure 7.24 Comparison between experimental and analytical strain profiles of longitudinal reinforcement along the web of Specimen HP5D0

Figure 7.25 Comparison between experimental and analytical strain profiles of longitudinal reinforcement along the web of Specimen TP5D0

Figure 7.26 Comparison between experimental and analytical strain profiles of longitudinal reinforcement along the web of Specimen TP5D45

Figure 7.27 Front and side views of the model of Specimen DP1 [P2] 259 
Figure 7.28 Web reinforcement and flange reinforcement in the model 259 of Specimen DP1 [P2]

Figure 7.29 Loads and boundary constraints in the model of Specimen DP1 [P2]

Figure 7.30 Comparison between experimental and analytical lateral load-displacement curves of Specimen DP1 [P2]

Figure 7.31 L-shaped models with $700 \mathrm{~mm}$ overhanging flange length 263

Figure 7.32 H-shaped models with $350 \mathrm{~mm}$ overhanging flange length 263

Figure 7.33 T-shaped models with $350 \mathrm{~mm}$ overhanging flange length 264

Figure 7.34 The peak shear strength of L-shaped RC squat walls under 269 lateral loading from various directions

Figure 7.35 The peak shear strength of H-shaped RC squat walls under 270 lateral loading from various directions

Figure 7.36 The peak shear strength of T-shaped RC squat walls under lateral loading from various directions 


\section{LIST OF SYMBOLS}

A Constraints of linear inequality in the mincing nonlinear solver

$A_{c w} \quad$ Area of the concrete section of the individual vertical wall segment, $\mathrm{mm}^{2}$

$A_{c v} \quad$ Gross area of the concrete section bounded by web thickness and length of section in the direction of the shear force, $\mathrm{mm}^{2}$

$A_{\text {eff }} \quad$ Total wall area of barbell walls and the effective area for flanged walls, in $^{2}$

$A_{f} \quad$ Total area of the longitudinal reinforcement in the flange, $\mathrm{mm}^{2}$

$A_{g} \quad$ Gross area of section, $\mathrm{mm}^{2}$

$A_{h} \quad$ Total area of vertical and horizontal reinforcement, $\mathrm{mm}^{2}$

$A_{t} \quad$ Total area of wall, in ${ }^{2}$

$A_{v} \quad$ Area of shear reinforcement within spacing $s$ or total area of longitudinal reinforcement ratio in the wall, $\mathrm{mm}^{2}$

$A_{w} \quad$ Gross area of the concrete section bounded by web thickness and length of section in the direction of the shear force, $\mathrm{mm}^{2}$

Aeq Constraints of linear equality in the fmincon nonlinear solver

ARIL Average relative interval length

$a_{s} \quad$ Depth of the diagonal strut or horizontal length of the compression zone, $\mathrm{mm}$

$a_{w} \quad$ Depth of the compression zone at the base of the wall, $\mathrm{mm}$

$b \quad$ Constraints of linear inequality the fmincon nonlinear solver

beq Constraints of linear equality the fmincon nonlinear solver 
$C_{d} \quad$ Resultant compressive force of the flange and web concrete, $\mathrm{N}$

$c(x) \quad$ Function that returns vectors the fmincon nonlinear solver

$\operatorname{ceq}(x) \quad$ Function that returns vectors the fmincon nonlinear solver

$\operatorname{cov}\left[\theta_{i}, \theta_{j}\right]$ Covariance of random variables $\theta_{i}$ and $\theta_{j}$

$d \quad$ Distance from extreme compression fiber to the center of force of all reinforcement in tension or length of the wall section, $\mathrm{m}$ or $\mathrm{mm}$

$d_{w} \quad$ Distance from extreme compression fiber to the location of the resultant of tensile force of longitudinal reinforcement or horizontal length between the tensile force $T_{d}$ and compressive force $C_{d}, \mathrm{~m}$

$E_{c} \quad$ Elastic modulus of concrete, $\mathrm{MPa}$

F $\quad$ Function form of probabilistic equation for the shear strength

$F_{h} \quad$ Tensile force in the horizontal tie, $\mathrm{N}$

$F_{v} \quad$ Tensile force in the vertical tie, $\mathrm{N}$

$F_{v b e} \quad$ Force attributed to boundary element reinforcement, $1 \mathrm{~b}$

$F_{v w} \quad$ Force attributed to vertical web reinforcement, $\mathrm{lb}$

$f_{c}^{\prime} \quad$ Compressive strength of concrete, $\mathrm{MPa}$

$f_{t} \quad$ Tensile strength of concrete, $\mathrm{MPa}$

$f_{y} \quad$ Specified yield strength for reinforcement, $\mathrm{MPa}$

$f_{y f} \quad$ Yield strength of longitudinal reinforcement in the flange, $\mathrm{MPa}$

$f_{y h} \quad$ Yield stress of horizontal reinforcement, $\mathrm{MPa}$

$f_{y t} \quad$ Yield stress of horizontal reinforcement, $\mathrm{MPa}$

$f_{y v} \quad$ Yield strength of longitudinal reinforcement, MPa or psi 
$f(x) \quad$ Function that returns a scalar in the fmincon nonlinear solver

G Shear modulus, $\mathrm{N} / \mathrm{m}^{2}$

$G_{c} \quad$ Compressive fracture energy, $\mathrm{N}-\mathrm{mm} / \mathrm{mm}^{2}$

$G_{f} \quad$ Fracture energy, $\mathrm{N}-\mathrm{mm} / \mathrm{mm}^{2}$

$G^{c r} \quad$ Shear modulus after cracking, N/m ${ }^{2}$

$H_{w} \quad$ Distance from horizontal loading to the wall base, $\mathrm{m}$

$h \quad$ Thickness of the wall or crack bandwidth, $m$

$h_{w} \quad$ Height of wall or vertical distance from horizontal loading to the wall base, $\mathrm{m}$ or $\mathrm{mm}$

$I_{e} \quad$ Effective moment of inertia, $\mathrm{mm}^{4}$

$I_{g} \quad$ Moment of inertia of gross section, $\mathrm{mm}^{4}$

$K_{h} \quad$ Index of horizontal tie

$K_{v} \quad$ Index of vertical tie

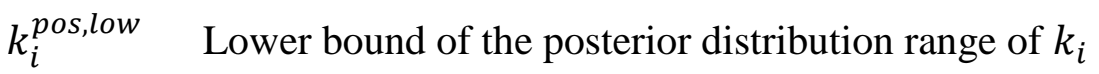

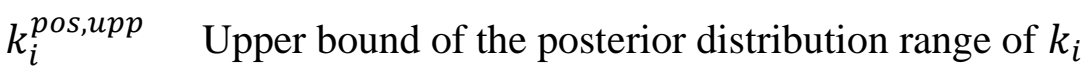

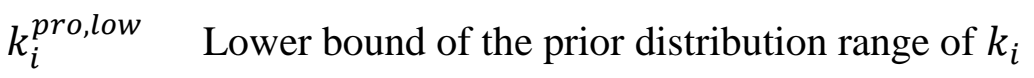

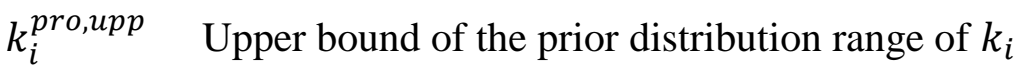

$k_{1} \quad$ Modification coefficient for longitudinal reinforcement in the flange or unknown coefficient or unknown model parameter

$k_{2} \quad$ Modification coefficient for longitudinal reinforcement in the web or unknown coefficient or unknown model parameter

$k_{3} \quad$ Modification coefficient for horizontal reinforcement in the web or unknown coefficient or unknown model parameter 
$k_{4} \quad$ Modification coefficient for the compressive force in the flange and the web or unknown coefficient or unknown model parameter

$L_{w} \quad$ Length of the wall, $\mathrm{m}$ or $\mathrm{mm}$

$l_{f} \quad$ Length of the flange, $\mathrm{m}$ or $\mathrm{mm}$

$l_{w} \quad$ Length of the wall, $\mathrm{m}$ or $\mathrm{mm}$

lb Lower boundary of variables in the fmincon nonlinear solver

$M \quad$ Number of experimental data

$M_{u} \quad$ Factored moment, N-mm

$N \quad$ Axial load or compression force of the diagonal strut or the number of the specimen in database or the number of the behavior parameter set, $\mathrm{N}$

$N_{A} \quad$ Axial load, lb

$N_{u} \quad$ Factored axial compressive force, $\mathrm{N}$

$P \quad$ Axial load, lb or $\mathrm{N}$

$P_{n} \quad$ Axial load, $\mathrm{N}$

$p(x) \quad$ Predicted PDF of shear strength

$p\left(\Theta_{i}\right) \quad$ Probability of the $\mathrm{i}^{\text {th }}$ behavior parameter set

$R_{i} \quad$ Reduction coefficient for $\mathrm{i}^{\text {th }}$ unknown model parameter

$r(i) \quad$ Ratio of calculated shear strength to peak shear strength of specimens in the database

$s \quad$ Center-to-center spacing of horizontal reinforcement, $\mathrm{m}$

$T_{d} \quad$ Tensile force from the longitudinal reinforcement in the flange, $\mathrm{N}$

$t_{f} \quad$ Thickness of the flange, $\mathrm{mm}$

$t_{n} \quad$ Thickness of the wall, in 
$t_{w} \quad$ Thickness of the web, $\mathrm{mm}$

$u b \quad$ Upper boundary of variables in the fmincon nonlinear solver

$V \quad$ Predicted quantity or the lateral reaction force, $\mathrm{kN}$

$V_{A C I} \quad$ Calculated shear strength using modified equations in Section 18.10 of ACI 318-14, N

$V_{A S C E} \quad$ Calculated shear strength using modified equations in ASCE 43-05, N

$V_{\text {avg }} \quad$ Average experimental shear strength of all specimens, $\mathrm{N}$

$V_{B E} \quad$ Peak shear strength of squat walls with boundary elements, $\mathrm{lb}$

$V_{\text {cal }} \quad$ Calculated shear strength, $\mathrm{N}$

$V_{c} \quad$ Nominal shear strength provided by concrete, $\mathrm{N}$

$V_{d} \quad$ Shear strength provided by the diagonal compression strut and flange, $\mathrm{N}$

$V_{\text {exp }} \quad$ Measured shear strength of $\mathrm{k}^{\text {th }}$ specimen for the $\mathrm{i}^{\text {th }}$ parameter set $\Theta_{i}, \mathrm{~N}$

$V_{h} \quad$ Shear strength of squat walls, $\mathrm{N}$

$V_{i}^{L} \quad$ Lower confidence limit for the $\mathrm{i}^{\text {th }}$ specimen in the database

$V_{i}^{U} \quad$ Upper confidence limit for the $\mathrm{i}^{\text {th }}$ specimen in the database

$V_{k} \quad$ Predicted shear strength of $\mathrm{k}^{\text {th }}$ specimen for the $\mathrm{i}^{\text {th }}$ parameter set $\Theta_{i}, \mathrm{~N}$

$V_{n} \quad$ Nominal shear strength, $\mathrm{N}$

$V_{n 1} \quad$ Calculated shear strength using equations in Section 11.5 of ACI 318-14, $\mathrm{N}$

$V_{n 2} \quad$ Calculated shear strength using equations in Section 18.10 of ACI 318$14, \mathrm{~N}$

$V_{n 3} \quad$ Calculated shear strength using equations in ASCE 43-05, $\mathrm{N}$

$V_{n 4} \quad$ Calculated shear strength using equations proposed by Barda et al., $\mathrm{N}$ 
$V_{n 5} \quad$ Calculated shear strength using equations proposed by Wood, $\mathrm{N}$

$V_{n 6} \quad$ Calculated shear strength using equations proposed by Hwang et al., $\mathrm{N}$

$V_{n 7} \quad$ Calculated shear strength using equations proposed by Gulec and Whittaker, N

$V_{n 8} \quad$ Calculated shear strength using equations proposed by Kassem, $\mathrm{N}$

$V_{\text {peak }} \quad$ Peak shear strength of specimens in database, $\mathrm{N}$

$V_{s} \quad$ Nominal shear strength provided by shear reinforcement, $\mathrm{N}$

$V_{u} \quad$ Factored shear force or total shear capacity, $\mathrm{N}$

$V_{w} \quad$ Shear strength provided the web reinforcement, $\mathrm{N}$

$v_{c} \quad$ Poisson's ratio of concrete

$v_{n} \quad$ Shear stress, psi

$v_{R k} \quad$ Ultimate shear stress, $\mathrm{MPa}$

$v_{u} \quad$ Ultimate shear stress, psi

$X \quad$ Design parameters in the probabilistic equation for the shear strength

$x \quad$ Variable in functions in the fmincon nonlinear solver or distance from the base to the top, $\mathrm{mm}$

$z_{w} \quad$ Distance between two flanges, $\mathrm{mm}$

$\alpha \quad$ Inclination angle of the diagonal strut

$\alpha_{c} \quad$ Coefficients defining the ratio of concrete contribution to the nominal shear strength

$\beta \quad$ Reduction factor varying from 1 to 0 for uncracked concrete and completely cracked concrete 
$\gamma_{h} \quad$ Fraction of diagonal compression carried by the horizontal tie in the absence of the vertical tie

$\gamma_{v} \quad$ Fraction of diagonal compression carried by the vertical tie in the absence of the horizontal tie

$\Delta_{\omega} \quad$ Deformation incompatibility between web and flange, $\mathrm{mm}$

$\Theta \quad$ Set of unknown parameters

$\Theta_{i} \quad i^{\text {th }}$ set of unknown parameters

$\theta \quad$ Angle of inclination of the diagonal compression strut

$\theta_{i} \quad$ Random variables

$\theta_{j} \quad$ Random variables

$\theta_{1} \quad$ Angle of inclination of steep strut in vertical mechanism

$\lambda \quad$ Modification factor to reflect the reduced mechanical properties of lightweight concrete relative to normal weight concrete of the same compressive strength

$\xi \quad$ Softening coefficient

$\rho_{f} \quad$ Longitudinal reinforcement ratio in the flange

$\rho_{h} \quad$ Horizontal reinforcement ratio

$\rho_{l} \quad$ Ratio of area of distributed longitudinal reinforcement to gross concrete area perpendicular to that reinforcement

$\rho_{n} \quad$ Longitudinal reinforcement ratio

$\rho_{s e} \quad$ Combined reinforcement ratio calculated using horizontal reinforcement ratio, longitudinal reinforcement ratio and constants $A$ and $B$

$\rho_{t} \quad$ Ratio of area of distributed horizontal reinforcement to gross concrete area perpendicular to that reinforcement 
$\rho_{v} \quad$ Longitudinal reinforcement ratio or longitudinal reinforcement ratio in the web

$\sigma_{0} \quad$ Standard deviation of model error

$\phi \quad$ Capacity reduction factor

$\psi \quad$ Nondimensional function identical to a minor modification of the European code

$\omega_{f} \quad$ Deformation of the flange along the wall height, $\mathrm{mm}$

$\omega_{h} \quad$ Index of horizontal reinforcement

$\omega_{v} \quad$ Index of longitudinal reinforcement

$\omega_{w} \quad$ Deformation of the web along the wall height, $\mathrm{mm}$ 


\section{CHAPTER 1 \\ INTRODUCTION}

\subsection{General Background}

Reinforced concrete (RC) walls with a shear span ratio of less than or equal to two are widely used nowadays, especially in safety-related nuclear facilities (Figure 1.1), where they play a pivotal role in providing the lateral strength and stiffness to the structures [S9]. These walls are described as squat, short or low-rise walls in the literature.

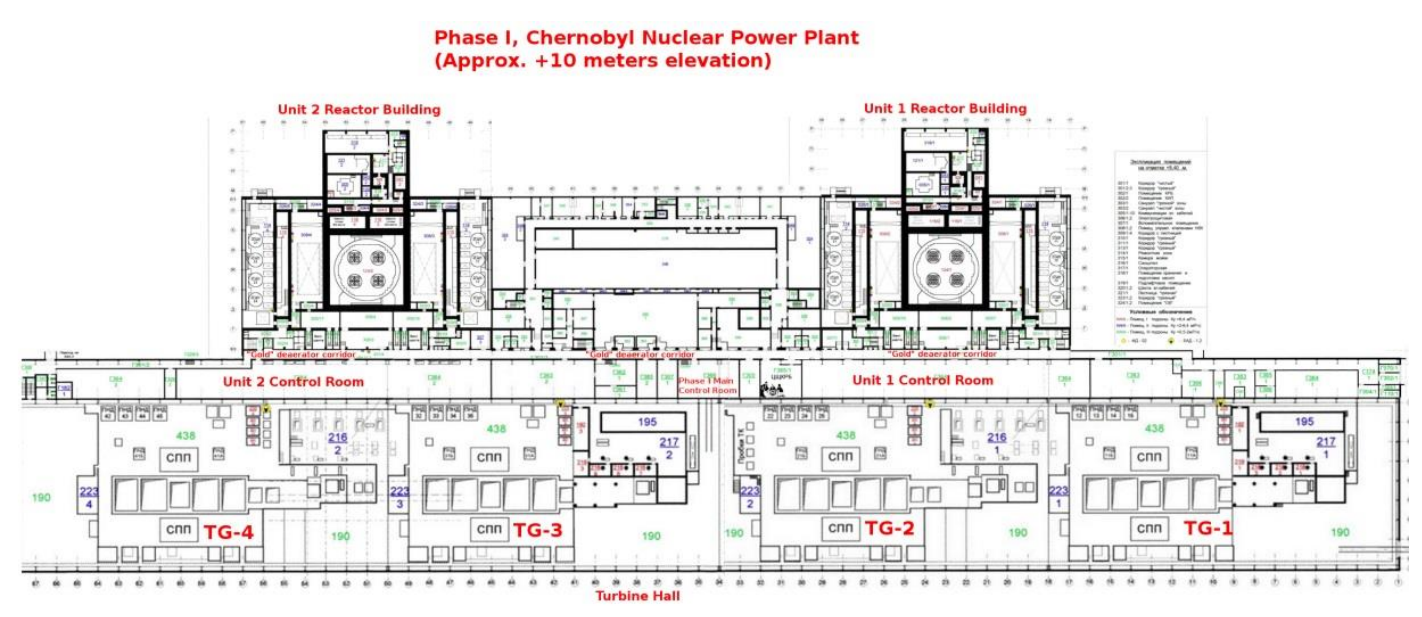

Figure 1.1 Floor plan of the Chernobyl nuclear power plant (courtesy of Carl Willis)

Squat walls can be divided into two groups according to the plan geometry, namely rectangular squat walls and non-rectangular squat walls. The latter are commonly adopted by engineers because they possess significant stiffness in both principal directions, which contributes to their resistance to biaxial bending actions during earthquakes. Moreover, to minimize their impacts on the floor plan (Figure 1.2), architects prefer walls to be concentrated around hallways or elevator cores, which necessitates walls to be designed into non-rectangular shapes. This design is commonly witnessed in the existing buildings in Singapore and other countries in South Asia. 


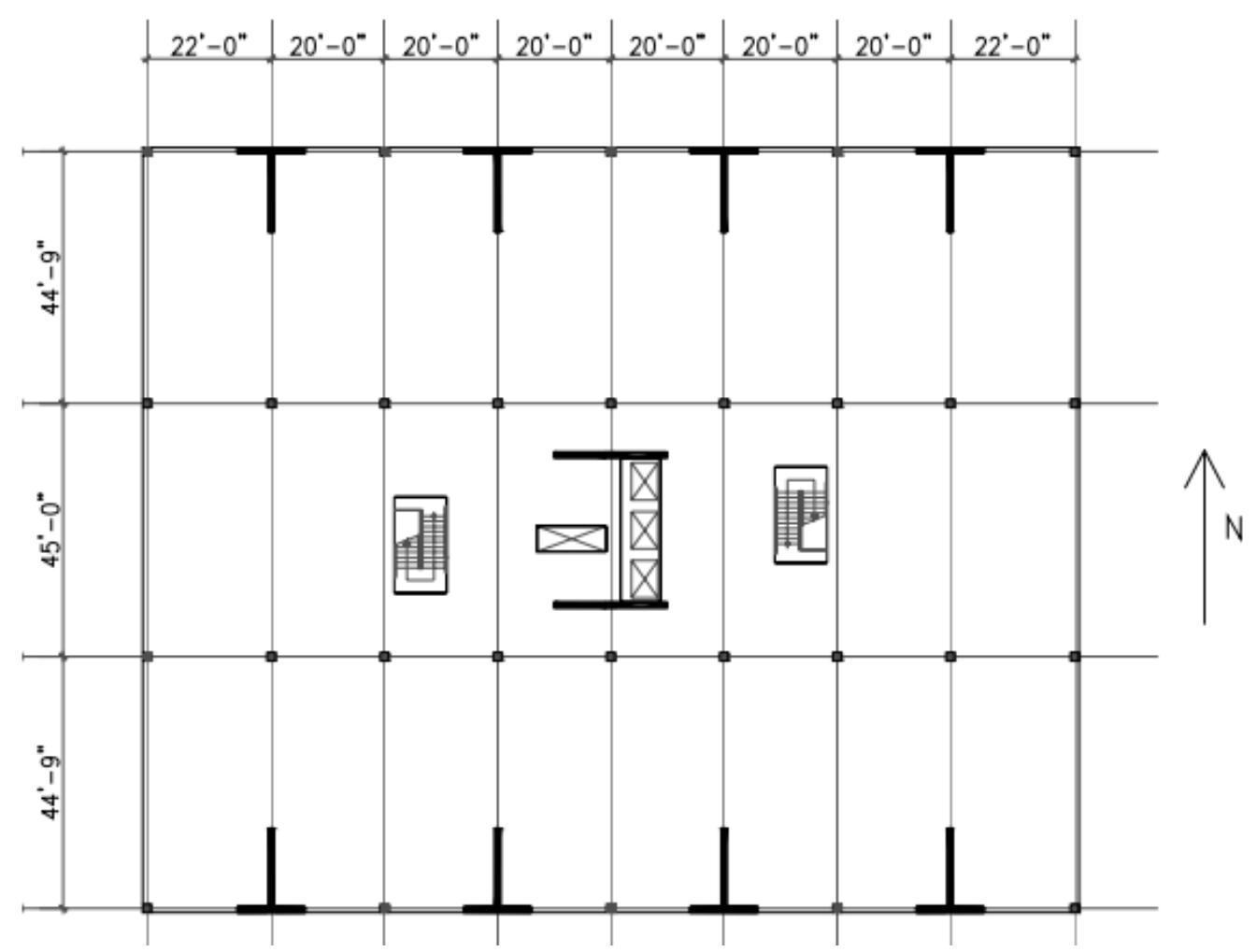

Figure 1.2 Typical story framing plan [B5]

The irregular configuration of non-rectangular squat walls, on the other hand, tends to complicate the interaction between wall segments, which means that it is no longer reasonable to assume that wall segments resist lateral loads in each direction independently [B5]. Moreover, the uniaxial behavior of these walls is also affected due to their non-symmetric sections. When the loading direction is not parallel to the wall segment, the interaction is expected to be further complicated. Besides, unlike rectangular slender walls, which have a tendency for flexural failure, nonrectangular squat walls are mainly controlled by shear, which has a relatively complex failure mechanism.

Scholars were also aware of these problems and conducted a number of experiments in the past decades. The first cyclic tests on squat walls with boundary elements was carried out by Barda et al. [B1] in 1972. Since then, numerous experiments have been conducted on squat shear walls [L2, S7, S10, X1]. In the meantime, many tests have also been done on non-rectangular RC walls [B5, T1, Z1]. Among all these studies, however, there are only a few tests [K1, P1, S2, S3] on L-shaped, H-shaped 
or T-shaped squat walls, let alone experiments in which the lateral loading direction is not parallel to the wall segment.

Furthermore, current design procedures [A1, A3] are developed for rectangular slender walls, and these have remained largely unchanged for many years. Wood [W7] discovered that equations in ACI 318-83 did not correlate well with the measured peak shear strength of squat shear walls. Gulec et al. [G5] and Kassem [K1] found that design procedures in building codes yielded substantial scatters in the prediction of the peak shear strength of squat walls with flanged cross section. Thus research is needed to improve the performance of current design procedures on the prediction of the peak shear strength of non-rectangular RC squat walls.

Demand for further studies on the behavior of structural walls was also highlighted in recent earthquakes. Damages on shear walls were observed in the 2010 Chile Earthquake [M2]. The crisis of Fukushima Daiichi nuclear power plant also sounded the alarm on the safety design of shear walls in the nuclear facilities. Although major earthquakes are rare, Singapore and nearby Peninsular Malaysia are still under the seismic risk, since they lie close to the active earthquake belt, which has caused devastating earthquakes and tsunamis in recent years. Furthermore, RC structural walls in Singapore are mostly designed in accordance with the British Standard, in which the seismic loading is not considered, meaning that these walls are vulnerable to earthquakes. Hence it is necessary to conduct research on structural walls imposed with seismic loads. The authorities also realized this necessity and introduced the Eurocode that contains seismic clauses as the new design standard in 2013.

The above discussions demonstrate the necessity of further research on the behavior of non-rectangular RC squat walls under different lateral loading directions. 


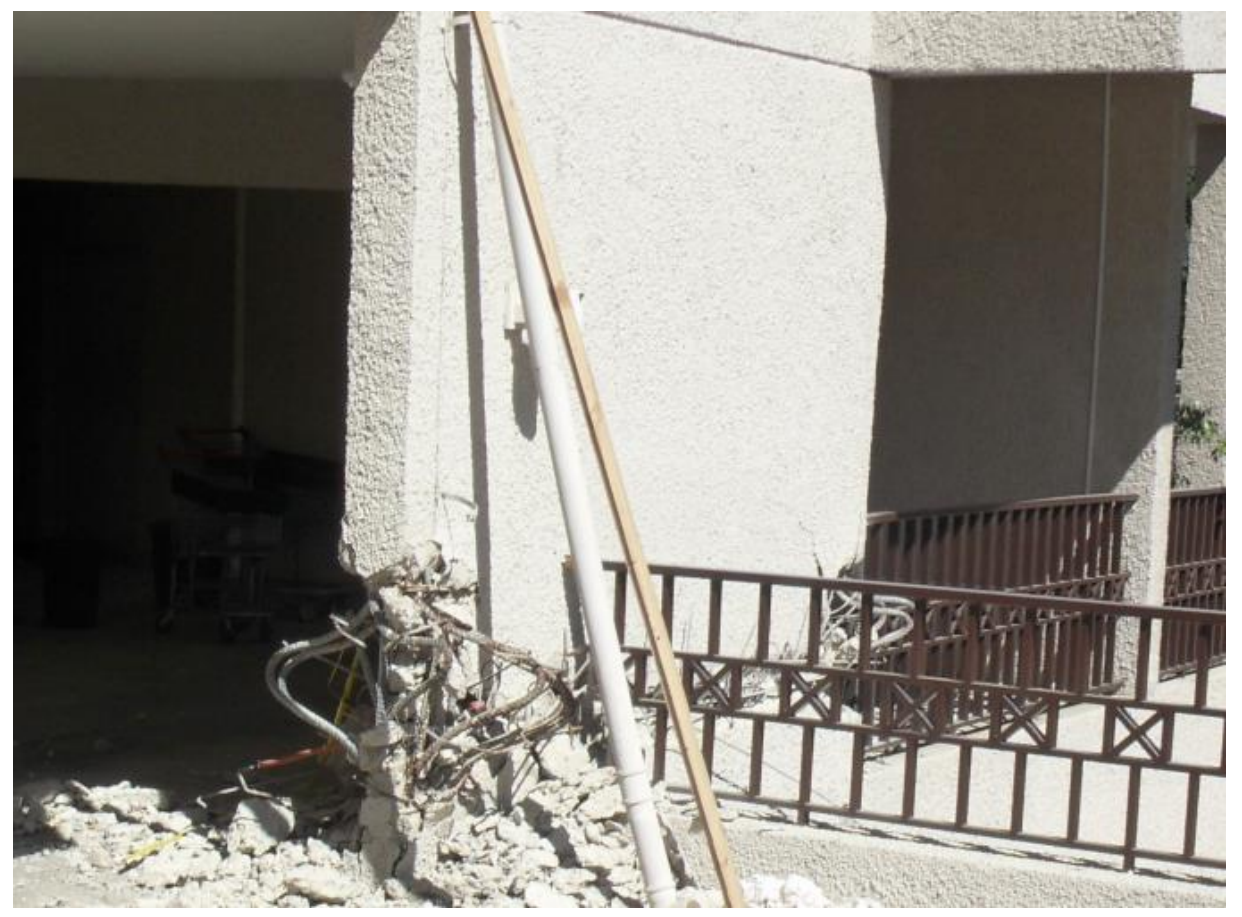

Figure 1.3 Failure of a shear wall in 2010 Chile earthquake [M2]

\subsection{Objectives and Scope}

This study aims to improve the understanding of the seismic behavior of nonrectangular RC squat walls. It also intends to consummate the database of nonrectangular RC squat walls. Furthermore, the purpose of this study also includes evaluating and improving the existing design procedures for non-rectangular RC squat walls.

This study is divided into three stages. First, an experimental program including twelve non-rectangular RC squat walls is designed and carried out. Second, the results obtained from the tests are discussed and analyzed from various aspects. Third, an analytical model is developed, and used as the basis for proposing the equation for predicting the peak shear strength of non-rectangular RC squat walls. The scope of this study consists of the following task:

1) Conducting a series of experiments to study the seismic behavior of nonrectangular RC squat walls. 
2) Evaluating the experimental results using nonlinear section analyses and methods available in the literature

3) Developing a reasonable strut-and-tie model accounting for the forceresisting mechanism of non-rectangular RC squat walls, and proposing equations for the peak shear strength of non-rectangular RC squat walls.

4) Undertaking a parametric study using finite element analyses to further investigate the influence of lateral loading direction as well as other important parameters on the peak shear strength of non-rectangular RC squat walls.

\subsection{Organization of the Thesis}

This report is organized into eight chapters and a list of references. Chapter 1 provides the background, identification of gaps in the past research, objectives and the scope of this study. Chapter 2 summarizes current design procedures and equations proposed in the literature concerning the peak shear strength of squat walls. Several renowned experiments on non-rectangular RC squat walls are also reported. In Chapter 3, the experimental program is introduced in detail. Chapter 4 presents the test results in terms of cracking patterns, failure mechanisms, hysteretic responses, displacement components and strain profiles. These results are compared and discussed in Chapter 5 from various perspectives, such as failure mechanisms, load-displacement relationships, shear deformations, strains in horizontal reinforcement, shear lag effects and effects of design parameters. In Chapter 6, a strut-and-tie model describing the force-resisting mechanism in non-rectangular RC squat walls is developed, based on which equations for the prediction of peak shear strength are established. Moreover, the probabilistic approach is utilized to reconcile the large scatter and to avoid the biased estimate found in the deterministic prediction of the peak shear strength of non-rectangular RC squat walls. The influence of lateral loading direction on the peak shear strength of nonrectangular RC squat walls is further explored through a parametric study using finite element analyses in Chapter 7. Finally, the main findings of this study are summarized in Chapter 8, followed with suggestions for future research. 


\section{CHAPTER 2 \\ LITERATURE REVIEW}

\subsection{Introduction}

This chapter firstly summarizes design procedures [A1, A3] and equations [B1, G3, $\mathrm{H} 4, \mathrm{~K} 1, \mathrm{~W} 7 \mathrm{]}$ available in the literature regarding the peak shear strength of squat shear walls. Furthermore, a review of experiments [B1, F1, K2, M4, P2, S10] concerning non-rectangular RC squat walls is presented, followed by three tables summarizing key features of existing experimental programs on non-rectangular RC squat walls. Finally, lessons learned from the literature review are concluded.

\subsection{Estimation of the Peak Shear Strength of RC Squat Walls}

Peak shear strength, among other characteristics, plays a key role in the seismic design of RC structural walls as it is closely related to the performance of walls under earthquakes. Generally, limited information is provided by codes and standards on the peak shear strength of non-rectangular RC squat walls. Three codes which can be used to these walls are: Section 11.5 of ACI 318-14 [A1], which is applicable for both slender and squat walls, Section 18.10 of ACI 318-14 [A1], which is specifically written for the seismic resistance of structural walls, and ASCE 43-05 [A3], which is used for structures in nuclear facilities. Moreover, equations proposed by Barda et al. [B1], Wood [W7], Hwang et al. [H4], Gulec and Whittaker [G3], and Kassem [K1] for the peak shear strength of squat shear walls are also reviewed in this section.

\subsubsection{Section 11.5 of ACI 318-14}

In Section 11.5 of ACI 318-14 [A1], the nominal shear strength of structural walls is expressed as the combination of contributions from the concrete and shear reinforcement as:

$$
V_{n}=V_{c}+V_{s}
$$


in which the concrete contribution is chosen from the smaller value of the following two equations:

$$
\begin{gathered}
V_{c}=0.27 \lambda \sqrt{f_{c}^{\prime}} h d+\frac{N_{u} d}{4 l_{w}} \\
V_{c}=\left[0.05 \lambda \sqrt{f_{c}^{\prime}}+\frac{l_{w}\left(0.1 \lambda \sqrt{f_{c}^{\prime}}+0.2 \frac{N_{u}}{l_{w} h}\right)}{\frac{M_{u}}{V_{u}}-\frac{l_{w}}{2}}\right] h d
\end{gathered}
$$

where $f_{c}^{\prime}(\mathrm{MPa})$ is the compressive strength of concrete, $h(\mathrm{~m})$ is the thickness of the wall, $d(\mathrm{~m})$ is the distance from the extreme compression fiber to the centroid of tensile reinforcement, which shall be taken equal to $0.8 l_{w}$. A larger value of $d$ shall be permitted if the center of tension is calculated by a strain compatibility analysis. $l_{w}(\mathrm{~m})$ is the length of the wall, $\lambda$ is the modification factor concerning concrete weight, which shall be taken as 1 for normal weight concrete, $N_{u}(\mathrm{~N})$ is the factored axial compressive force, $M_{u}(\mathrm{~N}-\mathrm{mm})$ is the factored moment, $V_{u}(\mathrm{~N})$ is the factored shear force. It should be noted that if $M_{u} / V_{u}-l_{w} / 2$ is negative, Equation 2.3 is not applicable.

The resistance provided by shear reinforcement is:

$$
V_{s}=\frac{A_{v} f_{y t} d}{s}
$$

where $A_{v}\left(\mathrm{~mm}^{2}\right)$ is the area of shear reinforcement within spacing $s, f_{y t}(\mathrm{MPa})$ is the yield stress of horizontal reinforcement, $s(\mathrm{~mm})$ is the center-to-center spacing of horizontal reinforcement, which must not exceed the smallest of $l_{w} / 5,3 h$ and 450 $\mathrm{mm}$.

To prevent diagonal compression failure in shear walls, a limit of shear strength is imposed by:

$$
V_{n} \leq 0.83 \sqrt{f_{c}^{\prime}} h d
$$

Also, both horizontal and vertical shear reinforcement in the walls are required as:

$$
\rho_{l}=0.0025+0.5\left(2.5-\frac{h_{w}}{l_{w}}\right)\left(\rho_{t}-0.0025\right)
$$




$$
\rho_{t} \geq 0.0025
$$

The spacing of longitudinal reinforcement should not exceed the smallest of $l_{w} / 3$, $3 h$ and $450 \mathrm{~mm}$.

\subsubsection{Section 18.10 of ACI 318-14}

In Section 18.10 of ACI 318-14 [A1], the nominal shear strength of structural walls is calculated based on the modified truss analogy proposed by Wood [W7], as shown below:

$$
V_{n}=A_{c v}\left(\alpha_{c} \lambda \sqrt{f_{c}^{\prime}}+\rho_{t} f_{y}\right)
$$

where $A_{c v}\left(\mathrm{~mm}^{2}\right)$ is the gross area of the concrete section bounded by web thickness and length of the section in the direction of the shear force, $\alpha_{c}$ is a coefficient: 0.25 for $h_{w} / l_{w} \leq 1.5,0.17$ for $h_{w} / l_{w} \geq 2.0$, and varies linearly between 0.25 and 0.17 for $h_{w} / l_{w}$ between 1.5 and $2.0, \lambda$, as mentioned above, is the modification factor concerning concrete weight, which is 1 for normal weight concrete, $f_{c}^{\prime}(\mathrm{MPa})$ is the compressive strength of concrete, $\rho_{t}$ is the ratio of horizontal reinforcement, $f_{y}$ (MPa) is the yield strength of reinforcement.

Similar to the equations in Section 11.5, a limit of shear strength is imposed by:

$$
V_{n} \leq 0.83 A_{c w} \sqrt{f_{c}^{\prime}}
$$

where $A_{c w}\left(\mathrm{~mm}^{2}\right)$ is the area of the concrete section of the individual vertical wall segment. Section 18.10 also requires that the ratio of horizontal reinforcement $\rho_{t}$ shall be at least the value of longitudinal reinforcement $\rho_{l}$ for squat shear walls.

\subsubsection{ASCE 43-05}

In ASCE 43-05 [A3], the shear strength of low-rise RC shear walls is given as:

$$
\begin{gathered}
v_{u}=\phi\left(8.3 \sqrt{f_{c}^{\prime}}-3.4 \sqrt{f_{c}^{\prime}}\left(\frac{h_{w}}{l_{w}}-0.5\right)+\frac{N_{A}}{4 l_{w} t_{n}}+\rho_{s e} f_{y}\right) \leq \phi 20 \sqrt{f_{c}^{\prime}} \\
\rho_{s e}=A \rho_{v}+B \rho_{h} \\
V=v_{u} d t_{n}
\end{gathered}
$$


where $v_{u}$ (psi) is the ultimate shear stress, $\phi$ is the capacity reduction factor which is assumed to be $0.8, f_{c}^{\prime}$ (psi) is the concrete compressive strength, $h_{w}$ (in) is the wall height, $l_{w}$ (in) is the wall length, $N_{A}$ (lb) is the axial load, $t_{n}$ (in) is the wall thickness, $\rho_{s e}$ is the combined reinforcement ratio calculated by horizontal reinforcement ratio $\rho_{h}$, longitudinal reinforcement ratio $\rho_{v}$ and constants $A$ and $B$ presented in Table 2.1, $f_{y}$ (psi) is the yield strength of reinforcement, $V_{u}(\mathrm{lb})$ is the total shear capacity, $d$ (in) is the distance from the extreme compression fiber to the center of force of all reinforcement in tension, which can be determined from a strain compatibility analysis (or $0.6 l_{w}$ ).

Table 2.1 Constants $A$ and $B$ for the calculation of $\rho_{\text {se }}$ [A3]

\begin{tabular}{|c|c|c|}
\hline Aspect Ratio & $A$ & $B$ \\
\hline$h_{w} / l_{w} \leq 0.5$ & 1 & 0 \\
\hline $0.5 \leq h_{w} / l_{w} \leq 1.5$ & $-h_{w} / l_{w}+1.5$ & $h_{w} / l_{w}-0.5$ \\
\hline$h_{w} / l_{w} \geq 1.5$ & 0 & 1 \\
\hline
\end{tabular}

\subsubsection{Barda et al. (1977)}

Barda et al. [B1] proposed a shear strength equation based on the test of eight lowrise $\mathrm{RC}$ walls with heavily reinforced flanges, which is given by:

$$
V_{u}=\left(8.3 \sqrt{f_{c}^{\prime}}-2.5 \sqrt{f_{c}^{\prime}} \frac{h_{w}}{l_{w}}+\frac{N_{u}}{4 l_{w} h}+\rho_{n} f_{y}\right) h d
$$

where $V_{u}(\mathrm{lb})$ is the ultimate shear strength, $f_{c}^{\prime}$ (psi) is the concrete compressive strength, $h_{w}$ (in) is the wall height, $l_{w}$ (in) is the wall length, $N_{u}$ (lb) is the axial load, $h$ (in) is the wall thickness, $\rho_{n}$ is the longitudinal reinforcement ratio, $f_{y}$ (psi) is the yield strength of reinforcement, $d$ (in) is the distance from extreme compression fiber to the centroid of tensile reinforcement.

\subsubsection{Wood (1990)}

Wood [W7] proposed a semi-empirical equation for the peak shear strength of squat shear walls based on the shear friction and evaluations of experimental data obtained from 143 structural walls with low aspect ratio, which is expressed as: 


$$
6 \sqrt{f_{c}^{\prime}} \leq v_{n}=\frac{A_{v} f_{y v}}{4 A_{c v}} \leq 10 \sqrt{f_{c}^{\prime}}
$$

where $v_{n}$ (psi) is the shear stress. $f_{c}^{\prime}$ (psi) is the concrete compressive strength, $A_{c v}$ $\left(\mathrm{in}^{2}\right)$ is the gross area of the concrete section bounded by web thickness and length of section, $A_{v}\left(\mathrm{in}^{2}\right)$ is the total area of longitudinal reinforcement in the wall, $f_{y v}$ (psi) is the yield strength of longitudinal reinforcement.

\subsubsection{Hwang et al. (2001)}

Hwang et al. [H4] utilized a soften strut-and-tie model to study the equilibrium, compatibility and constitutive laws of squat walls and proposed shear strength equations as demonstrated below:

$$
\begin{gathered}
V_{h}=\left(K_{h}+K_{v}-1\right) \xi f_{c}^{\prime} A_{s t r} \cos \theta \\
K_{h}=\frac{1}{1-0.2\left(\gamma_{h}+\gamma_{h}^{2}\right)} \\
K_{v}=\frac{1}{1-0.2\left(\gamma_{v}+\gamma_{v}^{2}\right)} \\
\gamma_{h}=\frac{2 \tan \theta-1}{3} \text { for } 0 \leq \gamma_{h} \leq 1 \\
\gamma_{v}=\frac{2 \cot \theta-1}{3} \text { for } 0 \leq \gamma_{v} \leq 1 \\
\xi=\frac{3.35}{\sqrt{f_{c}^{\prime}}} \leq 0.52 \\
A_{s t r}=a_{s} \times t_{w} \\
\theta=\left(0.25+0.85 \frac{N}{A_{w} f_{c}^{\prime}}\right) l_{w} \\
a_{s}=a_{w} \frac{h_{w}}{l_{w}}
\end{gathered}
$$

where $V_{h}(\mathrm{~N})$ is the shear strength of RC squat walls, $K_{h}$ and $K_{v}$ are the index of horizontal tie and vertical tie, $\gamma_{h}$ is the fraction of diagonal compression carried by the horizontal tie in the absence of the vertical tie, $\gamma_{v}$ is the fraction of diagonal 
compression carried by the vertical tie in the absence of the horizontal tie, $\theta$ is the angle of inclination of the diagonal compression strut, $\xi$ is the softening coefficient, $f_{c}^{\prime}(\mathrm{MPa})$ is the concrete compressive strength, $a_{s}(\mathrm{~mm})$ is the depth of the diagonal strut, $t_{w}(\mathrm{~mm})$ is the thickness of the web, $a_{w}(\mathrm{~mm})$ is the depth of the compression zone at the base of the wall, $N(\mathrm{~N})$ is the axial load, $A_{w}\left(\mathrm{~mm}^{2}\right)$ is the net area of the concrete section bounded by web thickness and length of section in the direction of shear force, $h_{w}(\mathrm{~mm})$ is the height of the wall, $l_{w}(\mathrm{~mm})$ is the length of the web.

\subsubsection{Gulec and Whittaker (2011)}

Gulec and Whittaker [G3] summarized a comprehensive database of squat walls with flanged cross section, and proposed an empirical equation for the peak shear strength specifically for barbell and flanged shear walls with low aspect ratio, which is presented below:

$$
V_{B E}=\frac{0.04 f_{c}^{\prime} A_{e f f}+0.40 F_{v w}+0.15 F_{v b e}+0.35 P}{\sqrt{h_{w} / l_{w}}} \leq 15 \sqrt{f_{c}^{\prime}} A_{t}
$$

where $V_{B E}(\mathrm{lb})$ is the peak shear strength of squat walls with boundary elements, $f_{c}^{\prime}$ (psi) is the concrete compressive strength, $A_{\text {eff }}\left(\mathrm{in}^{2}\right)$ is the total wall area of barbell walls and the effective area for flanged walls, which is equal to the sum of areas of the web plus the effective flange area, where effective flange area is the production of the flange thickness and half of the wall height minus the web thickness, $F_{v w}(\mathrm{lb})$ is the force attributed to vertical web reinforcement, $F_{v b e}(\mathrm{lb})$ is the force attributed to boundary element reinforcement, $P$ (lb) is the axial load, $h_{w}$ (in) is the wall height, $l_{w}$ (in) is the wall length, $A_{t}\left(\mathrm{in}^{2}\right)$ is the total area of wall.

\subsubsection{Kassem (2014)}

Kassem [K1] further complemented the database of squat walls with flanged cross section summarized by Gulec and Whittaker [G3]. After studying the strut-and-tie model established by Hwang et al. [H4], Kassem proposed peak shear strength equations as shown below:

$$
v_{R k}=0.47 f_{c}^{\prime}\left[\psi k_{s} \sin 2 \alpha+0.15 \omega_{h} \frac{H_{w}}{d_{w}}+1.76 \omega_{v} \cot \alpha\right] \leq 1.25 \sqrt{f_{c}^{\prime}}
$$




$$
\begin{gathered}
\psi=0.95-\frac{f_{c}^{\prime}}{250} \\
k_{s}=\frac{a_{s}}{d_{w}} \\
\omega_{h}=\frac{\rho_{h} f_{y h}}{f_{c}^{\prime}} \\
\omega_{v}=\frac{\rho_{v} f_{y v}}{f_{c}^{\prime}} \\
\alpha=\tan ^{-1} \frac{H_{w}}{d_{w}} \\
d_{w}=d-\frac{a_{s}}{3} \\
a_{s}=\left(0.25+0.85 \frac{P_{n}}{A_{w} f_{c}^{\prime}}\right) L_{w}
\end{gathered}
$$

where $v_{R k}(\mathrm{MPa})$ is the ultimate shear stress, $f_{c}^{\prime}(\mathrm{MPa})$ is the concrete compressive strength, $\psi$ is the nondimensional function which is identical to the minor modification of the European code, $\omega_{h}$ and $\omega_{v}$ are the index of horizontal and longitudinal reinforcement, $\rho_{h}$ and $\rho_{v}$ are the horizontal and longitudinal reinforcement ratio, $f_{y h}(\mathrm{MPa})$ and $f_{y v}(\mathrm{MPa})$ are yield stresses of horizontal and longitudinal reinforcement, $\alpha$ is the diagonal strut angle for inclination, $H_{w}(\mathrm{~mm})$ is the distance from horizontal loading to the wall base, $d(\mathrm{~mm})$ is the horizontal length between the tensile and compressive force in boundary elements, $d_{w}(\mathrm{~mm})$ is the distance from extreme compression fiber to the location of the resultant tensile force in longitudinal reinforcement, $a_{s}(\mathrm{~mm})$ is the depth of the diagonal strut, $P_{n}$ $(\mathrm{N})$ is the axial load, $A_{w}\left(\mathrm{~mm}^{2}\right)$ is the net area of the concrete section bounded by web thickness and length of section in the direction of shear force, $L_{w}(\mathrm{~mm})$ is the length of the web.

\subsection{Previously Research on Non-rectangular RC Squat Walls}

In this section, a series of experimental programs on non-rectangular RC squat walls are introduced, which includes: eight walls with boundary elements tested by 
Barda et al. [B1], two flanged walls tested by Synge [S10], twenty framed shear walls tested by Mo and Chan [M4], two large-scale H-shaped walls tested by Kitada et al. [K2], two H-shaped walls tested by Palermo and Vecchio [P2], seven flanged walls tested by Farvashany et al. [F1].

\subsubsection{Research conducted by Barda et al. (1972)}

Realizing the lack of studies on low-rise walls, Barda et al. [B1] conducted an experimental program comprising of eight squat walls with heavily-reinforced flanges to investigate the shear strength of these walls.

The principal variables were longitudinal reinforcement ratio, horizontal reinforcement ratio, and shear span ratio. The longitudinal reinforcement ratio varied from $1.8 \%$ to $6.4 \%$ in flange and 0 to $0.5 \%$ in web. The horizontal reinforcement ratio varied from 0 to $0.5 \%$ in the wall. Six specimens had a shear span ratio of 0.5 , while the remaining two had shear span ratios of 0.25 and 1 respectively. Besides, the concrete compressive strength varied between 16 to 29 $\mathrm{MPa}$ and the nominal yield strength of steel bars was $420 \mathrm{MPa}$. The test setup can be seen in Figure 2.1.

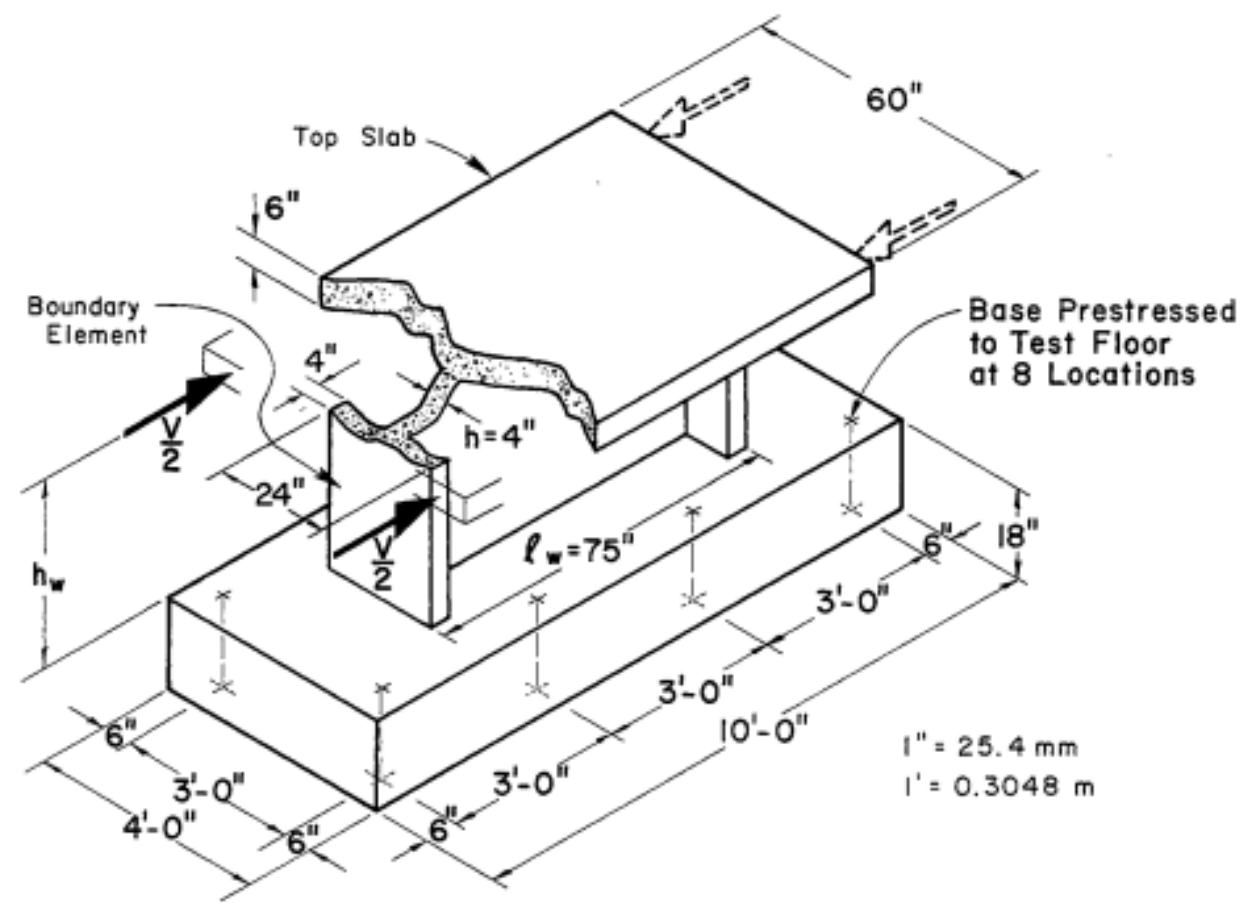

Figure 2.1 Details of specimens tested by Barda et al. [B1] 
Lessons learned from this study are:

- In addition to the horizontal reinforcement, a significant portion of shear introduced at the top of a cantilever wall was transmitted directly to the foundations by diagonal compression.

- The shear strength of the test specimens was not affected by differences in the amount of flexural reinforcement, as long as all bars were properly anchored to the foundation.

- For specimens with a shear span ratio of 0.5 or less, it was found that horizontal wall reinforcement did not contribute much to the shear strength. However, in ACI 318-14 [A1], only horizontal reinforcement is considered in resisting the shear force.

- Longitudinal reinforcement was effective as shear reinforcement in the specimens with aspect ratios of $1 / 2$ and $1 / 4$. However, it was less effective in the specimen with an aspect ratio of 1 . Longitudinal reinforcement was also effective in producing a more distributed cracking pattern and in reducing crack widths. The observations led to the recommendation that minimum longitudinal reinforcement as required should be provided in all walls.

\subsubsection{Research conducted by Synge (1980)}

To explore the mechanism of flexural and shear resistance of squat shear walls with an emphasis on the aspect of sliding shear, Synge [S10] conducted an experimental program on four squat walls under simulated seismic loading. Among them, the two flanged walls are presented here.

Figure 2.2 presents the geometry details and reinforcement contents of Wall 3. The most distinguishing difference between the flanged walls was that one (Wall 4) was reinforced with diagonal steel bars to reduce excessive sliding shear displacements under cyclic reversed loading. Other minor differences were the concrete compressive strength (26.0 $\mathrm{MPa}$ and $30.2 \mathrm{MPa}$ respectively) and horizontal reinforcement ratio $(1.608 \%$ and $0.670 \%$ respectively). The two specimens had the same shear span ratio of 0.57 . 


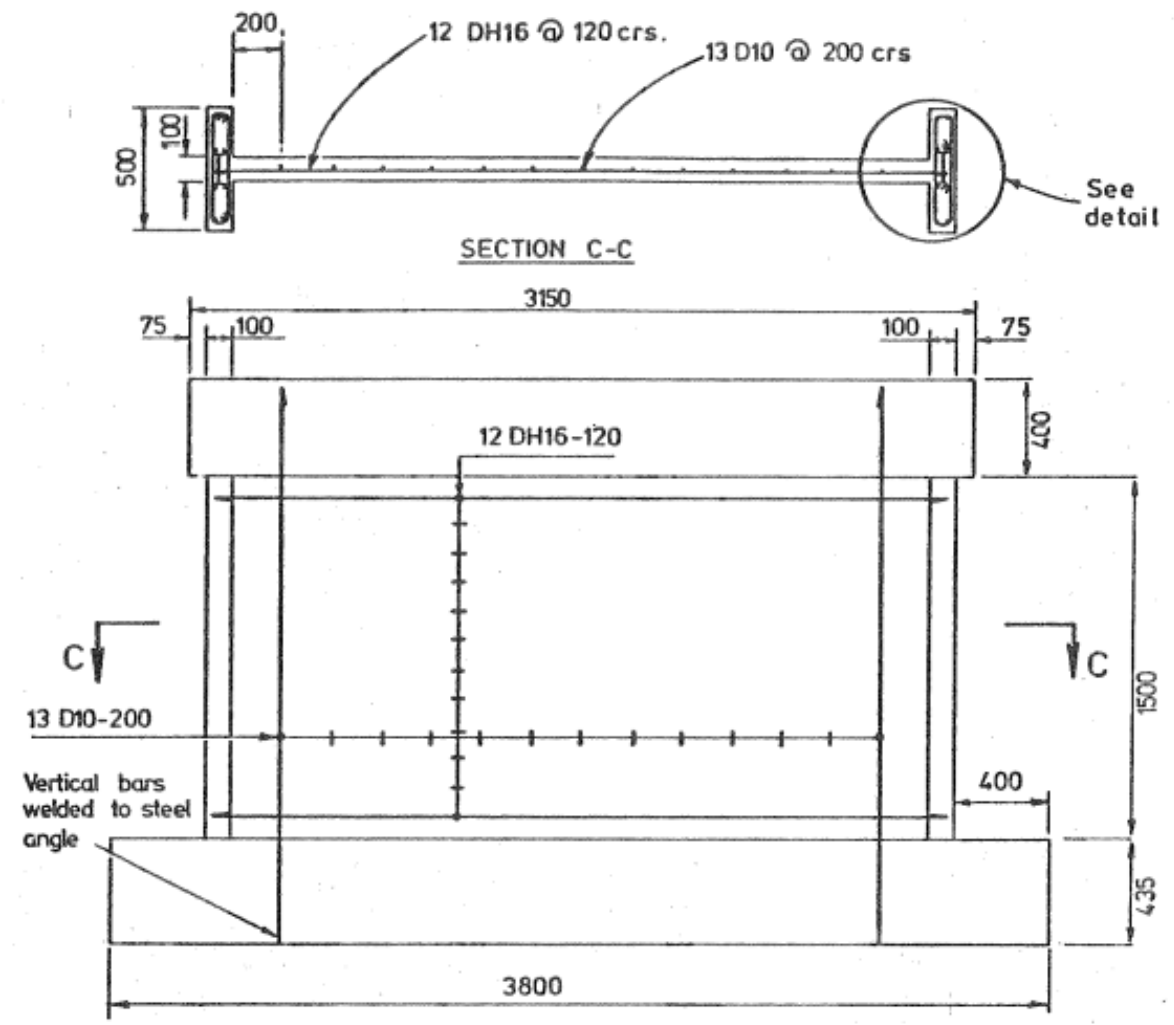

Figure 2.2 Details of Wall 3 tested by Synge [S10]

Conclusions drawn from this research are:

- The sliding shear along the base was the most significant cause of loss in stiffness and strength and consequent reduction in the ability to dissipate energy for squat shear walls. Unless specially detailed or subjected to high axial loads, all squat shear walls were likely to fail in this mode.

- Because of the reduction in flexural compression depth in the web of the critical base section, flanged walls were more seriously affected by sliding shear along interconnecting flexural cracks than walls with a rectangular cross section.

- The severity of sliding shear was considered to increase significantly with expected ductility demand, with decreasing longitudinal reinforcement providing dowel shear resistance, and with a decrease of the flexural compression zone of the wall section over which the bulk of the shear would need to be transferred by shear friction. 


\subsubsection{Research conducted by Mo and Chan (1996)}

To examine the applicability of the truss model theory proposed by Hsu and Mo [H3], Mo and Chan [M4] conducted an experiment including twenty framed shear walls with approximately one-half of the length used in the test program carried out by Barda et al. [B1].

All dimensions were kept constant in all specimens as presented in Figure 2.3. Variables in this study included concrete compressive strength, steel yield stress, longitudinal reinforcement ratio, and cyclic loading history. Lateral loads were applied by a 50 ton MTS dynamic actuator that transferred its force to the specimen through a steel bearing plate. Three types of loading histories were applied to the specimens.

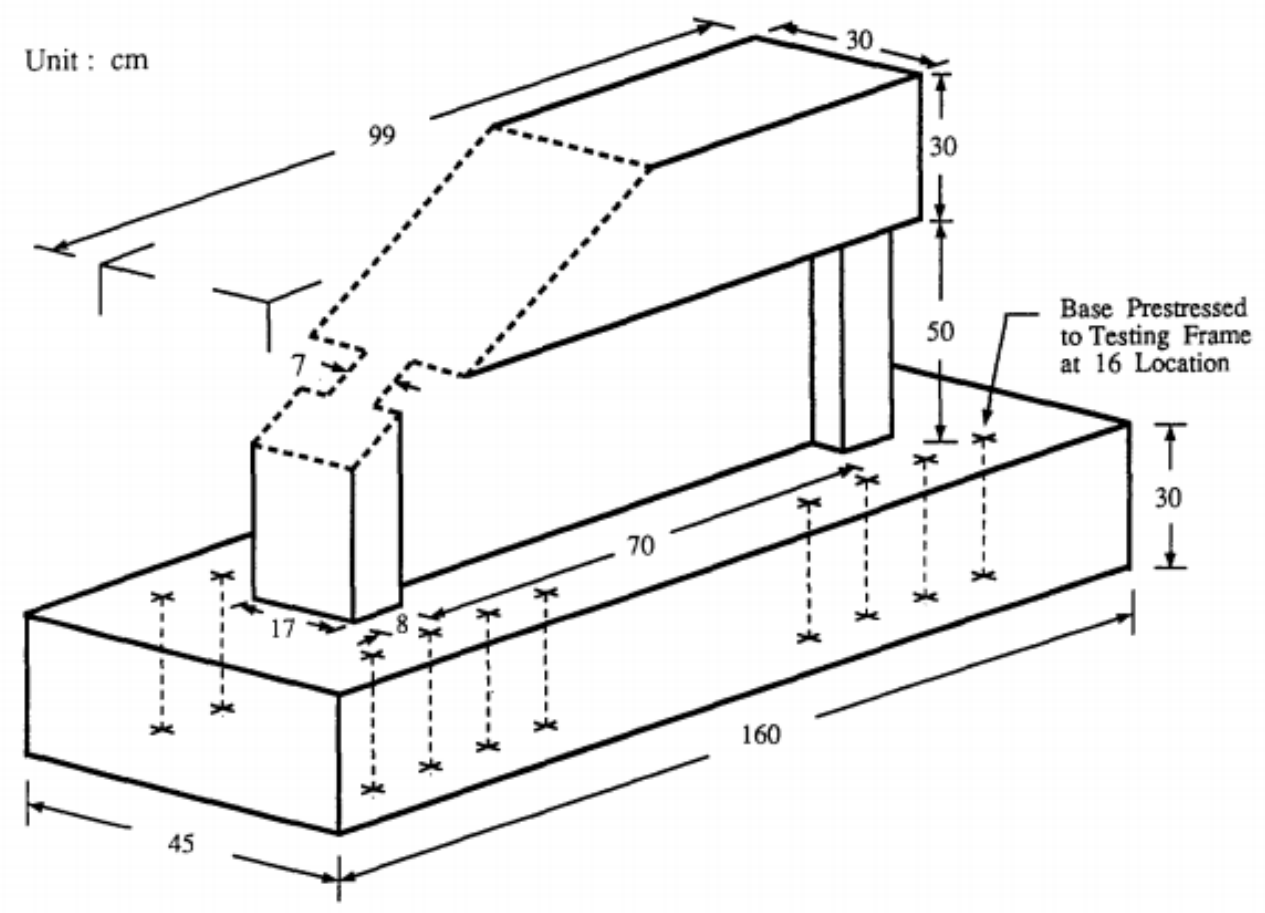

Figure 2.3 Details of specimens tested by Mo and Chan [M4]

Findings from this experiment are:

- When the softening parameter for normal and high-strength concrete was employed by the truss model theory, the calculated ultimate shear force was 
generally greater than the test value for low-rise shear walls subjected to dynamic shear forces with low frequency.

- The effect of the cyclic load history was significant on the framed shear wall behaviors.

- Hysteretic loops developed during the increase in force were different from those developed during the decrease in force.

- The maximum shear force that resulted from reversed cyclic loads was less than that resulting from monotonic loads. The main reason was that transverse tensile strains and intersecting diagonal cracks considerably reduced the concrete's compressive strength when reversed cyclic loads were applied.

\subsubsection{Research conducted by Kitada et al. (1997)}

This research program was carried out by NUPEC [K2] (Nuclear Power Engineering Corporation) for the evaluation of the seismic behavior of reactor buildings, aiming to verify and improve the existing seismic response analysis codes to enhance their reliability for the seismic safety design of reactor buildings. As a part of the program, the "Seismic Ultimate Dynamic Response Test" was conducted using the world's largest high-performance shaking table at the Tadotsu Engineering Laboratory in 1991.

Two specimens (U-1 and U-2) with the same design specifications were prepared and tested by applying the same input wave to ascertain the reproducibility of the test. Figure $\mathbf{2 . 4}$ below demonstrates the dimensions of the test specimens. The web wall had a thickness of $75 \mathrm{~mm}$, a flange center to center length of $3000 \mathrm{~mm}$, the clear height of $2020 \mathrm{~mm}$, and a shear span ratio of 0.8 . The flange walls had a thickness of $100 \mathrm{~mm}$ and a length of $2980 \mathrm{~mm}$. The steel percentage in the wall was $1.2 \%$, and D6 steel bars with a diameter of $6.35 \mathrm{~mm}$ were used in the web at an interval of $70 \mathrm{~mm}$ for both vertical and horizontal reinforcement. D6 steel bars were also used for the longitudinal reinforcement of flange walls at an interval of 175 $\mathrm{mm}$. The compressive strength of concrete was 29.2 MPa. The yield stress of D6 steel bars was $390 \mathrm{MPa}$. 


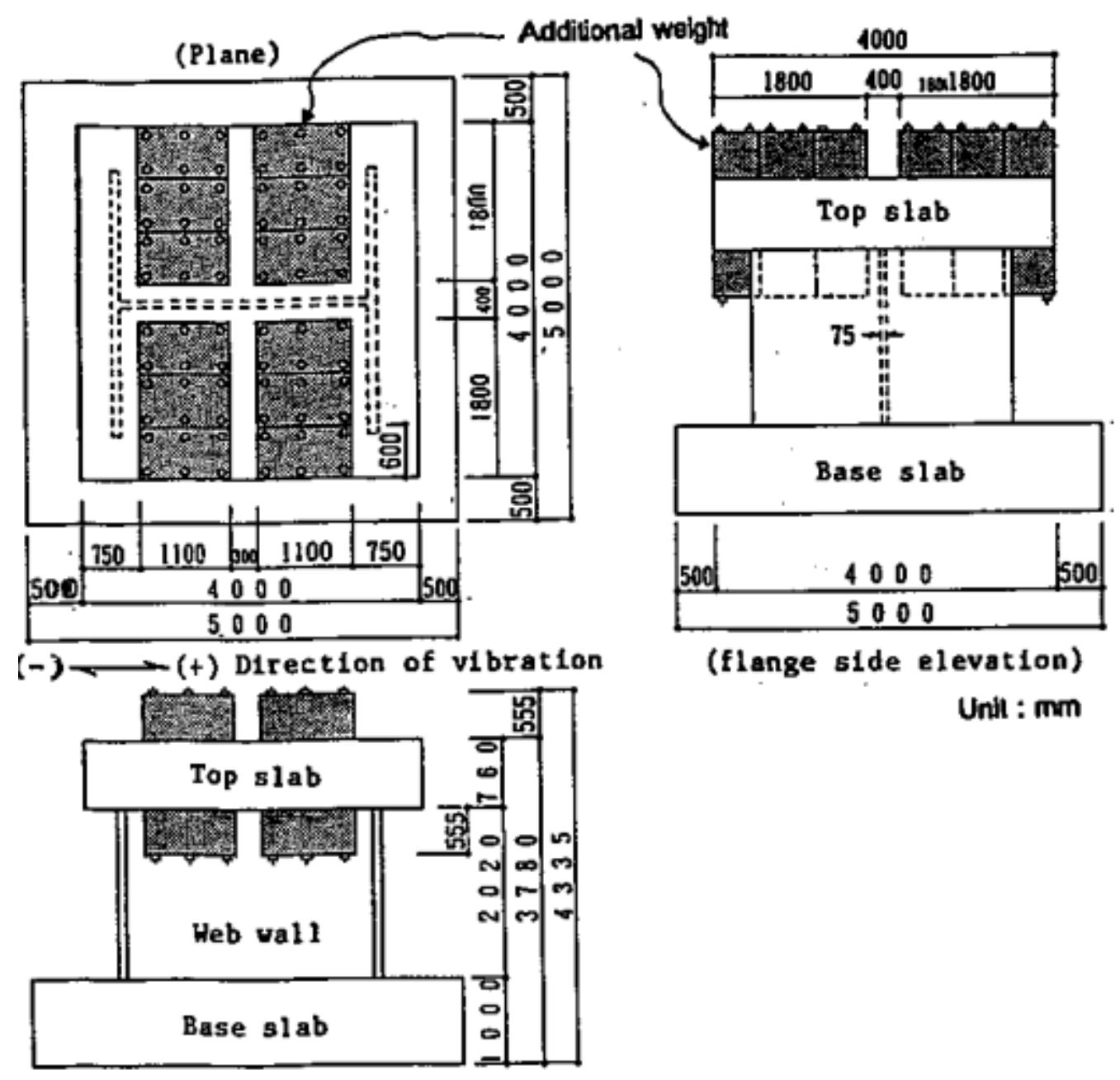

(veb side elevation)

Figure 2.4 Details of specimens tested by Kitada et al. [K2]

From this experiment, it can be concluded that:

- The effective width of the flange wall increased with the increase of the horizontal deformation.

- The out-of-plane stiffness of the flange wall should be taken into account because it had considerable influence on the location of failure in the web wall.

- There were some controversies over whether sliding shear failure appeared only under cyclic loading or whether they also appeared under monotonic loading. Further study on the difference between the behavior under monotonic and cyclic loading should be carried out. 
- The simplified model had been developed mainly to find the ultimate strength. It was hard to obtain local information by simplified models.

\subsubsection{Research conducted by Palermo and Vecchio (2002)}

To improve the understanding of the behavior of non-rectangular shear walls under cyclic lateral loading, and to refine the constitutive model of reinforced concrete, Palermo and Vecchio [P2] conducted an experiment on two large-scale H-shaped walls under static reversed loading in 2002.

The designs of the two specimens were similar to the NUPEC specimen [K2] with the same geometrical and reinforcement properties (Figure 2.5). DP1 was applied with an axial load of $940 \mathrm{kN}$, while the axial load was removed from DP2. The web wall was reinforced with D6 reinforcing bars spaced $140 \mathrm{~mm}$ horizontally and 130 $\mathrm{mm}$ vertically in two parallel layers. The horizontal reinforcement with provided in the flange using the same rebar at a spacing of $140 \mathrm{~mm}$. The longitudinal reinforcement was arranged at the spacing of $130 \mathrm{~mm}$ near the web and $355 \mathrm{~mm}$ near the tips of the flanges.
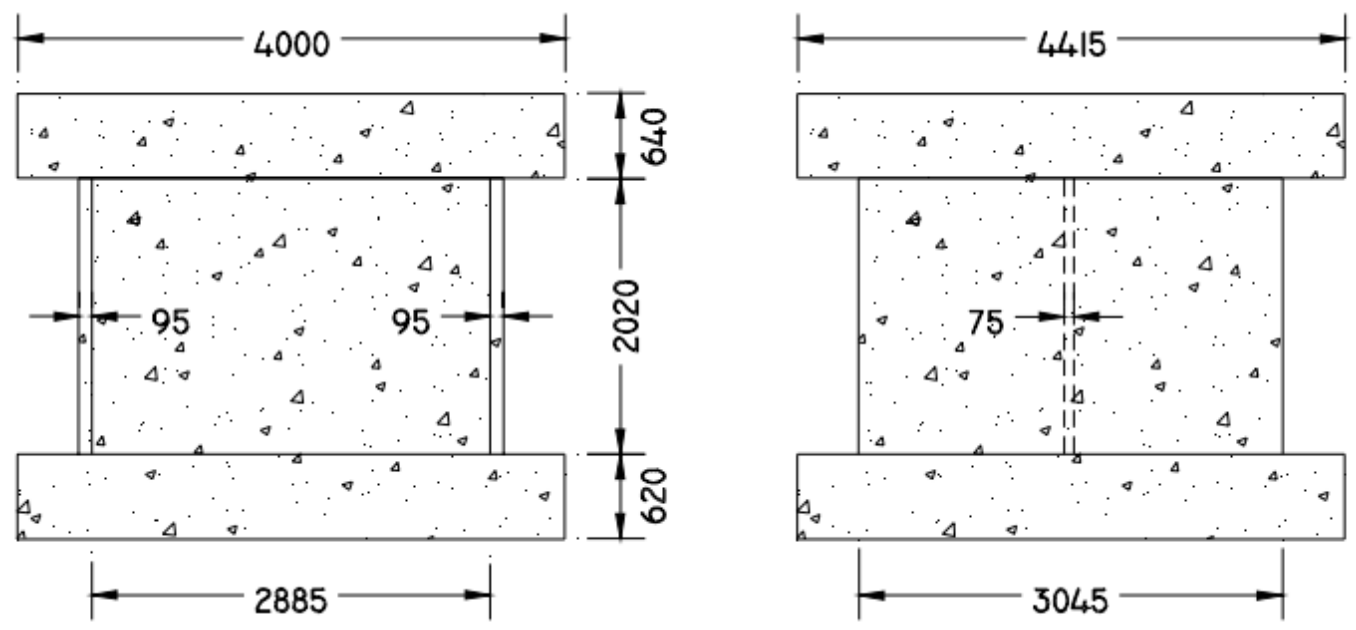

Figure 2.5 Details of specimens tested by Palermo and Vecchio [P2]

Several key findings from this research are:

- Imposed axial loads, and wall configurations consisting of end flange walls, significantly influenced the ultimate strength and failure modes of squat shear walls. 
- Squat shear walls showed highly pinched load-displacement curves with little energy dissipation in comparison to slender walls and were more influenced by shear-related mechanisms.

- The stiffness of the flange walls restrained the opening of web shear cracks and caused the formation of vertical slip planes in DP1.

- Second-order effects, namely the elongation of the flange walls and horizontal expansion of the web wall, were key indicators in determining the extent of damage and significantly affected the lateral resistance and failure mechanisms of squat shear walls.

\subsubsection{Research conducted by Farvashany et al. (2008)}

The seven specimens conducted by Farvashany et al. [F1] were approximately 1/3 scale of a prototype wall. The aim was to study the behavior of high-strength concrete shear walls. Moreover, the experimental results of the test specimens, as well as those reported by other researchers were used to compare with the ultimate resistance predicted by an interactive event simulator and design procedures from ACI 318 code.

Each specimen consisted of two beams, one at the top and one at the bottom, and a shear wall panel bounded by two edge columns. The shear span ratio of the test specimens was selected to be 1.25 . The shear wall was $700 \mathrm{~mm}$ in length, $1100 \mathrm{~mm}$ in height and $75 \mathrm{~mm}$ in thickness. The dimensions of edge columns are $375 \times 90 \mathrm{~mm}$ with the same height of $1100 \mathrm{~mm}$. The top beam was designed to be stiff enough to transfer loads to the shear wall without any cracking or considerable deformation.

The primary parameter, concrete compressive strength, varied from approximately $80 \mathrm{MPa}$ to $100 \mathrm{MPa}$ for different specimens. Axial compressive stresses of approximately $8 \mathrm{MPa}, 4 \mathrm{MPa}$ and zero were applied to the specimens. The horizontal reinforcement ratios of $0.47 \%$ and $0.75 \%$ and longitudinal reinforcement ratios of 0.75 and $1.26 \%$ were chosen for the wall panels. The details of specimens are presented below in Figure 2.6. 


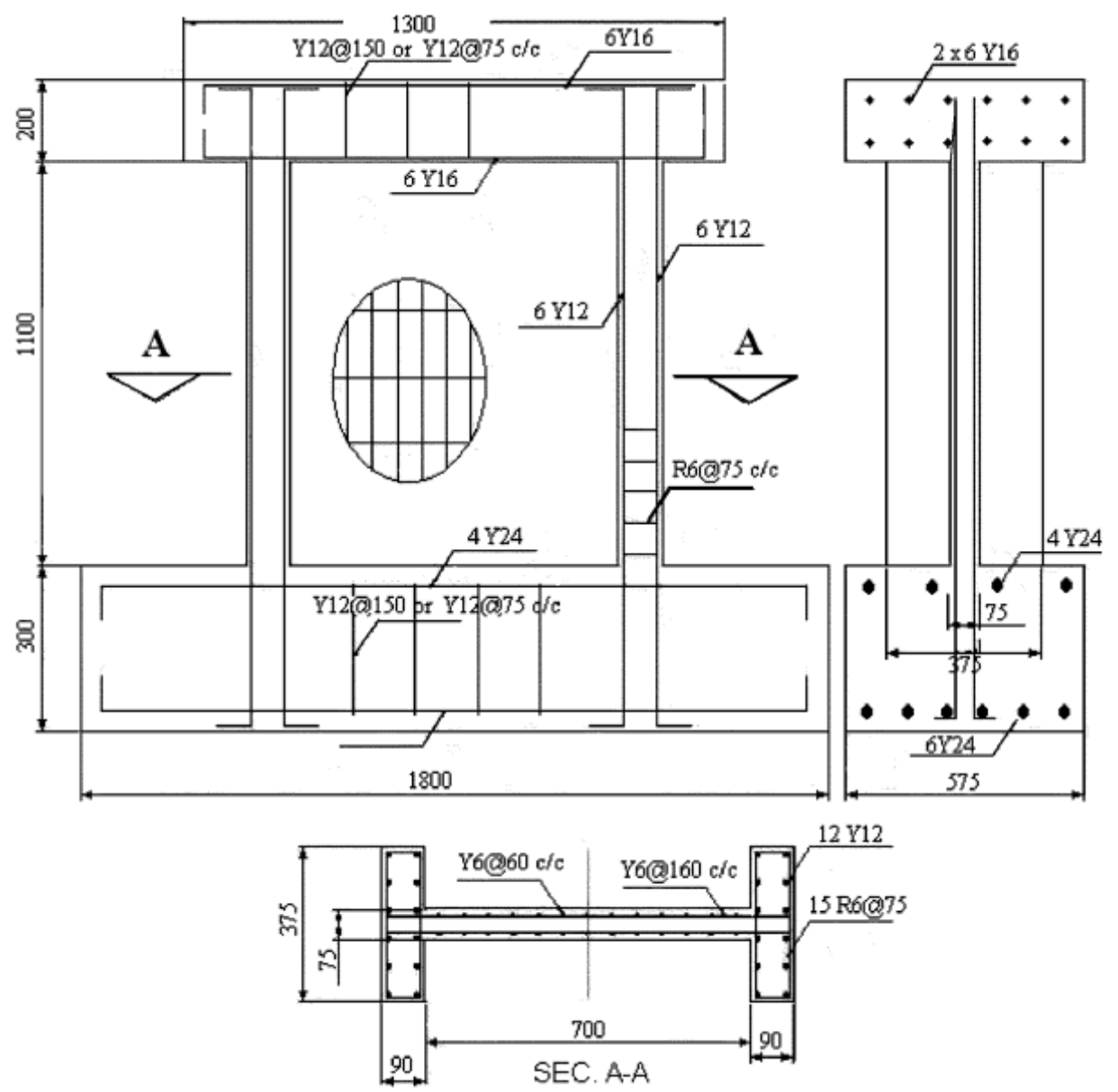

Figure 2.6 Details of specimens tested by Farvashany et al. [F1]

Lessons can be learned from this experiment are:

- An increase in vertical steel ratio increased the horizontal failure load. The effect of vertical steel was only marginal for higher vertical steel ratios in medium and low-rise walls.

- The effect of horizontal steel ratio was not as significant as the vertical steel ratio. The shear strength increased only marginally with an increase in horizontal steel ratio.

- The mean value and the standard deviation of the measured to predicted ultimate shear strength by ACI 318 code were 1.96 and 0.60 respectively, which indicated that the ACI 318 largely underestimated the peak shear strength of the tested non-rectangular RC squat walls. 


\subsubsection{Database of non-rectangular RC squat walls}

Gulec [G2] summarized a comprehensive database of squat shear walls with barbell and boundary elements. Among them, eighty-seven non-rectangular RC squat walls are selected and summarized in the following tables below, which include the research work of Barda et al. [B1], Synge [S10], Saito et al. [S2], Sato et al. [S3], Seki et al. [S4], Mo and Chan [M4], Kitada et al. [K2], Naze and Sidaner [N2], Palermo and Vecchio [P2] and Farvashany et al. [F1]. Key features of these experiments are categorized as geometric properties, reinforcement information and performance information.

Table 2.2 Geometric properties of non-rectangular RC squat walls

\begin{tabular}{|c|c|c|c|c|c|c|c|}
\hline Researcher & Specimen & $t_{w}(\mathrm{~mm})$ & $l_{w}(\mathrm{~mm})$ & $h_{w}(\mathrm{~mm})$ & $t_{f}(\mathrm{~mm})$ & $l_{f}(\mathrm{~mm})$ & $h_{w} / l_{w}$ \\
\hline Barda et al. (1972) & B1-1 & 102 & 1905 & 953 & 102 & 610 & 0.50 \\
\hline Barda et al. (1972) & B2-1 & 102 & 1905 & 953 & 102 & 610 & 0.50 \\
\hline Barda et al. (1972) & B3-2 & 102 & 1905 & 953 & 102 & 610 & 0.50 \\
\hline Barda et al. (1972) & B4-3 & 102 & 1905 & 953 & 102 & 610 & 0.50 \\
\hline Barda et al. (1972) & B5-4 & 102 & 1905 & 953 & 102 & 610 & 0.50 \\
\hline Barda et al. (1972) & B6-4 & 102 & 1905 & 953 & 102 & 610 & 0.50 \\
\hline Barda et al. (1972) & B7-5 & 102 & 1905 & 477 & 102 & 610 & 0.25 \\
\hline Barda et al. (1972) & B8-5 & 102 & 1905 & 1905 & 102 & 610 & 1.00 \\
\hline Synge (1980) & Wall 3 & 100 & 3000 & 1500 & 100 & 500 & 0.50 \\
\hline
\end{tabular}




\begin{tabular}{|c|c|c|c|c|c|c|c|}
\hline Researcher & Specimen & $t_{w}(\mathrm{~mm})$ & $l_{w}(\mathrm{~mm})$ & $h_{w}(\mathrm{~mm})$ & $t_{f}(\mathrm{~mm})$ & $l_{f}(\mathrm{~mm})$ & $h_{w} / l_{w}$ \\
\hline Saito et al. (1989) & W12-1 & 120 & 2120 & 750 & 120 & 120 & 0.35 \\
\hline Saito et al. (1989) & W12-2 & 120 & 2120 & 750 & 120 & 120 & 0.35 \\
\hline Saito et al. (1989) & W12-3 & 120 & 2120 & 750 & 120 & 120 & 0.35 \\
\hline Saito et al. (1989) & W12-4 & 120 & 2120 & 750 & 120 & 120 & 0.35 \\
\hline Saito et al. (1989) & W12-5 & 120 & 2120 & 750 & 120 & 120 & 0.35 \\
\hline Saito et al. (1989) & W12-6 & 120 & 2120 & 1750 & 120 & 120 & 0.83 \\
\hline Saito et al. (1989) & W12-7 & 120 & 2120 & 1750 & 120 & 120 & 0.83 \\
\hline Saito et al. (1989) & W15-1 & 150 & 2120 & 759 & 120 & 120 & 0.36 \\
\hline Saito et al. (1989) & W15-2 & 150 & 2120 & 1750 & 120 & 120 & 0.83 \\
\hline Sato et al. (1989) & $18 \mathrm{M} 12-40$ & 150 & 2150 & 2200 & 150 & 1000 & 1.02 \\
\hline Sato et al. (1989) & $24 \mathrm{M} 8-30$ & 150 & 2150 & 1400 & 150 & 1000 & 0.65 \\
\hline Sato et al. (1989) & $24 \mathrm{M} 8-40$ & 150 & 2150 & 1400 & 150 & 1000 & 0.65 \\
\hline Sato et al. (1989) & $24 \mathrm{M} 8-50$ & 150 & 2150 & 1400 & 150 & 1000 & 0.65 \\
\hline Sato et al. (1989) & $24 \mathrm{M} 6-30$ & 150 & 2150 & 1000 & 150 & 1000 & 0.47 \\
\hline Sato et al. (1989) & $24 \mathrm{M} 6-40$ & 150 & 2150 & 1000 & 150 & 1000 & 0.47 \\
\hline Sato et al. (1989) & $36 \mathrm{M} \mathrm{12-30}$ & 150 & 2150 & 2200 & 150 & 1000 & 1.02 \\
\hline Sato et al. (1989) & $36 \mathrm{M} \mathrm{12-40}$ & 150 & 2150 & 2200 & 150 & 1000 & 1.02 \\
\hline Sato et al. (1989) & $36 \mathrm{M} 12-50$ & 150 & 2150 & 2200 & 150 & 1000 & 1.02 \\
\hline
\end{tabular}




\begin{tabular}{|c|c|c|c|c|c|c|c|}
\hline Researcher & Specimen & $t_{w}(\mathrm{~mm})$ & $l_{w}(\mathrm{~mm})$ & $h_{w}(\mathrm{~mm})$ & $t_{f}(\mathrm{~mm})$ & $l_{f}(\mathrm{~mm})$ & $h_{w} / l_{w}$ \\
\hline Sato et al. (1989) & 36L 8-30 & 150 & 2150 & 1400 & 150 & 1000 & 0.65 \\
\hline Sato et al. (1989) & 36L 8-40 & 150 & 2150 & 1400 & 150 & 1000 & 0.65 \\
\hline Sato et al. (1989) & $36 \mathrm{M} \mathrm{8-30}$ & 150 & 2150 & 1400 & 150 & 1000 & 0.65 \\
\hline Sato et al. (1989) & $36 \mathrm{M} \mathrm{8-40}$ & 150 & 2150 & 1400 & 150 & 1000 & 0.65 \\
\hline Sato et al. (1989) & $36 \mathrm{M} \mathrm{8-50}$ & 150 & 2150 & 1400 & 150 & 1000 & 0.65 \\
\hline Sato et al. (1989) & $36 \mathrm{M} 6-30$ & 150 & 2150 & 1000 & 150 & 1000 & 0.47 \\
\hline Sato et al. (1989) & $36 \mathrm{M} 6-40$ & 150 & 2150 & 1000 & 150 & 1000 & 0.47 \\
\hline Sato et al. (1989) & $48 \mathrm{M} \mathrm{8-30}$ & 150 & 2150 & 1400 & 150 & 1000 & 0.65 \\
\hline Sato et al. (1989) & $48 \mathrm{M} \mathrm{8-40}$ & 150 & 2150 & 1400 & 150 & 1000 & 0.65 \\
\hline Sato et al. (1989) & $48 \mathrm{M} \mathrm{8-50}$ & 150 & 2150 & 1400 & 150 & 1000 & 0.65 \\
\hline Sato et al. (1989) & $48 \mathrm{H} 8-30$ & 150 & 2150 & 1400 & 150 & 1000 & 0.65 \\
\hline Sato et al. (1989) & $48 \mathrm{H} 8-40$ & 150 & 2150 & 1400 & 150 & 1000 & 0.65 \\
\hline Sato et al. (1989) & $48 \mathrm{H} 8-50$ & 150 & 2150 & 1400 & 150 & 1000 & 0.65 \\
\hline Seki et al. (1995) & RA-00P & 75 & 3075 & 1420 & 75 & 1500 & 0.46 \\
\hline Seki et al. (1995) & RA-15P & 75 & 3075 & 1420 & 75 & 1500 & 0.46 \\
\hline Seki et al. (1995) & RB-00P & 75 & 3075 & 2020 & 75 & 1500 & 0.66 \\
\hline Seki et al. (1995) & RB-15P & 75 & 3075 & 2020 & 75 & 1500 & 0.66 \\
\hline Seki et al. (1995) & RC-00P & 75 & 3075 & 2620 & 75 & 1500 & 0.85 \\
\hline
\end{tabular}




\begin{tabular}{|c|c|c|c|c|c|c|c|}
\hline Researcher & Specimen & $t_{w}(\mathrm{~mm})$ & $l_{w}(\mathrm{~mm})$ & $h_{w}(\mathrm{~mm})$ & $t_{f}(\mathrm{~mm})$ & $l_{f}(\mathrm{~mm})$ & $h_{w} / l_{w}$ \\
\hline Seki et al. (1995) & RC-15P & 75 & 3075 & 2620 & 75 & 1500 & 0.85 \\
\hline Mo and Chan (1996) & HN4-1 & 70 & 880 & 500 & 80 & 170 & 0.57 \\
\hline Mo and Chan (1996) & HN4-2 & 70 & 880 & 500 & 80 & 170 & 0.57 \\
\hline Mo and Chan (1996) & HN4-3 & 70 & 880 & 500 & 80 & 170 & 0.57 \\
\hline Mo and Chan (1996) & HN6-1 & 70 & 880 & 500 & 80 & 170 & 0.57 \\
\hline Mo and Chan (1996) & HN6-2 & 70 & 880 & 500 & 80 & 170 & 0.57 \\
\hline Mo and Chan (1996) & HN6-3 & 70 & 880 & 500 & 80 & 170 & 0.57 \\
\hline Mo and Chan (1996) & HM4-1 & 70 & 880 & 500 & 80 & 170 & 0.57 \\
\hline Mo and Chan (1996) & HM4-2 & 70 & 880 & 500 & 80 & 170 & 0.57 \\
\hline Mo and Chan (1996) & HM4-3 & 70 & 880 & 500 & 80 & 170 & 0.57 \\
\hline Mo and Chan (1996) & LN4-1 & 70 & 880 & 500 & 80 & 170 & 0.57 \\
\hline Mo and Chan (1996) & LN4-2 & 70 & 880 & 500 & 80 & 170 & 0.57 \\
\hline Mo and Chan (1996) & LN4-3 & 70 & 880 & 500 & 80 & 170 & 0.57 \\
\hline Mo and Chan (1996) & LN6-1 & 70 & 880 & 500 & 80 & 170 & 0.57 \\
\hline Mo and Chan (1996) & LN6-2 & 70 & 880 & 500 & 80 & 170 & 0.57 \\
\hline Mo and Chan (1996) & LN6-3 & 70 & 880 & 500 & 80 & 170 & 0.57 \\
\hline Mo and Chan (1996) & LM6-1 & 70 & 880 & 500 & 80 & 170 & 0.57 \\
\hline Mo and Chan (1996) & LM6-2 & 70 & 880 & 500 & 80 & 170 & 0.57 \\
\hline
\end{tabular}




\begin{tabular}{|c|c|c|c|c|c|c|c|}
\hline Researcher & Specimen & $t_{w}(\mathrm{~mm})$ & $l_{w}(\mathrm{~mm})$ & $h_{w}(\mathrm{~mm})$ & $t_{f}(\mathrm{~mm})$ & $l_{f}(\mathrm{~mm})$ & $h_{w} / l_{w}$ \\
\hline Mo and Chan (1996) & LM6-3 & 70 & 880 & 500 & 80 & 170 & 0.57 \\
\hline Mo and Chan (1996) & LM4-2 & 70 & 880 & 500 & 80 & 170 & 0.57 \\
\hline Mo and Chan (1996) & LM4-3 & 70 & 880 & 500 & 80 & 170 & 0.57 \\
\hline Kitada et al. (1997) & U-1 & 75 & 3000 & 2020 & 100 & 2980 & 0.67 \\
\hline Kitada et al. (1997) & U-2 & 75 & 3000 & 2020 & 100 & 2980 & 0.67 \\
\hline Naze and Sidaner (2001) & T03 & 160 & 3000 & 1200 & 160 & 800 & 0.40 \\
\hline Naze and Sidaner (2001) & T04 & 160 & 3000 & 1200 & 160 & 800 & 0.40 \\
\hline Naze and Sidaner (2001) & T05 & 200 & 3000 & 1200 & 160 & 800 & 0.40 \\
\hline Naze and Sidaner (2001) & T06 & 200 & 3000 & 1200 & 160 & 800 & 0.40 \\
\hline Naze and Sidaner (2001) & T07 & 200 & 3000 & 1200 & 160 & 800 & 0.40 \\
\hline Naze and Sidaner (2001) & T08 & 200 & 3000 & 1200 & 160 & 800 & 0.40 \\
\hline Naze and Sidaner (2001) & T09 & 200 & 3000 & 1200 & 160 & 800 & 0.40 \\
\hline Naze and Sidaner (2001) & T10 & 200 & 3000 & 1200 & 160 & 800 & 0.40 \\
\hline Naze and Sidaner (2001) & T11 & 200 & 3000 & 1200 & 160 & 800 & 0.40 \\
\hline Naze and Sidaner (2001) & T12 & 200 & 3000 & 1200 & 160 & 800 & 0.40 \\
\hline Palermo and Vecchio (2002) & DP1 & 75 & 3075 & 2020 & 95 & 3045 & 0.66 \\
\hline Palermo and Vecchio (2002) & DP2 & 75 & 3075 & 2020 & 100 & 3045 & 0.66 \\
\hline Farvashany et al. (2008) & HSCW1 & 75 & 880 & 1100 & 90 & 375 & 1.25 \\
\hline
\end{tabular}




\begin{tabular}{|c|c|c|c|c|c|c|c|}
\hline Researcher & Specimen & $t_{w}(\mathrm{~mm})$ & $l_{w}(\mathrm{~mm})$ & $h_{w}(\mathrm{~mm})$ & $t_{f}(\mathrm{~mm})$ & $l_{f}(\mathrm{~mm})$ & $h_{w} / l_{w}$ \\
\hline Farvashany et al. $(2008)$ & HSCW2 & 75 & 880 & 1100 & 90 & 375 & 1.25 \\
\hline Farvashany et al. $(2008)$ & HSCW3 & 75 & 880 & 1100 & 90 & 375 & 1.25 \\
\hline Farvashany et al. (2008) & HSCW4 & 75 & 880 & 1100 & 90 & 375 & 1.25 \\
\hline Farvashany et al. (2008) & HSCW5 & 75 & 880 & 1100 & 90 & 375 & 1.25 \\
\hline Farvashany et al. (2008) & HSCW6 & 75 & 880 & 1100 & 90 & 375 & 1.25 \\
\hline Farvashany et al. (2008) & HSCW7 & 75 & 880 & 1100 & 90 & 375 & 1.25 \\
\hline
\end{tabular}

Table 2.3 Material properties of non-rectangular RC squat walls

\begin{tabular}{|c|c|c|c|c|c|c|c|c|c|c|}
\hline Researcher & Specimen & $f_{c}^{\prime}(\mathrm{MPa})$ & $f_{y v}(\mathrm{MPa})$ & $f_{y h}(\mathrm{MPa})$ & $f_{y f}(\mathrm{MPa})$ & $\rho_{v}(\%)$ & $\rho_{h}(\%)$ & $\rho_{f}(\%)$ & $s_{v}(\mathrm{~mm})$ & $s_{h}(\mathrm{~mm})$ \\
\hline Barda et al. (1972) & B1-1 & 29.0 & 543.3 & 495.7 & 525.4 & 0.50 & 0.50 & 1.80 & 280 & 110 \\
\hline Barda et al. (1972) & B2-1 & 16.3 & 551.6 & 499.2 & 486.8 & 0.50 & 0.50 & 6.40 & 280 & 110 \\
\hline Barda et al. (1972) & B3-2 & 27.0 & 544.7 & 513.0 & 413.7 & 0.50 & 0.50 & 4.10 & 280 & 110 \\
\hline Barda et al. (1972) & B4-3 & 23.5 & 535.0 & N/A & 527.4 & 0.50 & 0 & 4.10 & 280 & N/A \\
\hline Barda et al. (1972) & B5-4 & 19.0 & N/A & 495.0 & 526.8 & 0 & 0.50 & 4.10 & N/A & 110 \\
\hline Barda et al. (1972) & B6-4 & 21.2 & 496.4 & 496.4 & 528.8 & 0.25 & 0.50 & 4.10 & 210 & 110 \\
\hline Barda et al. (1972) & B7-5 & 25.7 & 530.9 & 501.2 & 539.2 & 0.50 & 0.50 & 4.10 & 280 & 130 \\
\hline Barda et al. (1972) & B8-5 & 23.4 & 527.4 & 495.7 & 488.8 & 0.50 & 0.50 & 4.10 & 280 & 110 \\
\hline
\end{tabular}




\begin{tabular}{|c|c|c|c|c|c|c|c|c|c|c|}
\hline Researcher & Specimen & $f_{c}^{\prime}(\mathrm{MPa})$ & $f_{y v}(\mathrm{MPa})$ & $f_{y h}(\mathrm{MPa})$ & $f_{y f}(\mathrm{MPa})$ & $\rho_{v}(\%)$ & $\rho_{h}(\%)$ & $\rho_{f}(\%)$ & $s_{v}(\mathrm{~mm})$ & $s_{h}(\mathrm{~mm})$ \\
\hline Synge (1980) & Wall 3 & 26.0 & 315.1 & 380.0 & 300.0 & 0.37 & 1.61 & 1.81 & 200 & 120 \\
\hline Saito et al. (1989) & W12-1 & 35.0 & 369.0 & 369.0 & 369.0 & 1.32 & 1.32 & 4.72 & 90 & 90 \\
\hline Saito et al. (1989) & W12-2 & 38.0 & 369.0 & 369.0 & 369.0 & 0.90 & 0.90 & 4.72 & 130 & 130 \\
\hline Saito et al. (1989) & W12-3 & 36.0 & 369.0 & 369.0 & 369.0 & 1.69 & 1.69 & 4.72 & 70 & 70 \\
\hline Saito et al. (1989) & W12-4 & 36.0 & 369.0 & 369.0 & 369.0 & 1.32 & 1.32 & 4.72 & 90 & 90 \\
\hline Saito et al. (1989) & W12-5 & 40.0 & 369.0 & 369.0 & 369.0 & 1.32 & 1.32 & 4.72 & 90 & 90 \\
\hline Saito et al. (1989) & W12-6 & 33.0 & 369.0 & 369.0 & 369.0 & 1.32 & 1.32 & 4.72 & 90 & 90 \\
\hline Saito et al. (1989) & W12-7 & 34.0 & 369.0 & 369.0 & 369.0 & 1.32 & 1.32 & 4.72 & 90 & 90 \\
\hline Saito et al. (1989) & W15-1 & 25.0 & 369.0 & 369.0 & 369.0 & 1.06 & 1.06 & 4.72 & 90 & 90 \\
\hline Saito et al. (1989) & W15-2 & 26.0 & 369.0 & 369.0 & 369.0 & 1.06 & 1.06 & 4.72 & 90 & 90 \\
\hline Sato et al. (1989) & $18 \mathrm{M} 12-40$ & 43.1 & 422.0 & 422.0 & 422.0 & 0.45 & 0.45 & 2.25 & 200 & 200 \\
\hline Sato et al. (1989) & $24 \mathrm{M} \mathrm{8-30}$ & 40.1 & 296.0 & 296.0 & 296.0 & 0.80 & 0.80 & 0.80 & 120 & 120 \\
\hline Sato et al. (1989) & $24 \mathrm{M} 8-40$ & 41.0 & 422.0 & 422.0 & 422.0 & 0.60 & 0.60 & 0.60 & 160 & 160 \\
\hline Sato et al. (1989) & $24 \mathrm{M} 8-50$ & 38.0 & 296.0 & 528.0 & 528.0 & 0.48 & 0.48 & 0.48 & 200 & 200 \\
\hline Sato et al. (1989) & 24M 6-30 & 35.8 & 422.0 & 296.0 & 296.0 & 0.80 & 0.80 & 0.80 & 120 & 120 \\
\hline Sato et al. (1989) & $24 \mathrm{M} 6-40$ & 35.0 & 530.0 & 422.0 & 422.0 & 0.60 & 0.60 & 0.60 & 160 & 160 \\
\hline Sato et al. (1989) & $36 \mathrm{M} \mathrm{12-30}$ & 24.5 & 296.0 & 296.0 & 296.0 & 1.20 & 1.20 & 5.80 & 80 & 80 \\
\hline Sato et al. (1989) & $36 \mathrm{M} 12-40$ & 27.8 & 422.0 & 422.0 & 422.0 & 0.90 & 0.90 & 4.50 & 105 & 105 \\
\hline
\end{tabular}




\begin{tabular}{|c|c|c|c|c|c|c|c|c|c|c|}
\hline Researcher & Specimen & $f_{c}^{\prime}(\mathrm{MPa})$ & $f_{y v}(\mathrm{MPa})$ & $f_{y h}(\mathrm{MPa})$ & $f_{y f}(\mathrm{MPa})$ & $\rho_{v}(\%)$ & $\rho_{h}(\%)$ & $\rho_{f}(\%)$ & $s_{v}(\mathrm{~mm})$ & $s_{h}(\mathrm{~mm})$ \\
\hline Sato et al. (1989) & $36 \mathrm{M} 12-50$ & 36.3 & 296.0 & 528.0 & 528.0 & 0.72 & 0.72 & 3.60 & 130 & 130 \\
\hline Sato et al. (1989) & $36 \mathrm{~L} 8-30$ & 34.4 & 422.0 & 296.0 & 296.0 & 1.20 & 1.20 & 1.16 & 80 & 80 \\
\hline Sato et al. (1989) & $36 \mathrm{~L} 8-40$ & 37,2 & 530.0 & 422.0 & 422.0 & 0.90 & 0.90 & 0.90 & 110 & 110 \\
\hline Sato et al. (1989) & $36 \mathrm{M} \mathrm{8-30}$ & 33.4 & 296.0 & 296.0 & 296.0 & 1.20 & 1.20 & 1.16 & 80 & 80 \\
\hline Sato et al. (1989) & $36 \mathrm{M} 8-40$ & 34.6 & 422.0 & 422.0 & 422.0 & 0.90 & 0.90 & 0.90 & 110 & 110 \\
\hline Sato et al. (1989) & $36 \mathrm{M} \mathrm{8-50}$ & 39.3 & 296.0 & 528.0 & 528.0 & 0.72 & 0.72 & 0.72 & 130 & 130 \\
\hline Sato et al. (1989) & $36 \mathrm{M} \mathrm{6-30}$ & 38.8 & 422.0 & 296.0 & 296.0 & 1.20 & 1.20 & 1.16 & 80 & 80 \\
\hline Sato et al. (1989) & $36 \mathrm{M} \mathrm{6-40}$ & 37.5 & 530.0 & 422.0 & 422.0 & 0.90 & 0.90 & 0.90 & 120 & 120 \\
\hline Sato et al. (1989) & $48 \mathrm{M} \mathrm{8-30}$ & 41.8 & 296.0 & 296.0 & 296.0 & 1.60 & 1.60 & 1.60 & 60 & 60 \\
\hline Sato et al. (1989) & $48 \mathrm{M} \mathrm{8-40}$ & 43.1 & 422.0 & 422.0 & 422.0 & 1.20 & 1.20 & 1.16 & 80 & 80 \\
\hline Sato et al. (1989) & $48 \mathrm{M} 8-50$ & 44.6 & 530.0 & 528.0 & 528.0 & 0.96 & 0.96 & 0.96 & 100 & 100 \\
\hline Sato et al. (1989) & $48 \mathrm{H} 8-30$ & 27.4 & 296.0 & 296.0 & 296.0 & 1.60 & 1.60 & 1.60 & 60 & 60 \\
\hline Sato et al. (1989) & $48 \mathrm{H} 8-40$ & 27.5 & 422.0 & 422.0 & 422.0 & 1.20 & 1.20 & 1.16 & 80 & 80 \\
\hline Sato et al. (1989) & $48 \mathrm{H} 8-50$ & 28.0 & 530.0 & 528.0 & 528.0 & 0.96 & 0.96 & 0.96 & 100 & 100 \\
\hline Seki et al. (1995) & RA-00P & 31.6 & 350.0 & 350.0 & 350.0 & 1.08 & 1.08 & 1.10 & 70 & 70 \\
\hline Seki et al. (1995) & RA-15P & 29.5 & 350.0 & 350.0 & 350.0 & 1.08 & 1.08 & 1.10 & 70 & 70 \\
\hline Seki et al. (1995) & RB-00P & 28.9 & 380.0 & 380.0 & 380.0 & 1.08 & 1.08 & 1.10 & 70 & 70 \\
\hline Seki et al. (1995) & RB-15P & 28.9 & 380.0 & 380.0 & 380.0 & 1.08 & 1.08 & 1.10 & 70 & 70 \\
\hline
\end{tabular}




\begin{tabular}{|c|c|c|c|c|c|c|c|c|c|c|}
\hline Researcher & Specimen & $f_{c}^{\prime}(\mathrm{MPa})$ & $f_{y v}(\mathrm{MPa})$ & $f_{y h}(\mathrm{MPa})$ & $f_{y f}(\mathrm{MPa})$ & $\rho_{v}(\%)$ & $\rho_{h}(\%)$ & $\rho_{f}(\%)$ & $s_{v}(\mathrm{~mm})$ & $s_{h}(\mathrm{~mm})$ \\
\hline Seki et al. (1995) & RC-00P & 30.1 & 350.0 & 350.0 & 350.0 & 1.08 & 1.08 & 1.10 & 70 & 70 \\
\hline Seki et al. (1995) & RC-15P & 29.2 & 350.0 & 350.0 & 350.0 & 1.08 & 1.08 & 1.10 & 70 & 70 \\
\hline Mo and Chan (1996) & HN4-1 & 32.2 & 302.3 & 302.3 & 302.3 & 0.73 & 0.92 & 2.10 & 120 & 110 \\
\hline Mo and Chan (1996) & HN4-2 & 32.2 & 302.3 & 302.3 & 302.3 & 0.73 & 0.92 & 2.10 & 120 & 110 \\
\hline Mo and Chan (1996) & HN4-3 & 32.1 & 302.3 & 302.3 & 302.3 & 0.73 & 0.92 & 2.10 & 120 & 110 \\
\hline Mo and Chan (1996) & HN6-1 & 29.5 & 443.2 & 443.2 & 443.2 & 0.73 & 0.92 & 2.10 & 120 & 110 \\
\hline Mo and Chan (1996) & HN6-2 & 29.5 & 443.2 & 443.2 & 443.2 & 0.73 & 0.92 & 2.10 & 120 & 110 \\
\hline Mo and Chan (1996) & HN6-3 & 31.0 & 443.2 & 443.2 & 443.2 & 0.73 & 0.92 & 2.10 & 120 & 110 \\
\hline Mo and Chan (1996) & HM4-1 & 37.5 & 302.3 & 302.3 & 302.3 & 0.73 & 0.92 & 2.10 & 120 & 110 \\
\hline Mo and Chan (1996) & HM4-2 & 37.5 & 302.3 & 302.3 & 302.3 & 0.73 & 0.92 & 2.10 & 120 & 110 \\
\hline Mo and Chan (1996) & HM4-3 & 39.9 & 302.3 & 302.3 & 302.3 & 0.73 & 0.92 & 2.10 & 120 & 110 \\
\hline Mo and Chan (1996) & LN4-1 & 18.0 & 302.3 & 302.3 & 302.3 & 0.58 & 0.92 & 2.10 & 140 & 110 \\
\hline Mo and Chan (1996) & LN4-2 & 18.0 & 302.3 & 302.3 & 302.3 & 0.58 & 0.92 & 2.10 & 140 & 110 \\
\hline Mo and Chan (1996) & LN4-3 & 29.7 & 302.3 & 302.3 & 302.3 & 0.58 & 0.92 & 2.10 & 140 & 110 \\
\hline Mo and Chan (1996) & LN6-1 & 30.7 & 443.2 & 443.2 & 443.2 & 0.58 & 0.92 & 2.10 & 140 & 110 \\
\hline Mo and Chan (1996) & LN6-2 & 30.2 & 443.2 & 443.2 & 443.2 & 0.58 & 0.92 & 2.10 & 140 & 110 \\
\hline Mo and Chan (1996) & LN6-3 & 30.2 & 443.2 & 443.2 & 443.2 & 0.58 & 0.92 & 2.10 & 140 & 110 \\
\hline Mo and Chan (1996) & LM6-1 & 39.3 & 443.2 & 443.2 & 443.2 & 0.58 & 0.92 & 2.10 & 140 & 110 \\
\hline
\end{tabular}




\begin{tabular}{|c|c|c|c|c|c|c|c|c|c|c|}
\hline Researcher & Specimen & $f_{c}^{\prime}(\mathrm{MPa})$ & $f_{y v}(\mathrm{MPa})$ & $f_{y h}(\mathrm{MPa})$ & $f_{y f}(\mathrm{MPa})$ & $\rho_{v}(\%)$ & $\rho_{h}(\%)$ & $\rho_{f}(\%)$ & $s_{v}(\mathrm{~mm})$ & $s_{h}(\mathrm{~mm})$ \\
\hline Mo and Chan (1996) & LM6-2 & 37.0 & 443.2 & 443.2 & 443.2 & 0.58 & 0.92 & 2.10 & 140 & 110 \\
\hline Mo and Chan (1996) & LM6-3 & 34.5 & 443.2 & 443.2 & 443.2 & 0.58 & 0.92 & 2.10 & 140 & 110 \\
\hline Mo and Chan (1996) & LM4-2 & 66.0 & 302.3 & 302.3 & 302.3 & 0.58 & 0.92 & 2.10 & 140 & 110 \\
\hline Mo and Chan (1996) & LM4-3 & 66.0 & 302.3 & 302.3 & 302.3 & 0.58 & 0.92 & 2.10 & 140 & 110 \\
\hline Kitada et al. (1997) & U-1 & 29.2 & 390.0 & 390.0 & 390.0 & 1.20 & 1.20 & 0.47 & 70 & 70 \\
\hline Kitada et al. (1997) & $\mathrm{U}-2$ & 29.2 & 390.0 & 390.0 & 390.0 & 1.20 & 1.20 & 0.47 & 70 & 70 \\
\hline Naze and Sidaner (2001) & T03 & 36.2 & 570.0 & 570.0 & 570.0 & 0.80 & 0.80 & 2.41 & 125 & 125 \\
\hline Naze and Sidaner (2001) & T04 & 43.4 & 570.0 & 570.0 & 570.0 & 0.80 & 0.80 & 2.41 & 125 & 125 \\
\hline Naze and Sidaner (2001) & T05 & 43.4 & 570.0 & 570.0 & 570.0 & 0.80 & 0.80 & 2.41 & 125 & 125 \\
\hline Naze and Sidaner (2001) & T06 & 43.4 & 600.0 & 600.0 & 600.0 & 0.40 & 0.60 & 1.77 & 125 & 125 \\
\hline Naze and Sidaner (2001) & T07 & 43.4 & 600.0 & 600.0 & 600.0 & 0.40 & 0.60 & 1.77 & 125 & 125 \\
\hline Naze and Sidaner (2001) & T08 & 43.4 & 600.0 & 600.0 & 600.0 & 0.40 & 0.40 & 1.23 & 125 & 125 \\
\hline Naze and Sidaner (2001) & T09 & 43.4 & 600.0 & 600.0 & 600.0 & 0.40 & 0.40 & 1.23 & 125 & 125 \\
\hline Naze and Sidaner (2001) & T10 & 43.4 & 570.0 & 570.0 & 570.0 & 0.60 & 0.60 & 1.77 & 125 & 125 \\
\hline Naze and Sidaner (2001) & T11 & 43.4 & 600.0 & 600.0 & 600.0 & 0.40 & 0.40 & 1.23 & 125 & 125 \\
\hline Naze and Sidaner (2001) & $\mathrm{T} 12$ & 43.4 & 560.0 & 560.0 & 560.0 & 0.11 & 0.11 & 1.77 & 250 & 250 \\
\hline Palermo and Vecchio (2002) & DP1 & 21.7 & 605.0 & 605.0 & 605.0 & 0.82 & 0.73 & 0.37 & 130 & 140 \\
\hline Palermo and Vecchio (2002) & DP2 & 18.8 & 605.0 & 605.0 & 605.0 & 0.82 & 0.73 & 0.35 & 130 & 140 \\
\hline
\end{tabular}




\begin{tabular}{|c|c|c|c|c|c|c|c|c|c|c|}
\hline Researcher & Specimen & $f_{c}^{\prime}(\mathrm{MPa})$ & $f_{y v}(\mathrm{MPa})$ & $f_{y h}(\mathrm{MPa})$ & $f_{y f}(\mathrm{MPa})$ & $\rho_{v}(\%)$ & $\rho_{h}(\%)$ & $\rho_{f}(\%)$ & $s_{v}(\mathrm{~mm})$ & $s_{h}(\mathrm{~mm})$ \\
\hline Farvashany et al. (2008) & HSCW1 & 104.0 & 400.0 & 400.0 & 400.0 & 1.26 & 0.47 & 4.02 & 60 & 160 \\
\hline Farvashany et al. (2008) & HSCW2 & 93.0 & 400.0 & 400.0 & 400.0 & 1.26 & 0.47 & 4.02 & 60 & 160 \\
\hline Farvashany et al. (2008) & HSCW3 & 86.0 & 400.0 & 400.0 & 400.0 & 0.75 & 0.47 & 4.02 & 100 & 160 \\
\hline Farvashany et al. (2008) & HSCW4 & 91.0 & 400.0 & 400.0 & 400.0 & 0.75 & 0.47 & 4.02 & 100 & 160 \\
\hline Farvashany et al. (2008) & HSCW5 & 84.0 & 400.0 & 400.0 & 400.0 & 1.26 & 0.75 & 4.02 & 60 & 100 \\
\hline Farvashany et al. (2008) & HSCW6 & 90.0 & 400.0 & 400.0 & 400.0 & 1.26 & 0.75 & 4.02 & 60 & 100 \\
\hline Farvashany et al. (2008) & HSCW7 & 102.0 & 400.0 & 400.0 & 400.0 & 0.75 & 0.75 & 4.02 & 100 & 100 \\
\hline
\end{tabular}

Table 2.4 Performance of non-rectangular RC squat walls

\begin{tabular}{|c|c|c|c|c|c|}
\hline Researcher & Specimen & Loading Type & $P / A_{t} f_{c}^{\prime}(\%)$ & $V_{\text {peak }}(\mathrm{kN})$ & Failure \\
\hline Barda et al. (1972) & B1-1 & Monotonic & 0.0 & 1218.4 & Shear \\
\hline Barda et al. (1972) & B2-1 & Monotonic & 0.0 & 978.6 & Shear \\
\hline Barda et al. (1972) & B3-2 & Cyclic & 0.0 & 1108.1 & Shear \\
\hline Barda et al. $(1972)$ & B4-3 & Cyclic & 0.0 & 1017.3 & Shear \\
\hline Barda et al. (1972) & B5-4 & Cyclic & 0.0 & 698.8 & Shear \\
\hline Barda et al. (1972) & B6-4 & Cyclic & 0.0 & 876.3 & Shear \\
\hline Barda et al. (1972) & B7-5 & Cyclic & 0.0 & 1139.6 & Shear \\
\hline
\end{tabular}




\begin{tabular}{|c|c|c|c|c|c|}
\hline Researcher & Specimen & Loading Type & $P / A_{t} f_{c}^{\prime}(\%)$ & $V_{\text {peak }}(\mathrm{kN})$ & Failure \\
\hline Barda et al. (1972) & B8-5 & Cyclic & 0.0 & 885.6 & Shear \\
\hline Synge (1980) & Wall 3 & Cyclic & 0.0 & 742.4 & Shear \\
\hline Saito et al. (1989) & W12-1 & Cyclic & 5.6 & 2657.4 & Shear \\
\hline Saito et al. (1989) & W12-2 & Cyclic & 5.1 & 2510.6 & Shear \\
\hline Saito et al. (1989) & W12-3 & Cyclic & 5.5 & 2510.6 & Shear \\
\hline Saito et al. (1989) & W12-4 & Cyclic & 2.7 & 2481.2 & Shear \\
\hline Saito et al. (1989) & W12-5 & Cyclic & 4.9 & 2667.6 & Shear \\
\hline Saito et al. (1989) & W12-6 & Cyclic & 5.9 & 1755.3 & Shear \\
\hline Saito et al. (1989) & W12-7 & Cyclic & 2.9 & 1647.6 & Shear \\
\hline Saito et al. (1989) & W15-1 & Cyclic & 7.9 & 2186.7 & Shear \\
\hline Saito et al. (1989) & W15-2 & Cyclic & 7.6 & 1814.4 & Shear \\
\hline Sato et al. (1989) & $18 \mathrm{M} 12-40$ & Cyclic & 4.6 & 2250.0 & Shear \\
\hline Sato et al. (1989) & 24M 6-30 & Cyclic & 5.0 & 2100.0 & Shear \\
\hline Sato et al. (1989) & $24 \mathrm{M} \mathrm{6-40}$ & Cyclic & 4.9 & 2190.0 & Shear \\
\hline Sato et al. (1989) & $24 \mathrm{M} \mathrm{8-30}$ & Cyclic & 5.3 & 1680.0 & Shear \\
\hline Sato et al. (1989) & $24 \mathrm{M} 8-40$ & Cyclic & 5.6 & 1740.0 & Shear \\
\hline Sato et al. (1989) & $24 \mathrm{M} \mathrm{8-50}$ & Cyclic & 5.7 & 1740.0 & Shear \\
\hline Sato et al. (1989) & $36 \mathrm{~L} 8-30$ & Cyclic & 8.2 & 1800.0 & Shear \\
\hline
\end{tabular}




\begin{tabular}{|c|c|c|c|c|c|}
\hline Researcher & Specimen & Loading Type & $P / A_{t} f_{c}^{\prime}(\%)$ & $V_{\text {peak }}(\mathrm{kN})$ & Failure \\
\hline Sato et al. (1989) & $36 \mathrm{~L} 8-40$ & Cyclic & 7.2 & 1830.0 & Shear \\
\hline Sato et al. (1989) & $36 \mathrm{M} \mathrm{12-30}$ & Cyclic & 5.5 & 2490.0 & Shear \\
\hline Sato et al. (1989) & $36 \mathrm{M} 12-40$ & Cyclic & 5.8 & 2490.0 & Shear \\
\hline Sato et al. (1989) & $36 \mathrm{M} 12-50$ & Cyclic & 5.4 & 2430.0 & Shear \\
\hline Sato et al. (1989) & $36 \mathrm{M} \mathrm{6-30}$ & Cyclic & 6.0 & 2250.0 & Shear \\
\hline Sato et al. (1989) & $36 \mathrm{M} \mathrm{6-40}$ & Cyclic & 5.8 & 2370.0 & Shear \\
\hline Sato et al. (1989) & $36 \mathrm{M} \mathrm{8-30}$ & Cyclic & 5.1 & 1935.0 & Shear \\
\hline Sato et al. (1989) & $36 \mathrm{M} \mathrm{8-40}$ & Cyclic & 5.2 & 2040.0 & Shear \\
\hline Sato et al. (1989) & $36 \mathrm{M} \mathrm{8-50}$ & Cyclic & 5.3 & 1980.0 & Shear \\
\hline Sato et al. (1989) & $48 \mathrm{H} 8-30$ & Cyclic & 4.8 & 2280.0 & Shear \\
\hline Sato et al. (1989) & $48 \mathrm{H} 8-40$ & Cyclic & 4.6 & 2340.0 & Shear \\
\hline Sato et al. (1989) & $48 \mathrm{H} 8-50$ & Cyclic & 4.5 & 2430.0 & Shear \\
\hline Sato et al. (1989) & $48 \mathrm{M} \mathrm{8-30}$ & Cyclic & 7.3 & 1980.0 & Shear \\
\hline Sato et al. (1989) & $48 \mathrm{M} \mathrm{8-40}$ & Cyclic & 7.3 & 2040.0 & Shear \\
\hline Sato et al. (1989) & $48 \mathrm{M} \mathrm{8-50}$ & Cyclic & 7.1 & 2040.0 & Shear \\
\hline Seki et al. (1995) & RA-00P & Cyclic & 0.0 & 1470.0 & Shear \\
\hline Seki et al. (1995) & RA-15P & Cyclic & 3.4 & 1670.0 & Shear \\
\hline Seki et al. (1995) & RB-00P & Cyclic & 0.0 & 1260.0 & Shear \\
\hline
\end{tabular}




\begin{tabular}{|c|c|c|c|c|c|}
\hline Researcher & Specimen & Loading Type & $P / A_{t} f_{c}^{\prime}(\%)$ & $V_{\text {peak }}(\mathrm{kN})$ & Failure \\
\hline Seki et al. (1995) & RB-15P & Cyclic & 3.5 & 1465.0 & Shear \\
\hline Seki et al. (1995) & RC-00P & Cyclic & 0.0 & 1030.0 & Shear \\
\hline Seki et al. (1995) & RC-15P & Cyclic & 3.5 & 1170.0 & Shear \\
\hline Mo and Chan (1996) & HN4-1 & Cyclic & 0.0 & 205.0 & Flexure \\
\hline Mo and Chan (1996) & HN4-2 & Cyclic & 0.0 & 247.0 & Flexure \\
\hline Mo and Chan (1996) & HN4-3 & Cyclic & 0.0 & 202.0 & Flexure \\
\hline Mo and Chan (1996) & HN6-1 & Cyclic & 0.0 & 255.0 & Shear \\
\hline Mo and Chan (1996) & HN6-2 & Cyclic & 0.0 & 204.0 & Shear \\
\hline Mo and Chan (1996) & HN6-3 & Cyclic & 0.0 & 205.0 & Shear \\
\hline Mo and Chan (1996) & HM4-1 & Cyclic & 0.0 & 223.0 & Flexure \\
\hline Mo and Chan (1996) & HM4-2 & Cyclic & 0.0 & 231.0 & Flexure \\
\hline Mo and Chan (1996) & HM4-3 & Cyclic & 0.0 & 250.0 & Flexure \\
\hline Mo and Chan (1996) & LN4-1 & Cyclic & 0.0 & 193.0 & Flexure \\
\hline Mo and Chan (1996) & LN4-2 & Cyclic & 0.0 & 217.0 & Flexure \\
\hline Mo and Chan (1996) & LN4-3 & Cyclic & 0.0 & 203.0 & Flexure \\
\hline Mo and Chan (1996) & LN6-1 & Cyclic & 0.0 & 246.0 & Shear \\
\hline Mo and Chan (1996) & LN6-2 & Cyclic & 0.0 & 200.0 & Shear \\
\hline Mo and Chan (1996) & LN6-3 & Cyclic & 0.0 & 210.0 & Shear \\
\hline
\end{tabular}




\begin{tabular}{|c|c|c|c|c|c|}
\hline Researcher & Specimen & Loading Type & $P / A_{t} f_{c}^{\prime}(\%)$ & $V_{\text {peak }}(\mathrm{kN})$ & Failure \\
\hline Mo and Chan (1996) & LM6-1 & Cyclic & 0.0 & 219.0 & Shear \\
\hline Mo and Chan (1996) & LM6-2 & Cyclic & 0.0 & 205.0 & Shear \\
\hline Mo and Chan (1996) & LM6-3 & Cyclic & 0.0 & 210.0 & Shear \\
\hline Mo and Chan (1996) & LM4-2 & Cyclic & 0.0 & 250.0 & Flexure \\
\hline Mo and Chan (1996) & LM4-3 & Cyclic & 0.0 & 227.0 & Flexure \\
\hline Kitada et al. (1997) & U-1 & ST & 3.9 & 1636.1 & Shear \\
\hline Kitada et al. (1997) & U-2 & ST & 3.8 & 1618.3 & Shear \\
\hline Naze and Sidaner (2001) & T03 & Cyclic & 0.0 & 3620.0 & Shear \\
\hline Naze and Sidaner (2001) & T04 & Cyclic & 0.0 & 5300.0 & Shear \\
\hline Naze and Sidaner (2001) & T05 & Cyclic & 0.0 & 5400.0 & Shear \\
\hline Naze and Sidaner (2001) & T06 & Cyclic & 2.3 & 5180.0 & Shear \\
\hline Naze and Sidaner (2001) & T07 & Cyclic & 2.3 & 7060.0 & Shear \\
\hline Naze and Sidaner (2001) & T08 & Cyclic & 0.0 & 3920.0 & Shear \\
\hline Naze and Sidaner (2001) & T09 & Cyclic & 0.0 & 4170.0 & Shear \\
\hline Naze and Sidaner (2001) & T10 & Cyclic & 0.0 & 5640.0 & Shear \\
\hline Naze and Sidaner (2001) & T11 & Cyclic & 0.0 & 4100.0 & Shear \\
\hline Naze and Sidaner (2001) & T12 & Cyclic & 2.3 & 4000.0 & Shear \\
\hline Palermo and Vecchio (2002) & DP1 & Cyclic & 7.0 & 1298.0 & Shear \\
\hline
\end{tabular}




\begin{tabular}{|c|c|c|c|c|c|}
\hline Researcher & Specimen & Loading Type & $P / A_{t} f_{c}^{\prime}(\%)$ & $V_{\text {peak }}(\mathrm{kN})$ & Failure \\
\hline Palermo and Vecchio (2002) & DP2 & Cyclic & 1.4 & 904.0 & Shear \\
\hline Farvashany et al. (2008) & HSCW1 & Cyclic & 4.3 & 735.0 & Shear \\
\hline Farvashany et al. (2008) & HSCW2 & Cyclic & 8.5 & 845.0 & Shear \\
\hline Farvashany et al. (2008) & HSCW3 & Cyclic & 9.2 & 625.0 & Shear \\
\hline Farvashany et al. (2008) & HSCW4 & Cyclic & 21.6 & 866.0 & Shear \\
\hline Farvashany et al. (2008) & HSCW5 & Cyclic & 9.5 & 801.0 & Shear \\
\hline Farvashany et al. (2008) & HSCW6 & Cyclic & 5.1 & 745.0 & Shear \\
\hline Farvashany et al. (2008) & HSCW7 & Cyclic & 7.8 & 800.0 & Shear \\
\hline
\end{tabular}




\subsection{Summary}

The design procedures [A1, A3] together with equations proposed by various researchers [B1, G3, H4, K1, W7] for the peak shear strength of squat shear walls were reviewed first. Then previous experiments regarding non-rectangular RC squat walls were presented. Finally, key features of the existing experiments on nonrectangular RC squat walls were summarized.

Above all, the remaining problems are identified as follows:

- Current codes and standards are developed for rectangular slender walls. The performance-affected factors such as the large flanges of the non-rectangular RC squat walls are not well explained in these codes. An improved analytical model should be developed and design procedures should be refined for nonrectangular RC squat walls.

- Contradictions are found between building codes and experimental results. For instance, Section 11.5 and 18.10 of ACI 318-14 [A1] only recognize the contribution of the horizontal reinforcement as the shear resisting component. However, Barad et al. concluded that the vertical web reinforcement was more effective than the horizontal reinforcement on the shear strength of squat walls. Further research is needed to study the influence of important parameters on the shear strength.

- Experiments on non-rectangular RC squat walls are rather sparse in the literature. Most tests on L-shaped and T-shaped walls are primarily focused on slender walls. Thus more experimental data is needed for squat walls with Lshaped and T-shaped cross sections.

- Most tests on non-rectangular RC squat walls are conducted with lateral loading direction parallel to the wall segment. However, in real earthquakes, seismic waves can reach structures from any direction, which necessitates experiments studying the behavior of these walls under various lateral loading directions. 
- The shear lag effect in the tension flange distinctly affected the performance of flanged walls [K2, P2]. The effective flange width increased as the drift ratio rose [K2], which was not addressed in the codes and standards. Thus further investigations are warranted. 


\section{CHAPTER 3}

\section{EXPERIMENTAL PROGRAM OF NON-RECTANGULAR RC SQUAT WALLS}

\subsection{Introduction}

Non-rectangular RC squat walls, as introduced in Chapter 1, are widely used in existing buildings as well as safety-related nuclear facilities. However, current experimental data of non-rectangular RC squat walls is sparse [G2], and issues regarding their performance at various aspects have not been well-addressed. Moreover, due to the complexity of materials as well as the limits of technique, numerical studies are unable to fully represent the real structures. To improve the understanding of the behavior of non-rectangular RC squat walls, it is necessary to conduct experiments in this area.

Therefore, an experimental program comprising a total of twelve specimens was conducted at the Protective Engineering Lab in Nanyang Technological University (NTU). These specimens were designed based on the provisions of ACI 318-14 [A1] and subjected to the simulated seismic loading. The influence of axial loads, lateral loading directions, and section shapes were investigated in the experiment.

In this chapter, the design of test specimens, including dimensions and the reinforcement detailing, is first described. Then, the test setup is illustrated with loading history being presented. Finally, the instruments used to monitor the seismic behavior of specimens are introduced.

\subsection{Design of Specimens}

\subsubsection{Specimen details}

The aim of this experimental program was to investigate the seismic behavior of non-rectangular RC squat walls, which required research carried out on different section shapes. In this study, H-shaped, L-shaped, and T-shaped sections were selected for test specimens since these sections represented the typical non- 
rectangular sections of RC squat walls. The design of test specimens was based on the provisions of ACI 318-14 [A1]. As reviewed in Chapter 2, six vertical slip planes were observed on the web of a H-shaped wall tested by Palermo and Vecchio [P2], which had been barely presented in other experiments and lacked deep analyses. To examine this phenomenon and provide insight into the seismic response of structural walls, the configuration of the H-shaped specimens in this study were designed to be similar to the H-shaped wall tested by Palermo and Vecchio [P2]. However, in consideration of the limits of the lab, the scale of test specimens was diminished to approximately half of the prototype, but shear span ratio was kept the same as 0.67. Therefore, as illustrated in Figure 3.1, all H-shaped specimens were $1000 \mathrm{~mm}$ high, $1500 \mathrm{~mm}$ long for the web and flange, and $100 \mathrm{~mm}$ thick. T10 and T20 steel bars with high tensile strength were used to reinforce the base of the specimens. D10 deformed bars with a nominal yield strength of 554 MPa were arranged at an interval of $150 \mathrm{~mm}$ in the web and $160 \mathrm{~mm}$ in the flange as the longitudinal reinforcement, which contributed to reinforcement ratios of $0.97 \%$ and $1.26 \%$ respectively. R6 round bars with a nominal yield strength of $355 \mathrm{MPa}$ were arranged at a spacing of $80 \mathrm{~mm}$ as the horizontal reinforcement, which yielded a reinforcement ratio of $0.71 \%$. To prevent the buckling of the boundary longitudinal reinforcement and maintain the integrity of wall segments, confined boundary elements were provided at the edges of the flange and the juncture of the web and flange by R6 round bars, based on the requirements of ACI 318-14 [A1] and the suggestions from Thomsen IV and Wallace [T1]. Grade 40 normal weight concrete was used to cast all specimens.

A total of four H-shaped specimens were constructed. The differences between each specimen were axial loads and lateral loading directions. The influence of axial loads on the seismic behavior of structural walls has been observed by numerous researchers $[\mathrm{F} 1, \mathrm{~N} 2, \mathrm{P} 2, \mathrm{~S} 4]$ in the literature. To simulate the loading condition of squat walls in nuclear plants and low-rise buildings, two levels of axial loads were imposed in the test: no external axial loads besides the weight of a top slab and axial loads slightly above $0.05 f_{c}^{\prime} A_{g}$. Two lateral loading directions $\left(0^{\circ}\right.$ and $45^{\circ}$ from the web segment) were adopted in consideration of real earthquakes, where seismic waves can reach structures from any direction. Moreover, this arrangement was 
intended to more comprehensively understand the behavior of these walls, since most tests performed on non-rectangular RC squat walls were conducted with the lateral loading direction parallel to wall segments. Each specimen was tagged with a name indicating the varying parameters. For example, Specimen HP0D45 meant the specimen was imposed with lateral horizontal displacements which were $45^{\circ}$ from the web and no external axial loads.

In order to facilitate the construction as well as to accommodate the test setup, similar configurations were also adopted for the L-shaped and T-shaped specimens, which are demonstrated in Figure 3.2 and Figure 3.3. Identical arrangements of reinforcement were also applied to these specimens as presented in Figure 3.4. However, due to the different section shapes, longitudinal reinforcement ratios were slightly different as demonstrated in Table 3.1. Steel bars and concrete of the same properties were continuously used to construct the remaining specimens. A total of eight specimens were built for the L-shaped and T-shaped groups. The design parameters and principles of naming were also consistent with the $\mathrm{H}$-shaped specimens mentioned above.

To effectively transfer lateral displacements, a heavily-reinforced top slab with a dimension of $2700 \times 2700 \times 400 \mathrm{~mm}$ and a self-weight of $73.5 \mathrm{kN}$ was attached to the specimens by prestressing rods. The concrete slab was reinforced with a doublelayer grid consisting of T28 high tensile strength steel bars at a spacing of $150 \mathrm{~mm}$ in each direction. Grade 40 normal weight concrete was used to cast the top slab. Photos of test specimens are demonstrated in Figure 3.5 to Figure 3.7. 
Table 3.1 Summary of specimens

\begin{tabular}{|c|c|c|c|c|c|c|c|c|c|c|c|}
\hline Specimen & $\begin{array}{l}\text { Section } \\
\text { shape }\end{array}$ & $\begin{array}{l}\text { Wall } \\
\text { height } \\
(\mathrm{mm})\end{array}$ & $\begin{array}{l}\text { Area of } \\
\text { cross } \\
\text { section } \\
\left(\mathrm{m}^{2}\right)\end{array}$ & $\begin{array}{l}\text { longitudinal } \\
\text { reinforceme } \\
\text { nt in the } \\
\text { web }\end{array}$ & $\begin{array}{l}\text { longitudinal } \\
\text { reinforceme } \\
\text { nt in the } \\
\text { flange }\end{array}$ & $\begin{array}{l}\text { Horizontal } \\
\text { reinforcem } \\
\text { ent of the } \\
\quad \text { wall }\end{array}$ & $\begin{array}{c}\text { longitudinal } \\
\text { reinforceme } \\
\text { nt ratio of } \\
\text { the web } \\
(\%)\end{array}$ & $\begin{array}{c}\text { longitudinal } \\
\text { reinforceme } \\
\text { nt ratio of } \\
\text { the flange } \\
(\%)\end{array}$ & $\begin{array}{l}\text { Horizontal } \\
\text { reinforcem } \\
\text { ent ratio } \\
(\%)\end{array}$ & $\begin{array}{l}\text { Axial } \\
\text { load } \\
\text { ratio } \\
(\%)\end{array}$ & $\begin{array}{c}\text { Lateral } \\
\text { loading } \\
\text { direction } \\
\left(^{\circ}\right)\end{array}$ \\
\hline HP0D0 & \multirow{4}{*}{ H-shaped } & 1000 & 0.43 & D10@150 & D10@160 & R6@80 & 0.97 & 1.26 & 0.71 & 0.49 & 0 \\
\hline HP0D45 & & 1000 & 0.43 & D10@150 & D10@160 & R6@80 & 0.97 & 1.26 & 0.71 & 0.49 & 45 \\
\hline HP5D0 & & 1000 & 0.43 & D10@150 & D10@160 & R6@80 & 0.97 & 1.26 & 0.71 & 5.14 & 0 \\
\hline HP5D45 & & 1000 & 0.43 & D10@150 & D10@160 & R6@80 & 0.97 & 1.26 & 0.71 & 5.14 & 45 \\
\hline LP0D0 & \multirow{4}{*}{ L-shaped } & 1000 & 0.29 & D10@150 & D10@150 & R6@80 & 1.12 & 1.26 & 0.71 & 0.72 & 0 \\
\hline LP0D45 & & 1000 & 0.29 & D10@150 & D10@150 & R6@80 & 1.12 & 1.26 & 0.71 & 0.72 & 45 \\
\hline LP5D0 & & 1000 & 0.29 & D10@150 & D10@150 & R6@80 & 1.12 & 1.26 & 0.71 & 5.65 & 0 \\
\hline LP5D45 & & 1000 & 0.29 & D10@150 & D10@150 & R6@80 & 1.12 & 1.26 & 0.71 & 5.65 & 45 \\
\hline TP0D0 & \multirow{4}{*}{ T-shaped } & 1000 & 0.29 & D10@150 & D10@160 & R6@80 & 1.12 & 1.26 & 0.71 & 0.72 & 0 \\
\hline TP0D45 & & 1000 & 0.29 & D10@150 & D10@160 & R6@80 & 1.12 & 1.26 & 0.71 & 0.72 & 45 \\
\hline TP5D0 & & 1000 & 0.29 & D10@150 & D10@160 & R6@80 & 1.12 & 1.26 & 0.71 & 5.65 & 0 \\
\hline TP5D45 & & 1000 & 0.29 & D10@150 & D10@160 & R6@80 & 1.12 & 1.26 & 0.71 & 5.65 & 45 \\
\hline
\end{tabular}




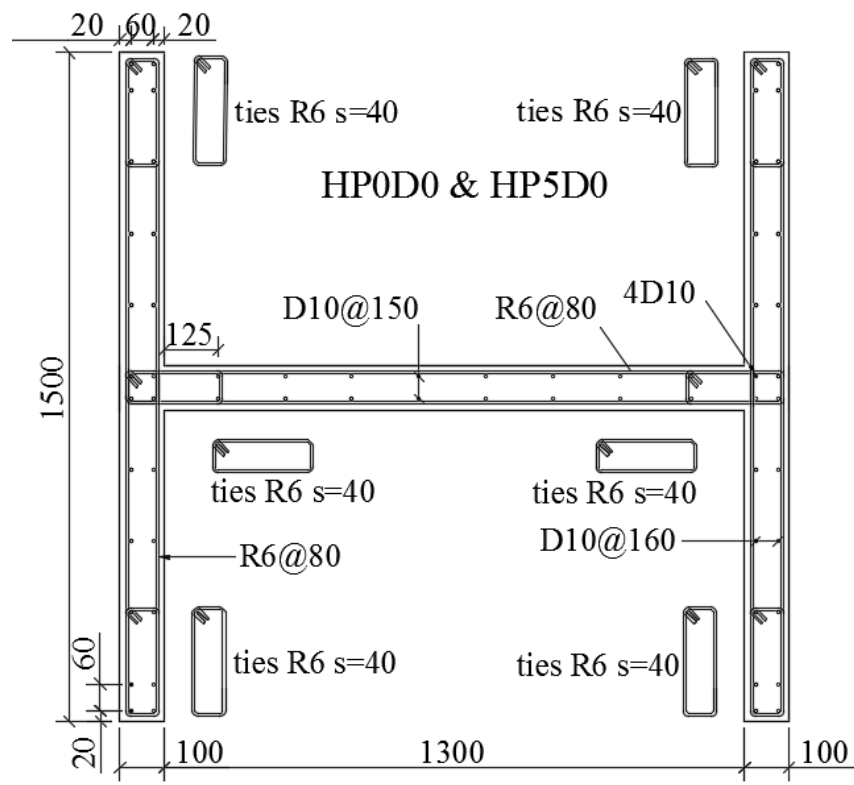

$->+$

Loading Direction

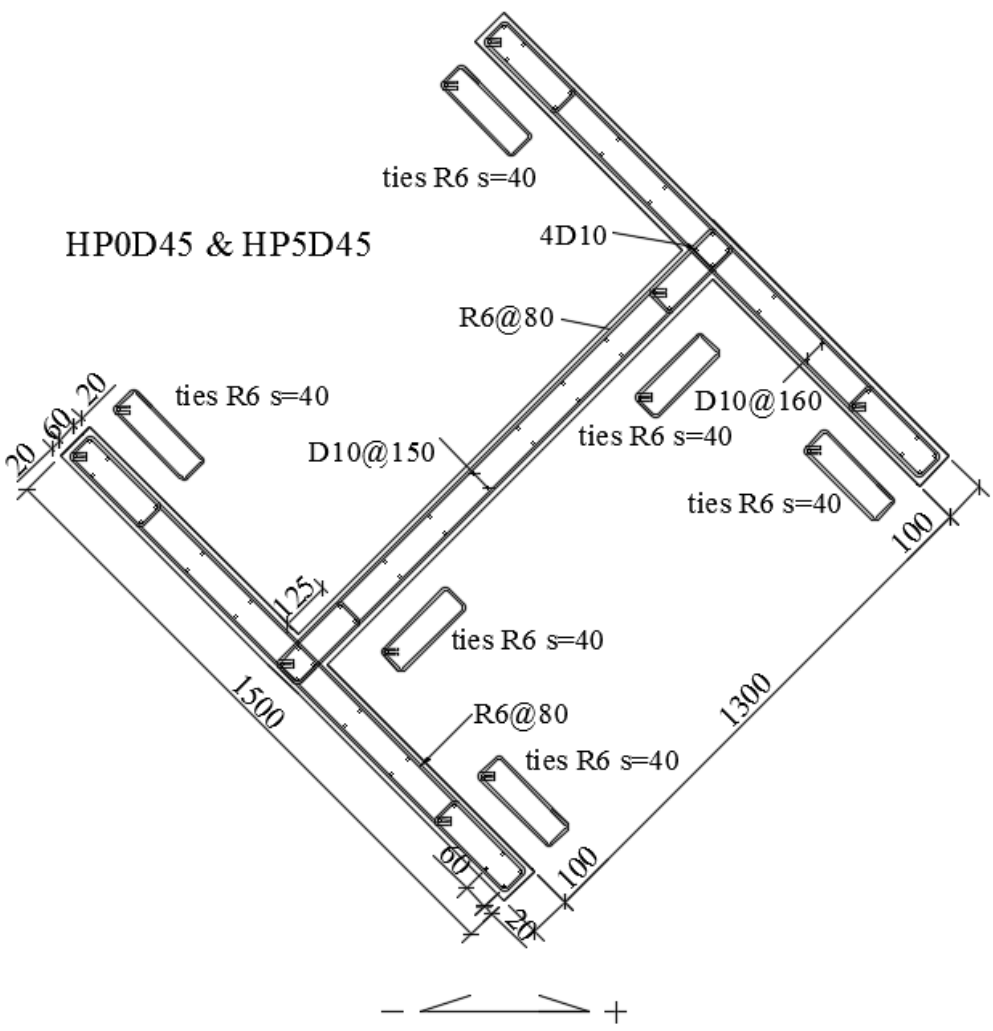

Loading Direction

Figure 3.1 Details of the H-shaped specimens in plan view 
Chapter 3

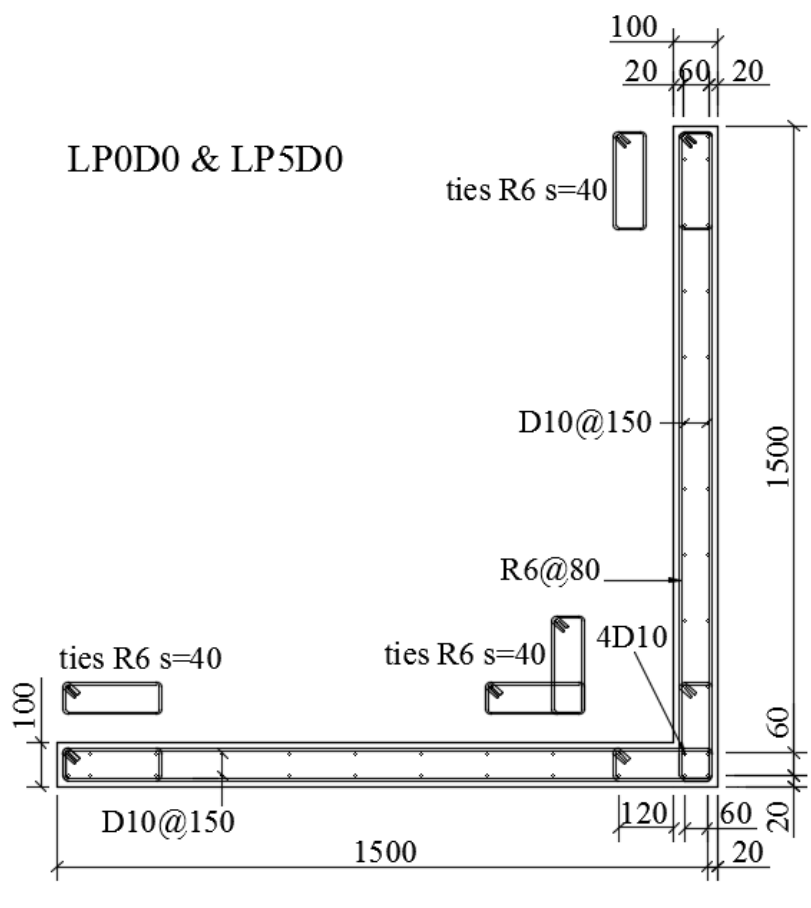

Loading Direction

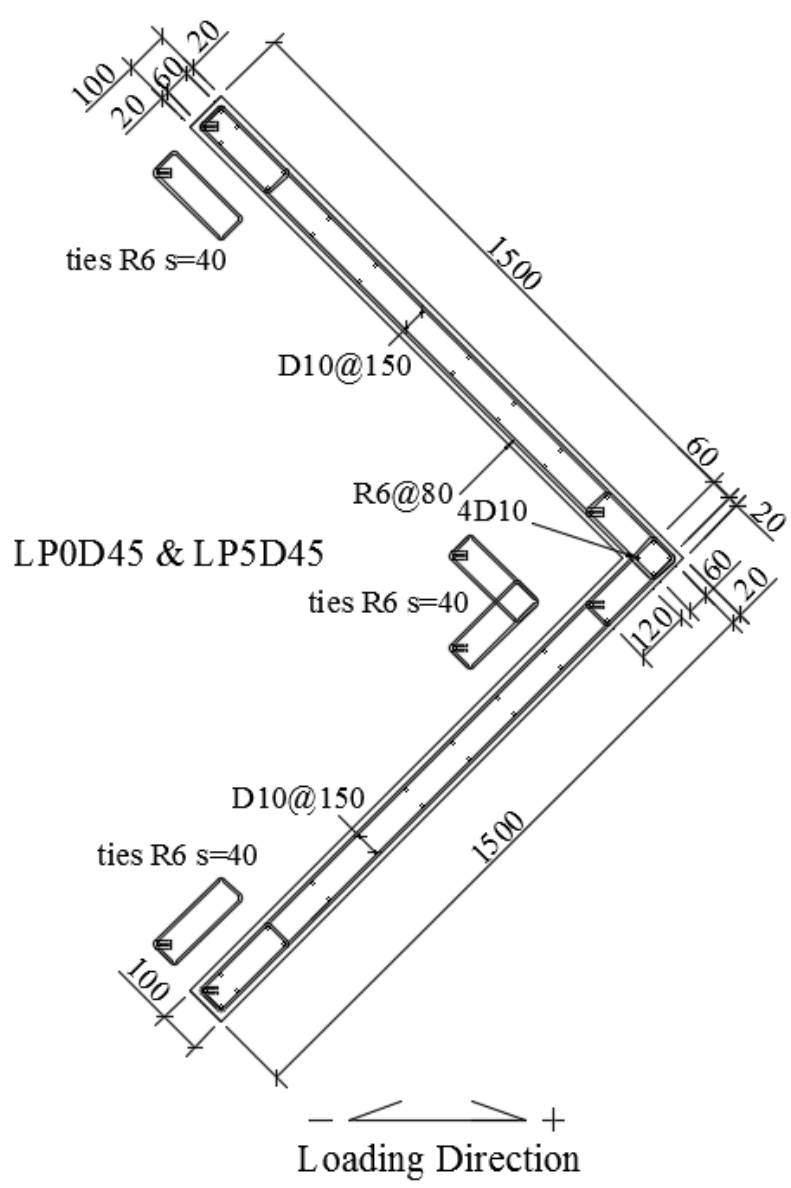

Figure 3.2 Details of the L-shaped specimens in plan view 
Chapter 3

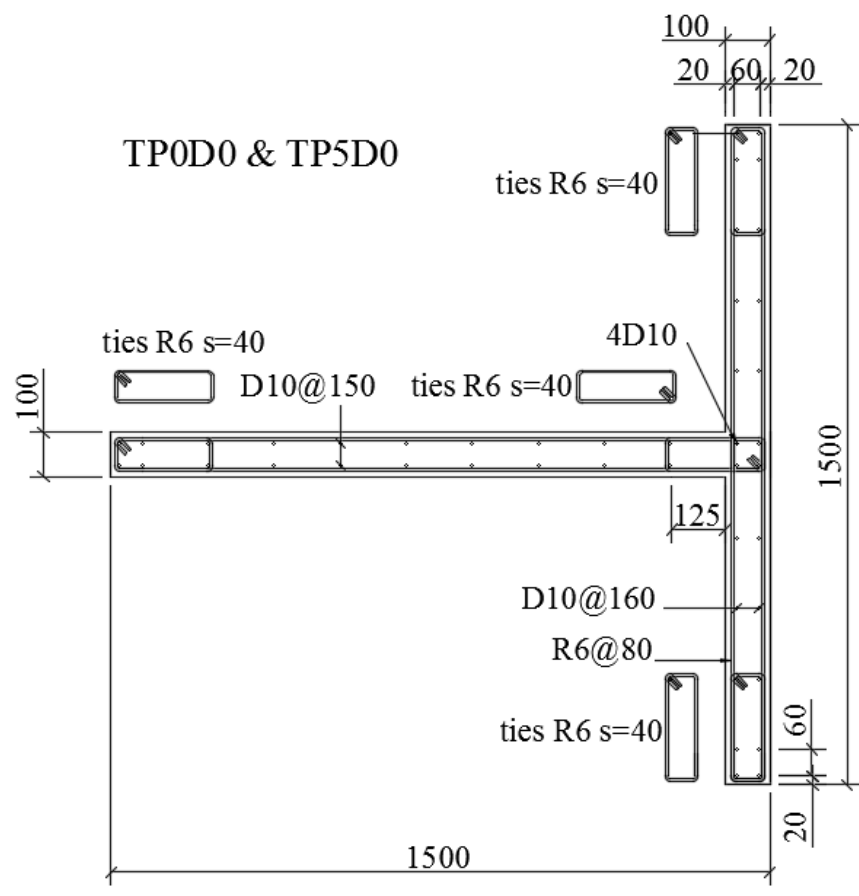

$\underset{\text { Loading Direction }}{+}$

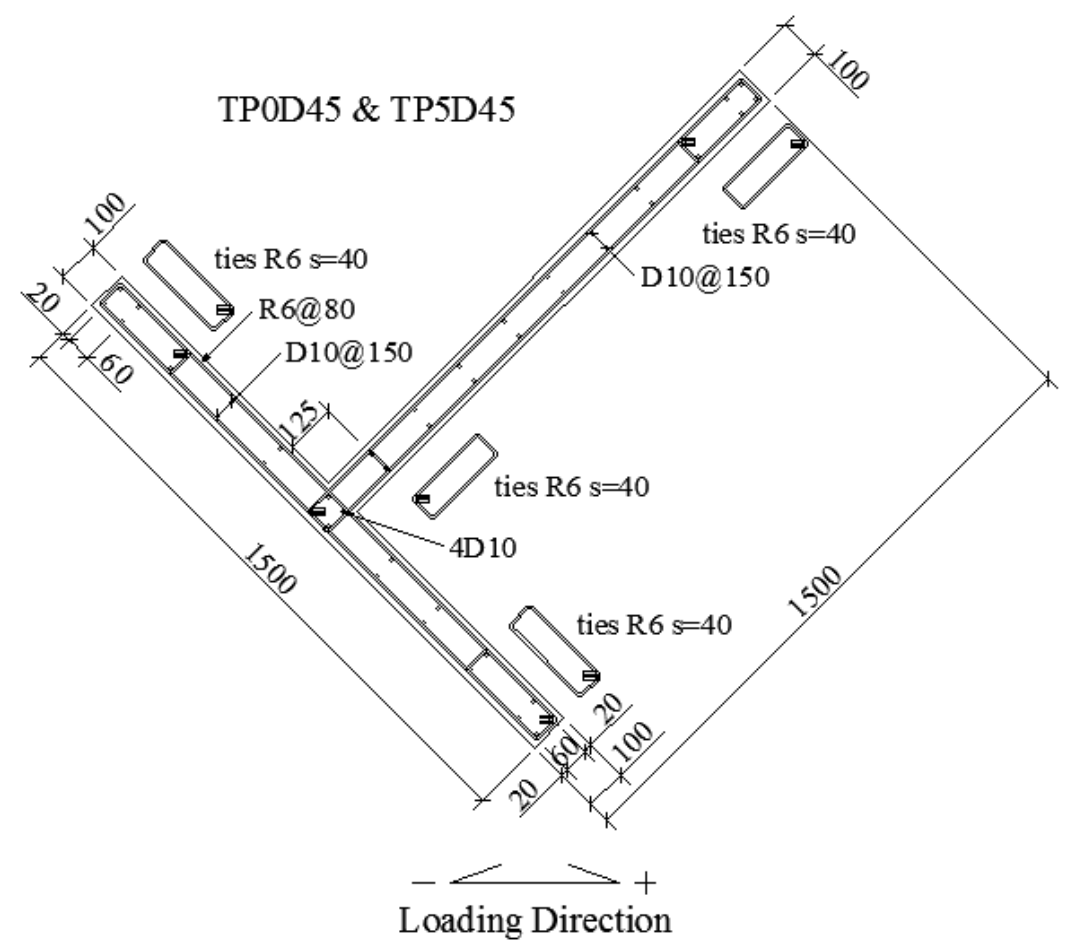

Figure 3.3 Details of the T-shaped specimens in plan view 
Chapter 3

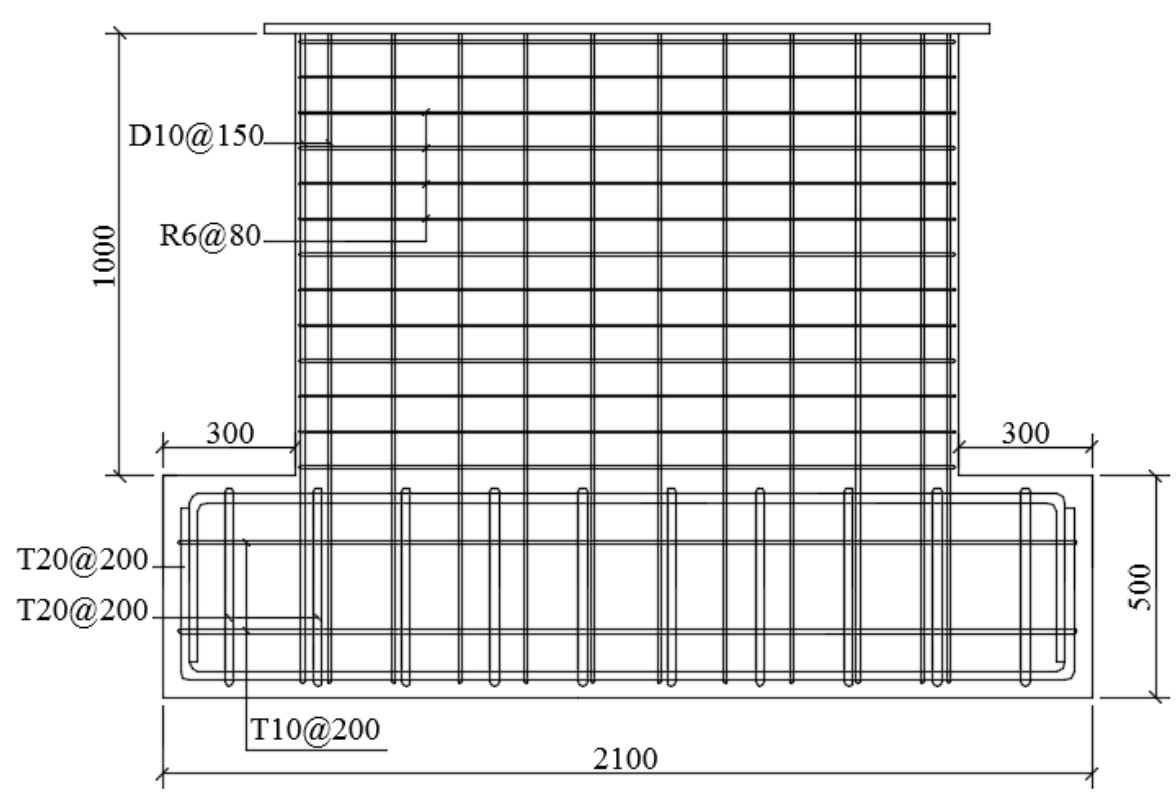

Figure 3.4 Details of specimens in front view

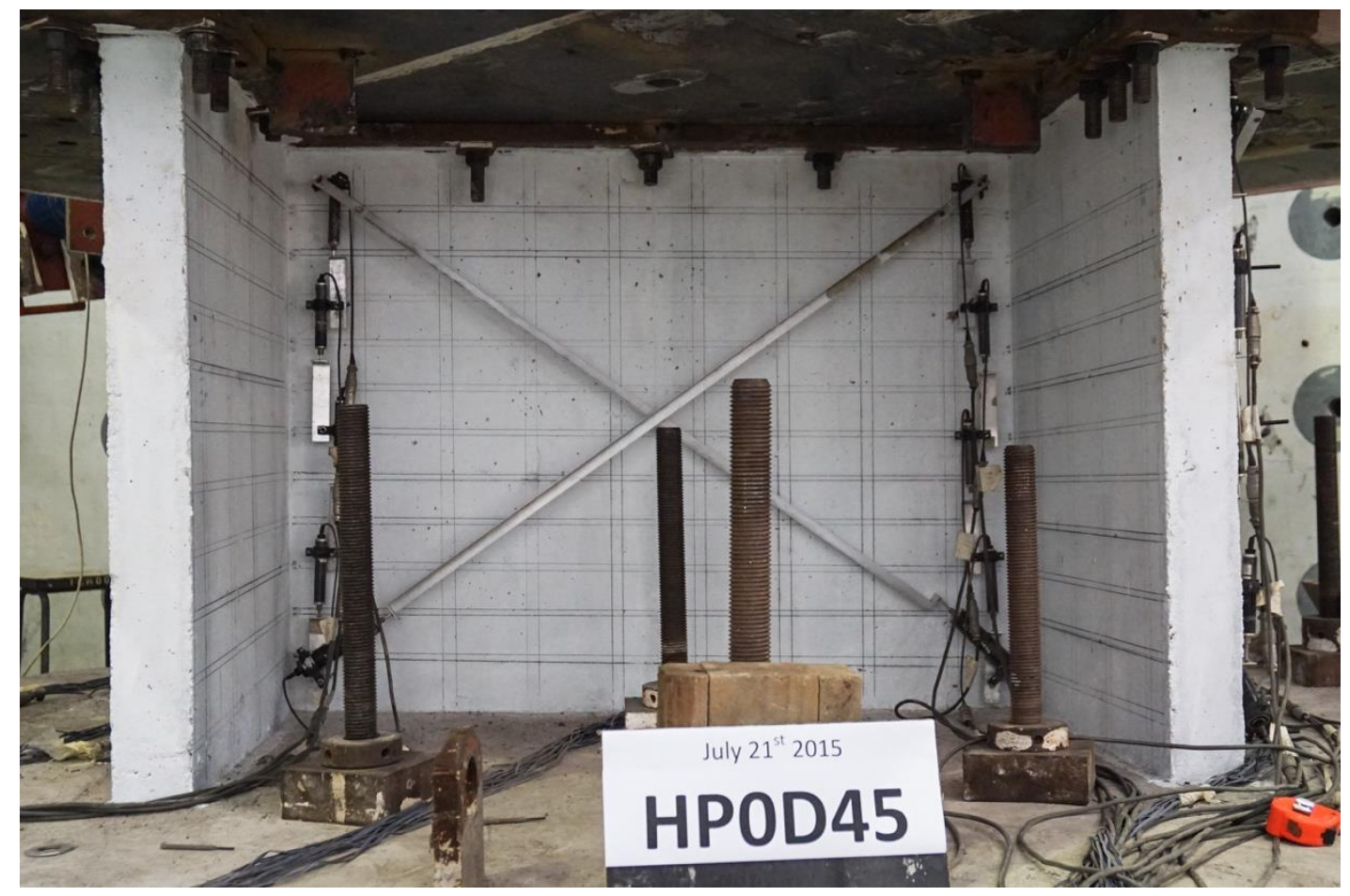

Figure 3.5 Photo of a H-shaped specimen 
Chapter 3

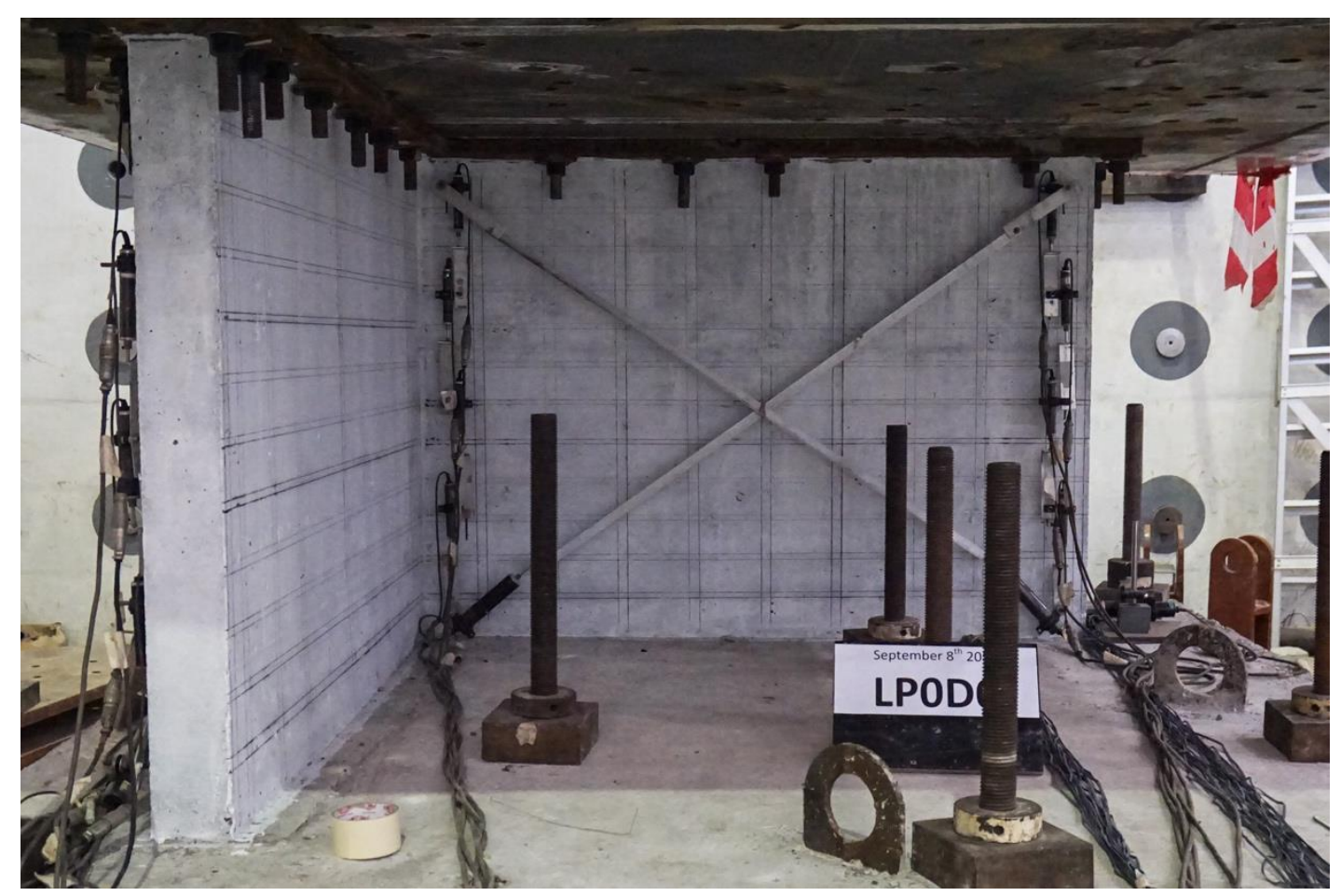

Figure 3.6 Photo of a L-shaped specimen

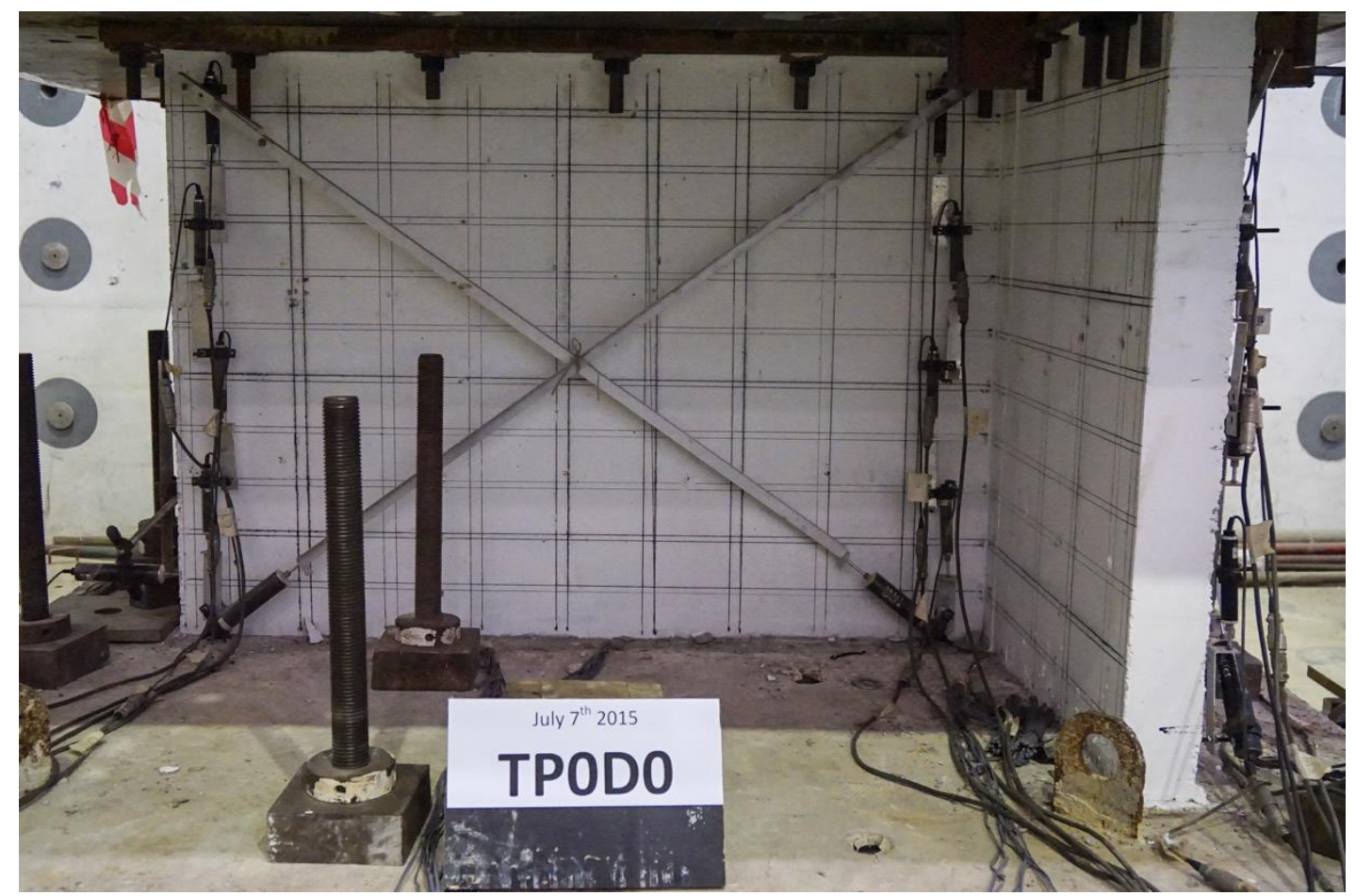

Figure 3.7 Photo of a T-shaped specimen 


\subsubsection{Material properties}

Six batches of Grade 40 ready-mix concrete with $13 \mathrm{~mm}$ maximum size aggregate were used to cast the twelve specimens. A total of thirty-six $150 \times 300 \mathrm{~mm}$ cylinders with six in each batch were cast and cured. The average cylinder compressive strength of different specimens was obtained by testing the cylinder samples 28 days after the cast, which are presented in Table 3.2.

Table 3.2 Concrete properties

\begin{tabular}{|c|c|c|}
\hline Specimens & $\begin{array}{c}\text { Age at test day } \\
\text { (days) }\end{array}$ & $\begin{array}{c}\text { Cylinder compressive } \\
\text { strength (MPa) }\end{array}$ \\
\hline HP0D0 and HP5D0 & 28 & 35.9 \\
\hline HP0D45 and HP5D45 & 29 & 33.8 \\
\hline LP0D0 and LP5D0 & 28 & 35.0 \\
\hline LP0D45 and LP5D45 & 30 & 34.9 \\
\hline TP0D0 and TP5D0 & 30 & 35.0 \\
\hline TP0D45 and TP5D45 & 28 & 36.2 \\
\hline
\end{tabular}

R6 round reinforcing bars with a diameter of $6 \mathrm{~mm}$ were used as the horizontal reinforcement and the transverse reinforcement in the confined boundary elements. D10 deformed reinforcing bars with a diameter of $10 \mathrm{~mm}$ were used as the longitudinal reinforcement. Tensile tests were performed on samples of these reinforcing bars in the lab. The average yield strength and other properties of these bars are revealed in Table 3.3.

Table 3.3 Reinforcement properties

\begin{tabular}{|c|c|c|c|c|c|}
\hline Type & $\begin{array}{c}\text { Nominal } \\
\text { diameter } \\
(\mathrm{mm})\end{array}$ & $\begin{array}{c}\text { Yield } \\
\text { strength } f_{y} \\
(\mathrm{MPa})\end{array}$ & $\begin{array}{c}\text { Yield strain } \\
\varepsilon_{y}\end{array}$ & $\begin{array}{c}\text { Elastic } \\
\text { modulus } E_{s} \\
(\mathrm{GPa})\end{array}$ & $\begin{array}{c}\text { Ultimate } \\
\text { strength } f_{u} \\
(\mathrm{MPa})\end{array}$ \\
\hline R6 & 6 & 335 & 0.0015952 & 220 & 507 \\
\hline D10 & 10 & 554 & 0.0025182 & 220 & 642 \\
\hline
\end{tabular}




\subsubsection{Design considerations}

As presented in Chapter 2, squat walls are mainly controlled by shear, which has a more complex mechanism than slender walls controlled by flexure. To explore the seismic performance of squat walls, it was necessary to design the specimens to be shear critical. In other words, the flexural strength of specimens should be larger than the shear strength. Conventional section analyses were performed for the flexural strength, in which the reinforcement hardening was considered. It could be seen that for L-shaped and T-shaped specimens, the flexural strength were different in positive and negative loading directions, due to their unsymmetrical section shapes. The shear strength was calculated based on Section 11.5 of ACI 318-14 [A1] and Section 18.10 of ACI 318-14 [A1], the results of which are presented in Table 3.4. It was found that the shear capacity of all specimens met the anticipations and the requirements of the building codes.

Table 3.4 Flexural and shear capacities of specimens $(\mathrm{kN})$

\begin{tabular}{ccccc}
\hline Specimens & \multicolumn{2}{c}{ Flexural strength } & ACI 318-14 & ACI 318-14 \\
& Positive & Negative & $\S 11.5$ [A1] & $\S 18.10$ [A1] \\
\hline HP0D0 & 1668 & 1668 & 497 & 581 \\
\hline HP0D45 & 1668 & 1668 & 491 & 574 \\
\hline HP5D0 & 1668 & 1668 & 597 & 581 \\
\hline HP5D45 & 1668 & 1668 & 580 & 574 \\
\hline LP0D0 & 662 & 1294 & 495 & 578 \\
\hline LP0D45 & 662 & 1294 & 495 & 577 \\
\hline LP5D0 & 662 & 1294 & 590 & 578 \\
\hline LP5D45 & 662 & 1294 & 589 & 577 \\
\hline TP0D0 & 662 & 1294 & 495 & 578 \\
\hline TP0D45 & 662 & 1294 & 498 & 582 \\
\hline TP5D0 & 662 & 1294 & 590 & 578 \\
\hline TP5D45 & 662 & 1294 & 598 & 582 \\
\hline
\end{tabular}




\subsection{Test Setup and Loading Sequence}

All test specimens were subjected to quasi-static horizontal loads to simulate earthquakes. Among them, six specimens were imposed with a constant axial load around $0.05 f_{c}^{\prime} A_{g}$, which were $700 \mathrm{kN}$ for the $\mathrm{H}$-shaped walls and $500 \mathrm{kN}$ for the Lshaped and T-shaped walls. The level of the axial load was designed to represent the load condition of squat walls in nuclear facilities and low-rise buildings. Axial loads were applied by a hydraulic jack of $1000 \mathrm{kN}$ loading capacity, with its end connecting to the steel bracket, which was further connected to a triangular steel frame mounted on the reaction wall of the lab. The load transfer system consisted of three steel beams, a steel spreader plate, several steel rollers, four triangular steel plates and twelve square steel plates, which can be seen in Figure 3.8. This setup was conservatively designed to ensure uniform axial load distribution on the wall cross section area, and more importantly, to accommodate horizontal movements of wall specimens due to lateral displacements. External axial loads were removed in the other six specimens to evaluate the effect of axial loads on the seismic behavior of squat walls.

The reversible lateral loads were applied to the top slab by a hydraulic jack with $2000 \mathrm{kN}$ capacity and $1000 \mathrm{~mm}$ stroke, which was connected to a load cell by high strength steel plates. The lateral loads further transferred to the top slab by a series of steel brackets. It should be noted that steel rods of high yield strength were utilized for the connection to ensure lateral loads were properly transferred. The loading history with cycles versus displacements is illustrated in Figure 3.10. The lateral displacement increased by $1 \mathrm{~mm}$ every two cycles and terminated when the lateral strength dropped to less than $80 \%$ of the recorded maximum strength.

The bottom of the base was fixed to the laboratory strong floor by prestressing rods, which aimed to prevent horizontal movements and overturning moments between the specimen and the strong floor. Lateral braces consisting of several steel plates, rods, and load cells were mounted on the reaction wall to prevent out-of-plane deformations. All specimens shared the same loading apparatus. 
Chapter 3

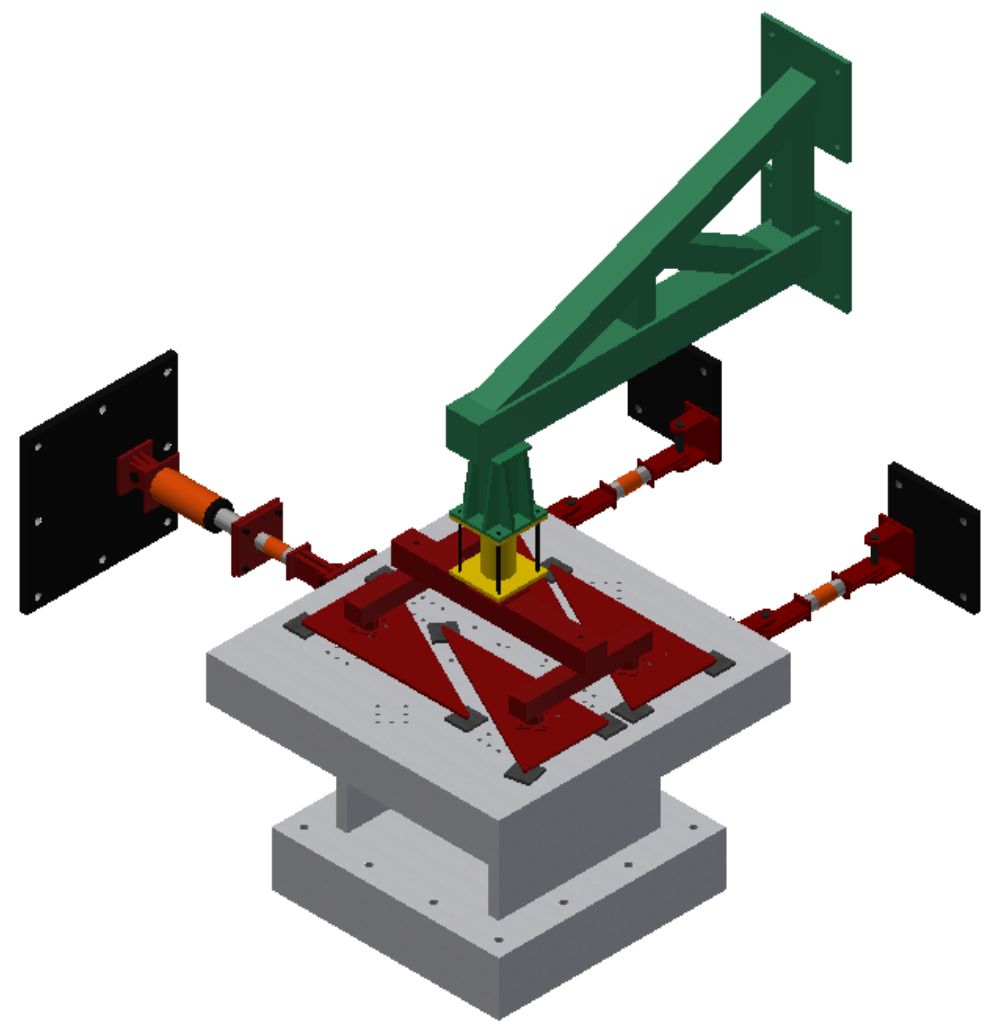

Figure 3.8 Test setup in isometric view

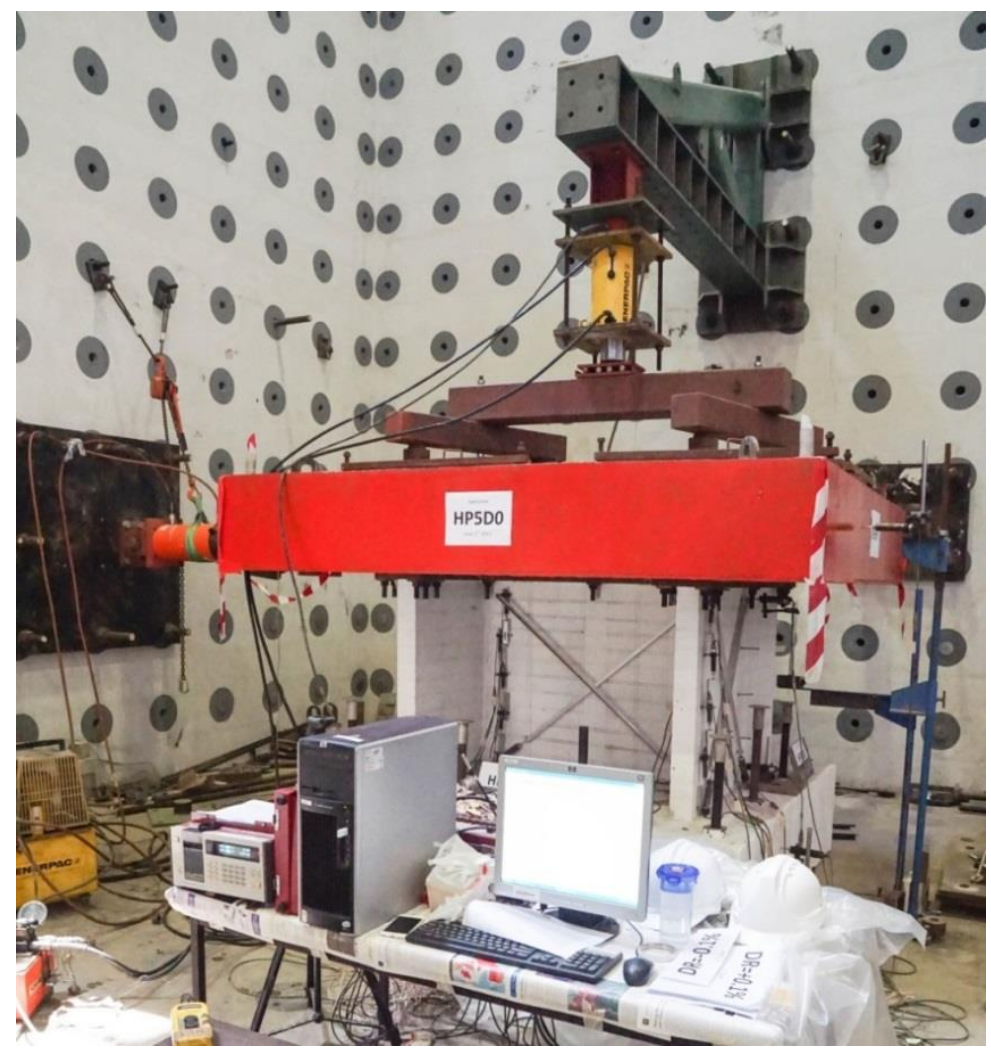

Figure 3.9 Photo of the test setup 


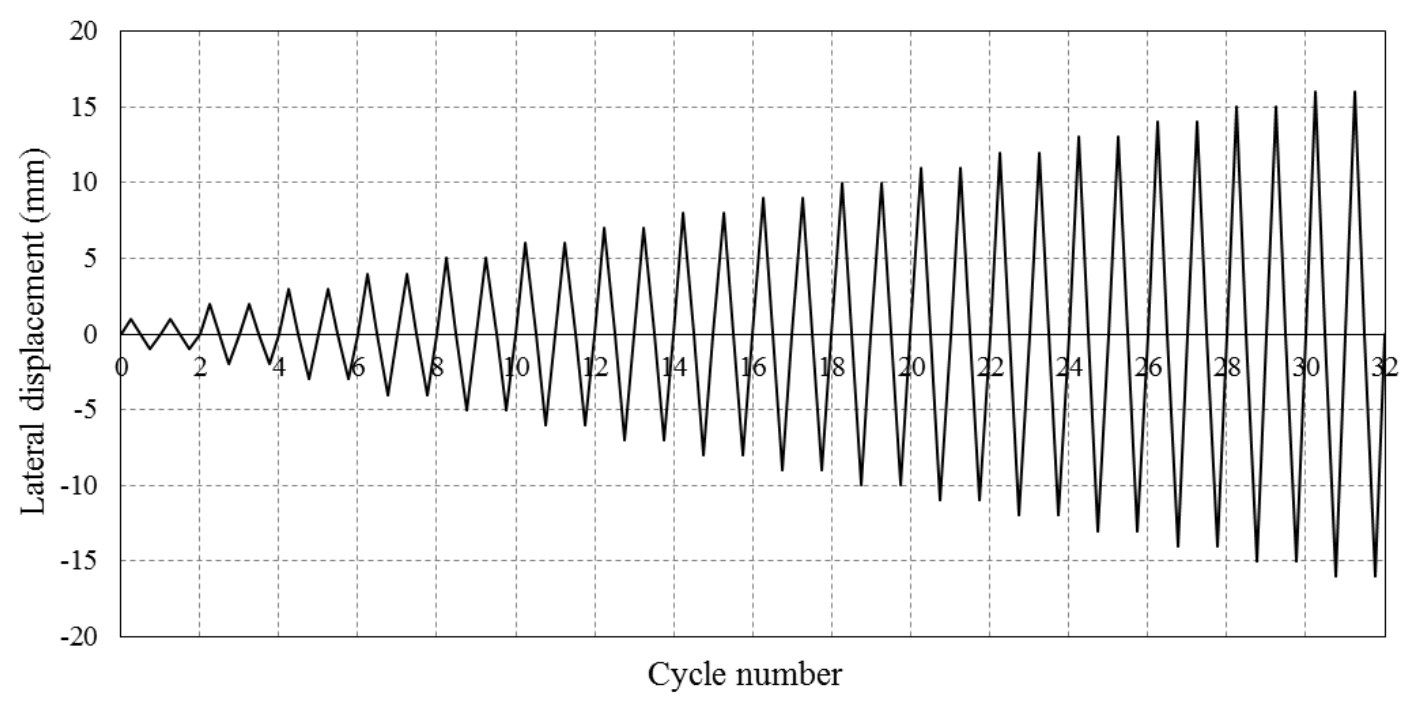

Figure 3.10 Loading history

\subsection{Instrumentation}

Several parameters closely related to the seismic performance of structural walls required accurate measurements. These were: forces applied by hydraulic jacks, lateral displacements at the top of walls, flexural deformations of wall segments, shear deformations of wall segments, sliding shear deformations of wall segments, and strains of reinforcement. In this experimental program, lateral forces were measured by load cells as demonstrated in Figure 3.8. Deformations were measured by linear variable differential transducers (LVDTs) mounted on the wall surfaces. Strains of steel bars were measured using strain gauges. Besides, cracking patterns on each surface at every drift ratio were captured by cameras fixed on tripods.

\subsubsection{Measurement of loads}

To measure the in-plane lateral reaction, a double-acting (tension and compression) load cell with a capacity of $2000 \mathrm{kN}$ was connected to the lateral hydraulic jack using high strength steel plates and rods as illustrated in Figure 3.8. A load cell with a capacity of $1000 \mathrm{kN}$ was connected to the vertical hydraulic jack to measure the applied axial loads. In addition, two load cells with a capacity of $1000 \mathrm{kN}$ were connected to the lateral braces to monitor out-of-plane reactions and possible torsion. 


\subsubsection{Measurement of strains in reinforcing bars}

KFG type $3 \mathrm{~mm}$-gauge length strain gauges with $5 \mathrm{~m}$ lead wires were utilized to measure local strains of reinforcing bars. Strain gauges were arranged in consideration of several critical zones such as the bottom of walls and the diagonal concrete strut. Figure 3.11 to Figure 3.16 below show the locations of strain gauges in all specimens in detail. As can be seen from the figure, two layers of strain gauges were attached to the longitudinal reinforcement, which were $60 \mathrm{~mm}$ and $460 \mathrm{~mm}$ above the wall base. The bottom of all longitudinal reinforcing bars in the web was mounted with strain gauges, and a staggered distribution was adopted for the middle layer. As for the horizontal reinforcement, two reinforcing bars which were $20 \mathrm{~mm}$ and $500 \mathrm{~mm}$ above the wall base were selected with strain gauges attached at three locations.

The strain gauges were tagged with two letters together with two numbers for identification. The first letter indicated the section in which strain gauges were located: $\mathrm{N}$ and $\mathrm{S}$ representing the north and south steel grids in the web, and A, B, C, $\mathrm{D}$ representing the steel grids in flanges. The second letter, $\mathrm{V}$ and $\mathrm{H}$, revealed the types of reinforcement: vertical and horizontal. The remaining two numbers suggested the exact location of strain gauges. Among them, the first number indicated the bar number, as seen in the figures. The second number referred to the position number. As demonstrated in Figure 3.11 to Figure 3.16, there were two positions for the longitudinal reinforcement, which were the bottom and the middle of the rebar ( $60 \mathrm{~mm}$ and $460 \mathrm{~mm}$ above the wall base), indicated by number 1 and 2 respectively. There were three positions for the horizontal reinforcement, which were the left, the middle and the right of the rebar, indicated by number 1, 2 and 3 respectively. For instance, tag NV61 meant the strain gauge was mounted on the bottom of No. 6 longitudinal rebar which was located at the north grid of the web. The data of strain gauges was collected by an automatic data logger in the test. 

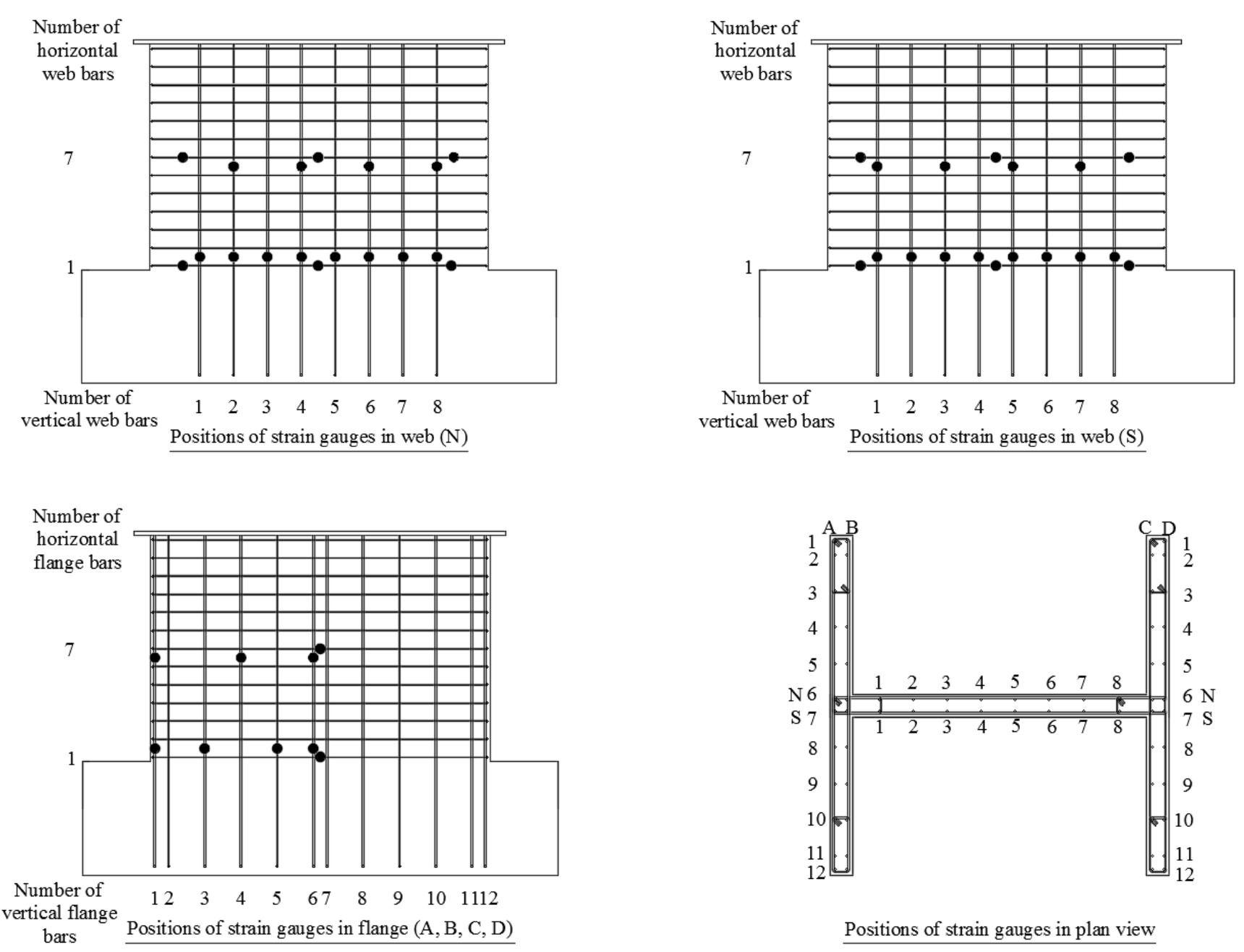

Positions of strain gauges in plan view

Figure 3.11 Positions of strain gauges in Specimens HP0D0 and HP5D0 

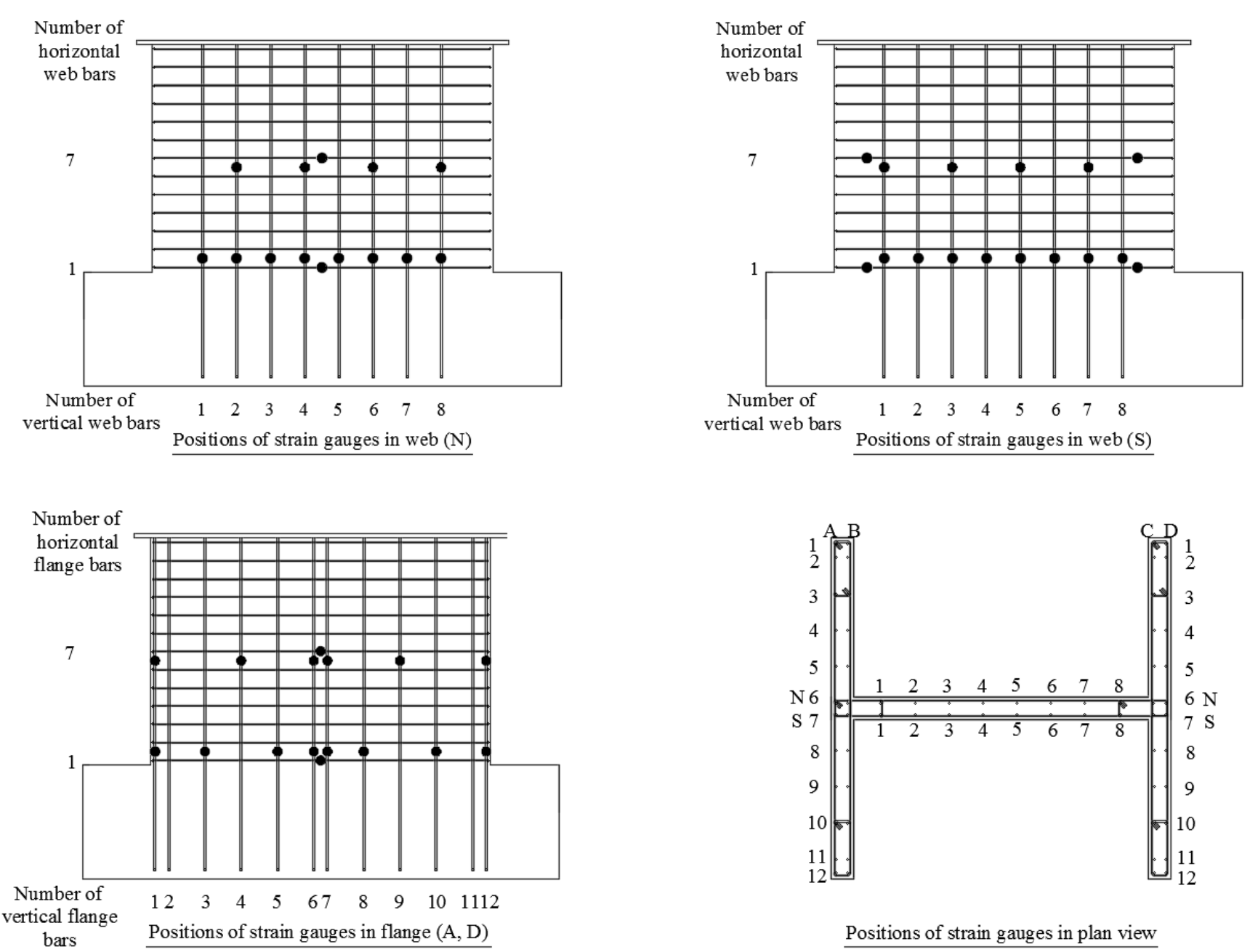

Positions of strain gauges in plan view

Figure 3.12 Positions of strain gauges in Specimens HP0D45 and HP5D45 

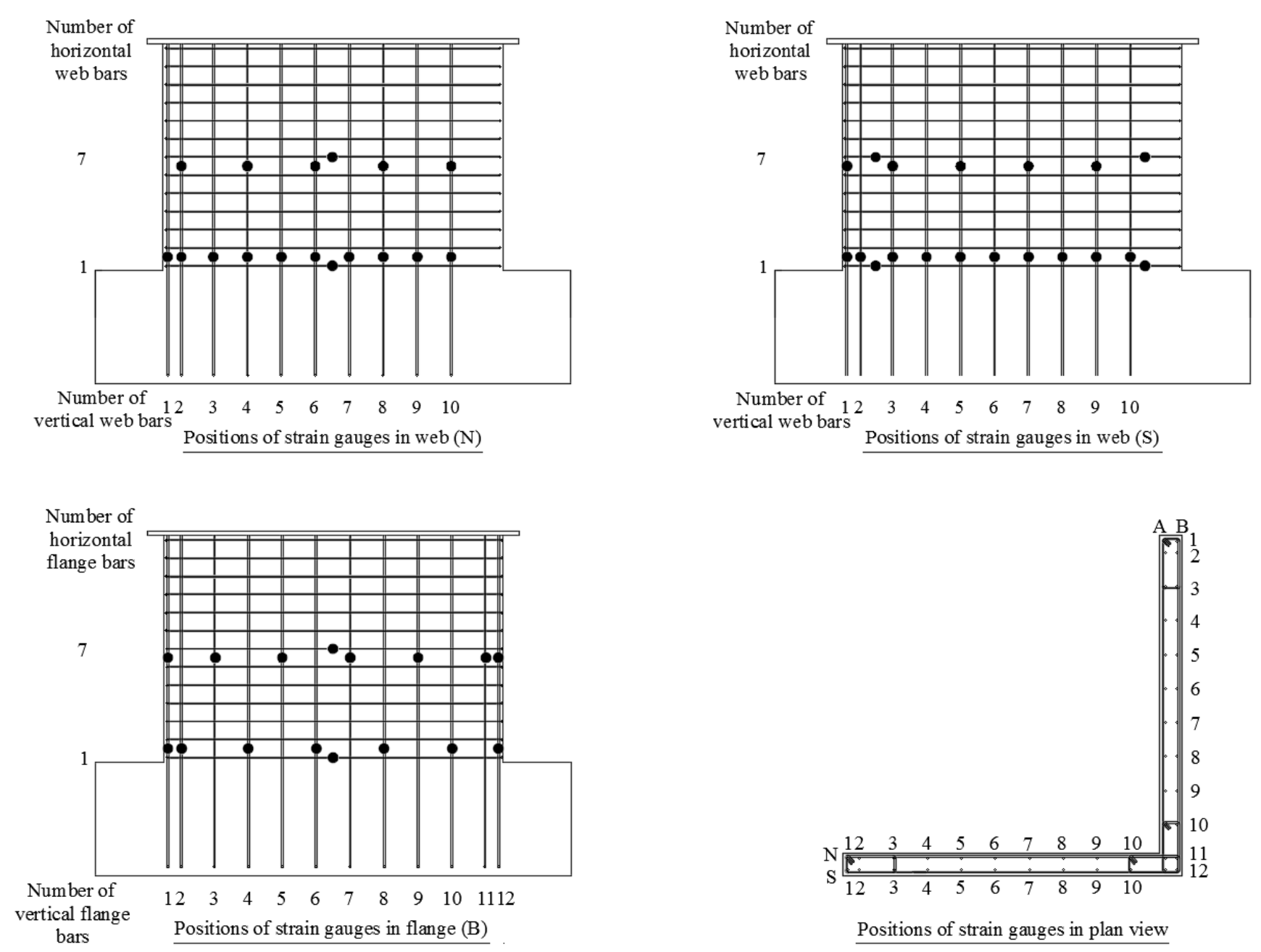

Figure 3.13 Positions of strain gauges in Specimens LP0D0 and LP5D0 

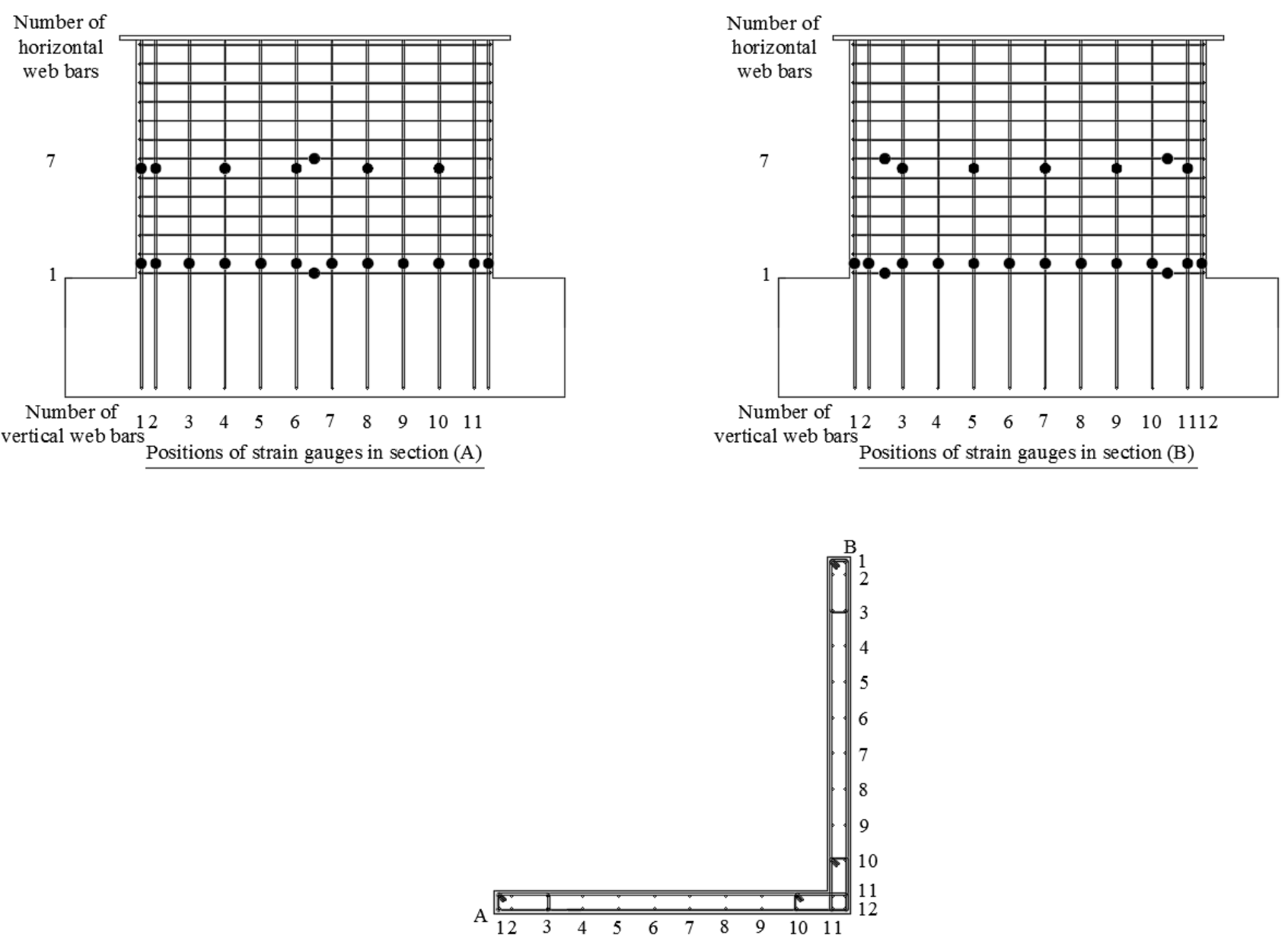

Positions of strain gauges in plan view

Figure 3.14 Positions of strain gauges in Specimens LP0D45 and LP5D45 

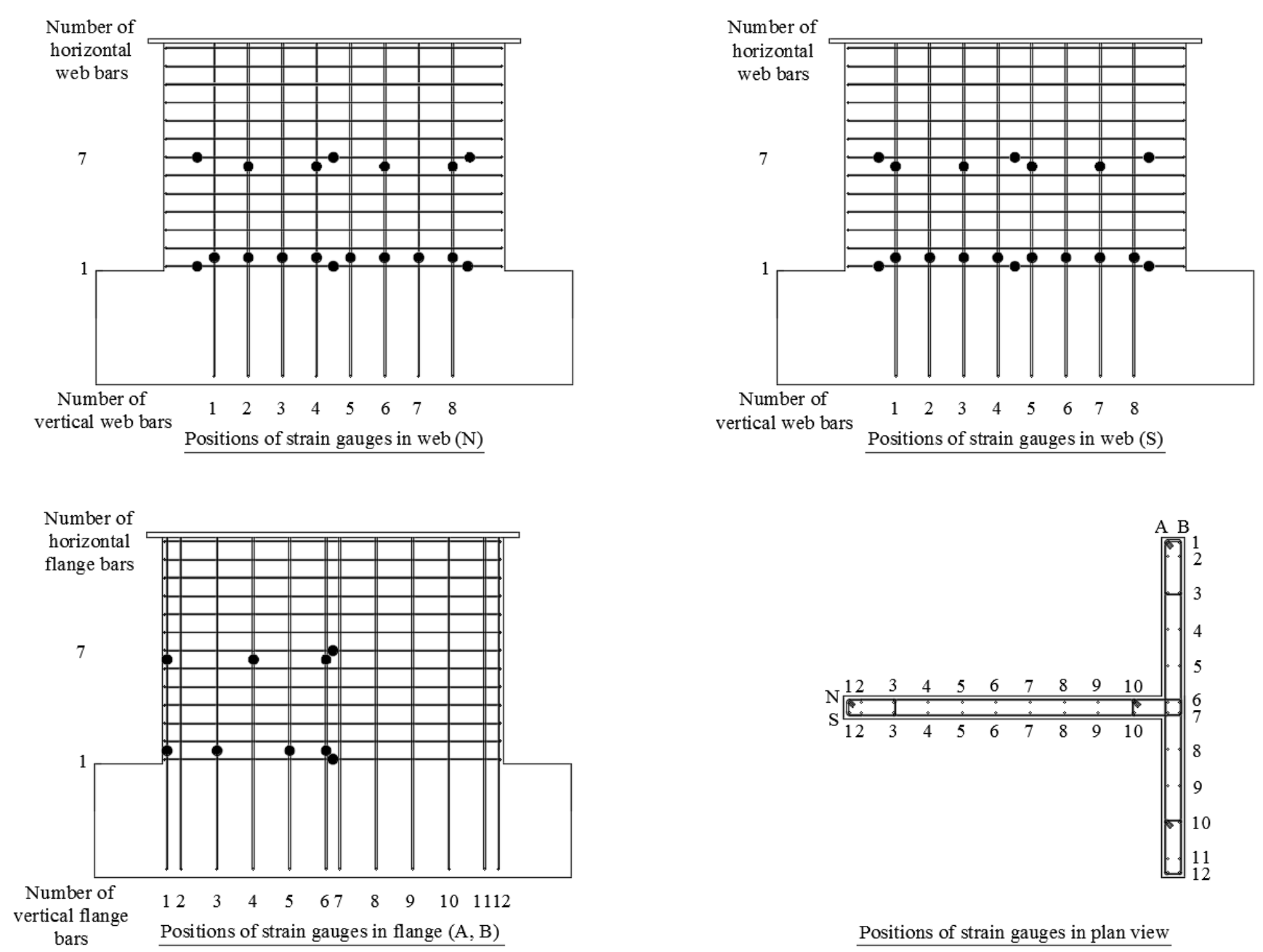

Positions of strain gauges in plan view

Figure 3.15 Positions of strain gauges in Specimens TP0D0 and TP5D0 

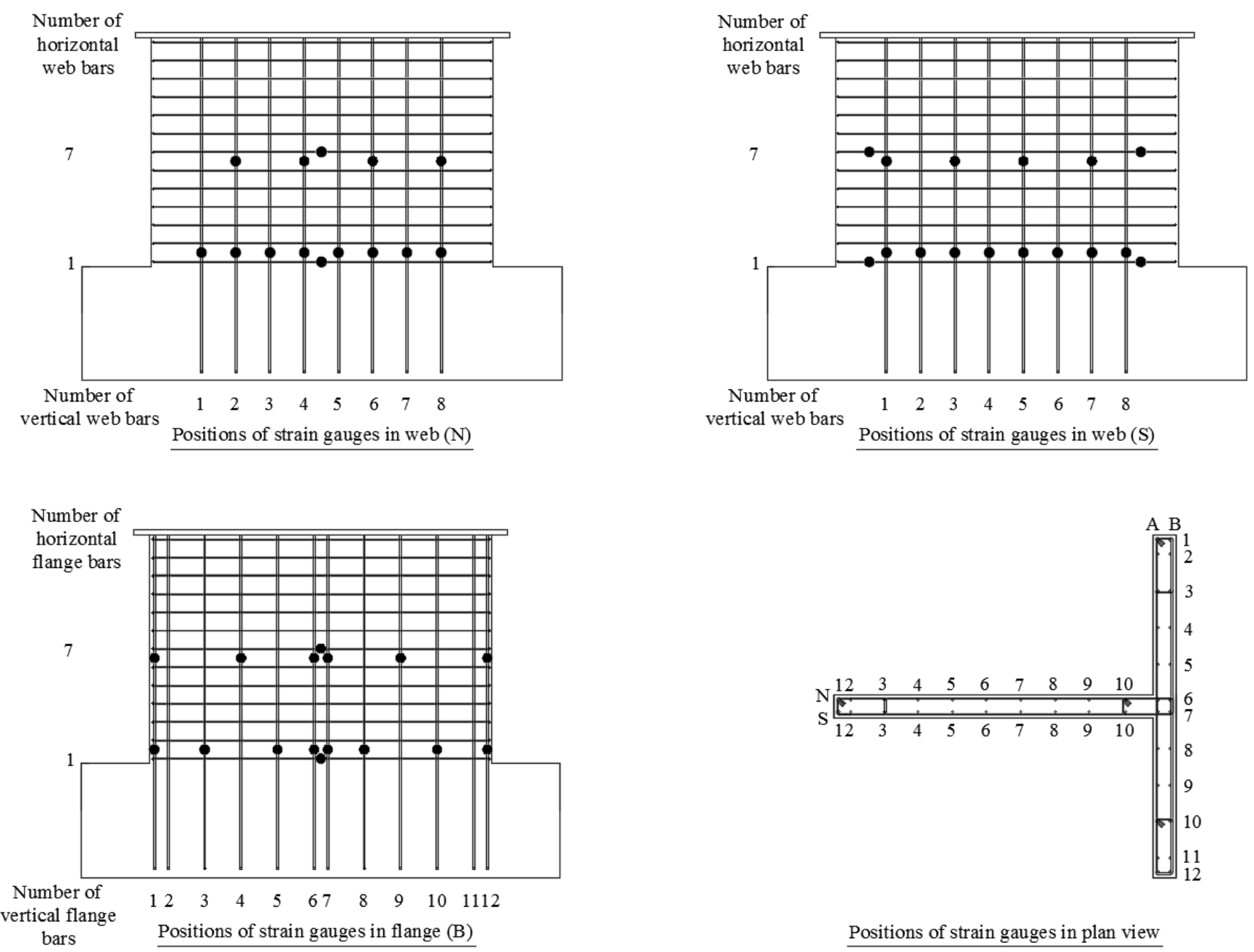

Positions of strain gauges in plan view

Figure 3.16 Positions of strain gauges in Specimens TP0D45 and TP5D45 


\subsubsection{Measurement of lateral displacements and deformations}

In order to measure lateral displacements at the wall top, flexural deformations and shear deformations of wall units, three types of Linear Variable Differential Transducers (LVDTs), with $50 \mathrm{~mm}$ travel, $25 \mathrm{~mm}$ travel, and $10 \mathrm{~mm}$ travel, were introduced as illustrated in Figure 3.17.

As the figure shows, four pairs of LVDTs with $10 \mathrm{~mm}$ travel were mounted along the two vertical edges of wall segments to detect the flexural deformation. The panel shear deformations were measured by two LVDTs with $25 \mathrm{~mm}$ travel distributed along the diagonal directions of the panel. The sliding of wall segment was captured by two LVDTs, with $25 \mathrm{~mm}$ travel horizontally installed slightly above the wall base. The sliding of wall base was recorded by one LVDT with 50 $\mathrm{mm}$ travel magnetically connected to the steel plate on the strong floor. The rotation of wall base was recorded by two LVDT with $50 \mathrm{~mm}$ travel mounted at the base. The flange shared a similar arrangement of LVDTs as the web. In this study, flexural deformations were computed based on average curvatures captured by the aforementioned LVDTs. Shear deformations were calculated according to the method proposed by Hiraishi [H2].

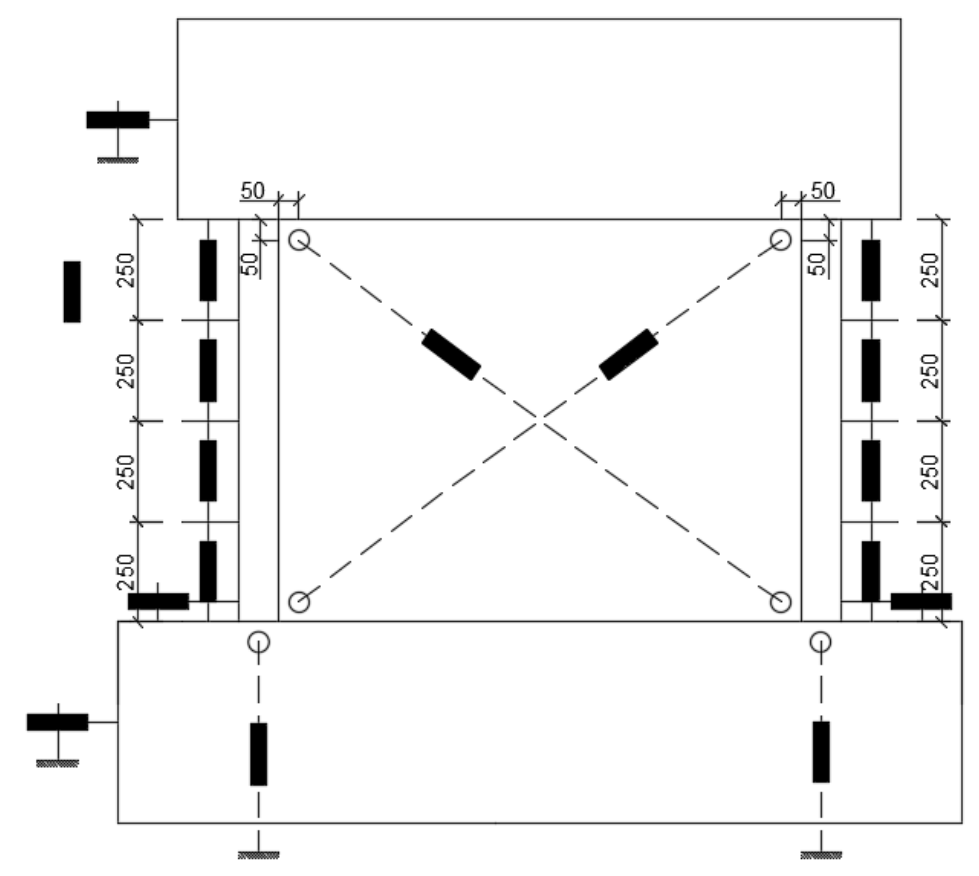

Figure 3.17 Arrangements of LVDTs in specimens 


\subsection{Summary}

An experimental program including twelve RC squat wall specimens with nonrectangular section shapes was presented in this chapter in detail. The specimens were described in terms of dimensions, reinforcement contents, and material properties. After that, the test setup was illustrated together with the loading history. Finally, the instruments used for the measurement of the seismic responses of test specimens were demonstrated. 


\section{CHAPTER 4 PRESENTATION OF THE EXPERIMENTAL RESULTS}

\subsection{Introduction}

Twelve non-rectangular RC squat walls with details described in Chapter 3 were tested at the Protective Engineering Laboratory in NTU. Cyclic lateral displacements and constant axial loads were imposed on these specimens to simulate the seismic loading. The experimental results were presented and discussed in terms of cracking patterns, failure mechanisms, hysteretic responses, displacement components and strain profiles. Furthermore, comparisons were conducted among each group and the influences of axial loads and loading directions on the seismic performance of these specimens were also reported.

\subsection{Experimental Results of the L-shaped Specimens}

\subsubsection{Cracking patterns}

The cracking pattern is not only a revelation of failure modes but also important in studying the force transfer mechanism and seismic performance of RC structures. Figure 4.1 to Figure 4.4 demonstrate the development of cracks on each wall surface with outlines of reinforcement. The blue lines represent the cracks formed during the positive loading cycles. The red lines represent the cracks formed during the negative loading cycles.

Figure 4.1 illustrates the development of cracks on each surface of Specimen LPOD0 at drift ratios of $0.3 \%, 0.9 \%$ and $1.6 \%$ (displacements of $3 \mathrm{~mm}, 9 \mathrm{~mm}$ and $16 \mathrm{~mm}$ ), which corresponded to the initial stage, the crack development stage, and the final failure stage respectively. As seen from the figure, a diagonal crack first appeared on the web $700 \mathrm{~mm}$ above the ground when the drift ratio rose to $0.3 \%$ and the flange was in compression. As the test progressed, a long diagonal crack occurred at the center of the flange at the first cycle of the displacement of $6 \mathrm{~mm}$. The outermost longitudinal reinforcement in the junction of the web and flange 
experienced yielding at the drift ratio of $0.7 \%$ when the flange was in tension. As lateral loads continued to increase, cracks spread to other areas. Dense diagonal cracks were found at the displacement of $9 \mathrm{~mm}$, and most of them were inclined at $45^{\circ}$. Similar cracks also appeared on the flange and most of them were created when the flange was in tension. As the displacement increased, more diagonal cracks formed on each surface. In the positive direction, a maximum load of $592 \mathrm{kN}$ was recorded at a displacement of $14 \mathrm{~mm}$, and a load of $517 \mathrm{kN}$ at a displacement of $15 \mathrm{~mm}$ was attained in the negative direction. The corner of the web segment severely crushed at the drift ratio of $1.6 \%$, leading to a sudden drop of lateral strength and the subsequent failure of the specimen.
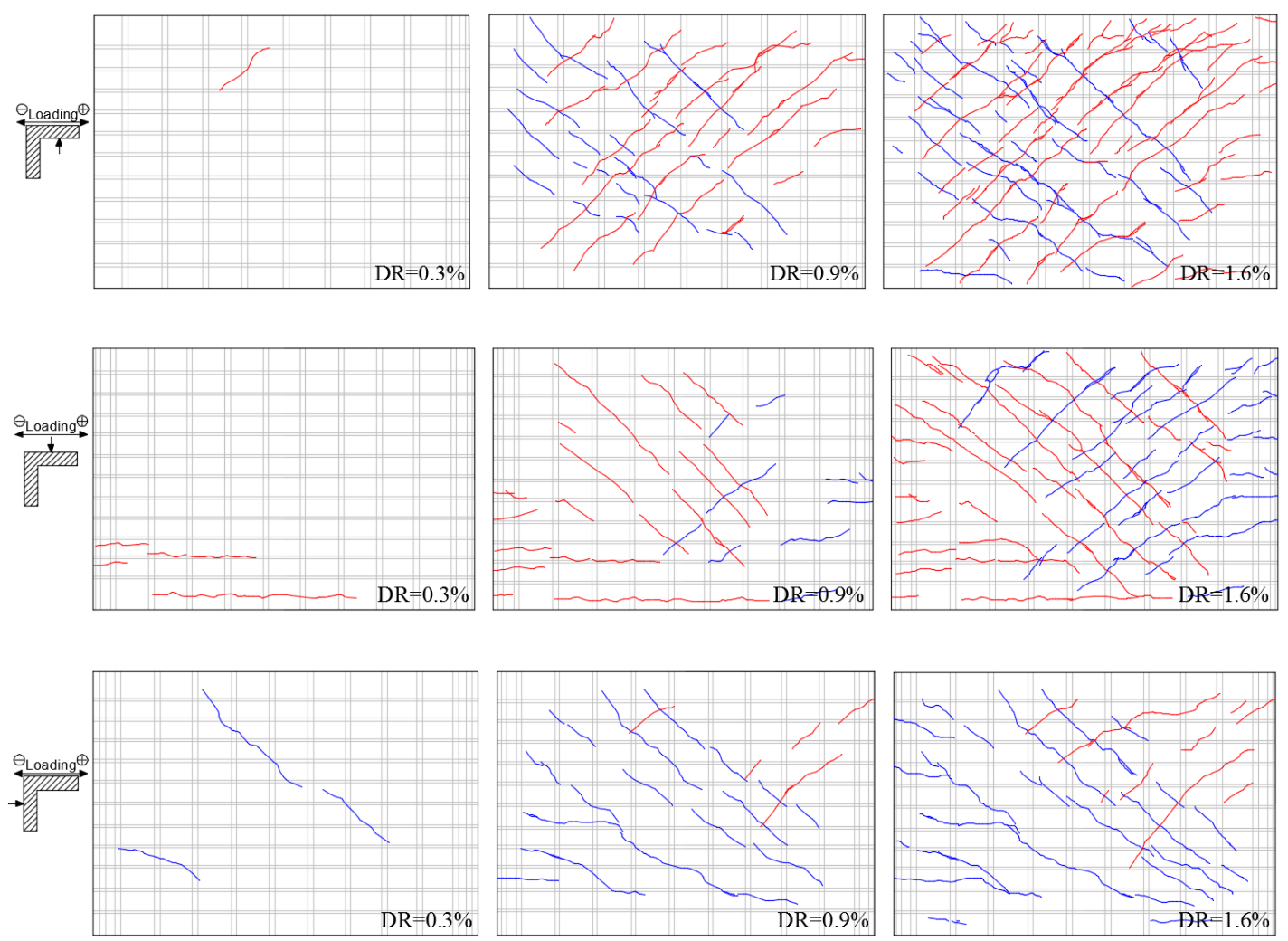

Figure 4.1 Cracking patterns of Specimen LP0D0

In Specimen LP0D45, cracks first appeared at the displacement of $2 \mathrm{~mm}$, when a few diagonal cracks were found at the upper right part of the web and the right part of the flange. With increases in lateral displacements, more diagonal cracks occurred on all surfaces with their orientations parallel to the diagonal of the wall. Besides, the specimen also experienced a few horizontal cracks at the corner of the web. Increasing the lateral displacement to $6 \mathrm{~mm}$ in the positive direction resulted 
in the first yielding of the longitudinal reinforcement in the juncture of the web and flange. As the test progressed, cracks with a smaller angle of inclination gradually appeared near the corner of the juncture of two segments. The maximum capacities, $573 \mathrm{kN}$ in the positive loading direction and $656 \mathrm{kN}$ in the negative loading direction, were reached at displacements of $12 \mathrm{~mm}$ and $11 \mathrm{~mm}$, respectively. With further increases of horizontal displacements, gradual and slight crushing of concrete was observed near the base, which led to the development of a wide horizontal crack in the same area. The specimen eventually failed at the first cycle of the displacement of $14 \mathrm{~mm}$, when its shear strength gradually dropped below $80 \%$ of the maximum.
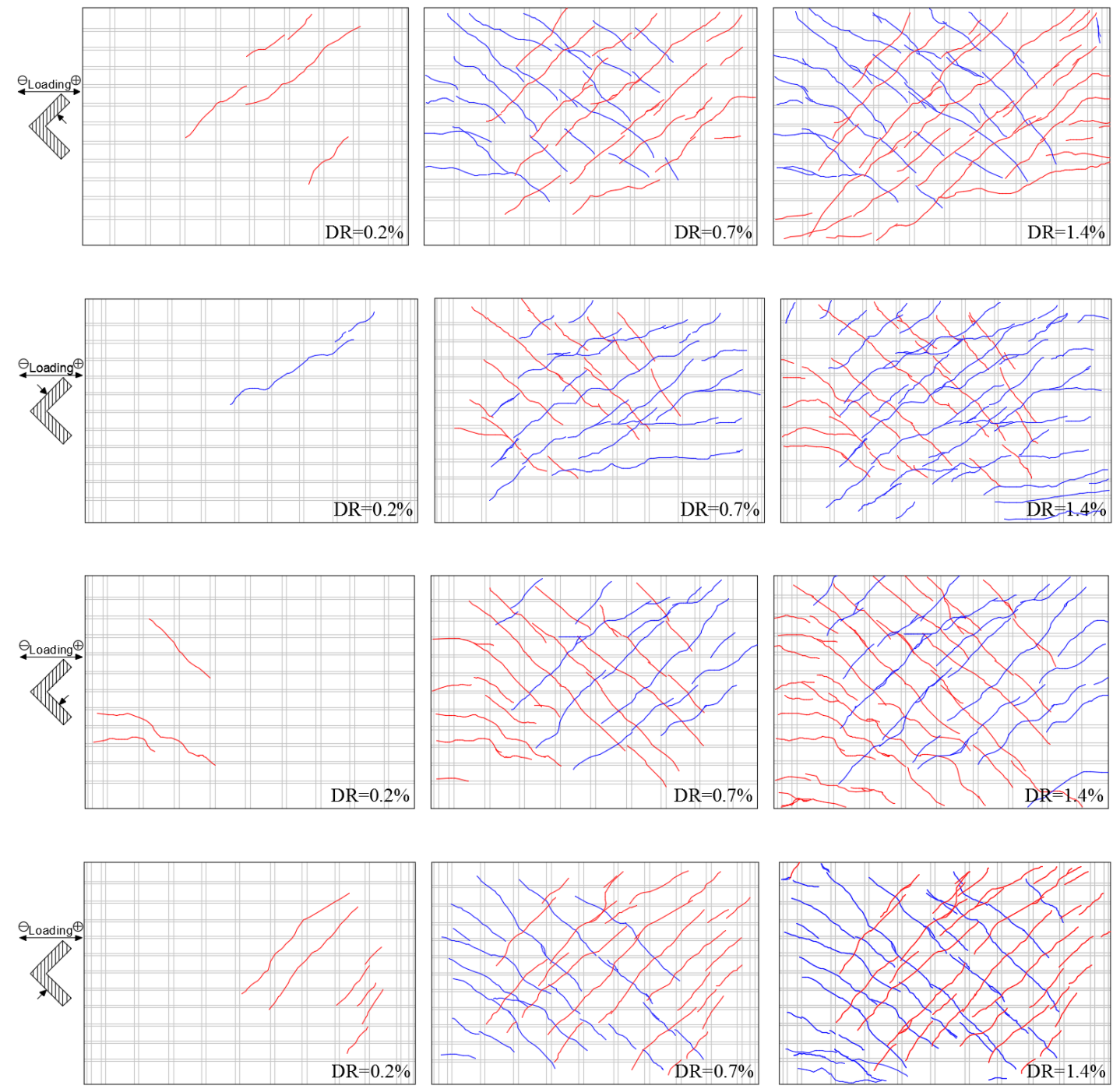

Figure 4.2 Cracking patterns of Specimen LP0D45 
The plots of cracking patterns on each surface of Specimen LP5D0 at different loading stages are presented in Figure 4.3. A few diagonal cracks were detected when the drift ratio reached $0.4 \%$. After that, cracks grew relatively slowly and did not begin to spread across the wall surface until the drift ratio reached $0.6 \%$. The first yielding of the longitudinal rebar occurred in the web at the drift ratio of $0.8 \%$ when the flange was in tension, with a corresponding shear foce of $328 \mathrm{kN}$. Compared with the specimens with lower axial loads, Specimen LP5D0 had fewer cracks at the same displacement. In the subsequent loading cycles, inclined cracks gradually spread to the entire surface of the web, while fewer diagonal cracks were discovered on the flange. Most of these cracks were formed when the flange was in tension. The specimen reached its maximum shear strength of approximately 739 $\mathrm{kN}$ in the positive direction and $650 \mathrm{kN}$ in the negative direction at the same displacement of $19 \mathrm{~mm}$. As the test continued, inclined cracks became denser and accumulated at the top of the web, forming a major horizontal crack. Due to the concrete spalling and the subsequent sliding along this slip plane, the specimen was unable to sustain the lateral strength and finally failed at the drift ratio of $2.1 \%$.
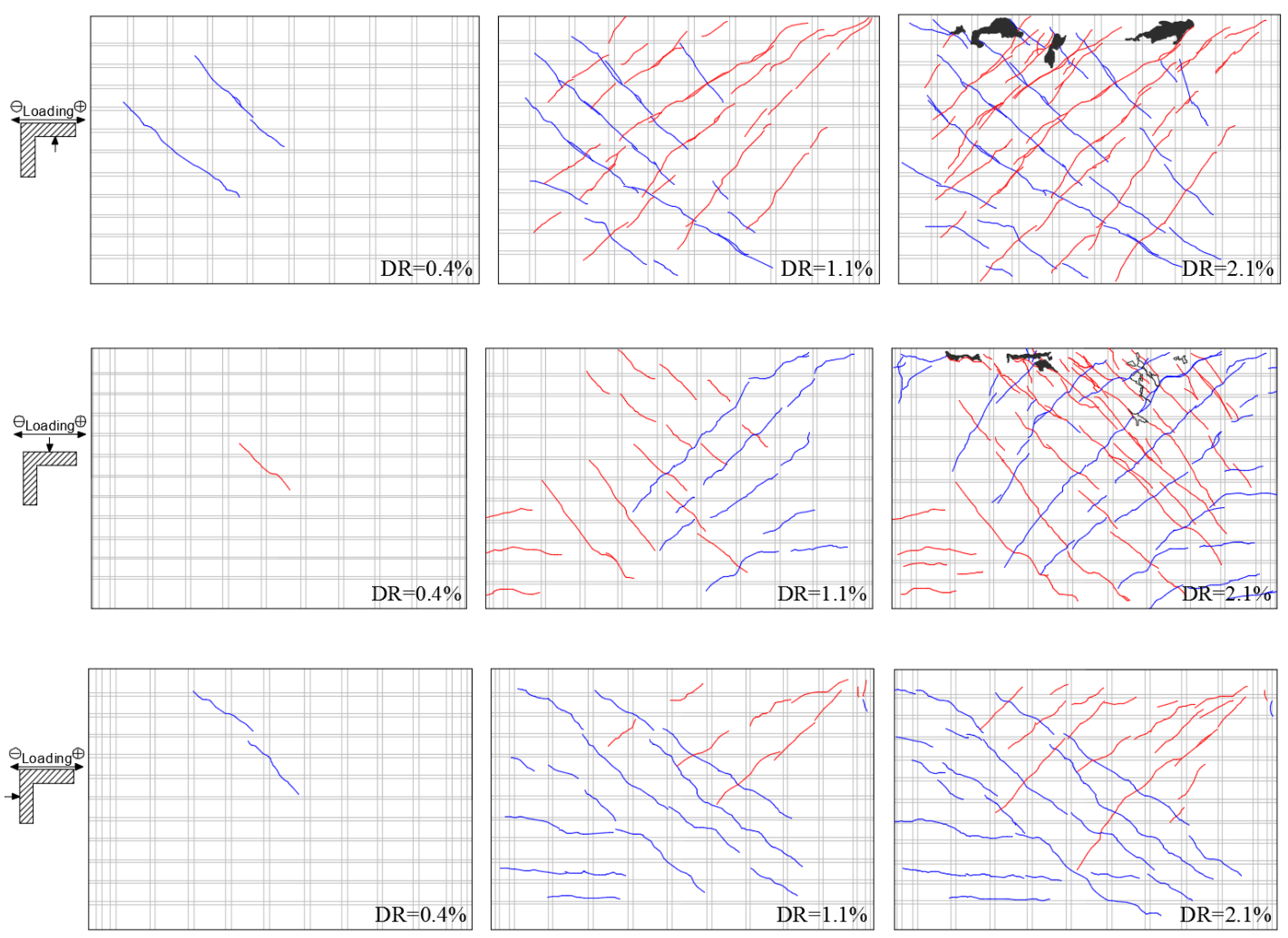

Figure 4.3 Cracking patterns of Specimen LP5D0 
As for Specimen LP5D45, the first cracks did not appear until the lateral displacement reached $3 \mathrm{~mm}$. As the test progressed, diagonal cracks occurred on all surfaces of the wall and intersected with each other. The two surfaces of the wall segments generally showed a similar distribution of cracks. Like Specimen LP5D0, axial loads restrained the development of cracks, thus fewer cracks were found in this specimen when compared with Specimen LP0D45. The longitudinal reinforcing bar in the web attained the first yielding at the displacement of $7 \mathrm{~mm}$, with a corresponding shear force of $587 \mathrm{kN}$. As lateral displacements further increased, the inclined cracks became denser, with the widest horizontal crack appearing at the upper part of the left segment. The drift ratio of $1.2 \%$ saw moderate concrete spalling along this major horizontal crack, as illustrated in Figure 4.8. The maximum shear strength and the corresponding displacement in the positive direction were $676 \mathrm{kN}$ and $12 \mathrm{~mm}$, respectively, and $714 \mathrm{kN}$ and $11 \mathrm{~mm}$ for the negative loading direction. Like Specimen LP5D0, Specimen LP5D45 quickly lost its lateral resistance after the concrete spalling and failed at the displacement of 13 $\mathrm{mm}$. 

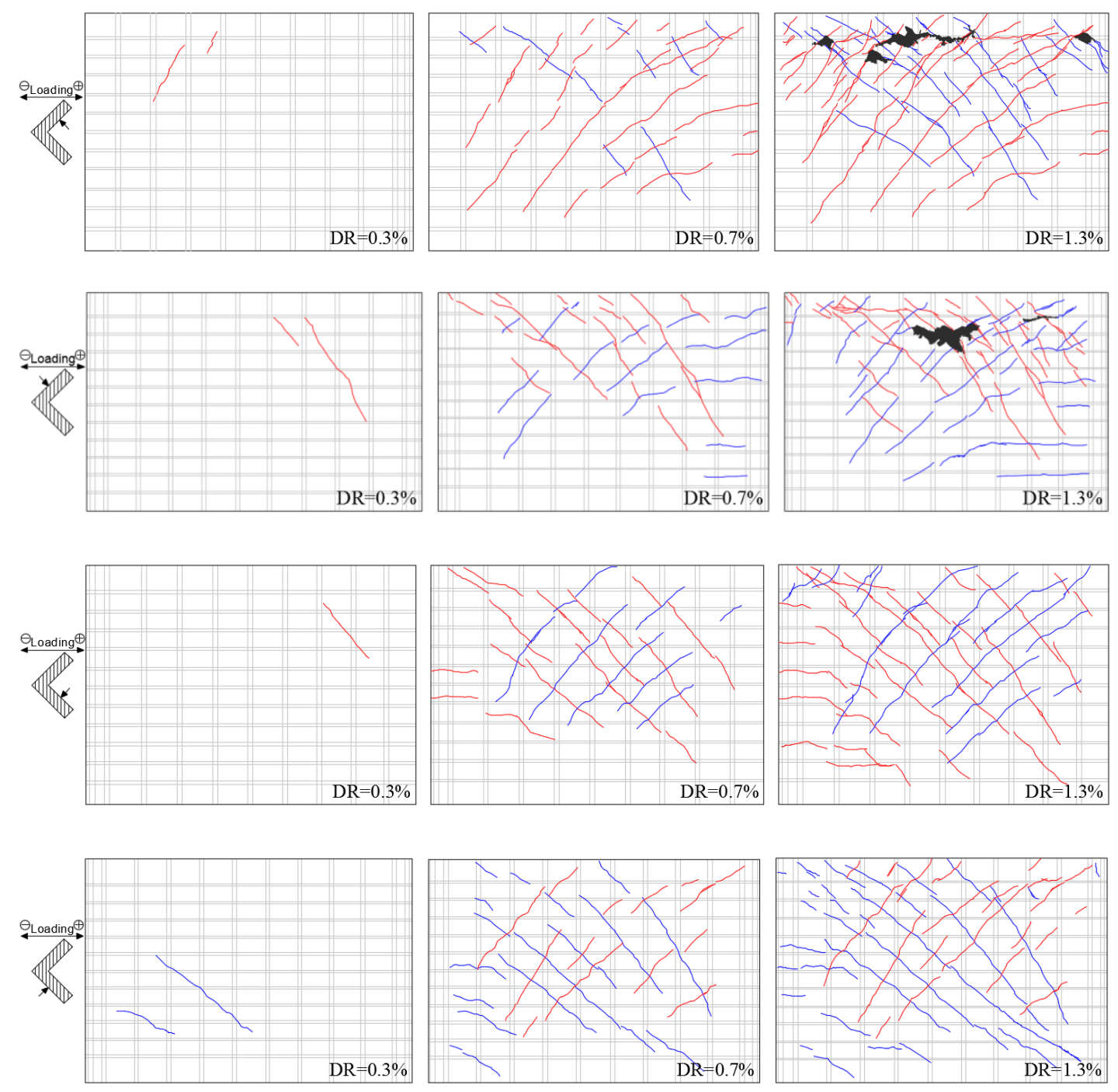

Figure 4.4 Cracking patterns of Specimen LP5D45

From the tests, it was observed that the initial shear cracks occurred at a drift ratio ranging from $0.2 \%$ to $0.4 \%$, which was prior to the yielding of longitudinal reinforcing bars. Those imposed with greater axial loads saw the delayed formation of initial cracks. As lateral displacements increased, more diagonal cracks appeared, intersected with each other and gradually spread to other parts of wall segments. Specimens with an higher axial load ratio showed a steeper angle of inclined cracks on the web. The phenomenon was more evident in the skew-loaded group, where the typical angles of cracks on the web were $48^{\circ}$ and $58^{\circ}$ for Specimens LP0D45 and LP5D45 respectively. Meanwhile, some flexural-shear cracks also developed at the bottom of walls, which were similar to those observed in the slender L-shaped walls $[\mathrm{Z2}]$. As for the cracks on the flange, Specimens LP0D0 and LP50D with 
orthogonal lateral loading saw more cracks appeared when the flange was in tension. Compared with the shear cracks on the web, most of these cracks were flexuralshear cracks. However, in specimens with skew lateral loads, the majority of cracks were shear cracks. Besides, almost equivalent amounts of cracks were found in positive and negative loading directions. Furthermore, the web showed denser crack distributions than the flange in specimens with lateral loads parallel to the web segment. As the test further progressed, diagonal cracks on the web became much wider and denser, and covered the entire surface. As a result, concrete spalling at the top of wall segments was discovered in specimens with extra axial loads. The ultimate drift ratios reached in the test were from $1.3 \%$ to $2.1 \%$.

\subsubsection{Failure mechanisms}

Figure 4.5 and Figure 4.6 depict the failure zones of Specimens LPOD0 and LP0D45 respectively. These two specimens were only imposed with the self-weight of the concrete slab. As seen from the figure, the drift ratio of $1.5 \%$ in the positive direction saw severe crushing of concrete at the bottom corner of the web segment in Specimen LP0D0. In the meantime, a sudden drop of lateral strength below $80 \%$ of the maximum was recorded. The specimen was unable to sustain its resistance afterward. As for specimen LP0D45, cracks with a smaller angle of inclination gradually appeared near the corner of the juncture of two wall segments from the drift ratio of $0.6 \%$. As the test progressed, these cracks became evidently wider and extended along the length of wall segments. Slight concrete crushing was witnessed in these areas at the drift ratio of $1.2 \%$, which led to the decline of the lateral strength. Further increases of horizontal displacements saw a wide horizontal crack developed near the base. At the same time, the specimens lost their lateral resistance by more than $30 \%$ of the maximum, and failed at the drift ratio of $1.4 \%$.

As demonstrated in Figure 4.7 and Figure 4.8, Specimens LP5D0 and LP5D45 with a higher axial load level shared a similar pattern of failure with a major horizontal crack developing at the upper part of wall segments. These two specimens experienced a drastic loss of the lateral strength once concrete spalling was spotted along the horizontal crack, and quickly failed in a few cycles of lateral displacements. This phenomenon was believed to be associated with the force 
transfer mechanism in the test. As stated previously, a large and stiff top slab was used to transfer lateral and axial loads to the wall segment. Ideally, the horizontal displacement would be transferred assuming that a perfect coordination existed between these two parts. In the real test, however, the existence of the stiff top slab and the subsequent incompatibility in transferring horizontal loads would cause stress concentration in the upper part of wall segments. As the test progressed, the inclined cracks formed in each loading direction intersected with each other near the wall top, narrowing the concrete struts between them. These struts were prone to crushing under the high stress in the wall. The concrete crushing further extended along the length of the wall segment, formed a weakened horizontal plane, and eventually led to the failure of specimens. Besides, the adopted weaker concrete and the nature of thin wall section were also contributed to the development of the major horizontal crack.

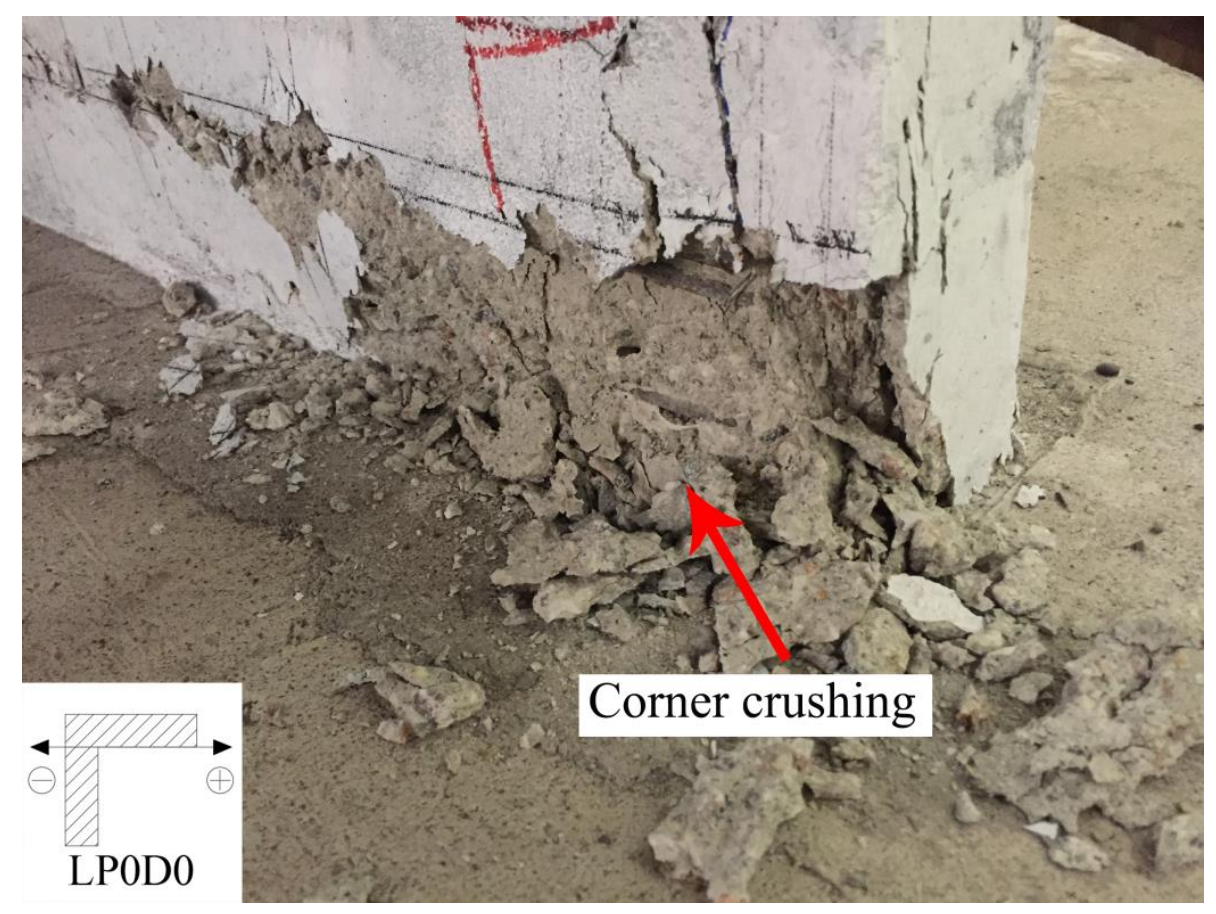

Figure 4.5 Failure mechanism of Specimen LP0D0 


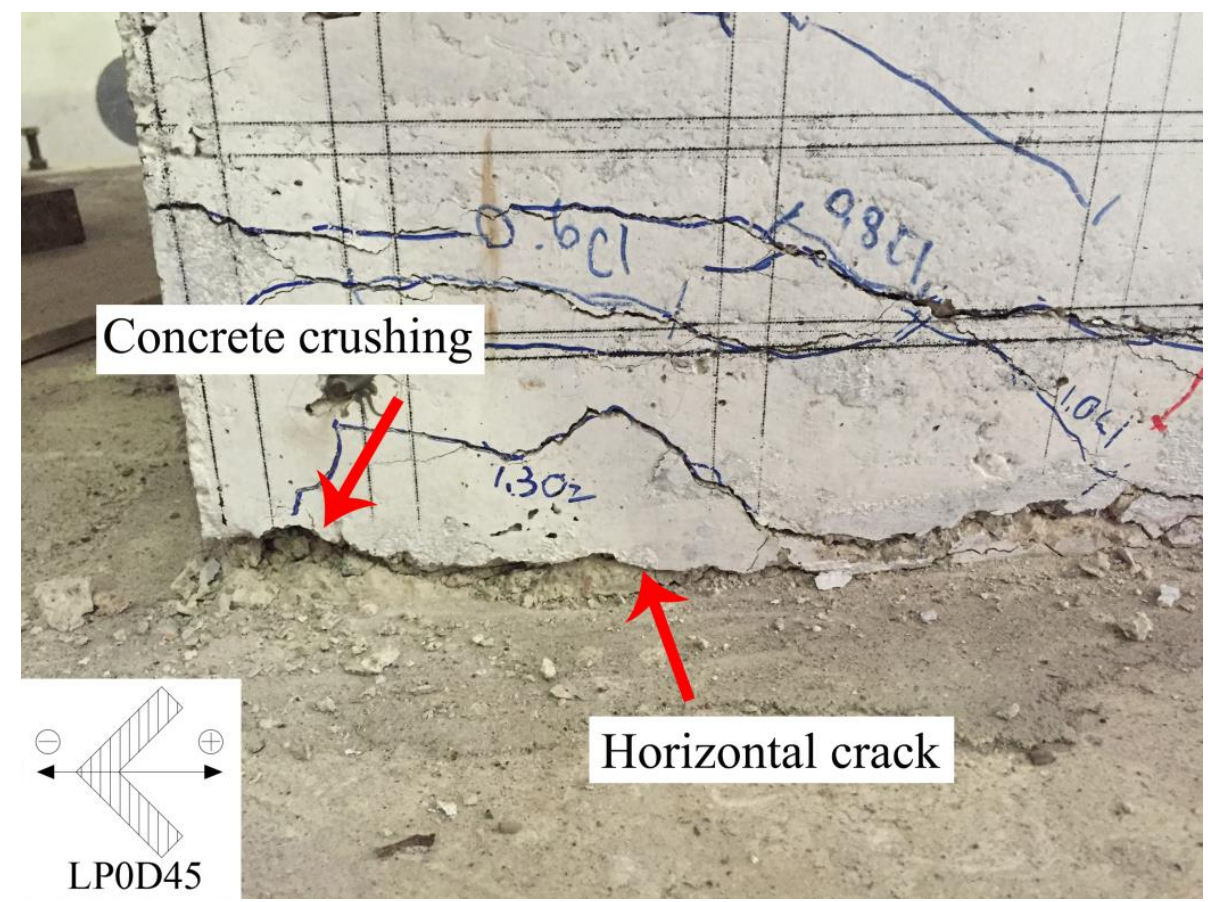

Figure 4.6 Failure mechanism of Specimen LP0D45

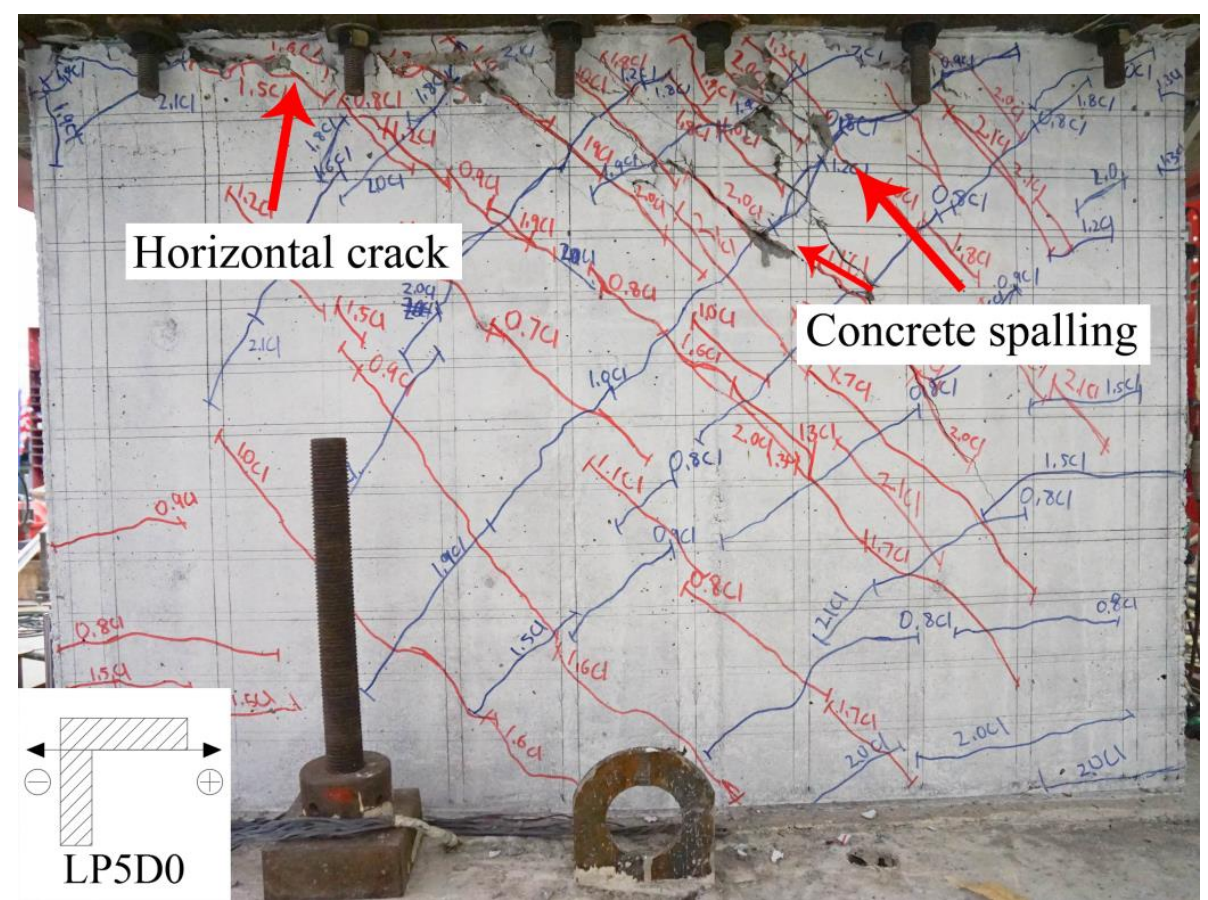

Figure 4.7 Failure mechanism of Specimen LP5D0 


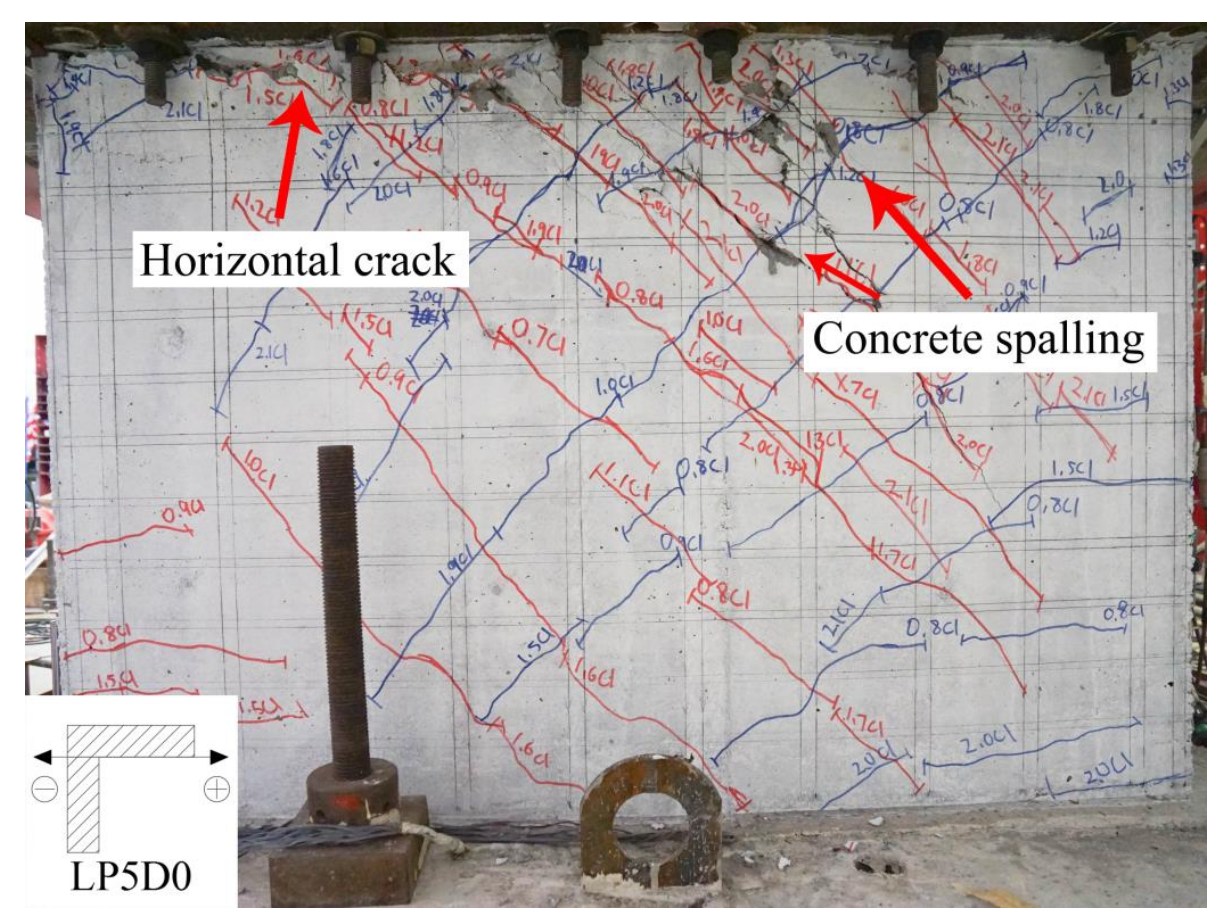

Figure 4.8 Failure mechanism of Specimen LP5D45

\subsubsection{Hysteretic responses}

Load-displacement curves play a pivotal role in evaluating the seismic performance of RC structures [S8, W6]. Data recorded by load cells and horizontally-mounted LVDTs were used to plot the load-displacement curves. Relevant aspects such as strength, secant stiffness and energy dissipation capacity were also depicted and analyzed in the following part. Table 4.1 summarizes some key features as well as the experimental results of the L-shaped specimens. Figure 4.9 to Figure 4.12 depict the lateral load versus top displacement curves of the L-shaped specimens. 
Table 4.1 Test results of the L-shaped specimens

\begin{tabular}{cccccccccc}
\hline \multirow{3}{*}{ Specimen } & $(1)$ & $(2)$ & $(3)$ & $(4)$ & $(5)$ & $(6)$ & $(7)$ & $(8)$ \\
& $f_{c}^{\prime}$ & $P / f_{c}^{\prime} A_{g}$ & $\theta$ & $V_{c r}$ & $V_{\max }$ & $\Delta_{y}$ & $K_{i}$ & $\delta_{\max }$ \\
\cline { 2 - 9 } & $\mathrm{MPa}$ & $\%$ & $\circ$ & $\mathrm{kN}$ & $\mathrm{kN}$ & $\mathrm{mm}$ & $\mathrm{kN} / \mathrm{mm}$ & $\%$ \\
\hline LP0D0 & 35.0 & 0.72 & 0 & 147 & 592 & 7.0 & 55 & 47 & 1.6 \\
\hline LP0D45 & 36.2 & 0.73 & 45 & 200 & 656 & 6.0 & 67 & 79 & 1.4 \\
\hline LP5D0 & 35.0 & 5.65 & 0 & 168 & 739 & 8.0 & 41 & 71 & 2.1 \\
\hline LP5D45 & 36.2 & 5.67 & 45 & 430 & 714 & 7.0 & 84 & 92 & 1.3 \\
\hline
\end{tabular}

Note: (1): Compressive strength of concrete; (2) Axial load ratio; (3) Angle between lateral loading and web segment; (4) Observed shear strength at the first cracking; (5) Maximum shear strength during the test; (6) Yield displacement; (7) Effective stiffness in positive and negative loading directions; (8) Maximum drift ratio.

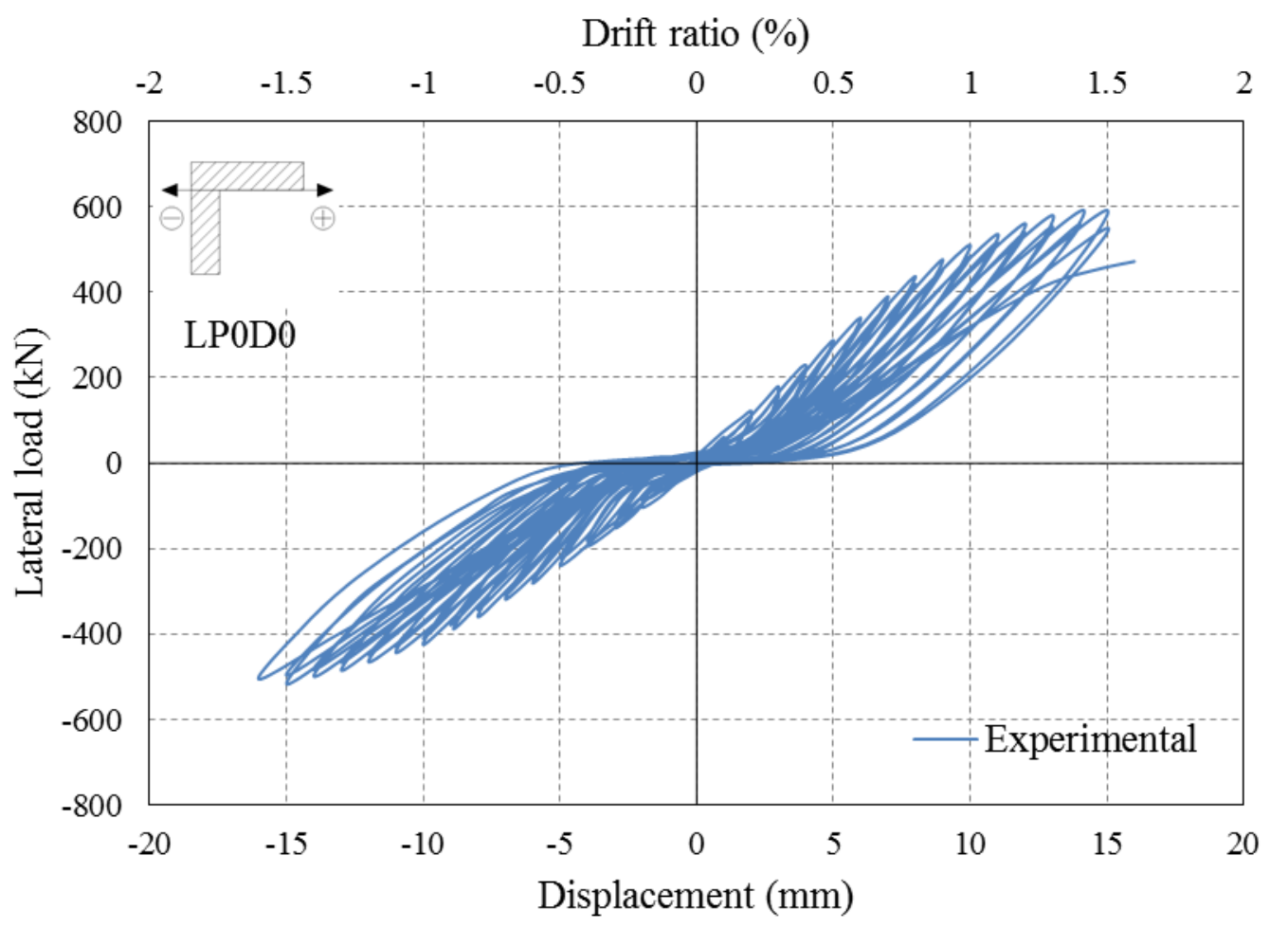

Figure 4.9 Lateral load-displacement curve of Specimen LP0D0 


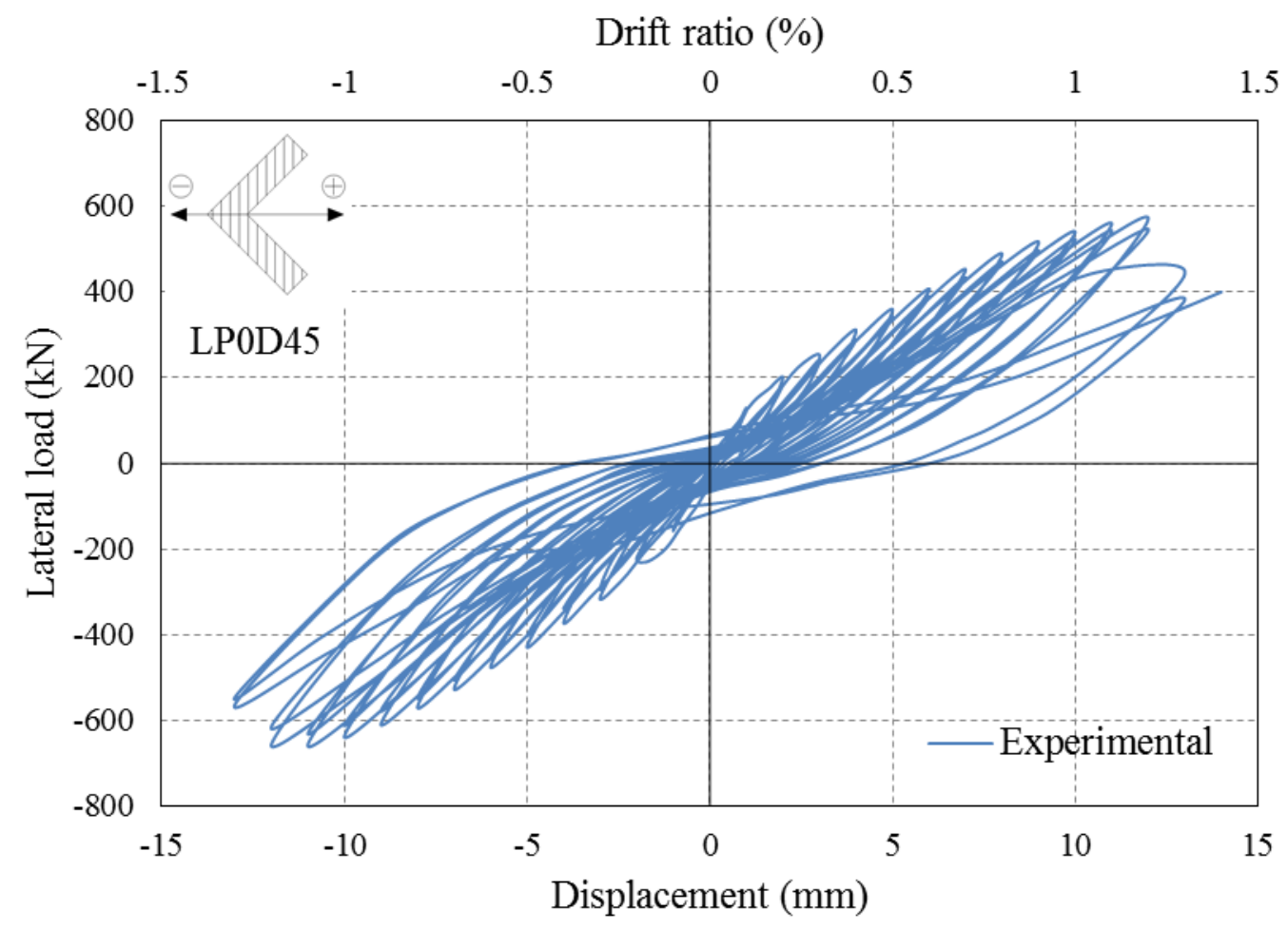

Figure 4.10 Lateral load-displacement curve of Specimen LP0D45

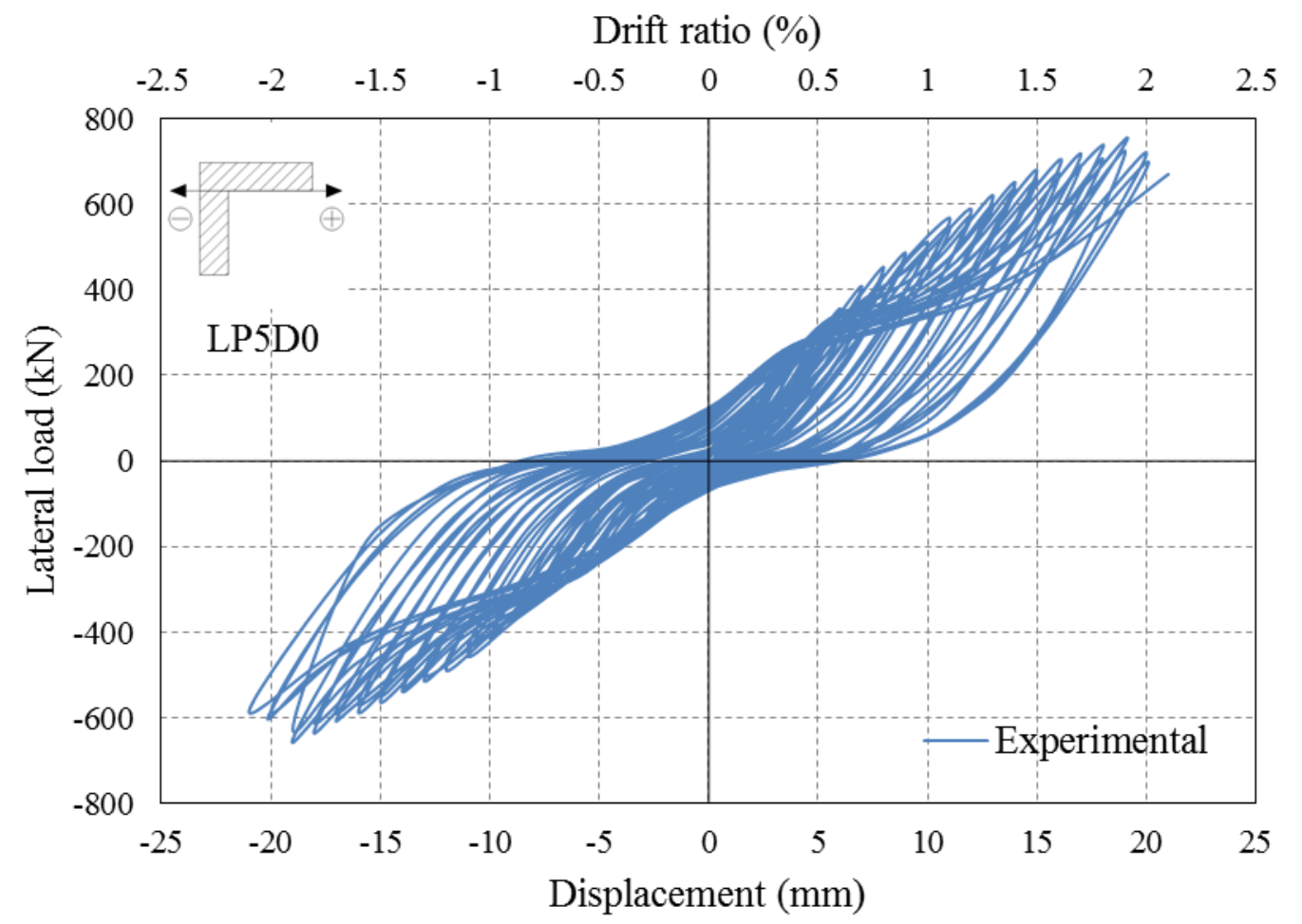

Figure 4.11 Lateral load-displacement curve of Specimen LP5D0 


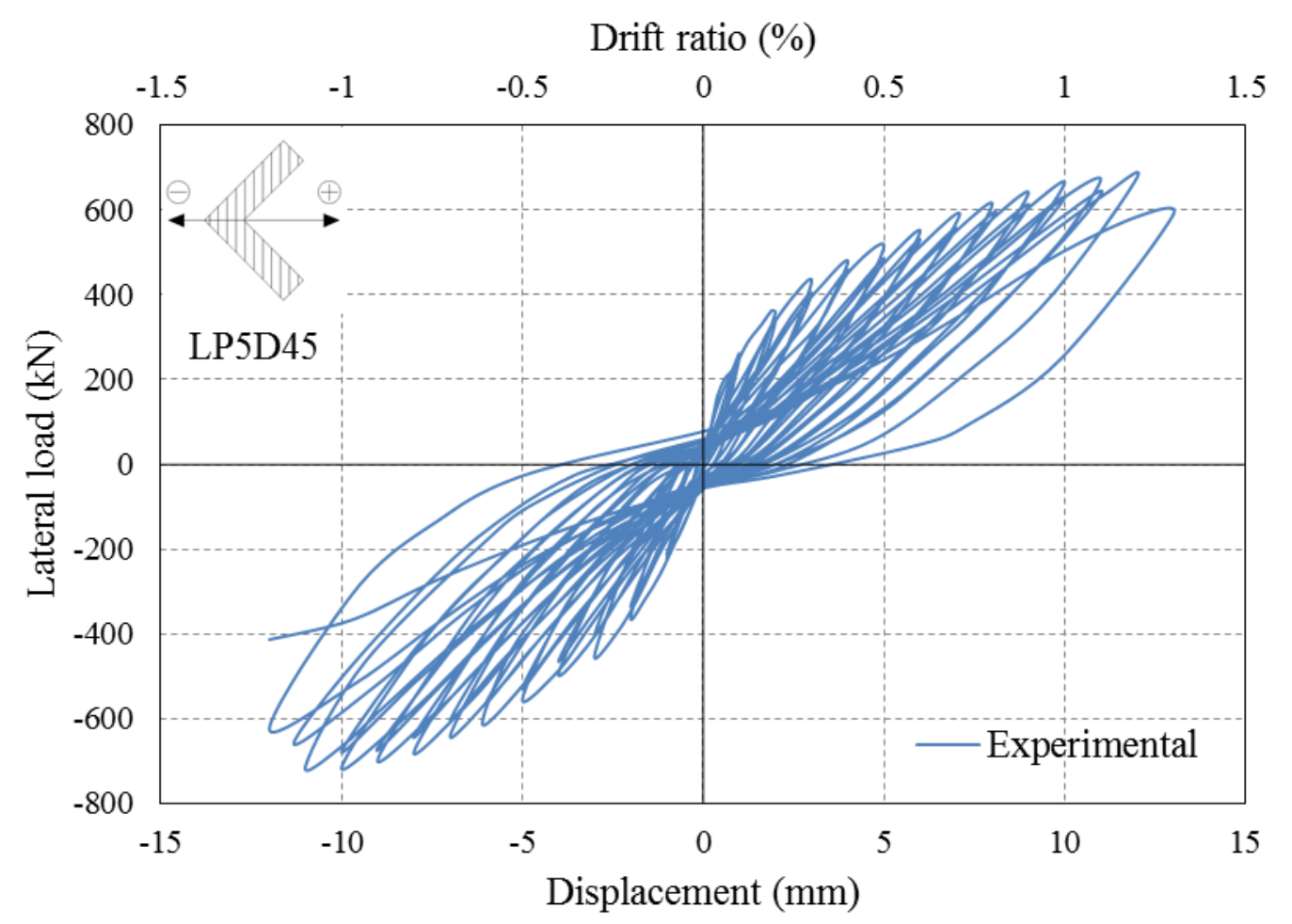

Figure 4.12 Lateral load-displacement curve of Specimen LP5D45

The backbone curve has long been recognized as an important feature when modeling the inelastic behavior of RC structural walls. Generally, the backbone curve is produced from monotonic experiments. But in this study, the peaks of lateral loads at every drift ratio were collected to construct the backbone envelopes as shown in Figure 4.13. As seen from the figure, all specimens demonstrated a rapid loss of strength in the last one or two cycles prior to the failure. It was evident that under skew horizontal loading, the specimen with more axial loads had larger lateral strength at a specific drift ratio. However, the increase of the lateral force brought by axial loads was not obvious in specimens under orthogonal lateral loading. As shown in Figure 4.13, specimens subjected to orthogonal loading outperformed the skew-loaded group in terms of the deformation capacity. This phenomenon was most obvious between Specimens LP5D0 and LP5D45, in which the former achieved a drift ratio of $2.1 \%$ while its counterpart only had the number as $1.3 \%$. The axial load, however, did not seem to play an important role on deformation capacity, which was different from observations in past experiments [Z2]. 
Secant stiffness is important in modeling and analyzing reinforcement concrete structures since it is deeply related to the iterative method and acted as an indicator of material properties. Figure 4.14 presents the variation of secant stiffness during the whole experiments. As expected, all specimens experienced a reduction in stiffness as the drift ratio increased. Among them, the most dramatic decline of secant stiffness was witnessed in specimens with skew horizontal loading. Specimen LP5D45 rapidly lost half of its stiffness when the drift ratio reached $0.4 \%$. Specimens under orthogonal loading, on the other hand, only showed moderate stiffness reduction during the experiment. As horizontal displacements rose, the secant stiffness of each specimen further declined and remained at a comparatively low value at the final stage. Moreover, axial loads were witnessed as an active factor in increasing the secant stiffness. This was attributed to the fact axial loads could effectively constrain the diagonal cracks and limit the development of the sliding shear, thereby enhancing the reaction force at a specific drift ratio.

The energy dissipation of wall specimens is calculated from the inner area of lateral load-displacement loops, which has long been recognized for its significance in evaluating the seismic performance of RC structures. Figure 4.15 below depicts the energy dissipation capacity of each specimen with respect to the drift ratio. In the initial stage, a rather small portion of energy was dissipated. As top displacement rose, the dissipation of energy steadily increased and experienced a drastic growth at the final stage. It was clear that the group with more axial loads showed a better energy dissipation capacity than its counterpart. Furthermore, the skew horizontal loading was observed to have a more favorable effect in increasing the energy dissipation, which was reflected in a $162 \%$ discrepancy between Specimens LP0D45 and LP0D0. This was due to the fact that when the L-shaped wall was arranged at $45^{\circ}$ to the horizontal loading, more evenly distributed compression zones could be introduced in both loading directions, thereby increasing the flexural capacity. 


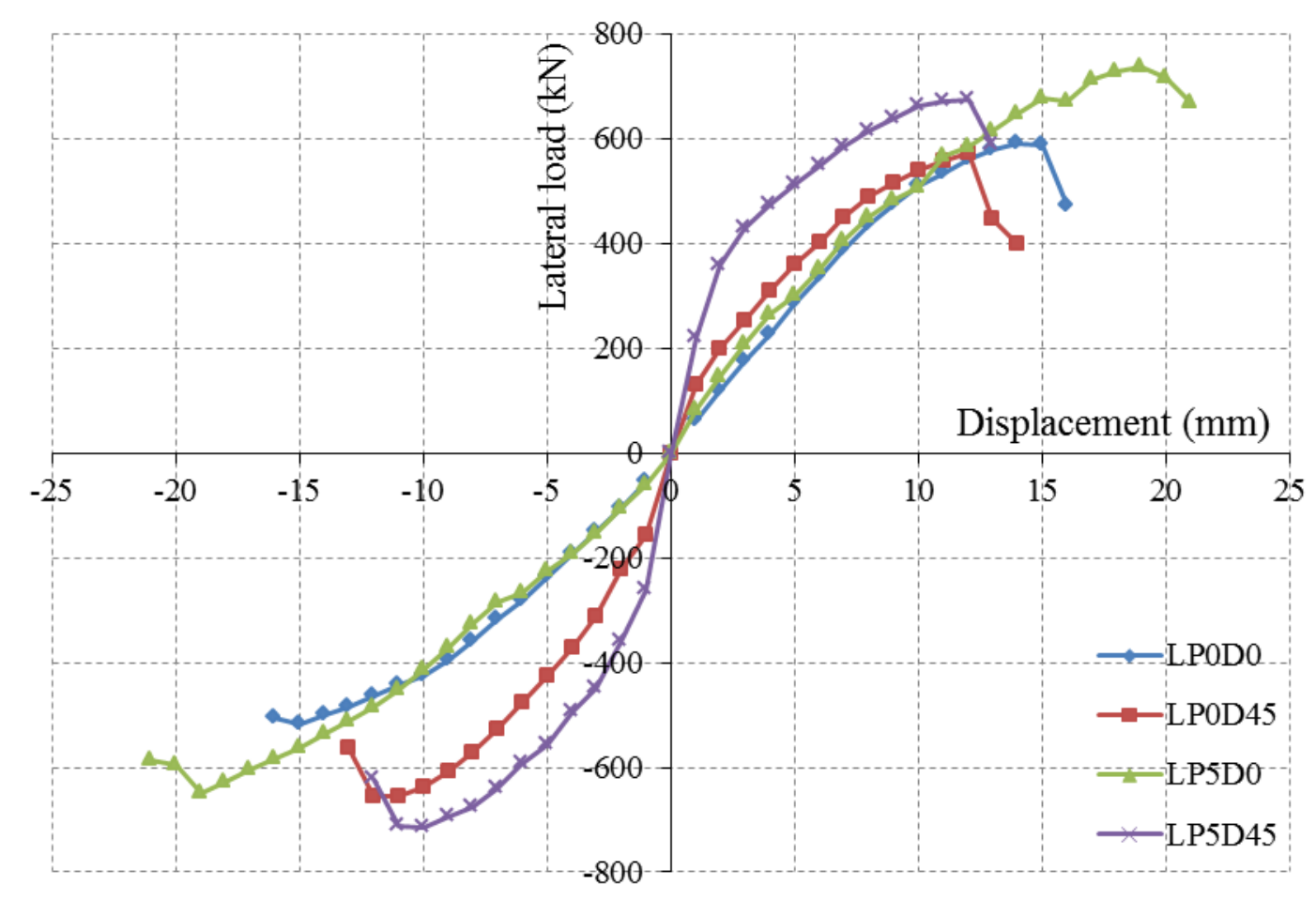

Figure 4.13 Backbone curves of the L-shaped specimens

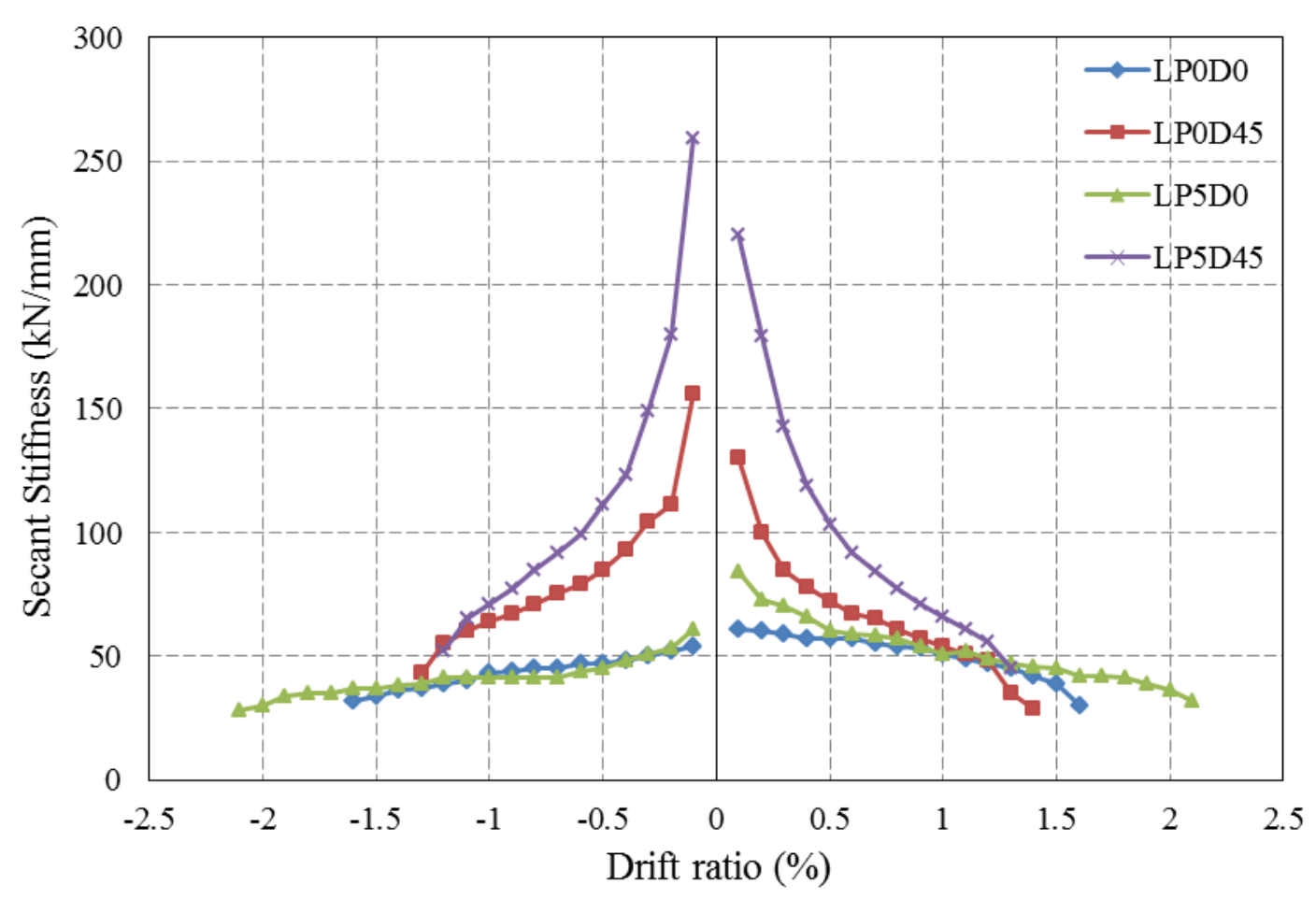

Figure 4.14 Secant stiffness of the L-shaped specimens 


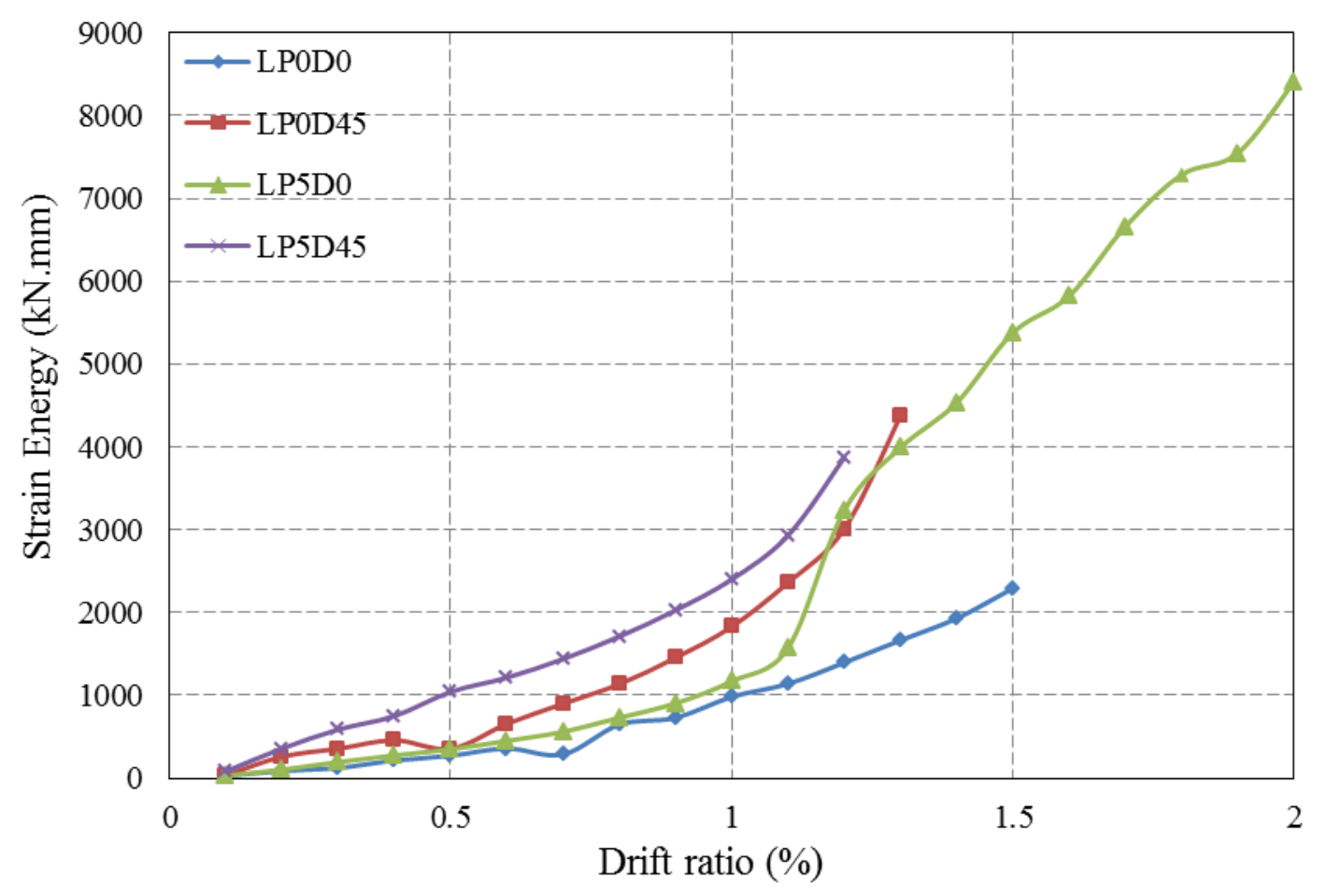

Figure 4.15 Energy dissipation capacities of the L-shaped specimens

\subsubsection{Displacement components}

Top deformations of structural walls mainly consist of three components: flexural deformation, shear deformation and sliding deformation. LVDTs were mounted at wall surfaces to provide data concerning these three deformations. Figure 4.16 to Figure 4.19 depicts the ratios of these three components to the total top displacement at different drift ratios, in which an unaccounted portion was also recorded since the summation of the other three could not always reach a perfect $100 \%$.

In Specimen LPOD0, the sliding displacement showed an increasing trend in both loading directions with its value doubled from around 5\% to $10 \%$. Flexural and shear displacements, nonetheless, revealed different varying patterns with regard to the loading directions. In the positive cycles, the shear portion experienced a slight decline while the flexural portion showed the opposite trend. Their difference also dwindled from $10 \%$ to $2 \%$ as the drift ratio increased. In the negative cycles, the shear deformation was evidently larger than its counterpart. The former rose from 
$47 \%$ to $53 \%$ while the latter diminished from $36 \%$ to $31 \%$. The unaccounted part experienced a slight decline throughout the test.

A similar rising trend of the sliding deformation was also observed in Specimen LP0D45, where the number ended up at approximately $10 \%$ in both directions. The shear deformation, throughout the whole test, preserved a dominant component of the top displacement. Despite an initial increase, the value of the shear displacement remained almost the same when the specimen failed in the positive loading direction. A similar trend was also witnessed in the negative loading direction. The flexural deformation, on the other hand, diminished in both directions, with the numbers dropping from $38 \%$ and $43 \%$ to $30 \%$ and $35 \%$ in two loading directions. The unaccounted part varied slightly but its value remained small.

The contribution of the sliding shear in Specimen LP5D0 also grew as the drift ratio increased. In the positive loading direction, the shear contribution rose from $43 \%$ to $53 \%$ while the flexural contribution decreased from $46 \%$ to $35 \%$. In the negative loading direction, however, the shear deformation experienced a moderate decline from $63 \%$ to $53 \%$ whereas the flexural deformation increased making their contribution equal when the wall failed. The unaccounted part showed various trends in different loading directions but its value remained relatively small.

With regard to Specimen LP5D45, the sliding shear component made up to $3 \%$ in the beginning and ended up at around 6\% in both loading directions. The shear displacement was constantly and evidently larger than the flexural displacement during the whole test. In the negative loading direction, the portion of the two components scarcely changed whereas in the positive loading direction, the shear contribution rose and the flexural contribution dwindled. The unaccounted part decreased as the drift ratio rose to $7 \%$, and then slightly increased toward the end.

From these four figures, it was found that in general, the shear deformation dominated the whole response because it accounted for the largest part of top displacements. This phenomenon was more obvious in the specimens with skew lateral loading. Moreover, the sliding shear component increased during all the tests, in which the specimens with no additional axial loads had a more rapid growth of 
sliding shear deformation. For instance, the portion of the sliding displacement of Specimen LP0D45 was around 10\% at the end of the experiment, which doubled the value of Specimen LP5D45. The same phenomenon was also observed between Specimens LP0D0 and LP5D0, showing that axial loads played a favorable role in controlling the sliding shear.

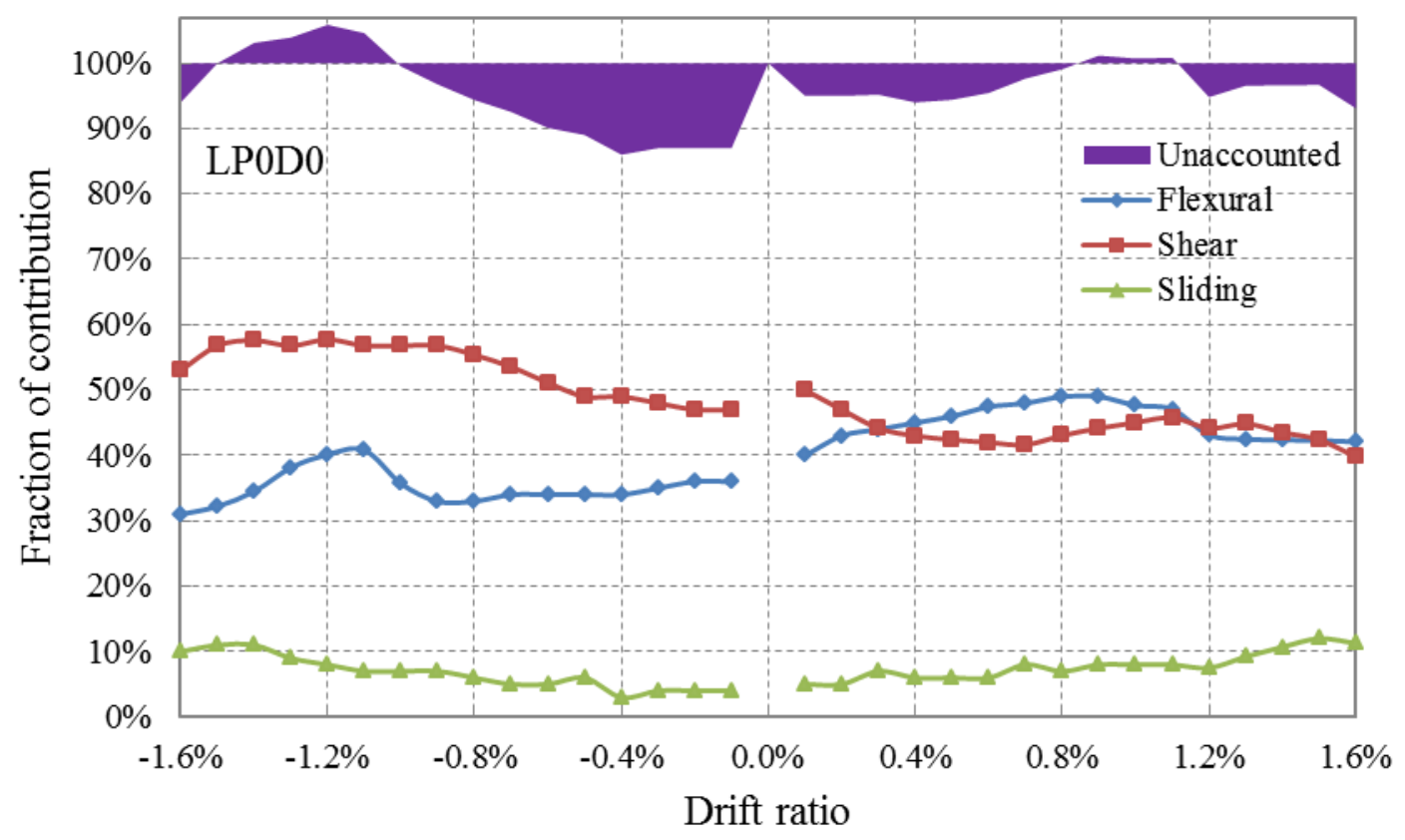

Figure 4.16 Displacement components of Specimen LP0D0

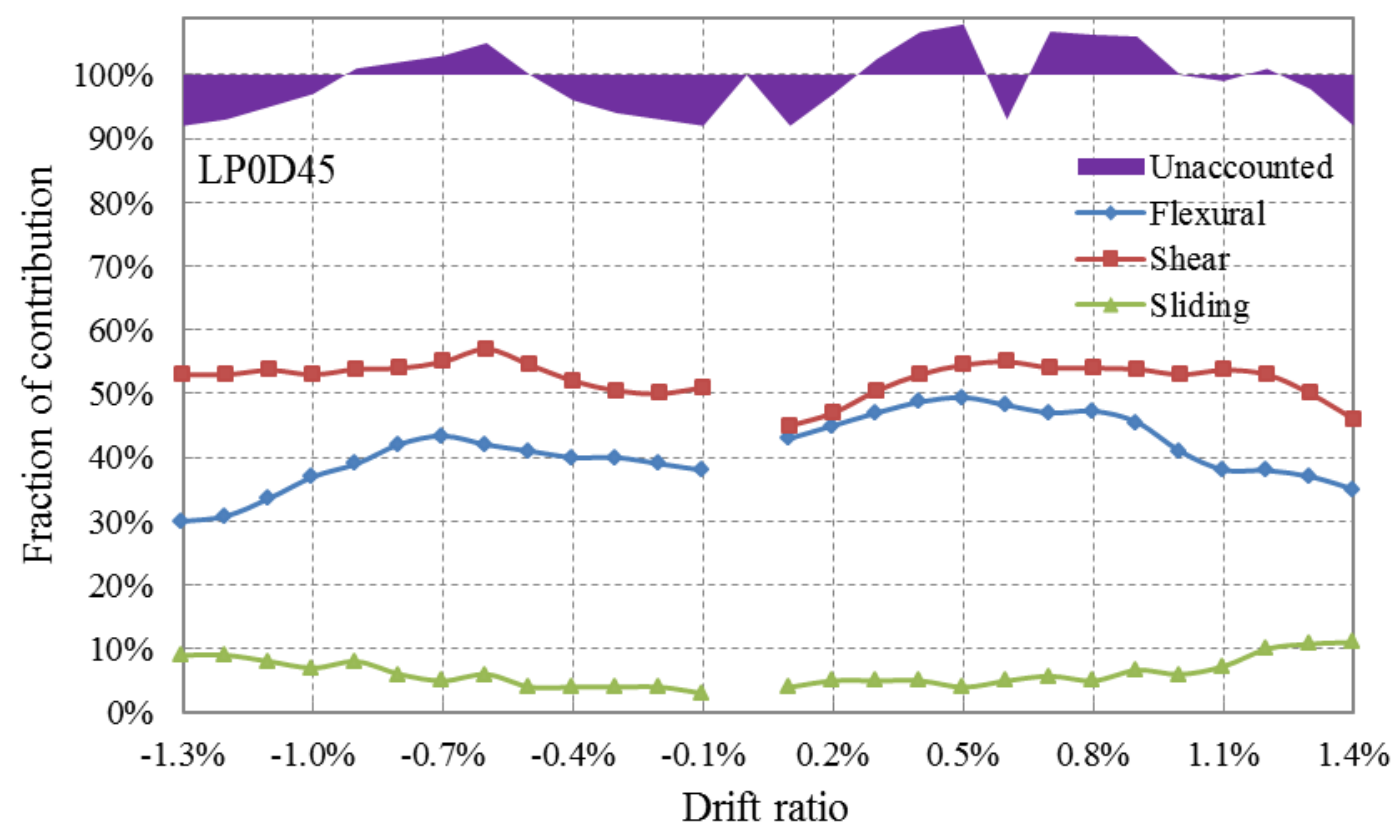

Figure 4.17 Displacement components of Specimen LP0D45 


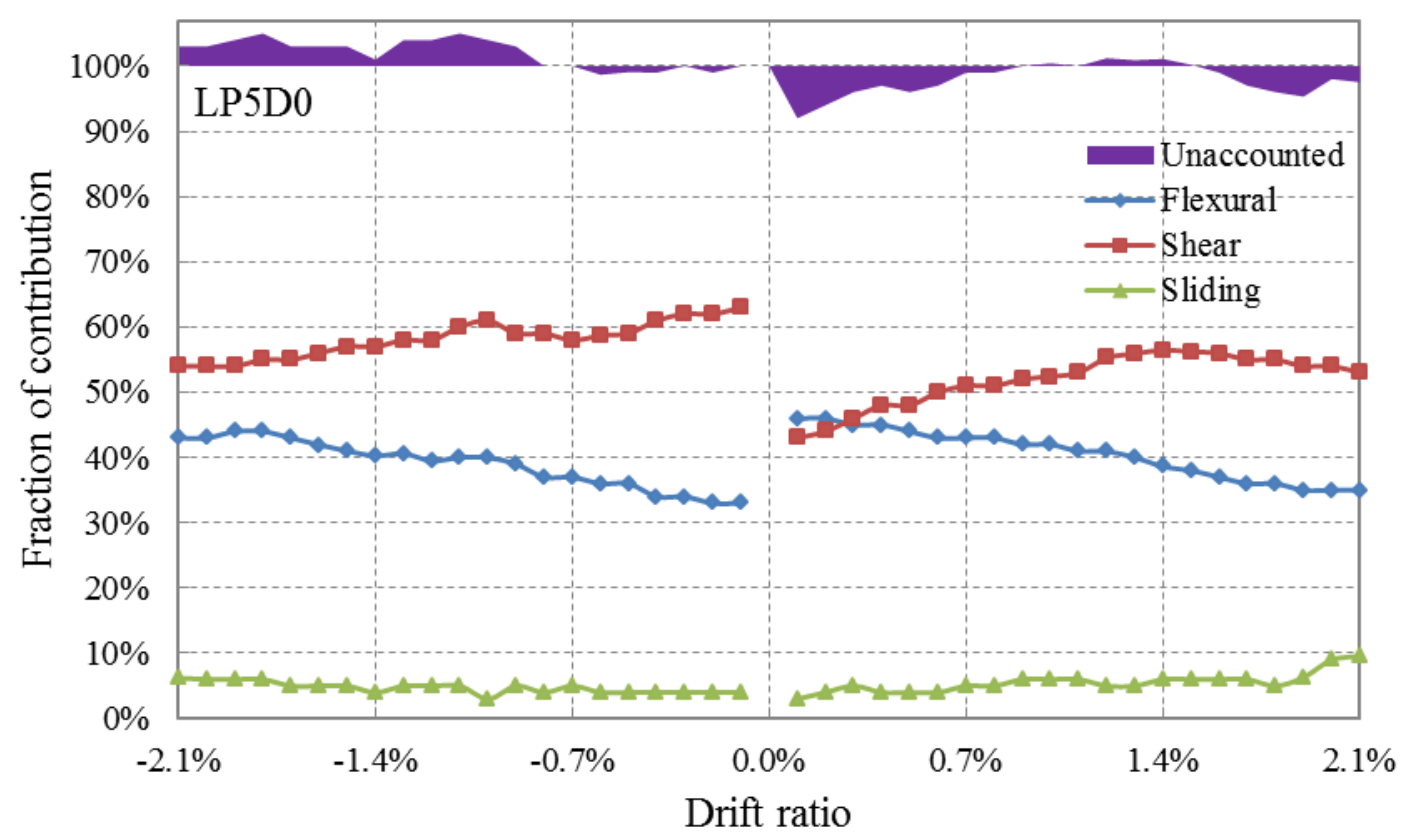

Figure 4.18 Displacement components of Specimen LP5D0

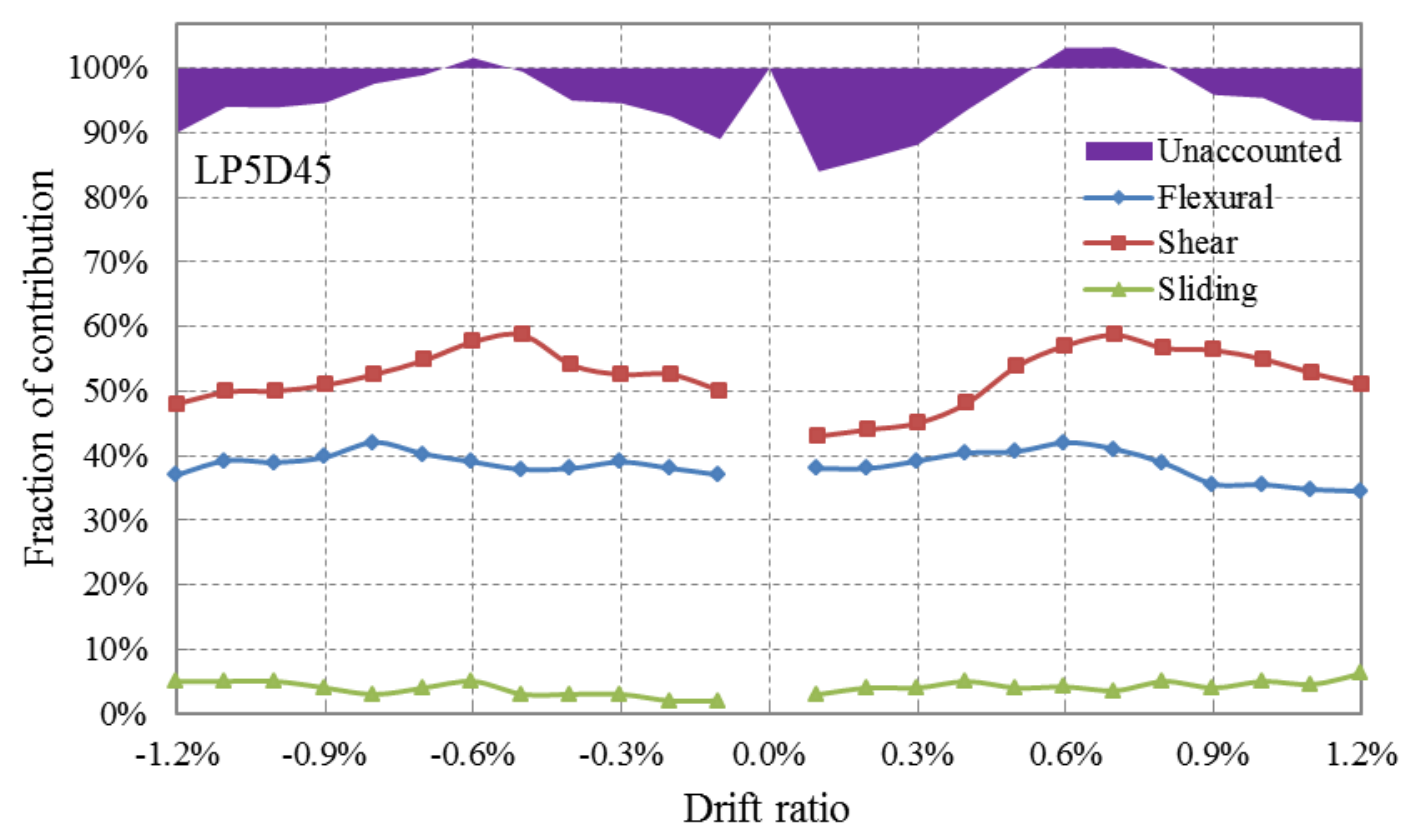

Figure 4.19 Displacement components of Specimen LP5D45

\subsubsection{Strain profiles}

Strain gauges, with their locations being illustrated in Chapter 3, were attached to the surface of reinforcing bars to monitor their reactions during the test. Data was compiled and plotted to show strain variations from the initial stage of the test to the 
wall failure at several critical sections, which were important for the study of the internal force transfer mechanism.

Figure 4.20 to Figure 4.27 demonstrate strains at the bottom of longitudinal reinforcement along the web of specimens at various drift ratios in both loading directions. Yielding of the vertical web reinforcement was detected in all specimens except Specimen LP5D45. For Specimen LP0D0, it was observed that strains remained almost linear until drift ratio reached $0.4 \%$, which indicated the plane section assumption was valid at this stage. Also, it was found that the neural axial did not shift during the whole test. As for Specimen LP0D45, the neutral axial moved approximately $400 \mathrm{~mm}$ towards the tip of the wall segment in the positive loading direction, whereas the neutral axial hardly moved when the specimen was imposed with negative displacements. As for Specimen LP5D0, the strain distribution in the half away from the flange quick became nonlinear after the drift ratio of $0.5 \%$ in the positive loading direction. The location of the neutral axis could hardly be identified as there was an unusual surge of strains at the vertical web reinforcement $1250 \mathrm{~mm}$ away from the web. The neutral axis also shifted $400 \mathrm{~mm}$ towards the tip of the wall segment in the positive loading direction in Specimen LP5D45. In the negative loading direction, the strains were seemed to be able to maintain linear in the test.

The strain variations along the bottom of longitudinal reinforcement in the flange were presented from Figure 4.28 to Figure 4.31. As seen from the figure, longitudinal reinforcing bars in both specimens yielded during the test when the flange was in tension. Among them, Specimen LP5D0 with more axial loads experienced an earlier yielding at the drift ratio around $0.8 \%$. Strains of the longitudinal reinforcement generally showed a declining trend with their locations moving away from the web in both loading directions, which served as an indicator that a shear lag effect existed in the wall. This phenomenon was more evident in Specimen LP5D0, where stains of the outmost longitudinal reinforcing bar were almost zero when the flange was in tension, suggesting that the outer part of the flange was hardly involved in withstanding lateral loads. When the flange was in compression, the shear lag effect was also detected. As reflected in Specimen 
LP5D0, at the drift ratio of $2.1 \%$, the strain of the longitudinal reinforcing bar located closest to the web was approximately $200 \%$ of that of longitudinal reinforcement located furthest from the web.

Figure 4.32 to Figure 4.39 illustrate strains along two selected horizontal reinforcing bars which were located $20 \mathrm{~mm}$ and $500 \mathrm{~mm}$ above the wall base. It was clear that the horizontal reinforcement did not yield during the test except for No.7 rebar in Specimen LP0D45. Moreover, strains increased as the drift ratio rose in both loading directions. As for Specimen LPOD0, the reading of the strain gauge located at the middle of both reinforcing bars experienced the highest growth in both loading directions, indicating that there was a main diagonal compression strut passing through these places. A similar trend was also found in No.7 rebar of Specimen LP0D45, where the reinforcement yielded at the final stage of the test. However, it was shown in the figure that strains at the middle of No.1 rebar had the lowest reading in the same specimen. Specimen LP5D0 had identical strain variation as Specimen LP0D0. The only difference was that the strain in the middle of horizontal rebar remained the highest reading number throughout the experiment. In Specimen LP5D45, the same intense increase of strain was also observed in No.7 rebar. However, the strain in the middle of No.1 rebar showed the opposite trend with its value being the lowest in both loading directions. 


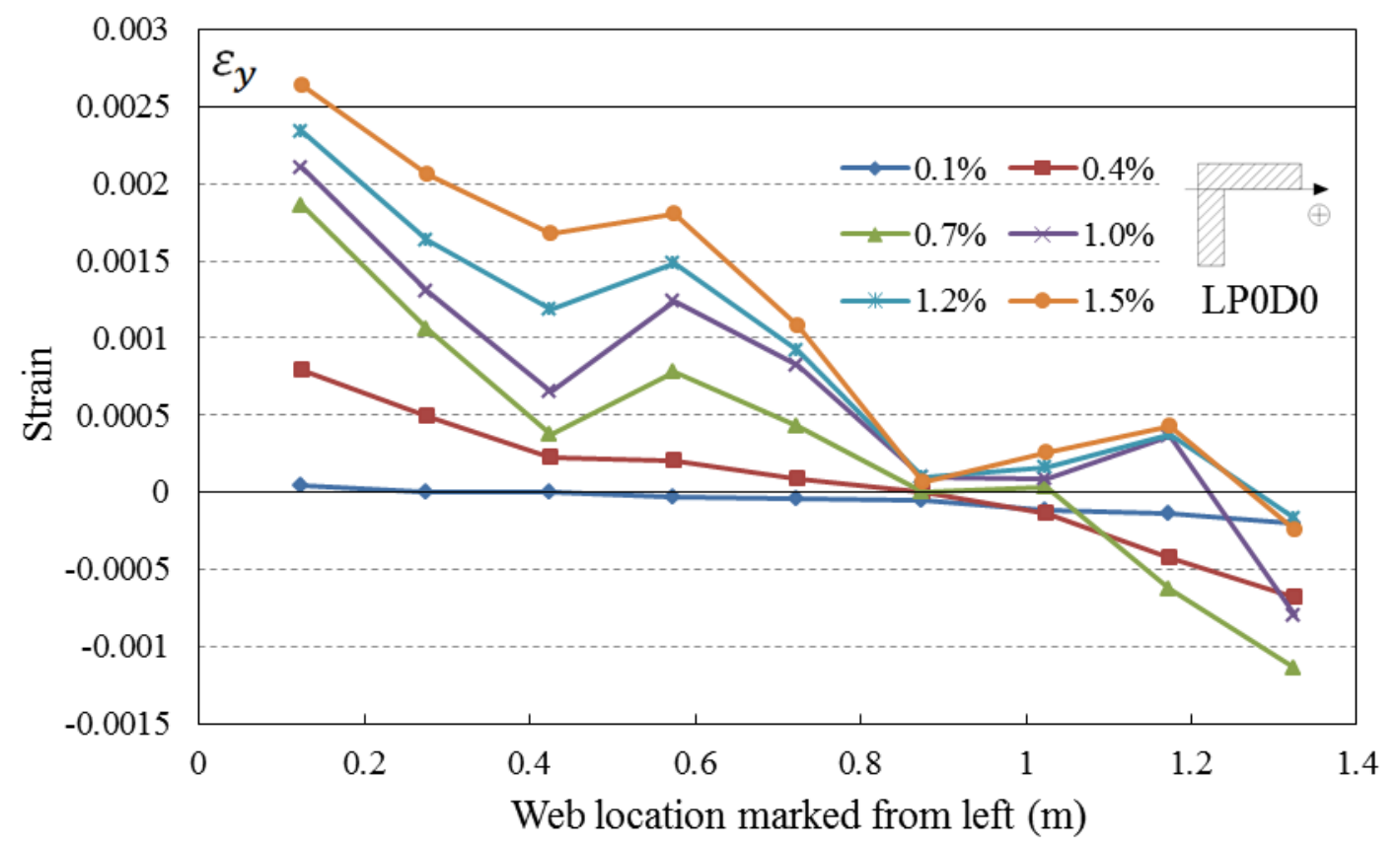

Figure 4.20 Strain profiles of longitudinal reinforcement along the web of Specimen LPOD0 in the positive loading direction

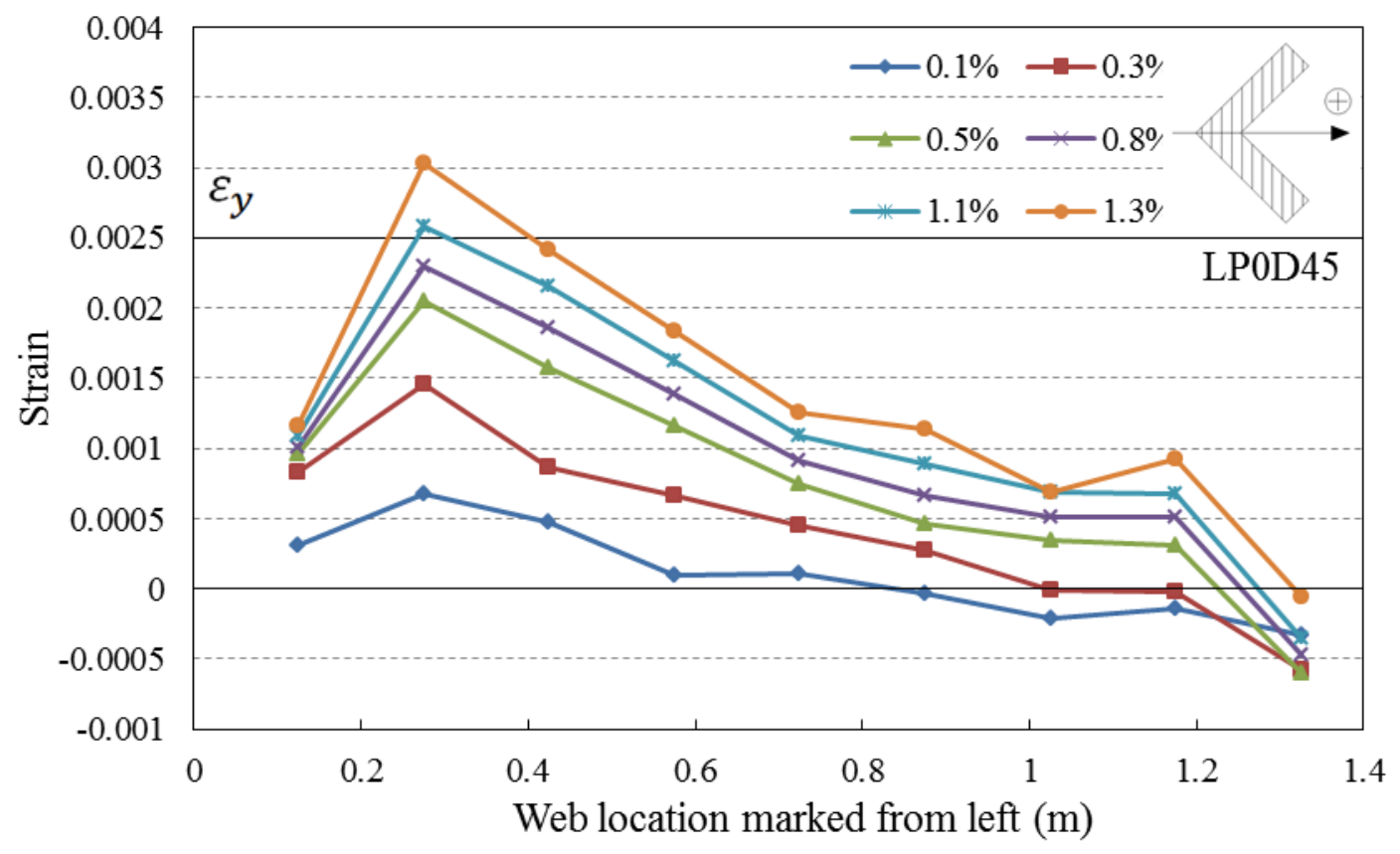

Figure 4.21 Strain profiles of longitudinal reinforcement along the web of Specimen LP0D45 in the positive loading direction 


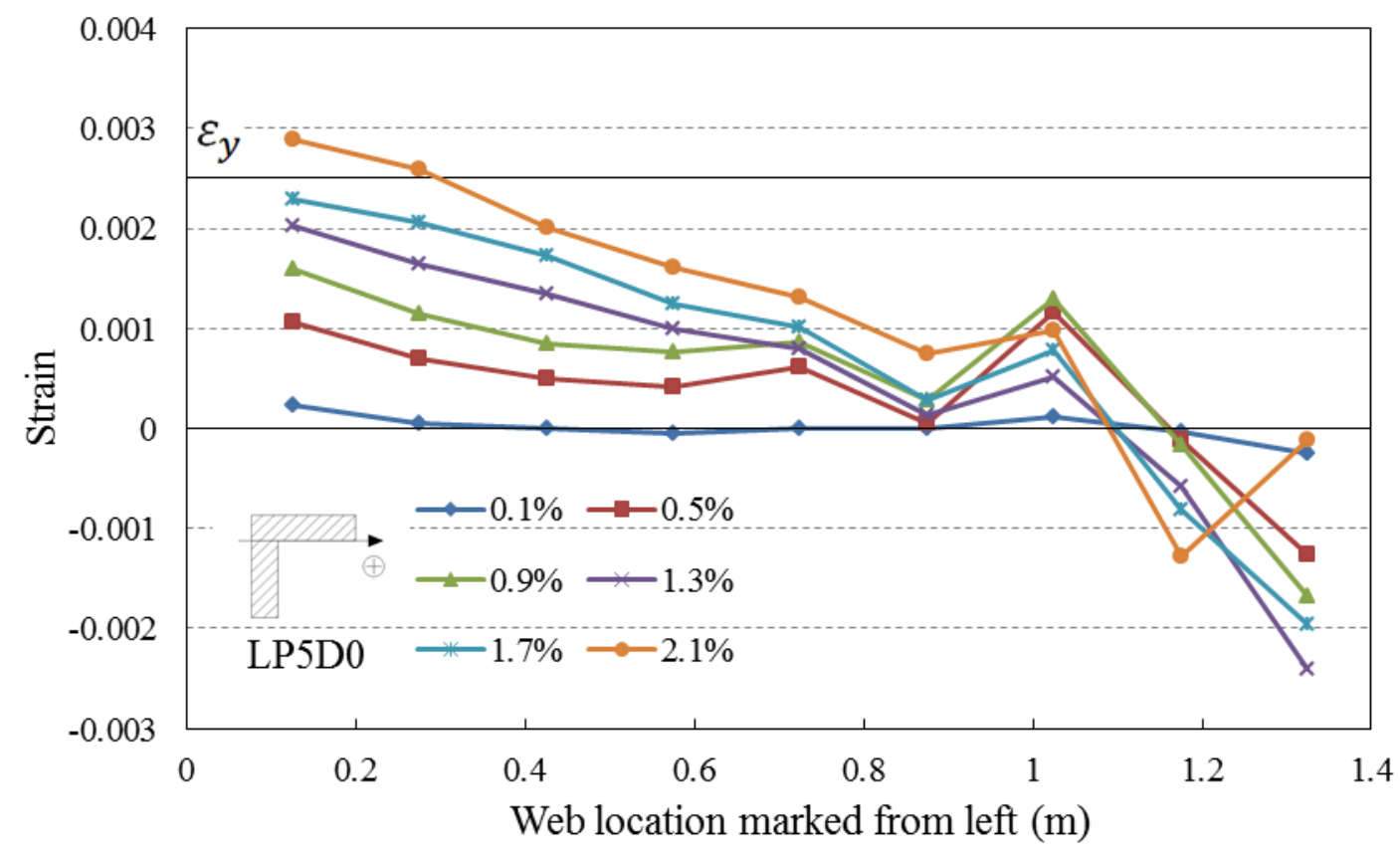

Figure 4.22 Strain profiles of longitudinal reinforcement along the web of Specimen LP5D0 in the positive loading direction

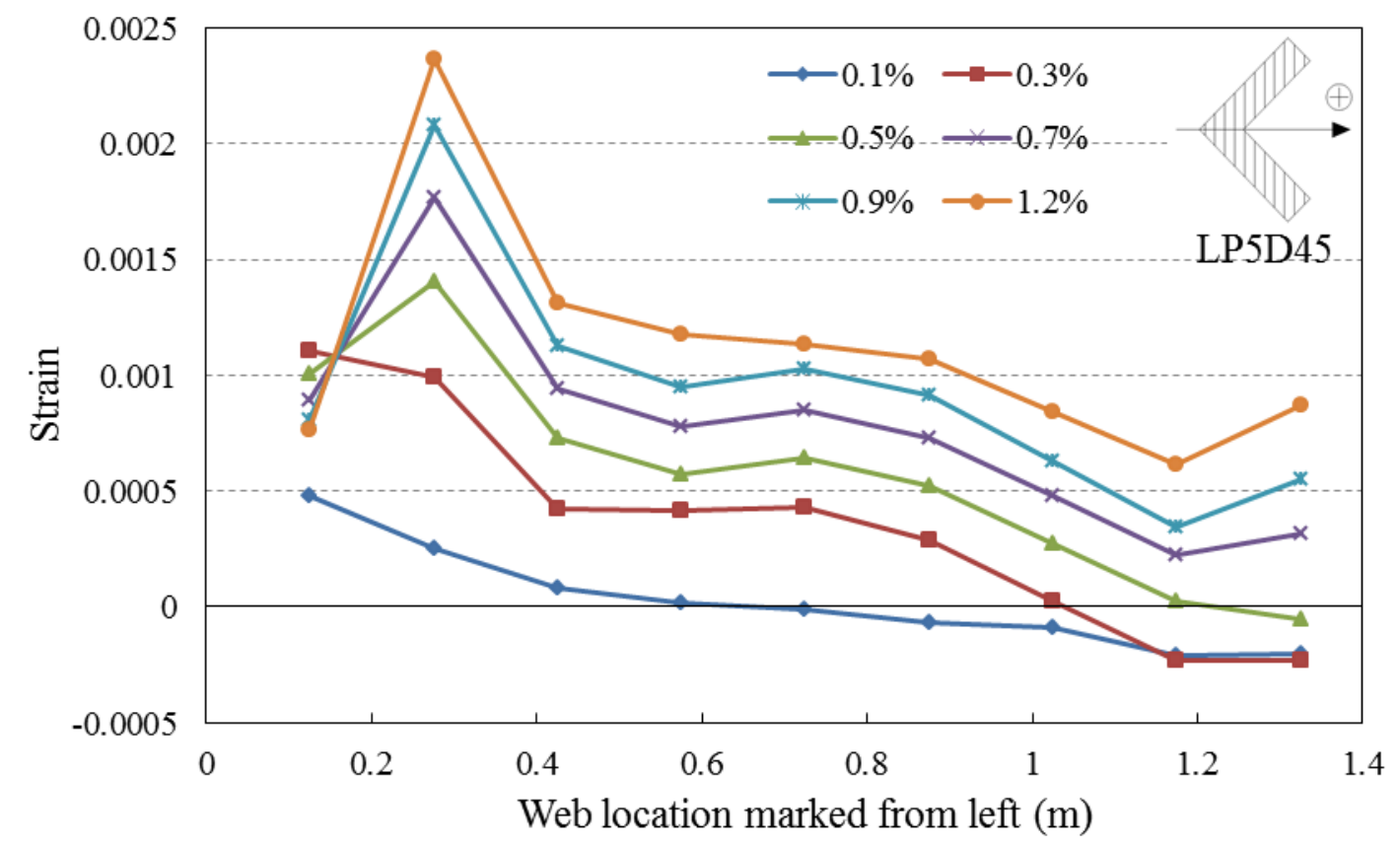

Figure 4.23 Strain profiles of longitudinal reinforcement along the web of Specimen LP5D45 in the positive loading direction 


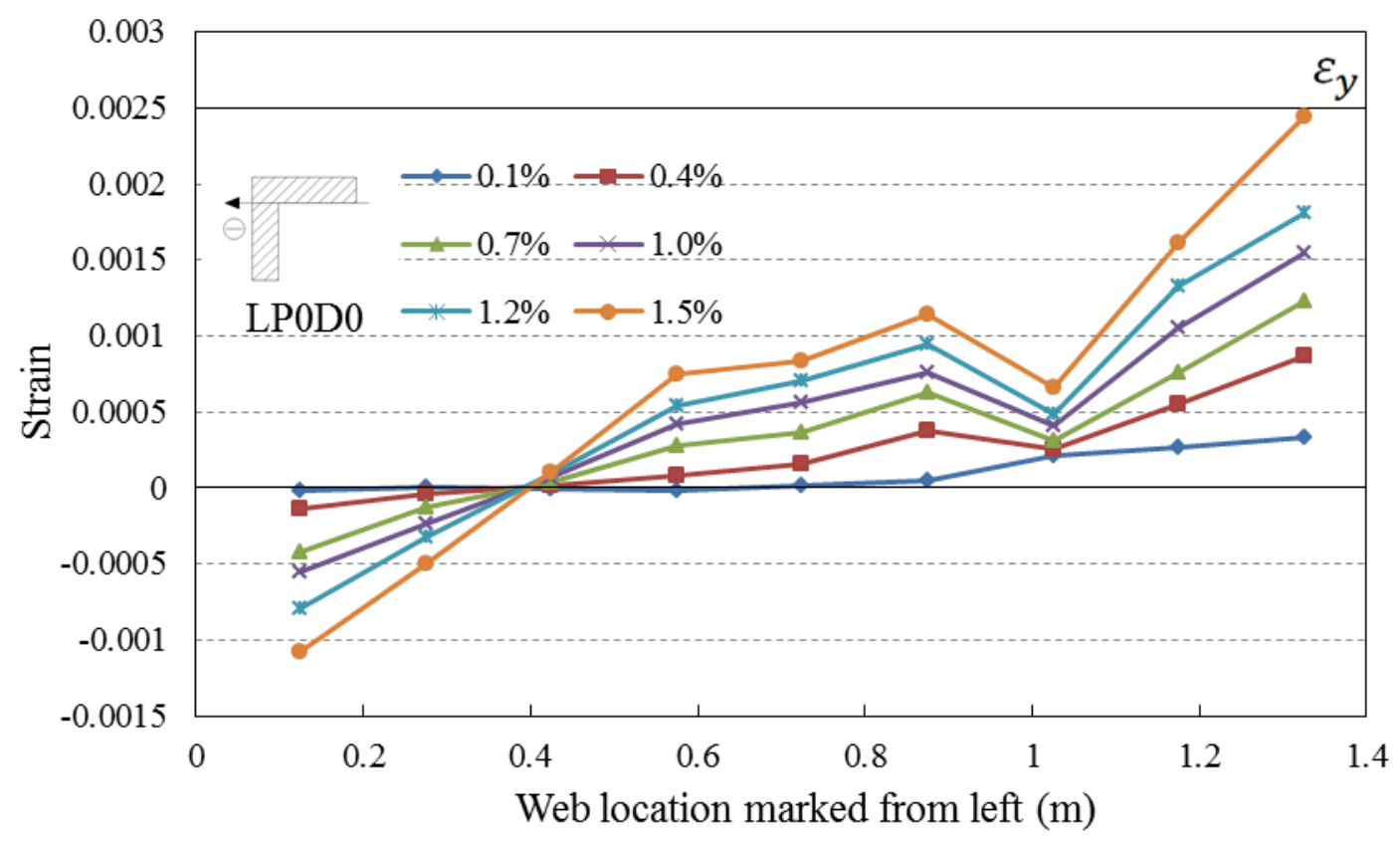

Figure 4.24 Strain profiles of longitudinal reinforcement along the web of Specimen LPOD0 in the negative loading direction

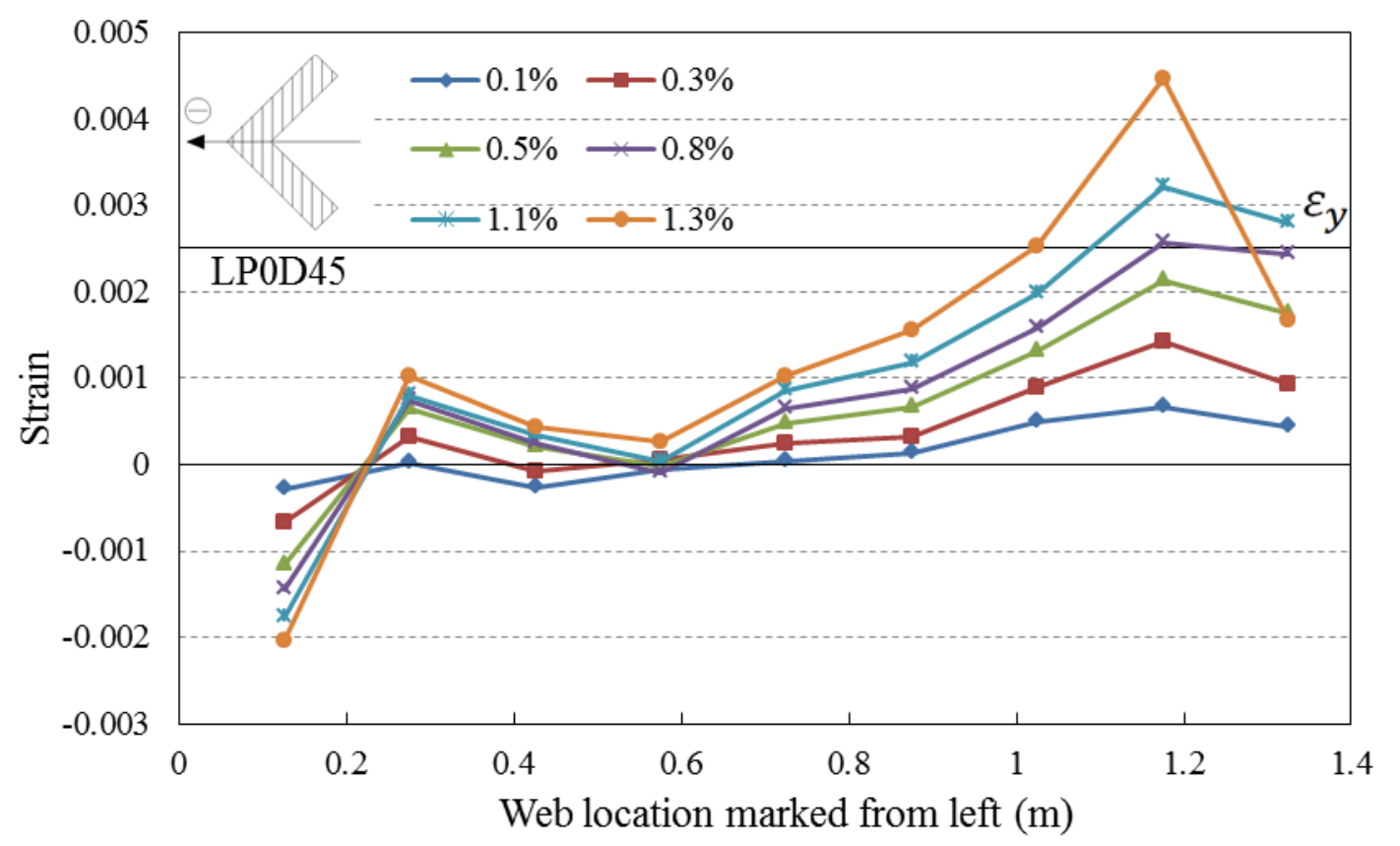

Figure 4.25 Strain profiles of longitudinal reinforcement along the web of Specimen LP0D45 in the negative loading direction 


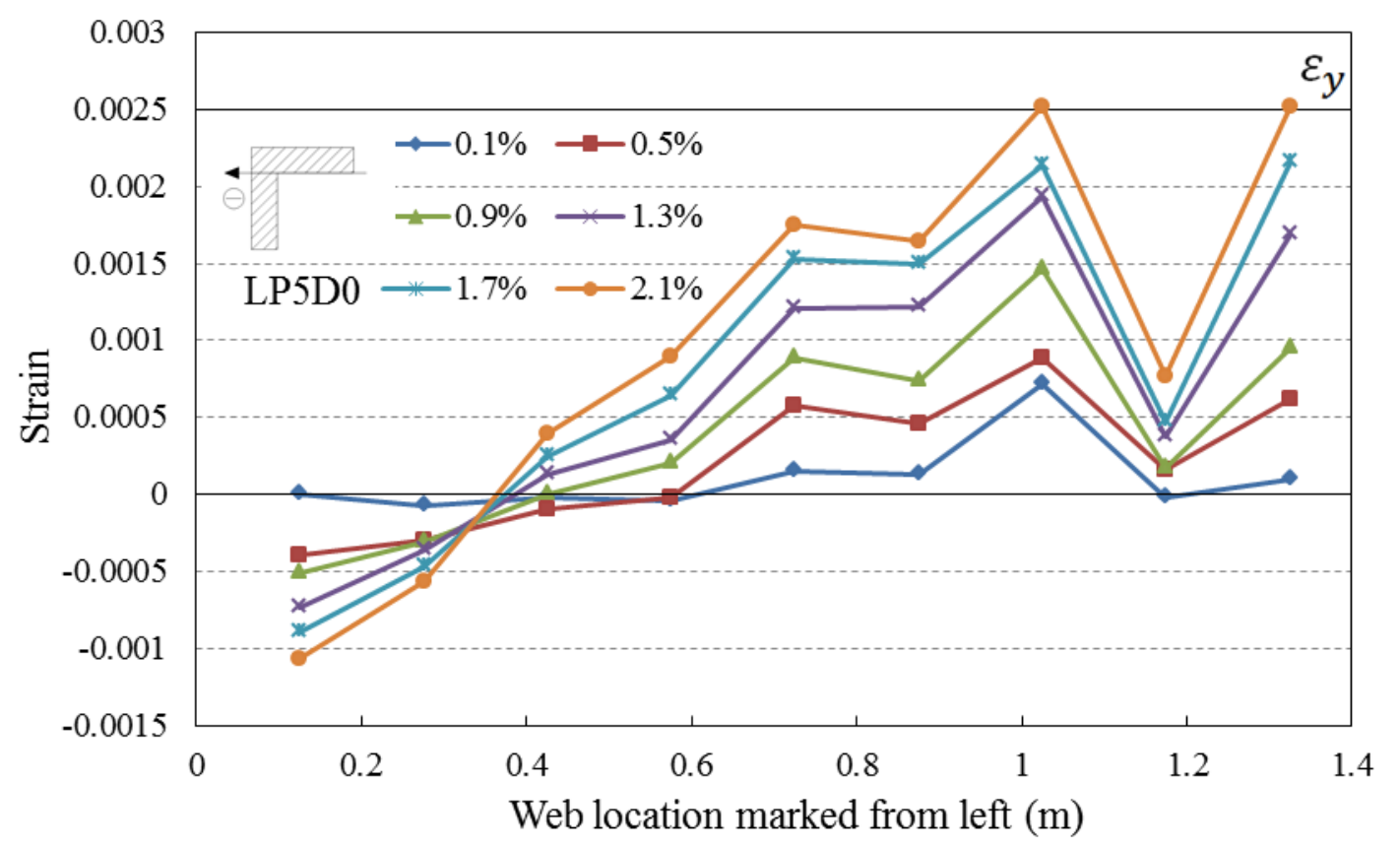

Figure 4.26 Strain profiles of longitudinal reinforcement along the web of Specimen LP5D0 in the negative loading direction

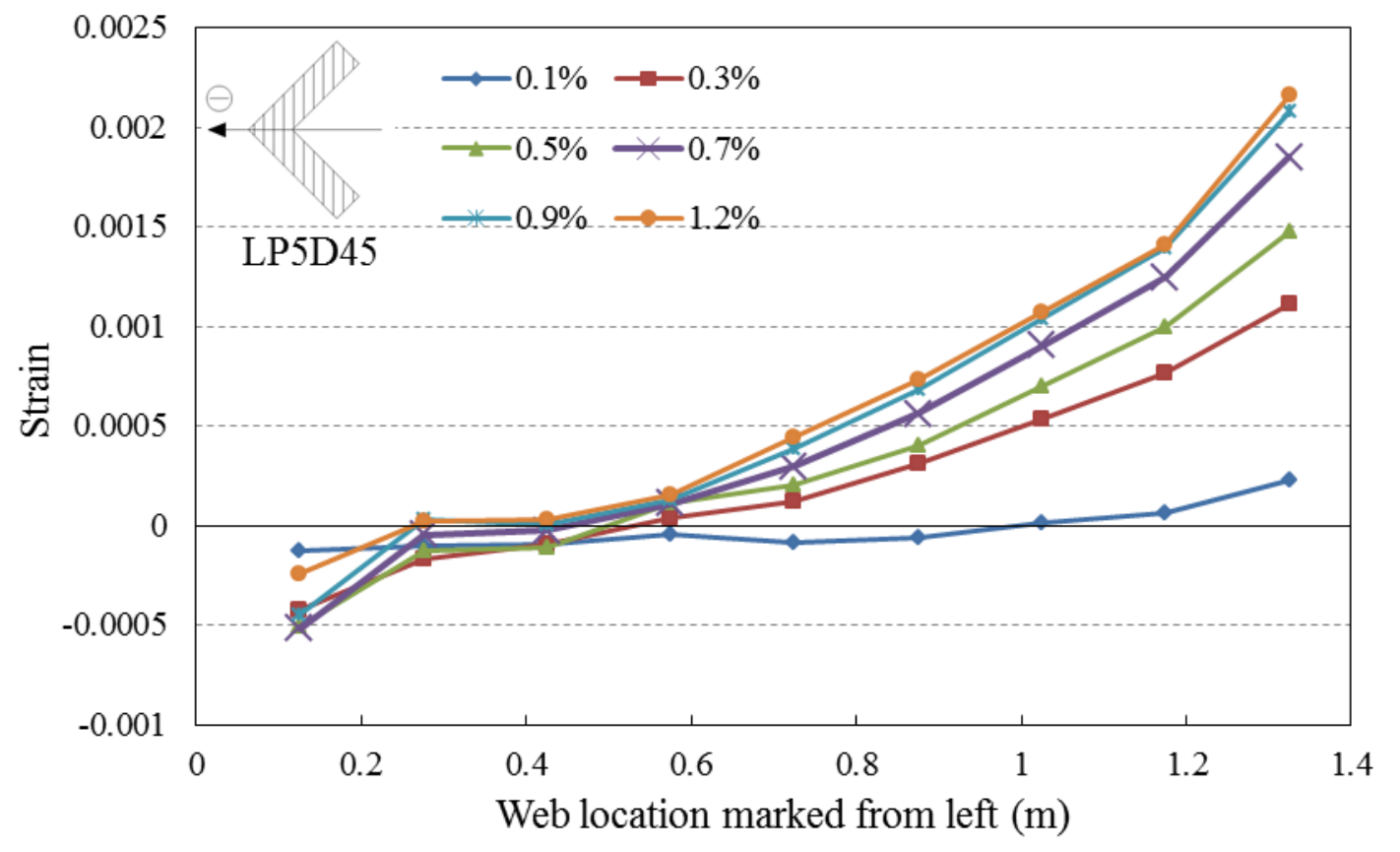

Figure 4.27 Strain profiles of longitudinal reinforcement along the web of Specimen LP5D45 in the negative loading direction 


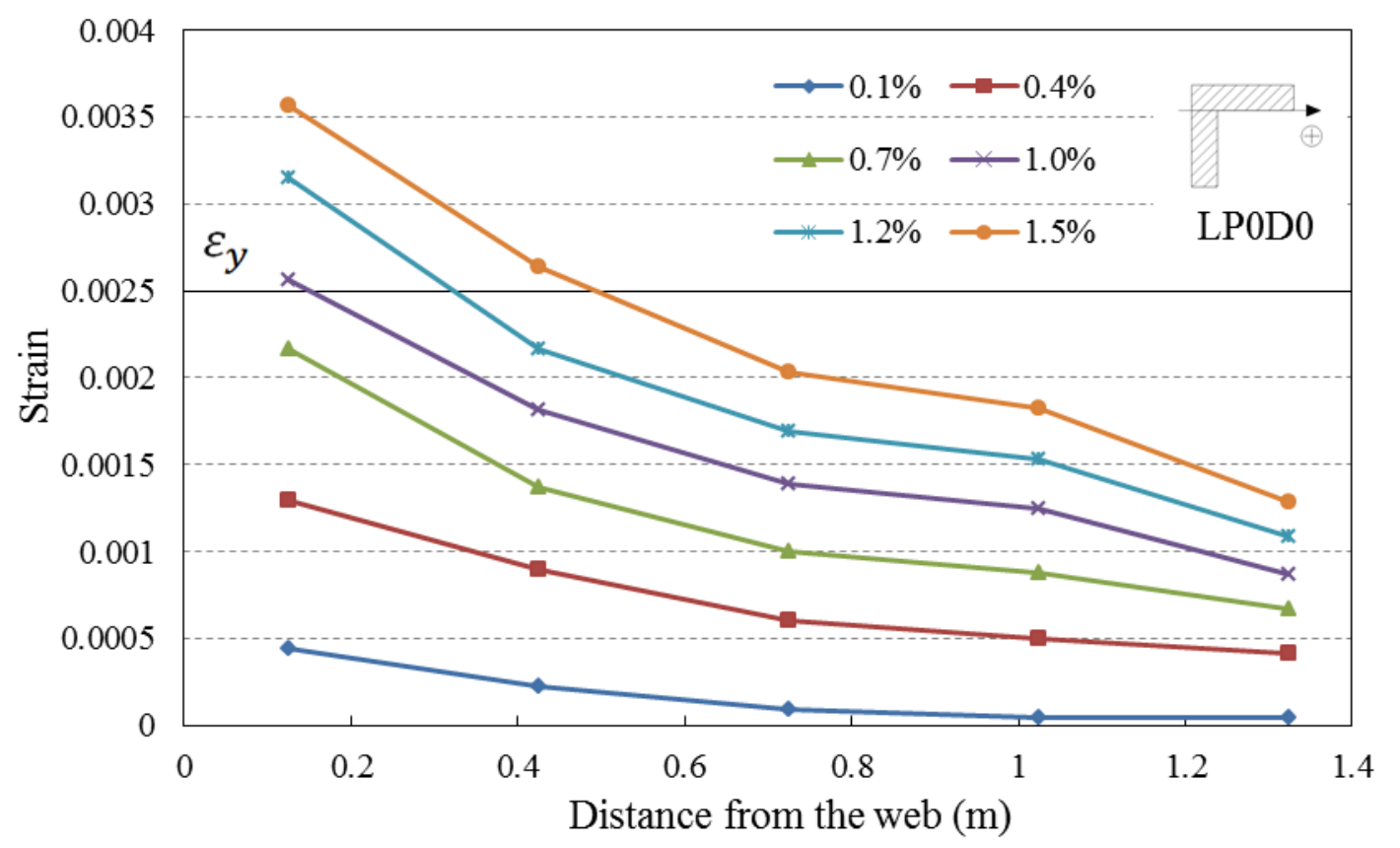

Figure 4.28 Strain profiles of longitudinal reinforcement along the flange of Specimen LPOD0 in the positive loading direction

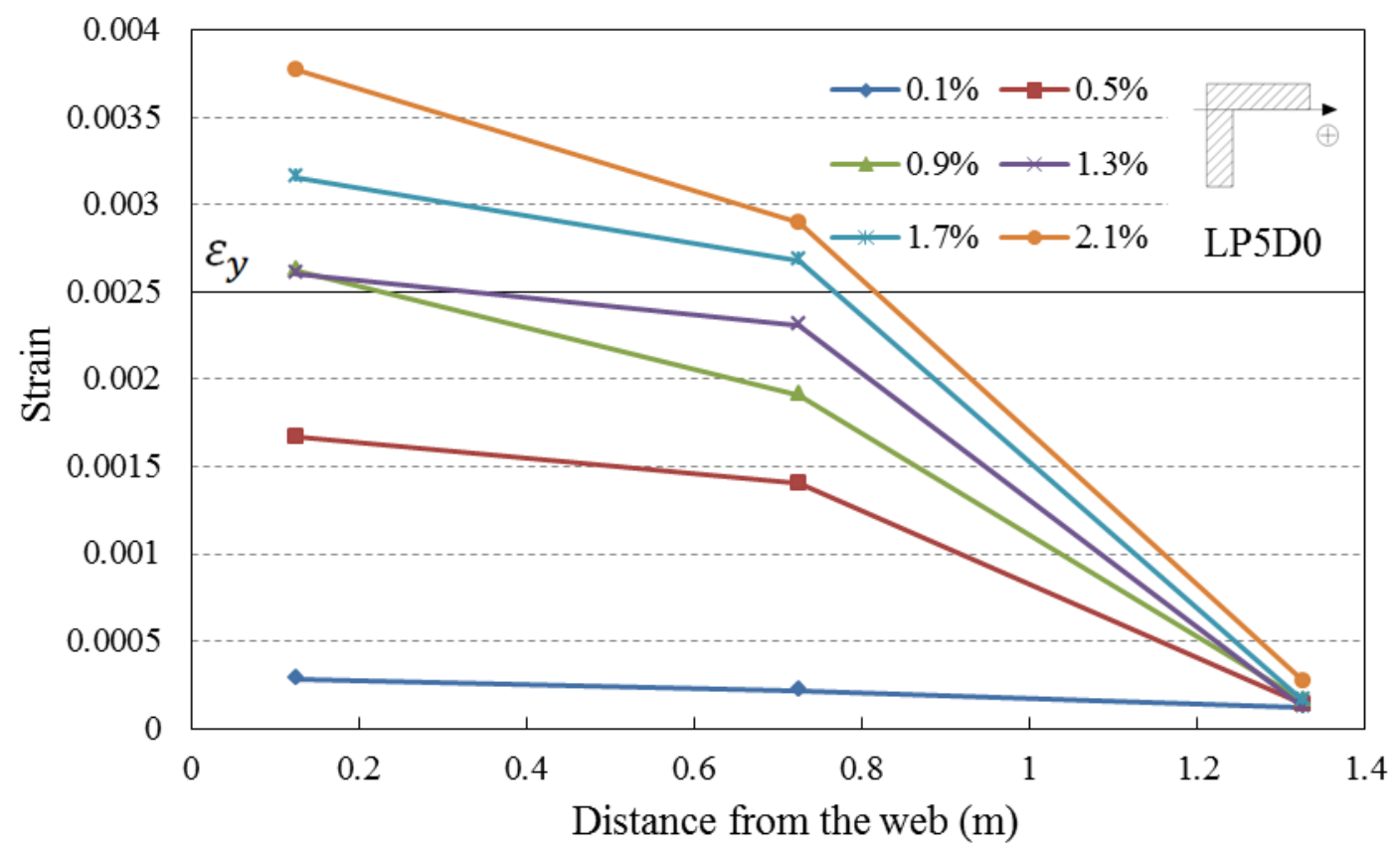

Figure 4.29 Strain profiles of longitudinal reinforcement along the flange of Specimen LP5D0 in the positive loading direction 


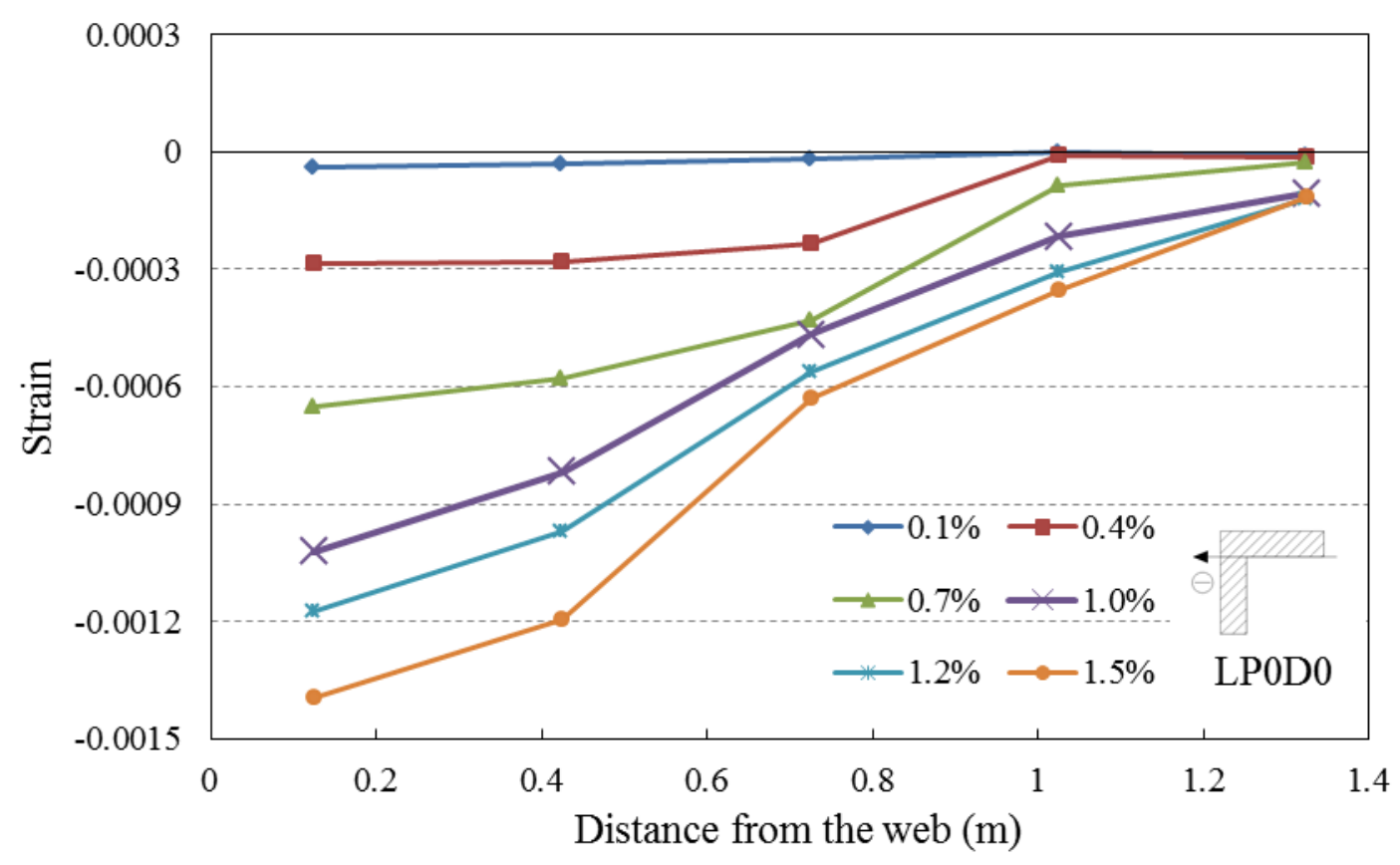

Figure 4.30 Strain profiles of longitudinal reinforcement along the flange of Specimen LPOD0 in the negative loading direction

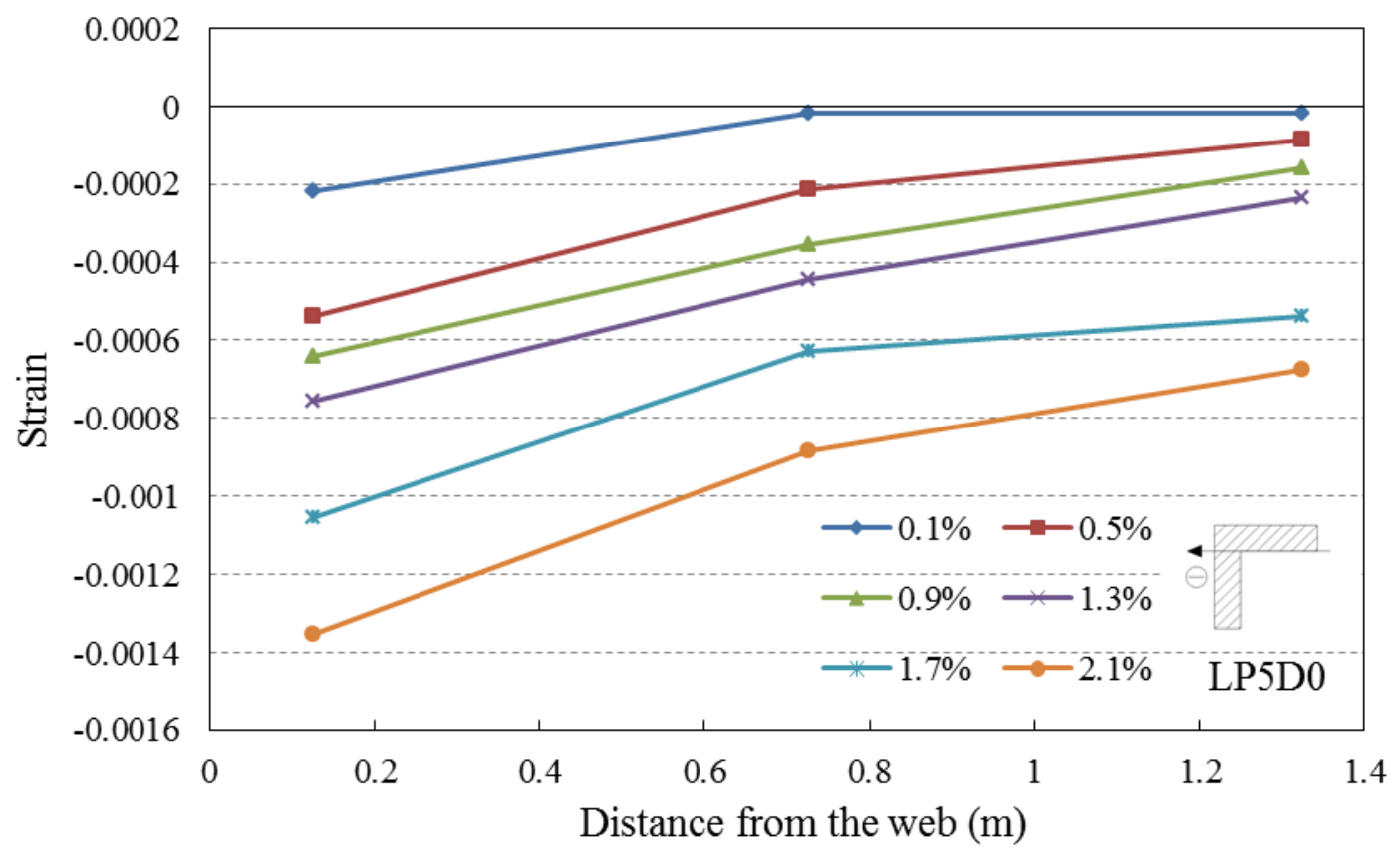

Figure 4.31 Strain profiles of longitudinal reinforcement along the flange of Specimen LP5D0 in the negative loading direction 


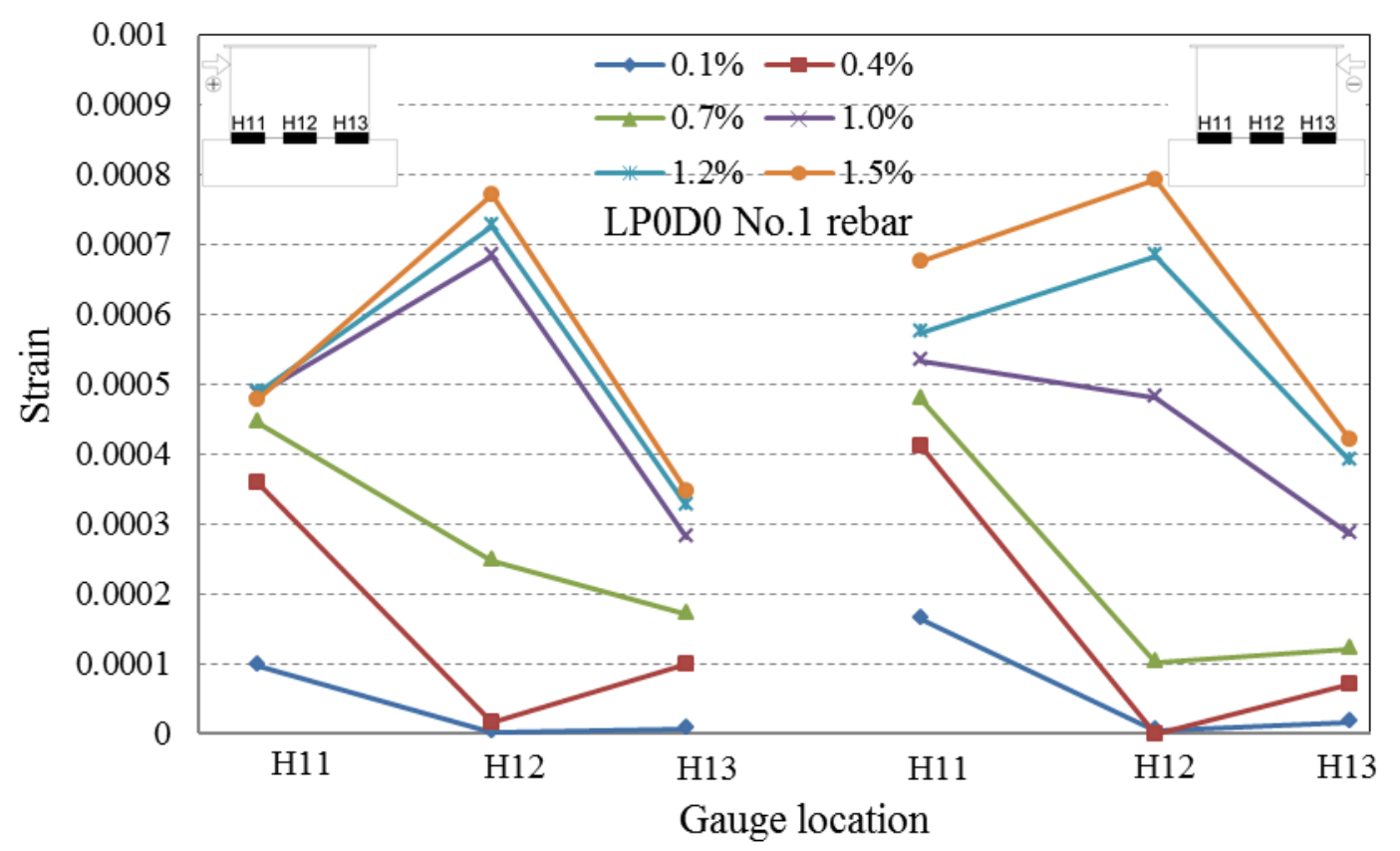

Figure 4.32 Strain profiles of No.1 horizontal rebar of Specimen LP0D0

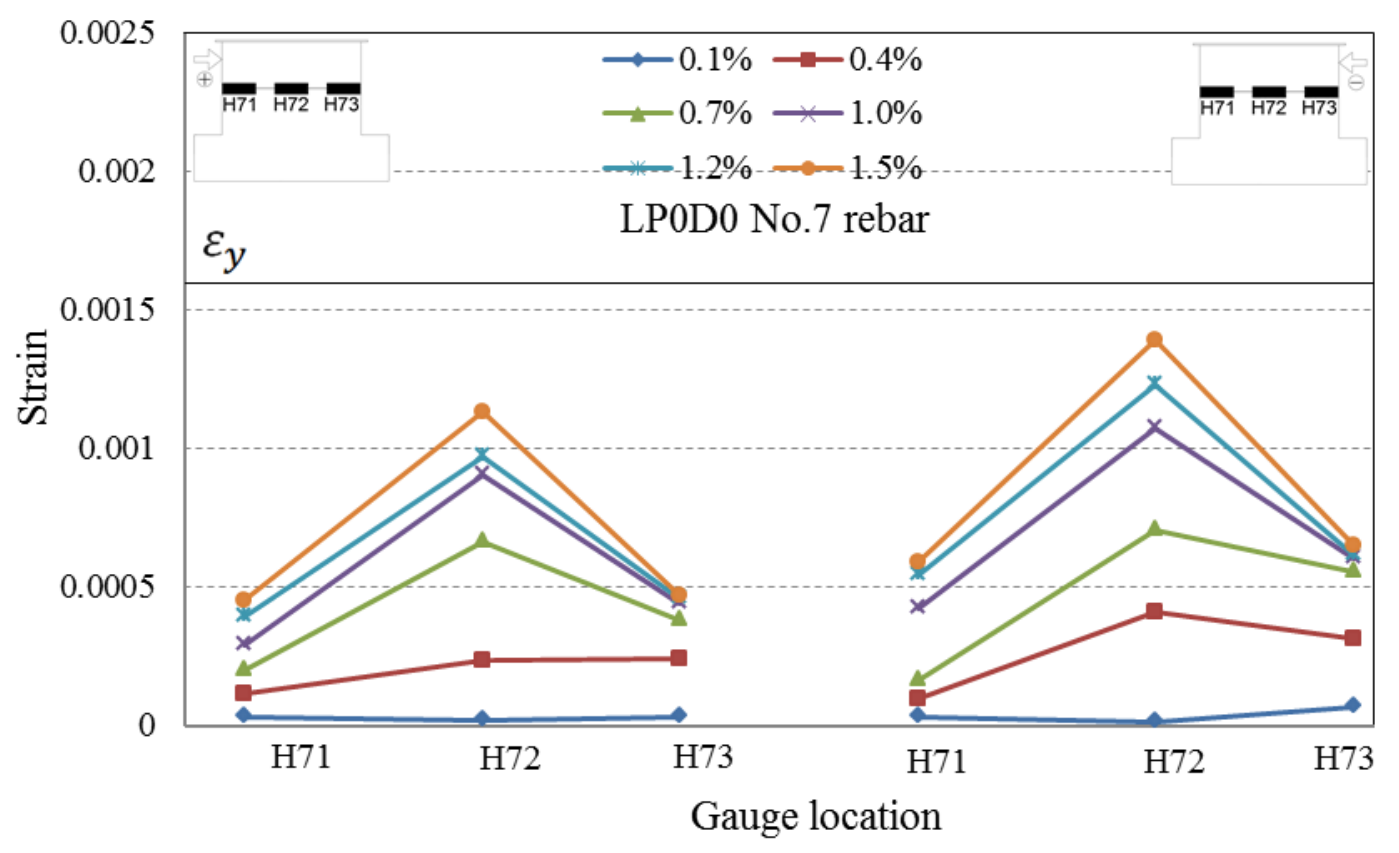

Figure 4.33 Strain profiles of No.7 horizontal rebar of Specimen LP0D0 


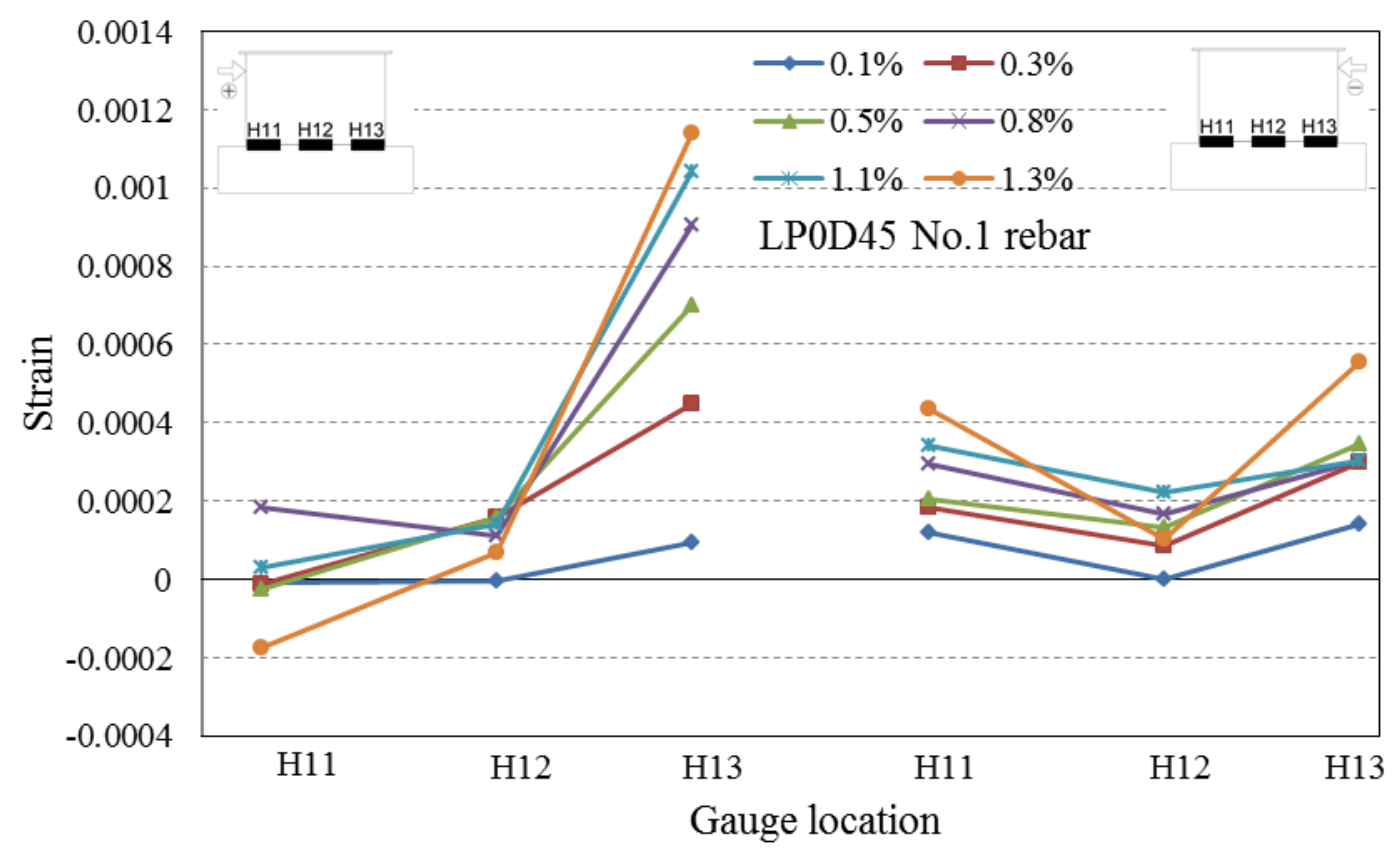

Figure 4.34 Strain profiles of No.1 horizontal rebar of Specimen LP0D45

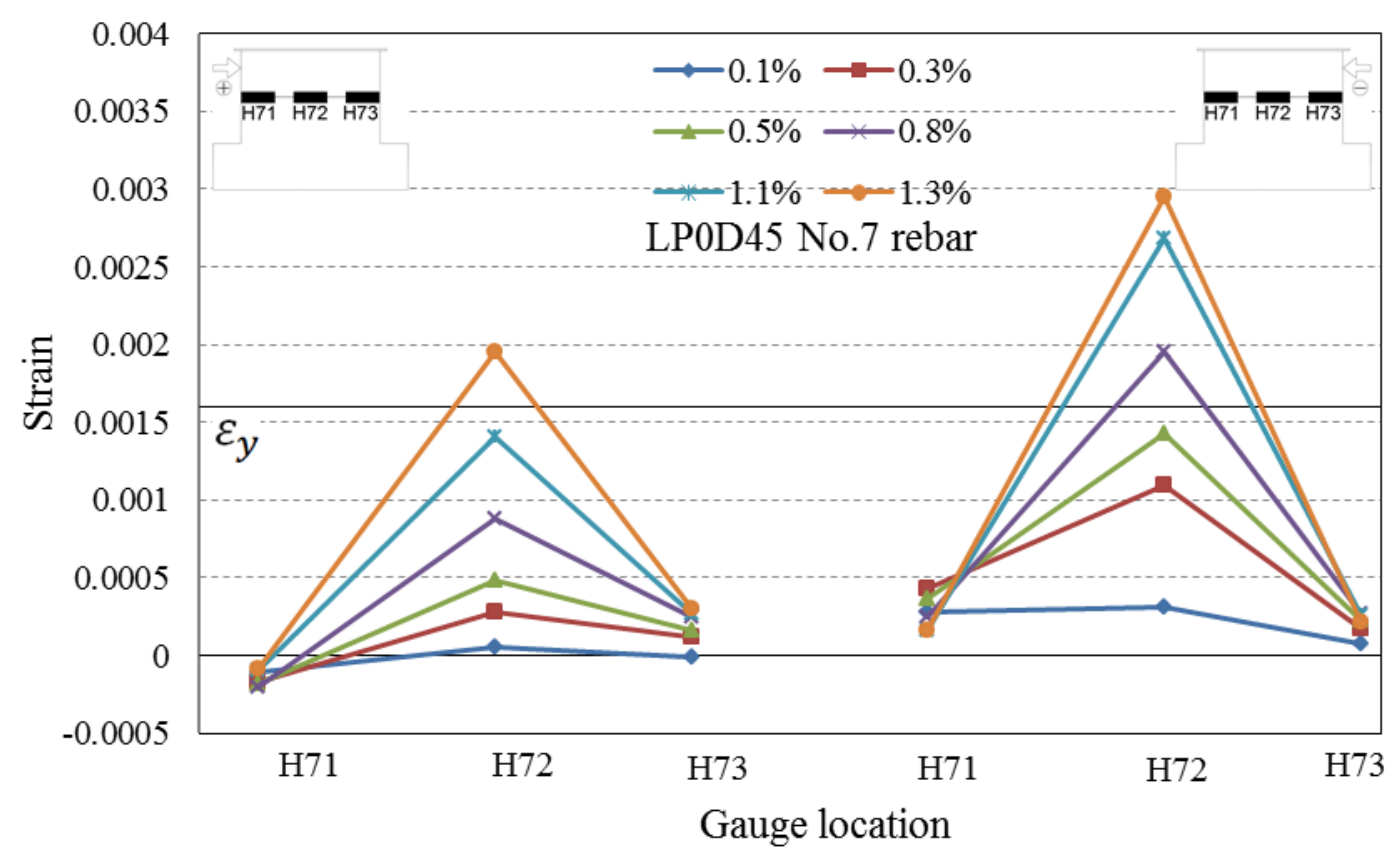

Figure 4.35 Strain profiles of No.7 horizontal rebar of Specimen LP0D45 


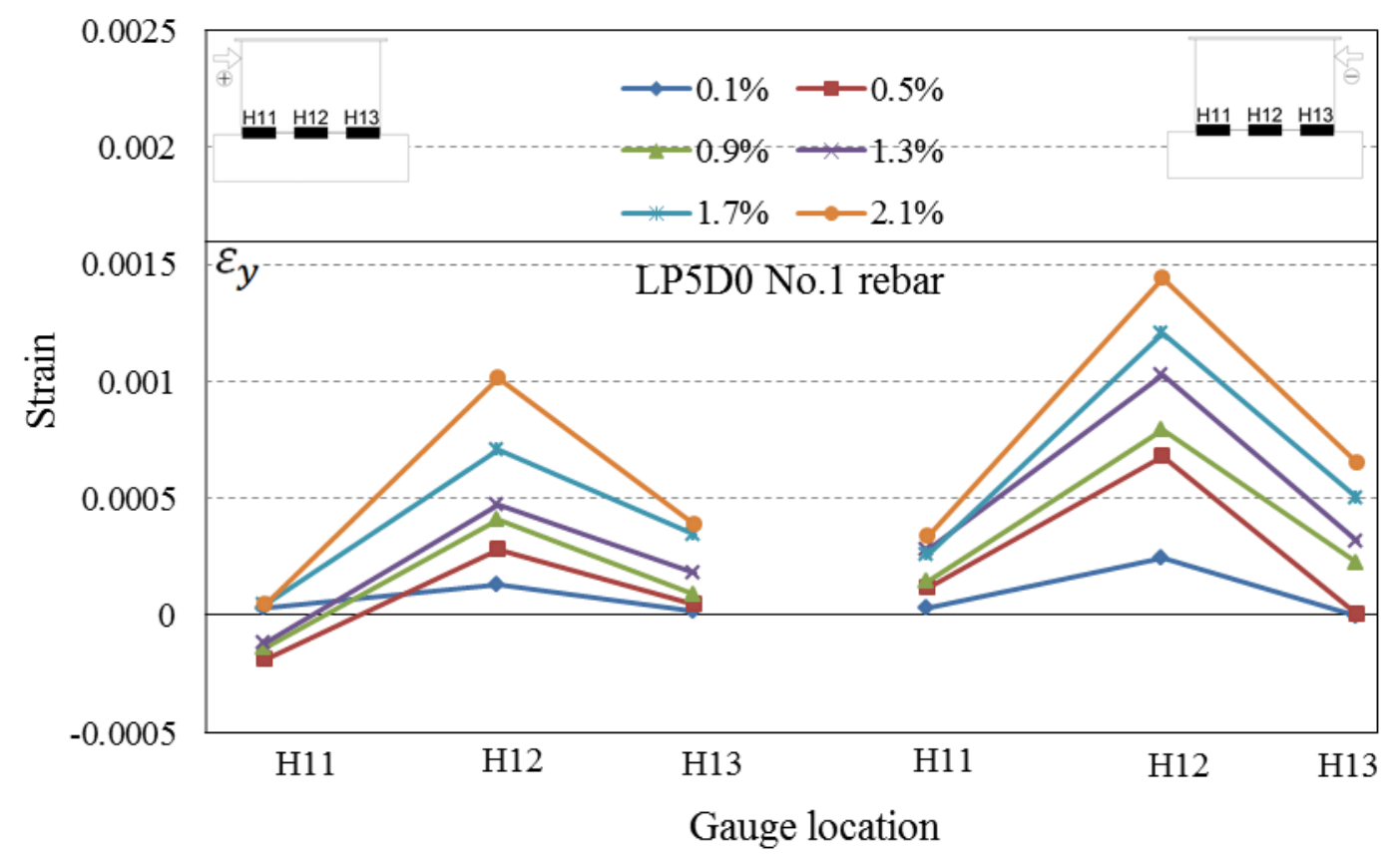

Figure 4.36 Strain profiles of No.1 horizontal rebar of Specimen LP5D0

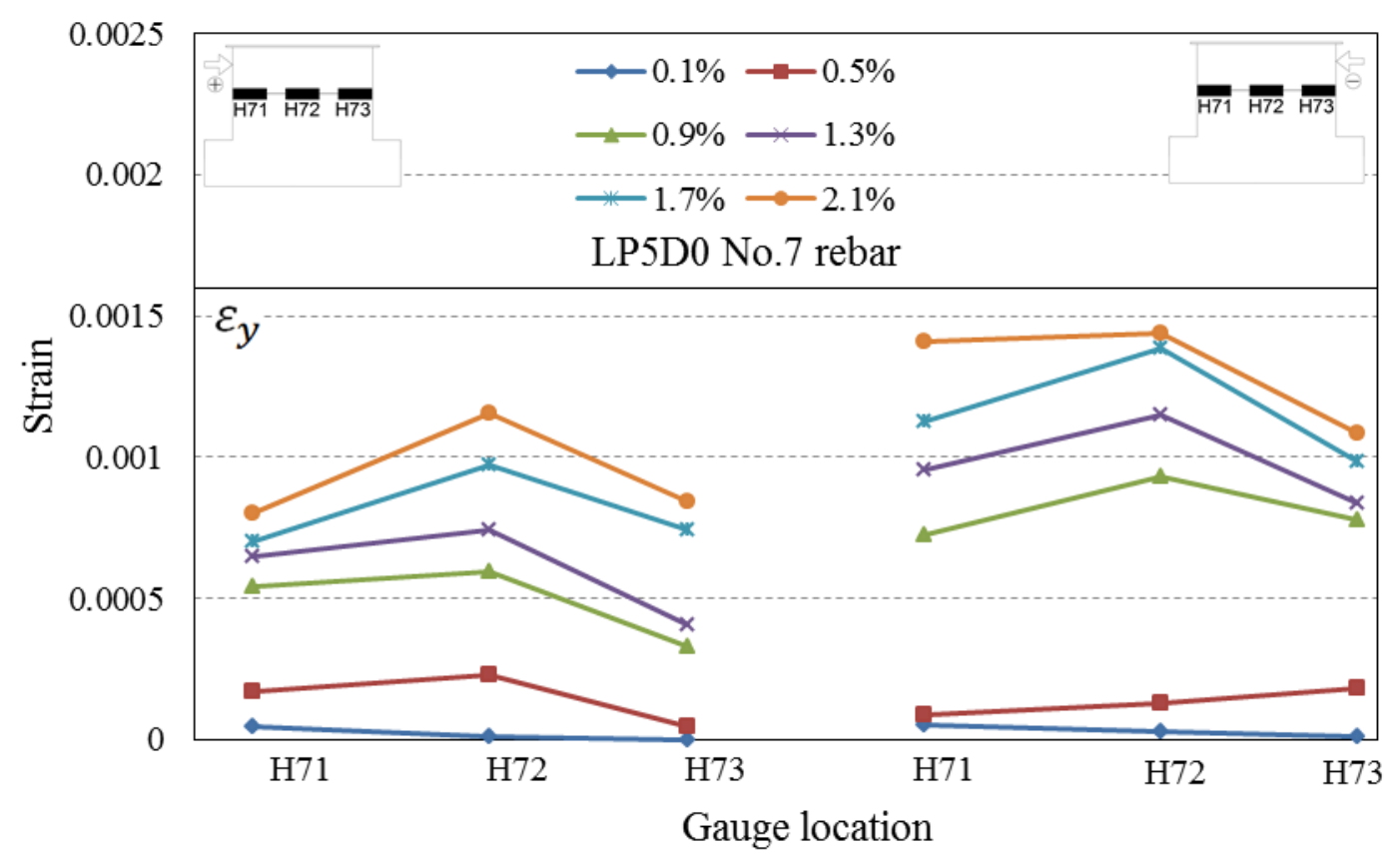

Figure 4.37 Strain profiles of No.7 horizontal rebar of Specimen LP5D0 


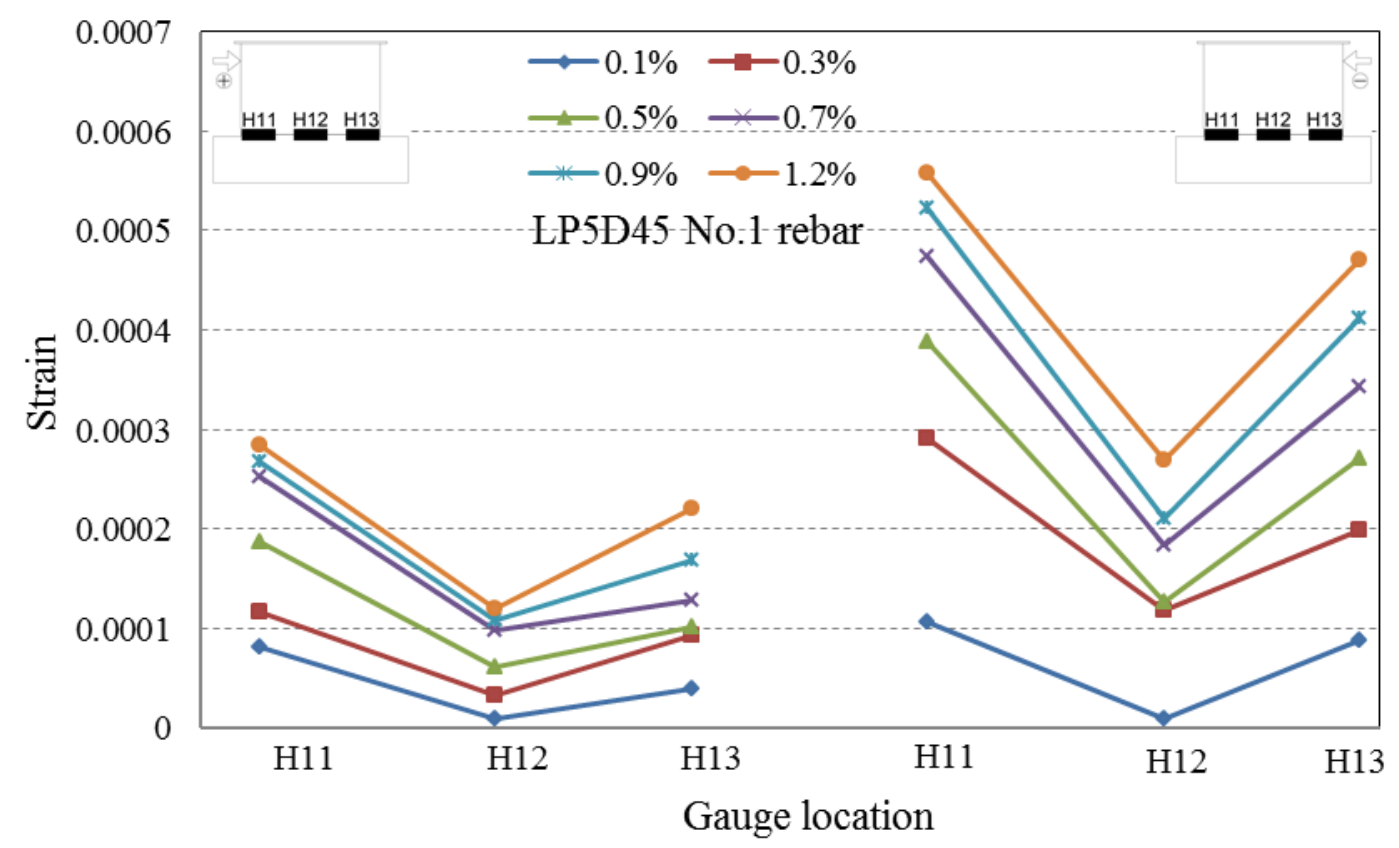

Figure 4.38 Strain profiles of No.1 horizontal rebar of Specimen LP5D45

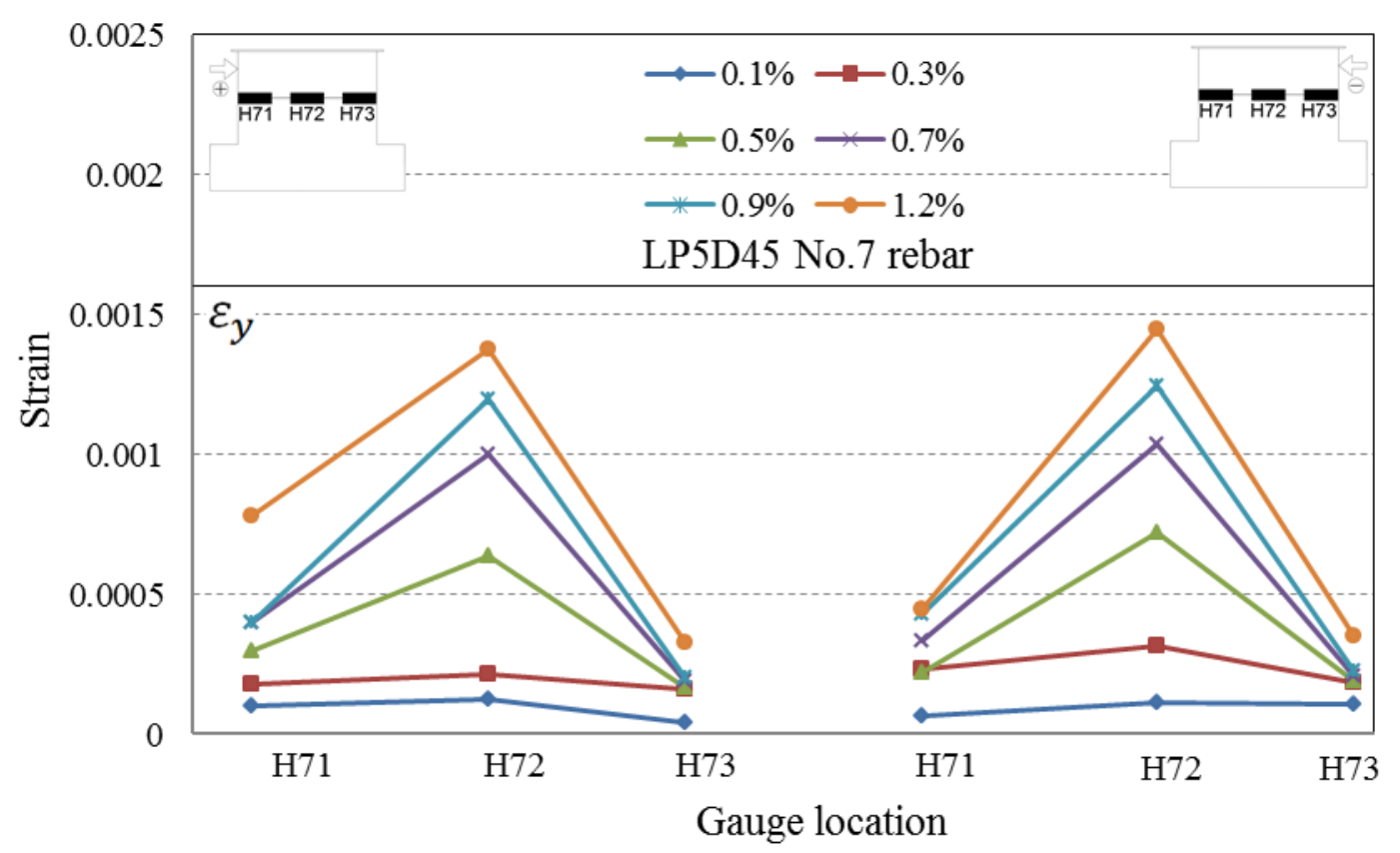

Figure 4.39 Strain profiles of No.7 horizontal rebar of Specimen LP5D45 


\subsection{Experimental Results of the H-shaped Specimens}

\subsubsection{Cracking patterns}

Figure 4.40 to Figure 4.43 demonstrate the development of cracks on each surface of the wall with outlines of reinforcement. The blue lines represent the cracks formed during the positive loading cycles. The red lines represent the cracks formed during the negative loading cycles.

Figure 4.40 below demonstrates the development of cracks on each surface of Specimen HPOD0 at drift ratios of $0.1 \%, 0.7 \%$ and $1.1 \%$ (displacements of $1 \mathrm{~mm}, 7$ $\mathrm{mm}$ and $11 \mathrm{~mm}$ ), which corresponded to the initial stage, the crack development stage, and the final failure stage respectively. As seen from the figure, a diagonal crack located at the web almost $650 \mathrm{~mm}$ above the base quickly appeared when the test began its first horizontal loading circle. The first yielding occurred in the outmost longitudinal reinforcement in the junction of the web and flange at the displacement of $7 \mathrm{~mm}$ in the positive loading direction, with a corresponding shear force of $680 \mathrm{kN}$. With the increasing of the drift ratio, more diagonal cracks occurred and intersected with each other at both faces of the web. Meanwhile, flexural cracks gradually developed on both flange surfaces. On the left flange, flexural cracks extended to nearly the full length of the flange and covered approximately $80 \%$ of the height, while fewer cracks were witnessed on the right flange. As the test progressed to the failure, diagonal cracks on the web became denser, spreading to the entire surfaces. The intersection of cracks caused severe concrete spalling at the upper part of the web, forming a major horizontal crack, and eventually leading to the failure of the specimen, when the lateral strength still showed an ascending trend. A similar premature failure was also observed in Specimen DP2 of Palermo and Vecchio's test [P2]. Causes of this kind of failure were discussed in the next section. On the other hand, both flanges remained relatively intact with a majority of cracks being flexural ones spreading all over the surface. The maximum capacities, $824 \mathrm{kN}$ in the positive and $857 \mathrm{kN}$ in the negative loading directions, were reached at the displacement of $11 \mathrm{~mm}$, respectively. As mentioned above, the specimen failed at the second cycle of 
displacement $11 \mathrm{~mm}$, as a result of concrete spalling and sliding along the major horizontal slip plane at the upper part of the web, which is illustrated in Figure 4.44.
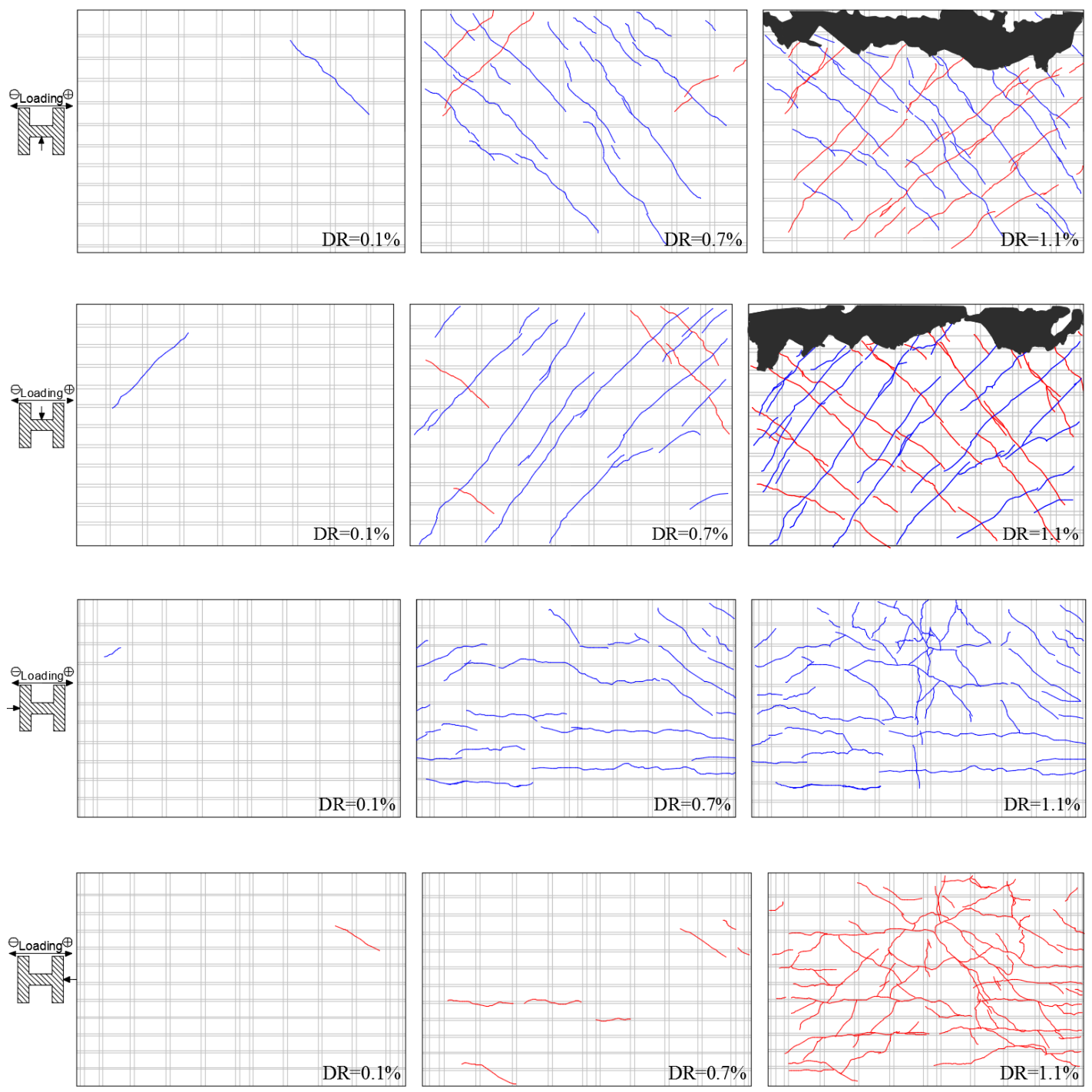

Figure 4.40 Cracking patterns of Specimen HP0D0

The plots of cracking patterns of each face of Specimen HP0D45 at different loading stages are presented in Figure 4.41. A few diagonal cracks appeared at both web and flanges after the first run of horizontal loading cycles. Increasing the drift ratio to $0.6 \%$ in the negative direction resulted in the first yielding of the longitudinal reinforcement near the tip of the flange. As the test progressed, the number of diagonal cracks increased and these cracks quickly intersected with each other on every surface of the specimen. It was noted that both surfaces of the web demonstrated a similar distribution of cracks, while the flange had more cracks 
when it was in tension. With a further increase in the lateral displacement, more diagonal cracks appeared on both sides of the web and extended all over the surface. On the other hand, cracks were mainly concentrated in the upper part of the two flanges. In the positive loading direction, a maximum load of $737 \mathrm{kN}$ was recorded at a corresponding displacement of $6 \mathrm{~mm}$, and a load of $672 \mathrm{kN}$ at a displacement of $7 \mathrm{~mm}$ was attained in the negative direction. After that, concrete spalling was observed at the upper left corner of the web, as a result of concentrated diagonal cracks formed in the negative loading direction. The specimen experienced a gradual drop of shear strength after the peak and finally failed at the drift ratio of $0.9 \%$.
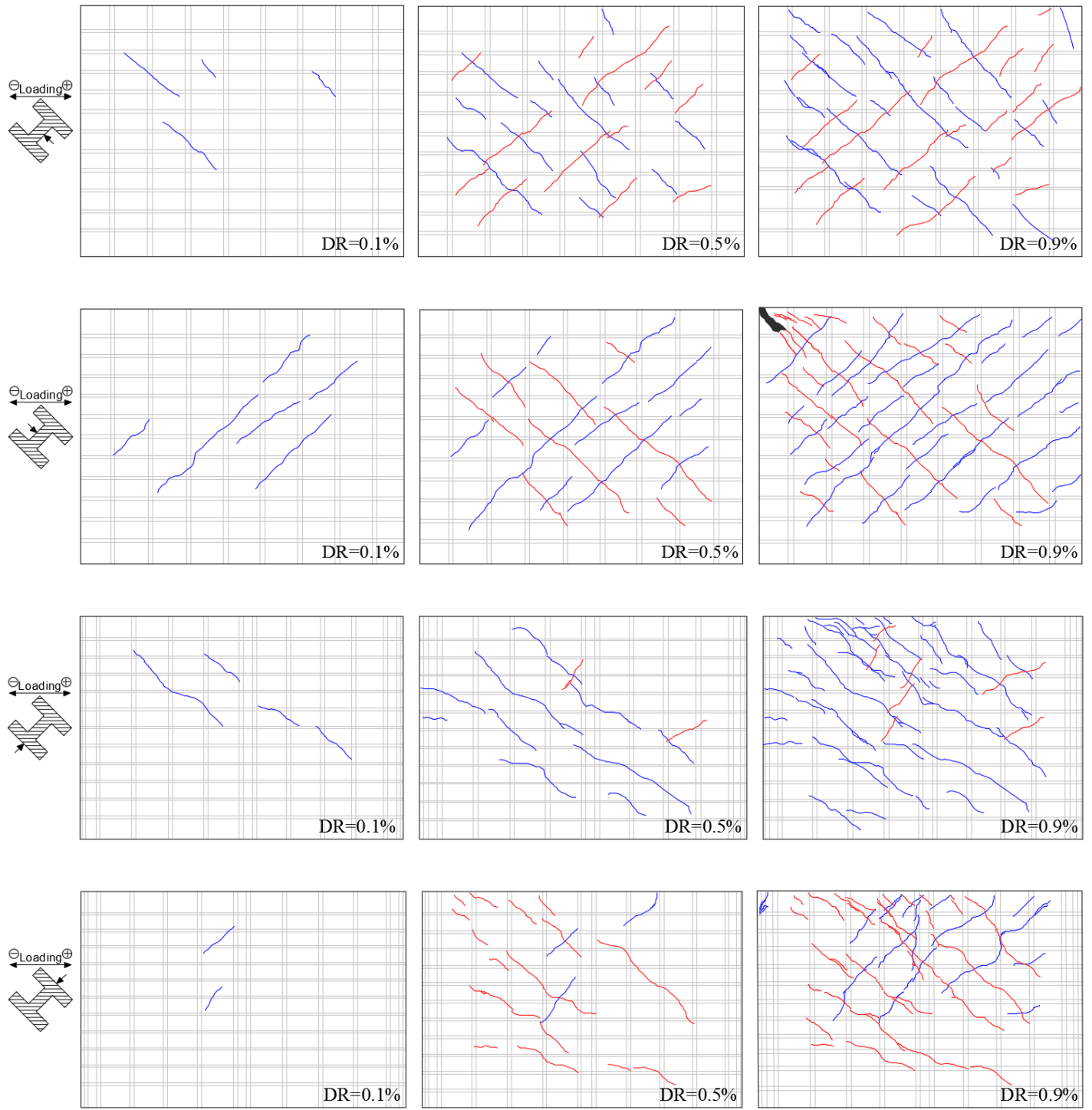

Figure 4.41 Cracking patterns of Specimen HP0D45 
As far as Specimen HP5D0 was concerned, the first cracks were also detected when the lateral displacement reached $1 \mathrm{~mm}$. As the test progressed, cracks grew quite fast and quickly spread all over the web surfaces before the drift ratio arrived at $0.7 \%$. It was clear that compared with Specimen HP0D0 with no axial load, the angle of diagonal cracks located on the web was apparently steeper in this specimen, which was in accordance with findings of past experiments [S10, Z2]. This phenomenon was attributed to the effect of vertical stress brought by the axial load, which changed the angle of the concrete strut to contribute more towards the vertical loads. Also, slight concrete spalling was witnessed at the middle of the web. The flanges of the specimen, meanwhile, demonstrated large numbers of horizontal cracks, which extended from the junction of wall segments through the entire width of the flange. After that, the longitudinal reinforcement located in the juncture of the web and flange experienced yielding at a drift ratio of $0.8 \%$. In the subsequent loading cycles, the development of cracks slowed down in terms of the number, while the width of cracks largely increased, causing severe concrete spalling at the upper part of the web and the following formation of a major horizontal slip plane. Moreover, from the drift ratio of $0.7 \%$, surface concrete spalling was witnessed at the middle of the web. During the unloading phase, crack surfaces were not able to re-align, which caused a grinding of two surfaces, and resulted in concrete crushing along the crack surfaces. As the test progressed, several vertical slip planes on both sides of the web gradually formed, which is presented in Figure 4.46. A similar observation was also recorded in Specimen DP1 of Palermo and Vecchio's test [P2]. The maximum shear strength and the corresponding displacement in the positive direction were $907 \mathrm{kN}$ and $8 \mathrm{~mm}$, and $995 \mathrm{kN}$ and $7 \mathrm{~mm}$ for the negative loading direction. The specimen shared a similar failure mode with Specimen HP0D0 and failed at a displacement of $11 \mathrm{~mm}$ due to sliding and concrete spalling in the top. 

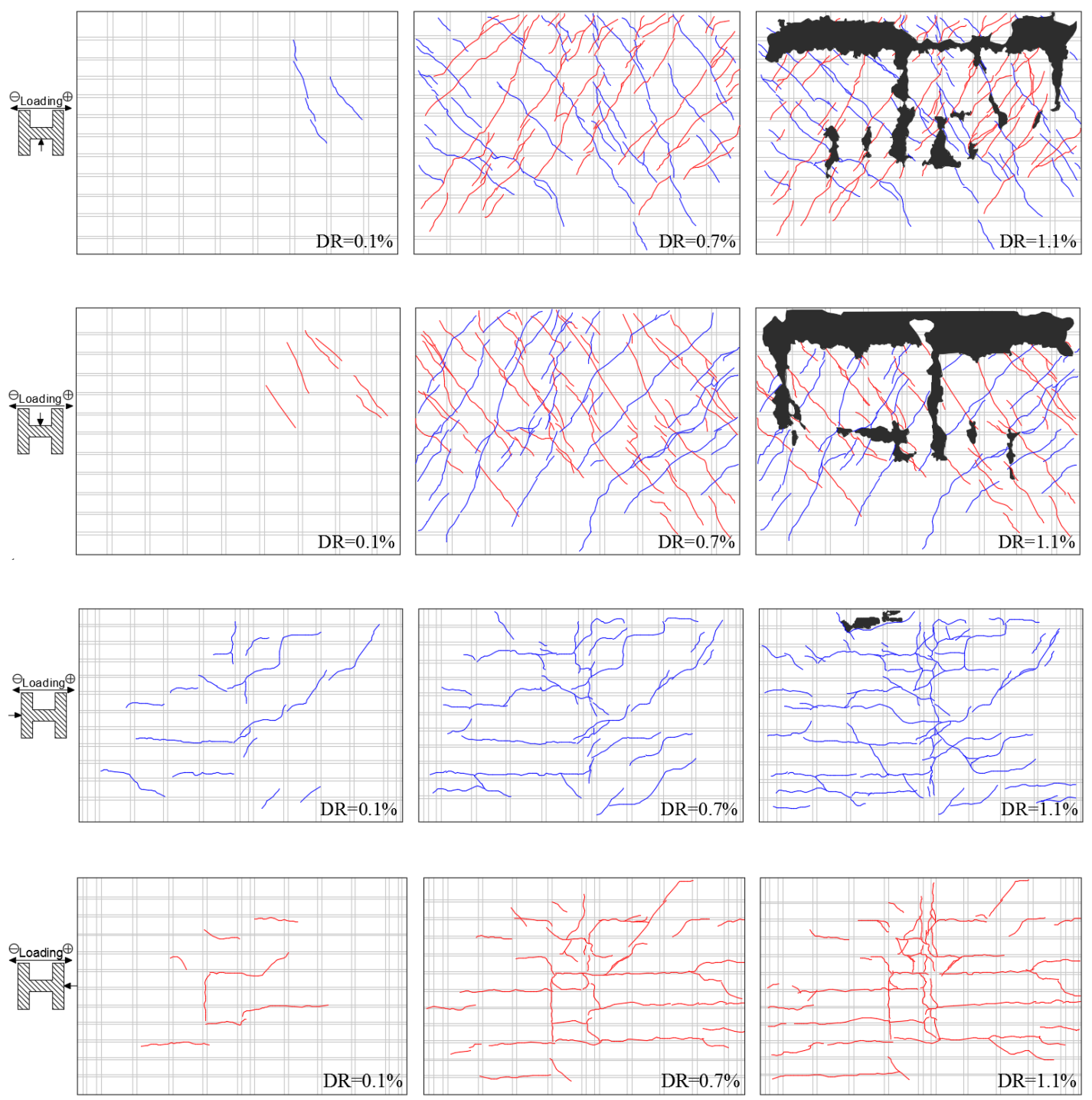

Figure 4.42 Cracking patterns of Specimen HP5D0

As for Specimen HP5D45, the lateral displacement of $1 \mathrm{~mm}$ caused cracks on each face of the wall. The longitudinal reinforcement in the flange attained its first yielding at the displacement of $7 \mathrm{~mm}$, corresponding to a shear force of $954 \mathrm{kN}$. With the increase in the drift ratio, more diagonal cracks occurred on the web and demonstrated a symmetric distribution. The two flanges, on the other hand, showed a slight different cracking pattern, with a majority of their cracks forming when it was in tension. The restraint brought by the axial load on the development of cracks was not evident in this specimen compared with the previous group. As the test was carried on, the specimen reached its maximum shear strength of approximately $1132 \mathrm{kN}$ in the positive loading direction, and $981 \mathrm{kN}$ in the negative loading 
direction at the lateral displacements of $10 \mathrm{~mm}$ and $12 \mathrm{~mm}$ respectively. With lateral loads further increasing, the inclined cracks continued to appear. The widest diagonal crack appeared at the juncture of the web and flange, as shown in Figure 4.45. Meanwhile, the shear strength of the wall experienced a steady drop. The specimen failed at the drift ratio of $1.5 \%$.
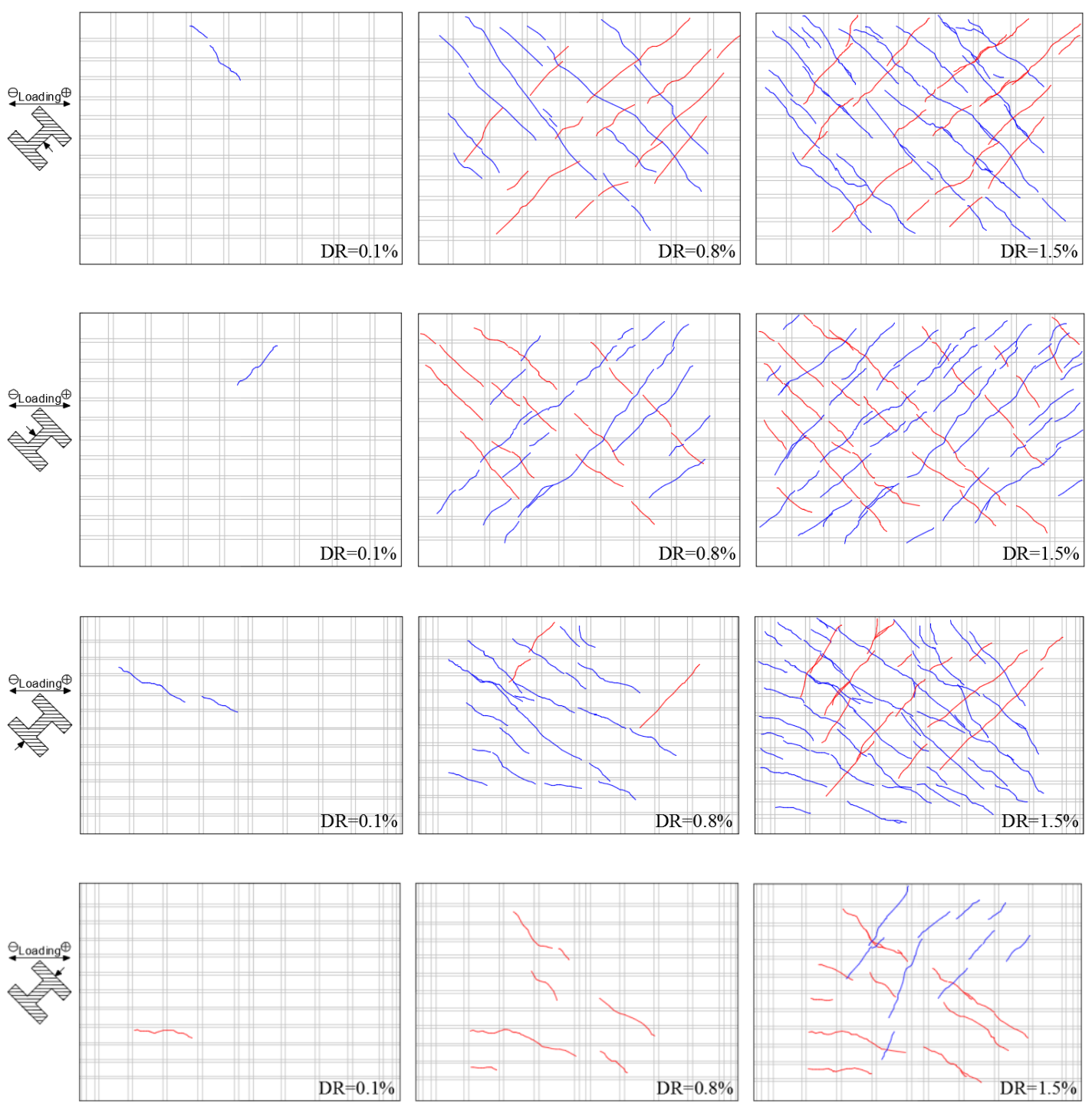

Figure 4.43 Cracking patterns of Specimen HP5D45

From the tests, it was clear that all specimens had their initial cracks on four surfaces after the first run of the lateral loads, which was equal to a displacement of $1 \mathrm{~mm}$. After that, more diagonal cracks appeared, intersected with each other and gradually spread all over the web. It was observed that Specimen HP5D0 with more axial loads had a steeper angle of inclined cracks on its web when compared with 
Specimen HP0D0. It was also witnessed that the two surfaces of the web generally showed a similar distribution of cracks. As for the cracking patterns on flanges, Specimens HP0D0 and HP5D0 with orthogonal lateral loading had most of their cracks as horizontal flexural cracks, which extended to the full length of the flange as lateral loads increased, while in Specimens HP0D45 and HP5D45 with skew lateral loading, more diagonal shear cracks occurred. Besides, cracks only formed in the flange when it was in tension. The specimens experienced their first yielding at a drift ratio ranging from $0.6 \%$ to $0.8 \%$. As the test progressed, denser cracks on the web caused severe concrete spalling at the upper part and the following formation of a major horizontal slip plane in walls with orthogonal lateral loading. As a result, Specimens HP0D0 and HP5D0 failed due to the sliding along the cracking plane. Moreover, several vertical slip planes were discovered in Specimen HP5D0, which further deteriorated the lateral resistance of the specimen. As for Specimens HP0D45 and HP5D45 with skew lateral loading, both specimens were able to sustain a ductile post-peak response before failure. The accumulated cracks on the web caused severe concrete spalling at the upper left corner of the web, leading to the failure of Specimen HPOD45. Reduced concrete spalling was witnessed in Specimen HP5D45, where the diagonal cracks in the junction of the web and flange became evidently wider than those around the center of the wall panel. The ultimate drift ratios of these specimens ranged from $0.9 \%$ to $1.5 \%$.

\subsubsection{Failure mechanisms}

As illustrated in Figure 4.44, The Specimen HPOD0 failed as severe concrete spalling at the upper part of the web formed a major horizontal sliding plane, when the lateral strength still showed an ascending trend. A similar premature failure was also observed in Specimen DP2 of Palermo and Vecchio's test [P2]. The formation of the horizontal sliding plane was believed to be associated with the weaker concrete adopted and the stress concentration caused by the stiff top slab, as presented in the last section. However, unlike the major horizontal crack developed in Specimens LP5D0 and LP5D45, the concrete crushing in Specimen HP0D0 extended to the full length of the web, which caused the top part to slide along the weakened horizontal plane, and eventually led to the failure of the specimen. 
Besides the horizontal sliding plane near the top slab, several distinguished vertical slip planes were found on both sides of the web of Specimen HP5D0, as a result of surface concrete spalling starting from the drift ratio of $0.7 \%$. A similar failure mechanism was also recorded in Specimen DP1 of Palermo and Vecchio's test [P2] as presented in Figure 4.42. Palermo and Vecchio concluded that axial loads and stiff flanges restrained the opening of the shear cracks. Thus large shear stresses were introduced in the web by the significant shear distortion, leading to the formation of vertical slip planes. This explanation was considered plausible. As presented previously, the existence of additional axial loads increased the vertical vector of the internal stress in the web, thereby ascending the inclination of diagonal shear cracks. More importantly, the flexure-dominated flange restrained the sheardominated web from moving, which meant a deformation incompatibility existed in this H-shaped squat wall. Under cyclic lateral loading, the web experienced tensile stresses in the opposite direction to the lateral displacements, which 'tear' the web along the weak part and caused the vertical slip planes. The model accounting for the deformation incompatibility will be illustrated in the next chapter.

As for Specimens HP0D45 and HP5D45 with skew lateral loading, both specimens were able to sustain a ductile post-peak response before failure. As lateral displacements further rose, a slight drop of shear strength was observed but in general, these two specimens maintained their strengths for several cycles. Meanwhile, the diagonal shear cracks became much wider, especially in the upper corner of the wall segment. As seen in Figure 4.45, inclined cracks resulted from the negative loading direction concentrated at the left upper corner of the web, causing moderate concrete spalling, leading to the failure of Specimen HP0D45 at the drift ratio of $0.9 \%$. Reduced concrete spalling was witnessed in Specimen HP5D45, where the diagonal crack in the junction of the web and flange became evidently wider than those around the center of wall panel (Figure 4.47). As the test progressed, the integrity of the web and flange continued to diminish, which impaired the wall's ability to resist horizontal loads. Hence the specimen gradually lost its lateral resistance by more than $30 \%$ of the maximum, and failed at the drift ratio of $1.5 \%$. 


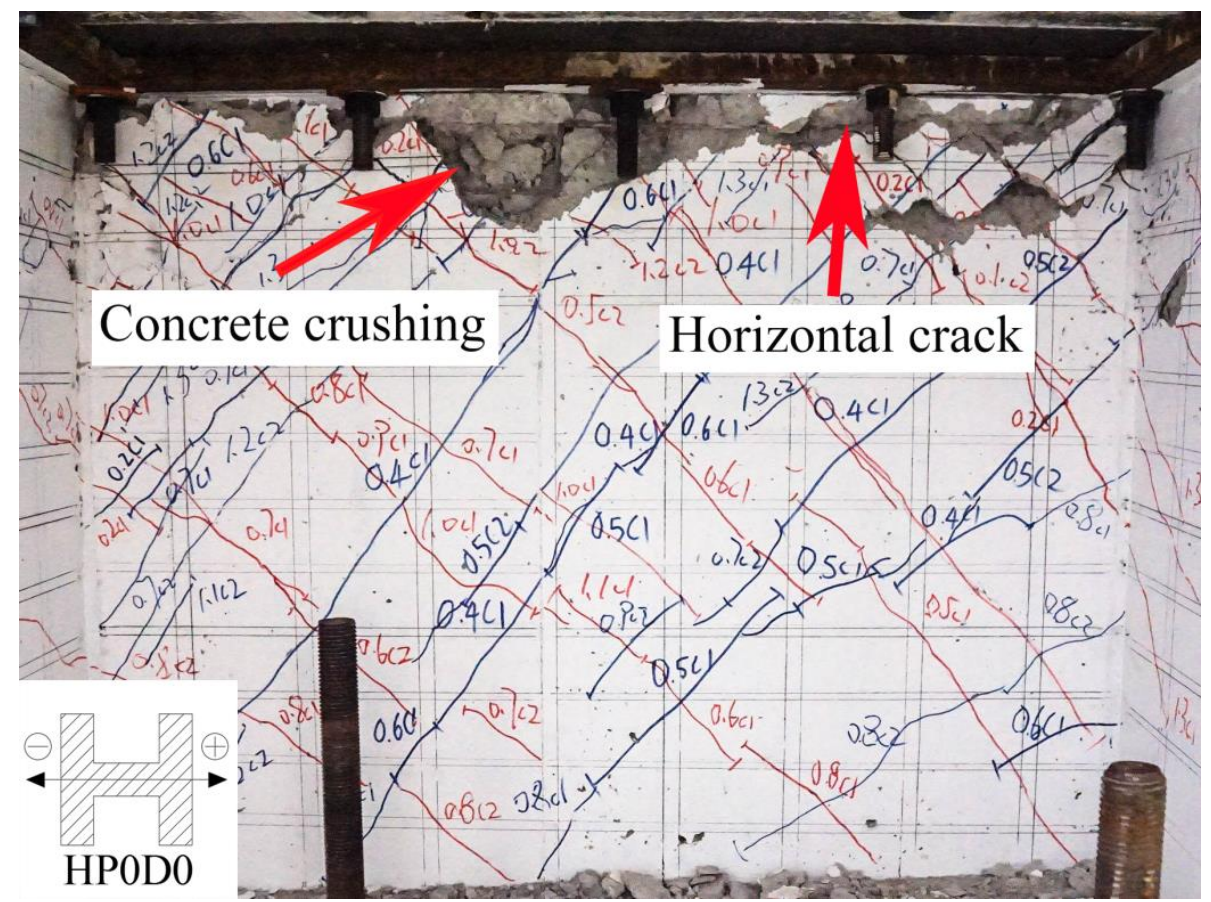

Figure 4.44 Failure mechanism of Specimen HP0D0

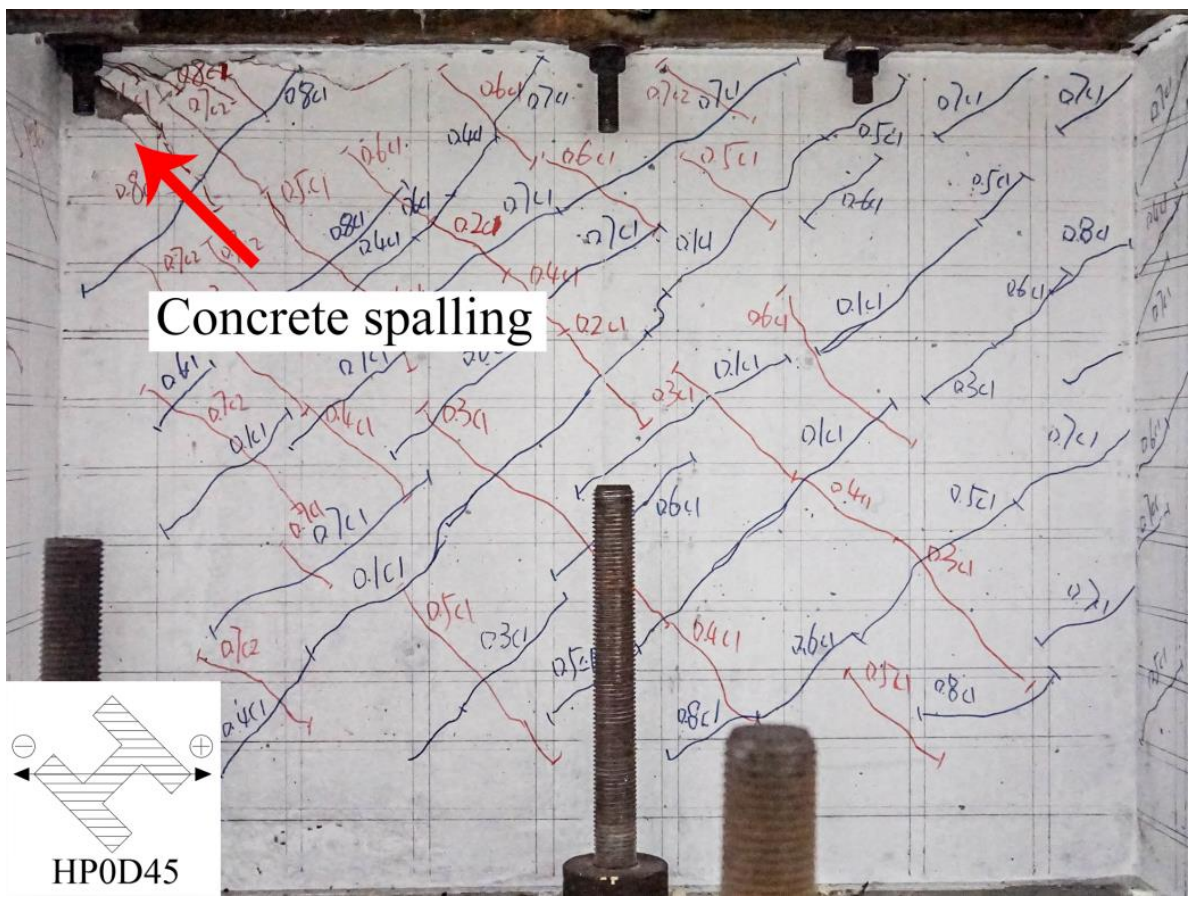

Figure 4.45 Failure mechanism of Specimen HP0D45 


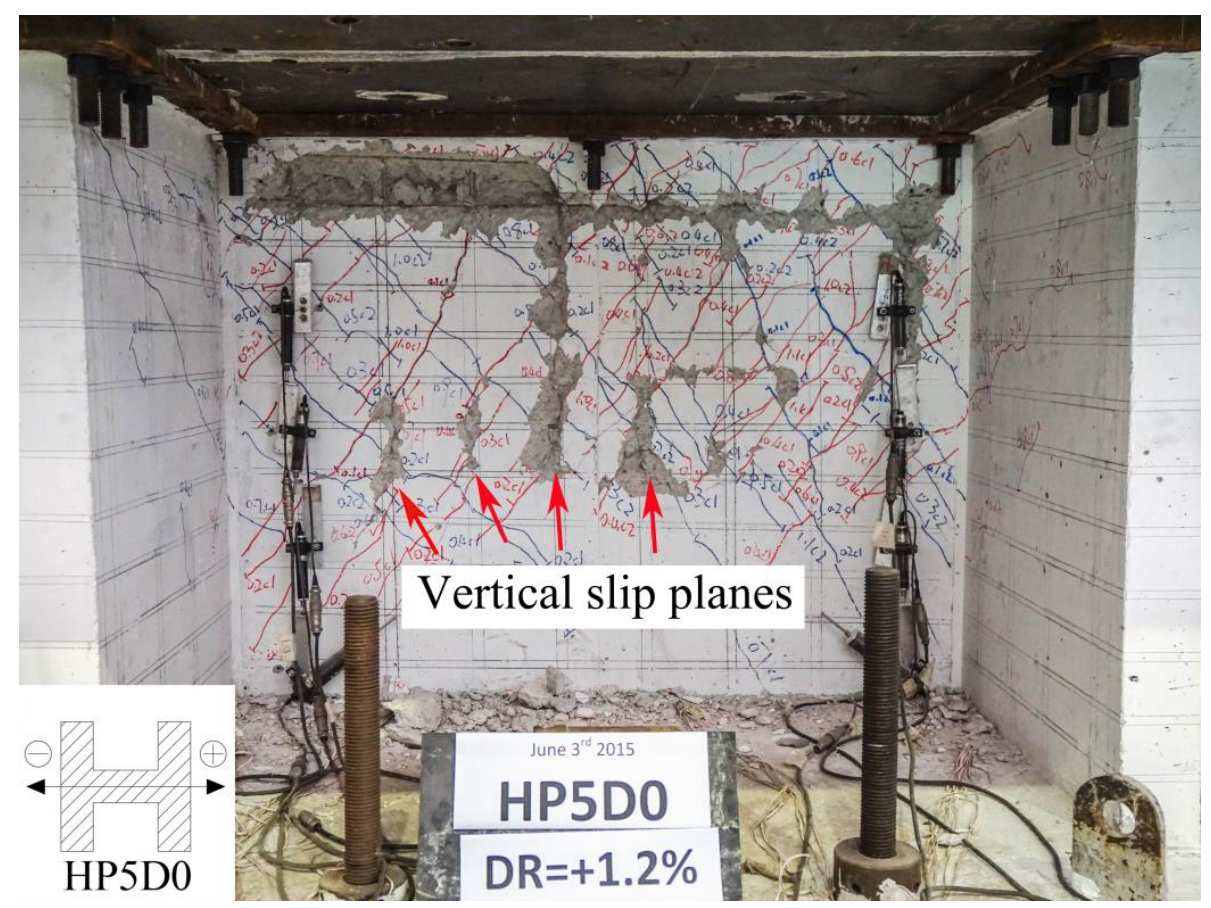

Figure 4.46 Failure mechanism of Specimen HP5D0

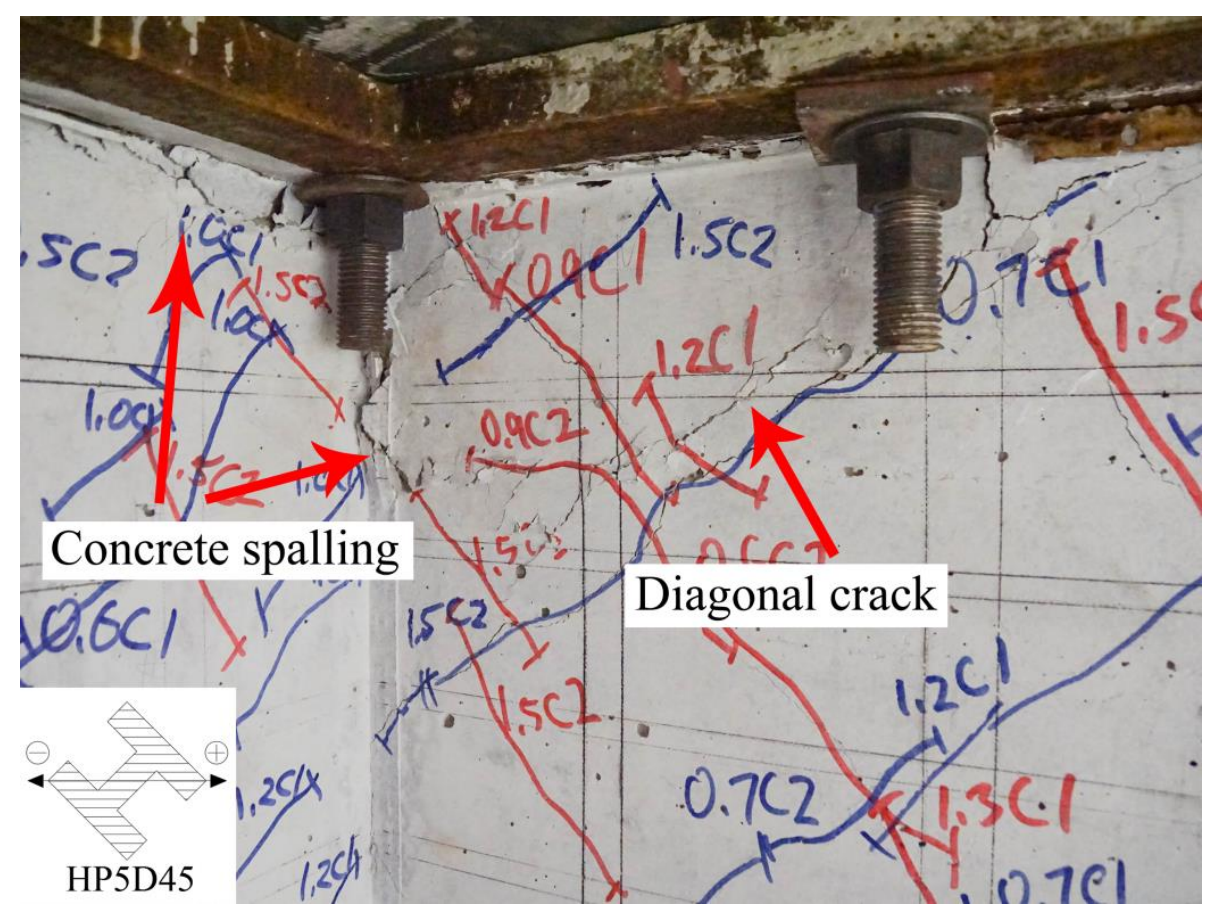

Figure 4.47 Failure mechanism of Specimen HP5D45

\subsubsection{Hysteretic responses}

As mentioned in the previous section, load-displacement curves play an important role in assessing the seismic performance of RC structures. Data recorded by load 
cells and horizontally-mounted LVDTs were used to plot load-displacement curves. Relevant aspects such as strength, secant stiffness and energy dissipation capacity were assessed and discussed in the following part. Table 4.2 summarizes some key features as well as the experimental results of the $\mathrm{H}$-shaped specimens. Figure 4.48 to Figure 4.51 depict the lateral load versus top displacement curves of the $\mathrm{H}$ shaped specimens.

Table 4.2 Test results of the H-shaped specimens

\begin{tabular}{|c|c|c|c|c|c|c|c|c|c|}
\hline \multirow{3}{*}{ Specimen } & (1) & (2) & (3) & (4) & (5) & (6) & \multicolumn{2}{|c|}{ (7) } & (8) \\
\hline & $f_{c}^{\prime}$ & $P / f_{c}^{\prime} A_{g}$ & $\theta$ & $V_{c r}$ & $V_{\max }$ & $\Delta_{y}$ & \multicolumn{2}{|c|}{$K_{i}$} & $\delta_{\max }$ \\
\hline & $\mathrm{MPa}$ & $\%$ & $\circ$ & $\mathrm{kN}$ & $\mathrm{kN}$ & $\mathrm{mm}$ & \multicolumn{2}{|c|}{$\mathrm{kN} / \mathrm{mm}$} & $\%$ \\
\hline HP0D0 & 35.9 & 0.48 & 0 & 155 & 857 & 7.0 & 97 & 90 & 1.1 \\
\hline HP0D45 & 33.8 & 0.51 & 45 & 107 & 737 & 6.0 & 123 & 96 & 0.9 \\
\hline HP5D0 & 35.9 & 5.01 & 0 & 217 & 994 & 8.0 & 113 & 123 & 1.1 \\
\hline HP5D45 & 33.8 & 5.32 & 45 & 262 & 1132 & 7.0 & 136 & 113 & 1.5 \\
\hline
\end{tabular}

Note: (1): Compressive strength of concrete; (2) Axial load ratio; (3) Angle between lateral loading and web segment; (4) Observed shear strength at the first cracking; (5) Maximum shear strength during the test; (6) Yield displacement; (7) Effective stiffness in positive and negative loading directions; (8) Maximum drift ratio. 


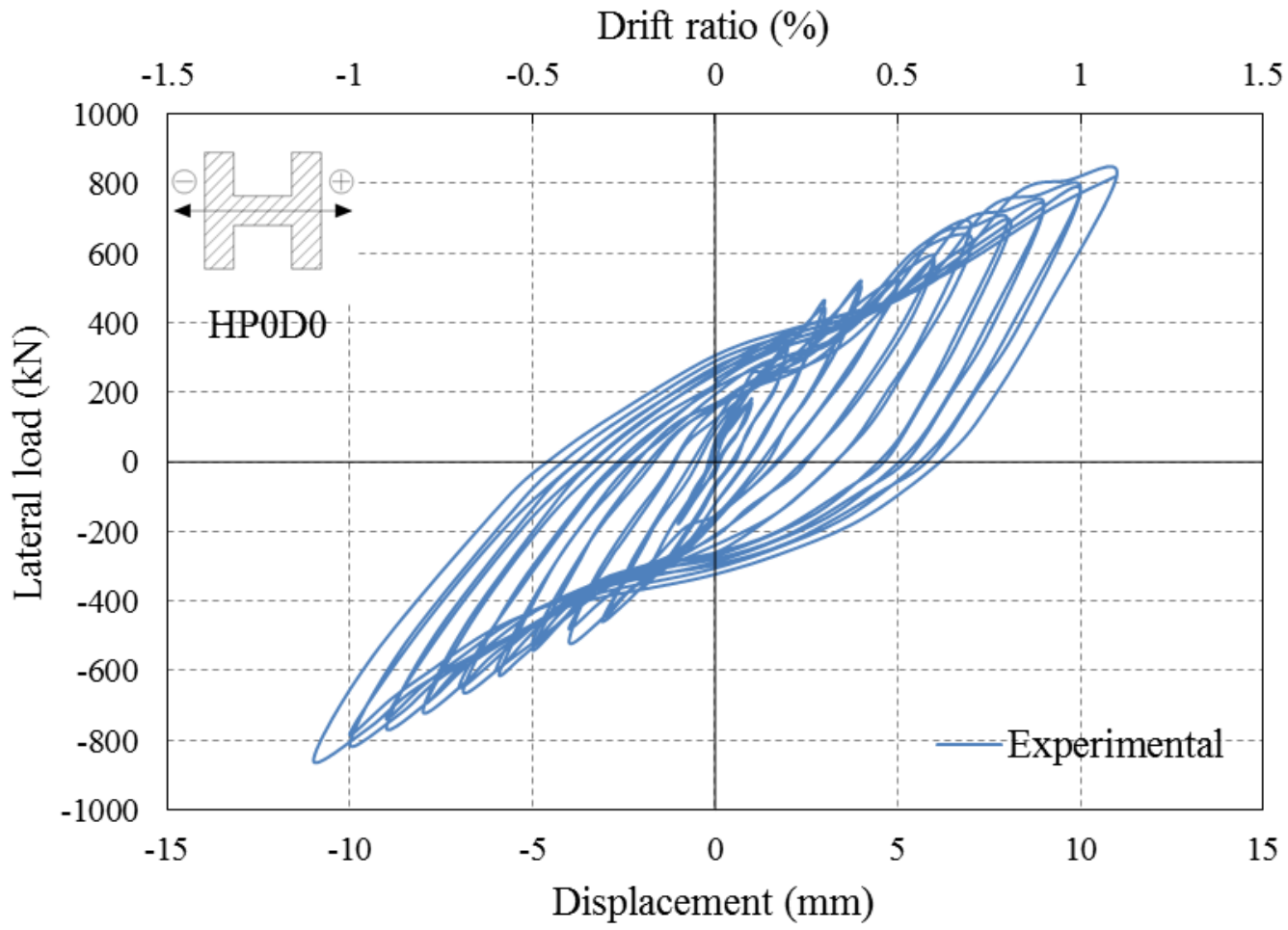

Figure 4.48 Lateral load-displacement curve of Specimen HP0D0

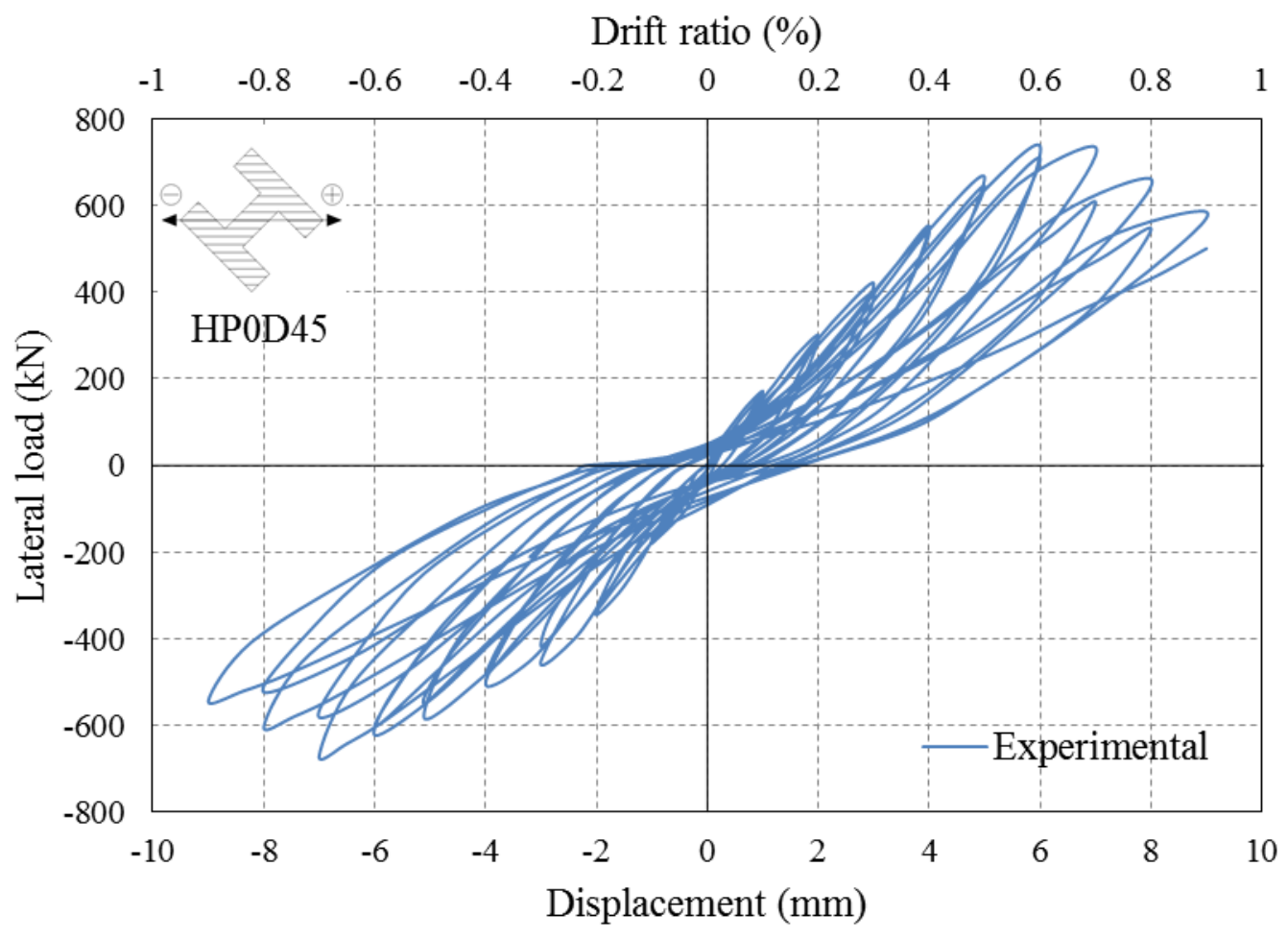

Figure 4.49 Lateral load-displacement curve of Specimen HP0D45 


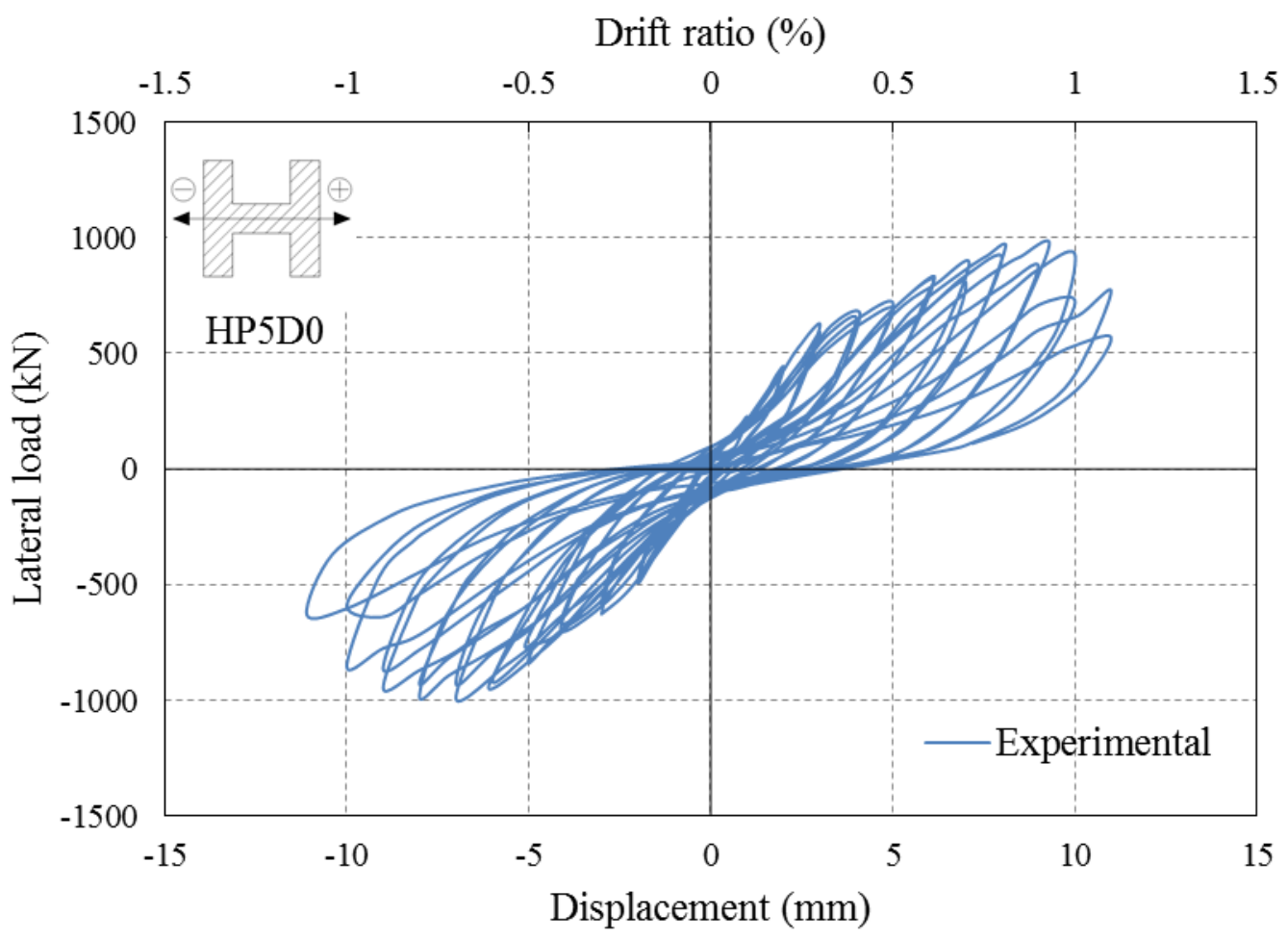

Figure 4.50 Lateral load-displacement curve of Specimen HP5D0

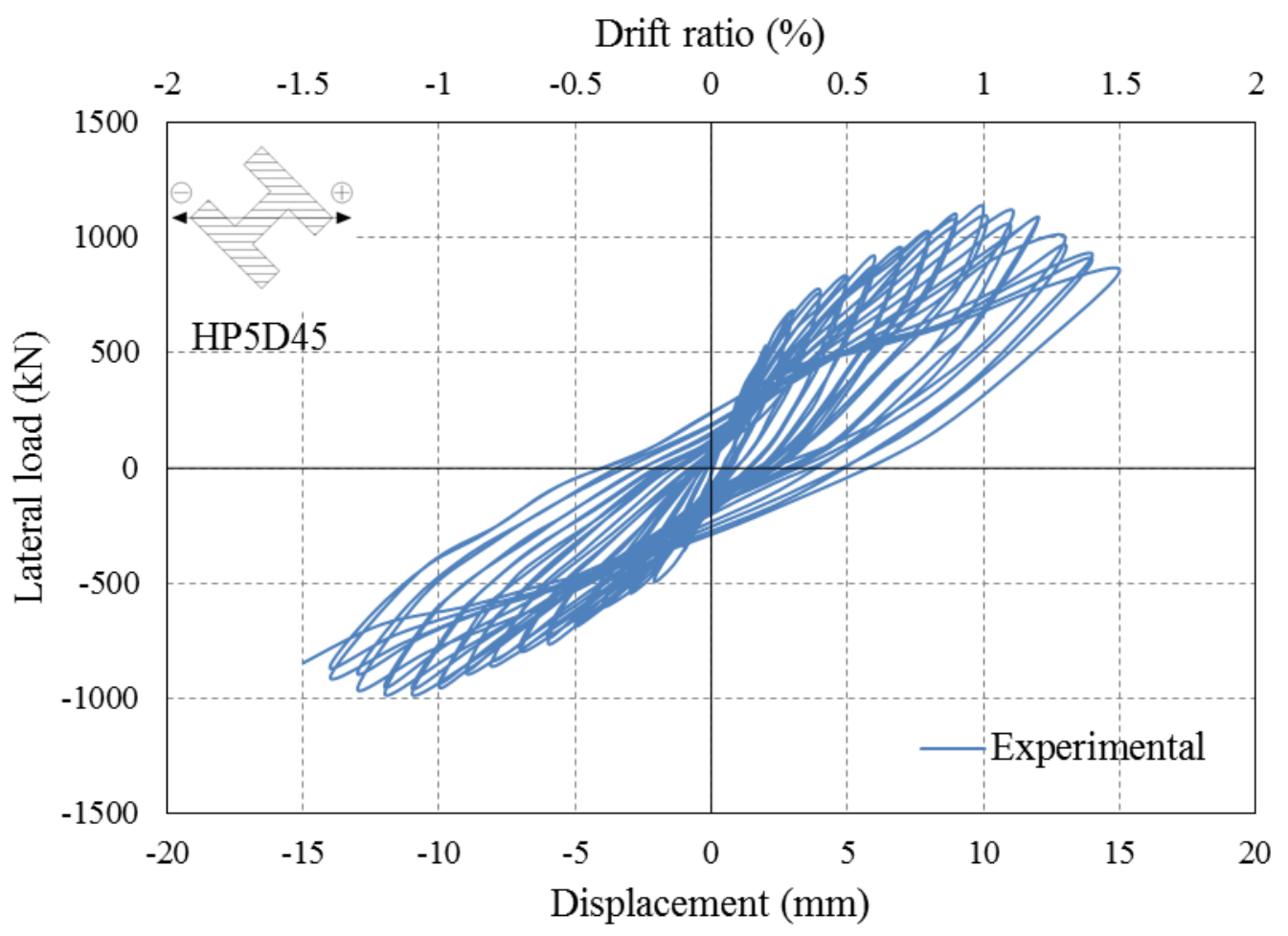

Figure 4.51 Lateral load-displacement curve of Specimen HP5D45 
Figure 4.52 shows the backbones curves of four H-shaped specimens. It was clear that all specimens were able to sustain a ductile post-peak response prior to the failure except for Specimen HP0D0, in which a premature failure happened as the upper part of the web slid. It was observed that the lateral strength of Specimen HP5D45 was larger than that of Specimen HP0D45 at the same displacement. A similar trend could also be traced in the group with orthogonal lateral loading, but their difference was less evident. When it comes to the deformation capacity, it was clear that Specimen HP5D45 outperformed Specimen HP0D45 with the number of $1.5 \%$ compared with $0.8 \%$. However, the specimens with orthogonal lateral loading demonstrated nearly the same capability of deformation.

Previous research [X1] in the literature has indicated that the stiffness of wall element is actually lower than the calculated stiffness using gross section properties. Further, stiffness acts as an important indicator of material properties and plays a key role in modeling RC structures. Hence it is crucial to evaluate the realistic stiffness of structural walls. Figure 4.53 presents the variation of secant stiffness during the whole experiment. As expected, all specimens experienced a reduction in stiffness throughout the test. At the initial stage, the stiffness of each specimen experienced a rapid decline, where the most dramatic drop occurred in Specimen HP5D45. With the increase of the drift ratio, the secant stiffness of each specimen further declined and remained at a comparatively low value at the final stage. It was evident that specimens with more axial loads had a bigger number of secant stiffness, which was attributed to the fact the axial load constrained the diagonal cracks and limited the development of the sliding shear, thereby enhancing the reaction at a specific drift ratio.

As stated previously, the energy dissipation of wall specimens is calculated from the inner area of lateral load-displacement loops. Figure 4.54 below illustrates the energy dissipation capacity of each specimen with respect to the drift ratio. In the initial stage, a rather small portion of energy was dissipated. As the drift ratio rose, the dissipation of energy steadily increased and experienced a drastic growth at the final stage except for Specimen HP5D0. In the groups with skew lateral loading, it was evident that the one with more axial loads demonstrated better energy 
dissipation capacity than its counterpart. However, this trend could not be detected in the specimens with orthogonal lateral loading. Specimen HPOD0 and Specimen HP5D0 had almost the same strain energy dissipated in the early stage of the test. But the number of the former continued to rise after the drift ratio of $7 \%$, whereas the latter experienced a drop of secant stiffness after reaching the maximum. Furthermore, the orthogonal horizontal loading was observed to have a more favorable effect in increasing the energy dissipation than the skew loading, which was revealed in the 195\% discrepancy between Specimens HP0D45 and HP0D0. This was mainly because $\mathrm{H}$-shaped walls were able to introduce a larger area as compression zones in both loading directions when the lateral loading was parallel to the web.

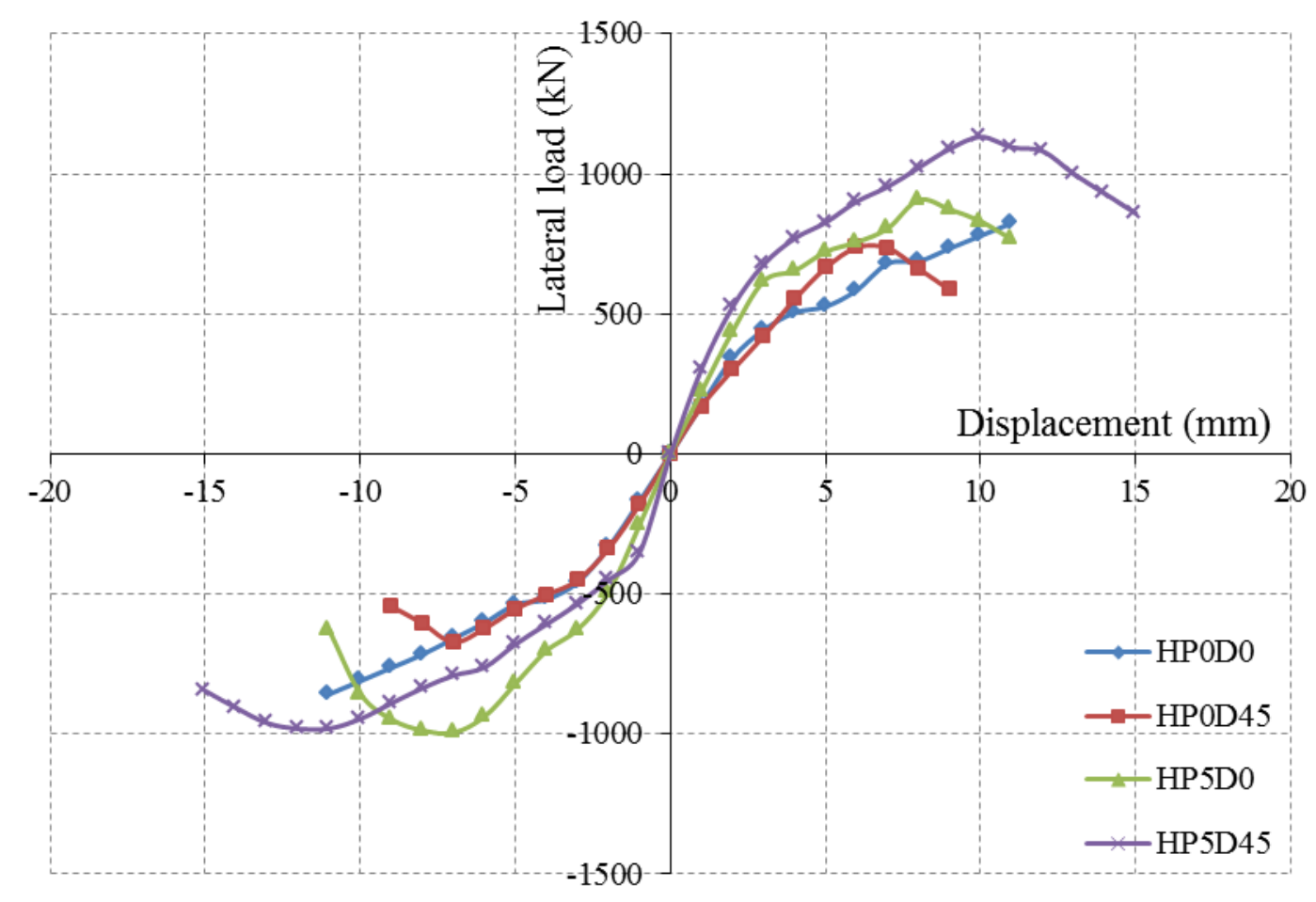

Figure 4.52 Backbone curves of the H-shaped specimens 


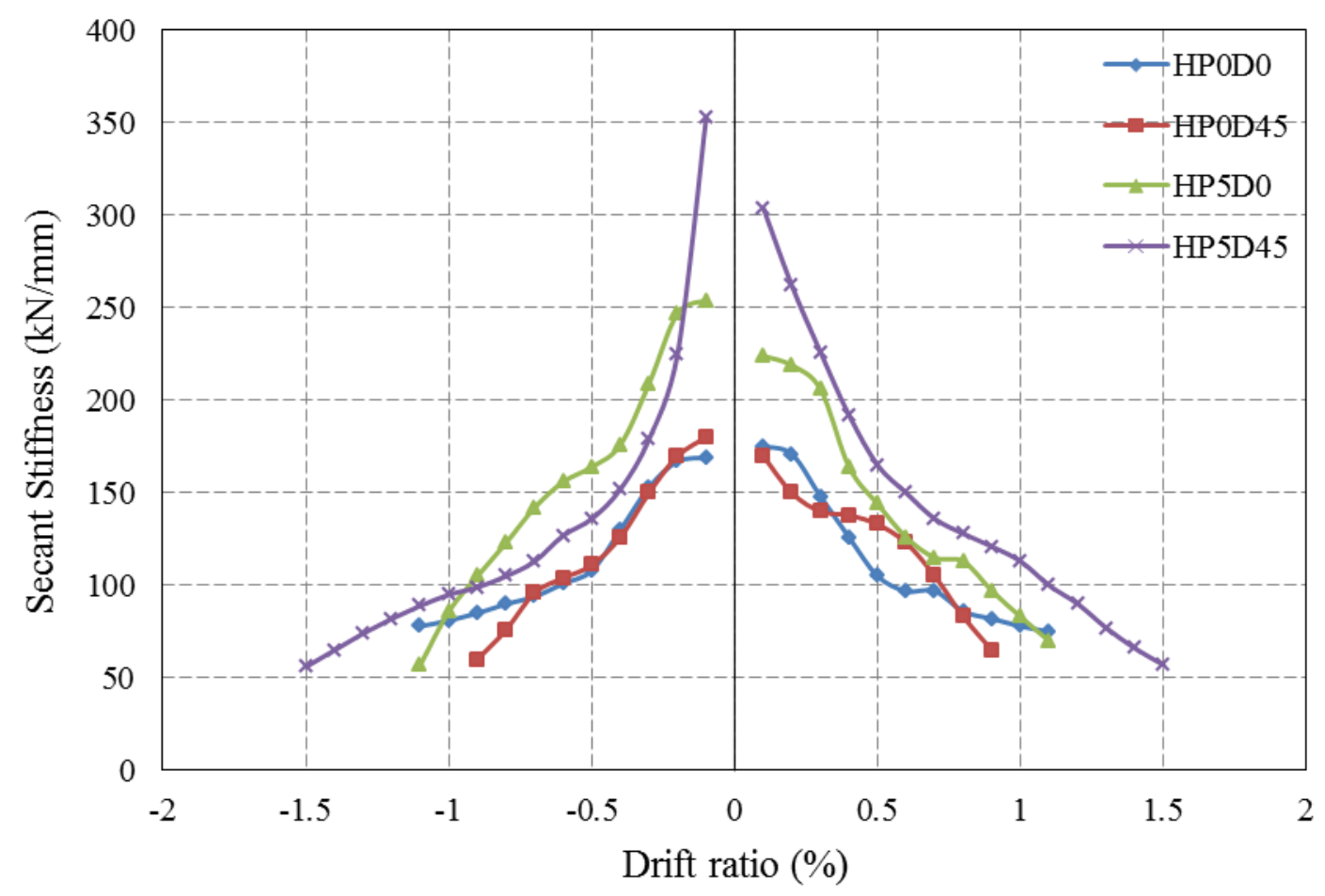

Figure 4.53 Secant stiffness of the H-shaped specimens

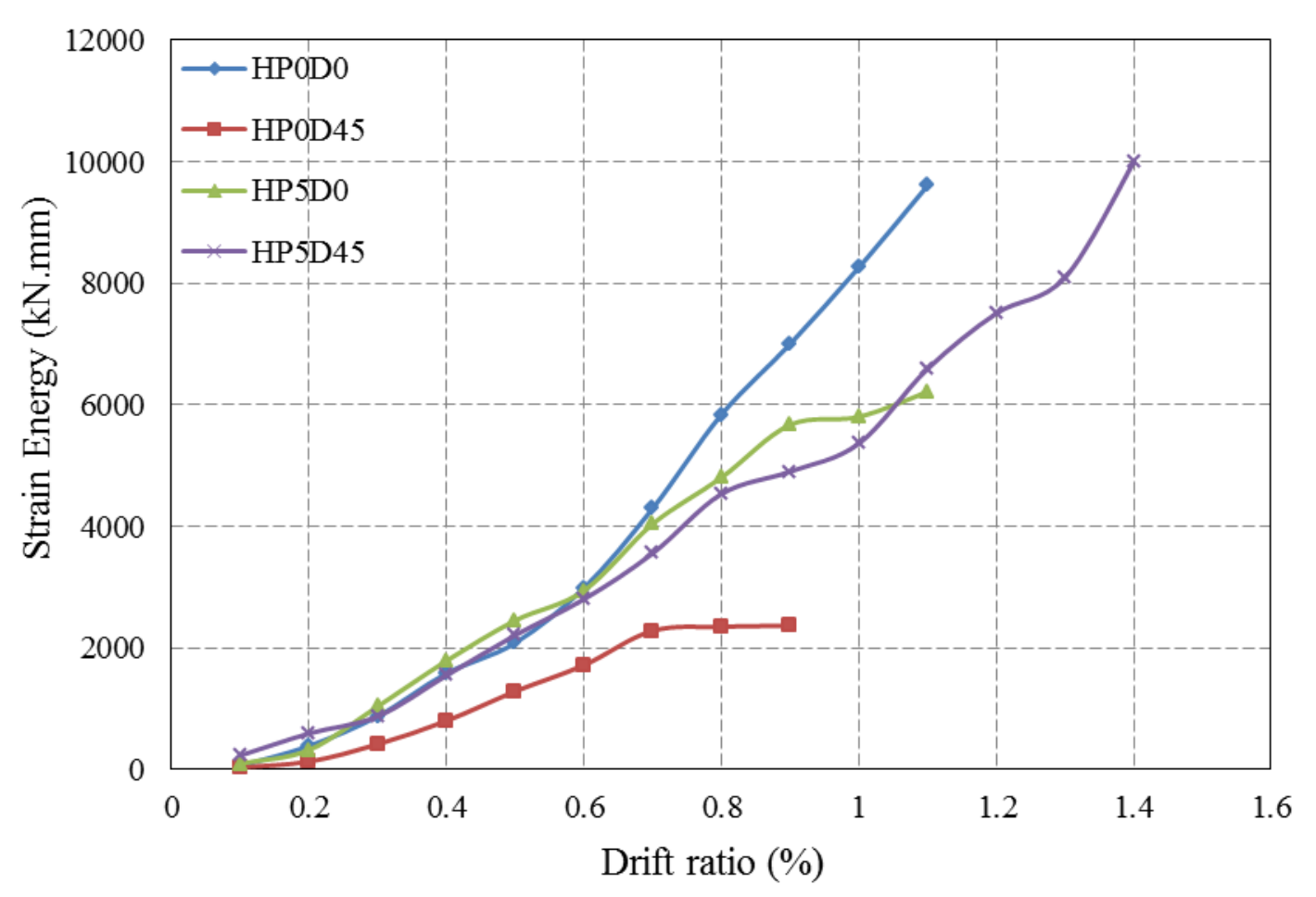

Figure 4.54 Energy dissipation capacities of the H-shaped specimens 


\subsubsection{Displacement components}

As presented in the previous section, top deformations of structural walls are mainly comprised of three components, which are flexural deformation, shear deformation and sliding deformation. LVDTs were mounted at wall surfaces to provide data concerning these three deformations. Figure 4.55 to Figure 4.58 illustrate the ratios of three components to the total top displacement at different drift ratios, in which an unaccounted portion was also recorded since the summation of the other three could not always reach a perfect $100 \%$.

In Specimen HP0D0, it was clear that the shear deformation accounted for the largest portion during the whole test, with its number experiencing a slight rise from $45 \%$ to $55 \%$. This phenomenon was also observed in other squat wall experiments [L5, X1]. The second largest portion, the flexural deformation, on the other hand, showed a declining trend as its number dropped from $40 \%$ to $35 \%$ in the positive loading direction. The sliding displacement experienced a slight increase in both loading directions with its values doubled from around $3 \%$ to $12 \%$. The unaccounted part showed a gradual decrease and remained at a low value in the test.

A similar rising trend of the sliding deformation was also observed in Specimen HP0D45, where the number ended up to approximately $12 \%$ in both directions. The shear deformation, which was lower than the flexural deformation in terms of its portion, showed a steady increase as the test progressed, making it the largest component at the end of the experiment. The flexural displacement demonstrated a moderate drop in both directions, with its figure dropped from 55\% to $35 \%$. The unaccounted part remained at a low value of approximately $2 \%$ during the test.

The contribution of the sliding shear in Specimen HP5D0 also grew as the drift ratio increased. But the extent of growth was smaller when compared with Specimen HP0D0. This was due to the constraining effect brought by axial loads. The shear deformation, throughout the whole experiment, preserved a dominant component of top displacements. In the positive loading direction, the shear contribution rose from $53 \%$ to $62 \%$, while in the negative loading direction, the growth was from $44 \%$ 
to $63 \%$. The flexural deformation, on the other hand, dwindled from $44 \%$ to $35 \%$. The unaccounted part varied slightly but its value remained relatively small.

As far as Specimen HP5D45 was concerned, the sliding shear component made up to $3 \%$ in the beginning and ended up at around $7 \%$ in both loading directions. The shear displacement, which was initially the second largest part, showed a moderate increase as the test progressed and equaled the flexural deformation at wall failure. The flexural displacement, consisting $55 \%$ of the top displacement in the beginning, dropped to $45 \%$ as the drift ratio rose. The unaccounted part remained at a low value below $10 \%$ throughout the test.

From the discussion above, it was clear that the portion of the sliding shear generally increased during the whole test. This phenomenon was more obvious in specimens with lower axial loads. The shear deformation, in all four tests, experienced a steady increase as lateral displacements rose, whereas the flexural deformation showed a decreasing trend. As for specimens with orthogonal lateral loading, the shear part was constantly and evidently larger than the flexural part and dominated the response of the wall. In specimens with skew lateral loading, the contribution of top deformations from the shear was initially smaller than that of the flexure, but ended up equal or higher than its counterpart.

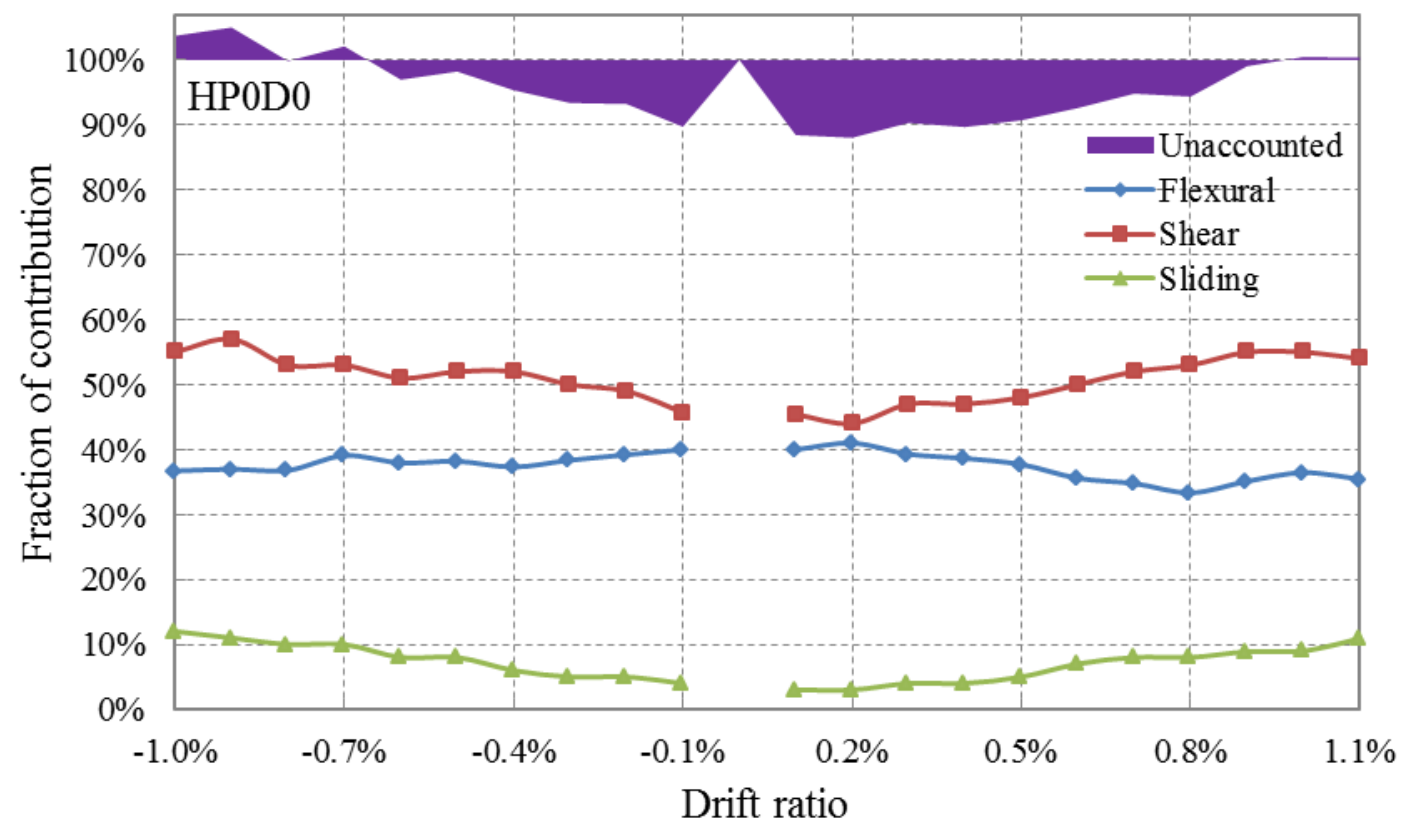

Figure 4.55 Displacement components of Specimen HP0D0 


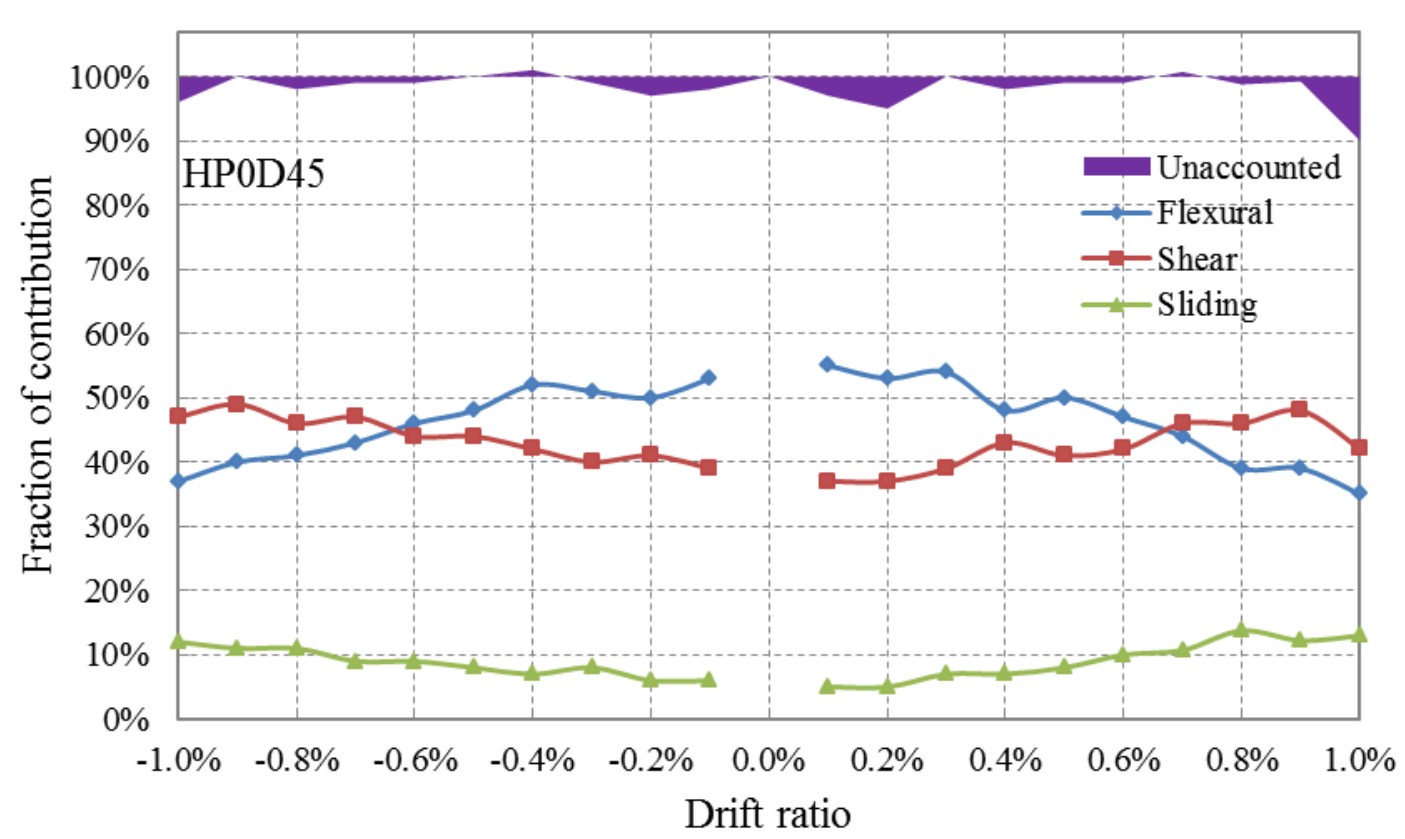

Figure 4.56 Displacement components of Specimen HP0D45

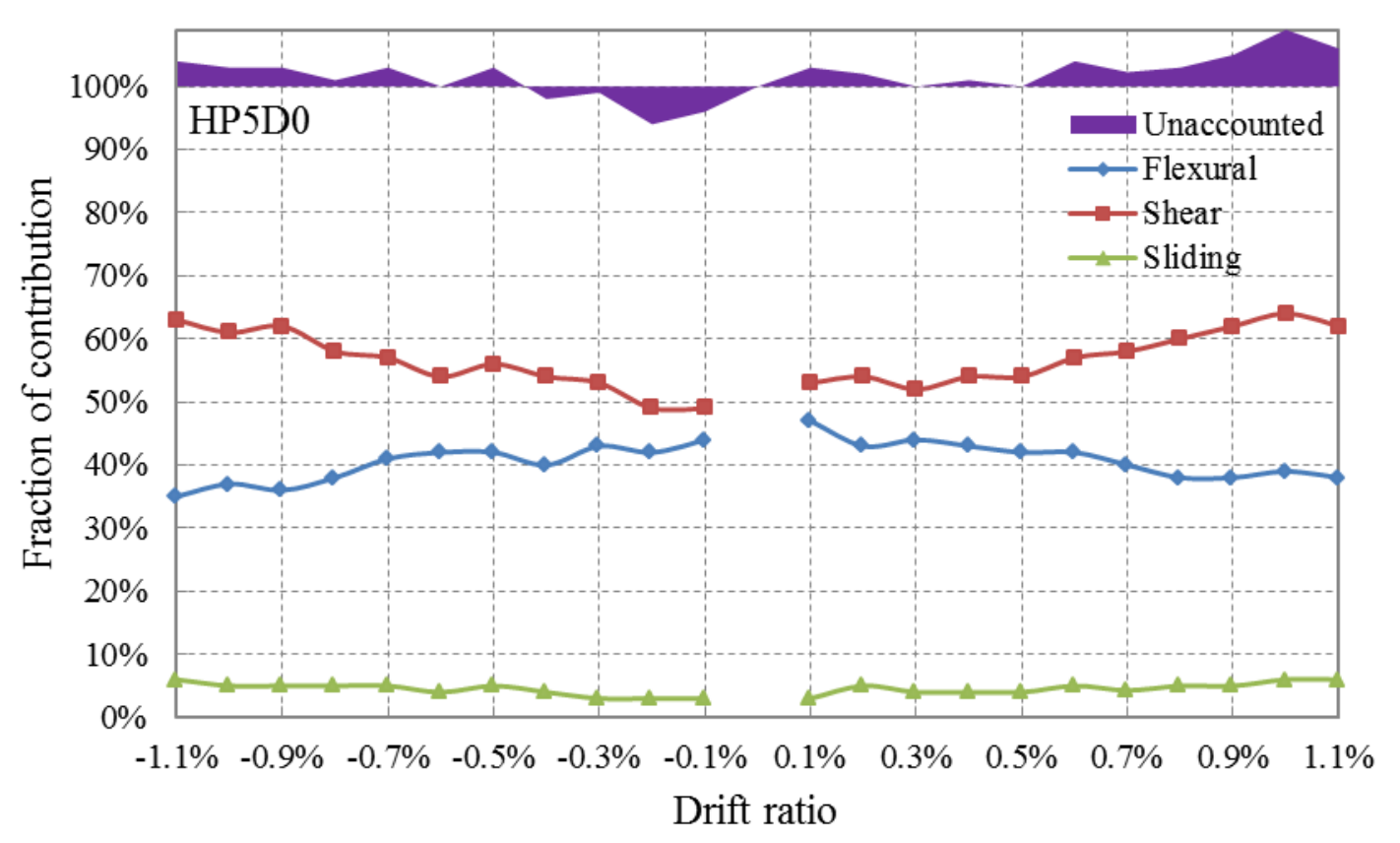

Figure 4.57 Displacement components of Specimen HP5D0 


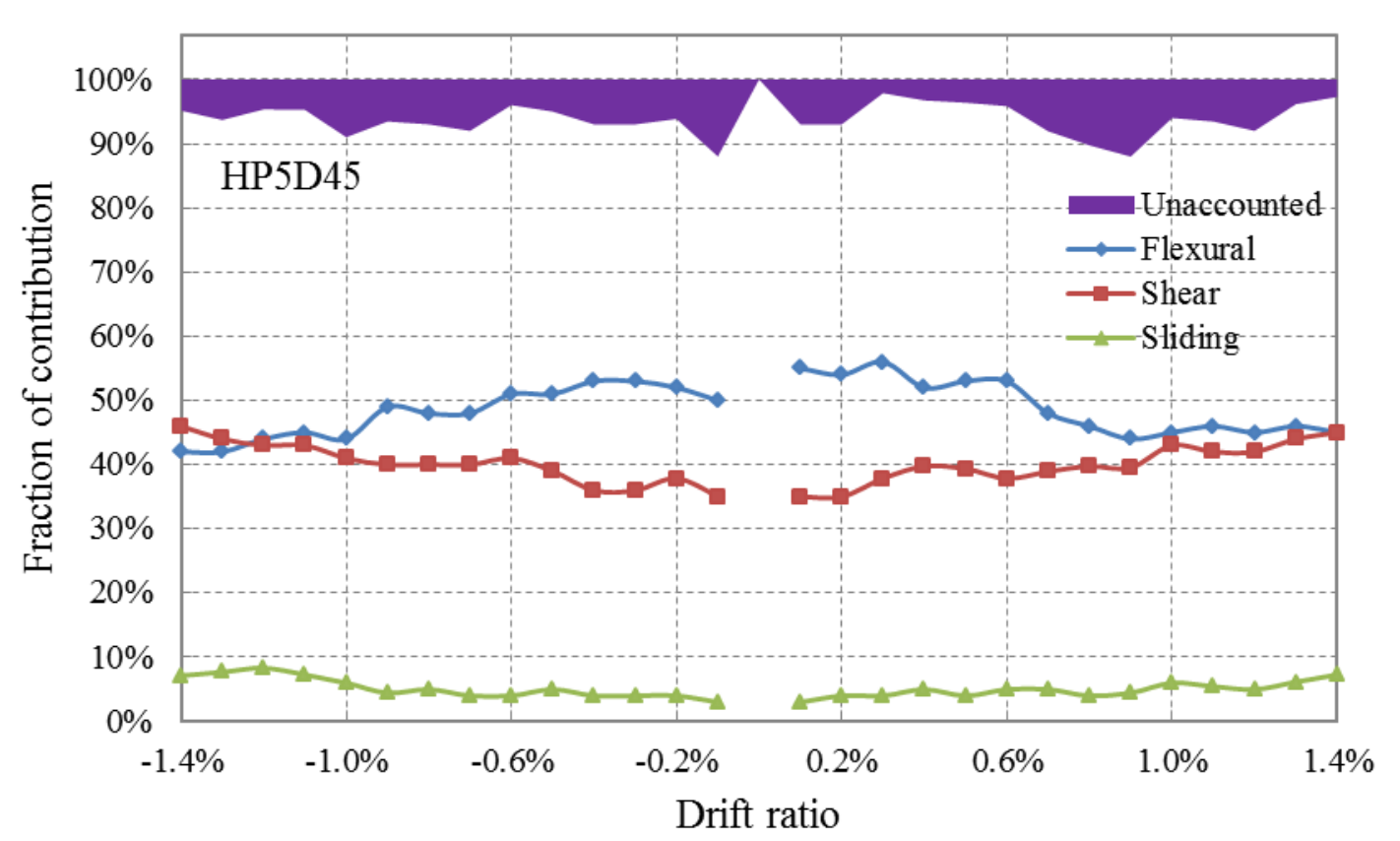

Figure 4.58 Displacement components of Specimen HP5D45

\subsubsection{Strain profiles}

Strain gauges, as illustrated in the previous chapter, were attached to the surface of reinforcing bars to monitor their reactions during the test. Data was compiled and plotted to show strain variations from the initial stage of the test to wall failure at several critical sections. It was found that several strain gauges had malfunctioned and showed error reading due to relevant damage. Hence strains of some reinforcing bars were not presented below.

Figure 4.59 to Figure 4.64 depict strains at the bottom of longitudinal reinforcement along the web in different specimens at various drift ratios in both loading directions. Yielding of vertical web reinforcement was not witnessed in all specimens. For Specimen HP0D0, it was observed that strains remained almost linear before the drift ratio reached $0.8 \%$, which indicated the plane section assumption was valid at this stage. Also, it was found that the neural axial did not shift during the whole test. For Specimen HP0D45, strains were unable to maintain linear after the drift ratio of $0.3 \%$. The neutral axis also hardly moved in the test. For Specimen HP5D0, the neutral axis shifted $100 \mathrm{~mm}$ towards the middle of the 
section in the negative loading direction. Moreover, the plane section assumption could not be used after the drift ratio of $0.5 \%$ since strains were no longer linear.

The strain variations along the bottom of longitudinal reinforcement in the flange are presented in Figure 4.65 to Figure 4.72. For those with orthogonal lateral loading, data of the left flange was collected; for those with skew lateral loading, data of the right flange was used. It was clear that the longitudinal rebar located in the juncture of the web and flange yielded in specimens with orthogonal loading. Meanwhile, in the group with skew loading, the outmost longitudinal rebar yielded at the end of the test. A significant shear lag effect was detected in Specimens HP0D0 and HP5D0. For instance, the strain of the rebar in the junction of the web and flange was as much as $320 \%$ of that of the longitudinal reinforcement located in the flange tip at the final test stage in Specimen HP0D0. This phenomenon was more evident in Specimen HP5D0, where stains of the outmost longitudinal reinforcement were almost zero when the flange was in tension, suggesting that the outer part of the flange was hardly involved in withstanding lateral loads. The shear lag effect was observed when the flange was in compression as well, which was seen from the larger strains of the rebar located closer to the web. When imposed with lateral displacements $45^{\circ}$ from the web, the shear lag effect was less obvious but still could be traced as tensile strains of longitudinal rebar showed an increasing trend towards the flange tip. However, it should be noticed that the maximum strain occurred $0.24 \mathrm{~m}$ from the flange tip, which was consistent with the section analysis.

The strain profiles of No.1 and No.7 horizontal reinforcing bars in the web are shown in Figure 4.73 to Figure 4.76. The two selected reinforcing bars were located $20 \mathrm{~mm}$ and $500 \mathrm{~mm}$ above the wall base respectively. It was evident that the No.7 rebar yielded in both tests. Moreover, continuous growth of strains was witnessed in both loading directions. As for Specimen HP0D45, the readings of strain gauge located at the right of No.1 rebar and the middle of No.7 rebar experienced the highest growth in the positive direction, indicating that there was a main diagonal compression strut passing through these places. However, when imposed with lateral loading in the negative direction, the difference of strains at different locations was not evident in No.1 rebar. In Specimen HP5D0, strains 
located at the left part of No.1 rebar were obviously larger than those in the other two locations in the negative loading direction. This phenomenon was also found in the middle of No.7 rebar throughout the test.

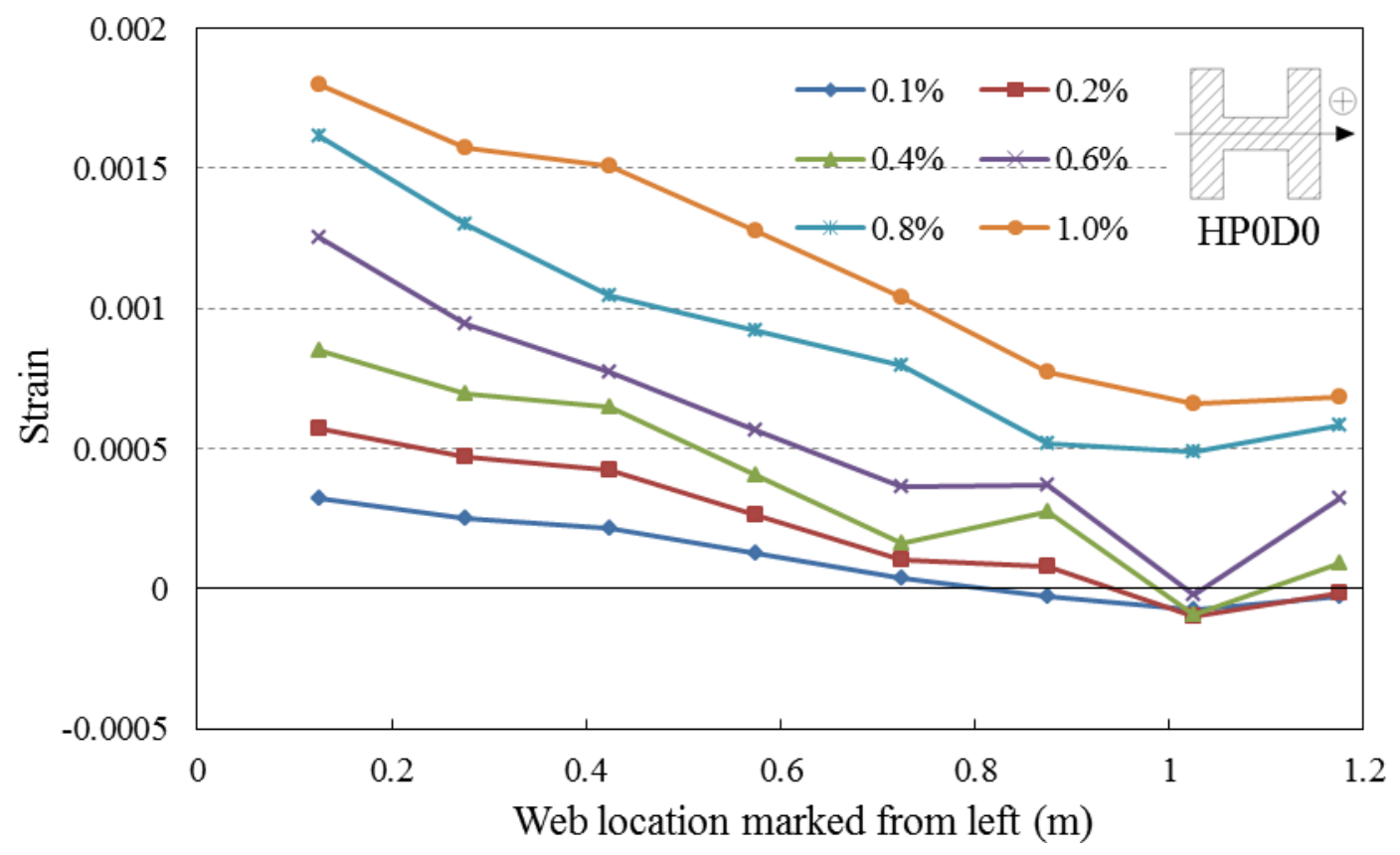

Figure 4.59 Strain profiles of longitudinal reinforcement along the web of Specimen HPOD0 in the positive loading direction

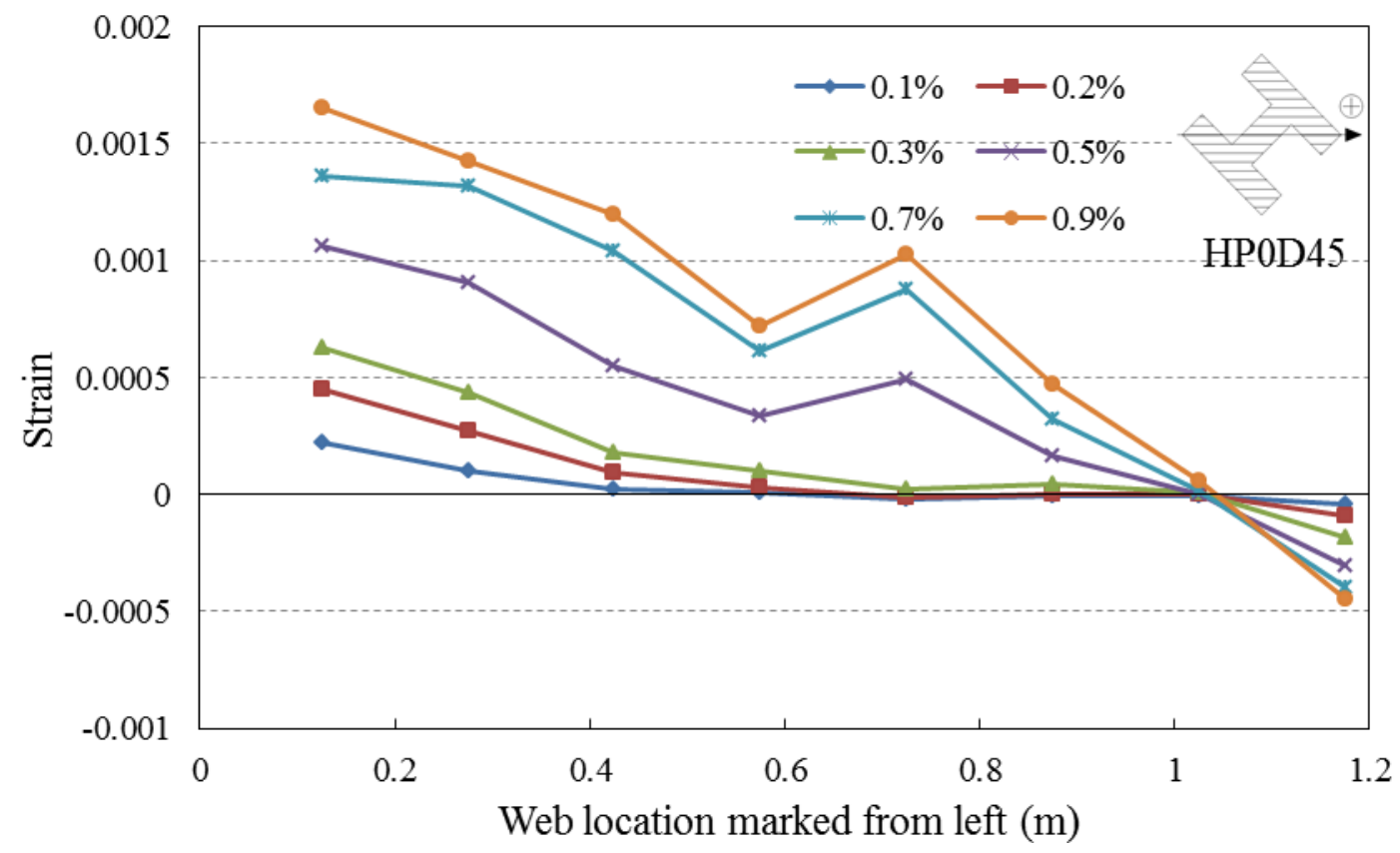

Figure 4.60 Strain profiles of longitudinal reinforcement along the web of Specimen HP0D45 in the positive loading direction 


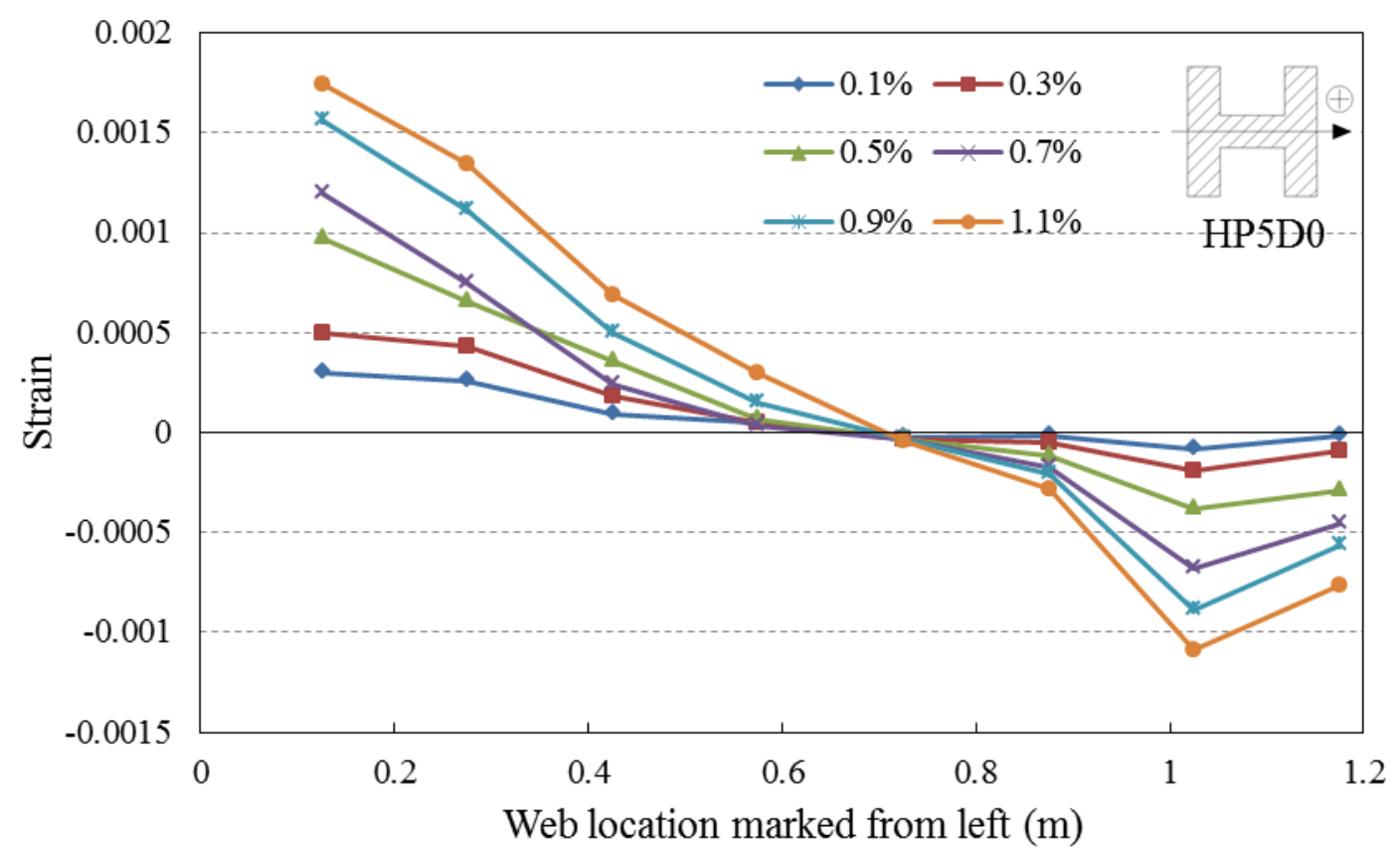

Figure 4.61 Strain profiles of longitudinal reinforcement along the web of Specimen HP5D0 in the positive loading direction

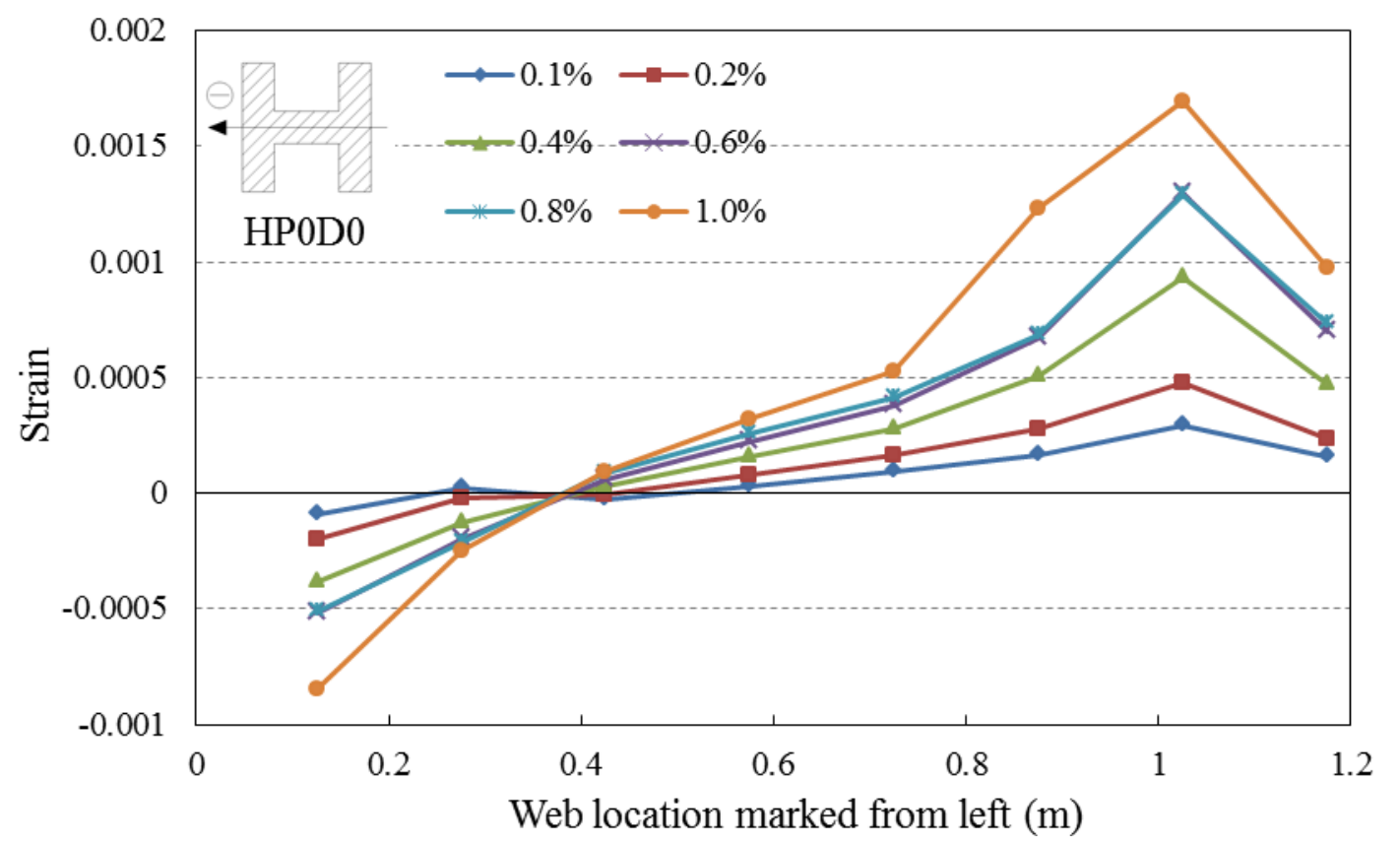

Figure 4.62 Strain profiles of longitudinal reinforcement along the web of Specimen HP0D0 in the negative loading direction 


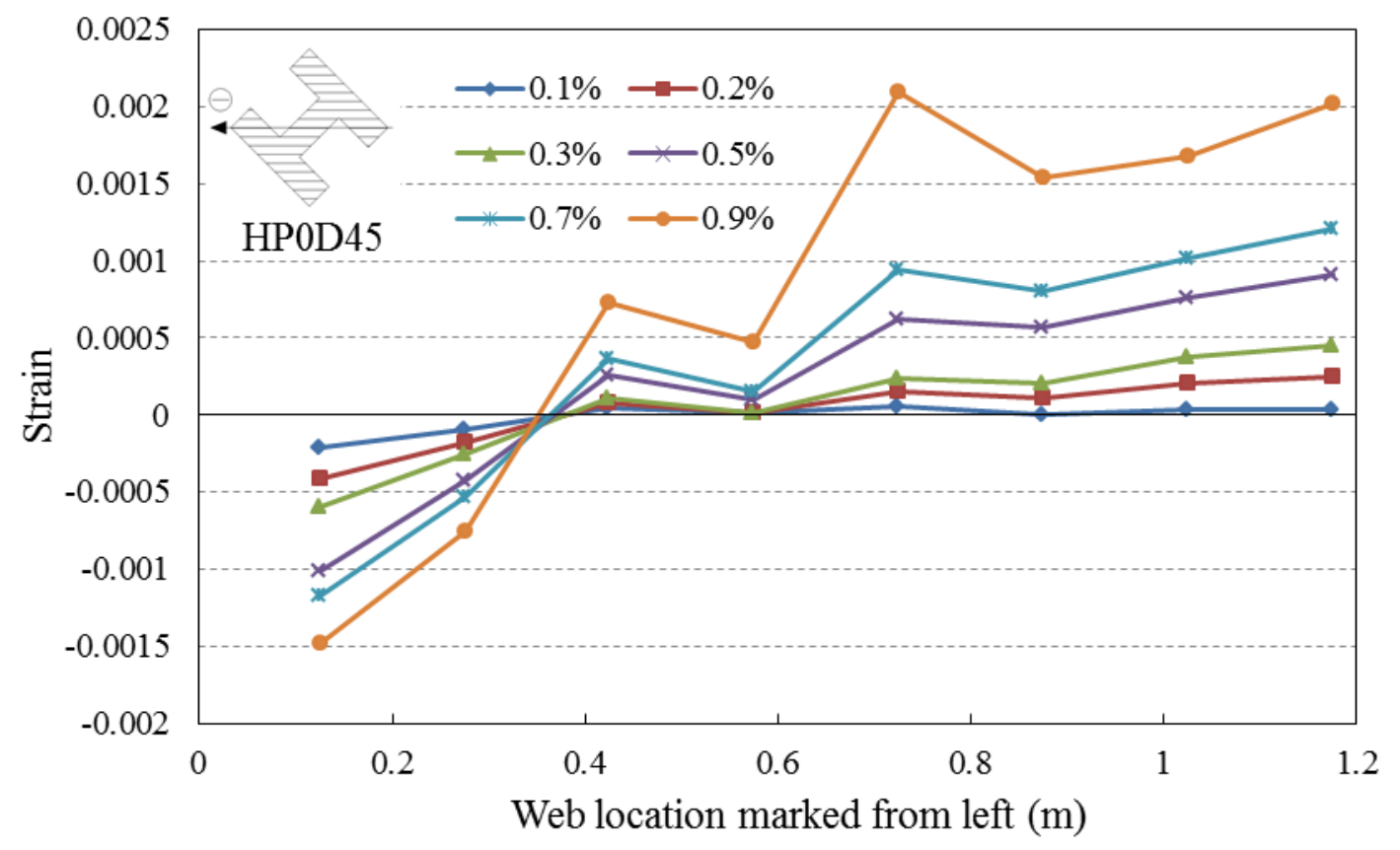

Figure 4.63 Strain profiles of longitudinal reinforcement along the web of Specimen HP0D45 in the negative loading direction

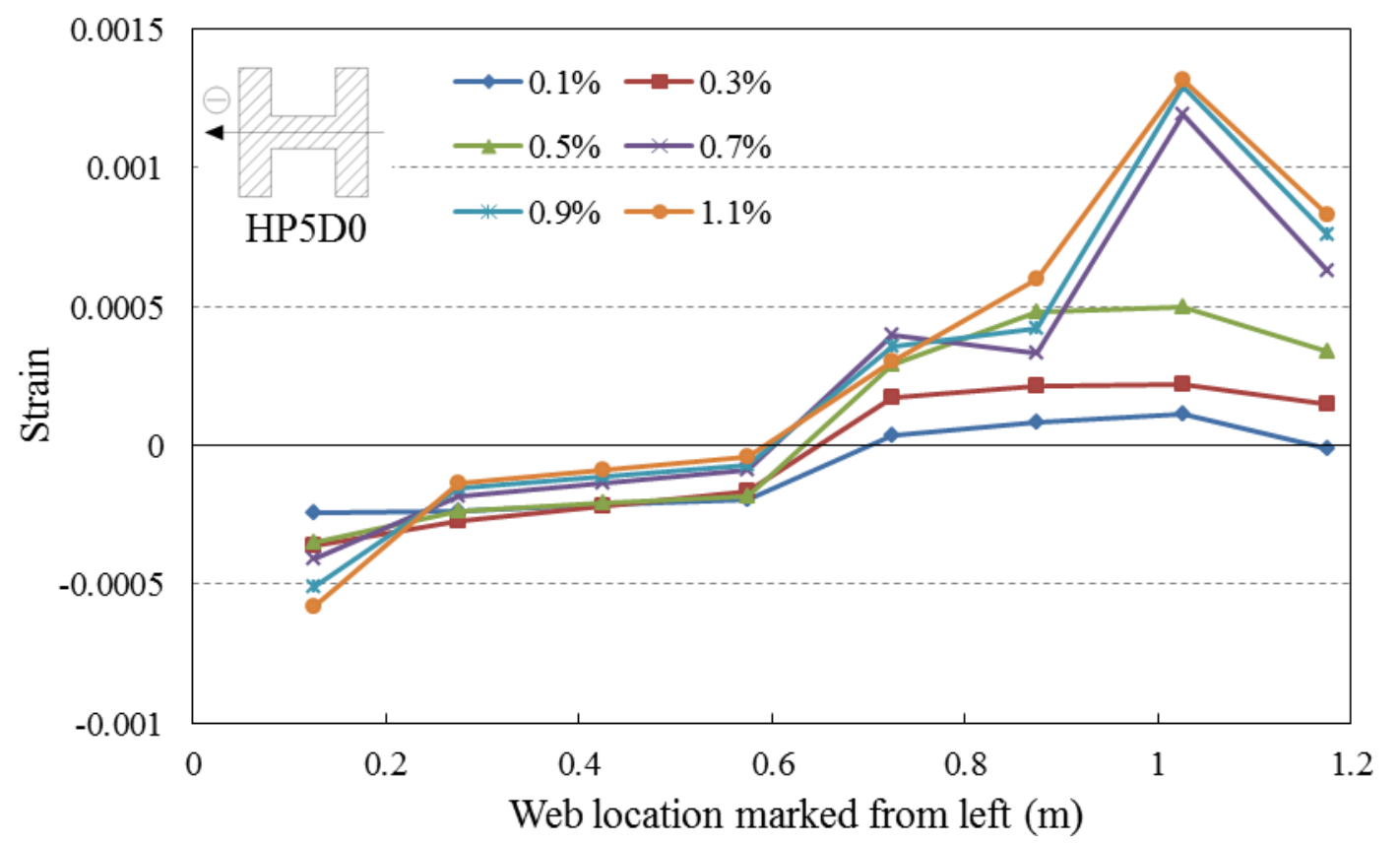

Figure 4.64 Strain profiles of longitudinal reinforcement along the web of Specimen HP5D0 in the negative loading direction 


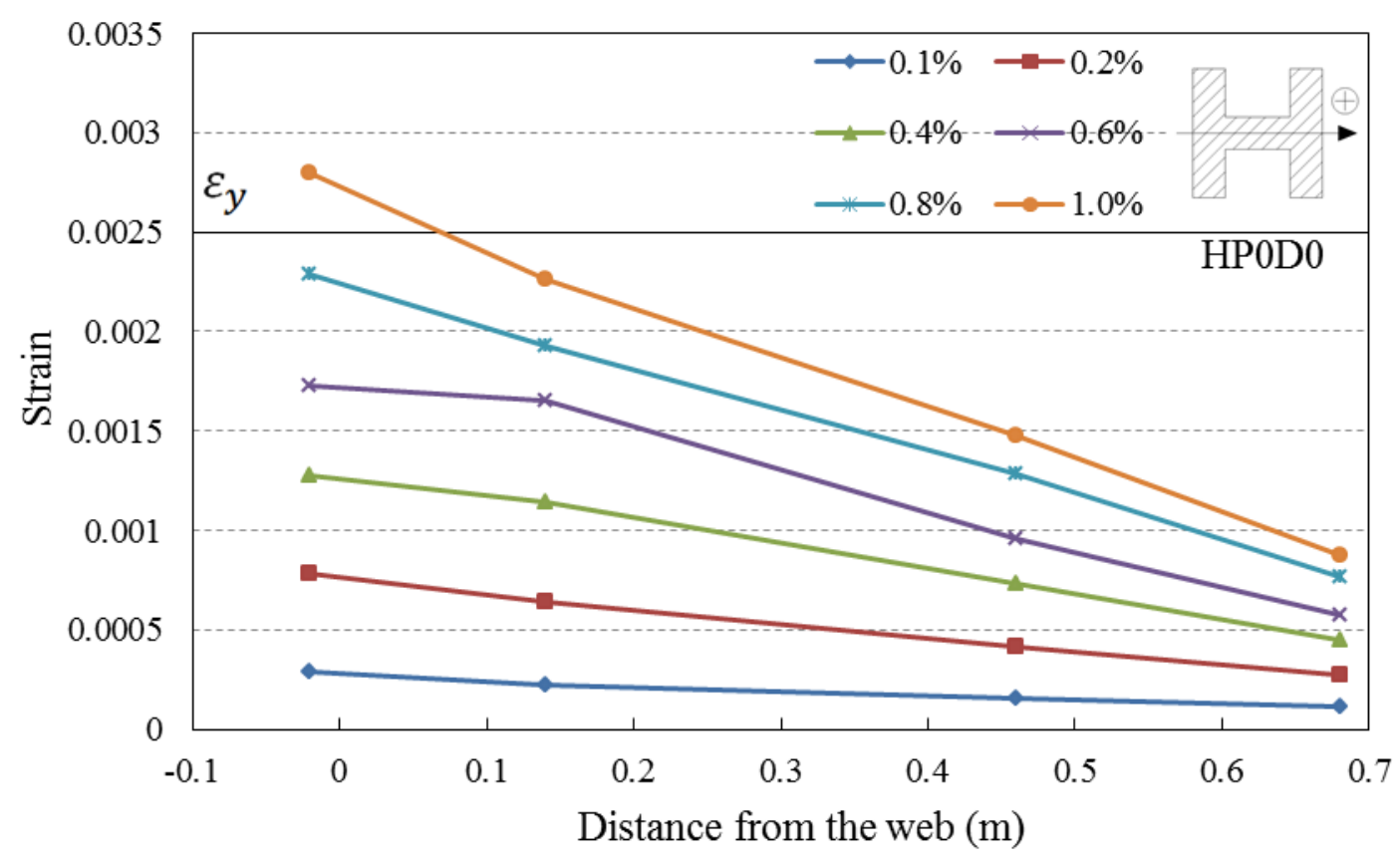

Figure 4.65 Strain profiles of longitudinal reinforcement along the flange of Specimen HP0D0 in the positive loading direction

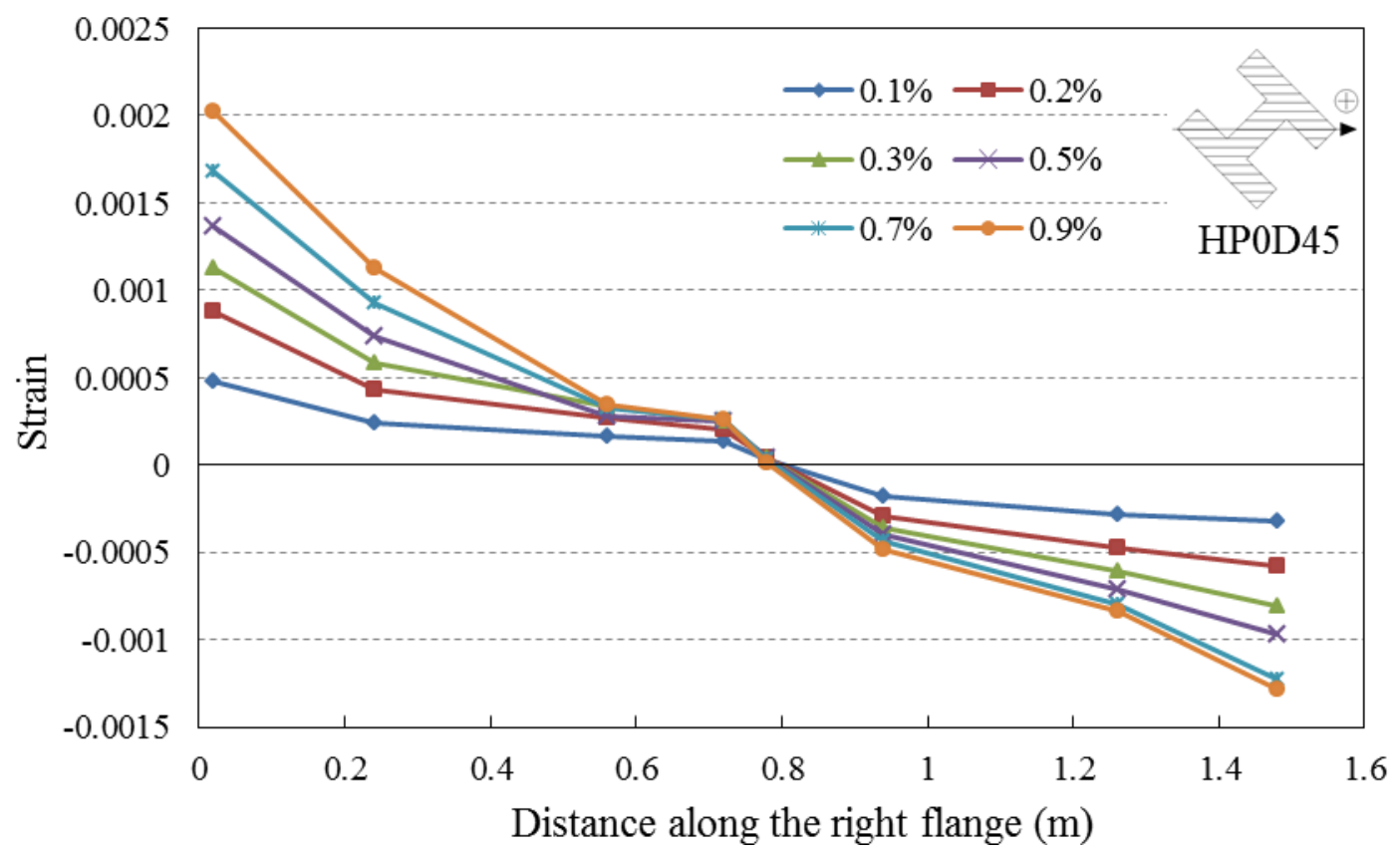

Figure 4.66 Strain profiles of longitudinal reinforcement along the flange of Specimen HP0D45 in the positive loading direction 


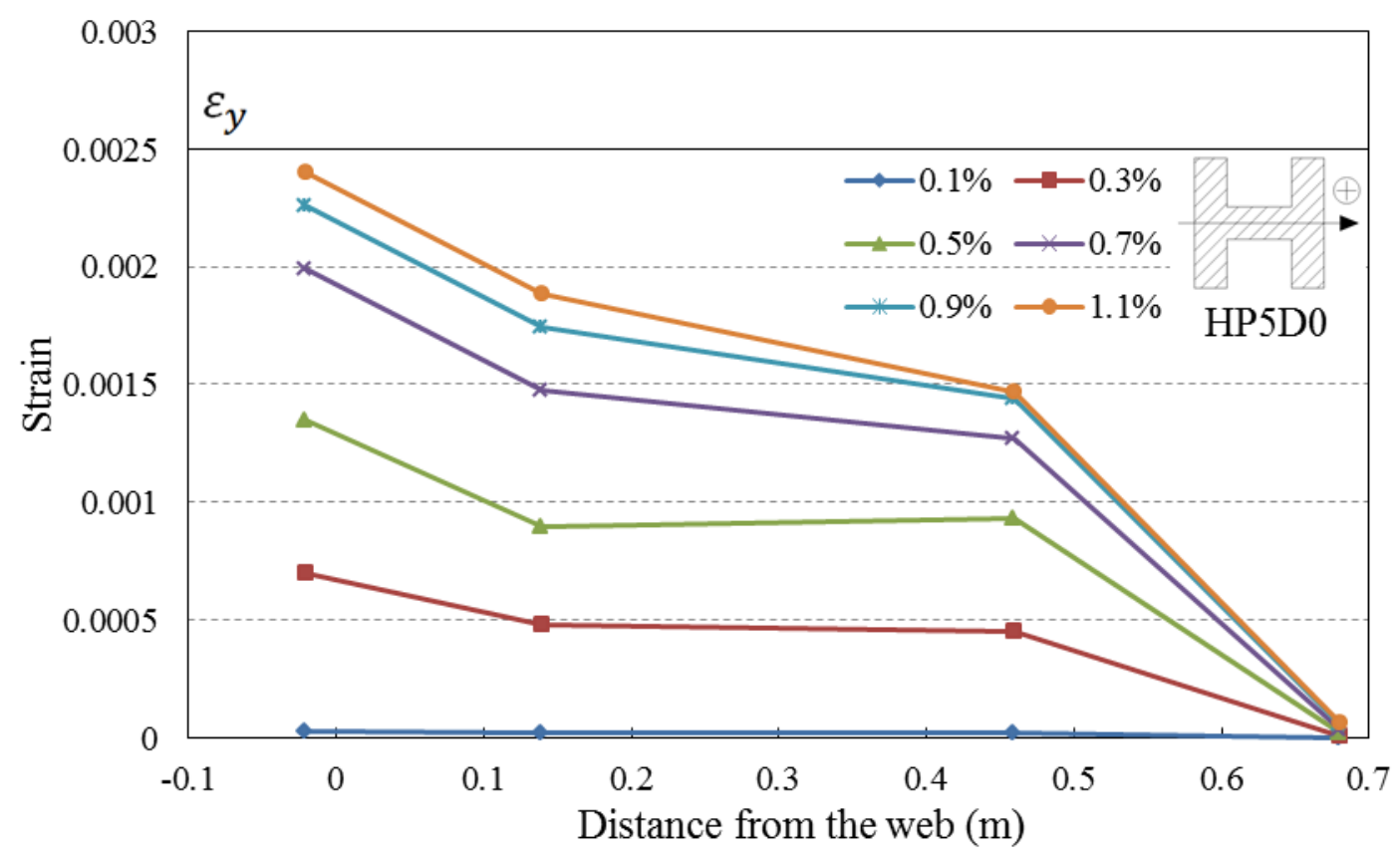

Figure 4.67 Strain profiles of longitudinal reinforcement along the flange of Specimen HP5D0 in the positive loading direction

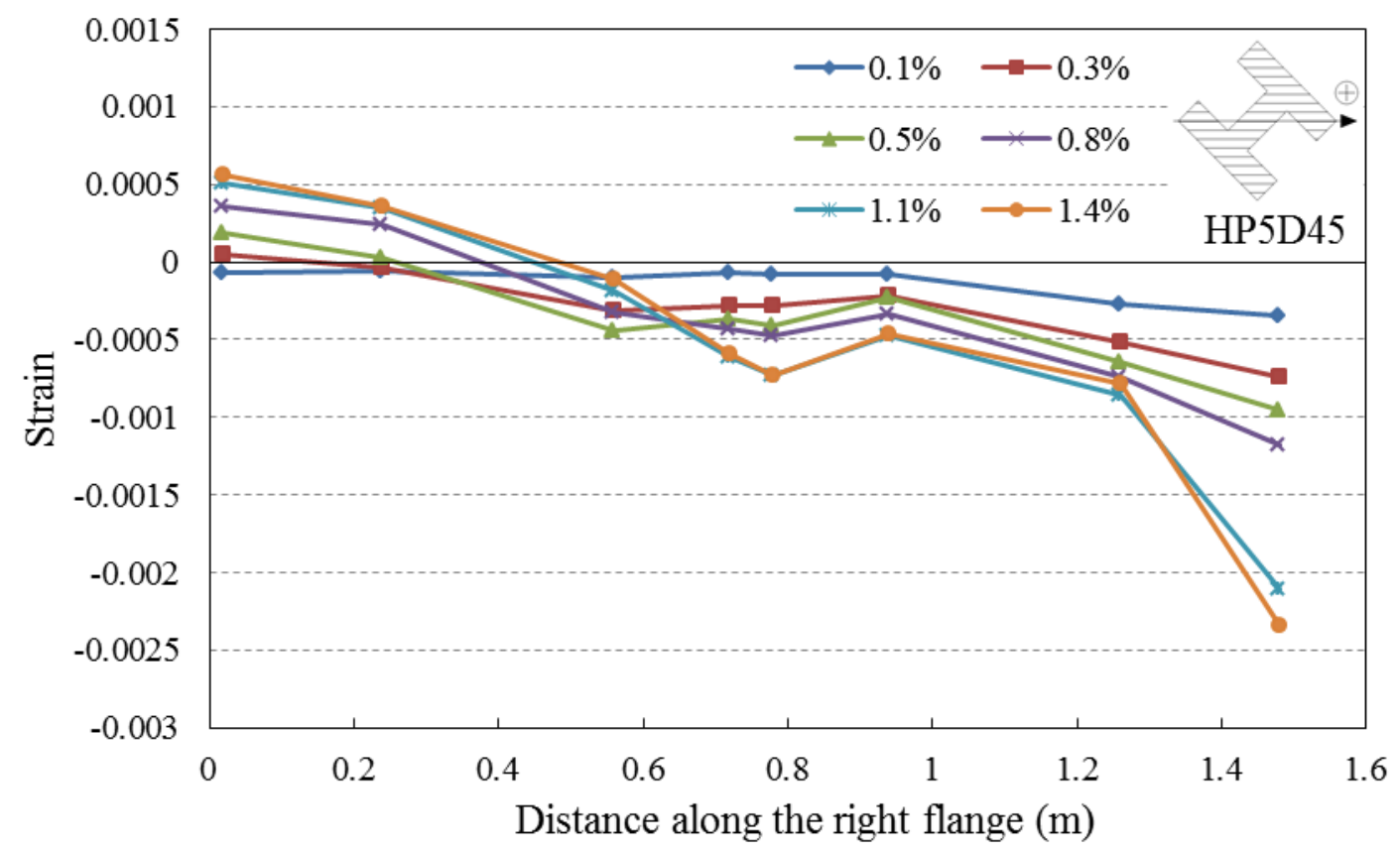

Figure 4.68 Strain profiles of longitudinal reinforcement along the flange of Specimen HP5D45 in the positive loading direction 


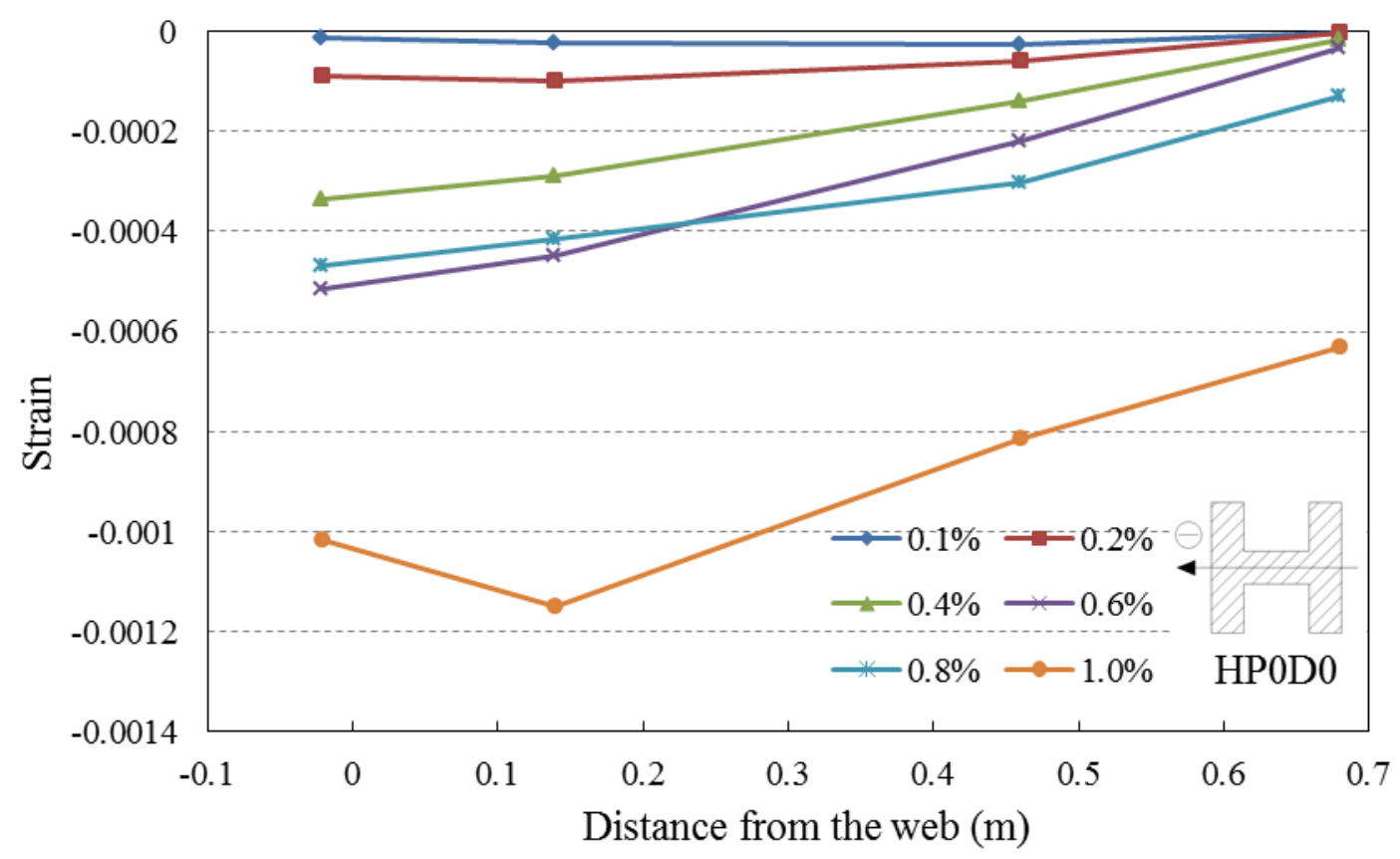

Figure 4.69 Strain profiles of longitudinal reinforcement along the flange of Specimen HPOD0 in the negative loading direction

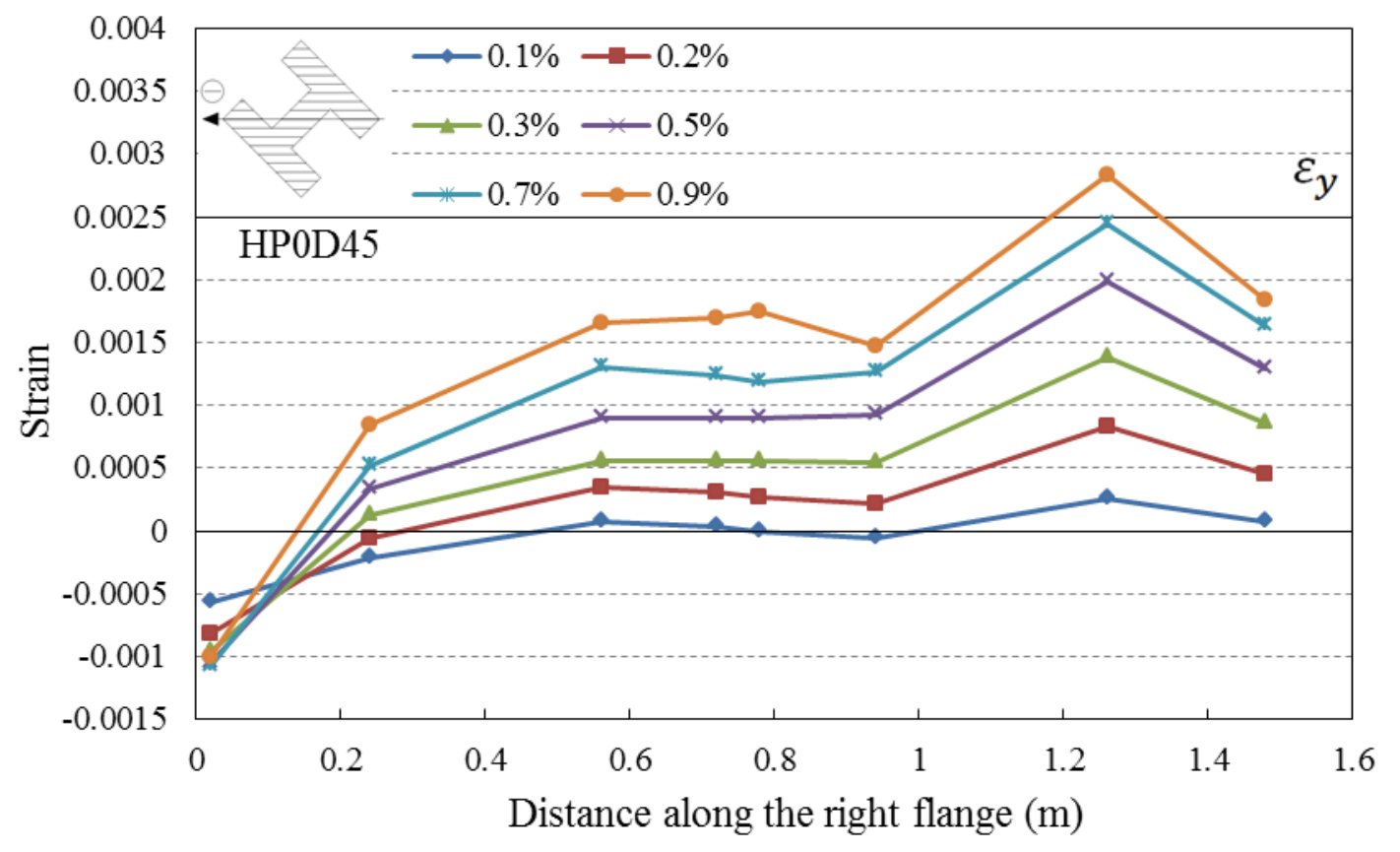

Figure 4.70 Strain profiles of longitudinal reinforcement along the flange of Specimen HP0D45 in the negative loading direction 


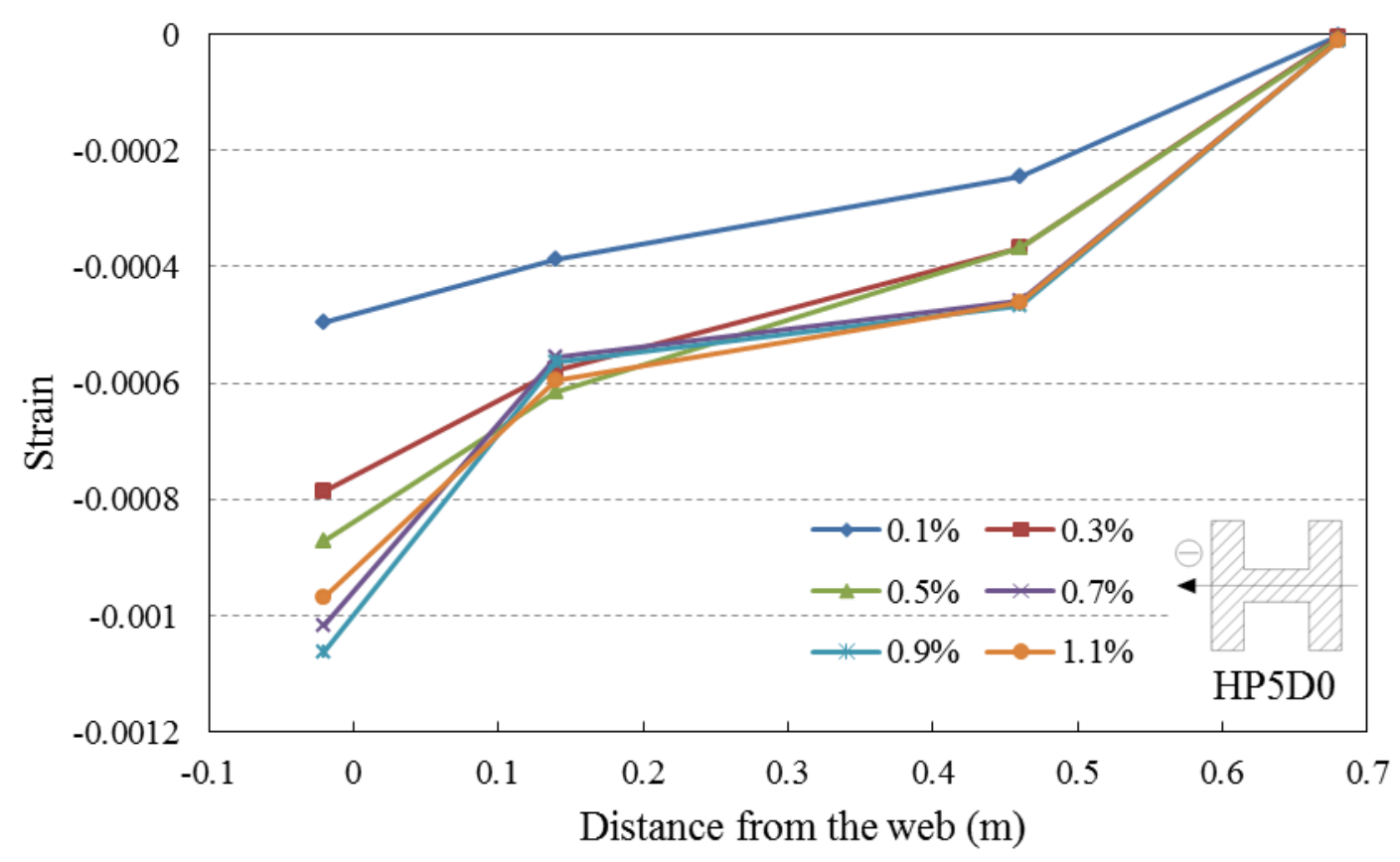

Figure 4.71 Strain profiles of longitudinal reinforcement along the flange of Specimen HP5D0 in the negative loading direction

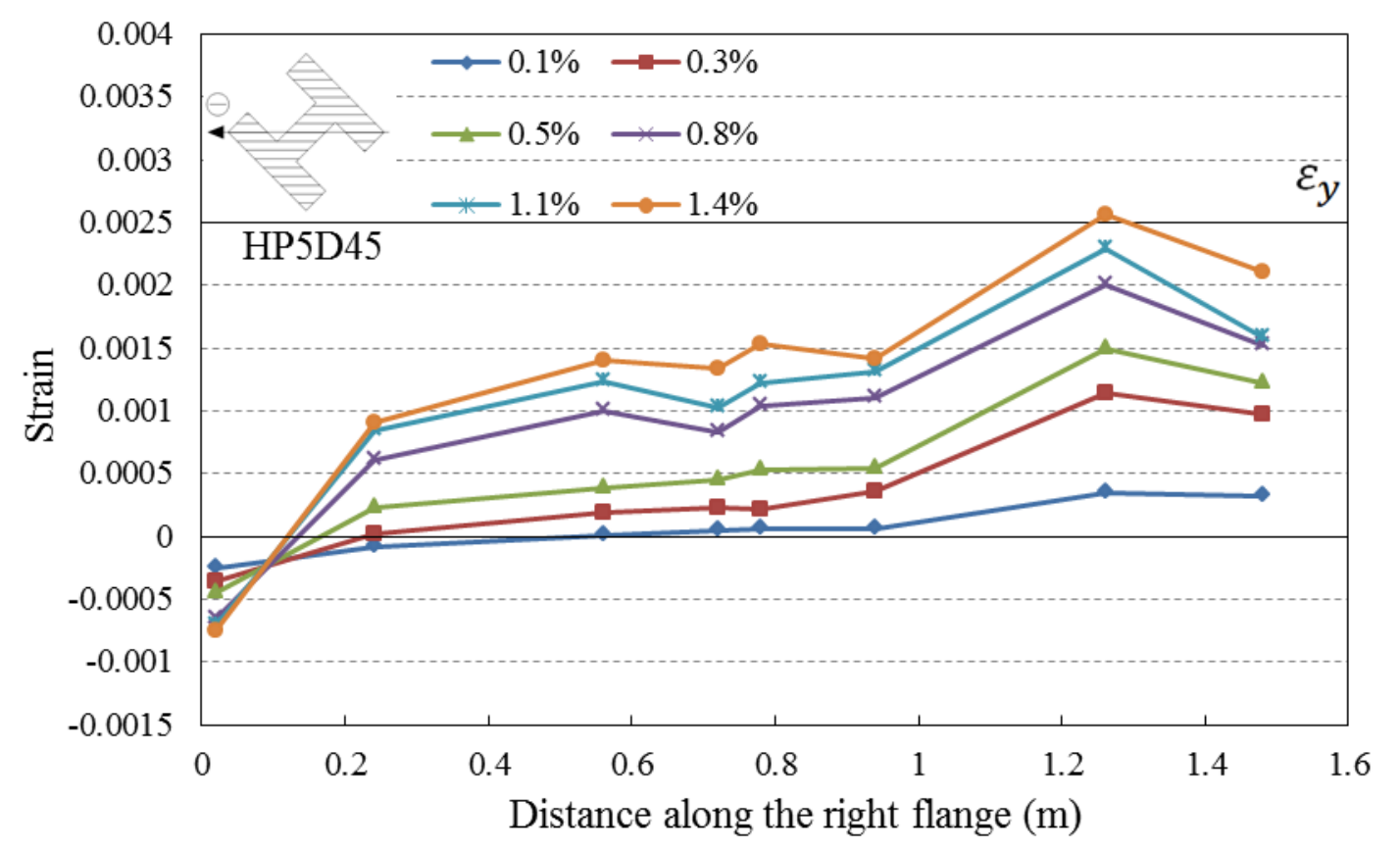

Figure 4.72 Strain profiles of longitudinal reinforcement along the flange of Specimen HP5D45 in the negative loading direction 


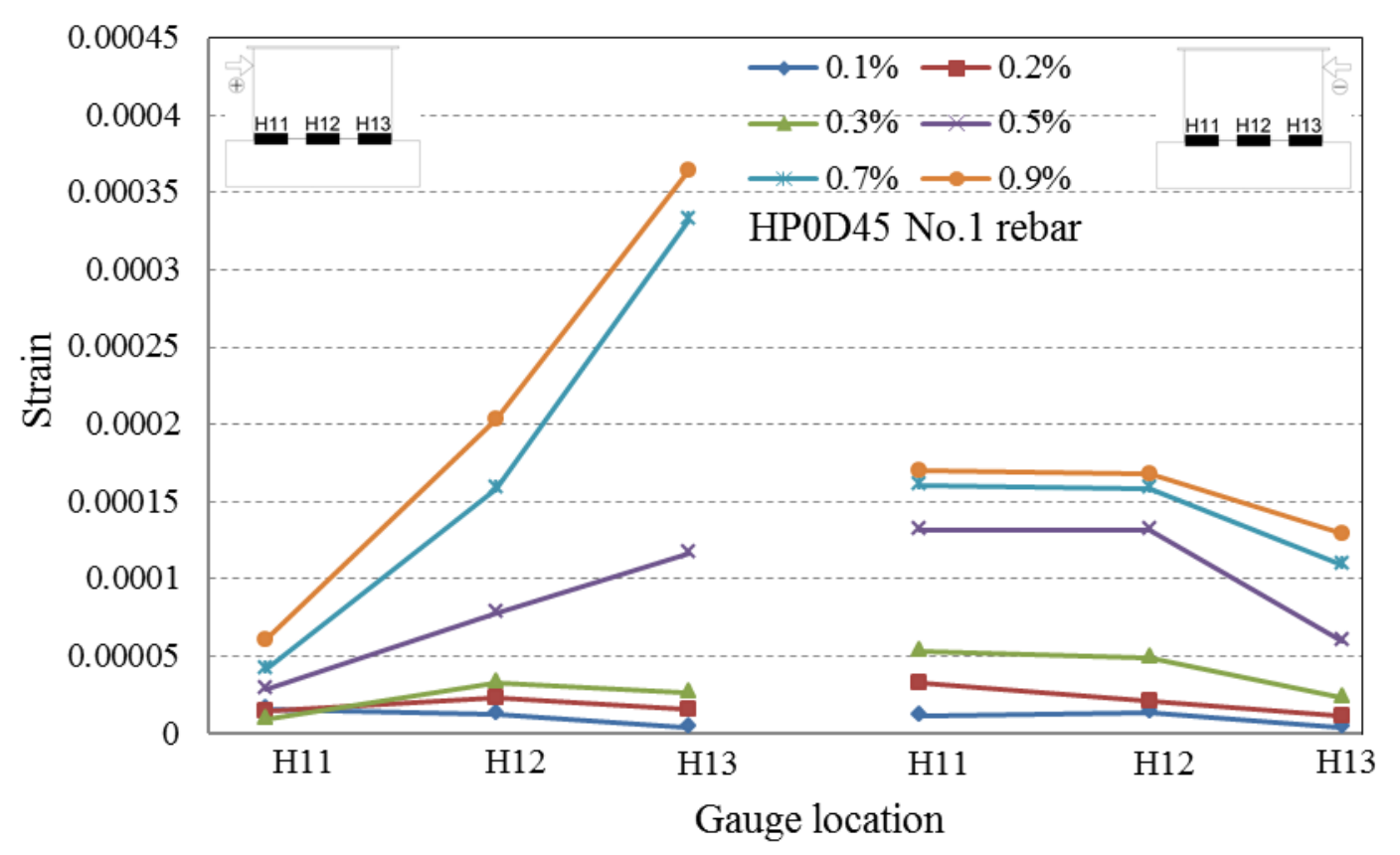

Figure 4.73 Strain profiles of No.1 horizontal rebar of Specimen HP0D45

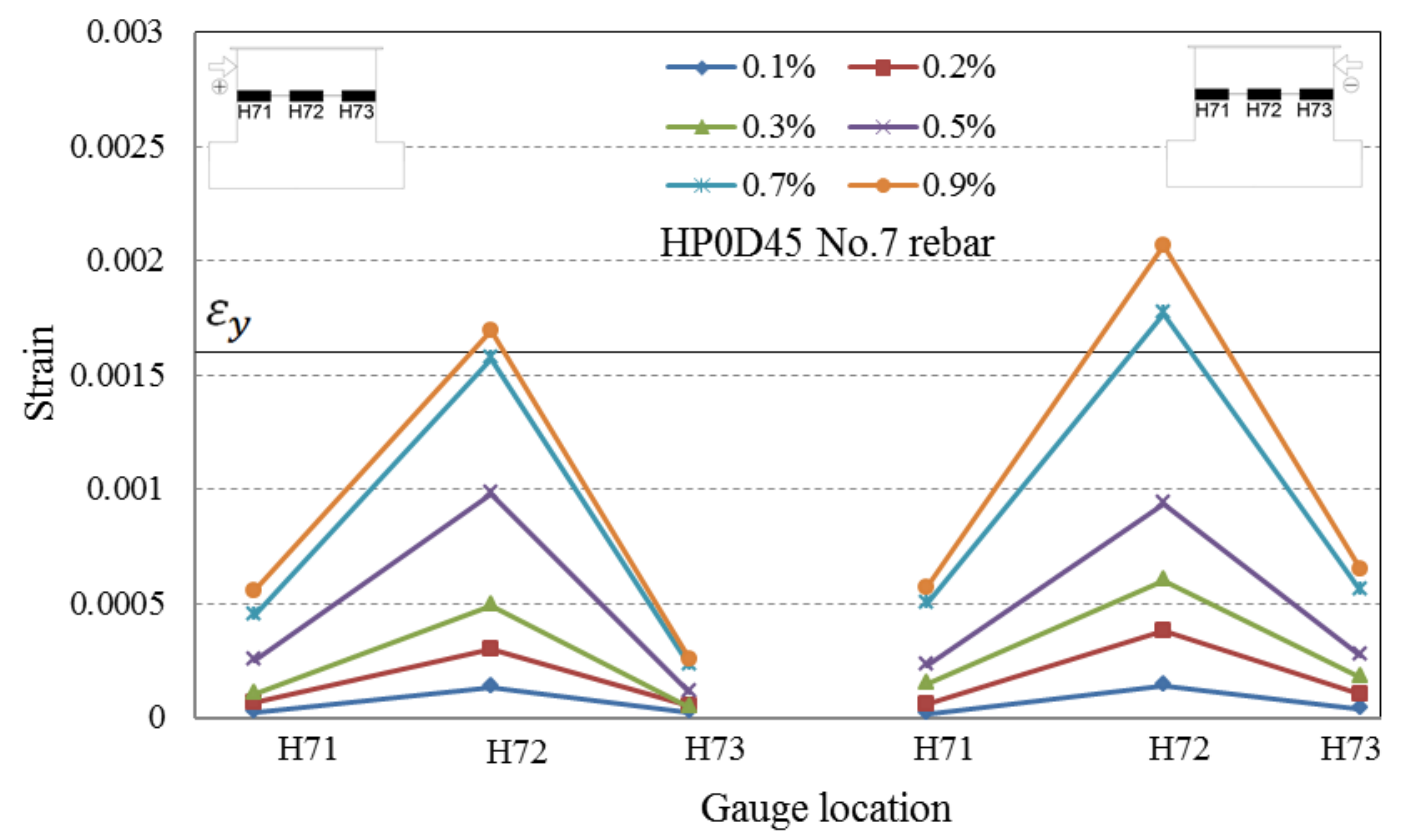

Figure 4.74 Strain profiles of No.7 horizontal rebar of Specimen HP0D45 


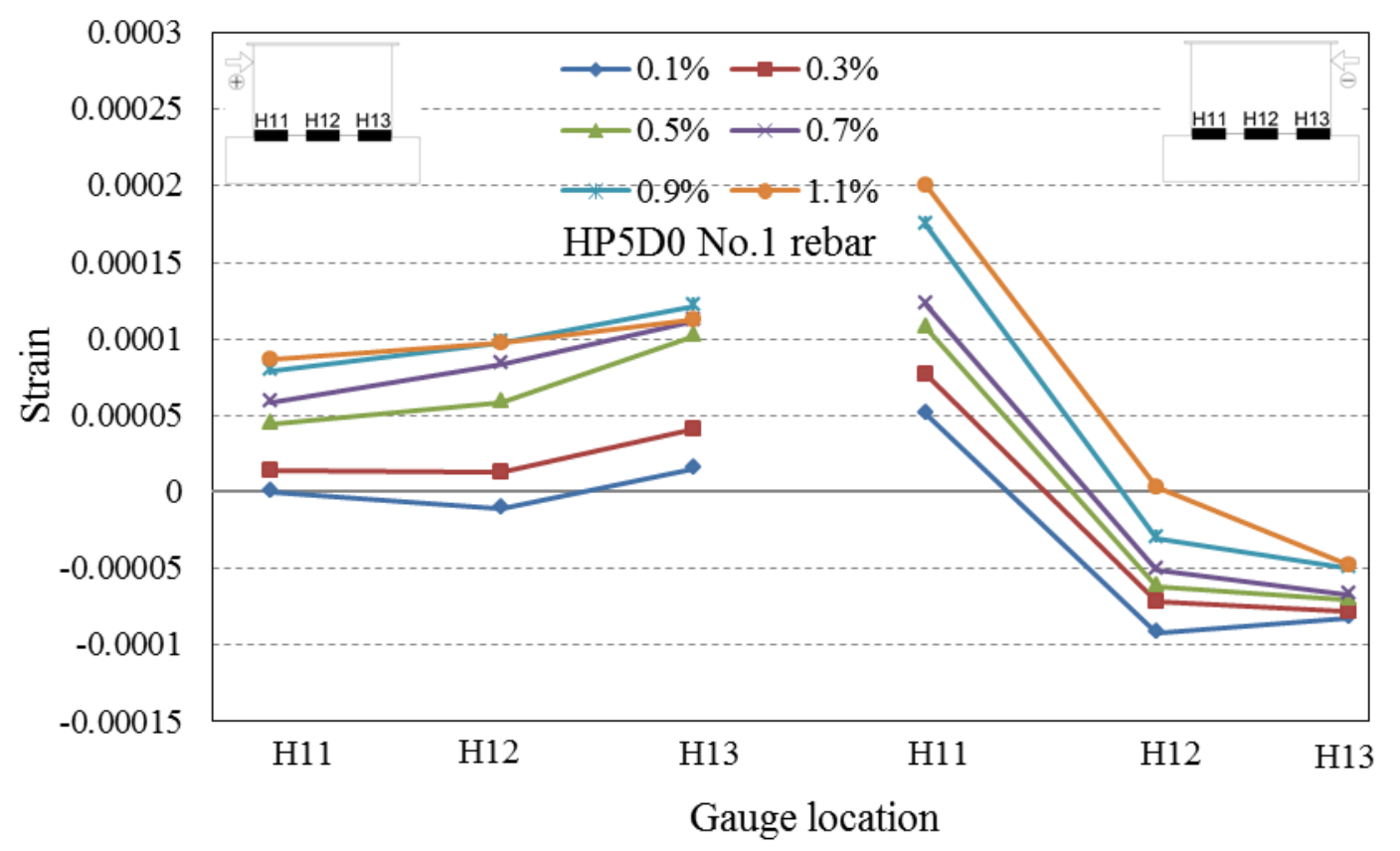

Figure 4.75 Strain profiles of No.1 horizontal rebar of Specimen HP5D0

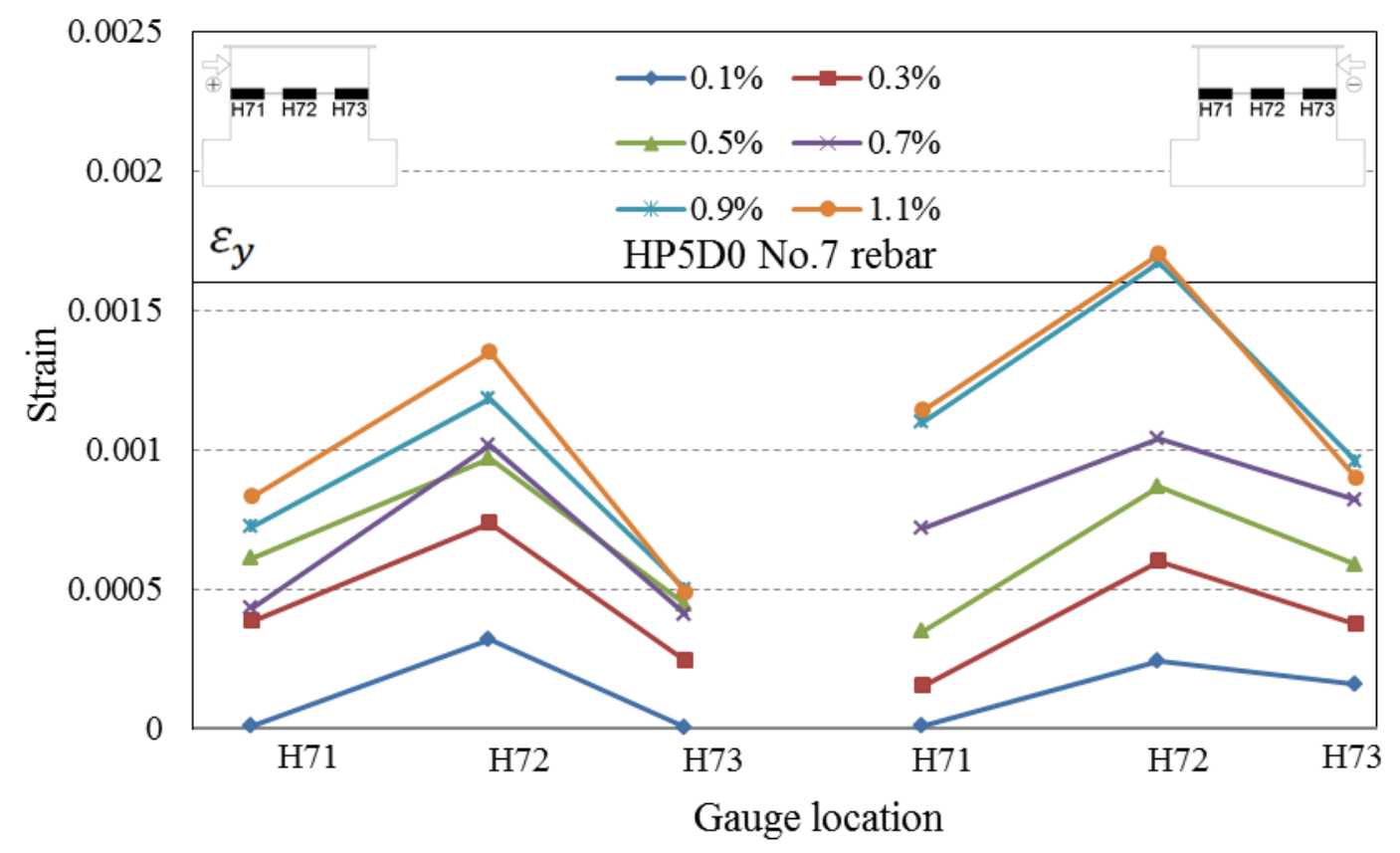

Figure 4.76 Strain profiles of No.7 horizontal rebar of Specimen HP5D0 


\subsection{Experimental Results of the T-shaped Specimens}

\subsubsection{Cracking patterns}

Figure 4.77 to Figure 4.80 demonstrate the development of cracks on each surface of the wall with outlines of reinforcement. The blue lines represent the cracks formed during the positive loading cycles. The red lines represent the cracks formed during the negative loading cycles.

Figure 4.77 below demonstrates the development of cracks on each surface of Specimen TP0D0 at drift ratios of $0.1 \%, 1.1 \%$ and $1.8 \%$ (displacements of $1 \mathrm{~mm}$, $11 \mathrm{~mm}$ and $18 \mathrm{~mm}$ ), which correspond to the initial stage, the crack development stage, and the final failure stage respectively. As seen from the figure, a diagonal crack located at the web almost $500 \mathrm{~mm}$ above the base quickly appeared when the test began its first horizontal loading circle. As the test progressed, the outermost longitudinal reinforcement in the junction of the web and flange experienced the first yielding at a drift ratio of $0.7 \%$, with a corresponding shear force of $406 \mathrm{kN}$. With lateral displacements rising to $11 \mathrm{~mm}$, more diagonal cracks occurred and intersected with each other on both faces of the web. Horizontal flexural cracks gradually developed and spread to the full length of the flange surface at the same time. In the positive direction, a maximum load of $637 \mathrm{kN}$ was recorded at a corresponding displacement of $17 \mathrm{~mm}$, and a load of $860 \mathrm{kN}$ at a displacement of $17 \mathrm{~mm}$ was attained in the negative direction. As illustrated in Figure 4.81, the specimens experienced a sudden drop of shear strength with moderate concrete spalling along the diagonal strut of the web and failed at the drift ratio of $1.8 \%$. 

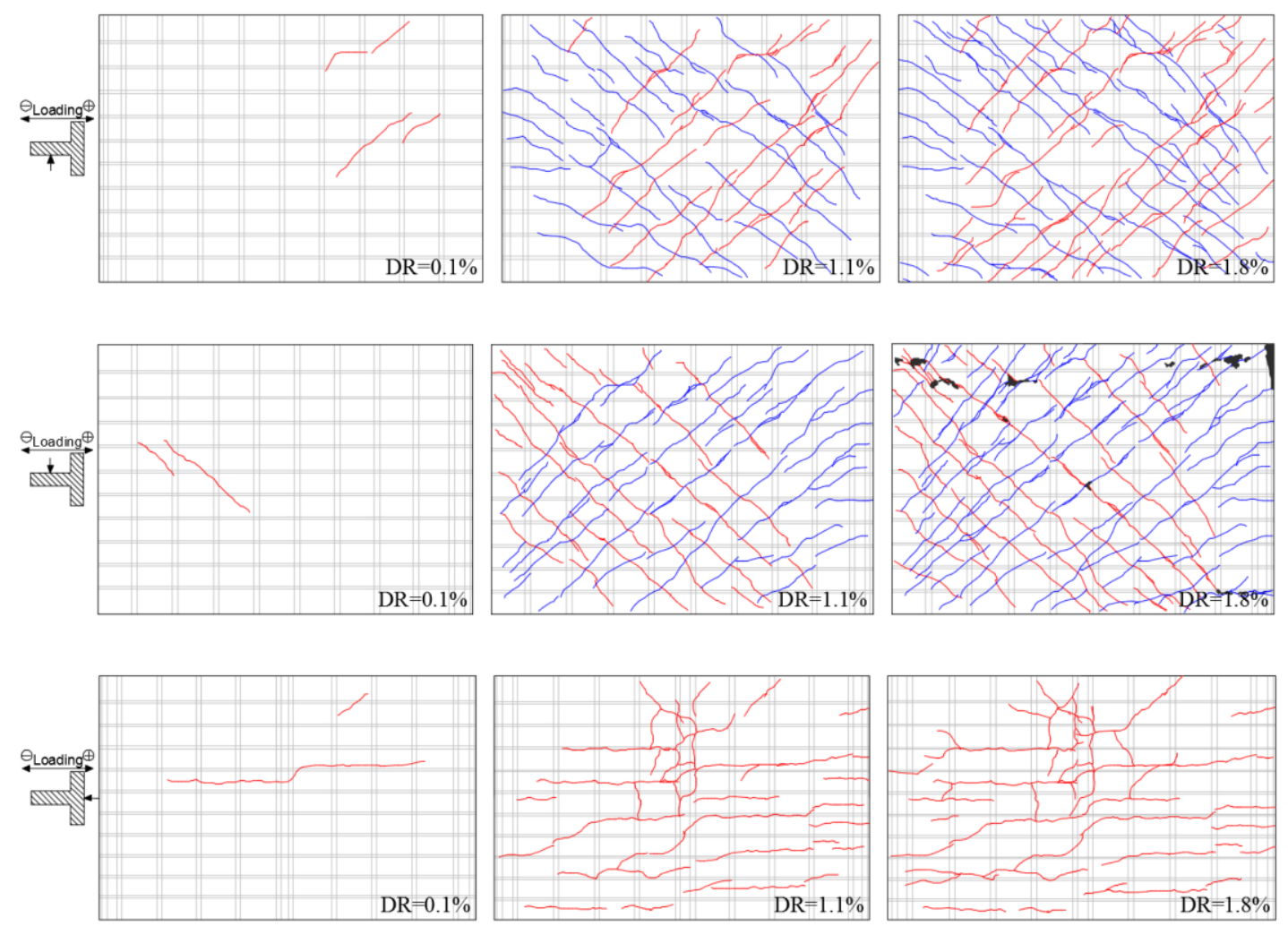

Figure 4.77 Cracking patterns of Specimen TP0D0

The plots of cracking patterns of each surface of Specimen TP0D45 at different loading stages are presented in Figure 4.78. A few diagonal cracks appeared at both web and flange after the first run of horizontal loading cycles. Increasing the drift ratio to $0.7 \%$ in the positive direction resulted in the first yielding of the longitudinal reinforcement near the tip of the flange. With lateral displacements further increasing to $8 \mathrm{~mm}$, diagonal cracks resulting from positive and negative loading grew slightly and intersected with each other on the web. It was witnessed that symmetric diagonal cracks from both loading directions occurred at the web, whereas the cracking patterns in two flanges showed a slight difference: the majority of their cracks formed when the flange was in tension. As the test proceeded to the failure, the number of cracks continued to rise and the cracks width also increased. The maximum capacities, $770 \mathrm{kN}$ in the positive and $874 \mathrm{kN}$ in the negative loading directions, were reached at displacements of $10 \mathrm{~mm}$ and 11 $\mathrm{mm}$. The specimen eventually failed at the first cycle of $15 \mathrm{~mm}$ displacement when the flange was in tension due to concrete crushing at the upper left corner of the web, as shown in Figure 4.82. 

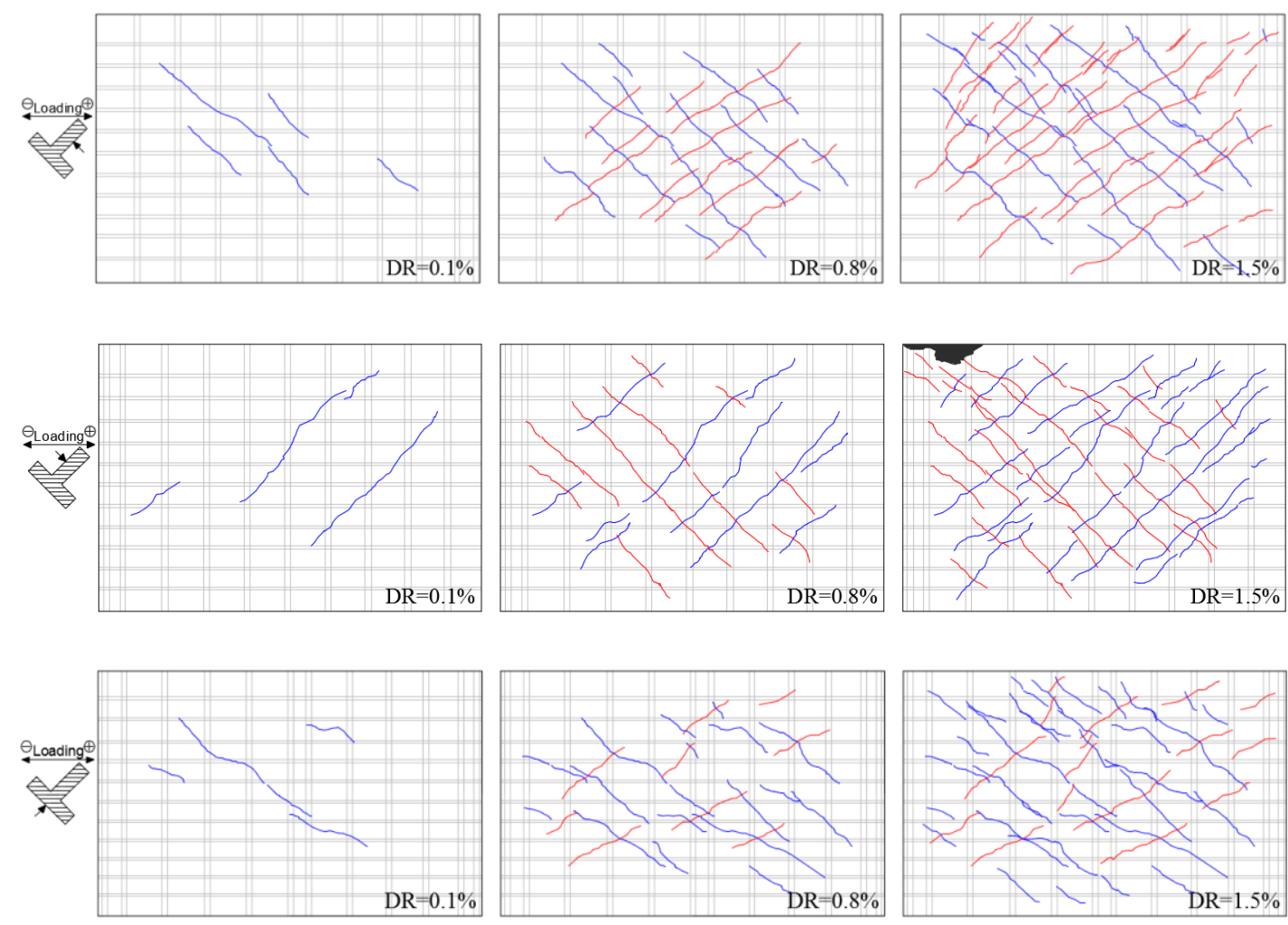

Figure 4.78 Cracking patterns of Specimen TP0D45

Regarding Specimen TP5D0, the first cracks were also detected when the lateral drift ratio reached $1 \%$. As the test continued, the outermost longitudinal reinforcement located in the juncture of the web and flange experienced yielding at the drift ratio of $0.8 \%$ with a corresponding shear of $650 \mathrm{kN}$. After that, cracks grew quite fast and quickly spread all over the web surfaces before the drift ratio reached $1.0 \%$. It was noted that compared with Specimen TP0D0 with no additional axial loads, the angle of diagonal cracks located on the web were apparently steeper in this specimen, which was in accordance with findings of past experiments [Z2]. This was attributed to the effect of vertical stress brought by the axial load, which changed the angle of the concrete strut. Meanwhile, however, only a few horizontal flexural cracks were witnessed on the flange, which was due to the restraint brought by axial loads. In the subsequent loading cycles, diagonal cracks on the web became denser, spreading to the entire surfaces, causing severe concrete spalling at the upper part of the web, forming a major horizontal crack, and eventually leading to the failure the specimen. This phenomenon was also found in the aforementioned $\mathrm{H}$-shaped squat walls. Furthermore, three vertical slip planes were observed on the 
web surface as presented in the Figure 4.83. A similar observation was also made in Specimen HP5D0 as presented previously. The maximum shear strength and the corresponding displacement in the positive direction were $748 \mathrm{kN}$ and $11 \mathrm{~mm}$, and $765 \mathrm{kN}$ and $10 \mathrm{~mm}$ for the negative loading direction. The specimen shared a similar failure mode with Specimen HP5D0 and failed at the lateral displacement of $14 \mathrm{~mm}$ due to sliding and concrete spalling in the top.
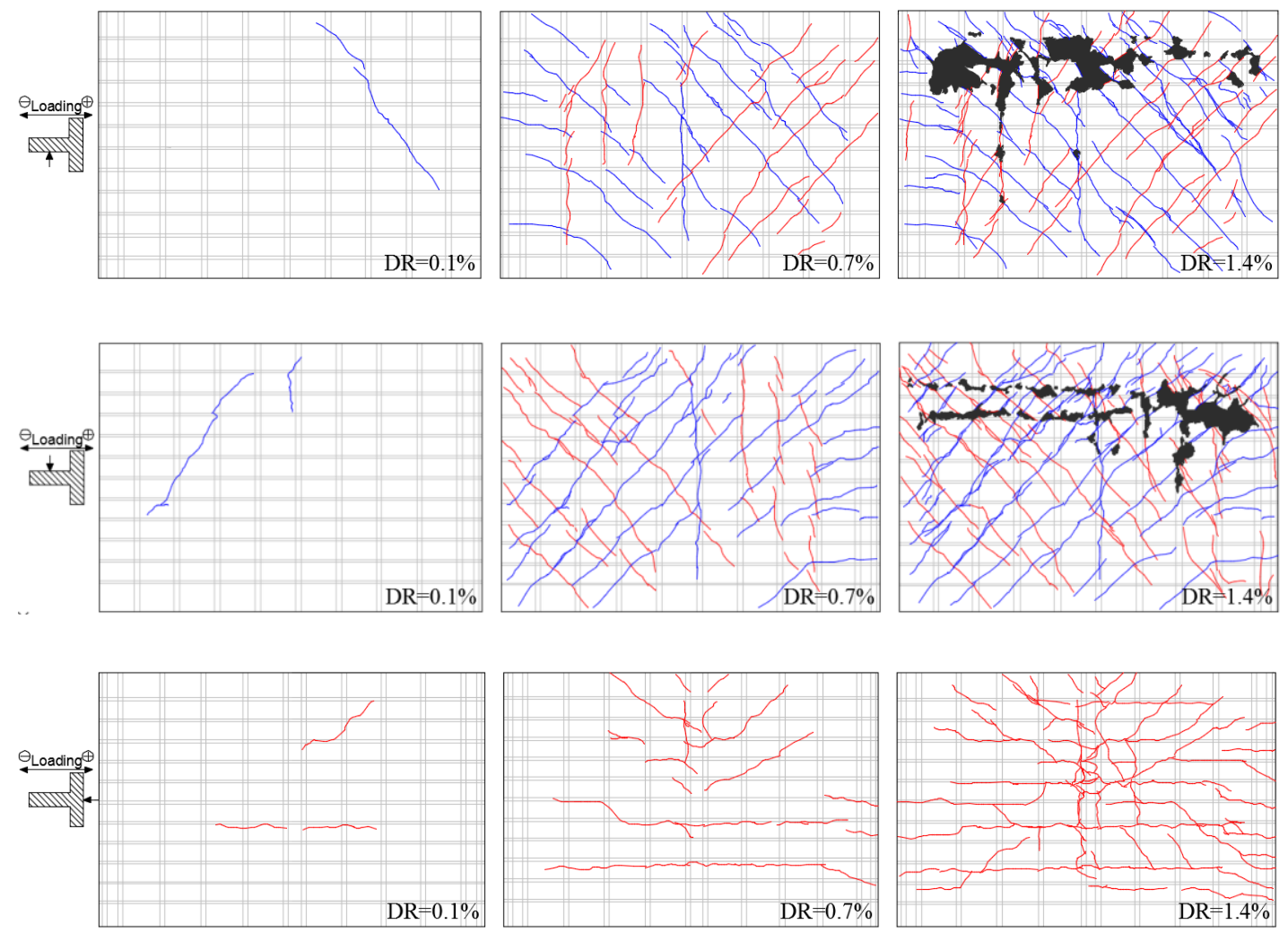

Figure 4.79 Cracking patterns of Specimen TP5D0

As for Specimen TP5D45, the lateral displacement of $1 \mathrm{~mm}$ caused cracks on each surface of the wall. The longitudinal reinforcement in the flange attained its first yielding at the displacement of $7 \mathrm{~mm}$, corresponding to a shear force of $879 \mathrm{kN}$. As lateral displacements rose, diagonal cracks continued to occur on all surfaces of the wall and intersected with each other. Similar to Specimen TP0D45, cracks resulting from positive and negative loading distributed evenly on both web surfaces, while the flange saw more cracks developed when it was in tension. The maximum shear strength and the corresponding displacement in the positive direction were $938 \mathrm{kN}$ and $10 \mathrm{~mm}$, respectively, and $1025 \mathrm{kN}$ and $11 \mathrm{~mm}$ for the negative loading 
direction. The specimen was able to maintain its lateral strength at drift ratios between $1.0 \%$ and $1.2 \%$. As seen in Figure 4.80, the specimen failed with a sudden drop of strength as a result of concrete crushing at the upper left corner of the web at the displacement of $14 \mathrm{~mm}$.
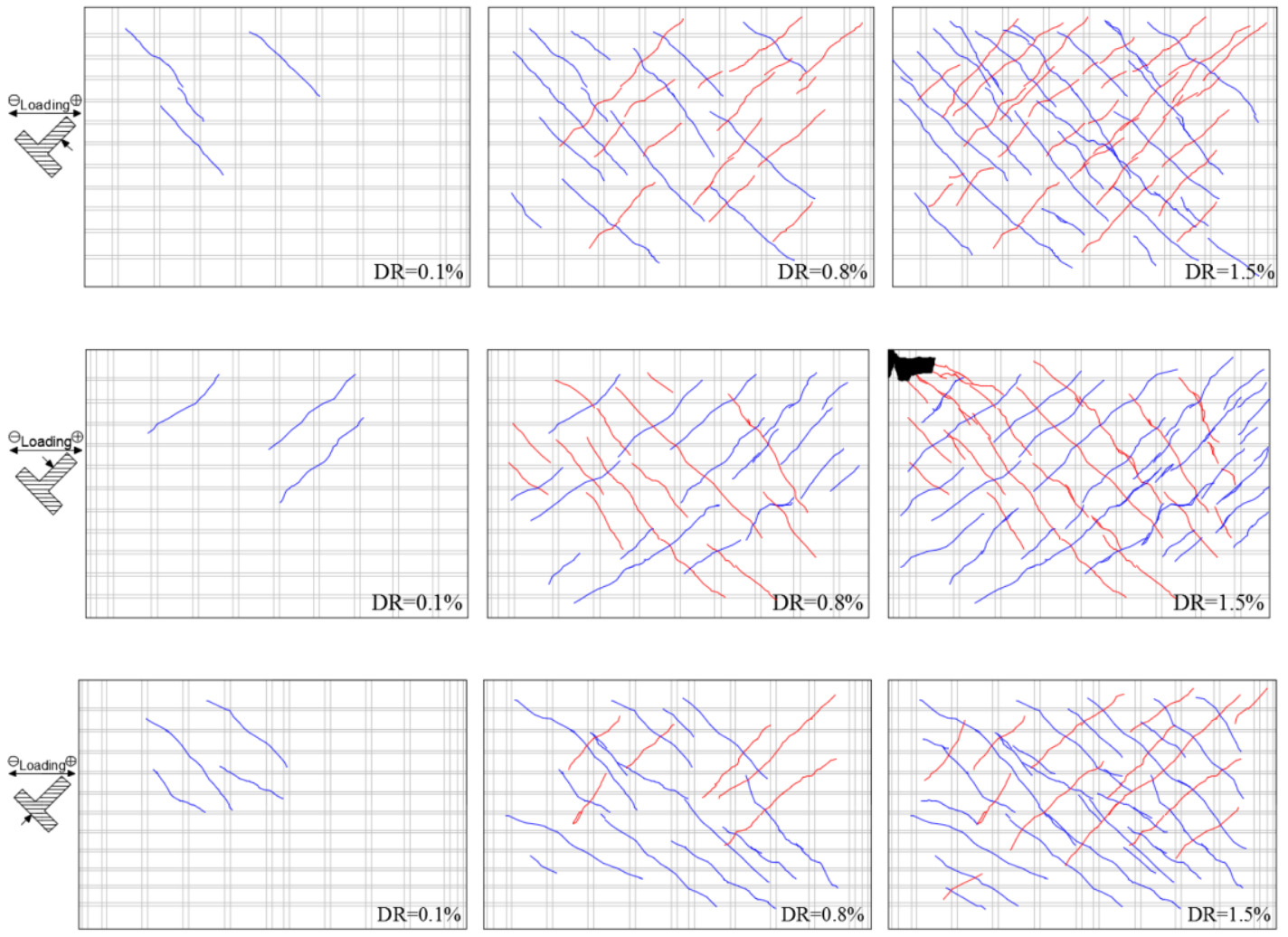

Figure 4.80 Cracking patterns of Specimen TP5D45

From the tests, it was clear that initial shear cracks located at the upper part of specimens occurred at a drift ratio of $0.1 \%$, which was prior to the yielding of longitudinal reinforcing bars. After that, more diagonal cracks appeared, intersected with each other and gradually spread all over the web. Compared with Specimen TP0D0, Specimen TP5D0 with more axial loads had a steeper angle of inclined cracks on its web. It was also witnessed that the two surfaces of the web generally showed a similar distribution of cracks. As for the cracking patterns on flanges, Specimens TP0D0 and TP5D0 with orthogonal lateral loading had most of their cracks as horizontal flexural cracks, which extended to the full length of the flange as lateral loads increased. In Specimens TP0D45 and TP5D45 with skew lateral loading, more shear cracks occurred. The specimens experienced their first yielding 
at a drift ratio ranging from $0.7 \%$ to $0.8 \%$. As the test progressed, denser cracks on the web caused severe concrete spalling at the upper part of the web in specimens with orthogonal lateral loading. Specimens TP0D0 failed with concrete spalling along the diagonal of the web and a drastic drop of the lateral strength. Specimens TP5D0, on the other hand, was able to develop its maximum shear strength before the formation of several vertical slip planes. As for Specimens TP0D45 and TP5D45, both walls were able to sustain a ductile post-peak response for several loading cycles. These specimens failed in a similar way as the concrete in the upper left corner of the web suddenly was crushed. The ultimate drift ratios reached in the test were from $1.4 \%$ to $1.8 \%$.

\subsubsection{Failure mechanisms}

Figure 4.81 depicts the web of Specimen TPOD0 at the ultimate drift ratio. The specimen failed in a similar way as the diagonal compression failure which has been proven common in walls with large flanges. With increases in the lateral displacements, the inclined cracks formed by the cyclic loading intersected with each other, causing moderate concrete spalling along the diagonal strut of the web. Unlike the typical diagonal compression failure characterized by concrete crushing at the toe of the web, Specimen TP0D0 failed when the right upper corner suddenly crushed and dropped, which resulted in a drastic loss of strength.

Similar to Specimen HP5D0 mentioned previously, Specimen TP5D0 failed with a major horizontal sliding plane at the upper part of the web and several vertical slip planes in the middle of the web, which was shown in Figure 4.83. The mechanism behind of the horizontal sliding plane was explained in the previous section, which was related to the weaker concrete adopted and the stress concentration caused by the stiff top slab. As for the vertical slip planes, the combined effect of axial loads, the stiff flange and the deformation incompatibility was believed to be the primary contributing factor. More detailed analytical investigations were carried out in the next chapter to account for the deformation incompatibility.

With regard to Specimens TP0D45 and TP5D45 with skew lateral loading, both specimens were able to sustain a ductile post-peak response before failure. As the 
drift ratio further increased, a slight drop of shear strength was witnessed but in general, the two specimens maintained their strength for several cycles. During this stage, the diagonal cracks became wider and slight concrete spalling was observed in the web. The drift ratio of $1.5 \%$ saw the failure of tested specimens. As seen in Figure 4.82, the crushing of concrete in the upper left corner of the web led to a sudden drop of lateral strength to approximately $30 \%$ of the maximum, which exceeded the failure criteria as 20\% decline. Hence Specimen TP0D45 was deemed as having failed. A similar failure mechanism was also observed in Specimen TP5D45, where the specimen was unable to provide adequate lateral strength after a corner of the diagonal concrete strut was crushed (Figure 4.84).

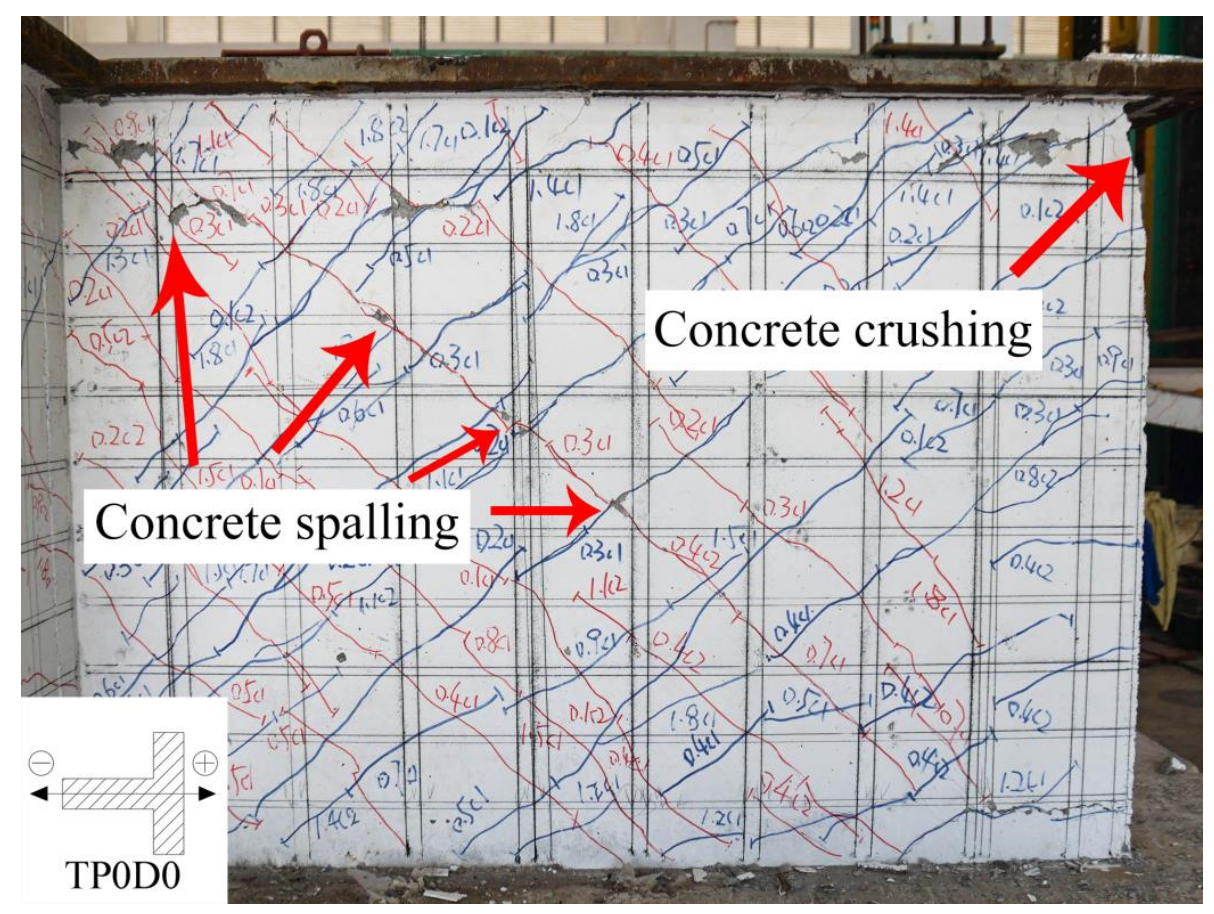

Figure 4.81 Failure mechanism of Specimen TP0D0 


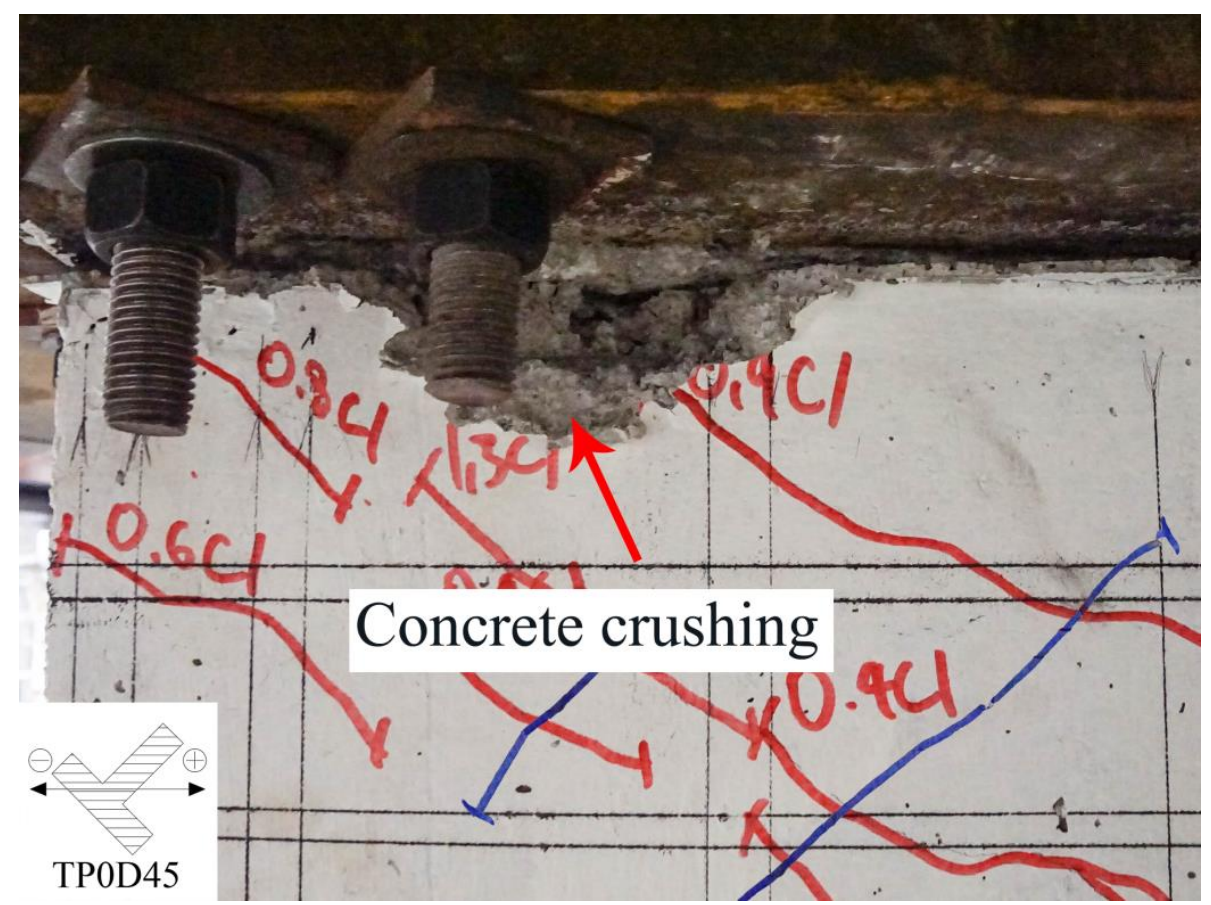

Figure 4.82 Failure mechanism of Specimen TP0D45

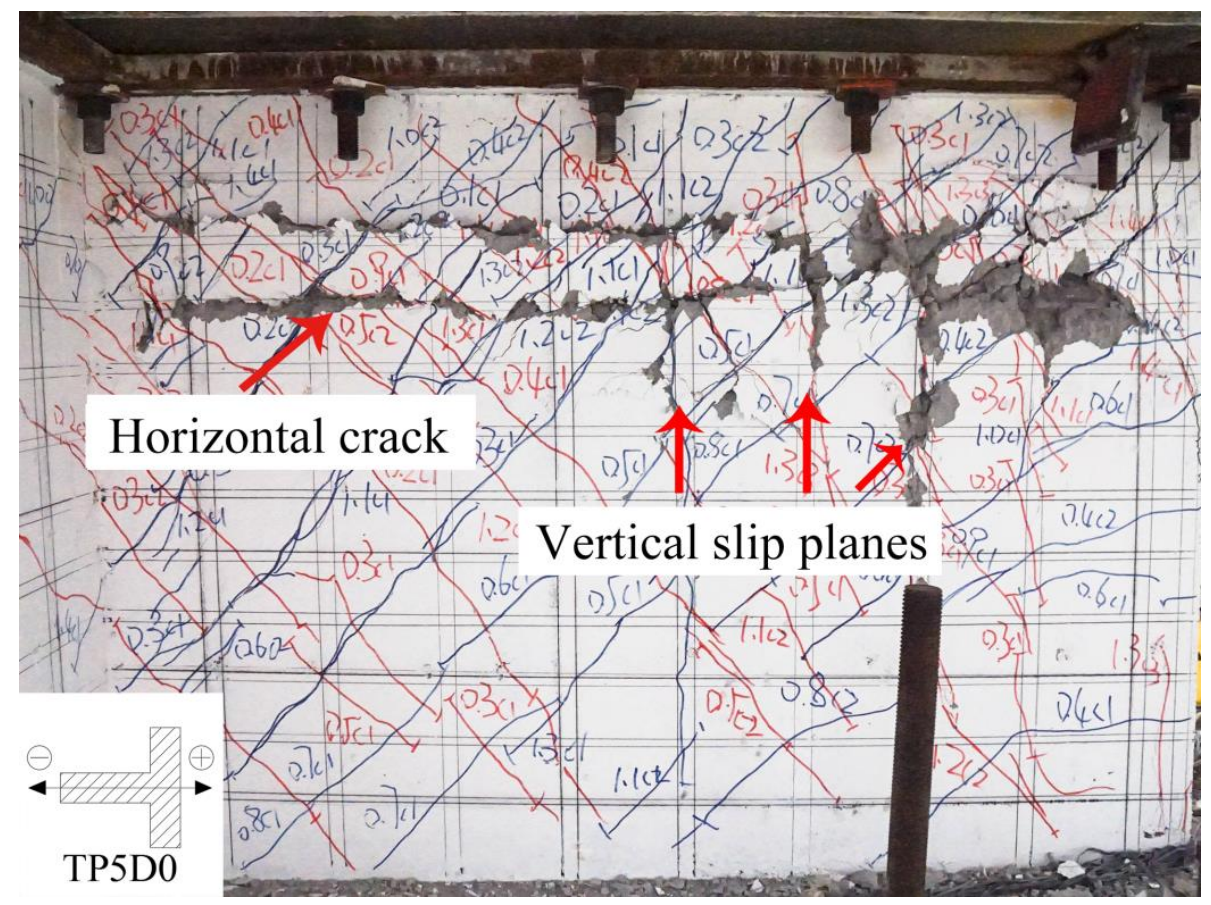

Figure 4.83 Failure mechanism of Specimen TP5D0 


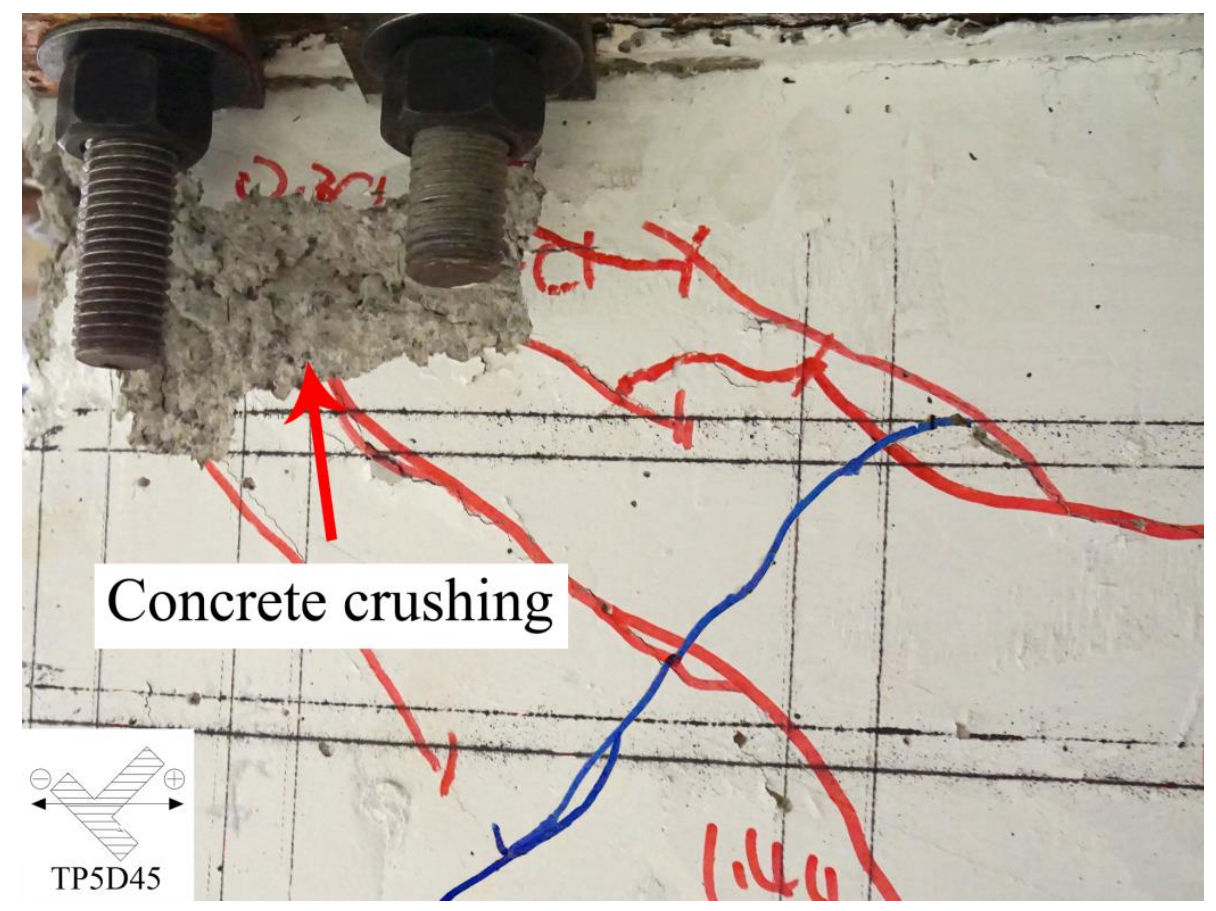

Figure 4.84 Failure mechanism of Specimen TP5D45

\subsubsection{Hysteretic responses}

As mentioned previously, load-displacement curves play a significant role in evaluating the seismic behavior of RC structures. Data recorded by load cells and horizontally-mounted LVDTs were used to plot load-displacement curves. Relevant aspects such as strength, secant stiffness and energy dissipation capacity were assessed and discussed in the following part. Table 4.3 summarizes some key features as well as the experimental results of the T-shaped specimens. Figure 4.85 to Figure 4.88 depict the lateral load versus top displacement curves of the Tshaped specimens. 
Table 4.3 Test results of the T-shaped specimens

\begin{tabular}{cccccccccc}
\hline \multirow{3}{*}{ Specimen } & $(1)$ & $(2)$ & $(3)$ & $(4)$ & $(5)$ & $(6)$ & $(7)$ & $(8)$ \\
& $f_{c}^{\prime}$ & $P / f_{c}^{\prime} A_{g}$ & $\theta$ & $V_{c r}$ & $V_{\max }$ & $\Delta_{y}$ & $K_{i}$ & $\delta_{\max }$ \\
\cline { 2 - 9 } & $\mathrm{MPa}$ & $\%$ & $\circ$ & $\mathrm{kN}$ & $\mathrm{kN}$ & $\mathrm{mm}$ & $\mathrm{kN} / \mathrm{mm}$ & $\%$ \\
\hline TP0D0 & 35.0 & 0.72 & 0 & 230 & 860 & 7.0 & 58 & 71 & 1.7 \\
\hline TP0D45 & 36.2 & 0.70 & 45 & 554 & 874 & 7.0 & 105 & 91 & 1.5 \\
\hline TP5D0 & 35.0 & 5.65 & 0 & 269 & 765 & 8.0 & 81 & 101 & 1.4 \\
\hline TP5D45 & 36.2 & 5.46 & 45 & 426 & 1025 & 7.0 & 128 & 109 & 1.5 \\
\hline
\end{tabular}

Note: (1): Compressive strength of concrete; (2) Axial load ratio; (3) Angle between lateral loading and web segment; (4) Observed shear strength at the first cracking; (5) Maximum shear strength during the test; (6) Yield displacement; (7) Effective stiffness in positive and negative loading directions; (8) Maximum drift ratio.

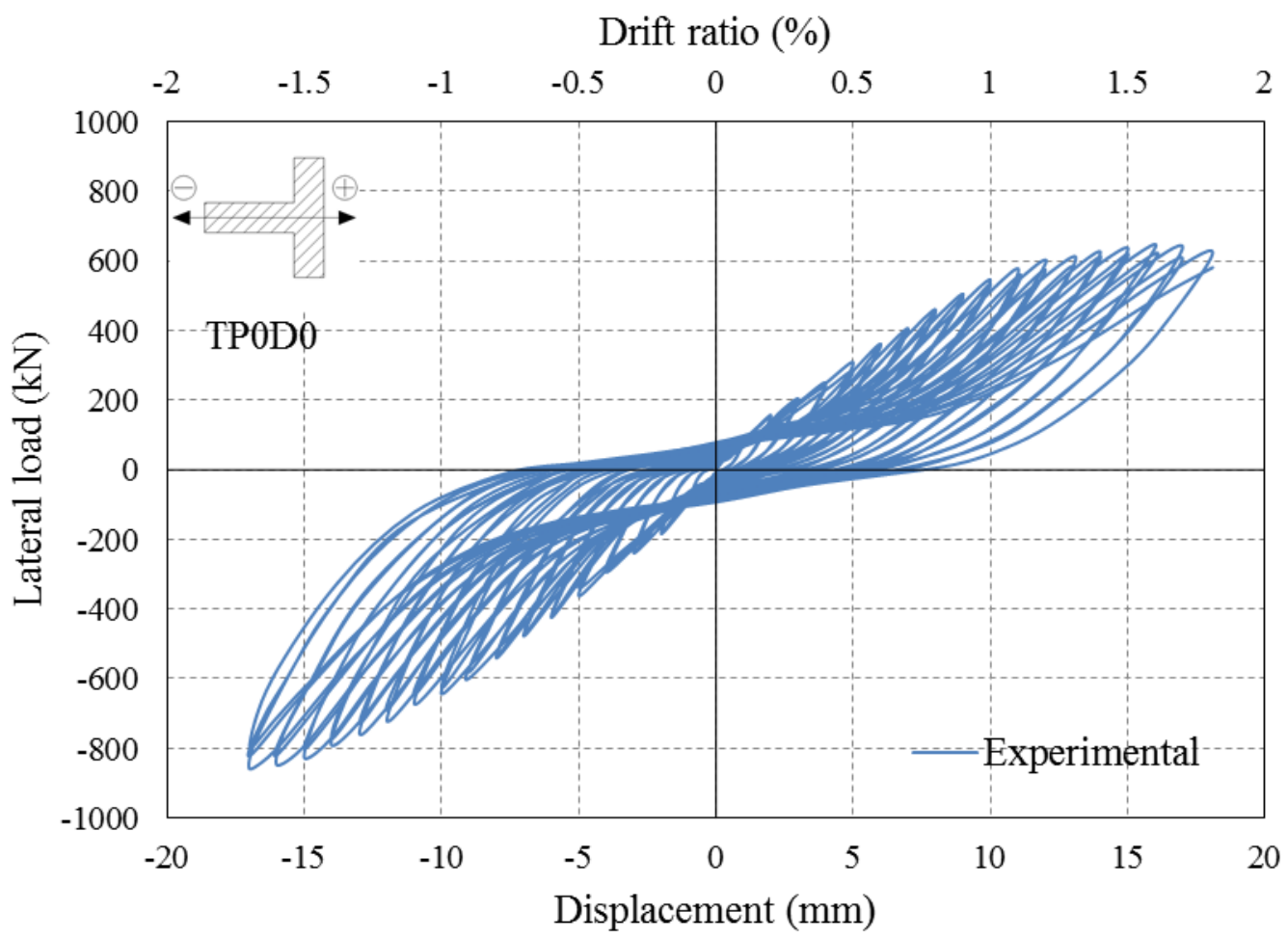

Figure 4.85 Lateral load-displacement curve of Specimen TP0D0 


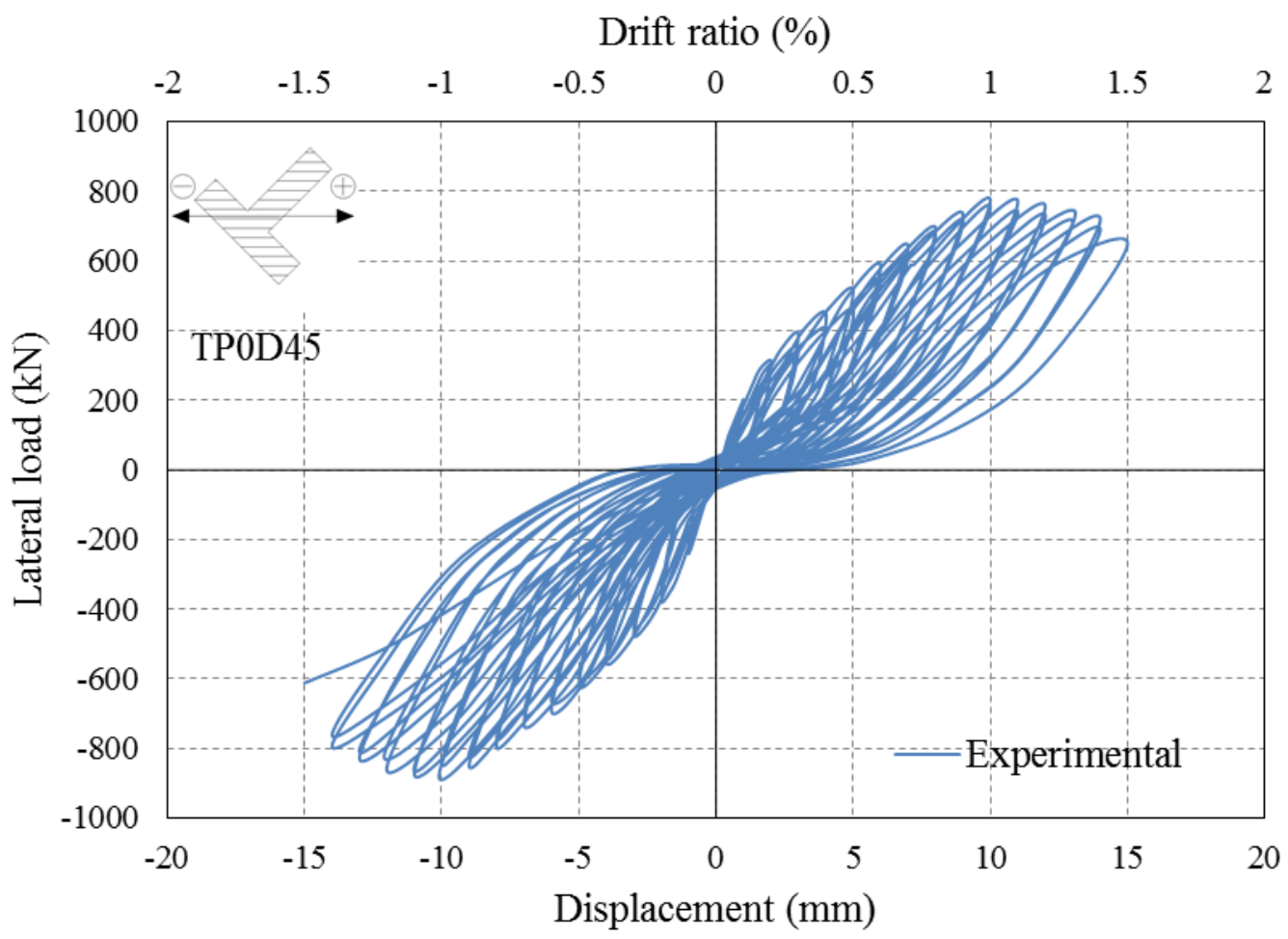

Figure 4.86 Lateral load-displacement curve of Specimen TP0D45

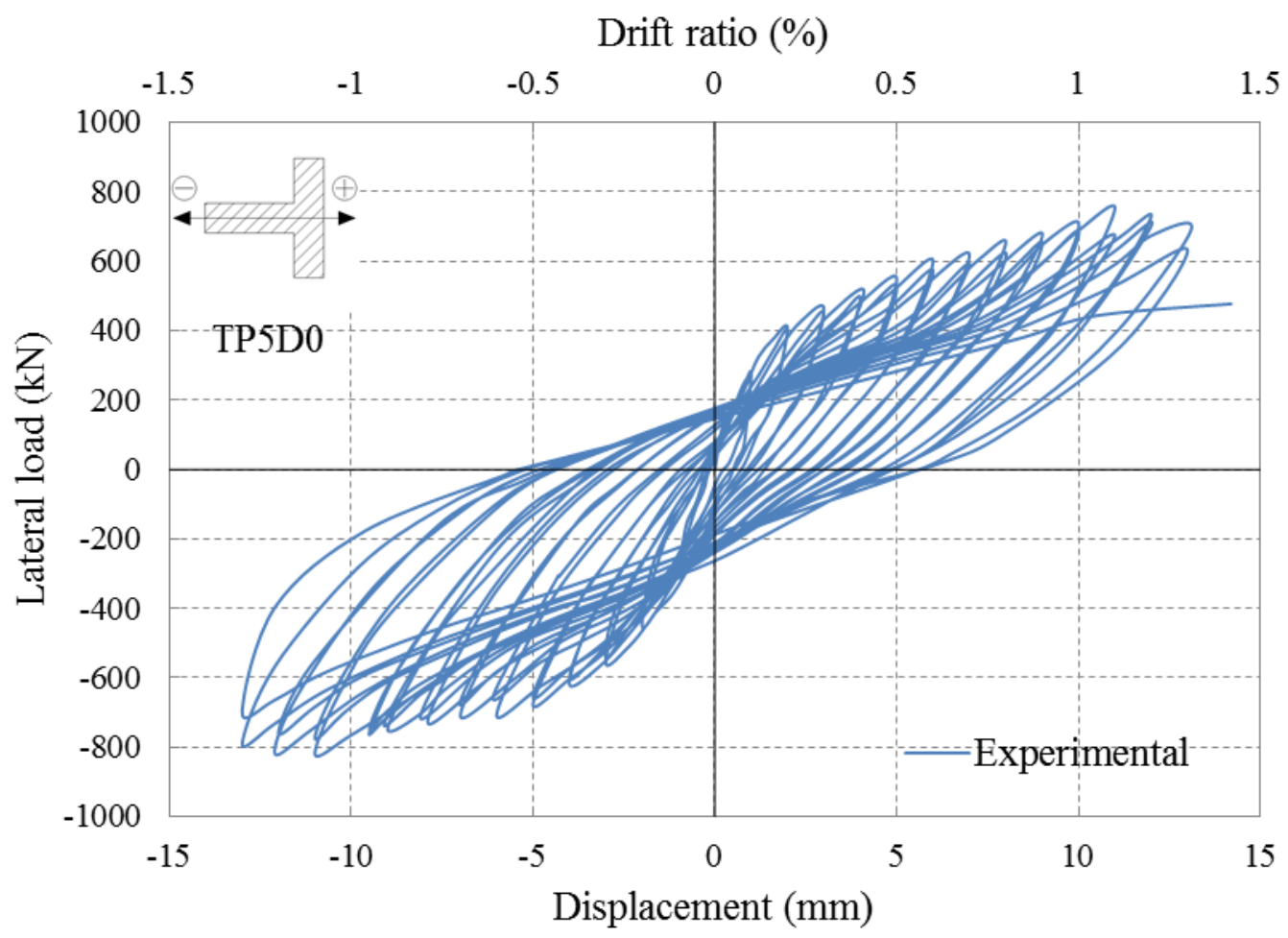

Figure 4.87 Lateral load-displacement curve of Specimen TP5D0 


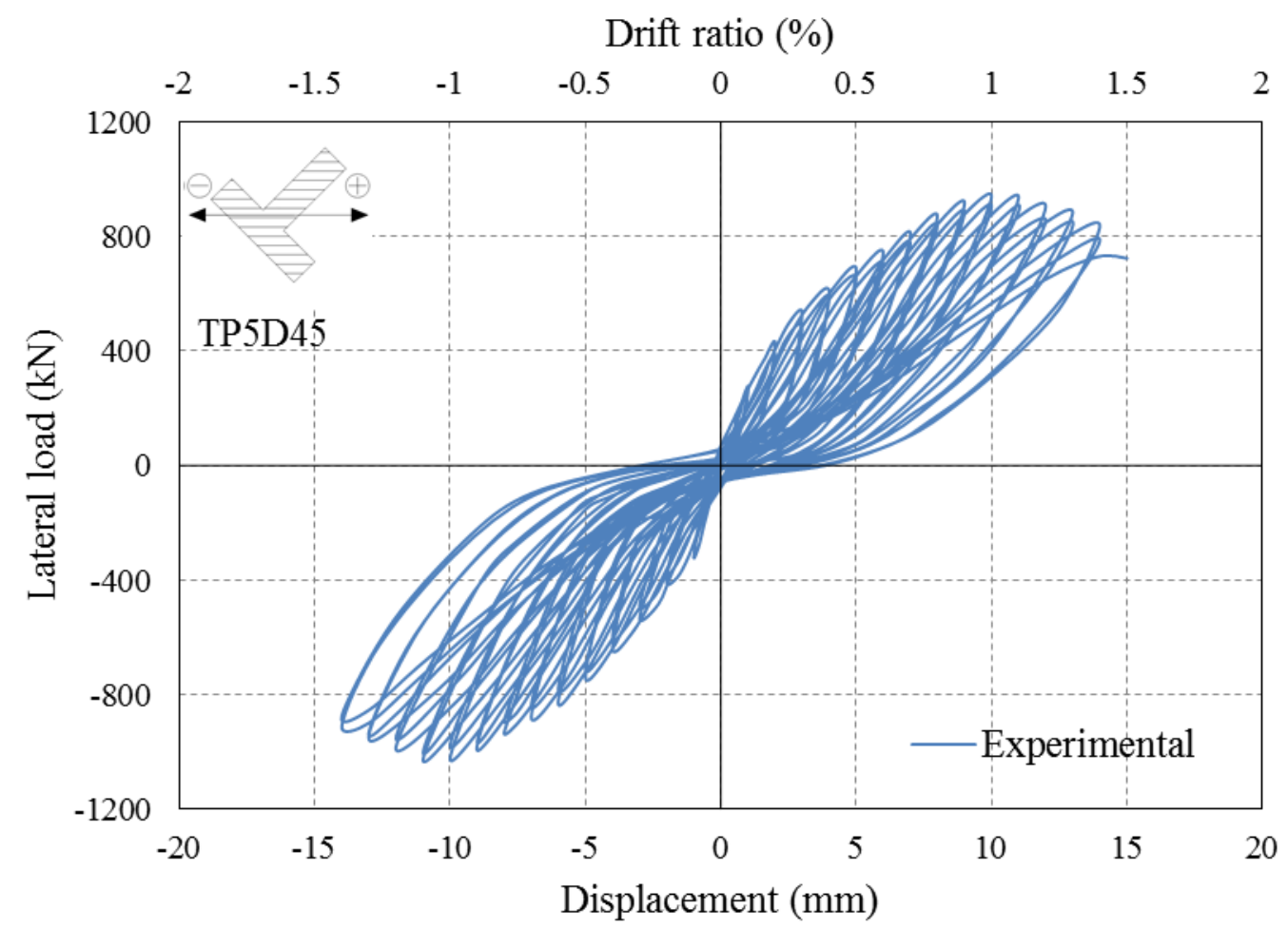

Figure 4.88 Lateral load-displacement curve of Specimen TP5D45

The backbone envelopes of T-shaped specimens were demonstrated in Figure 4.89. Although Specimen TP0D0 had a larger maximum shear strength than Specimen TP5D0, it was evident that specimens with additional axial loads had larger lateral reaction at the same drift ratio, which was consistent with the previous findings [Z2]. A similar trend could also be traced in the group with skew lateral loading. Moreover, it was clear that Specimen TP0D0 with lower axial loads outperformed its counterpart in terms of the deformation capacity, with their numbers of $1.7 \%$ and $1.4 \%$ respectively. However, the specimens with skew lateral loading demonstrated nearly the same capability of deformation.

As discussed in the previous sections, secant stiffness is crucial since it is deeply related to the iterative method and acted as an indicator of material properties. Figure 4.90 presents the variation of secant stiffness during the whole experiment. As expected, all specimens experienced a reduction in stiffness as the drift ratio increased. Among them, the most dramatic decline of secant stiffness was observed in Specimens TP5D0 and TP5D45 at the initial loading stage. With the increase of drift ratio, the secant stiffness of each specimen further declined and remained at a 
comparatively low value at the final stage. Moreover, axial loads could be seen as an active factor in increasing the secant stiffness. Its contribution was attributed to the fact that axial loads constrained the diagonal cracks and limited the development of the sliding shear, thereby enhancing the reaction at a specific drift ratio.

As presented in the previous section, the energy dissipation of wall specimens is calculated from the inner area of lateral load-displacement loops, which is important in assessing the seismic behavior of RC structures. Figure 4.91 below illustrates the energy dissipation capacity of each specimen with respect to the drift ratio. In the initial stage, a rather small portion of energy was dissipated. As the drift ratio rose, the dissipation of energy steadily increased and experienced a drastic growth at the final stage. It was evident in the figure that the group with more axial loads had a better energy dissipation capacity than its counterpart. Furthermore, the orthogonal horizontal loading was observed to have a more favorable effect in increasing the energy dissipation, which was reflected in the $59 \%$ discrepancy between Specimens TP5D0 and TP5D45. This phenomenon was in consistence with the findings of the H-shaped specimens.

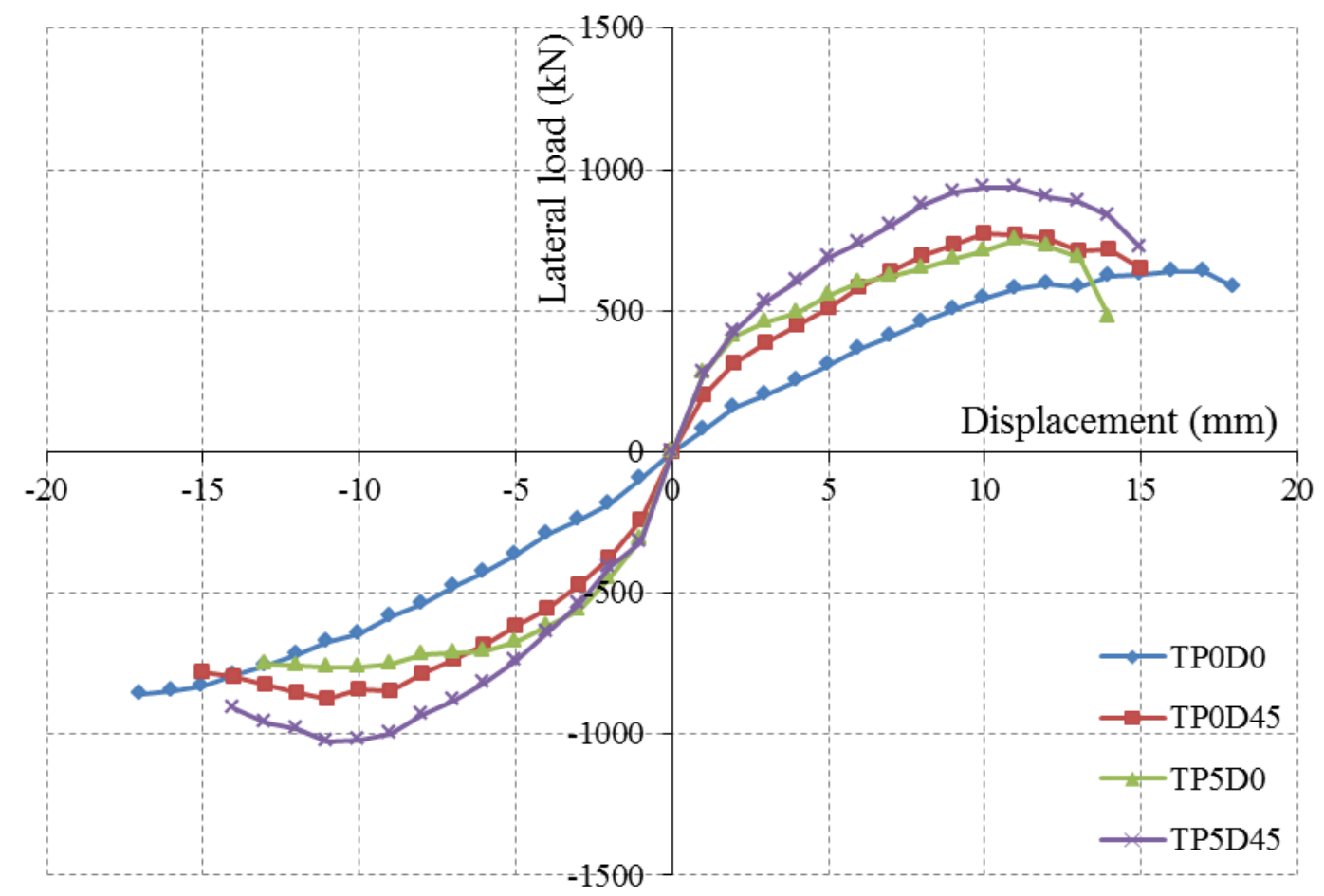

Figure 4.89 Backbone curves of the T-shaped specimens 


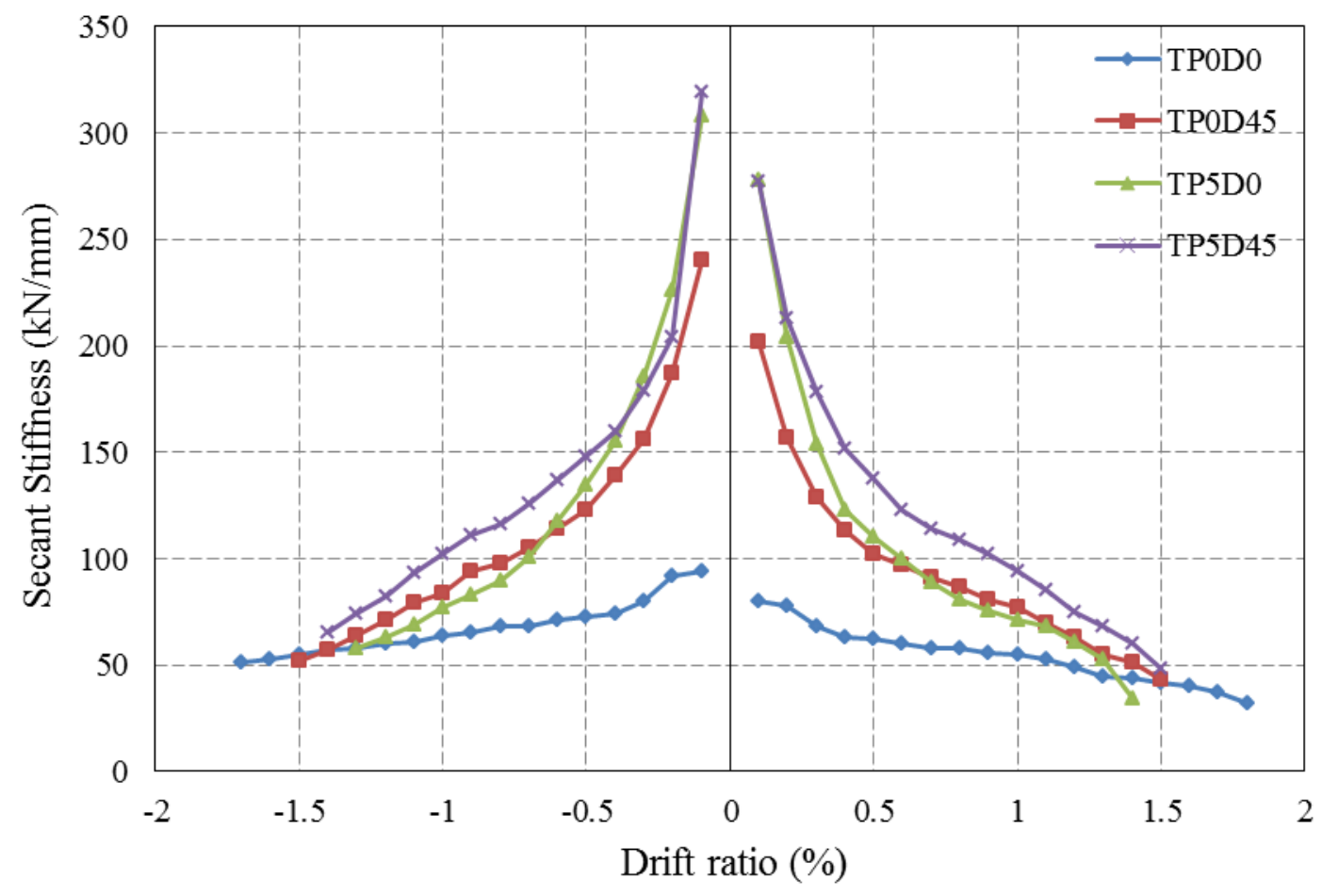

Figure 4.90 Secant stiffness of the T-shaped specimens

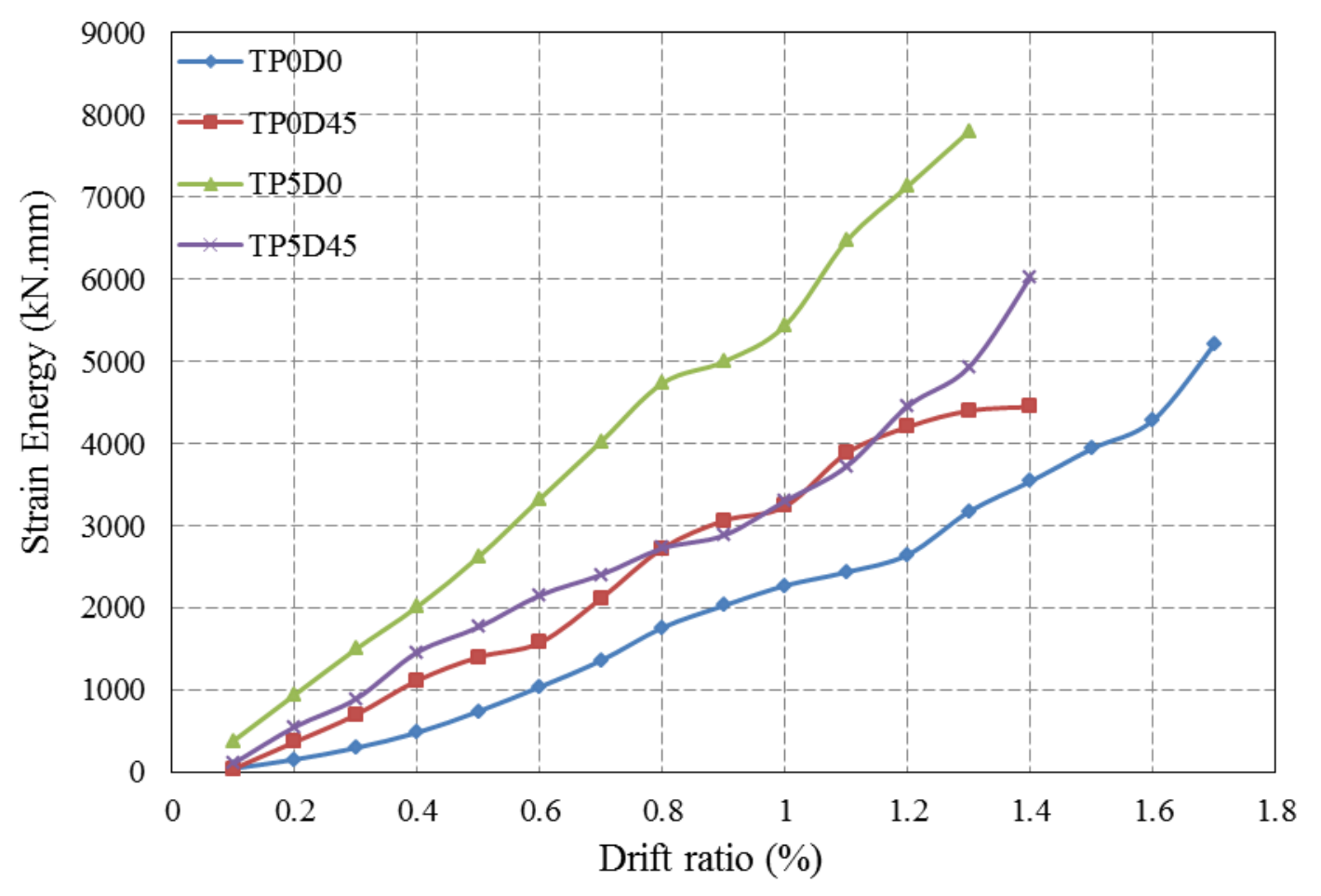

Figure 4.91 Energy dissipation capacities of the T-shaped specimens 


\subsubsection{Displacement components}

As discussed previously, top deformations of structural walls are mainly composed of three components, namely flexural deformation, shear deformation and sliding deformation. LVDTs were mounted at wall surfaces to provide data concerning these three deformations. Figure 4.92 to Figure 4.95 illustrates the ratios of three components to the total top displacement at different drift ratios, in which an unaccounted portion was also recorded since the summation of the other three could not always reach a perfect $100 \%$.

In Specimen TP0D0, the sliding displacement showed an increasing trend in both loading directions with its value rising from around 3\% to around $10 \%$. The percentages of flexural and shear displacements were identical at the beginning of the experiment. However, as the test progressed, the shear displacement gradually surpassed its counterpart in the positive loading direction. In the negative loading direction, in spite of a slight rise of the flexural component in the middle of the test, the shear deformation held its dominant position at wall failure. The unaccounted part remained at a relatively low value as the drift ratio rose in the negative direction, and experienced a slight growth in the positive loading direction.

A similar rising trend of the sliding deformation was also observed in Specimen TP0D45, where the number ended up at above $13 \%$ in both directions. The shear deformation, throughout the whole experiment, preserved a dominant component of the top displacement, with its number experiencing a slight rise from $50 \%$ to $53 \%$ in the negative loading direction. The second largest portion, the flexural deformation, on the other hand, showed a declining trend as its number dropped from around 39\% to $35 \%$ in both loading directions. The unaccounted part revealed a different varying pattern with regard to loading directions, with its number changing from 5\% to $-2 \%$ in the negative direction and $12 \%$ to $1 \%$ in opposite loading direction.

The contribution of the sliding shear in Specimen TP5D0 also grew as the drift ratio increased. In spite of a slight advantage at the beginning of the experiment, the percentages of the flexural deformation quickly decreased as the test progressed, with its number dropping from almost $50 \%$ to $35 \%$ in both loading directions. The 
shear deformation, on the other hand, showed a steady increasing trend as the test progressed, making it the largest component at the end of the experiment. The unaccounted part varied slightly but its value remained relatively small.

With regard to Specimen TP5D45, the sliding shear component took $3 \%$ in the beginning and ended up at around $10 \%$ in both loading directions. The shear displacement was constantly and evidently larger than the flexural displacement during the whole test. In the positive loading direction, the portion of these two components scarcely changed, while in the negative loading direction, only the flexural component maintained its value throughout the experiment. The shear contribution experienced a slight increase as the test progressed with its number varying from $50 \%$ to $53 \%$. The unaccounted part varied slightly but its value remained relatively small.

From these four figures, it was found that the shear deformation dominated the whole response because it accounted for the largest part of top displacements, which was consistent with the observation of the H-shaped and L-shaped squat walls. Moreover, the sliding shear component generally increased during the whole test. This phenomenon was more obvious in the specimens with lower axial loads. The contribution from the flexural displacement showed a decreasing trend in most tests. This phenomenon was more obvious in specimens with orthogonal lateral loading. 


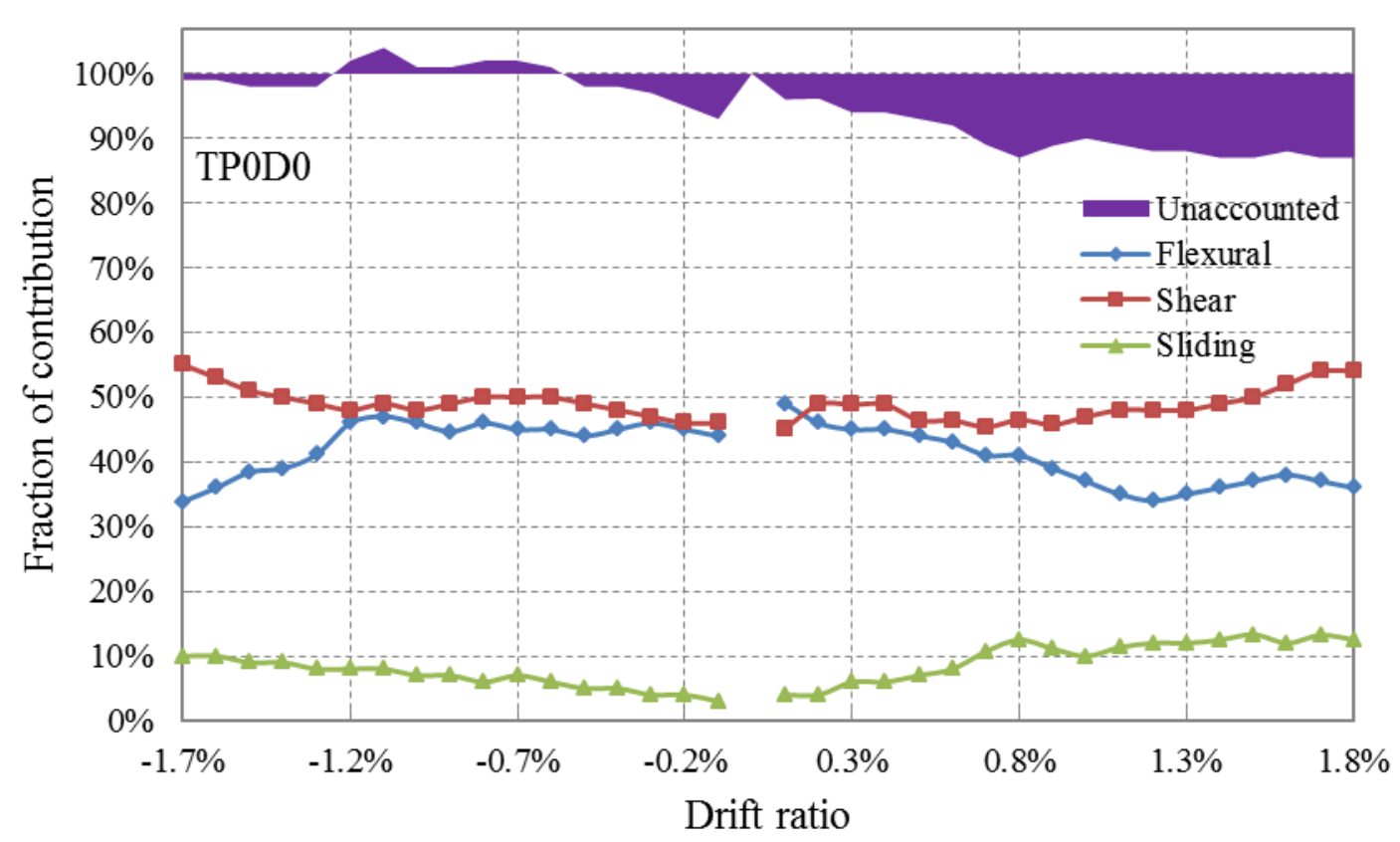

Figure 4.92 Displacement components of Specimen TP0D0

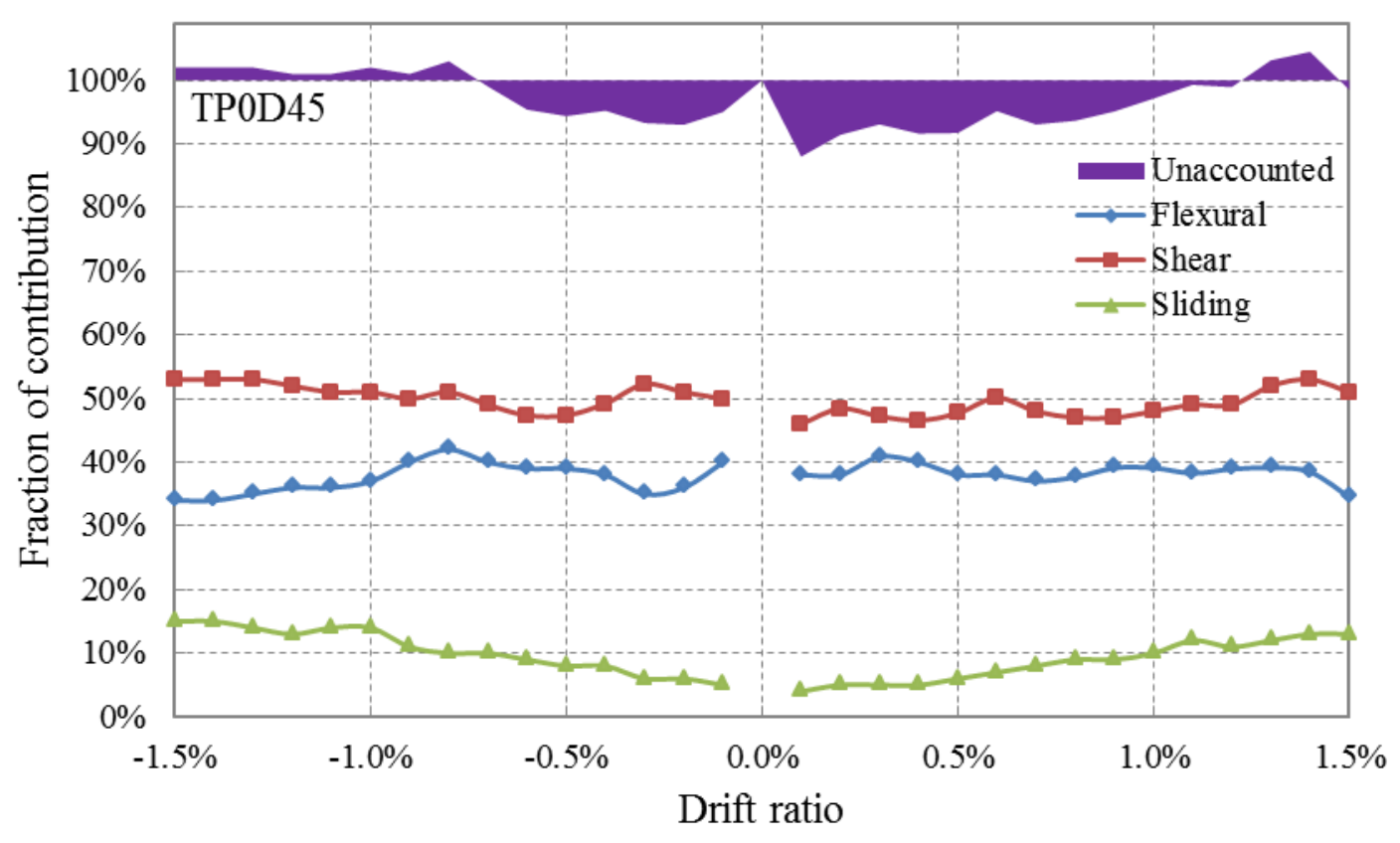

Figure 4.93 Displacement components of Specimen TP0D45 


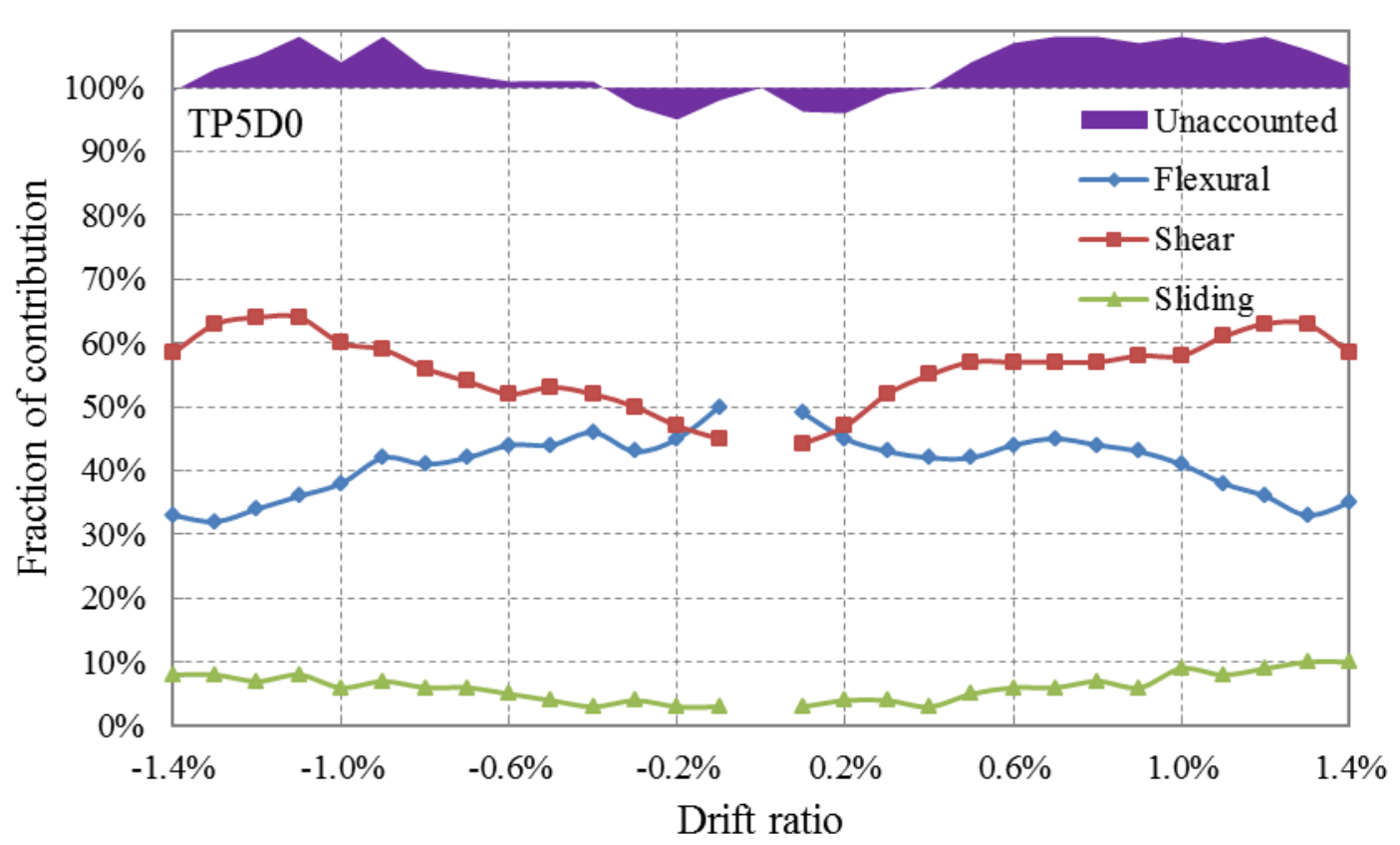

Figure 4.94 Displacement components of Specimen TP5D0

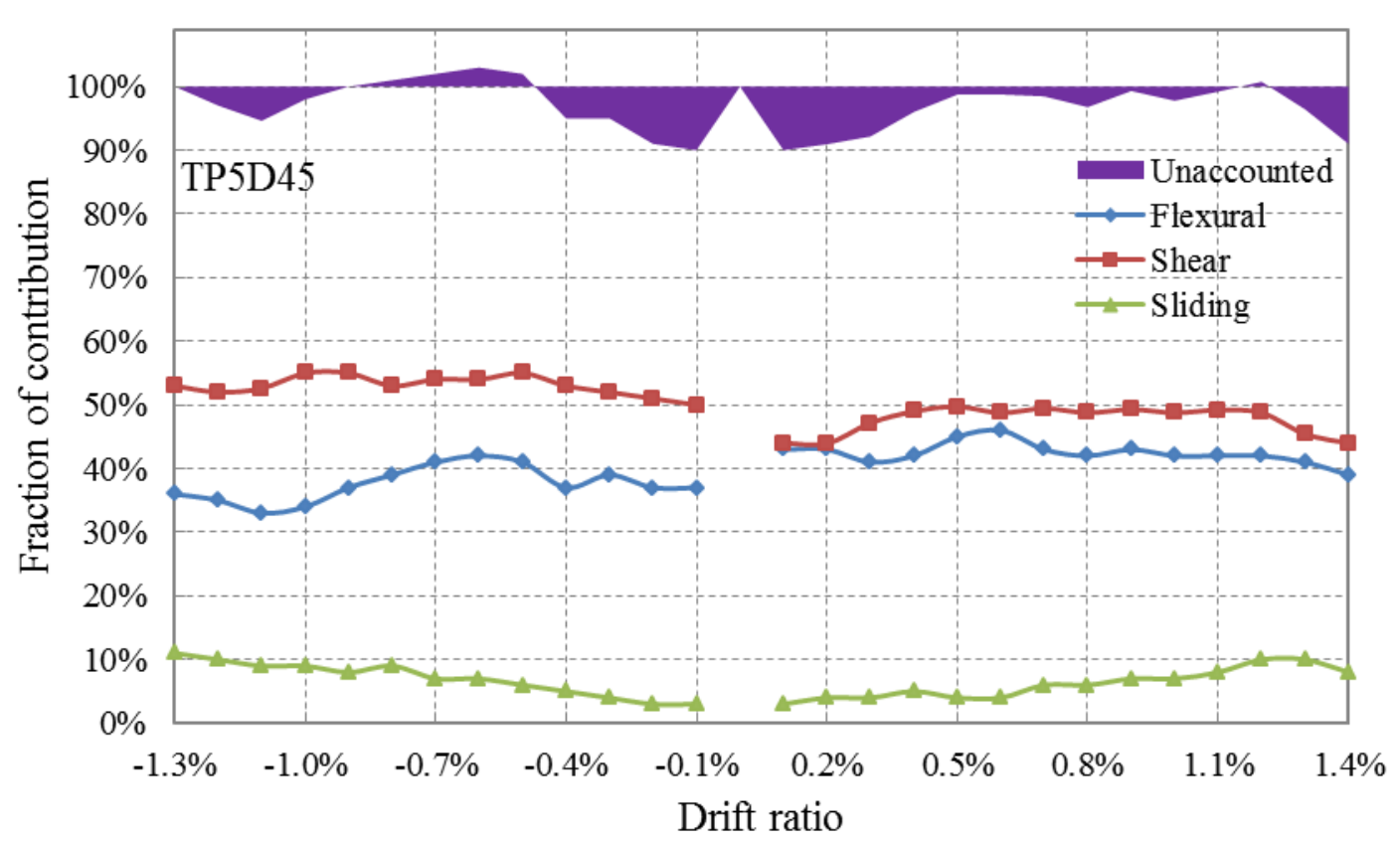

Figure 4.95 Displacement components of Specimen TP5D45

\subsubsection{Strain profiles}

As mentioned in previous section, strain gauges were attached to the surface of reinforcing bars to monitor their reactions during the test. Data was compiled and 
plotted to show strain variations from the initial stage of the test to the wall failure at several critical sections.

Figure 4.96 to Figure 4.103 show strains at the bottom of longitudinal reinforcement along the web in different specimens at various drift ratios in both loading directions. It was observed in specimens with orthogonal lateral loading that the vertical web reinforcement yielded at the final stage when the flange was in tension, while none of reinforcing bars in the web experienced yielding when the flange was in compression. As for specimens with diagonal loading direction, longitudinal rebar at the tip of the web approached to yielding at the end of the test. For Specimen TP0D0, it was observed that strains remained almost linear before the drift ratio reached $0.4 \%$, which indicated the plane section assumption was valid at this stage. The neutral axial was found shifting around $400 \mathrm{~mm}$ from the middle of the section towards the direction of loading. As for Specimen TP0D45, strains were not able to maintain linear after the drift ratio of $0.3 \%$. The neutral axial also hardly moved throughout the test. As for Specimen TP5D0, the neutral axial shifted 250 $\mathrm{mm}$ towards the middle of the section in the negative loading direction. Moreover, the plane section assumption could not be used after the drift ratio of $0.3 \%$ since strains were no long linear. With regard to Specimen TP5D45, it was found that the neural axial did not shift during the whole test. Furthermore, strains were seemingly able to maintain linear until drift ratio rose to $0.5 \%$ in the positive loading direction.

The strain variations along the bottom of longitudinal reinforcement in the flange are presented in Figure 4.104 to Figure 4.111. As seen from the figure, yielding was found during the tests in all specimens when the flange was in tension. A significant shear lag effect was detected in Specimens TP0D0 and TP5D0. For instance, the strain of the longitudinal reinforcement in the junction of the web and flange was as far as $225 \%$ of that of the longitudinal rebar located in the flange tip at the final stage in Specimen TP0D0. This phenomenon was more evident in Specimen TP5D0, in which stains of the outmost longitudinal reinforcement were almost zero when the flange was in tension, suggesting that the outer part of the flange was hardly involved in withstanding lateral loads. The shear lag effect was observed when the flange was in compression as well, which was seen from the 
larger strains of the rebar located closer to the web. In specimens with diagonal lateral loading, the shear lag effect was less obvious but still could be traced as the tensile strains of the longitudinal reinforcement showed an increasing trend towards the flange tip. However, it should be noticed that the maximum strain occurred 0.24 $\mathrm{m}$ from the flange tip, which was in accordance with the results of the section analysis.

The strain profiles of No.1 and No.7 horizontal reinforcing bars in the web are shown in Figure 4.112 to Figure 4.119. The two selected reinforcing bars were located $20 \mathrm{~mm}$ and $500 \mathrm{~mm}$ above the wall base respectively. Yielding was detected in the middle of No.7 rebar at wall failure in all specimens except Specimen TP5D0. On the other hand, No.1 rebar did not yield throughout the test. It was clear that strains increased as the drift ratio rose in both loading directions. As for Specimen TP0D0, the reading of strain gauge located at the right of No.1 rebar was evidently larger than its counterparts when the flange was in compression. However, when the flange was in tension, the strain gauge located at the left of the rebar had the largest reading. As for No.7 rebar, it was found that the variation of strains at three different locations was not significant before the wall failed. A similar strain distribution in No.1 rebar was also observed in Specimen TP5D0. But the strain in the middle of No.7 rebar experienced the highest growth in both loading directions, indicating that there was a main diagonal compression strut passing through these places. In Specimen TP0D45, the stain at the left part of No.1 rebar had a much lower reading compared with the other two in the positive loading direction. A similar trend was found in the right part of No.1 rebar in the negative loading direction. Moreover, the reading of strain gauge located in the middle of No.7 rebar had clearly larger increases in both loading directions. As for Specimen TP5D45, the similar phenomenon occurring in the other three specimens was also observed in No.1 rebar. An identical intense growth of strain was found in the middle of No.7 rebar. 


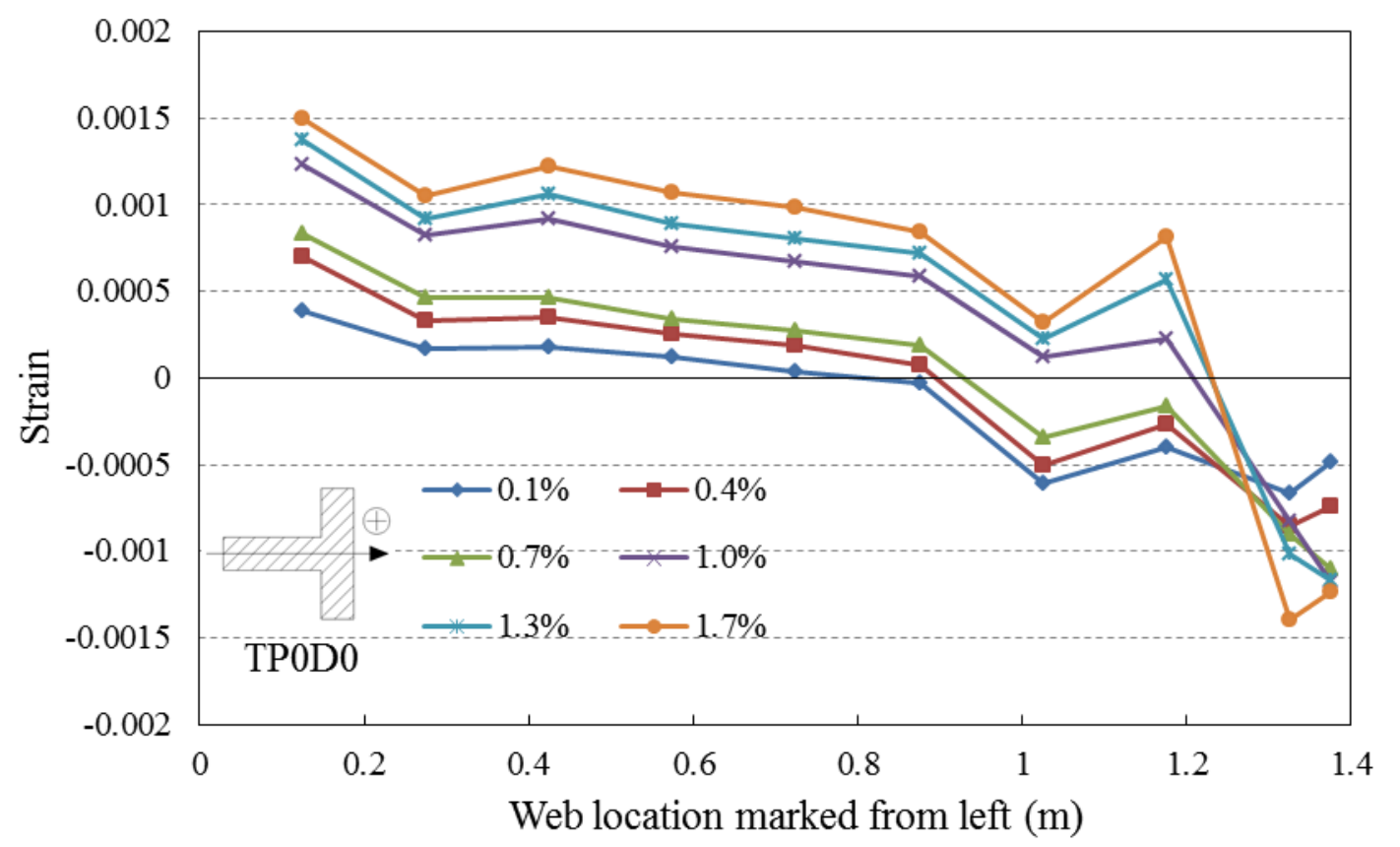

Figure 4.96 Strain profiles of longitudinal reinforcement along the web of Specimen TP0D0 in the positive loading direction

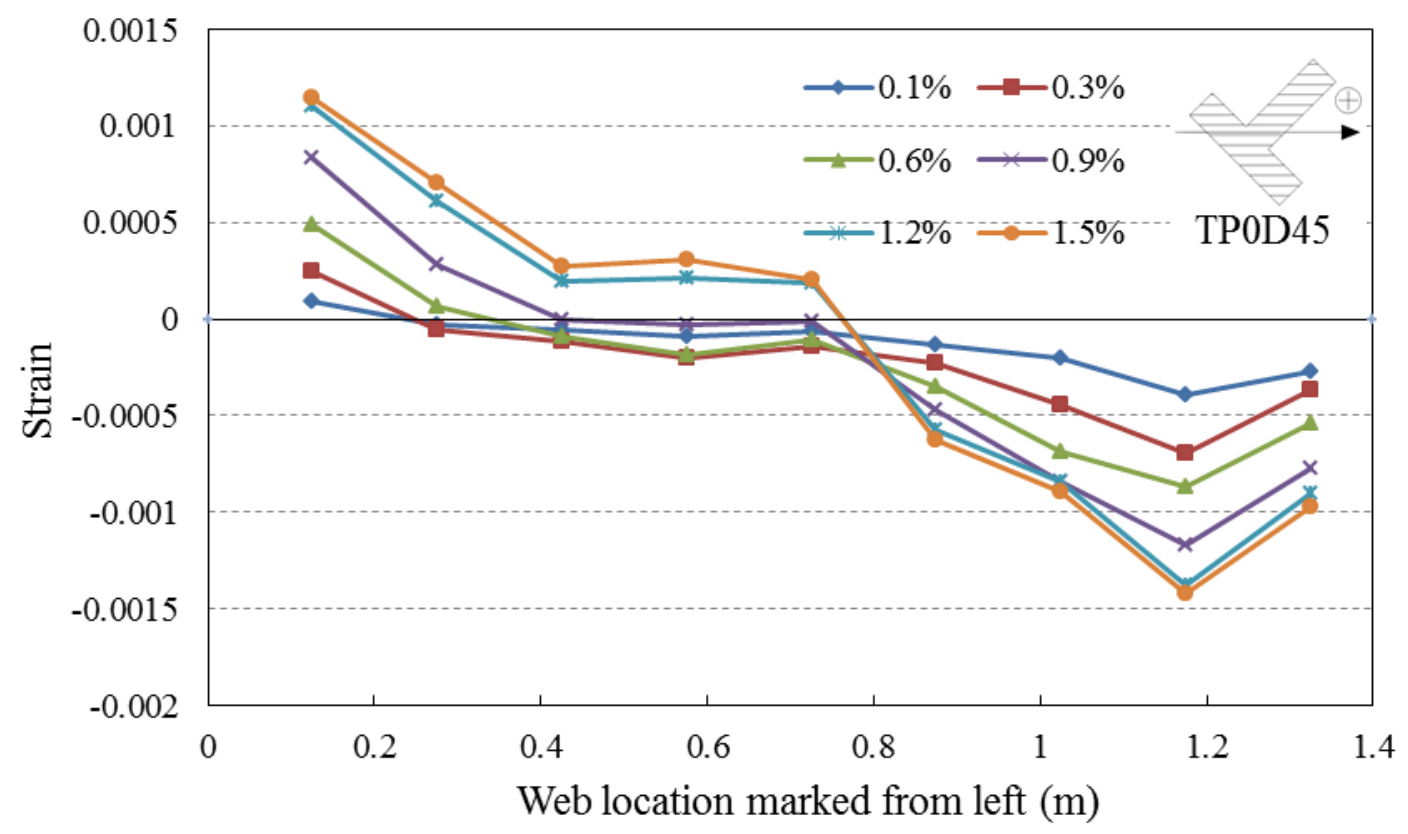

Figure 4.97 Strain profiles of longitudinal reinforcement along the web of Specimen TP0D45 in the positive loading direction 


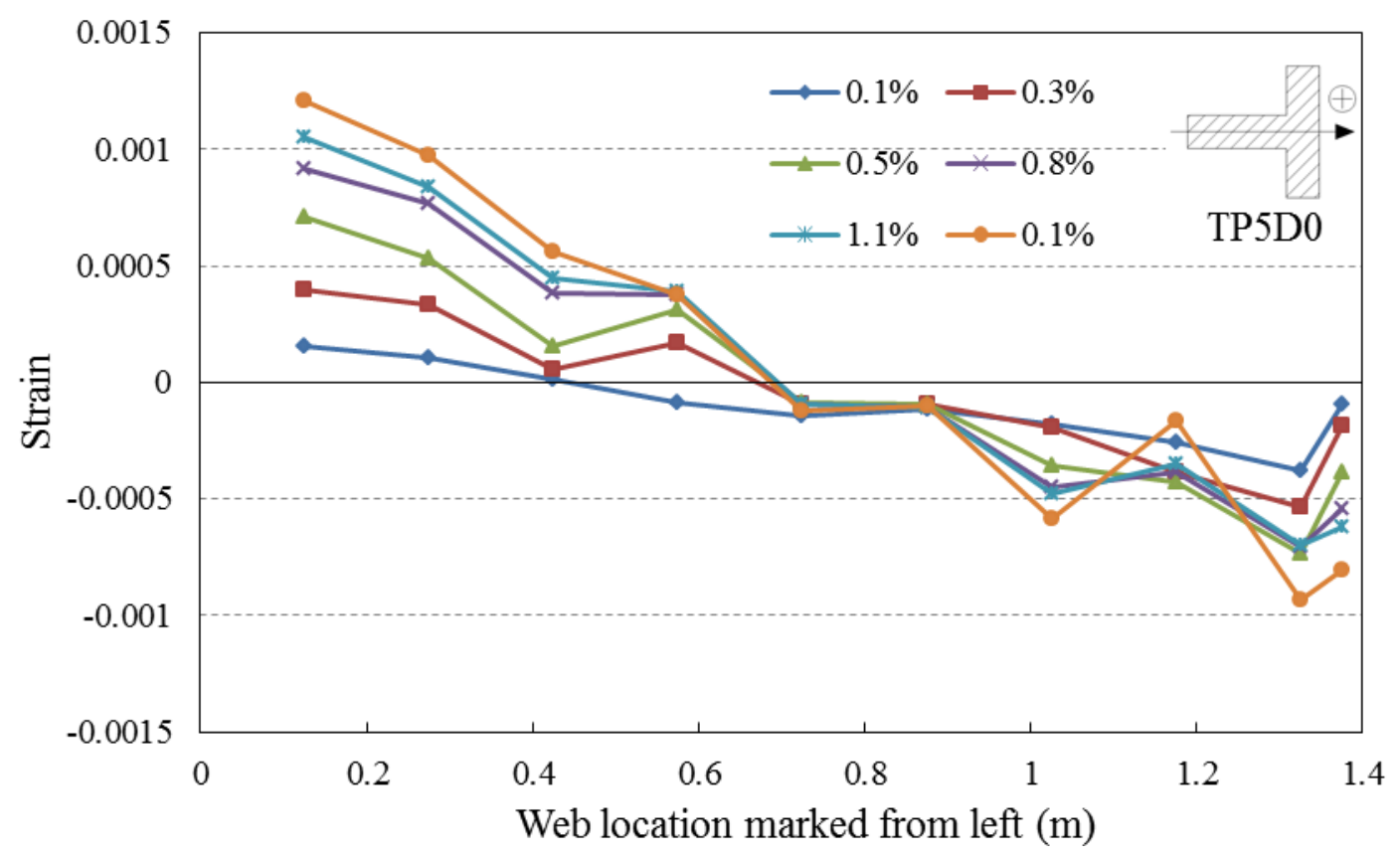

Figure 4.98 Strain profiles of longitudinal reinforcement along the web of Specimen TP5D0 in the positive loading direction

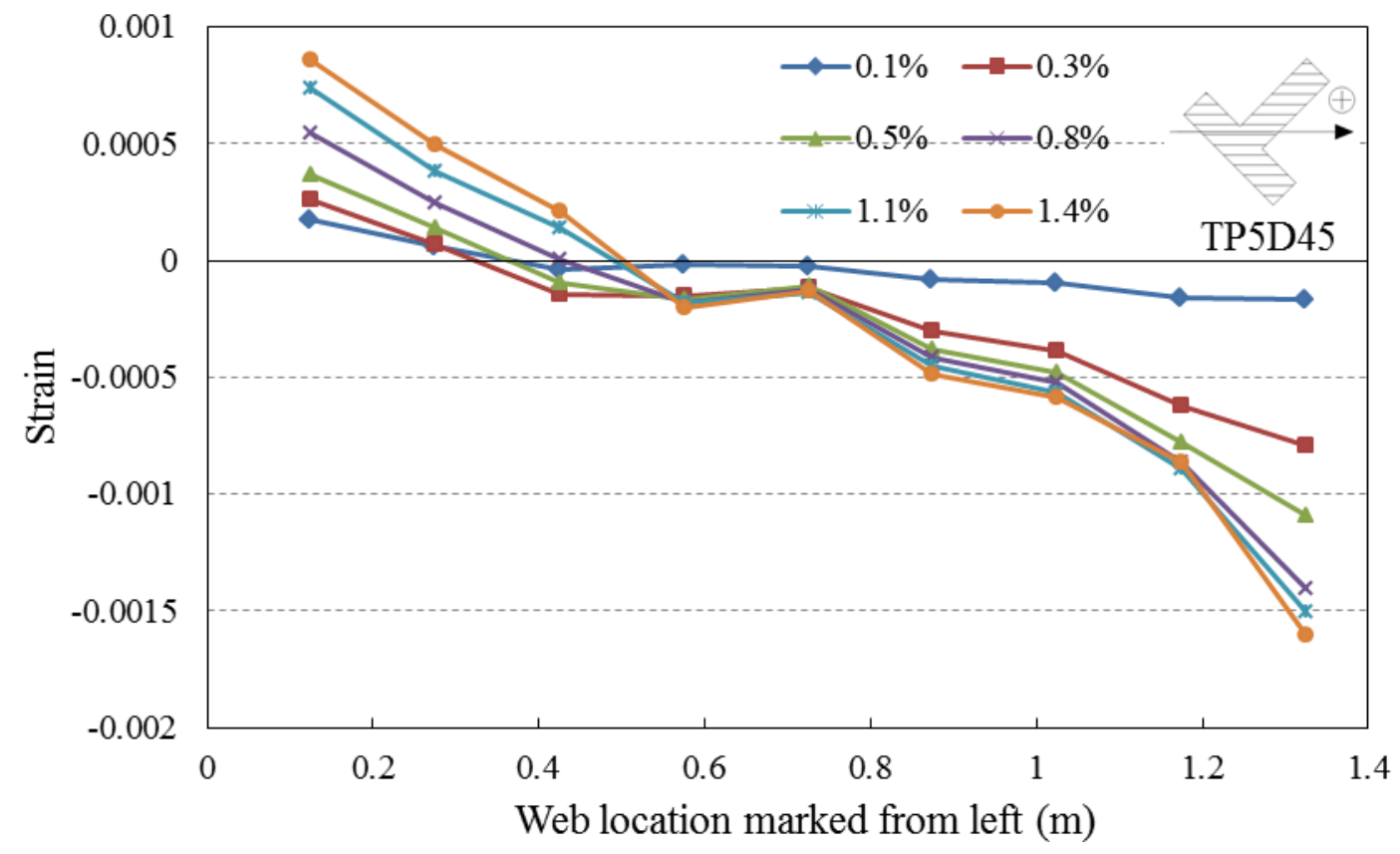

Figure 4.99 Strain profiles of longitudinal reinforcement along the web of Specimen TP5D45 in the positive loading direction 


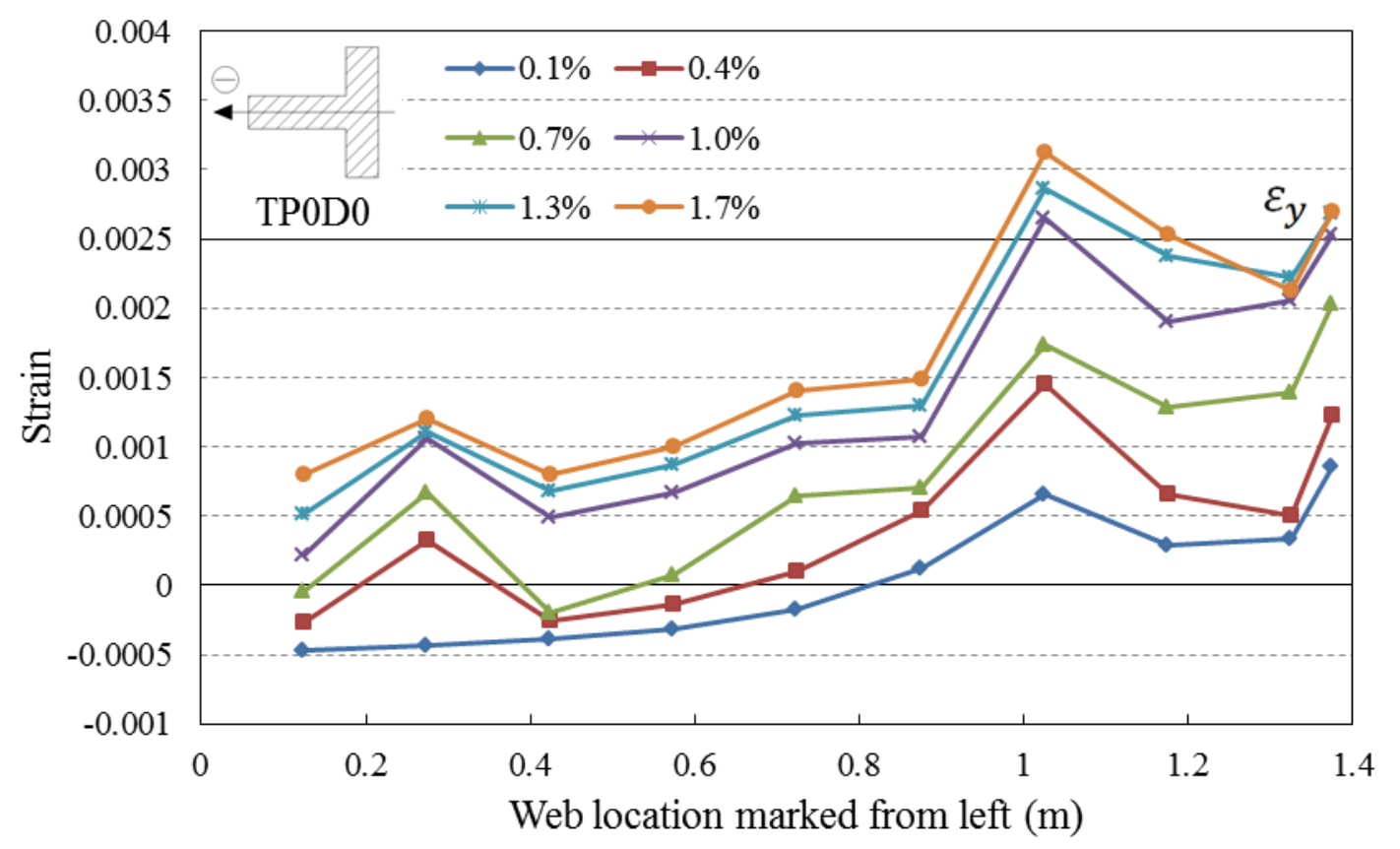

Figure 4.100 Strain profiles of longitudinal reinforcement along the web of Specimen TP0D0 in the negative loading direction

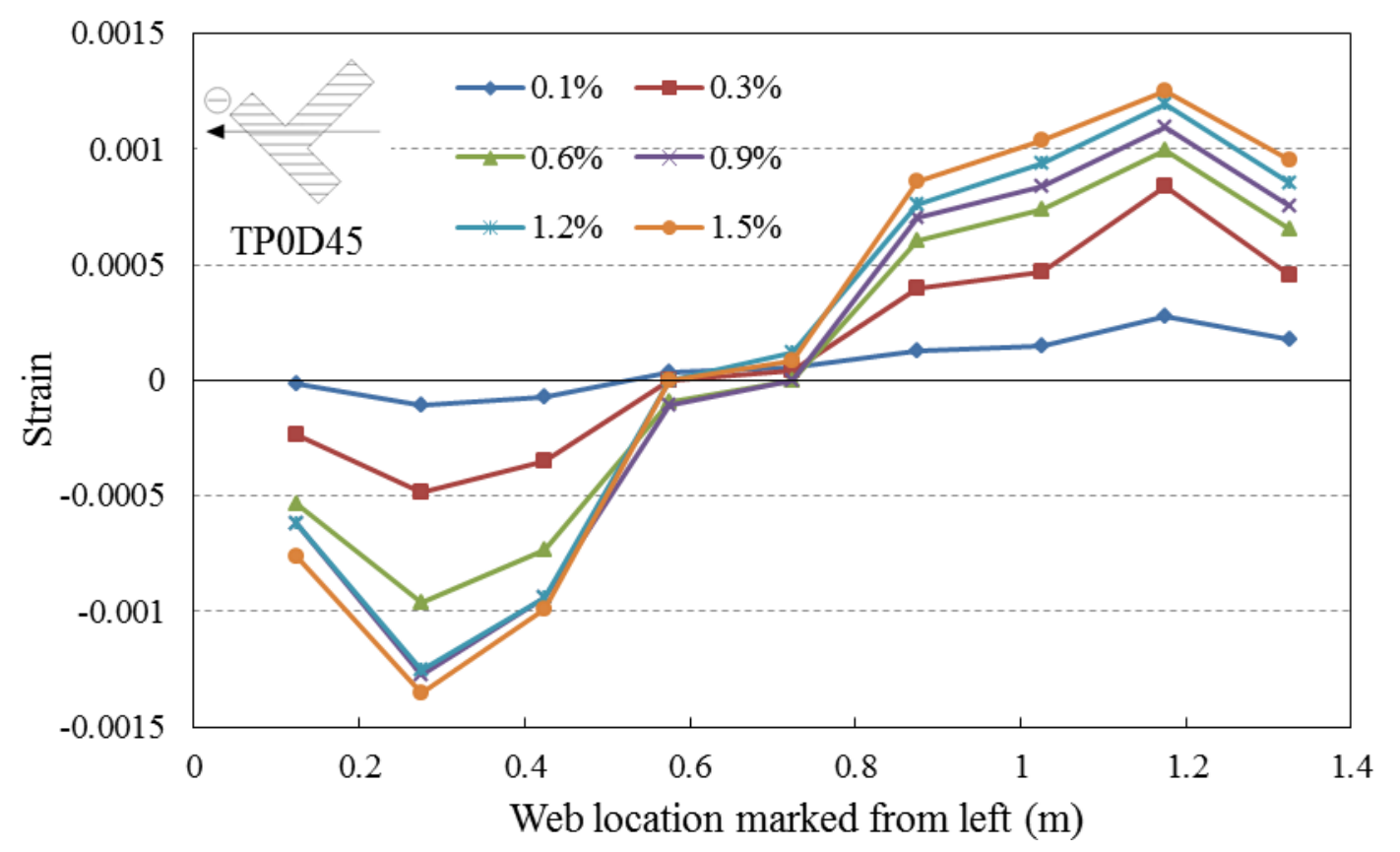

Figure 4.101 Strain profiles of longitudinal reinforcement along the web of Specimen TP0D45 in the negative loading direction 


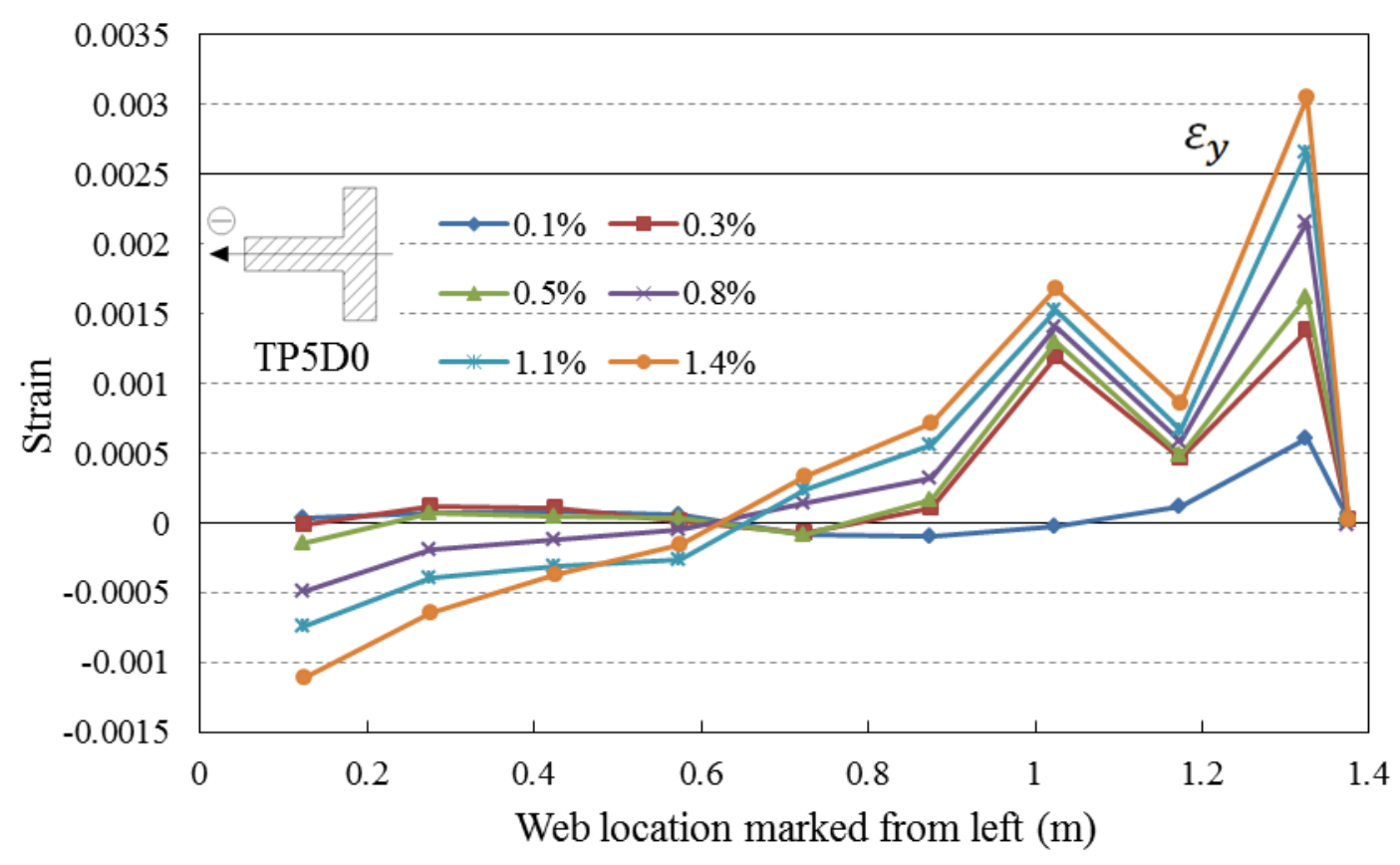

Figure 4.102 Strain profiles of longitudinal reinforcement along the web of Specimen TP5D0 in the negative loading direction

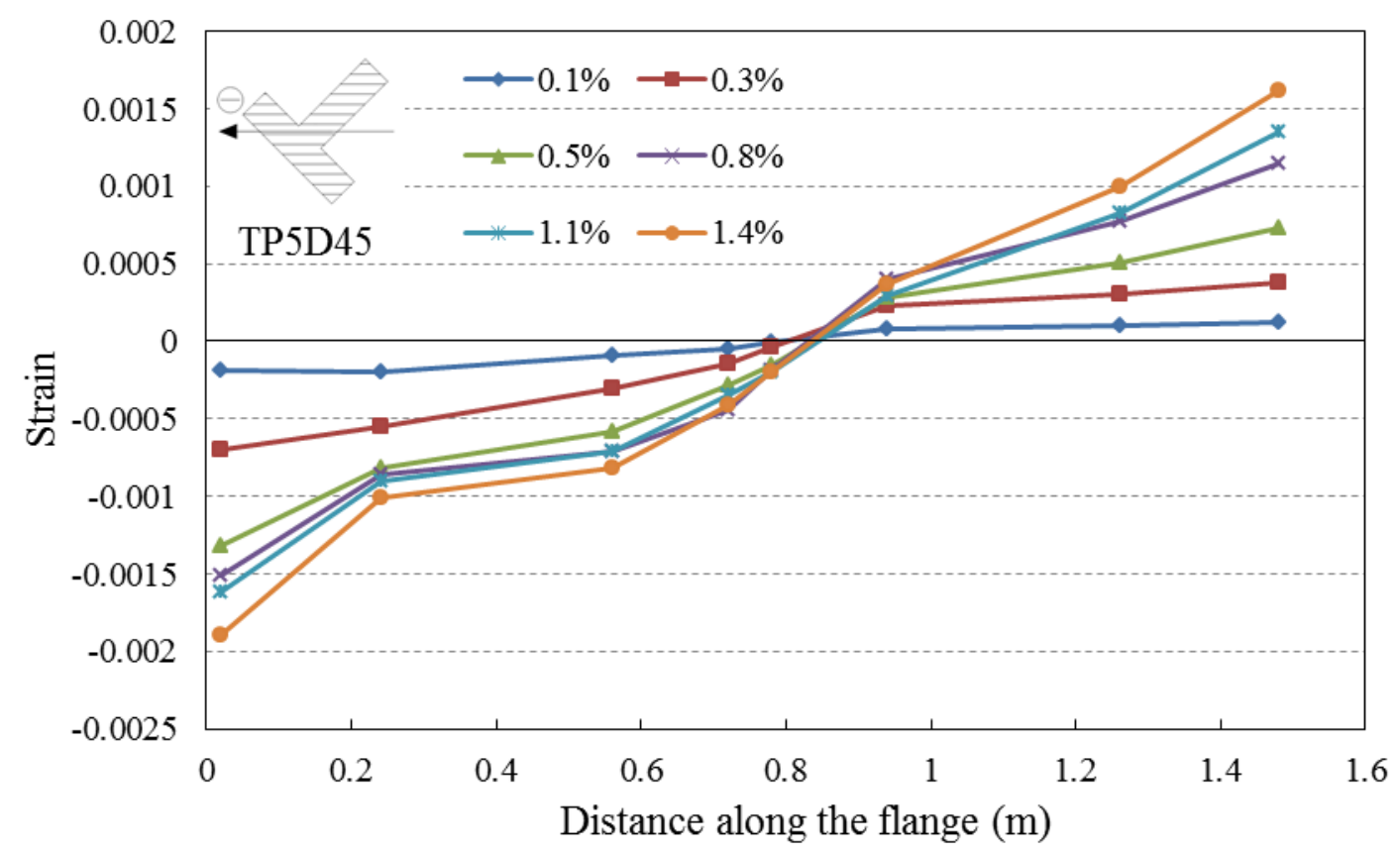

Figure 4.103 Strain profiles of longitudinal reinforcement along the web of Specimen TP5D45 in the negative loading direction 


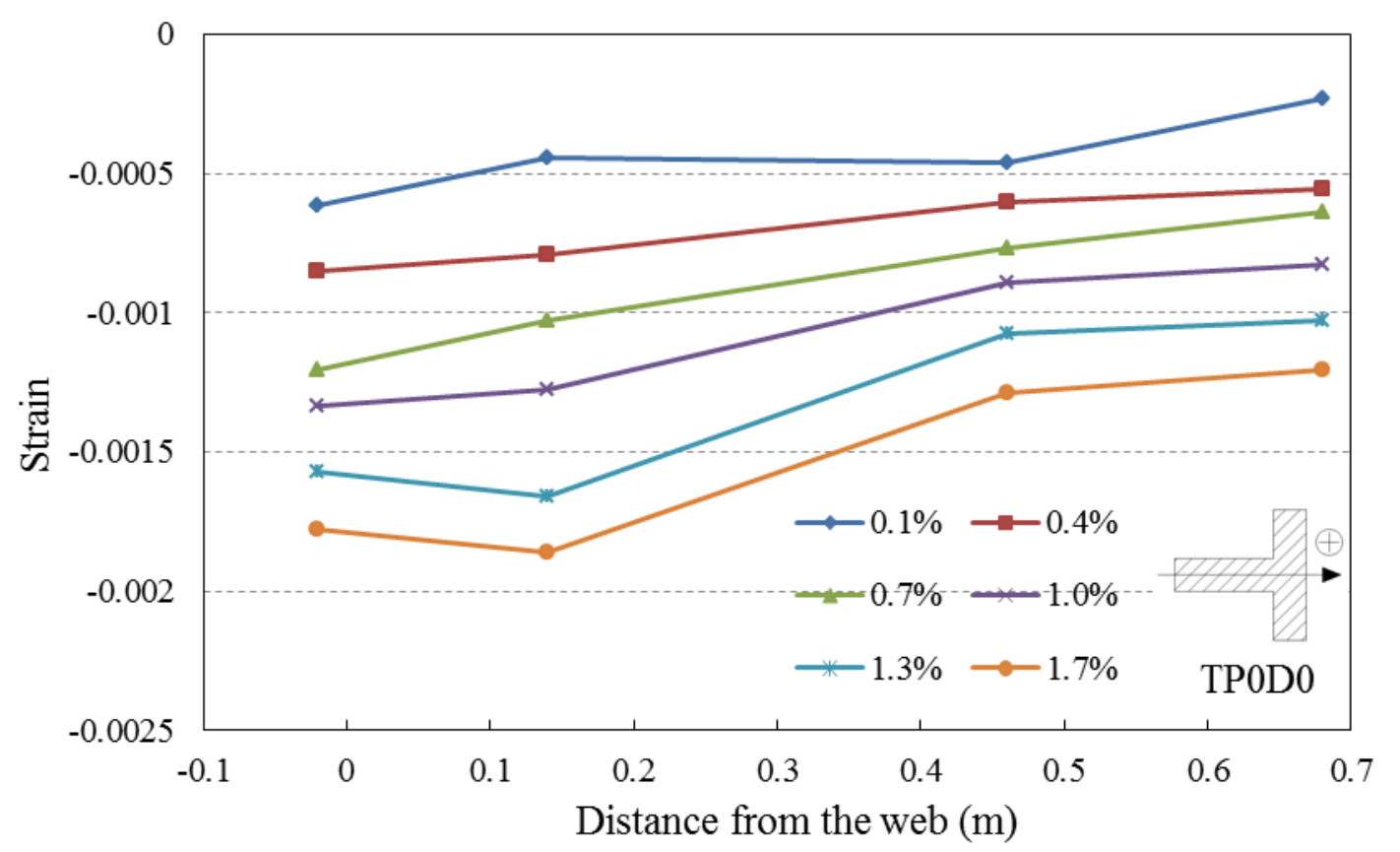

Figure 4.104 Strain profiles of longitudinal reinforcement along the flange of Specimen TP0D0 in the positive loading direction

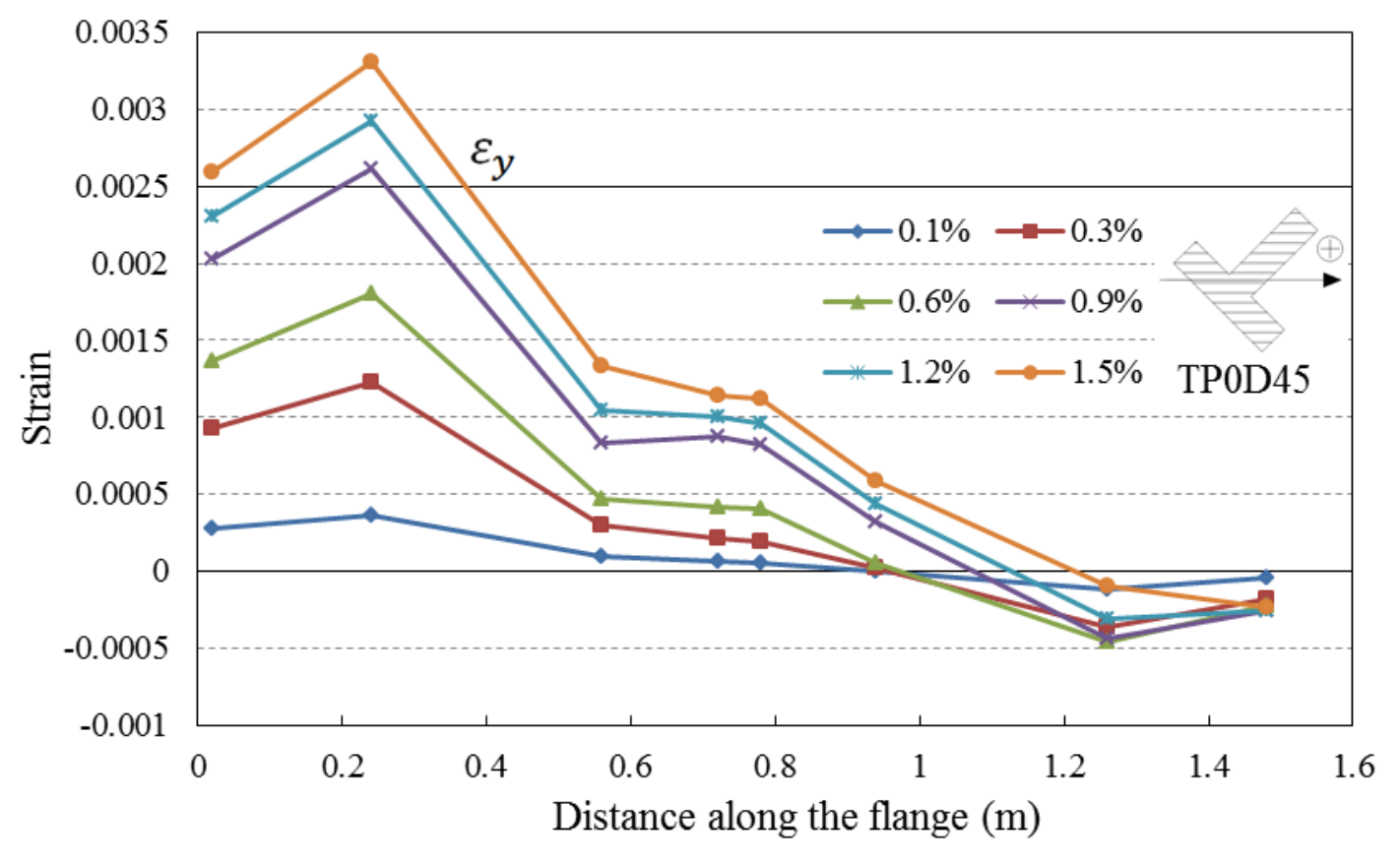

Figure 4.105 Strain profiles of longitudinal reinforcement along the flange of Specimen TP0D45 in the positive loading direction 


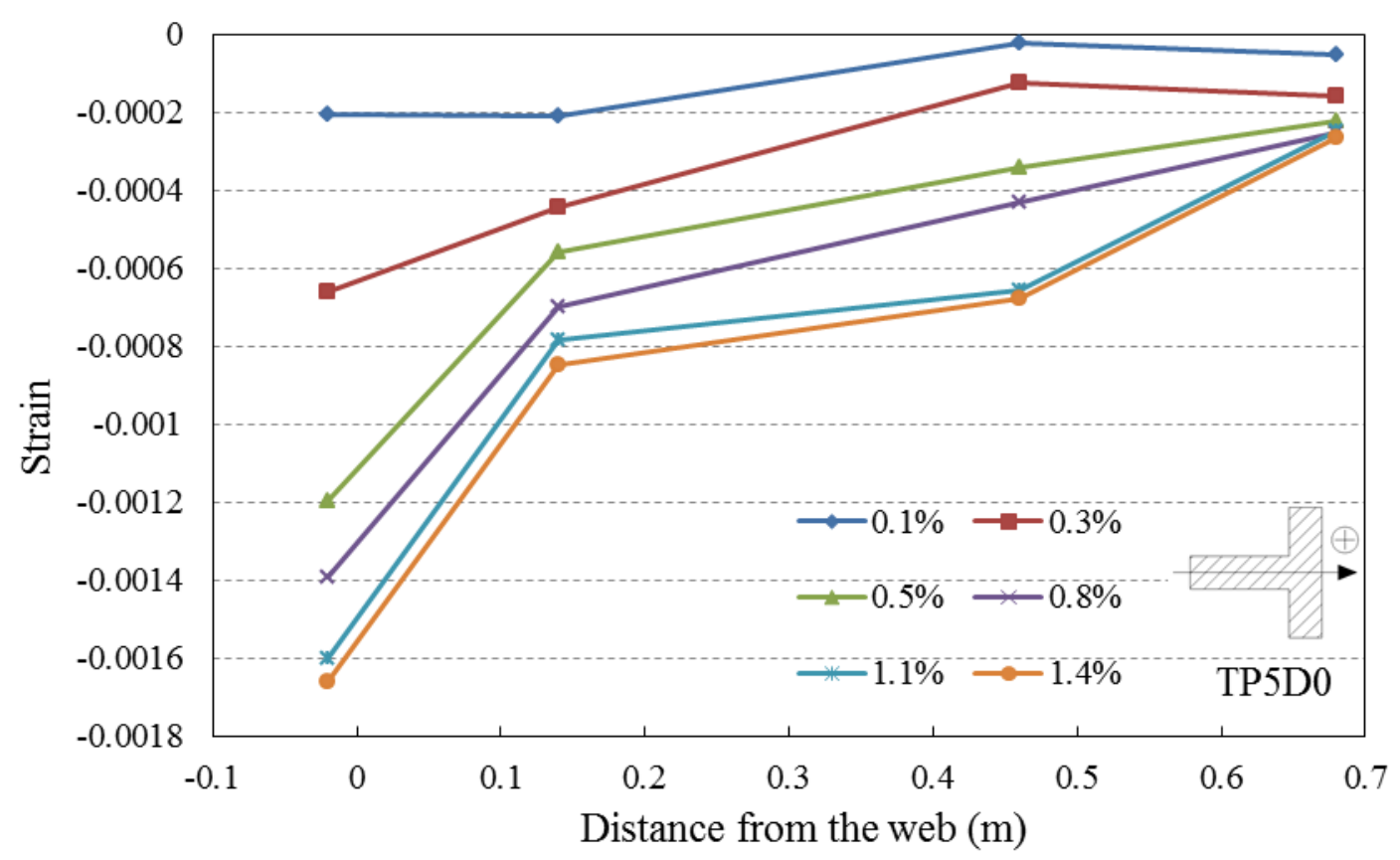

Figure 4.106 Strain profiles of longitudinal reinforcement along the flange of Specimen TP5D0 in the positive loading direction

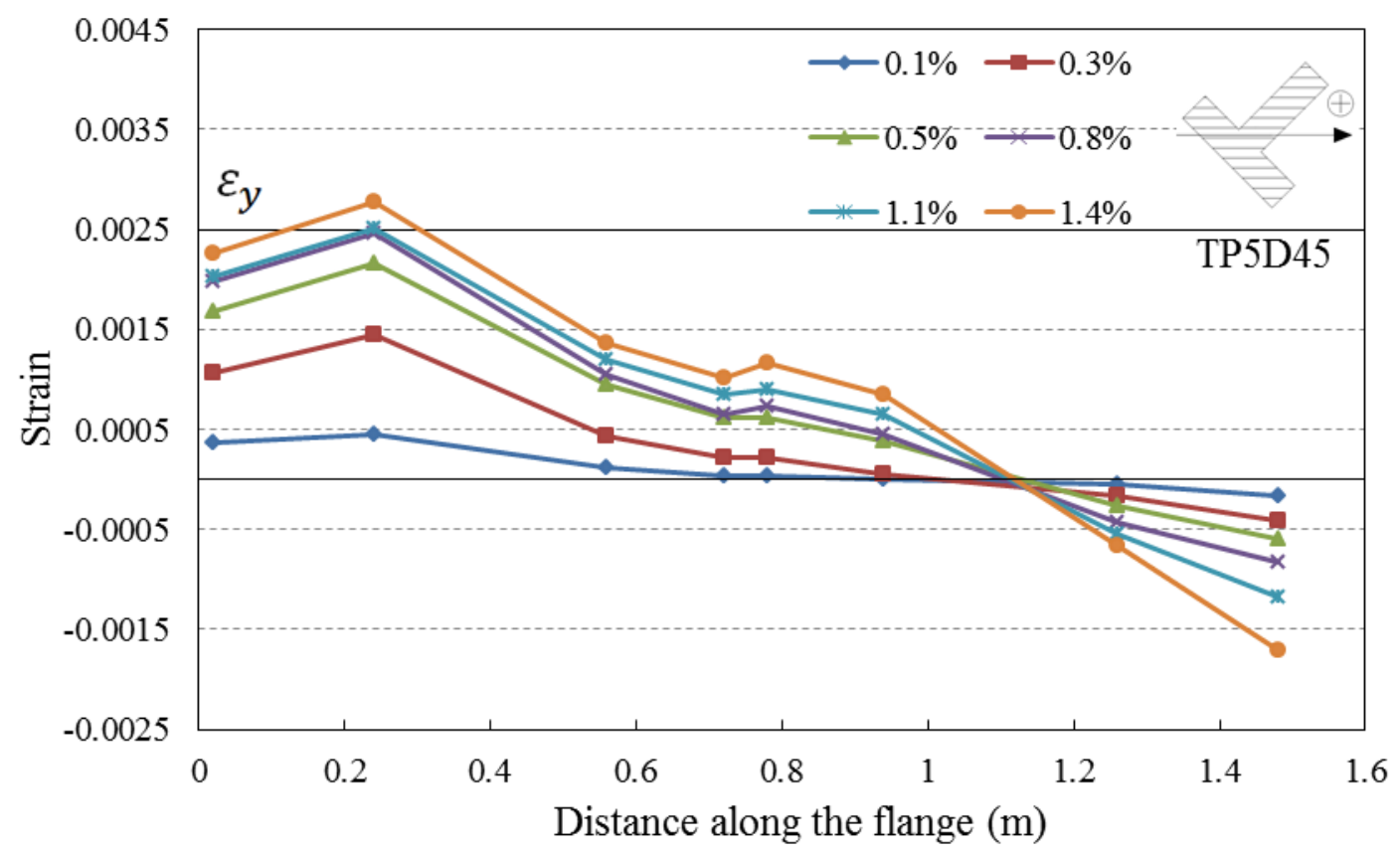

Figure 4.107 Strain profiles of longitudinal reinforcement along the flange of Specimen TP5D45 in the positive loading direction 


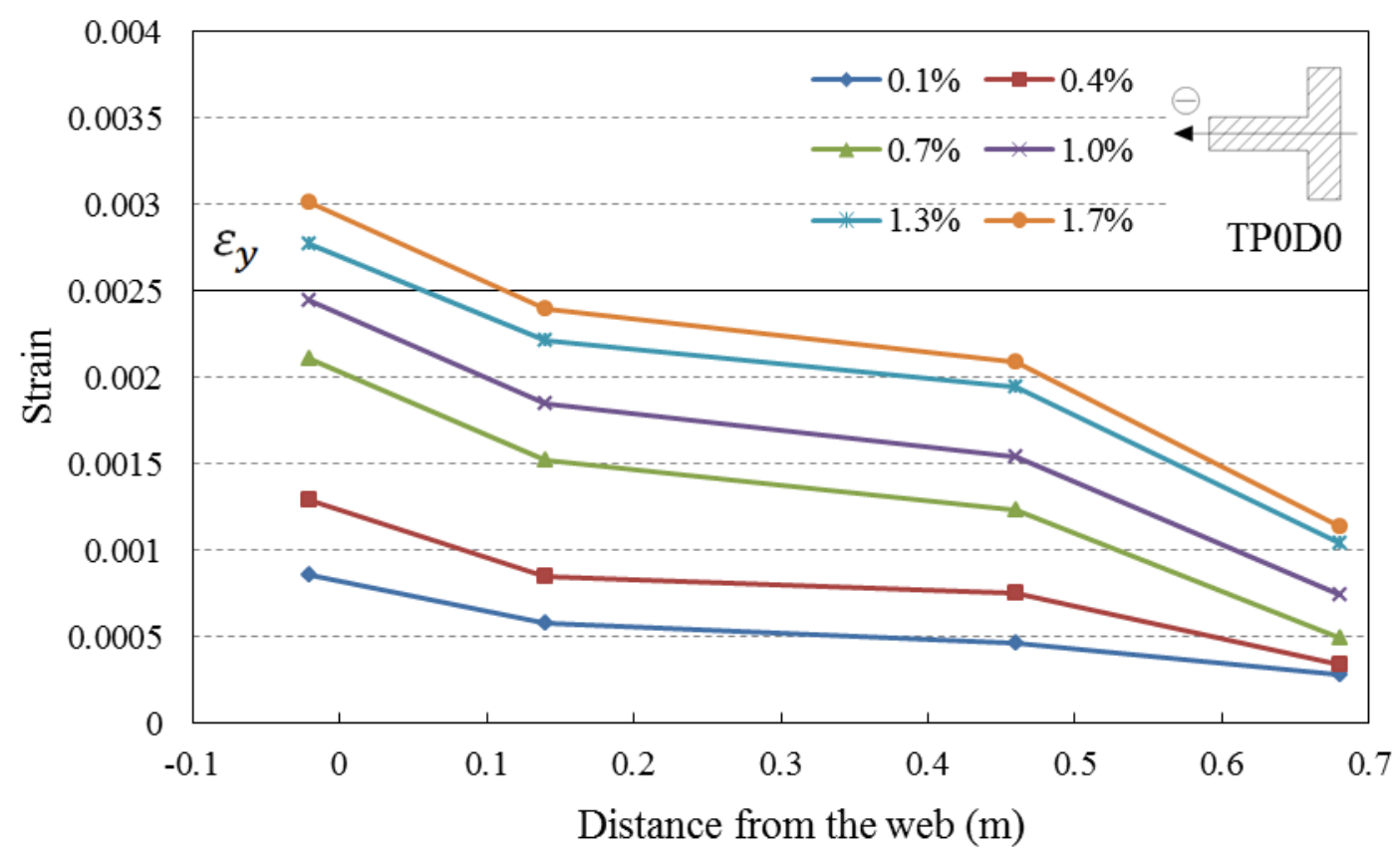

Figure 4.108 Strain profiles of longitudinal reinforcement along the flange of Specimen TP0D0 in the negative loading direction

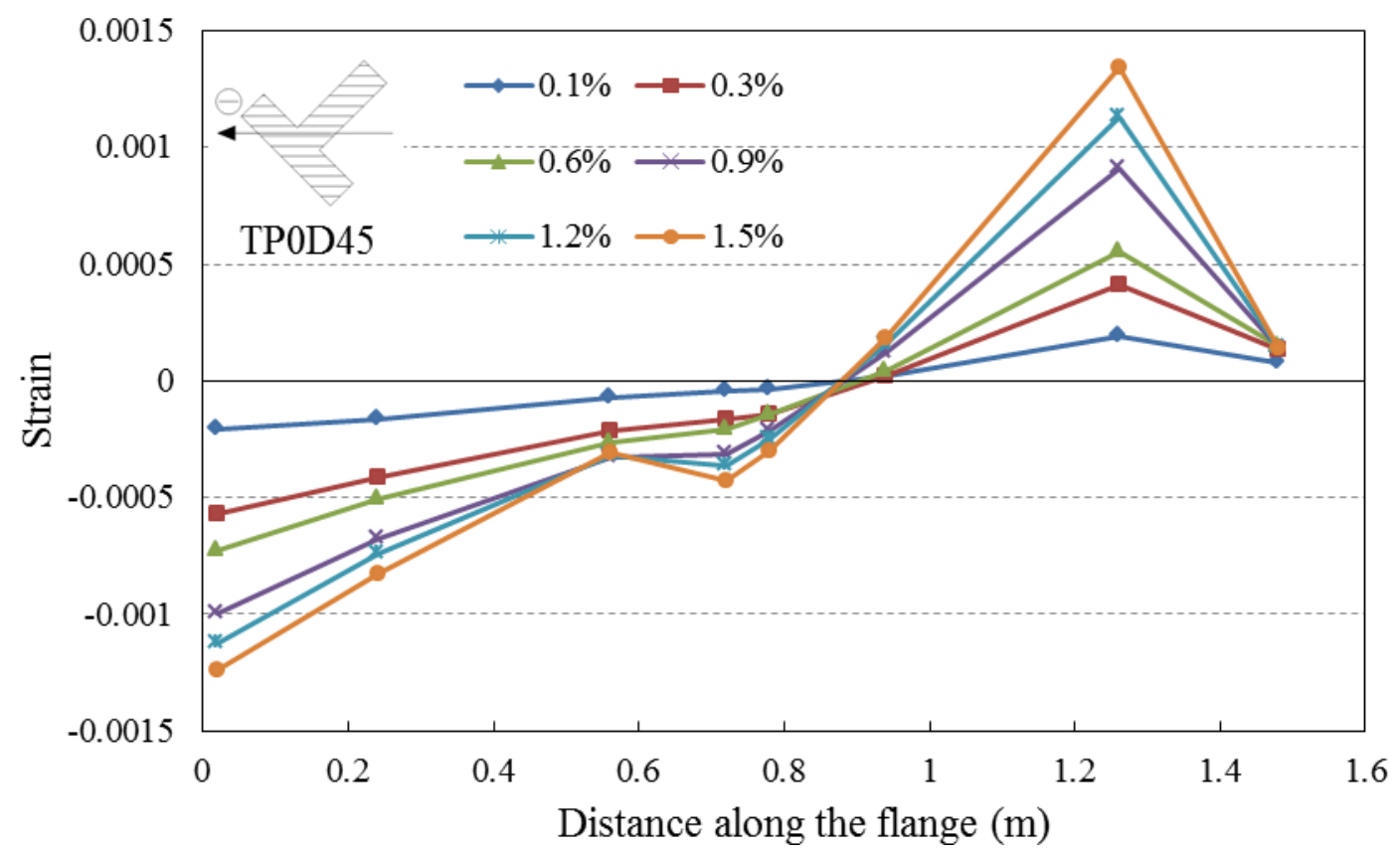

Figure 4.109 Strain profiles of longitudinal reinforcement along the flange of Specimen TP0D45 in the negative loading direction 


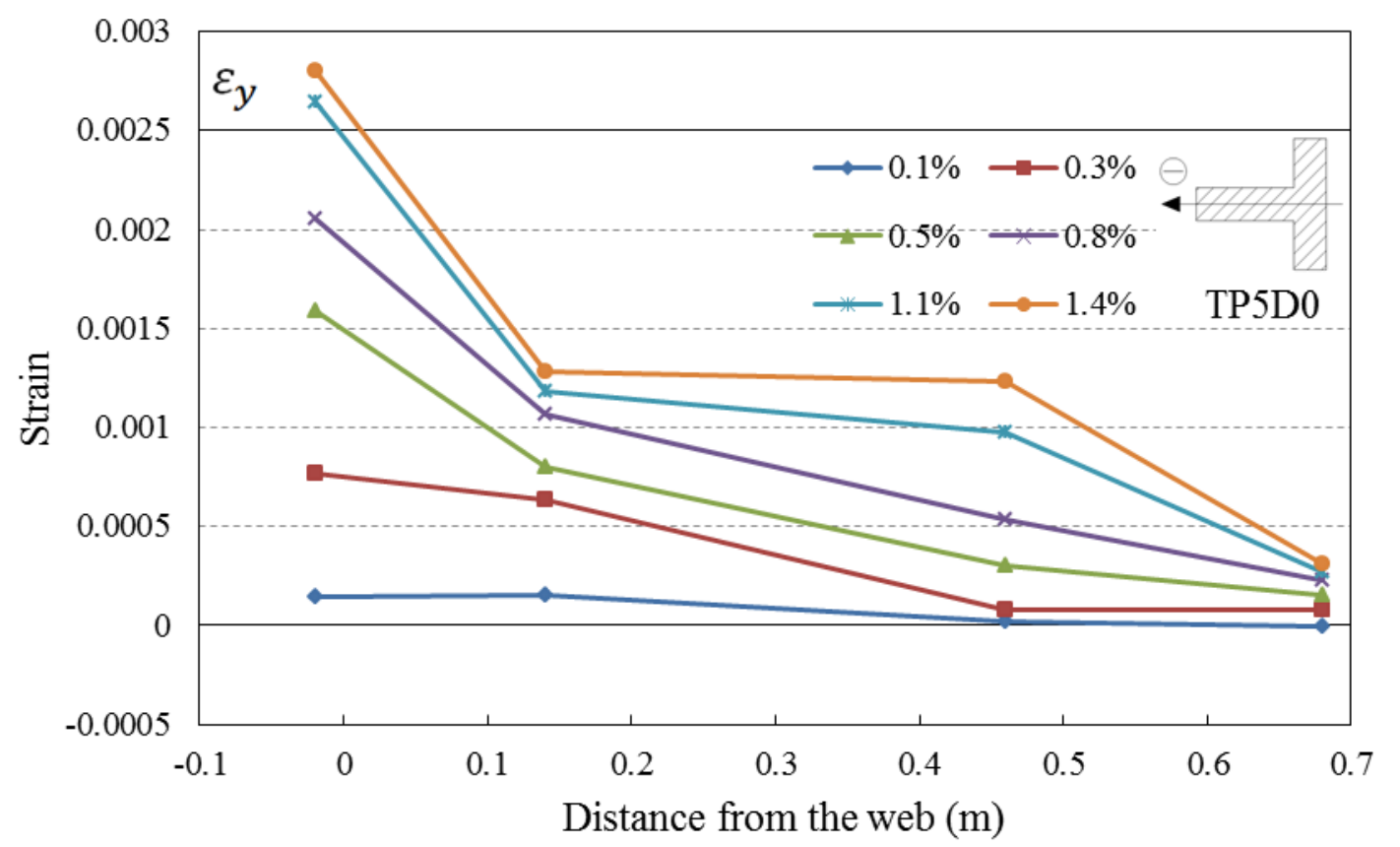

Figure 4.110 Strain profiles of longitudinal reinforcement along the flange of Specimen TP5D0 in the negative loading direction

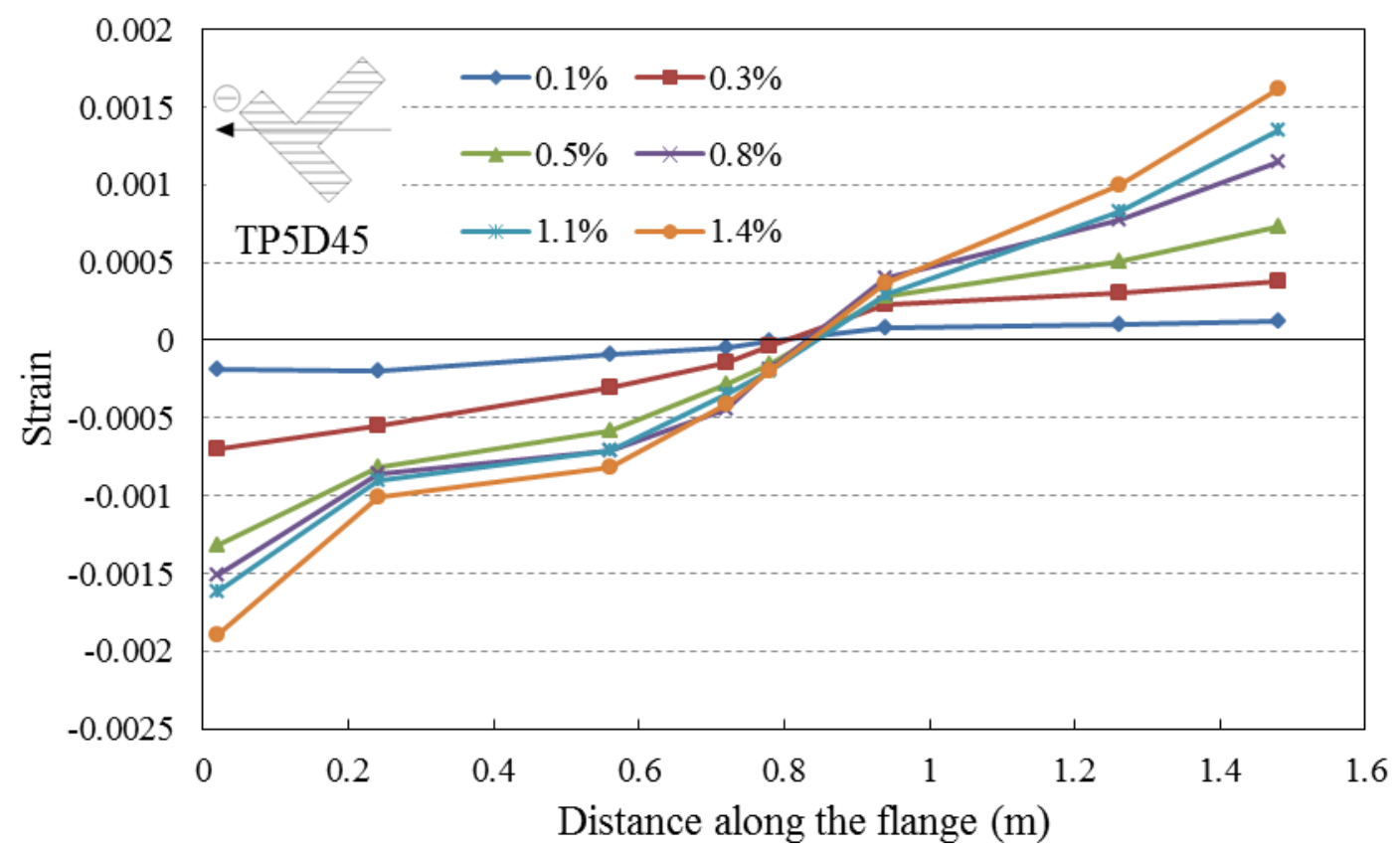

Figure 4.111 Strain profiles of longitudinal reinforcement along the flange of Specimen TP5D45 in the negative loading direction 


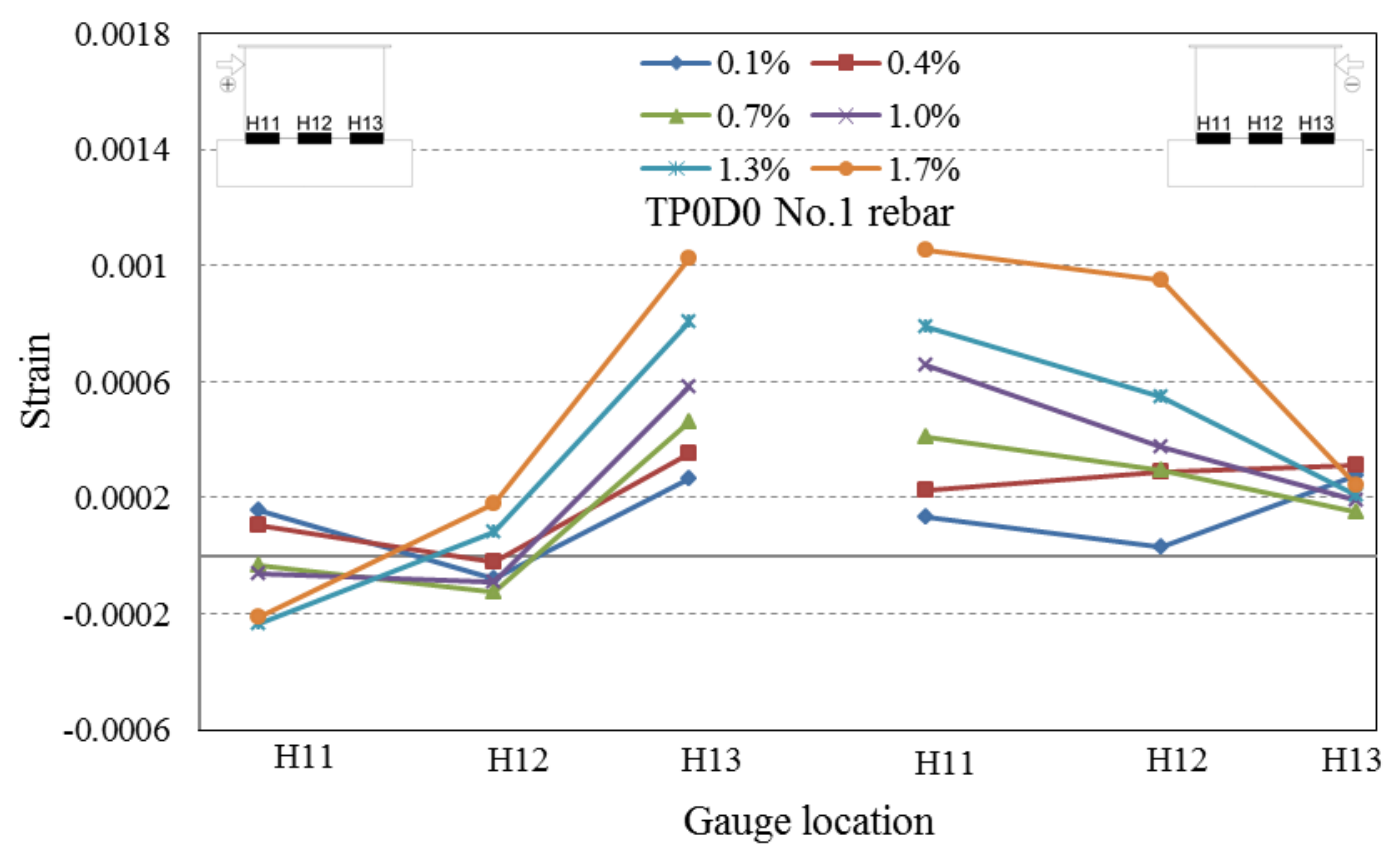

Figure 4.112 Strain profiles of No.1 horizontal rebar of Specimen TP0D0

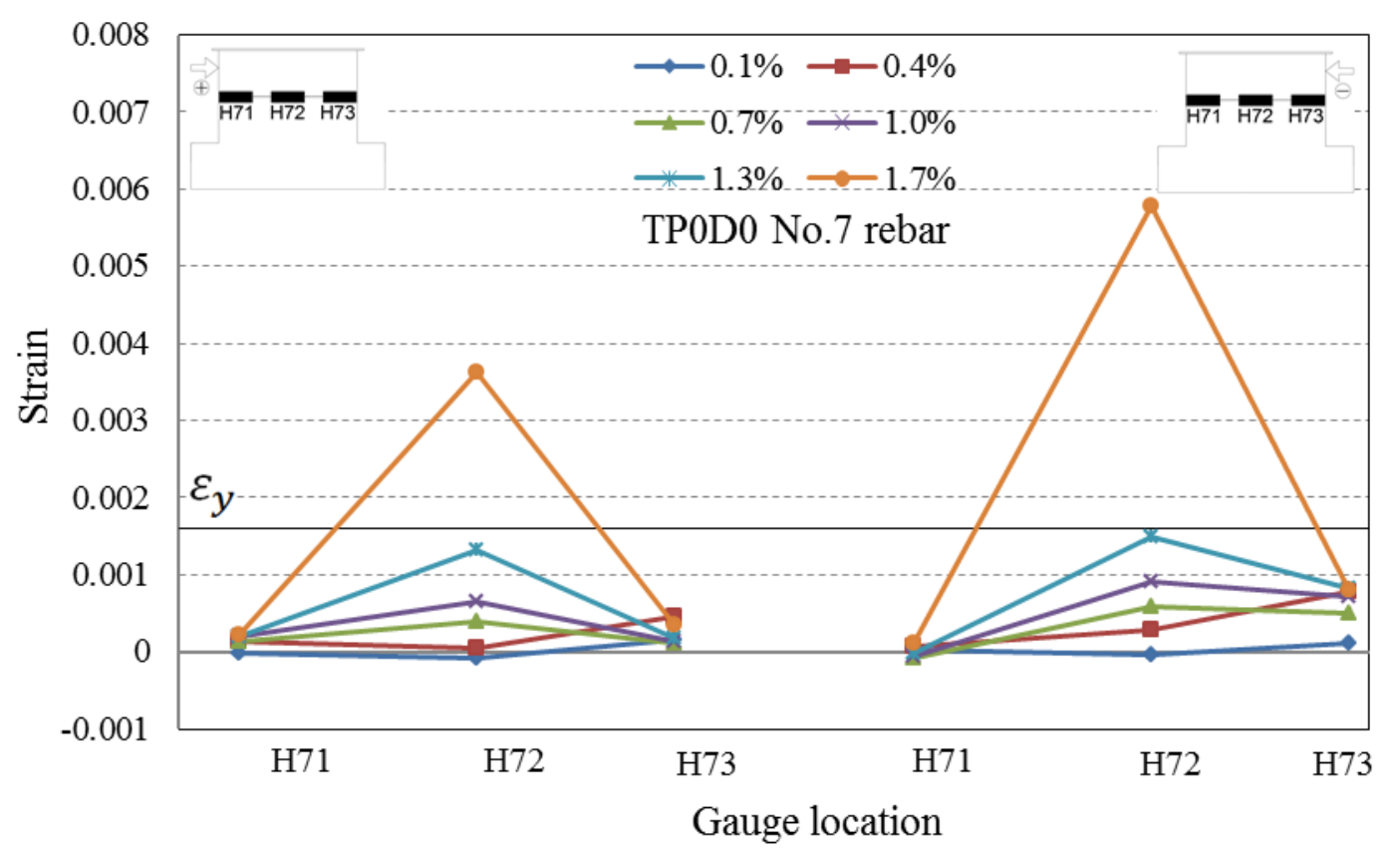

Figure 4.113 Strain profiles of No.7 horizontal rebar of Specimen TP0D0 


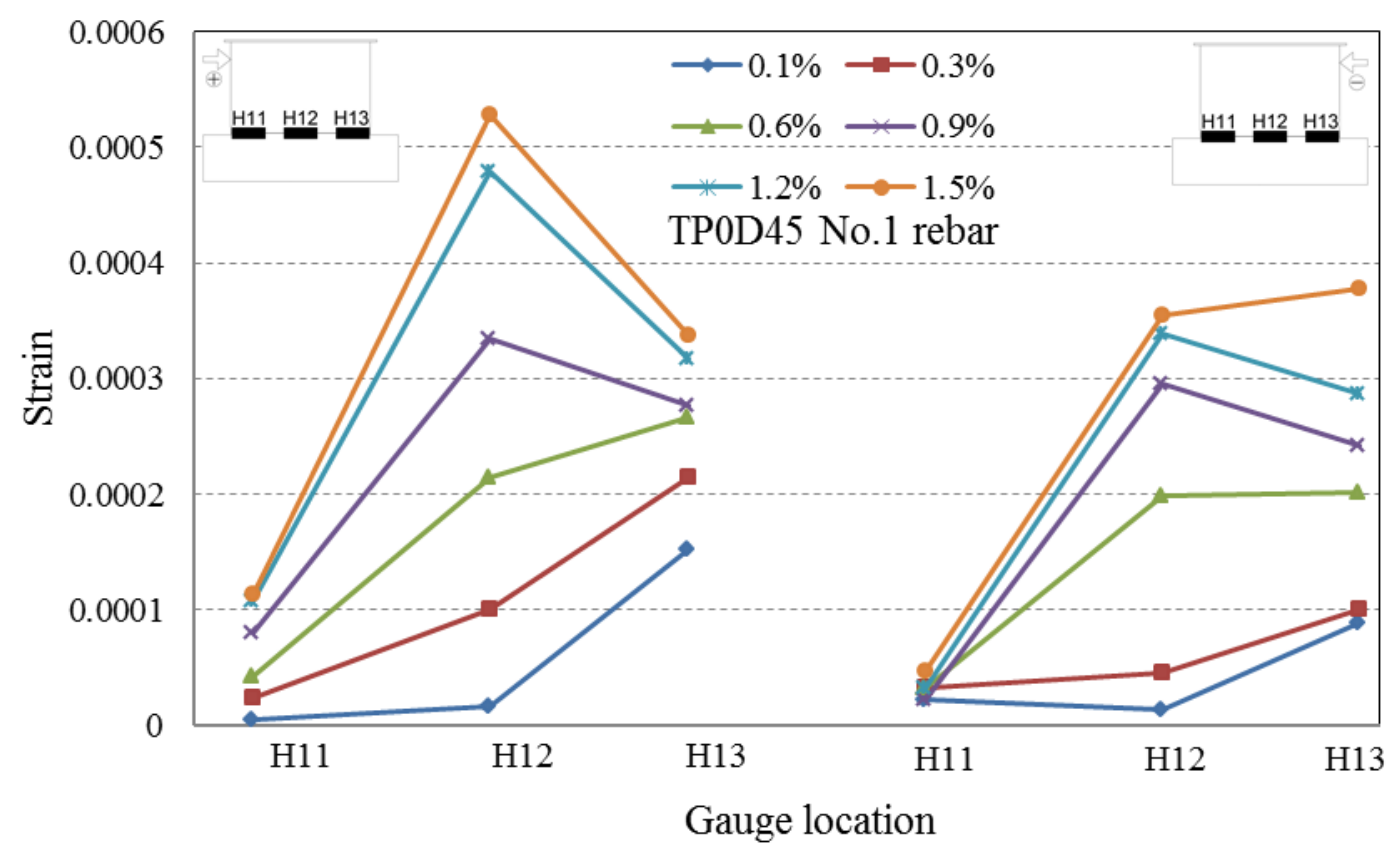

Figure 4.114 Strain profiles of No.1 horizontal rebar of Specimen TP0D45

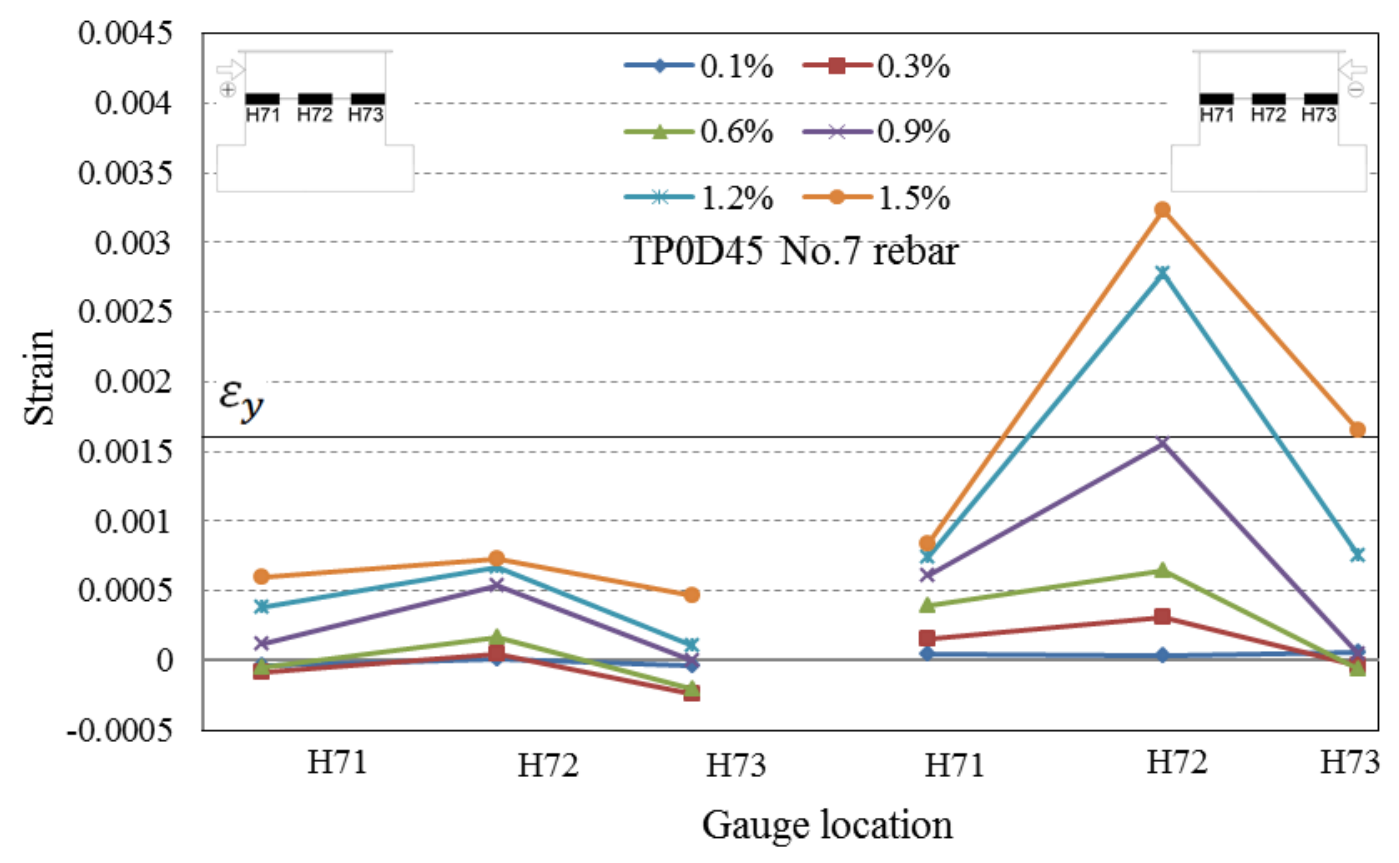

Figure 4.115 Strain profiles of No.7 horizontal rebar of Specimen TP0D45 


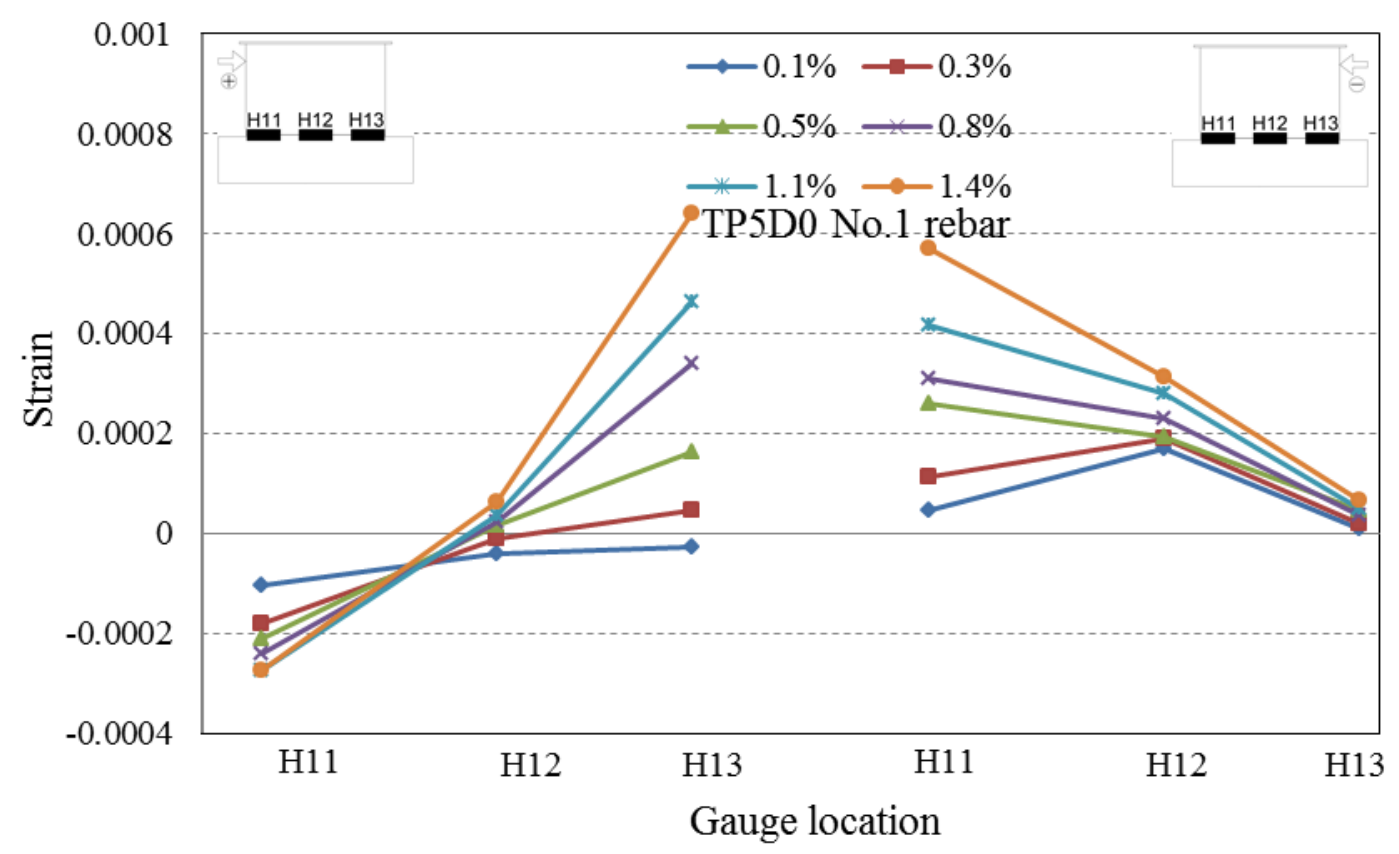

Figure 4.116 Strain profiles of No.1 horizontal rebar of Specimen TP5D0

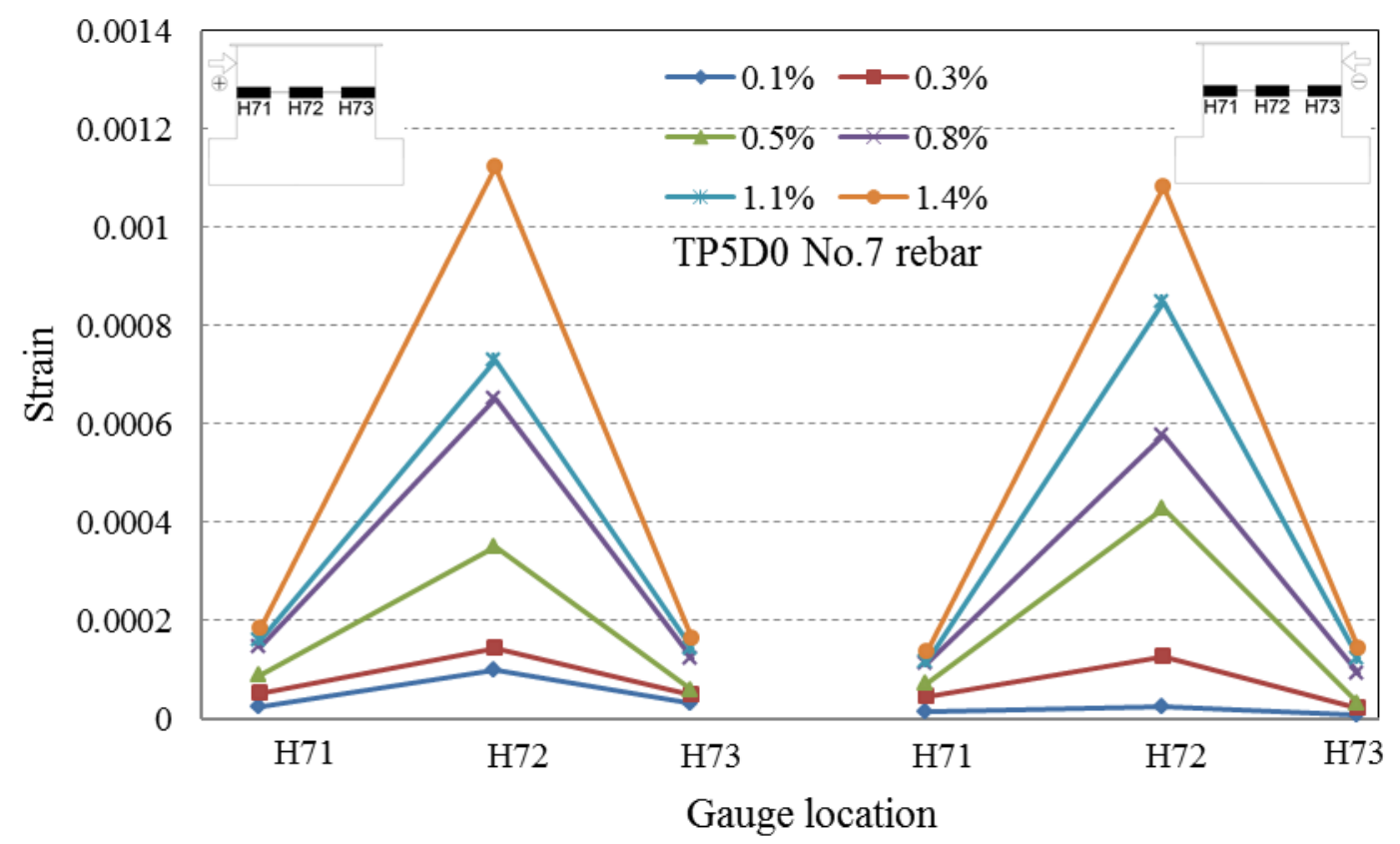

Figure 4.117 Strain profiles of No.7 horizontal rebar of Specimen TP5D0 


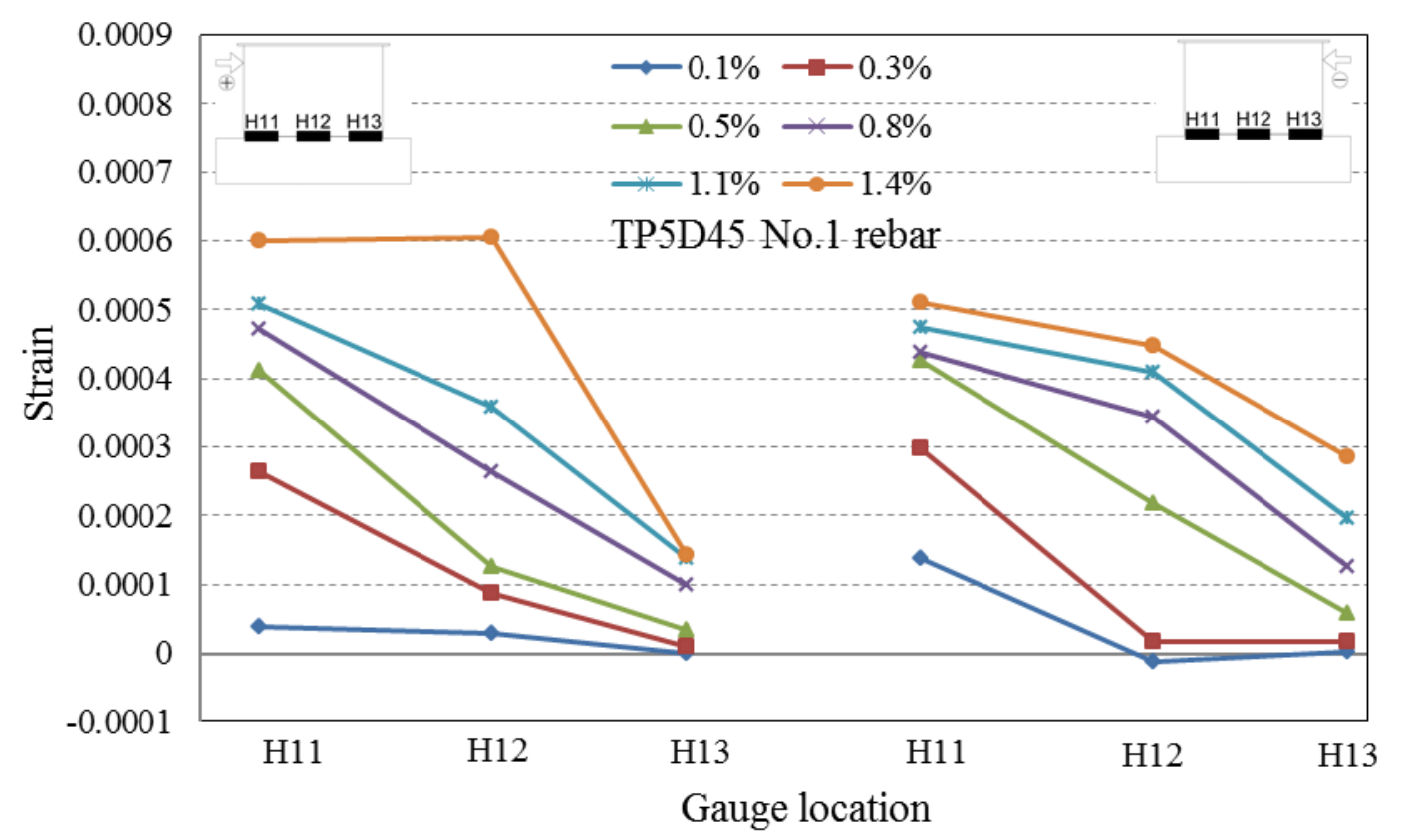

Figure 4.118 Strain profiles of No.1 horizontal rebar of Specimen TP5D45

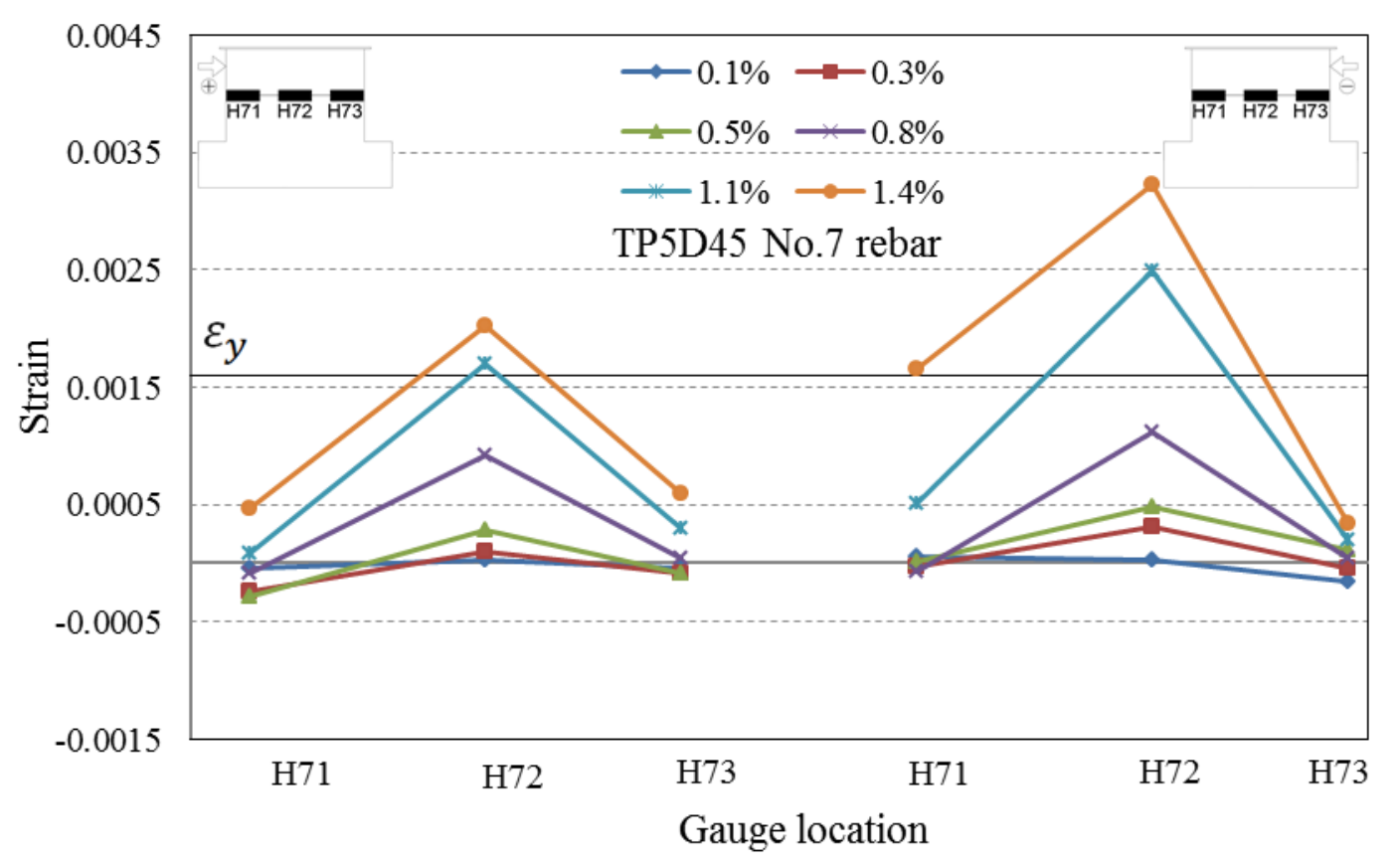

Figure 4.119 Strain profiles of No.7 horizontal rebar of Specimen TP5D45

\subsection{Summary}

An experimental program was carried out on twelve non-rectangular RC squat walls under simulated gravity and simulated seismic loading. The experimental results were presented and discussed in terms of cracking patterns, failure mechanisms, 
hysteretic responses, displacement components, and strain profiles. The main conclusions are drawn as follows:

- The initial crack of each specimen appeared at a drift ratio ranging from $0.1 \%$ to $0.4 \%$, which was prior to the yielding of longitudinal reinforcing bars. As the test progressed, diagonal cracks inclined from $30^{\circ}$ to $65^{\circ}$ spread to the other area of wall surfaces. Horizontal flexural cracks were found on the flange of the $\mathrm{H}$-shaped and $\mathrm{T}$-shaped specimens with orthogonal lateral loading, which extended to the full length of the flange as lateral displacements increased. Moreover, in the orthogonally-loaded group, fewer cracks were found on the flange when compared with those on the web. Most of these cracks were flexural horizontal cracks, which appeared when the flange was in tension. Concrete spalling and crushing were witnessed in the test, which led to the failure of specimens. Detailed discussions on the failure modes would be presented in the next chapter.

- Generally, axial loads contributed to the increase of shear strength and secant stiffness of test specimens, since it effectively constrained the diagonal cracks and limit the development of the sliding. Moreover, specimens with greater axial loads demonstrated better energy dissipation capacities. Its influence on the deformation capacity of specimens, on the other hand, remained unclear.

- The shear deformation which dominated the response of specimens was found to be more evident in those with orthogonal lateral loading. The maximum value of 64\% was recorded in Specimen TP5D0. Sustained and slight increases of the sliding shear displacement were observed in all specimens, in which more rapid growth was found in specimens with lower axial loads.

- Data concerning strains of longitudinal reinforcement in the flange showed that a significant shear lag effect existed in some specimens. Due to this effect, some longitudinal reinforcing bars did not yield during the whole test. This effect was more obvious in walls under orthogonal lateral loading. Moreover, the shear lag effect was also observed when the flange was in compression. 
- From the strain profiles, it was clear that the horizontal rebar did not yield in the L-shaped and $\mathrm{H}$-shaped specimens. In the $\mathrm{T}$-shaped group, yielding was detected at the failure in all specimens except Specimen TP5D0. Surges of strains were found in the middle of No.7 horizontal rebar and the left and right parts of No.1 horizontal rebar in most specimens, suggesting a diagonal compression strut passing through these places. 


\section{CHAPTER 5 \\ DISCUSSIONS AND COMPARISONS OF THE \\ EXPERIMENTAL RESULTS}

\subsection{Introduction}

The experimental results of the test specimens are presented in Chapter 4. To achieve an improved understanding of the seismic behavior of non-rectangular RC squat walls, it is necessary to further discuss, compare and analyze these experimental results. This chapter is composed of eight sections, in which failure mechanisms, load-displacement relationships, shear deformations, strains in horizontal reinforcement, shear lag effects and effects of design parameters are presented in turns.

\subsection{Cracking Patterns and Failure Mechanisms}

As presented in Chapter 4, various cracking patterns were witnessed in the test. Several common characteristics concerning the development of cracks can be summarized.

Generally, initial shear cracks occurred at the middle and upper part of wall segment at a drift ratio ranging from $0.1 \%$ to $0.4 \%$, which was prior to the yielding of longitudinal reinforcing bars. Those imposed with more axial loads saw the delayed formation of initial cracks. The specimens experienced their first yielding at a drift ratio ranging from $0.4 \%$ to $0.7 \%$. As the lateral displacement increased, diagonal cracks inclined from $30^{\circ}$ to $65^{\circ}$ quickly spread to the other area of the wall segments. Horizontal flexural cracks were found on the flange of the H-shaped and T-shaped specimens with orthogonal lateral loading, which extended to the full length of the flange as lateral loads increased, while diagonal shear cracks were observed to have a predominant position in the wall segments of specimens with skew lateral loading. The two surfaces of a wall segment generally showed a similar distribution of cracks. As the test progressed, the inclined cracks became denser and intersected with each other, which was the typical cracking pattern of squat walls. 
The cracks were observed to spread all over the surface of wall segments when specimens failed.

Due to the difference of section shapes, axial loads, and lateral loading directions, the twelve specimens also differed with each other in terms of cracking patterns. In the flanges of the $\mathrm{H}$-shaped walls with orthogonal lateral loading, horizontal flexural cracks were found running from the junction of wall segments to the entire width of the flange, while in the flange of the $\mathrm{H}$-shaped specimens with skew lateral loading, the majority of cracks were inclined shear cracks. A same phenomenon was also witnessed in the T-shaped specimens. As for the L-shaped specimens, no evident horizontal flexure cracks were observed in the flange, and the majority of the inclined cracks were formed when the flange was in tension.

Moreover, it was clear that specimens with higher axial load ratio tended to have steeper diagonal cracks on the web, which is demonstrated in the comparison between Specimens HP0D0 and HP5D0 as can be seen in Figure 5.1. This was because additional axial loads increased the vertical vector of the internal stress in the web, thereby ascending the angle of the inclined cracks. Furthermore, it was evident in the $\mathrm{H}$-shaped and $\mathrm{T}$-shaped groups that denser cracks appeared on the specimens with higher axial load ratio. Nonetheless, the opposite was found to be true in specimens with L-shaped section. This was because of the double effect of the axial force. On one hand, it limited the crack width; on the other hand, the large axial force also dramatically increased the compressive stresses in the wall segments, which accelerated the formation of cracks. Besides, at the final stage, moderate concrete spalling was observed in Specimens LP5D0 and LP5D45, which was accompanied with the sliding at the top. A major horizontal crack and more severe concrete spalling were found at the upper part of the web in Specimens HP0D0, HP5D0, and TP5D0. Several vertical slip planes were observed in Specimens HP5D0 and TP5D0. 

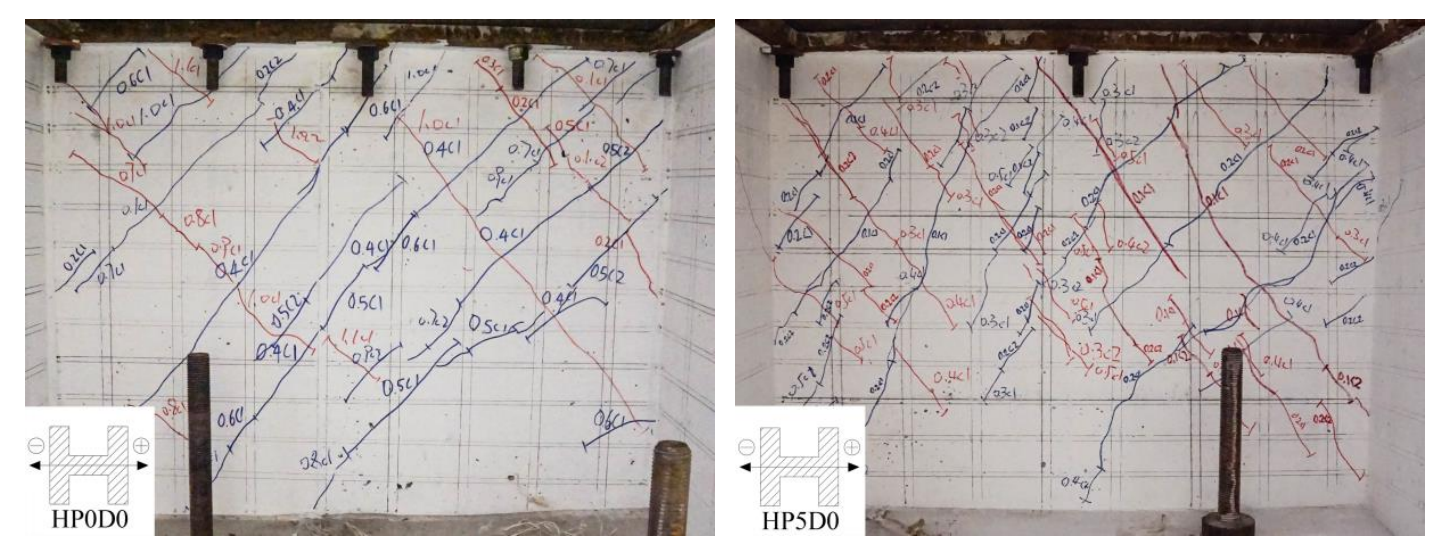

Figure 5.1 Comparison of the diagonal cracks on the web of Specimens HP0D0 and HP5D0

Generally, three types of shear failure are witnessed in squat walls, which are diagonal tension failure, diagonal compression failure and sliding shear failure [P3]. As presented in the design stage, the incorporated large flange extensively increases the flexural strength of specimens, indicating that the shear failure would occur before the wall obtains its full flexural capacity. Thus the mixed flexure-shear failure is not considered to happen in this experimental program, not to mention the flexural failure.

As reviewed by Paulay et al. [P3], the diagonal tension failure is generally observed in squat walls with light horizontal web reinforcement. It is characterized by one or more wide diagonal cracks in the web and cracks are not widespread. In this study, R6 round bars with a space of $80 \mathrm{~mm}$ was used as the horizontal rebar which contributed to a horizontal reinforcement ratio of $0.71 \%$ in all specimens. The design meets the requirement of ACI 318-14 [A1] and is considered to provide sufficient horizontal reinforcement. Accordingly, from the test results, no obvious single wide crack was witnessed and the diagonal cracks extended to the entire surface of the web, suggesting the diagonal tension failure did not happen in the test.

When provided with sufficient horizontal web reinforcement to prevent the diagonal tension failure, low-rise walls under axial loads and cyclic lateral loading are prone to the diagonal compression failure [P3]. This failure is characterized by crushing of concrete struts in the web and has been shown to occur easily in specimens with large flanges [G2]. In this experimental program, most specimens failed in the similar mode of the diagonal compression failure. Concrete spalling was found in 
Specimens HP0D45 and HP5D45. Both specimens were able to sustain a ductile post-peak response before failure. The former failed with moderated concrete spalling at the left upper corner of the web. The latter experienced slighter concrete spalling and failed as integrity of the web and flange was impaired by shear cracks. Specimens LP0D0, TP0D0, TP0D45, and TP5D45 shared a similar failure mode as the concrete in the corner of the web was crushed. In the former two specimens, the crushing of concrete was witnessed at the bottom corner, while the T-shaped specimen with skew lateral loads had the concrete crushing happening at the upper left corner. Specimens LPOD0 and TP0D0 experienced a sudden drop of lateral resistance when the strength was still in its ascending phase. Specimens TP0D45 and TP5D45, on the other hand, were able to sustain a ductile post-peak response before failure. The crushing of concrete was also detected in Specimen LP0D45, which was relatively slight in comparison with the corner crushing mentioned above. Specimen LP0D45 failed with a drop in its lateral resistance by more than $30 \%$ of the maximum as the concrete crushing extended along the length of wall segment.

The sliding shear failure shares several common characteristics with the diagonal compression failure. In this failure mode, concrete crushing resulted from the intersection of diagonal cracks spreads over the wall length in a narrow band, forming a weakened horizontal plane in the web and causing the remaining wall segment to slide along this plane. Heavily reinforced walls under cyclic lateral loads are prone to this failure. In the test, the sliding of wall segment occurred in Specimens HP0D0, HP5D0, and TP5D0. However, instead of typical sliding which usually happened near the wall base, all sliding was observed in the upper part of the wall. An identical phenomenon was also witnessed in the Specimen DP2 of Palermo and Vecchio's test [P2]. The comparison of the horizontal sliding planes between Specimens HPOD0 and DP2 is illustrated below in Figure 5.2. In both studies, this sliding caused a premature failure in which the specimens failed before attaining their full lateral strengths. Palermo and Vecchio suggested that the main reason of this failure could be the weaker concrete adopted in the upper part and the nature of thin wall section [P2], which is a reasonable assumption since these two factors both contributed to the crushing of concrete at the top. However, the main reason behind this phenomenon was more related to the force transfer mechanism in 
the test. In both experiments, a large and stiff concrete slab was used to transfer lateral loads to the wall segment. Ideally, the horizontal displacement was transferred assuming that a perfect coordination existed between these two sections. Nonetheless, in the test, the existence of the stiff top slab and the incompatibility in transferring horizontal loads would cause a stress concentration in the upper part of the web. Under the cyclic loading, inclined cracks formed in each loading direction intersected with each other near the top, causing the concrete struts to become narrow. These struts were prone to crushing under such high stress. The concrete crushing further spread along the wall length, formed a weakened horizontal plane, and eventually led to the sliding of the wall. A major horizontal crack was also observed at the upper part of the wall segment in Specimens LP5D0 and LP5D45. However, the horizontal crack did not extend to the full length of the wall segment. Thus no evident sliding was witnessed. These two specimens experienced a drastic loss of lateral strength once concrete spalling was spotted along the horizontal crack, and quickly failed after a few more cycles of loading.
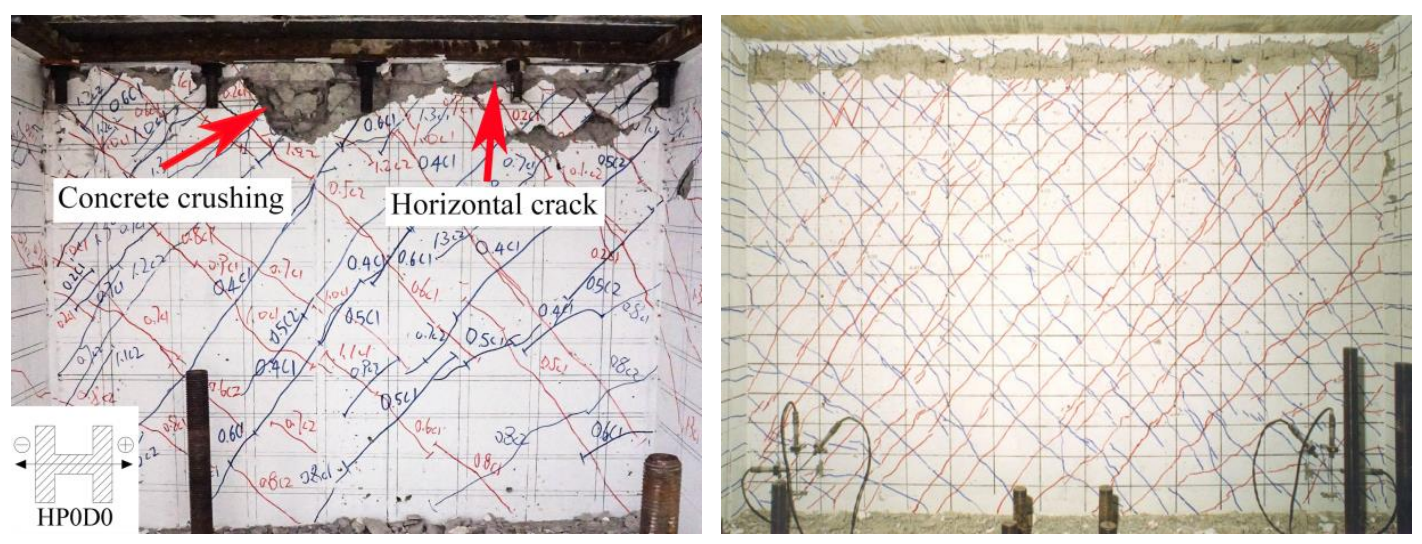

Figure 5.2 Failure mechanisms of Specimens HP0D0 and DP2

Furthermore, several vertical slip planes were observed in the web of Specimens HP5D0 and T50D0. An identical phenomenon was also detected in Specimen DP1 of Palermo and Vecchio's test $[\mathrm{P} 2]$, as demonstrated in Figure 5.3. Specimens HP5D0 and DP1 shared a similar section shape and reinforcement contents, and were both subjected to axial loads slightly above $0.05 f_{c}^{\prime} A_{g}$. The flange of both specimens remained relatively intact throughout the test. Palermo and Vecchio concluded that axial loads and stiff flanges restrained the opening of the shear 
cracks. Thus large shear stresses were introduced in the web by the significant shear distortion, leading to the formation of vertical slip planes [P2]. This explanation was considered plausible. As presented previously, the existence of additional axial loads tended to increase the vertical vector of the internal stress in the web, thereby ascending the inclination of diagonal shear cracks. In addition, and of more significance, the flexure-dominated flange restrained the shear-dominated web from moving, which meant a deformation incompatibility existed in the flanged walls. Under cyclic lateral loading, the web experienced tensile stresses in opposite directions to the lateral displacements which 'tear' the web at the weak part, causing the formation of vertical slip planes.

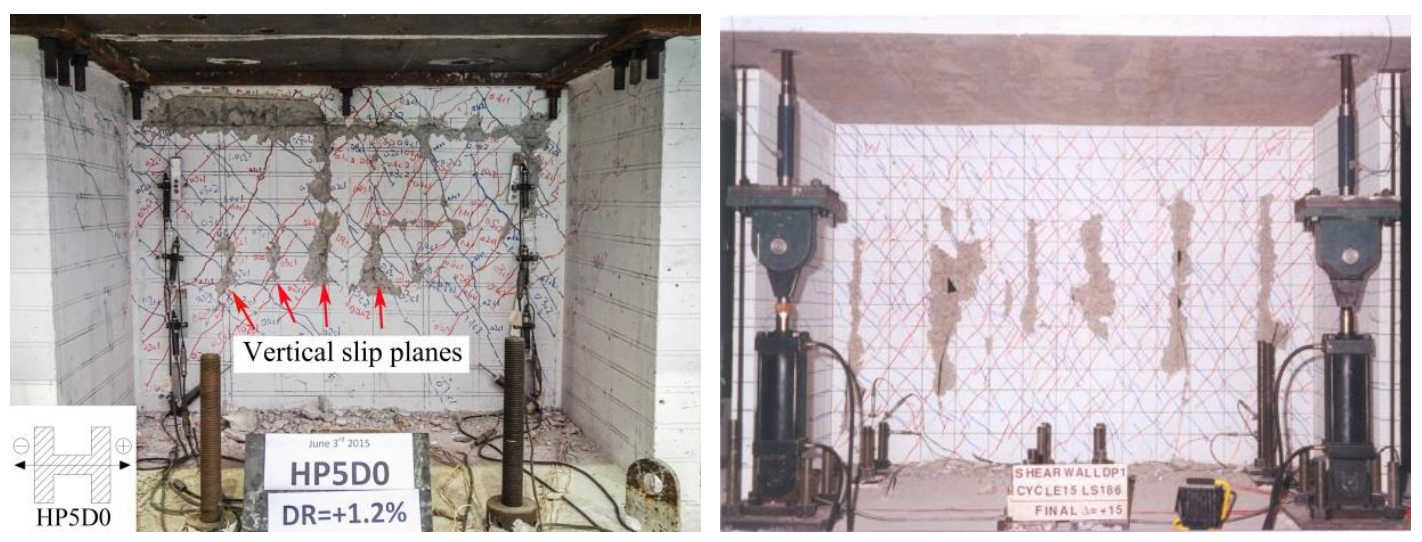

Figure 5.3 Failure mechanisms of Specimens HP5D0 and DP1

This deformation incompatibility was illustrated in Figure 5.4. In Specimens HP5D0 and DP1, the majority of cracks on flanges were flexural cracks, which extended to the full length of the flange. Thus, it could be concluded that the flexure dominated the responses of flanges. The web of these two specimens, on the other hand, was mainly controlled by shear. It was revealed not only in the cracking pattern as presented below, but also in the illustration of deformation components, where the shear deformation contributed to more than $60 \%$ of total deformation in both loading directions. As seen in the left, ideally, both flange and web segments experienced the same deformation at all heights. However, in reality, the flange was mainly subjected to the flexural distortion, while the shear distortion was concentrated in the web, which would inevitably cause an incompatibility in 
deformation between these two segments as marked in black. The deformation of the flange along the wall height is written as:

$$
\omega_{f}=\frac{V x^{2}}{6 E_{c} I_{g}}\left(3 h_{w}-x\right)
$$

where $V(\mathrm{kN})$ is the lateral reaction force, $x(\mathrm{~mm})$ is the distance from the base to the top, ranging from 0 to $h_{w}, E_{c}(\mathrm{MPa})$ is the elastic modulus of concrete, $I_{g}\left(\mathrm{~mm}^{4}\right)$ is the moment of inertia of the flange section, $h_{w}$ is the wall height $(\mathrm{mm})$. Since the flange and web had the same deformation at the top, assuming the web was only subjected to the shear distortion, the deformation of the web along the wall height is expressed as:

$$
\omega_{w}=\frac{V h_{w}^{2} x}{3 E_{c} I_{g}}
$$

Hence the deformation incompatibility is obtained by subtracting Equation 5.1 from Equation 5.2 as:

$$
\Delta_{\omega}=\omega_{w}-\omega_{f}=\frac{V}{6 E_{c} I_{g}}\left(x^{3}-3 h_{w} x^{2}+2 h_{w}^{2} x\right)
$$

In order to understand the variation of deformation incompatibility along the height, it was necessary to solve the derivative of the above function.

$$
\begin{aligned}
& \frac{d \Delta_{\omega}}{d x}=\frac{V}{6 E_{c} I_{g}}\left(3 x^{2}-6 h_{w} x+2 h_{w}^{2}\right)=0 \\
& x=\left(1 \pm \frac{\sqrt{3}}{3}\right) h_{w}=0.42 h_{w} \text { or } 1.58 h_{w}
\end{aligned}
$$

Since the predefined range of $x$ was from 0 to $h_{w}$, it was clear that the deformation incompatibility gradually increased from the wall base as the height rose, reaching its maximum at the height of $0.42 h_{w}$, and declined as the height further ascended. Accordingly, the horizontal tensile force created by the deformation incompatibility also gained its maximum around the middle height, which was in accordance with the position of vertical slip planes in both tests. Moreover, the imposed axial loads caused inclined shear cracks to become steeper. Under cyclic loading, more 
intersected cracks accumulated in the center of the web panel, as shown in Figure 5.4. Therefore, a weakened vertical plane would form in this particular area with concrete spalling as observed in the test.
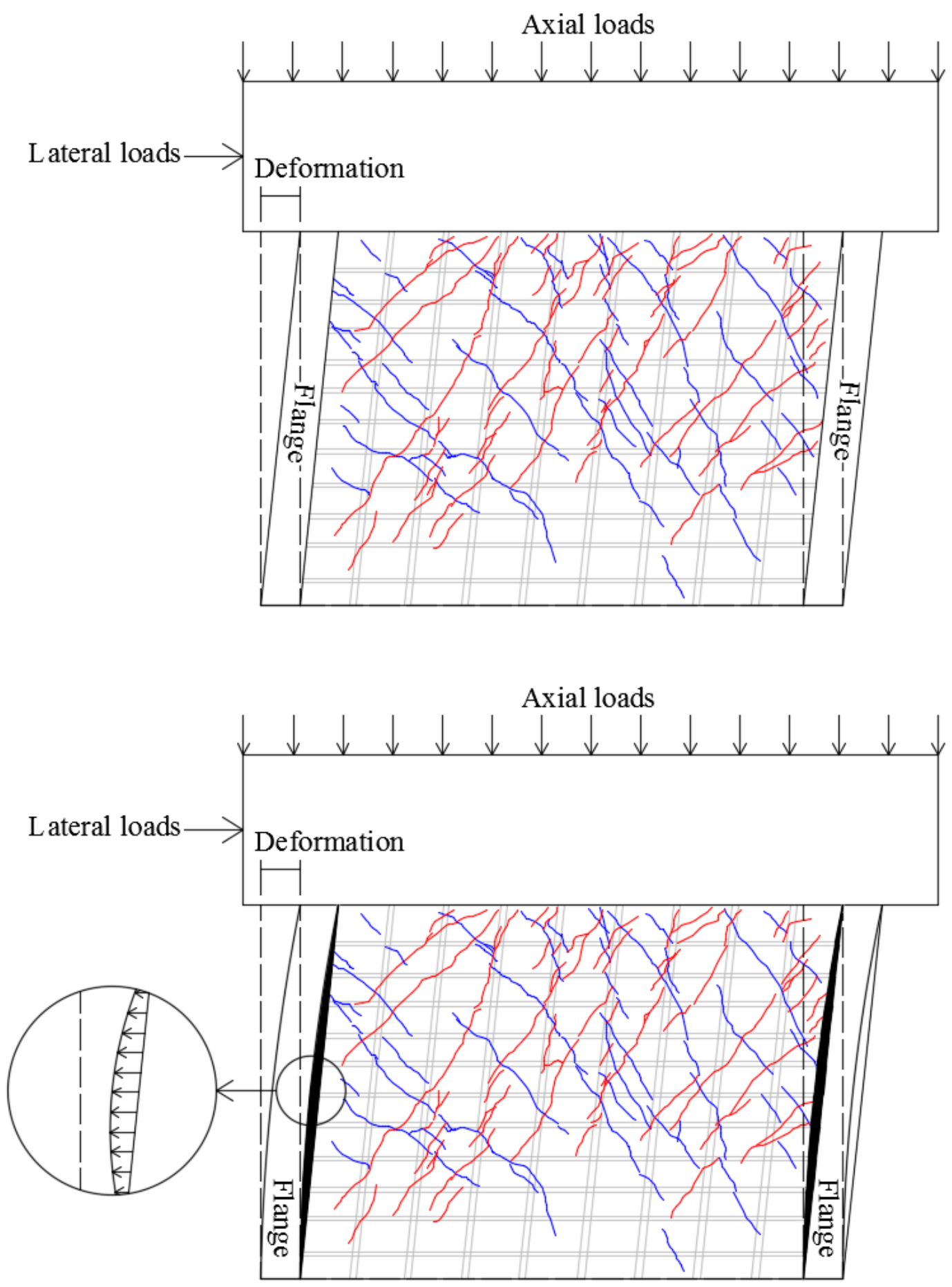

Figure 5.4 Deformation incompatibility in Specimen HP5D0 


\subsection{Load-displacement Relationships}

\subsubsection{Backbone curve}

As stated in the previous section, the backbone curve is significant in modeling the inelastic behavior of RC walls. In the test, the specimens only subjected to the weight of the top slab had axial load ratios ranging from $0.48 \%$ to $0.72 \%$, while the specimens with extra axial loads had ratios ranging from $5.14 \%$ to $5.65 \%$. The influence of axial loads on backbone curves was evident: it brought an increase in both lateral strength of secant stiffness in most specimens, which was in accordance with the finding of Palermo and Vecchio [P2]. For instance, at the drift ratio of $0.5 \%$, Specimen TP0D0 only attained around $50 \%$ of the lateral strength of Specimen TP5D0. As for the lateral loading direction, its impact on the peak shear strength remained unclear since the evidence was found on both sides. Furthermore, the peak shear strength of most specimens was larger than the calculated results from ACI 318-14 [A1], due to stiffness brought by the large flanges. An exception also existed, such as HPOD0, which experienced a sliding at the top part and failed before attaining its maximum lateral strength.

Figure 5.5 illustrates the comparison of backbone curves of specimens in the Lshaped and T-shaped groups when the flange was in tension, in which the L-shaped specimens are marked with solid lines and data markers while the T-shaped counterparts are marked with dotted lines of the same color but without data markers. It was clear that the T-shaped specimens processed larger stiffness and corresponding lateral strength at a specific drift ratio than the L-shaped specimens when imposed with orthogonal lateral loads. The primary reason for this phenomenon was that in the T-shaped specimens, the loading path was overlapped with the principle axis, where the wall segment was fully effective in resisting the lateral loads. Besides, the shear lag effect may also contribute to the loss stiffness in the L-shaped specimens. The overhanging flange width of the L-shaped walls was evidently longer than that of the T-shaped walls. Thus more longitudinal reinforcing bars in the flange were not active in resisting lateral loads, resulting in the reduction of the in-plane stiffness. However, under skew lateral loading, the opposite was true: the L-shaped specimens had larger stiffness. This was mainly because the 
symmetric section shape of the L-shaped walls brought more evenly distributed areas in tension and compression regions. Moreover, the principal shear force was less-concentrated as being transferred by two segments.

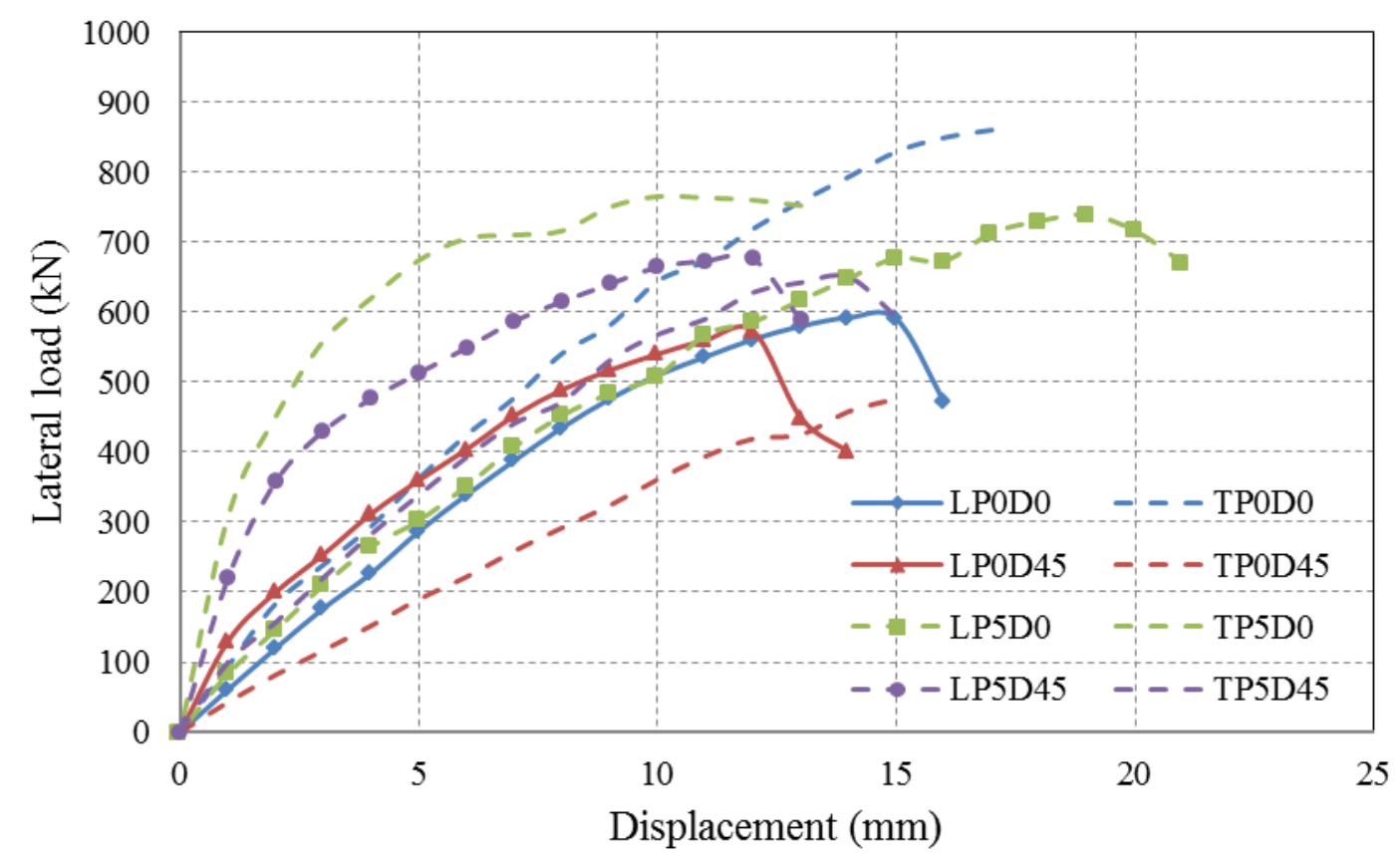

Figure 5.5 Comparison of the backbone curves of the L-shaped and T-shaped specimens in the direction of the flange in tension

\subsubsection{Effective stiffness}

The effective stiffness, known to be an important factor in the displacement-based design approach [W2, W4], plays a pivotal role in the seismic performance of RC structures, which can significantly affect the design of displacements, ductility factors and internal forces. With regard to squat shear walls, building codes offer reduction factors of gross moment of inertia to account for the loss of flexural and shear rigidities. In ACI 318-14 [A1], the reduction factors are recommended as 0.35 and 0.70 for cracked and uncracked concrete. As specified in ASCE 43-05 [A3], no reduction is recommended for uncracked concrete and 0.5 is recommended for cracked concrete.

This simplification was found by many researchers $[\mathrm{P} 4, \mathrm{X} 1]$ to overestimate the effective stiffness of squat walls. Hence numerous models and equations have been developed in the literature to improve the prediction of the effective stiffness of 
squat walls. Paulay and Priestley [P4] suggested that the effective stiffness should be closely related to axial loads and reinforcement contents as:

$$
\frac{I_{e}}{I_{g}}=\frac{100}{f_{y}}+\frac{P_{u}}{f_{c}^{\prime} A_{g}}
$$

Furthermore, to account for the effect of shear deformations on wall stiffness, the following expressions were also developed by Paulay and Priestley [P4] for structural walls with aspect ratios less than 4 :

$$
\begin{gathered}
I_{w}=\frac{I_{e}}{1.2+F} \\
F=\frac{30 I_{e}}{h_{w}^{2} b_{w} l_{w}}
\end{gathered}
$$

Fenwick and Bull [F2] later conducted a parametric study and included axial loads, the yield strength of longitudinal reinforcement and the concrete compressive strength to determine the effective stiffness. However, the equation proposed by Fenwick and Bull [F2] was found improper for the squat walls since it was derived from slender walls, in which flexure rather than shear dominated the responses. Li and Xiang [L3] noticed this imperfection and proposed an equation specifically for squat walls as shown below:

$$
\frac{I_{e}}{I_{g}}=0.19\left(\frac{100}{f_{y}}+\frac{N}{f_{c}^{\prime} A_{g}}\right)\left(0.53+0.37 \frac{h_{w}}{L_{w}}+0.31 \frac{h_{w}^{2}}{L_{w}^{2}}\right)
$$

where $I_{e}\left(\mathrm{~mm}^{4}\right)$ is the effective moment of inertia, $I_{g}\left(\mathrm{~mm}^{4}\right)$ is the moment of inertia of gross section, $f_{y}(\mathrm{MPa})$ is the yielding stress of reinforcement, $A_{g}\left(\mathrm{~mm}^{2}\right)$ is the gross area of section, $f_{c}^{\prime}(\mathrm{MPa})$ is the compressive strength of concrete, $N(\mathrm{~N})$ is the axial loads, $h_{w}(\mathrm{~mm})$ is the height of wall and $L_{w}(\mathrm{~mm})$ is the length of wall.

The evaluations of these two sets of equations mentioned above using data obtained from this study were shown in Table 5.1. In this study, the effective stiffness is defined as the stiffness of structure when either the first longitudinal rebar yields or the concrete reaches a compressive strain of 0.002 . 
Table 5.1 Comparisons between the predicted and experimental effective stiffness

\begin{tabular}{|c|c|c|c|c|c|c|c|c|}
\hline \multirow{3}{*}{ Specimen } & \multirow{3}{*}{$\begin{array}{l}\text { Axial } \\
\text { load } \\
\text { ratio }\end{array}$} & \multirow{3}{*}{$\begin{array}{c}\text { Direction } \\
\text { of lateral } \\
\text { loads }\end{array}$} & \multirow{3}{*}{$\begin{array}{l}\text { Loading } \\
\text { direction }\end{array}$} & \multicolumn{3}{|c|}{$E I_{e} / E I_{g}$} & \multicolumn{2}{|c|}{$\begin{array}{c}\text { Ratio (Predicted / } \\
\text { Test) }\end{array}$} \\
\hline & & & & (1) & (2) & (3) & 1) $(2)$ & (0) (2) \\
\hline & & & & Eq. (5.6) & Eq. (5.9) & Test & (1)/(3) & $(2) /(3)$ \\
\hline \multirow{2}{*}{ HP0D0 } & \multirow{2}{*}{0.48} & \multirow{2}{*}{ Orthogonal } & Positive & 0.010 & 0.032 & 0.010 & 3.31 & 3.15 \\
\hline & & & Negative & 0.009 & 0.032 & 0.009 & 3.59 & 3.41 \\
\hline \multirow{2}{*}{ HP0D45 } & \multirow{2}{*}{0.51} & \multirow{2}{*}{ Skew } & Positive & 0.020 & 0.032 & 0.020 & 2.31 & 1.64 \\
\hline & & & Negative & 0.015 & 0.032 & 0.015 & 2.96 & 2.09 \\
\hline \multirow{2}{*}{ HP5D0 } & \multirow{2}{*}{5.14} & \multirow{2}{*}{ Orthogonal } & Positive & 0.012 & 0.040 & 0.012 & 2.96 & 3.37 \\
\hline & & & Negative & 0.013 & 0.040 & 0.013 & 2.72 & 3.10 \\
\hline \multirow{2}{*}{ HP5D45 } & \multirow{2}{*}{5.46} & \multirow{2}{*}{ Skew } & Positive & 0.022 & 0.041 & 0.022 & 2.22 & 1.87 \\
\hline & & & Negative & 0.018 & 0.041 & 0.018 & 2.68 & 2.25 \\
\hline \multirow{2}{*}{ LP0D0 } & \multirow{2}{*}{0.72} & \multirow{2}{*}{ Orthogonal } & Positive & 0.015 & 0.033 & 0.015 & 4.27 & 2.13 \\
\hline & & & Negative & 0.013 & 0.033 & 0.013 & 5.08 & 2.53 \\
\hline \multirow{2}{*}{ LP0D45 } & \multirow{2}{*}{0.73} & \multirow{2}{*}{ Skew } & Positive & 0.046 & 0.033 & 0.046 & 2.17 & 0.71 \\
\hline & & & Negative & 0.054 & 0.033 & 0.054 & 1.85 & 0.60 \\
\hline \multirow{2}{*}{ LP5D0 } & \multirow{2}{*}{5.65} & \multirow{2}{*}{ Orthogonal } & Positive & 0.011 & 0.041 & 0.011 & 6.34 & 3.64 \\
\hline & & & Negative & 0.020 & 0.041 & 0.020 & 3.64 & 2.09 \\
\hline \multirow{2}{*}{ LP5D45 } & \multirow{2}{*}{5.67} & \multirow{2}{*}{ Skew } & Positive & 0.058 & 0.041 & 0.058 & 2.01 & 0.72 \\
\hline & & & Negative & 0.063 & 0.041 & 0.063 & 1.84 & 0.66 \\
\hline TPिDी & 072 & Orthogonal & Positive & 0.016 & 0.033 & 0.016 & 4.09 & 2.04 \\
\hline IFUDV & 0.12 & intuogonal & Negative & 0.020 & 0.033 & 0.020 & 3.34 & 1.66 \\
\hline TDOD45 & 070 & $\mathrm{C}$ & Positive & 0.025 & 0.033 & 0.025 & 2.60 & 1.28 \\
\hline 1PUD45 & 0.10 & SKEW & Negative & 0.029 & 0.033 & 0.032 & 2.27 & 1.12 \\
\hline TP5DO & 5.65 & $O r$ & Positive & 0.022 & 0.041 & 0.022 & 3.19 & 1.84 \\
\hline IPJDO & 5.05 & Urtnogonal & Negative & 0.028 & 0.041 & 0.028 & 2.56 & 1.47 \\
\hline TD5D15 & 546 & $\mathrm{Cl}$ & Positive & 0.030 & 0.041 & 0.030 & 2.38 & 1.35 \\
\hline 1РЈD4J & 5.40 & SKew & Negative & 0.036 & 0.041 & 0.036 & 2.03 & 1.15 \\
\hline & & & & & & Mean & 3.017 & 1.908 \\
\hline & & & & & & $\mathrm{COV}$ & 0.360 & 0.480 \\
\hline
\end{tabular}


As seen from the table, generally both methods overestimated the test results, especially for the H-shaped group. This was because the equation was developed mainly for rectangular or barbell squat walls, which did not consider the influence of large flanges in the non-rectangular walls. As known in the geometry, the introduced large flanges significantly increased the moment of inertia of gross section. Since an evident shear lag effect was found in the specimens, it was inappropriate to adopt the moment of inertia of the whole uncracked section in estimating the effective stiffness of non-rectangular walls. Zhang [Z2] also found this overestimation in a study of non-rectangular slender walls, and concluded that the results would be refined if the effective flange length of one-quarter of wall height as suggested by ACI 318-14 [A1] was used. Compared with the equation from Li and Xiang [L3], the equations proposed by Paulay and Priestley [P4] evidently deviated from the experimental results. This was because the latter was mainly developed for walls that were controlled by flexure, which was unsuitable for the shear-dominated squat specimens in this study. With regard to the parameters in tests, it was clear that the increase of axial loads brought growth on the effective stiffness, which was revealed in both test results and analytical methods. As for the influence of lateral loading direction, specimens with skew lateral loading had greater effective stiffness than specimens with orthogonal lateral loading, which was in accordance with the observations from Zhang [Z2]. Besides, it was observed that the equation yielded the same results for both loading directions, which was considered improper because, for the L-shaped and T-shaped specimens, the section shape was not symmetrical about the bending axis. Further research is required to investigate the effective stiffness of non-rectangular RC squat walls.

\subsubsection{Yield displacement and deformation capacity}

Table 5.2 presents complementary information regarding the load-displacement relationships, which includes shear strength at the first crack, peak shear strength, yield displacement, initial stiffness, and deformation capacity. 
Table 5.2 Summary of test results

\begin{tabular}{cccccccc}
\hline \multirow{2}{*}{ Specimen } & $(1)$ & $(2)$ & $(3)$ & $(4)$ & $(5)$ & $(6)$ & $(8)$ \\
& $f_{c}^{\prime}$ & $P / f_{c}^{\prime} A_{g}$ & $\theta$ & $V_{c r}$ & $V_{\max }$ & $\Delta_{y}$ & $\delta_{\max }$ \\
\cline { 2 - 8 } & $\mathrm{MPa}$ & $\%$ & $\circ$ & $\mathrm{kN}$ & $\mathrm{kN}$ & $\mathrm{mm}$ & $\%$ \\
\hline LP0D0 & 35.0 & 0.72 & 0 & 147 & 592 & 5.0 & 1.6 \\
\hline LP0D45 & 36.2 & 0.70 & 45 & 200 & 656 & 4.0 & 1.4 \\
\hline LP5D0 & 35.0 & 5.65 & 0 & 168 & 739 & 6.0 & 2.1 \\
\hline LP5D45 & 36.2 & 5.46 & 45 & 430 & 714 & 5.0 & 1.3 \\
\hline HP0D0 & 35.9 & 0.48 & 0 & 155 & 857 & 7.0 & 1.1 \\
\hline HP0D45 & 33.8 & 0.51 & 45 & 107 & 737 & 4.0 & 0.9 \\
\hline HP5D0 & 35.9 & 5.14 & 0 & 217 & 994 & 5.0 & 1.1 \\
\hline HP5D45 & 33.8 & 5.46 & 45 & 262 & 1132 & 5.0 & 1.5 \\
\hline TP0D0 & 35.0 & 0.72 & 0 & 230 & 860 & 5.0 & 1.7 \\
\hline TP0D45 & 36.2 & 0.70 & 45 & 187 & 515 & 4.0 & 1.5 \\
\hline TP5D0 & 35.0 & 5.65 & 0 & 269 & 765 & 6.0 & 1.4 \\
\hline TP5D45 & 36.2 & 5.46 & 45 & 157 & 650 & 4.0 & 1.5 \\
\hline
\end{tabular}

Note: (1): Compressive strength of concrete; (2) Axial load ratio; (3) Angle between lateral loading and web segment; (4) Observed shear strength at the first cracking; (5) Peak shear strength during the test; (6) Yield displacement; (7) Initial stiffness of specimen; (8) Maximum drift ratio.

Yielding is of importance in the displacement-based design of ductile RC structural walls [W1]. Even though squat walls are dominated by shear and less ductile compared with slender walls. Attention should also be paid to this since it is essential for the accurate evaluation of the seismic behavior of squat walls. As stated previously, yielding of reinforcement happened after the formation of the first crack in all specimens. The typical displacement at the first yielding ranged from 4 $\mathrm{mm}$ to $7 \mathrm{~mm}$, which meant the difference of yield displacement was insignificant among the specimens. 
Previous research on the non-rectangular slender walls [Z2] indicated that significant reductions of the deformation capacity was observed in specimens with higher axial load ratio. However, in this study, no obvious regularity could be summarized since the influence of axial loads could not even be determined within a single group. The premature failure that occurred in some specimens might contribute to this complexity. For instance, the Specimen HPOD0 failed at a drift ratio of $1.1 \%$ with severe concrete spalling at the top, when its lateral strength still had a tendency of increase. The specimen HP5D0 imposed with extra axial loads, on the other hand, was able to sustain a ductile post-peak response before failure. Thus these two specimens demonstrated identical deformation capacity. A similar phenomenon was also revealed in the experiments of Palermo and Vecchio [P2], in which the specimen with more axial loads even had a larger value of the maximum drift ratio than its counterpart.

\subsection{Shear Deformation}

Shear deformation was found to dominate the response since it accounted for the largest portion of total deformation in the majority of test specimens. The maximum value of $64 \%$ was witnessed in Specimen TP5D0 in both loading directions, in which the specimen failed due to the vertical slip planes and the horizontal crack in the web. As for the L-shaped specimens, the contribution of the shear deformation varied in each specimen as the test progressed, with slight declines being observed in the majority. Moreover, specimens with skew lateral loading had a slightly lower portion of the shear deformation compared with their counterparts loaded orthogonally. This was because the principal shear force was transferred by two segments in the former, while only one segment mainly functioned in the latter, which was in accordance with the finding of Zhang [Z2]. In the H-shaped specimens, the shear deformation increased during all tests. Since the section was symmetrical, almost equivalent portions of the shear deformation were found in both loading directions. As for the T-shaped specimens, the shear displacement was also found to have an increasing trend. However, the growth was less evident when compared with the H-shaped specimens, especially in the skew-loaded specimens. With regard to the influence of axial loads, a slight growth of the shear deformation 
was observed in the orthogonally-loaded specimens, whereas the impact was negligible in specimens with skew lateral loading. The identical phenomenon was also observed in the T-shaped specimens. Therefore, further research is required to comprehend the influence of axial loads on the shear deformation.

As for the composition of the sliding shear displacement, a continuous growth was observed in all specimens, with typical values rising from $3 \%$ to $5 \%$ to $8 \%$ to $13 \%$. A more rapid increase was found in specimens with no additional axial loads, which was reasonable since axial loads can effectively limit the width of cracks thereby slowing the development of the horizontal cracks. Moreover, there was no significant variation between the portions of sliding shear displacement in the orthogonally-loaded group and the skew-loaded group, indicating that the change of loading direction did not play a favorable role in controlling the sliding shear.

\subsection{Strain in Horizontal Reinforcement}

From the strain profiles of No.1 and No.7 horizontal reinforcing bars presented in Chapter 4, it was evident that strains increased in every location of these reinforcing bars as the test progressed. Among them, most significant growth was found in the middle of No.7 horizontal rebar located at $500 \mathrm{~mm}$ above the wall base, especially at the final stage of the test. However, only moderate increases were observed in the left and right corner of the same rebar. An identical rapid growth was also observed in the right and left parts of No.1 horizontal rebar in most specimens. This phenomenon can be served as evidence that a main diagonal compression strut in the web passing through these places, which is in accordance with the finding from twelve squat walls conducted by Luna et al. [L6].

Currently, two analytical models are widely used to account for the force-resisting mechanism in squat structural walls. The first is the softened truss model proposed by Hsu and Mo [H3], which was later modified by Gupta and Rangan [G6]. In this model, the state of stresses in the central panel (the web) was assumed to be uniform and the flow of compressive stresses was described by a series of parallel compressive struts. Based on the equilibrium and compatibility conditions, Hsu and Mo [H3] developed the equation for the shear strength and proved its applicability 
by calculating and comparing the shear strength of twenty-four walls available in the literature.

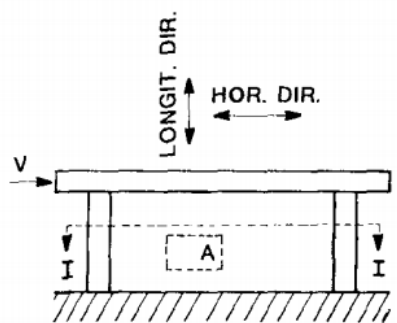

(a) GENERAL VIEW

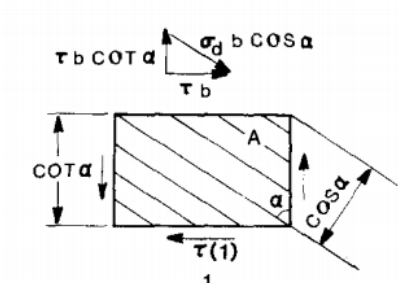

(b) WALL ELEMENT

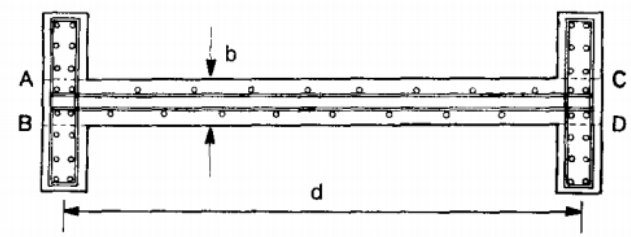

(c) SECTION I - I

Figure 5.6 Equilibrium of a shear wall in the softened truss model [H3]

The advantage of this model was that it included two types of stress-strain curves to describe the behaviors of softened and non-softened concrete under lateral loads respectively. Moreover, the truss model was developed for the shear-dominated structural walls, which were in accordance with the specimens in this study. Nonetheless, a closer examination revealed that the model was inappropriate. This was mainly because evenly distributed shear stresses were assumed in this model, yet this assumption was at odds with the observation of surges of strains in the middle of the horizontal rebar located $500 \mathrm{~mm}$ above the base and two corners of the horizontal rebar near the base.

Hwang et al. [H4] also noticed these deficiencies and developed a softened strutand-tie model to better describe the internal mechanism of squat shear walls. In this model, the contribution of concrete was incorporated into the diagonal mechanism, where a single diagonal compression strut with certain angle of inclination was assumed to resist the lateral force, as shown in Figure 5.7. The model was deemed more reasonable than the softened truss model. Because it not only comprised three mechanisms representing the contributions of concrete, horizontal reinforcement, 
and longitudinal reinforcement, but also matched the observation of strain profiles of horizontal rebar in test specimens. Therefore, the softened strut-and-tie model was adopted in the following analyses. However, it also needed to be noticed that the effect of large flanges was not incorporated in this model. Further studies should be carried out on the force-resisting mechanism of non-rectangular RC squat walls.

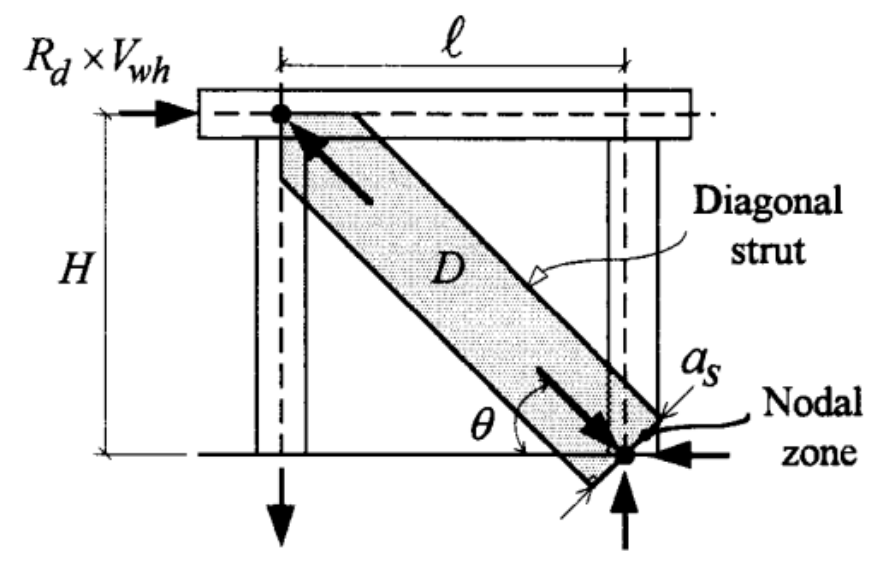

Figure 5.7 The diagonal mechanism of the softened strut-and-tie model [H4]

\subsection{Shear Lag Effect}

When a non-rectangular wall is exposed to lateral loads, the traditional theory assumes that the normal stress from the bending moment is uniformly distributed. However, in reality, a nonlinear distribution of longitudinal stress is usually found in the flange. This is because, under the law of Poisson's ratio, the flange becomes shorter and wider in compression, which further affects the shear deformation and causes a lag in activating the furthest fiber in the flange [S1]. To avoid direct consideration of the shape of nonlinear distributed stress, a reduction of the flange width is commonly used by engineers, in which a portion of the flange with uniformly distributed stress is considered effective in resisting loads as demonstrated in Figure 5.8. The importance of the effective flange width is fully appreciated in the research society. UBC 1994 required that the assumed effective flange width to be less than one-tenth of the wall height [U1]. Wallace [W3] later suggested that a low effective flange width would underestimate the flexural strength, which was not conservative and could cause inadequate shear reinforcement in the wall web. The ACI 318-14 [A1] adopted the advice from 
Wallace and defined the effective flange width as lesser of one-quarter of the wall height and half the distance to an adjacent wall web. To investigate influences of some important parameters on the effective flange width which were not addressed in ACI 318-14 [A1], Hassan and El-Tawil [H1] conducted a parametric study on a coupled flange wall system using the finite element software Diana. It was found in the finite element analysis that the effective flange width decreased with greater axial loads, while the increase of drift ratio contributed to the rapid growth of the effective flange width. Hassan and El-Tawil [H1] also concluded that wall length was more influential than wall height on the effective flange width. For walls in axial compression, the overhanging effective flange width was recommended as $0.6 d$ for the drift ratio of $0.5 \%, 0.95 d$ for the drift ratio of $1 \%$ and $1.15 d$ for the drift ratio of $2 \%$, where $d(\mathrm{~mm})$ was the length of the wall section.

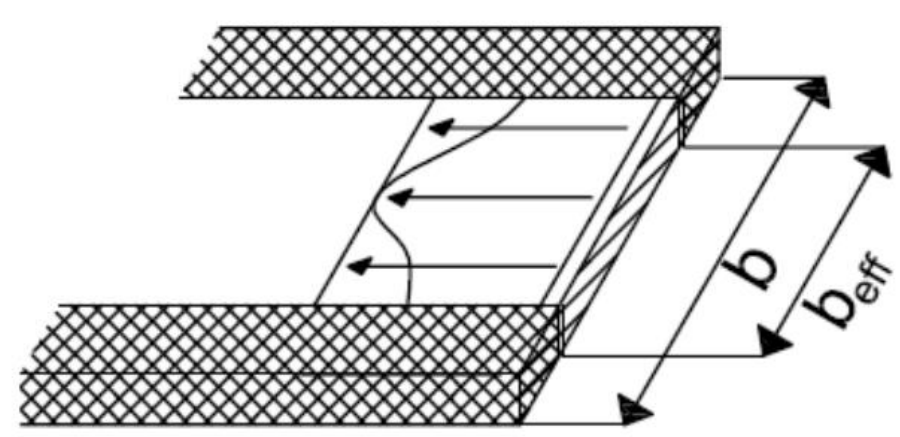

Figure 5.8 Effective flange width [S1]

As illustrated in Chapter 3, the overhanging flange width was $700 \mathrm{~mm}$ for the $\mathrm{H}$ shaped and T-shaped specimens and $1400 \mathrm{~mm}$ for the L-shaped specimens. According to ACI 318-14 [A1], the effective flange width is $250 \mathrm{~mm}$ for all specimens, which is apparently not proper for the L-shaped specimens since the strain of the longitudinal rebar in the middle of the flange revealed only a slight decrease compared with that of the rebar closest to the web. As recommended by Hassan and El-Tawil [H1], the effective flange width ranges from $780 \mathrm{~mm}$ to 1610 $\mathrm{mm}$ for different drift ratios, suggesting that the flange should be expected to be fully effective in tension for the T-shaped and $\mathrm{H}$-shaped specimens, and for the Lshaped specimens at the final loading stage. However, as observed in the strain profiles, a shear lag effect existed during all tests. Therefore, these two methods are 
considered inappropriate for the estimation of the effective flange width of nonrectangular RC squat walls.

To investigate the shear lag effect in the flange of test specimens, comparisons of base moments were made between measured data and results obtained from the nonlinear section analysis tool Xtract [X2] at various loading levels (Figure 5.9 to Figure 5.11), in which the material properties as stated in Chapter 3 were used. As shown in Table 5.3, the nonlinear section analyses overestimated the base moments of test specimens, due to the neglect of the shear lag effect, which served as proof that the shear lag effect should be carefully considered when calculating the lateral strength of non-rectangular RC squat walls. Furthermore, it was found that the shear lag effect was also affected by section shapes, loading directions, drift ratios and axial loads. Generally, the shear lag effect was more influential on the L-shaped specimens, due to the longer overhanging flanges. Besides, it was observed that the shear lag effect was more distinct on the T-shaped specimens with symmetrical loading direction. For instance, the average ratio of experimental to predicted moments was only 0.71 for the symmetrically-loaded Specimen TP0D0, while its counterpart Specimen TP0D45 had a number of 1.02. However, this phenomenon was not evident in the $\mathrm{H}$-shaped specimens. As for the drift ratio, the largest discrepancies were found in all specimens at initial loading stage, indicating that shear lag caused decreases of strains in the flange. As the drift ratio rose, the effect of shear lag on the lateral load capacity gradually diminished when more longitudinal reinforcing bars in the flange reached the yielding point, which is in accordance with the finding of Hassan and El-Tawil [H1]. This was because the large differences in strains could hardly lead to the equivalent disparity of stress in steel after the longitudinal rebar in the flange yielded, resulting in a slower growth of moment resisted by the section. It was clear that additional axial loads increased the impact of shear lag effects. Similar observations of the influence of axial loads and drift ratios were also recorded in the T-shaped slender walls of Zhang [Z2], indicating that the shear lag effect affected all kinds of T-shaped walls, regardless of the shear span ratio. 
In conclusion, the shear lag effect significantly affected the lateral load capacity of non-rectangular RC squat walls, and should be discreetly addressed in the design. Current methods available in the building codes [A1] or the literature [H1] were incapable of accurately evaluating the effective flange length. Furthermore, as demonstrated in the nonlinear section analyses, the effective flange length was evidently influenced by section shapes, axial loads, drift ratios and loading directions. Therefore, further research is needed to improve the accuracy in evaluating the impact of the shear lag effect.
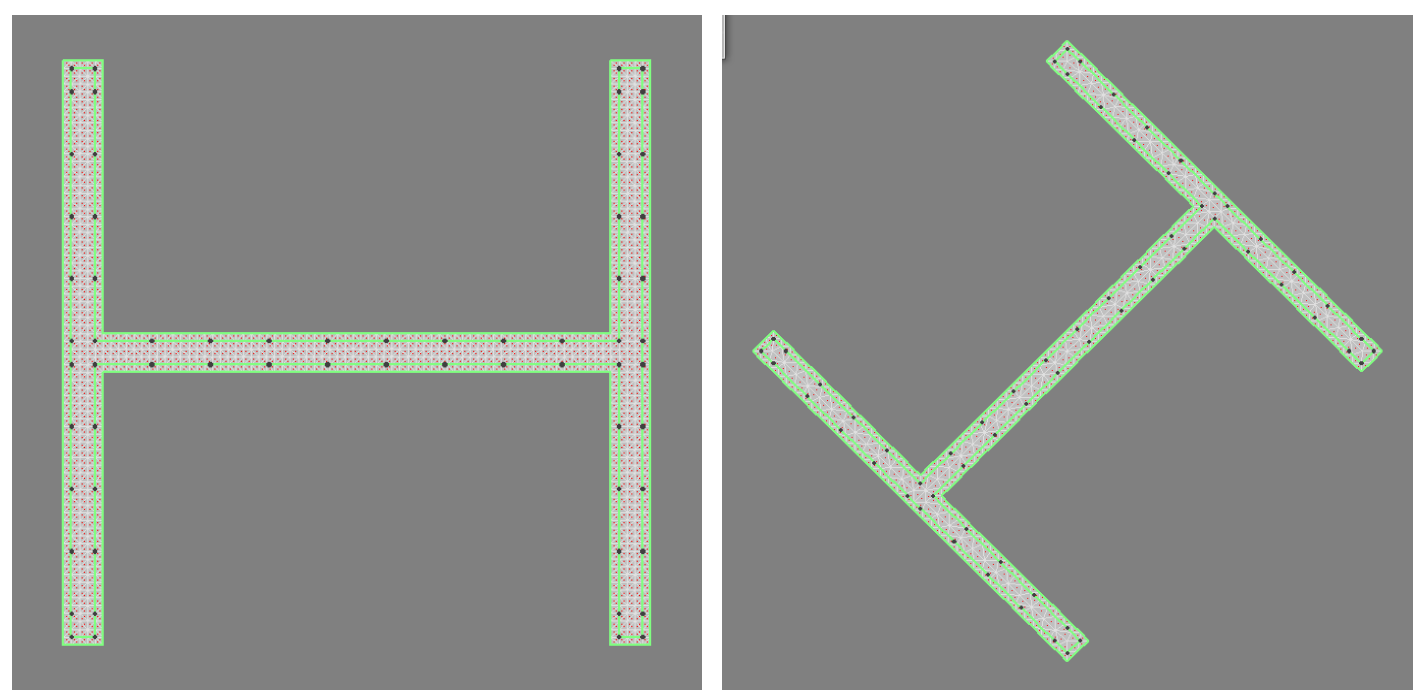

Figure 5.9 Models of the H-shaped specimens in Xtract [X2]
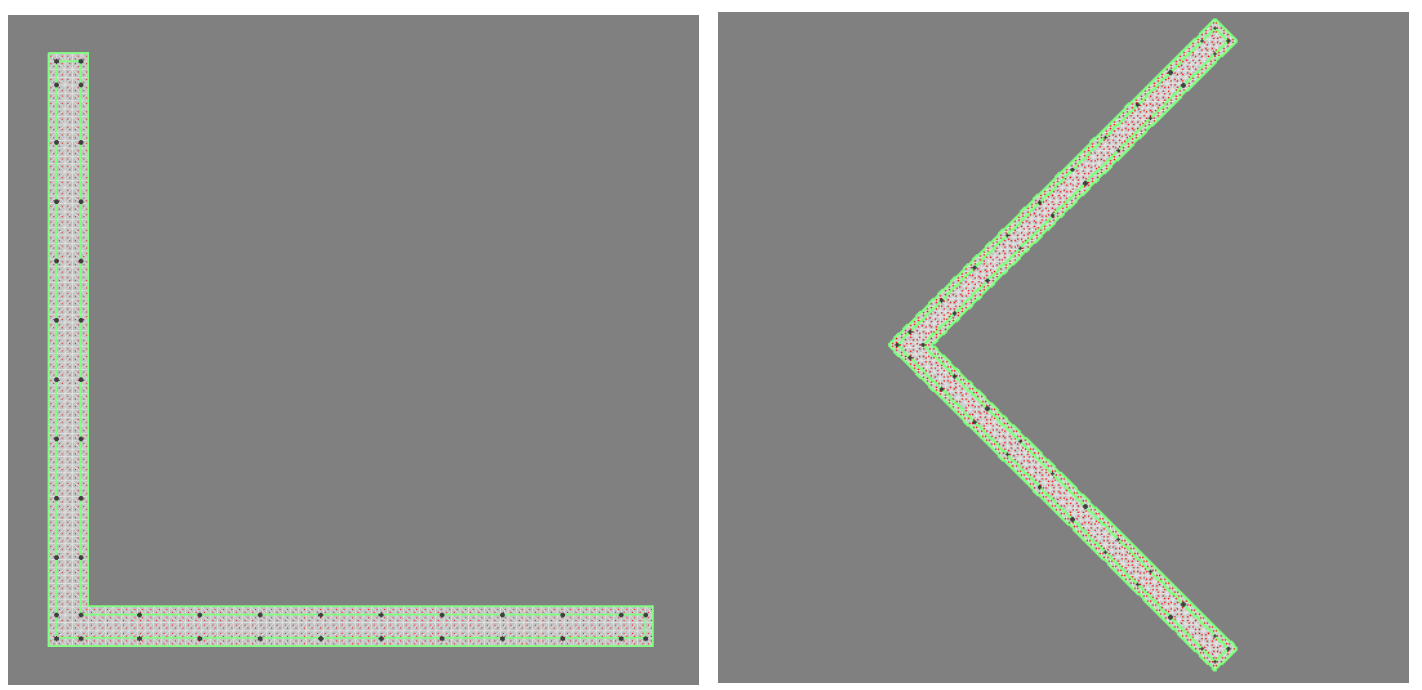

Figure 5.10 Models of the L-shaped specimens in Xtract [X2] 

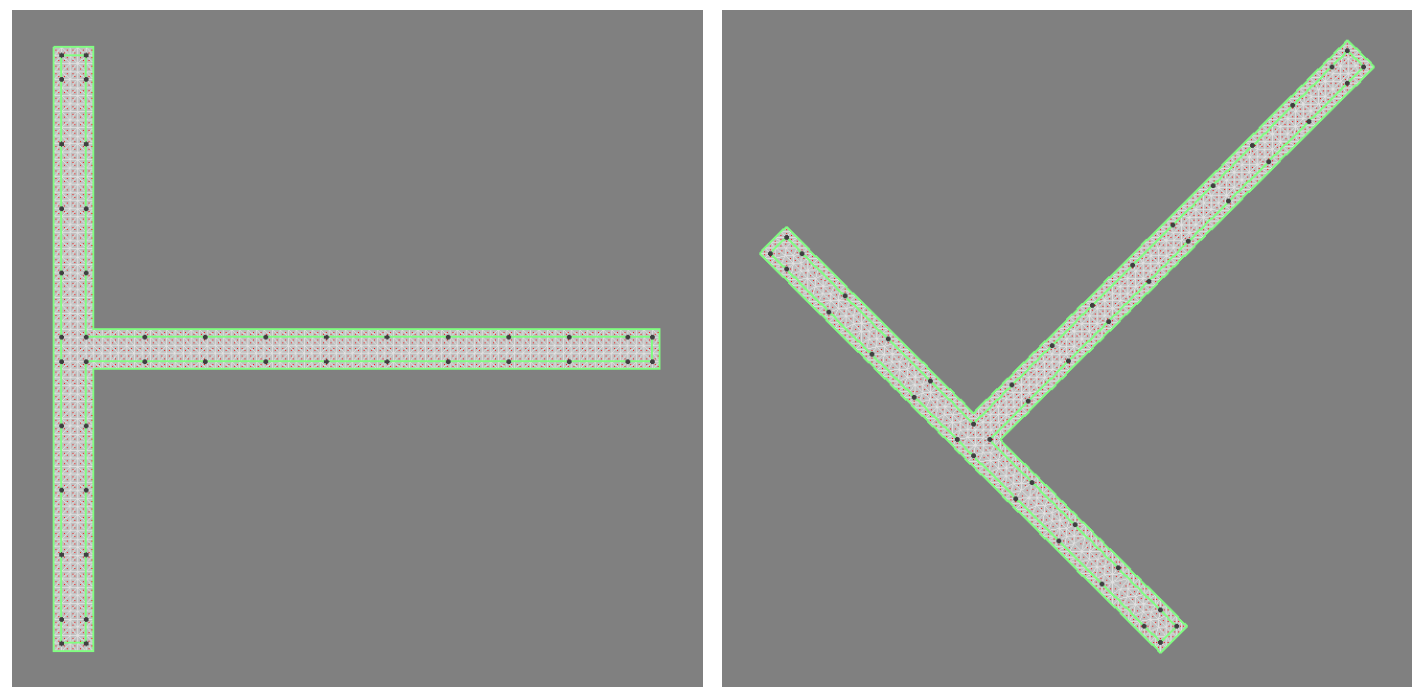

Figure 5.11 Models of the T-shaped specimens in Xtract [X2]

Table 5.3 Ratios of experimental to predicted base moments at various loading levels

\begin{tabular}{ccccccc}
\hline $\begin{array}{c}\text { Maximum } \\
\text { flange } \\
\text { reinforcement } \\
\text { strain }(\mu \varepsilon)\end{array}$ & 500 & 1000 & 1500 & 2000 & 2500 & 3000 \\
\hline HP0D0 & 0.93 & 0.94 & 1.01 & 0.98 & 1.02 & \\
\hline HP0D45 & 0.75 & 0.81 & 0.92 & 0.96 & 0.92 & \\
\hline HP5D0 & 0.68 & 0.52 & 0.71 & 0.73 & & \\
\hline HP5D45 & 0.64 & 0.54 & 0.62 & 0.64 & 0.72 & \\
\hline LP0D0 & 0.30 & 0.34 & 0.37 & 0.31 & 0.34 & 0.39 \\
\hline LP0D45 & 0.89 & 0.91 & 0.98 & 0.97 & 1.09 & 0.86 \\
\hline LP5D0 & 0.14 & 0.15 & 0.17 & 0.18 & 0.20 & 0.30 \\
\hline LP5D45 & 0.77 & 0.83 & 0.89 & 0.93 & & \\
\hline TP0D0 & 0.66 & 0.71 & 0.73 & 0.66 & 0.75 & 0.74 \\
\hline TP0D45 & 0.93 & 0.91 & 1.00 & 1.09 & 1.07 & 1.11 \\
\hline TP5D0 & 0.42 & 0.52 & 0.56 & 0.57 & 0.63 & \\
\hline TP5D45 & 0.71 & 0.77 & 0.81 & 0.84 & 0.98 & \\
\hline
\end{tabular}




\subsection{Effects of Design Parameters}

As stated in Chapter 3, two design parameters were investigated in the test, which are axial loads and loading directions. Half of the specimens were imposed with only the self-weight of the top slab of $73.5 \mathrm{kN}$, which contributed to axial load ratios of around $0.7 \%$ for the L-shaped and T-shaped specimens, and $0.5 \%$ for the H-shaped specimens. The other half were applied with constant axial loads of approximately $0.05 f_{c}^{\prime} A_{g}$, which were $700 \mathrm{kN}$ for the $\mathrm{H}$-shaped specimens and 500 $\mathrm{kN}$ for the L-shaped and T-shaped specimens. With regard to the loading direction, six specimens were subjected with lateral displacements parallel to the web, as the orthogonally-loaded group. The other six were under lateral displacements $45^{\circ}$ from the web, as the skew-loaded group. The effects of each parameter on the seismic behavior of non-rectangular RC squat walls are summarized and discussed in this section.

\subsubsection{Effects of axial loads}

The existence of axial loads has been shown to have a significant effect on the seismic behavior of structural walls by many researchers [B1, B5, Z2]. In this study, it was observed that the additional axial loads could postpone the formation of initial cracks, thereby increasing the lateral strength at first crack. Moreover, axial loads increased the degree of inclination of the diagonal cracks on the web. As for cracking patterns, it was evident that extra axial loads resulted in the crushing of concrete in the upper part of specimens, which was attributed to the double effect of axial force. On the one hand, it limited the crack widths, which would increase the peak shear strength. On the other hand, a large axial force also dramatically increased the compressive stresses in the wall segments, which led to a more severe failure of concrete struts. As for the hysteretic responses, it was found that the imposed axial loads played a favorable role in increasing the peak shear strength of specimens, which was in accordance with the past findings [B1, G2]. The influence of axial loads on the deformation capacity was not apparent, which is in contrast with observations in the past experiments [Z2]. Moreover, axial loads were seen as an active factor in increasing the secant stiffness. Its contribution was attributed to the fact that axial loads constrained the diagonal cracks and limited the development 
of the sliding shear, thereby enhancing the reaction at a specific drift ratio. As for the energy dissipation capacity, it was evident that the group with more axial loads displayed better energy dissipation capacity than its counterpart. As discussed

previously, axial loads can effectively limit the width of cracks, thereby controlling the development of the sliding shear. However, the effect of axial loads on control shear deformation remained unclear since evidence was found on both sides. As for strain profiles, the influence of axial loads was untraceable, and so required further investigation.

\subsubsection{Effects of loading directions}

In an earthquake event, seismic waves can reach the structure from any direction, which caused different responses of walls as witnessed by many researchers [B3, B5, Z2]. In this study, it was found that in the orthogonally-loaded specimens, the web saw denser cracks than the flanges. Besides, the majority of cracks appearing on the flange of walls with orthogonal lateral loading were horizontal flexural cracks, while the diagonal shear cracks had their predominant position in specimens with skew lateral displacement. It was also witnessed in specimens with orthogonal lateral loading that more cracks appeared when the flange was in tension. With regard to hysteretic responses, the influence of loading directions on the peak shear strength remained unclear since evidence was found on both sides. As for the deformation capacity, specimens subjected to the orthogonal lateral loading outperformed the skew-loaded group, which was most obvious in Specimens LP5D0 and LP5D45. Furthermore, specimens with skew horizontal loading seemed to have a more rapid loss of secant stiffness as the drift ratio rose. As for the energy dissipation capacity, specimens with skew horizontal loading demonstrated better capability. This phenomenon was most obvious in specimens with an L-shaped section. This was because when the L-shaped walls were arranged at $45^{\circ}$ to the horizontal loading, more evenly distributed compression zones could be introduced in both loading directions, thereby increasing the flexural capacity. However, the opposite was true in the $\mathrm{H}$-shaped specimens, which was closely related to the section shape; in other words, the $\mathrm{H}$-shaped specimens were able to introduce a larger area as compression zones in both loading directions when arranged as 
orthogonal to the lateral loading. Unlike axial loads, loading directions did not play a favorable role in the control of sliding shear. Nonetheless, when it comes to the shear deformation, it was found in the L-shaped group that specimens with skew lateral loading had a slightly lower portion than specimens with the orthogonal lateral loading. This is because the principal shear was carried by two segments in the former, while only one segment mainly functioned in the latter. As to the strain profiles, it was witnessed that the shear lag effect was more influential in specimens with orthogonal lateral loading.

\subsection{Summary}

The experimental results have been presented in Chapter 4 in detail. In this chapter, further comparisons and discussions on various aspects were carried out to improve the understanding of the seismic behavior of non-rectangular RC squat walls. Several conclusions are drawn as follows:

- Generally, initial shear cracks occurred at the middle and upper part of wall segments at a drift ratio ranging from $0.1 \%$ to $0.4 \%$, which was prior to the yielding of longitudinal reinforcing bars. The specimens experienced their first yielding at a drift ratio ranging from $0.4 \%$ to $0.7 \%$. As the lateral displacement increased, diagonal cracks inclined from $30^{\circ}$ to $65^{\circ}$ quickly spread to the other area of the wall segments. Horizontal flexural cracks were found on the flange of the H-shaped and T-shaped specimens with orthogonal lateral loading, which extended to the full length of the flange. Diagonal shear cracks were observed to have a predominant position in specimens with skew lateral loading.

- Most specimens failed in the similar mode as the diagonal compression failure with moderate or severe concrete spalling. Specimens LP0D45, LP5D45, HP0D45, HP5D0, HP5D45, TP0D45, and TP5D45 were able to sustain a ductile post-peak response before failure, whereas a sudden drop of the lateral strength happened in the other specimens.

- A major horizontal crack and severe concrete spalling were found at the upper part of the web in Specimens HP0D0, HP5D0 and TP5D0, due to the weaker 
concrete adopted and the stress concentration caused by the stiff top slab. Several vertical slip planes as found in Specimen DP1 of Palermo and Vecchio's test [P2] also occurred in Specimens HP5D0 and TP5D0, which were attributed to the combined effects of axial loads and stiff flanges.

- The web of Specimens HP5D0 and DP1 [P2] was dominated by shear, while their flanges were controlled by flexure. Thus a deformation incompatibility existed between the web and flange when the specimens were imposed with cyclic lateral displacements. Further analytical investigations revealed that the deformation incompatibility reached its maximum at a height of $0.42 h_{w}$, leading to the vertical slip planes forming at the same altitude along the weakened area.

- When the flange was in tension, T-shaped specimens with orthogonal lateral loading processed larger shear strength at a specific drift ratio than the L-shaped specimens, due to the shear lag effect and the loading path. The opposite was true in the skew-loaded groups, because the symmetric section of the L-shaped walls brought more evenly distributed areas in both tension and compression regions.

- The methods proposed by Pauley and Priestley [P4] and Li and Xiang [L3] overestimated the effective stiffness of test specimens, as a result of neglecting the non-rectangular section shape and the shear lag effect. Besides, these two methods yielded identical results for both loading directions, which was considered improper for the L-shaped and T-shaped specimens since their sections were not symmetrical about the bending axis. As corroborated by Zhang [Z2] and Xiang [X1], the increase of axial loads brought growth on the effective stiffness. Also, specimens with skew lateral loading had greater effective stiffness than specimens with orthogonal lateral loading.

- Yielding of reinforcement happened after the formation of the first crack in all specimens. The reduction of the deformation capacity brought by axial loads found in previous research was unclear in this experimental program, due to the premature failure in some specimens. 
- Shear deformation was found to dominate the response of test specimens since it accounted for more than 50 percent of the total deformation in most specimens. During the test, the shear deformation increased in all T-shaped and $\mathrm{H}$-shaped specimens, with a larger growth found in the latter. In the L-shaped specimens, a slightly lower portion of the shear deformation was witnessed in the group with skew lateral loading direction, as a result of more evenly distributed shear force in two segments.

- Continuous and slight increases of sliding shear displacements were observed in all specimens, in which a more rapid growth was found in specimens with lower axial loads. Besides, there was no significant variation between the portions of sliding shear displacements in groups with orthogonal lateral loading and groups with skew lateral loading.

- Rapid growths of strains were observed in the middle of No.7 horizontal rebar and in the right and left parts of No.1 horizontal rebar, which indicated that a main diagonal compression strut passing through these places. Hence, the strutand-tie model was considered appropriate for the non-rectangular RC squat walls.

- Significant shear lag effect was detected in some specimens, especially for the L-shaped specimens. Furthermore, the shear lag effect was more distinct on the T-shaped specimens under symmetrical lateral loading. It was found that axial loads increased the impact of shear lag effect, while less influence of the shear lag effect was witnessed as lateral displacements rose.

- Methods available in the building codes [A1] and the literature [H1] were found inappropriate for the estimation of the effective flange width of non-rectangular $\mathrm{RC}$ squat walls. Further research is required to improve the evaluation of the effective flange length. 


\section{CHAPTER 6 \\ PEAK SHEAR STRENGTH OF NON-RECTANGULAR RC SQUAT WALLS}

\subsection{Introduction}

Peak shear strength is a critical parameter in the evaluation of the seismic performance of structural walls. As reviewed in Chapter 2, different analytical models [B1, G3, H4, W7] have been proposed to predict the peak shear strength of structural walls. Despite the improved performance, current design procedures [A1, A3] are developed for rectangular slender walls, which have been found incompetent to address the force-resisting mechanism in squat walls [G2, G4]. Furthermore, the effect of flanges of non-rectangular RC squat walls on the peak shear strength has scarcely been considered in these analytical models. Therefore, it is essential to conduct research in this area to improve the prediction of the peak shear strength of non-rectangular RC squat walls.

In this chapter, the performance of current methods [A1, A3, B4, G3, H4, K1, W7] on the prediction of the peak shear strength of test specimens is evaluated first. After that, a modified strut-and-tie model is developed to derive the function form of the peak shear strength equation for non-rectangular RC squat walls. Then, nonlinear regression is used to obtain the values of unknown coefficients in the function form. Finally, the probabilistic approach is adopted to reconcile large scatters and to avoid the biased estimate found in the deterministic prediction of the peak shear strength of non-rectangular RC squat walls.

\subsection{Evaluation of Current Methods for the Peak Shear Strength of Test Specimens}

The peak shear strength of test specimens was presented previously. Equations from Section 11.5 and 18.1 of ACI 318-14 [A1], ASCE 43-05 [A3], Barda et al. [B1], Wood [W7], Hwang et al. [H4], Gulec and Whittaker [G3], Kassem [K1], as reviewed in Chapter 2, were used to predict the peak shear strength of test 
specimens. The results are presented in Table 6.1 below. Moreover, statistics for ratios of the predicted to experimental peak shear strength are summarized in Table 6.2 to provide insight into the accuracy of these equations. Values in the last row (over-prediction) in Table 6.2 report the percentage of overestimation for the twelve specimens.

It was evident from the table that significant scatters existed in the prediction of the peak shear strength of test specimens. Moreover, it seemed that none of these equations was particularly suitable to assess the performance of non-rectangular RC squat walls tested in this study.

Equations from Section 11.5 of ACI 318-14 [A1] underestimated the peak shear strength of test specimens. This was because these equations were developed for rectangular walls, which failed to reflect the effect of large flanges in nonrectangular walls. Besides, only horizontal reinforcement in the web was assumed to withstand shear force in Section 11.5 of ACI 318-14 [A1], which contradicted with the findings of Lefas et al. [L2]. Also, Wood [W7] has shown that, compared with horizontal reinforcement, the shear strength of squat walls was more dependent on the vertical web reinforcement. The equation from Section 18.10 of ACI 318-14 [A1], which was based on the modified truss analogy proposed by Wood [W7], performed slightly better than its counterpart in Section 11.5 [A1]. However, this equation still underestimated the peak shear strength, mainly due to the inclusion of insufficient parameters, such as axial loads and the horizontal reinforcement. Unlike equations in ACI 318-14 [A1], design procedures in ASCE 43-05 [A3] overestimated the specimens' peak shear strength. The same phenomenon was also witnessed in the equation provided by Barda et al. [B1], which had a similar function form as those in ASCE 43-05 [A3]. Neither of these two equations considered the contribution of flanges. The equations proposed by Hwang et al. [H4] were analytically reasonable, but the effect of flanges was not considered in the diagonal mechanism, the vertical mechanism or the horizontal mechanism. Gulec \& Whittaker [G3] and Kassem [K1] used nonlinear regression to determine the values of coefficient for influential parameters. The former used a simple free body diagram to obtain the function form of the equation and incorporated the 
contribution of the flange. However, this equation seemed to overestimate the peak shear strength by assuming all longitudinal reinforcement in the flange was effective in resisting lateral loads. The latter built the function form of the peak shear strength equation on the strut-and-tie model proposed by Hwang et al. [H4], and used a comprehensive database of squat walls with flanged cross section to obtain the final equation for the peak shear strength. Nonetheless, the contribution of both longitudinal reinforcement and concrete in the flange which has been proven to be influential on the lateral strength [B5] was not included in this equation.

From the discussion above, it was clear that some equations failed to include certain important parameters, such as axial loads, vertical web reinforcement and horizontal web reinforcement, which has been shown to have evident effects on the shear strength of squat walls [B1, S3]. Therefore, to obtain an accurate prediction of the peak shear strength of non-rectangular RC squat walls, it was essential to include these influential parameters in the proposed equation. Besides, except the equation proposed by Gulec and Whittaker [G3], all other equations ignored the effect of large flanges of non-rectangular walls in the prediction of the peak shear strength. Nonetheless, it has been observed in many experiments [P1, S10] that flanged walls usually possessed higher strengths than similar rectangular counterparts. Furthermore, even methods specifically developed for the squat walls with flanged cross section were unsuitable for walls with an unsymmetrical section shape, not to mention those walls imposed with lateral loading not parallel to the web. Thus, it was highly necessary to develop equations to better predict the peak shear strength of non-rectangular RC squat walls. 
Table 6.1 Predicted peak shear strength of test specimens $(\mathrm{kN})$

\begin{tabular}{|c|c|c|c|c|c|c|c|c|}
\hline Specimen & $\begin{array}{c}\text { ACI 318- } \\
14 \S 11.5 \\
{[\mathrm{~A} 1]}\end{array}$ & $\begin{array}{c}\text { ACI 318- } \\
14 \S 18.10 \\
{[\mathrm{~A} 1]}\end{array}$ & $\begin{array}{c}\text { ASCE } \\
43-05 \\
{[\mathrm{~A} 3]}\end{array}$ & $\begin{array}{c}\text { Barda } \\
\text { et al. } \\
{[\mathrm{B} 1]}\end{array}$ & $\begin{array}{c}\text { Wood } \\
{[\mathrm{W} 7]}\end{array}$ & $\begin{array}{c}\text { Hwang } \\
\text { et al. } \\
{[\mathrm{H} 4]}\end{array}$ & $\begin{array}{c}\text { Gulec and } \\
\text { Whittaker } \\
{[\mathrm{G} 3]}\end{array}$ & $\begin{array}{c}\text { Kassem } \\
{[\mathrm{K} 1]}\end{array}$ \\
\hline HP0D0 & 497 & 581 & 856 & 1036 & 696 & 923 & 966 & 1123 \\
\hline HP0D45 & 491 & 574 & 846 & 1024 & 696 & 872 & 943 & 1090 \\
\hline HP5D0 & 597 & 581 & 896 & 1180 & 696 & 1325 & 1274 & 1123 \\
\hline HP5D45 & 580 & 574 & 869 & 1168 & 696 & 1273 & 1250 & 1090 \\
\hline LP0D0 & 495 & 578 & 852 & 1133 & 478 & 901 & 973 & 1109 \\
\hline LP0D45 & 495 & 577 & 851 & 1133 & 478 & 899 & 972 & 1108 \\
\hline LP5D0 & 590 & 578 & 885 & 1233 & 478 & 1180 & 1186 & 1109 \\
\hline LP5D45 & 589 & 577 & 883 & 1233 & 478 & 1177 & 1186 & 1108 \\
\hline TP0D0 & 495 & 578 & 852 & 1133 & 478 & 901 & 973 & 1109 \\
\hline TP0D45 & 498 & 582 & 858 & 1139 & 478 & 931 & 984 & 1128 \\
\hline TP5D0 & 590 & 578 & 885 & 1233 & 478 & 1180 & 1186 & 1109 \\
\hline TP5D45 & 598 & 582 & 900 & 1239 & 478 & 1209 & 1198 & 1128 \\
\hline
\end{tabular}


Table 6.2 Statistics for ratios of the predicted to measured shear strength using different sets of equations

\begin{tabular}{|c|c|c|c|c|c|c|c|c|}
\hline Specimen & $\begin{array}{c}\text { ACI 318- } \\
14 \S 11.5 \\
{[\mathrm{~A} 1]}\end{array}$ & $\begin{array}{c}\text { ACI 318- } \\
14 \S 18.10 \\
{[A 1]}\end{array}$ & $\begin{array}{c}\text { ASCE } \\
43-05 \\
\text { [A3] }\end{array}$ & $\begin{array}{l}\text { Barda } \\
\text { et al. } \\
\text { [B1] }\end{array}$ & $\begin{array}{l}\text { Wood } \\
\text { [W7] }\end{array}$ & $\begin{array}{l}\text { Hwang } \\
\text { et al. } \\
{[\mathrm{H} 4]}\end{array}$ & $\begin{array}{c}\text { Gulec and } \\
\text { Whittaker } \\
\text { [G3] }\end{array}$ & $\begin{array}{c}\text { Kassem } \\
{[\mathrm{K} 1]}\end{array}$ \\
\hline HP0D0 & 0.58 & 0.68 & 1.00 & 1.21 & 0.81 & 1.08 & 1.13 & 1.31 \\
\hline HP0D45 & 0.67 & 0.78 & 1.15 & 1.39 & 0.94 & 1.18 & 1.28 & 1.48 \\
\hline HP5D0 & 0.60 & 0.58 & 0.90 & 1.19 & 0.70 & 1.33 & 1.28 & 1.13 \\
\hline HP5D45 & 0.51 & 0.51 & 0.77 & 1.03 & 0.61 & 1.12 & 1.10 & 0.96 \\
\hline LP0D0 & 0.84 & 0.98 & 1.44 & 1.91 & 0.81 & 1.52 & 1.64 & 1.87 \\
\hline LP0D45 & 0.75 & 0.88 & 1.30 & 1.73 & 0.73 & 1.37 & 1.48 & 1.69 \\
\hline LP5D0 & 0.80 & 0.78 & 1.20 & 1.67 & 0.65 & 1.60 & 1.61 & 1.50 \\
\hline LP5D45 & 0.82 & 0.81 & 1.24 & 1.73 & 0.67 & 1.65 & 1.66 & 1.55 \\
\hline TP0D0 & 0.58 & 0.67 & 0.99 & 1.32 & 0.56 & 1.05 & 1.13 & 1.29 \\
\hline TP0D45 & 0.57 & 0.67 & 0.98 & 1.30 & 0.55 & 1.07 & 1.13 & 1.29 \\
\hline TP5D0 & 0.77 & 0.76 & 1.16 & 1.61 & 0.63 & 1.54 & 1.55 & 1.45 \\
\hline TP5D45 & 0.58 & 0.57 & 0.88 & 1.21 & 0.47 & 1.18 & 1.17 & 1.10 \\
\hline Mean & 0.673 & 0.721 & 1.083 & 1.441 & 0.677 & 1.308 & 1.347 & 1.386 \\
\hline Median & 0.634 & 0.716 & 1.073 & 1.354 & 0.659 & 1.258 & 1.280 & 1.380 \\
\hline $\begin{array}{l}\text { Standard } \\
\text { deviation }\end{array}$ & 0.116 & 0.135 & 0.195 & 0.277 & 0.132 & 0.224 & 0.225 & 0.258 \\
\hline $\begin{array}{l}\text { Coefficient } \\
\text { of variation }\end{array}$ & 0.173 & 0.188 & 0.180 & 0.192 & 0.196 & 0.171 & 0.167 & 0.186 \\
\hline $\begin{array}{l}\% \text { Over- } \\
\text { prediction }\end{array}$ & 0.0 & 0.0 & 50.0 & 100.0 & 0.0 & 100.0 & 100.0 & 91.7 \\
\hline
\end{tabular}




\subsection{Proposed Peak Shear Strength Equation for Non-rectangular RC Squat Walls}

\subsubsection{Development of the function form}

As discussed previously, Hsu and Mo [H3] proposed a softened truss model to predict the shear strength of squat walls in 1990, which was later modified by Gupta and Rangan [G6]. In this model, the state of stress in the central panel was assumed to be uniform and the flow of compressive stresses was described by a series of parallel compressive struts. The competence of this model was demonstrated by calculating the shear strength of twenty-four walls available in the literature. However, the assumption of evenly distributed stresses was found inappropriate by Hwang et al. [H4], since the stress flow of the squat wall was always highly disturbed by the existence of concentrated loads on the top and at the bottom. Hwang et al. [H4] further developed a strut-and-tie model in which the flow of compressive stresses was represented by a diagonal compressive strut.

In the experiments of twelve RC squat walls, Luna [L5] recorded the surface strain fields of specimens and found the strain was highly concentrated along the major diagonal cracks, which showed that the truss model was unrealistic and failed to predict the correct failure mode of RC squat walls. An identical phenomenon was also found in the majority of test specimens presented in Chapter 4. Therefore, in this study, the strut-and-tie model was employed to develop the function form of the peak shear strength equation for non-rectangular RC squat walls.

Three force-resisting mechanisms could be separated from the strut-and-tie model: a diagonal mechanism, accounting for the contribution of the concrete strut; a horizontal mechanism, accounting for the contribution of horizontal web reinforcement; and a vertical mechanism, accounting for the contribution of vertical web reinforcement. However, since this model was originally developed for rectangular squat walls, the effect of large flanges as well as the internal reinforcement was not considered in this model, meaning it could not be directly used for non-rectangular RC squat walls. Therefore, the model was redesigned as seen in Figure 6.1. 

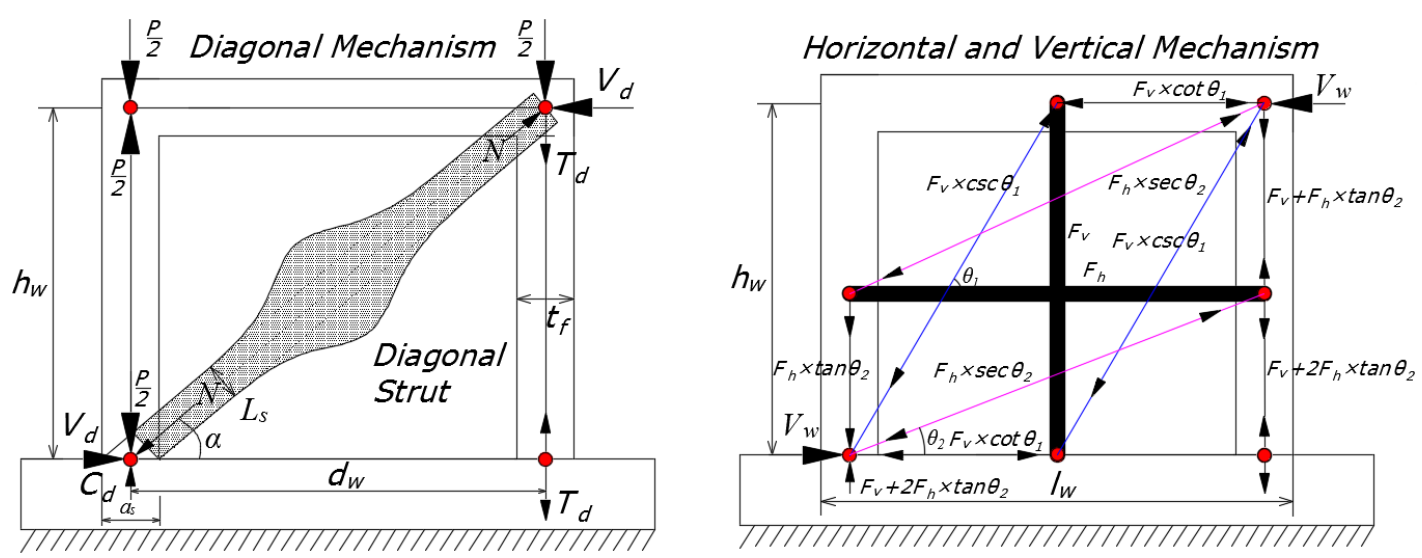

Figure 6.1 Diagonal mechanism, horizontal and vertical mechanism of the proposed strut-and-tie model

It was evident that the total shear strength was calculated as the summation of the lateral strength carried by the diagonal mechanism and the combination of the horizontal and vertical mechanism, which is expressed by:

$$
V_{c a l}=V_{d}+V_{w}
$$

where $V_{d}(\mathrm{~N})$ is the shear strength provided by the diagonal compression strut and flange, $V_{w}(\mathrm{~N})$ is the shear strength provided the web reinforcement.

The shear strength provided by the diagonal mechanism could be computed by the internal and external force equilibrium on the assumption that the flexural strength was greater than the shear strength in non-rectangular RC squat walls. As demonstrated in Figure 6.1, the diagonal mechanism comprised a single diagonal compression strut transferring force from the loading point to the opposite corner point, which normally had a bottle-shaped form in the panel zone. According to the horizontal equilibrium at the point of loading or the opposite corner point of the model, the shear strength carried by the diagonal mechanism can be estimated as:

$$
V_{d}=N \cos \alpha
$$

where $N(\mathrm{~N})$ is the compression force of the diagonal strut and $\alpha$ is the inclination angle of the diagonal strut, which is calculated as:

$$
\alpha=\tan ^{-1} \frac{h_{w}}{d_{w}}
$$


$h_{w}(\mathrm{~mm})$ is the vertical distance from the horizontal loading to the wall base, $d_{w}$ (mm) is the horizontal length between the tensile force $T_{d}$ and compressive force $C_{d}$. Then according to the vertical force equilibrium at the lower left point, the following equation can be obtained:

$$
C_{d}=\frac{P}{2}+N \sin \alpha
$$

where $C_{d}(\mathrm{~N})$ is the resultant compressive force of the flange and web concrete. Similarly, according to the vertical force equilibrium at lower right point, the following expression can be written:

$$
T_{d}=N \sin \alpha-\frac{P}{2}
$$

where $T_{d}(\mathrm{~N})$ is the resultant tensile force from the longitudinal reinforcement in the flange. Zhang [Z2] found that not all longitudinal reinforcement attained yielding at the peak shear strength. This phenomenon was also observed in the strain profiles of longitudinal reinforcement in the flange of the specimens presented in this study. Hence a reduction coefficient is adopted in the calculation of $T_{d}$ as presented below:

$$
T_{d}=k_{1} f_{y f} A_{f}
$$

where $k_{1}$ is a modification coefficient ranging from 0 to $1, f_{y f}(\mathrm{MPa})$ is the yield stress of longitudinal reinforcement in the flange, $A_{f}\left(\mathrm{~mm}^{2}\right)$ is the total area of the longitudinal reinforcement in the flange. By combing Equation 6.4 to Equation 6.6, the compressive force $C_{d}$ could be expressed as:

$$
C_{d}=k_{1} f_{y f} A_{f}+P
$$

$A_{f}$ could be written as $\rho_{f} t_{f} l_{f}$, where $\rho_{f}$ is the longitudinal reinforcement ratio in the flange, $t_{f}(\mathrm{~mm})$ is the thickness of the flange, $l_{f}(\mathrm{~mm})$ is the length of the flange. Therefore, $N$ could be substituted in Equation 6.2 and $V_{d}$ could be expressed as:

$$
V_{d}=\left(k_{1} f_{y f} \rho_{f} t_{f} l_{f}+\frac{P}{2}\right) \cot \alpha
$$

The presence of the horizontal and longitudinal reinforcement provided an additional loading path to transfer the lateral force in non-rectangular RC squat 
walls in addition to the diagonal mechanism. As demonstrated in Figure 6.1, the horizontal mechanism was comprised of one horizontal tie and two flat struts. The vertical mechanism was comprised of one vertical tie and two relatively steep struts. It should be noted that the vertical ties only represented the longitudinal reinforcement in the web and excluded the longitudinal reinforcement in the flange. According to the force equilibrium, $V_{w}$ could be expressed as:

$$
V_{w}=2 F_{v} \cot \theta_{1}+F_{h}
$$

where $\theta_{1}$ is the angle of inclination of the steep strut in the vertical mechanism, $F_{v}$ $(\mathrm{N})$ is the tensile force in the vertical tie and $F_{h}(\mathrm{~N})$ is the tensile force in the horizontal tie, which could be calculated as:

$$
\begin{aligned}
& F_{v}=k_{2} f_{y v} A_{v} \\
& F_{h}=k_{3} f_{y h} A_{h}
\end{aligned}
$$

where $k_{2}$ and $k_{3}$ are the modification coefficients for the vertical and horizontal web reinforcement, which range from 0 to $1, f_{y v}(\mathrm{MPa})$ and $f_{y h}(\mathrm{MPa})$ are the yield stress of the vertical and horizontal web reinforcement, $A_{v}\left(\mathrm{~mm}^{2}\right)$ and $A_{h}\left(\mathrm{~mm}^{2}\right)$ are the total area the vertical and horizontal web reinforcement, which could be represented by $\rho_{v} t_{w} z_{w}$ and $\rho_{h} t_{w} h_{w}$ respectively, where $\rho_{v}$ and $\rho_{h}$ are the vertical and horizontal reinforcement ratio in the web, $t_{w}(\mathrm{~mm})$ is the thickness of the web, $h_{w}(\mathrm{~mm})$ is the height of the wall, $z_{w}(\mathrm{~mm})$ is the distance between two flanges. By combing Equation 6.9 to Equation 6.11, $V_{w}$ could be expressed as:

$$
V_{w}=2 k_{2} f_{y v} \rho_{v} t_{w} z_{w} \cot \theta_{1}+k_{3} f_{y h} \rho_{h} t_{w} h_{w}
$$

As depicted in the horizontal and vertical mechanism shown in Figure 6.1, the angle of inclination of the steep strut in the vertical mechanism actually had a geometrical relationship with the angle of inclination of the strut in the diagonal mechanism, which is given as:

$$
\cot \alpha=2 \cot \theta_{1}
$$


Substituting $V_{d}$ and $V_{w}$ in Equation 6.1 using Equation 6.8 and Equation 6.12, then by combing the results of Equation 6.3 and Equation 6.13, the function form of peak shear strength equation for non-rectangular RC squat walls is obtained as:

$$
V_{c a l}=\left(k_{1} f_{y f} \rho_{f} t_{f} l_{f}+k_{2} f_{y v} \rho_{v} t_{w} z_{w}+\frac{P}{2}\right) \frac{d_{w}}{h_{w}}+k_{3} f_{y h} \rho_{h} t_{w} h_{w}
$$

As seen from the above equation, variable $d_{w}$ representing the horizontal length between the tensile force $T_{d}$ and compressive force $C_{d}$ needed further interpretation. For simplicity, it was reasonable to assume that the compressive stress was uniformly distributed in the junction of the web and flange as shown in Figure 6.2.

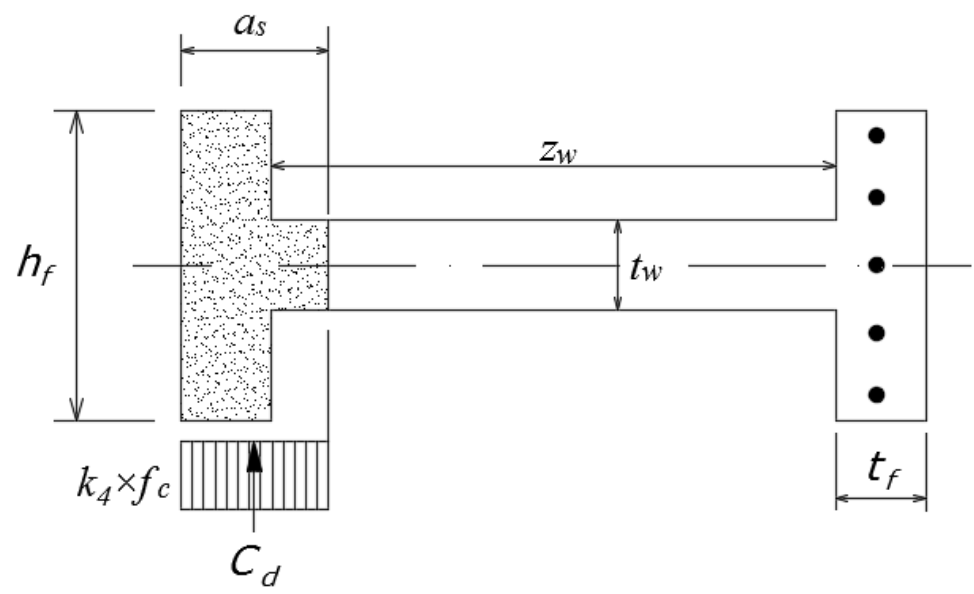

Figure 6.2 Force distribution in the proposed strut-and-tie model

Moreover, it was evident that the location of resultant tensile force from the longitudinal reinforcement in the flange lay in the middle of the juncture of the web and flange. Therefore, the following geometrical relationship can be attained:

$$
d_{w}=l_{w}-\frac{t_{f}}{2}-\frac{a_{s}}{2}
$$

where $a_{s}(\mathrm{~mm})$ is the horizontal length of the compression zone. The compressive force $C_{d}$ could be written as:

$$
C_{d}=k_{4} f_{c}^{\prime}\left[t_{f} l_{f}+\left(a_{s}-t_{f}\right) t_{w}\right]
$$


where $k_{4}$ is a modification coefficient for the compressive force in the flange and web, ranging from 0 to 1. Substituting $C_{d}$ in Equation 6.7 using Equation 6.16, $a_{s}$ could be determined as:

$$
a_{s}=\frac{1}{t_{w}}\left(\frac{k_{1} f_{y f} \rho_{f} t_{f} l_{f}+P}{k_{4} f_{c}^{\prime}}-t_{f} l_{f}\right)+t_{f}
$$

By combining Equation 6.15 and Equation 6.17, $d_{w}$ could be estimated as:

$$
d_{w}=l_{w}-t_{f}-\frac{1}{2}\left(\frac{k_{1} f_{y f} \rho_{f} t_{f} l_{f}+P}{k_{4} f_{c}^{\prime} t_{w}}-\frac{t_{f} l_{f}}{t_{w}}\right)
$$

\subsubsection{Nonlinear regression}

After attaining the proper function form of the peak shear strength equation, it was necessary to further determine the values of the four unknown coefficients. In this study, nonlinear regression was conducted using the fmincon nonlinear solver [M3] in MATLAB as recommended by Kassem [K1], Gulec and Whittaker [G3]. Fmincon was used to find the minimum of a constrained nonlinear multivariable function starting at initial values. A typical problem suited for Fmincon is described as:

$$
\min _{x} f(x) \text { such that }\left\{\begin{array}{c}
c(x) \leq 0 \\
c e q(x)=0 \\
A \cdot x \leq b \\
\text { Aeq } \cdot x=b e q \\
l b \leq x \leq u b
\end{array}\right.
$$

where $c(x)$ and $\operatorname{ceq}(x)$ are the functions that return vectors, $A$ and $b$ are the constraints of linear inequality, Aeq and beq are the constraints of linear equality, in which $A$ and $A e q$ are matrices, $b$ and $b e q$ are vectors, $l b$ and $u b$ are the lower boundary and upper boundary of variables, which can be passed as vectors or matrices. In this study, as stated in the last section, the lower boundary and the upper boundary were set as 0 and 1 respectively. A nonlinear constraint was set equal to 1 for the mean value of the calculated to experimental peak shear strength of non-rectangular RC squat walls. The unknown coefficients were obtained by 
minimizing the standard variation associated with ratios of the calculated to experimental peak shear strength as shown below:

$$
\begin{gathered}
r(i)=\frac{V_{\text {cal }}(i)}{V_{\text {peak }}(i)} \\
\text { nonlcon (constraint) } \rightarrow \operatorname{mean}[r(i)]=\frac{\sum_{1}^{n} r(i)}{n}=1 \\
\text { fun } \rightarrow \sqrt{\frac{1}{n} \sum_{1}^{n}[r(i)-\operatorname{mean}[r(i)]]^{2}}
\end{gathered}
$$

where $r(i)$ is the ratio of the calculated to experimental peak shear strength for number $i$ wall in the database, $n$ is the total number of walls in the database.

A database of non-rectangular RC squat walls was presented in Chapter 2. Among these walls, those imposed with quasi-static cyclic horizontal loading were selected together with Specimens HP0D0 and HP5D0 to calibrate the unknown coefficients. In total, sixty-nine walls were used in the nonlinear regression, which included the research work of Barda et al. [B1], Synge [S10], Saito et al. [S2], Sato et al. [S3], Mo and Chan [M4], Palermo and Vecchio [P2] and Farvashany et al. [F1].

\subsubsection{Equations for the peak shear strength of non-rectangular RC squat walls}

Besides the equations deduced in the last section, equations from Section 18.10 of ACI 318-14 [A1] and ASCE 43-05 [A3] were also selected for refinement and comparison using the same regression method. The formats of these two equations are presented below, in which $k_{1}$ to $k_{4}$ are unknown coefficients.

$$
\begin{gathered}
V_{A C I}=A_{c v}\left(k_{1} \sqrt{f_{c}^{\prime}}+k_{2} \rho_{t} f_{y}\right) \\
V_{A S C E}=\left(k_{1} \sqrt{f_{c}^{\prime}}-k_{2} \sqrt{f_{c}^{\prime}}\left(\frac{h_{w}}{l_{w}}-0.5\right)+k_{3} \frac{N_{A}}{l_{w} t_{n}}+k_{4} \rho_{s e} f_{y}\right) d t_{w}
\end{gathered}
$$

The calculated coefficients for these three equations are presented in Table 6.3. The statistics for ratios of the calculated to measured peak shear strength using the three equations mentioned above as well as equations from Section 11.5 of ACI 318-14 
$\left(V_{n 1}\right)$ [A1], 18.1 of ACI 318-14 $\left(V_{n 2}\right)$ [A1], ASCE 43-05 $\left(V_{n 3}\right)$ [A3], Barda et al. $\left(V_{n 4}\right)$ [B1], Wood $\left(V_{n 5}\right)$ [W7], Hwang et al. $\left(V_{n 6}\right)$ [H4], Gulec and Whittaker $\left(V_{n 7}\right)$ [G3], Kassem $\left(V_{n 8}\right)$ [K1] were listed in Table 6.4 below. Figure 6.3 to Figure 6.13 demonstrate the calculated peak shear strength using these eleven sets of equations versus the measured peak shear strength.

Table 6.3 Calculated coefficients for the proposed equation and the refined ACI and ASCE equations

\begin{tabular}{|c|c|c|c|c|}
\cline { 2 - 5 } \multicolumn{1}{c|}{} & $k_{1}$ & $k_{2}$ & $k_{3}$ & $k_{4}$ \\
\hline$V_{\text {cal }}$ & 0.26 & 0.13 & 1.00 & 0.31 \\
\hline$V_{A C I}$ & 0.91 & 0.01 & & \\
\hline$V_{A S C E}$ & 12.35 & 4.51 & 0.85 & 0.39 \\
\hline
\end{tabular}

Table 6.4 Statistics for ratios of the calculated to measured peak shear strength using the eleven sets of equations

\begin{tabular}{|c|c|c|c|c|c|c|c|}
\cline { 2 - 8 } \multicolumn{1}{c|}{} & Mean & Median & $\begin{array}{c}\text { Standard } \\
\text { deviation }\end{array}$ & COV & Min & Max & $\begin{array}{c}\text { \% Over- } \\
\text { prediction }\end{array}$ \\
\hline$V_{n 1} / V_{\text {peak }}$ & 0.710 & 0.617 & 0.235 & 0.331 & 0.238 & 1.293 & 17.4 \\
\hline$V_{n 2} / V_{\text {peak }}$ & 0.823 & 0.745 & 0.321 & 0.390 & 0.230 & 1.616 & 29.0 \\
\hline$V_{n 3} / V_{\text {peak }}$ & 0.898 & 0.835 & 0.290 & 0.323 & 0.500 & 1.527 & 31.9 \\
\hline$V_{n 4} / V_{\text {peak }}$ & 1.016 & 1.008 & 0.214 & 0.210 & 0.620 & 1.538 & 53.6 \\
\hline$V_{n 5} / V_{\text {peak }}$ & 0.662 & 0.689 & 0.186 & 0.281 & 0.300 & 1.073 & 1.4 \\
\hline$V_{n 6} / V_{\text {peak }}$ & 1.176 & 1.172 & 0.322 & 0.274 & 0.683 & 1.801 & 60.9 \\
\hline$V_{n 7} / V_{\text {peak }}$ & 0.966 & 0.984 & 0.165 & 0.170 & 0.617 & 1.435 & 43.5 \\
\hline$V_{n 8} / V_{\text {peak }}$ & 1.301 & 1.143 & 0.409 & 0.315 & 0.708 & 2.385 & 81.2 \\
\hline$V_{\text {cal }} / V_{\text {peak }}$ & 1.000 & 1.003 & 0.241 & 0.241 & 0.603 & 1.857 & 50.7 \\
\hline$V_{\text {ACI }} / V_{\text {peak }}$ & 1.013 & 0.859 & 0.352 & 0.348 & 0.520 & 1.968 & 37.7 \\
\hline$V_{\text {ASCE }} / V_{\text {peak }}$ & 1.002 & 0.995 & 0.242 & 0.242 & 0.559 & 1.788 & 47.8 \\
\hline
\end{tabular}




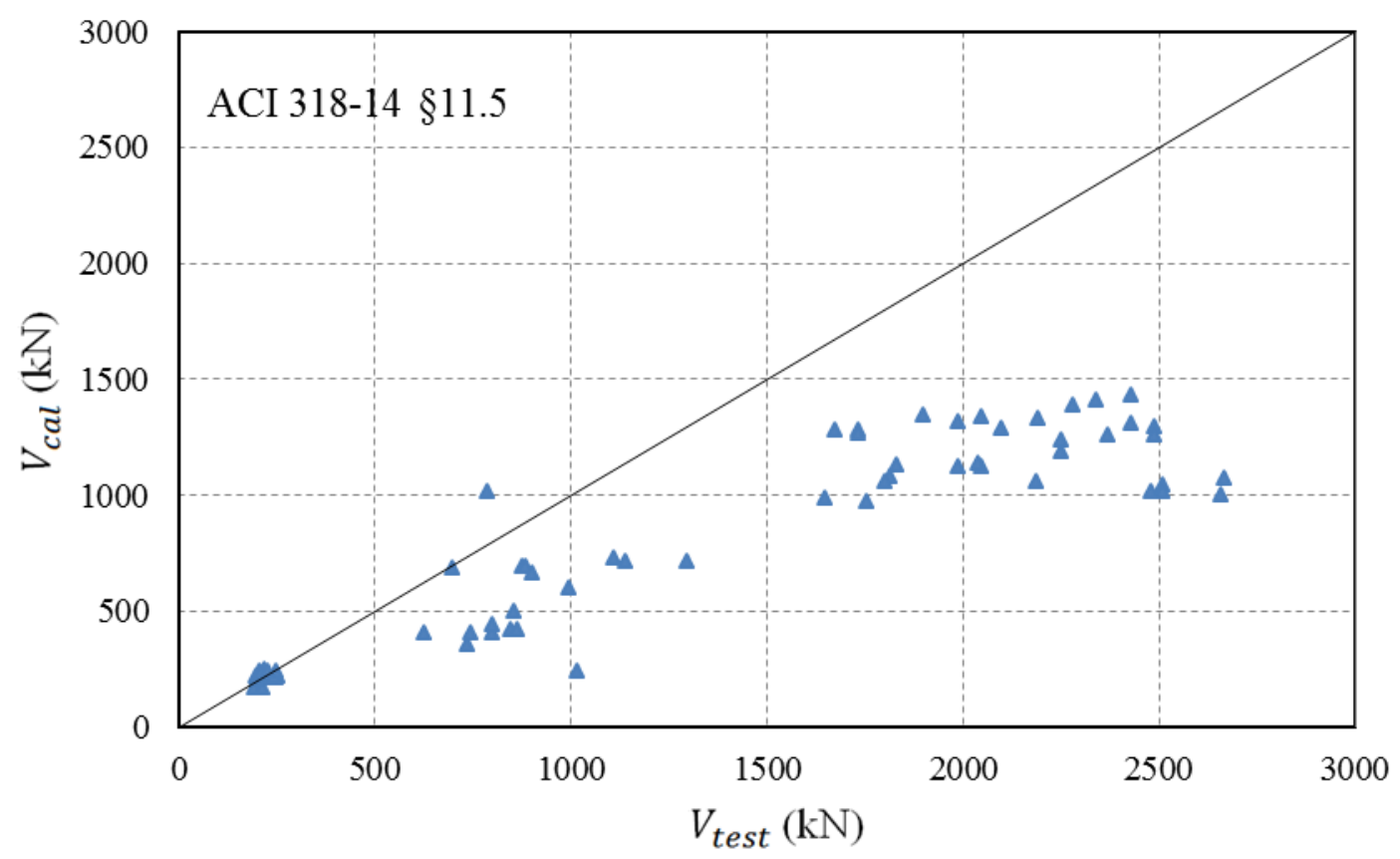

Figure 6.3 Calculated peak shear strength using equations from Section 11.5 of ACI 318-14 [A1] versus the measured peak shear strength

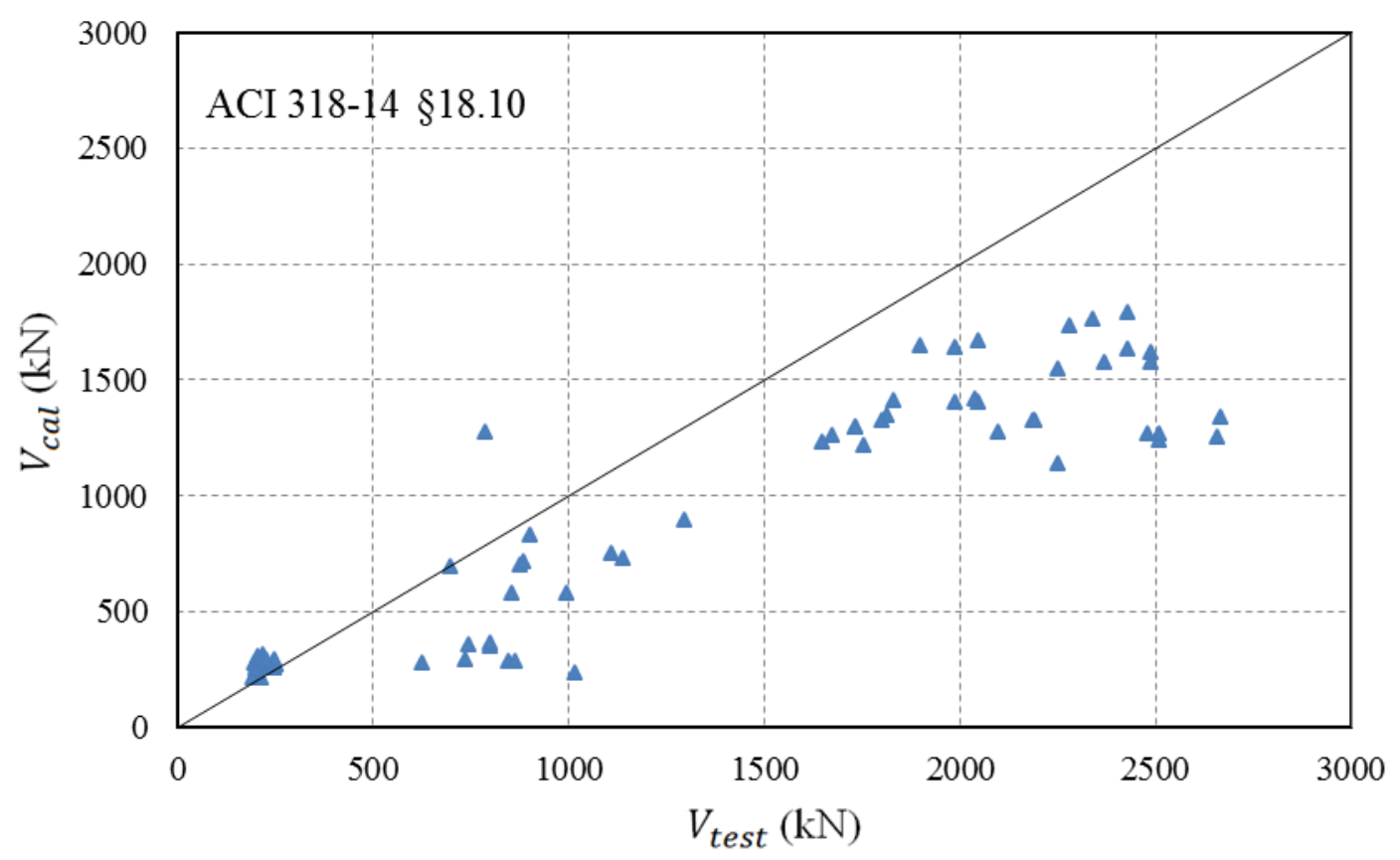

Figure 6.4 Calculated peak shear strength using the equation from Section 18.10 of ACI 318-14 [A1] versus the measured peak shear strength 


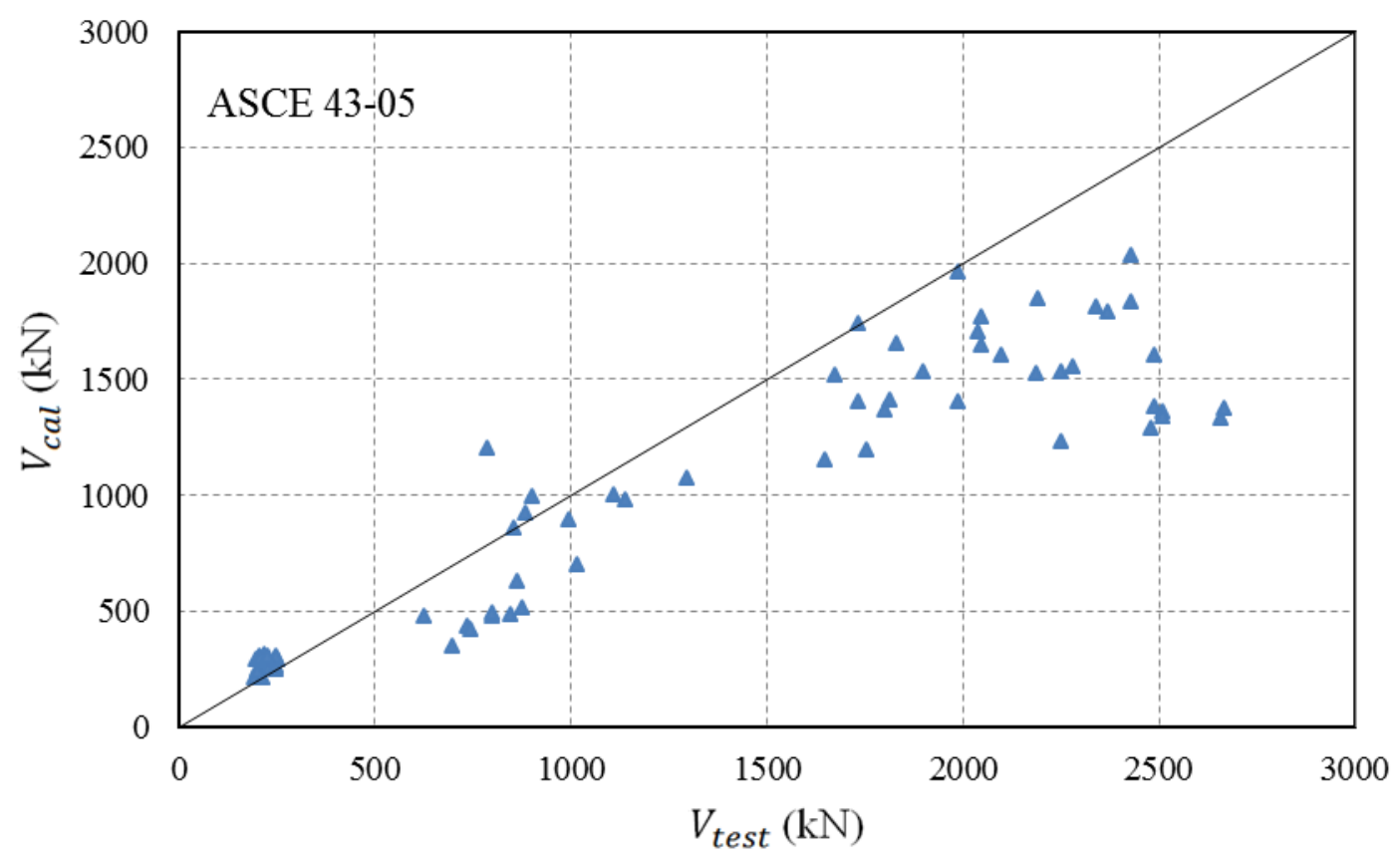

Figure 6.5 Calculated peak shear strength using the equation from ASCE 43-05 [A3] versus the measured peak shear strength

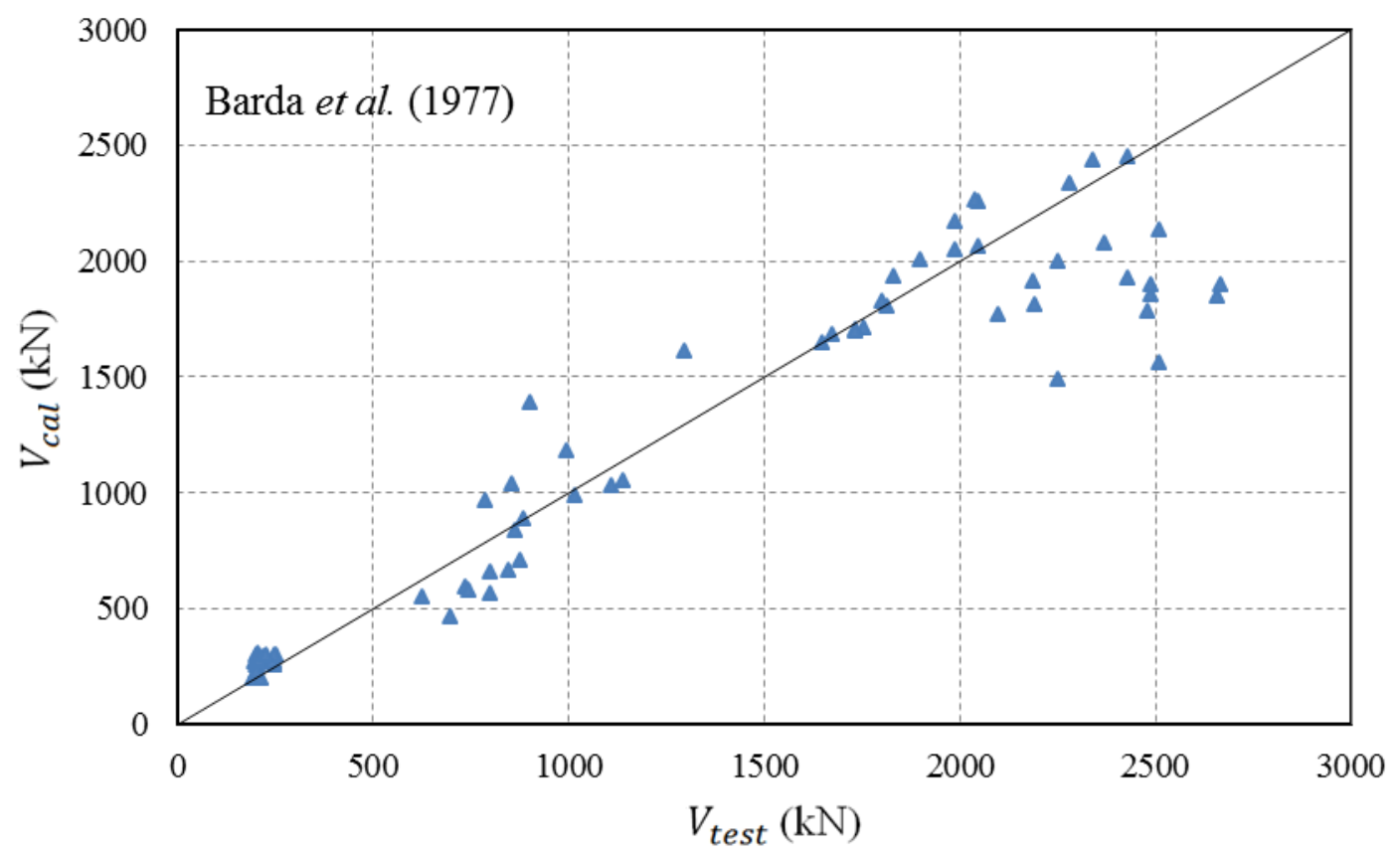

Figure 6.6 Calculated peak shear strength using the equation from Barda et al. [B1] versus the measured peak shear strength 


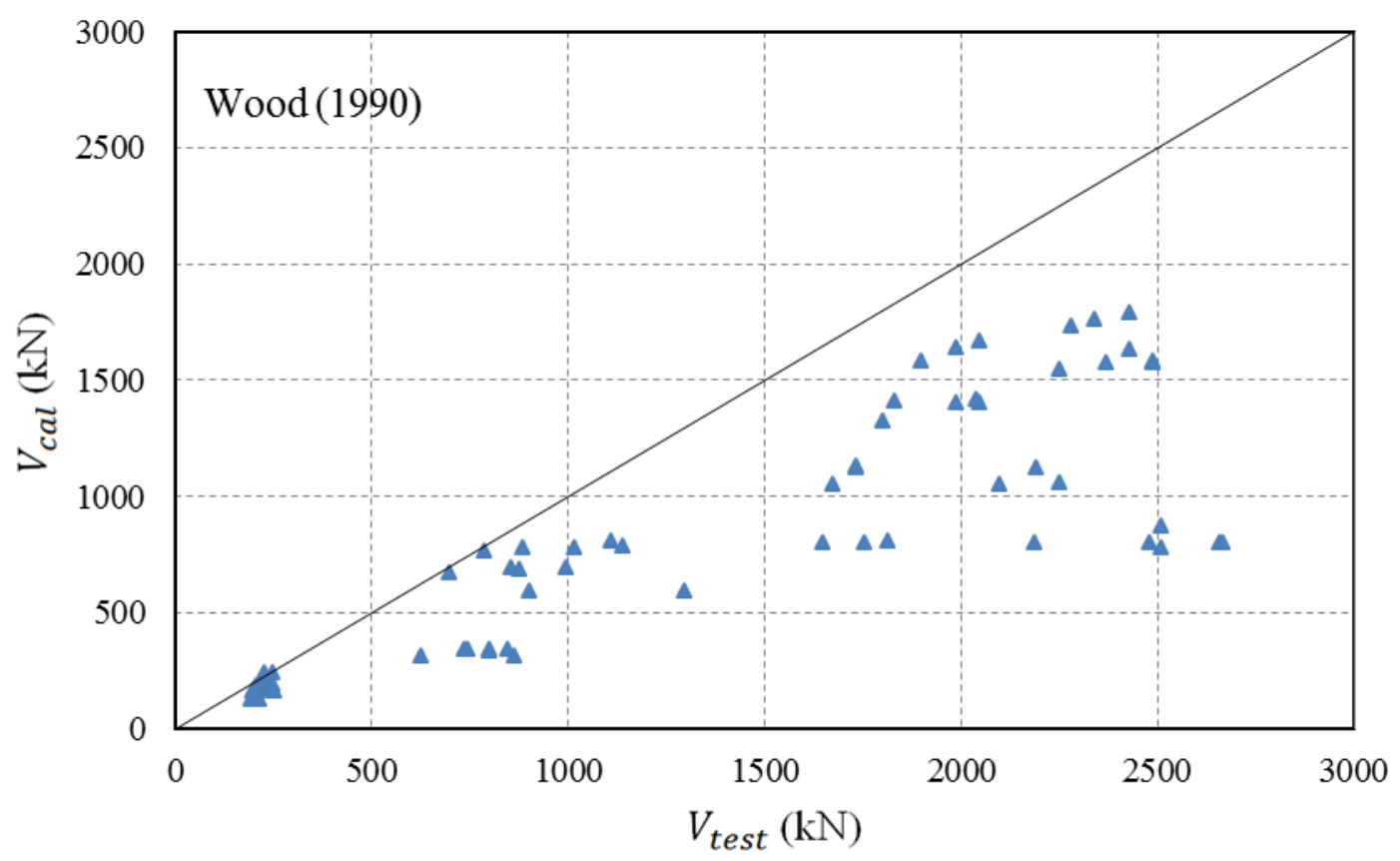

Figure 6.7 Calculated peak shear strength using the equation from Wood [W7] versus the measured peak shear strength

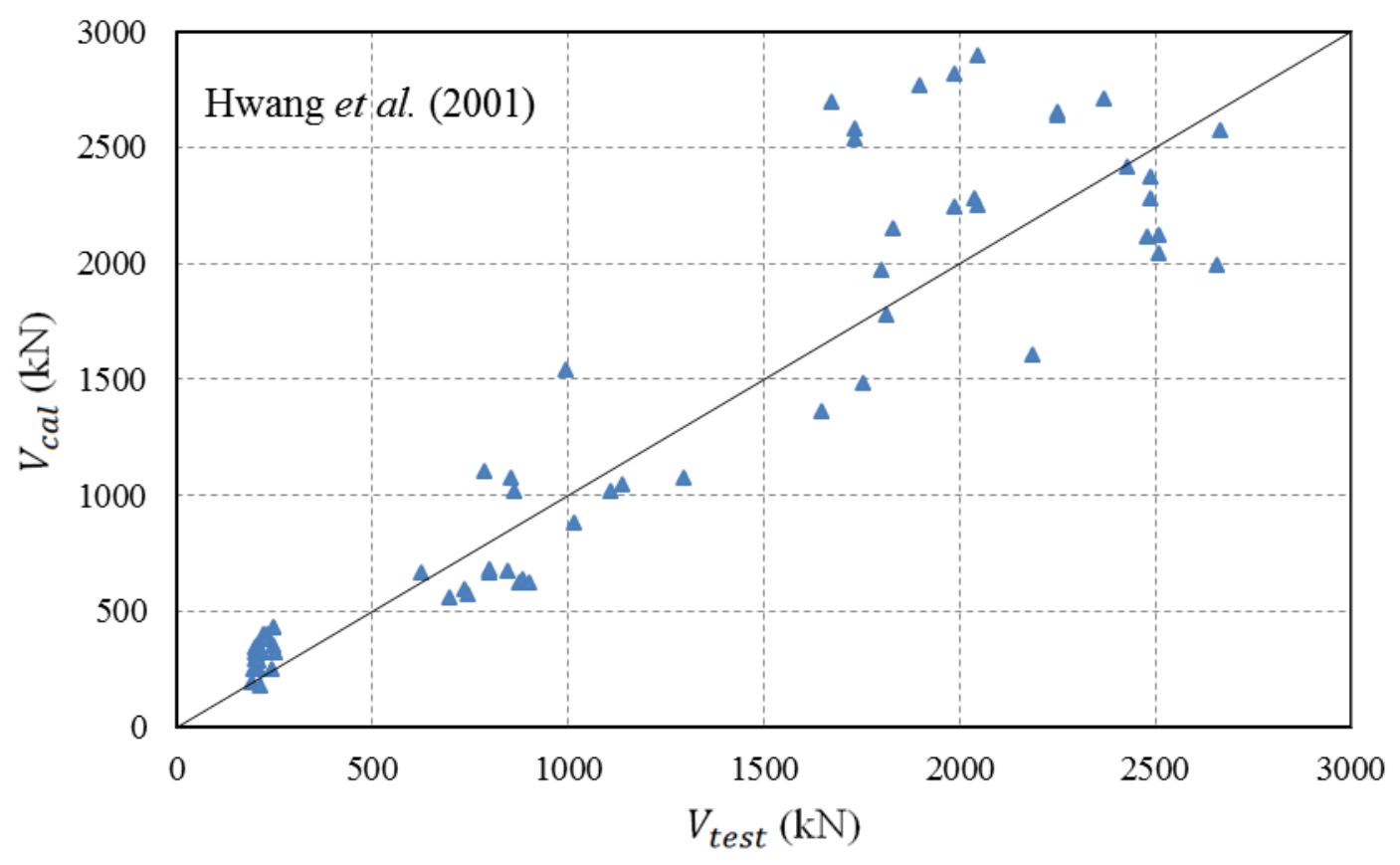

Figure 6.8 Calculated peak shear strength using equations from Hwang et al. [H4] versus the measured peak shear strength 


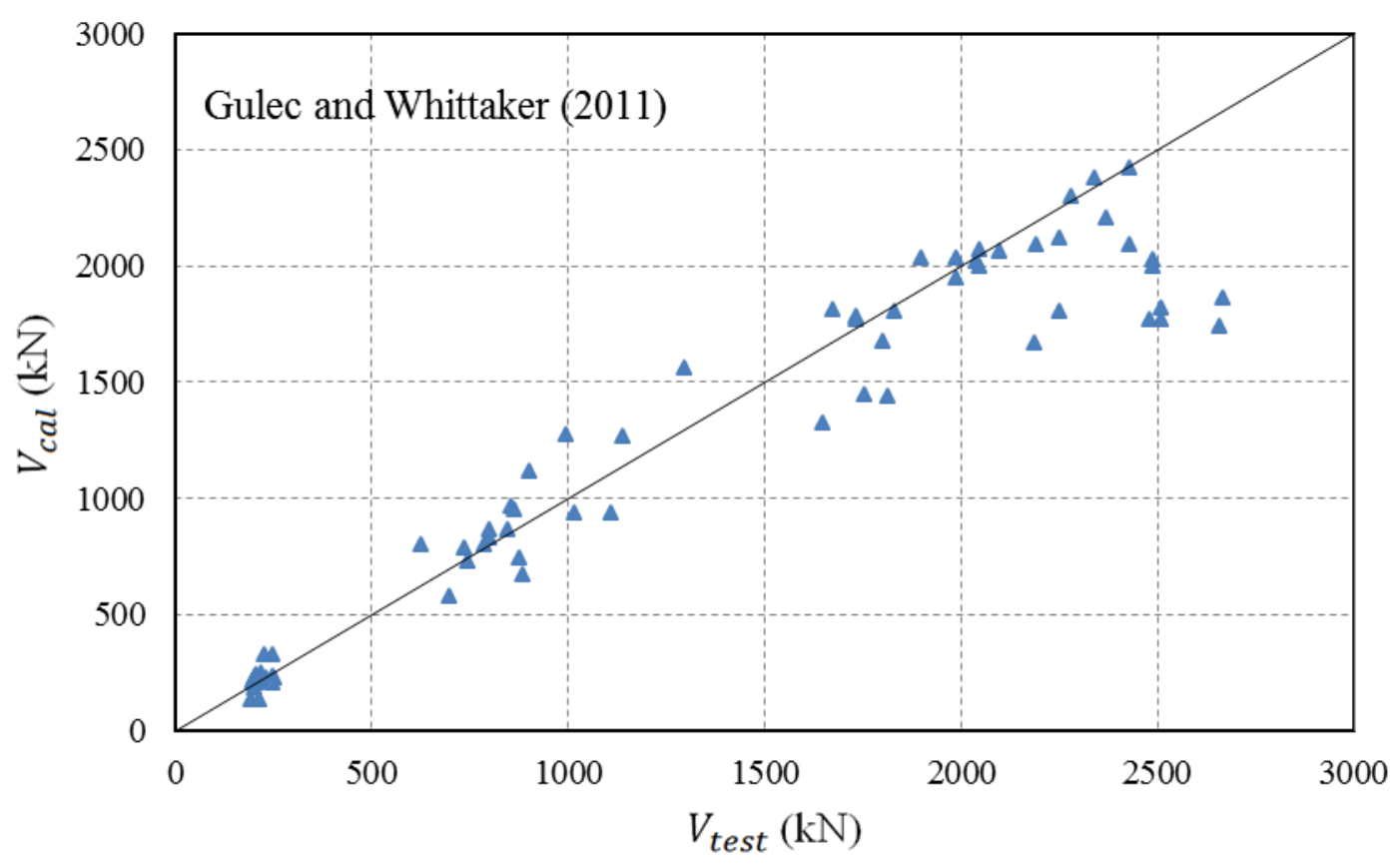

Figure 6.9 Calculated peak shear strength using the equation from Gulec and Whittaker [G3] versus the measured peak shear strength

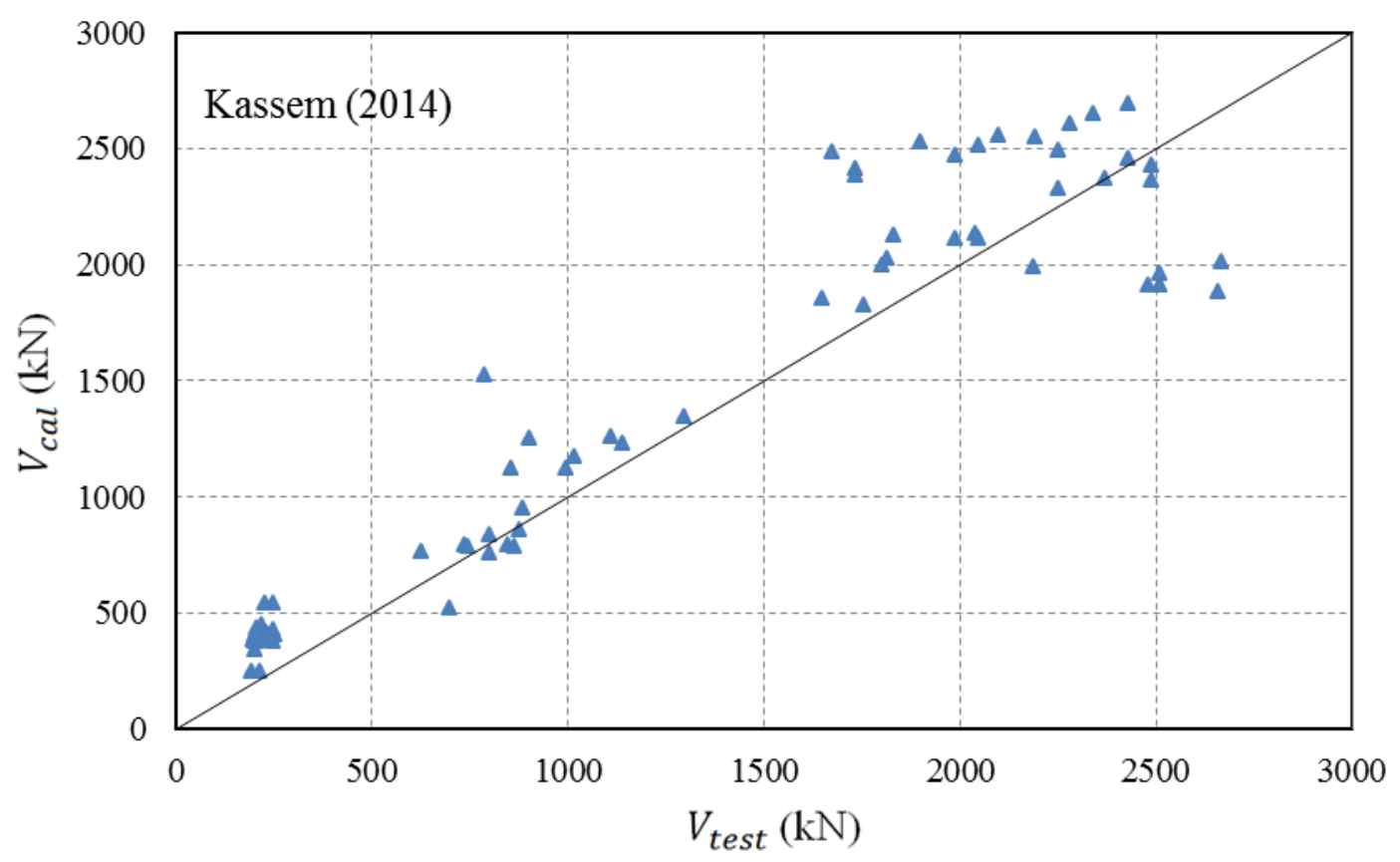

Figure 6.10 Calculated peak shear strength using equations from Kassem [K1] versus the measured peak shear strength 


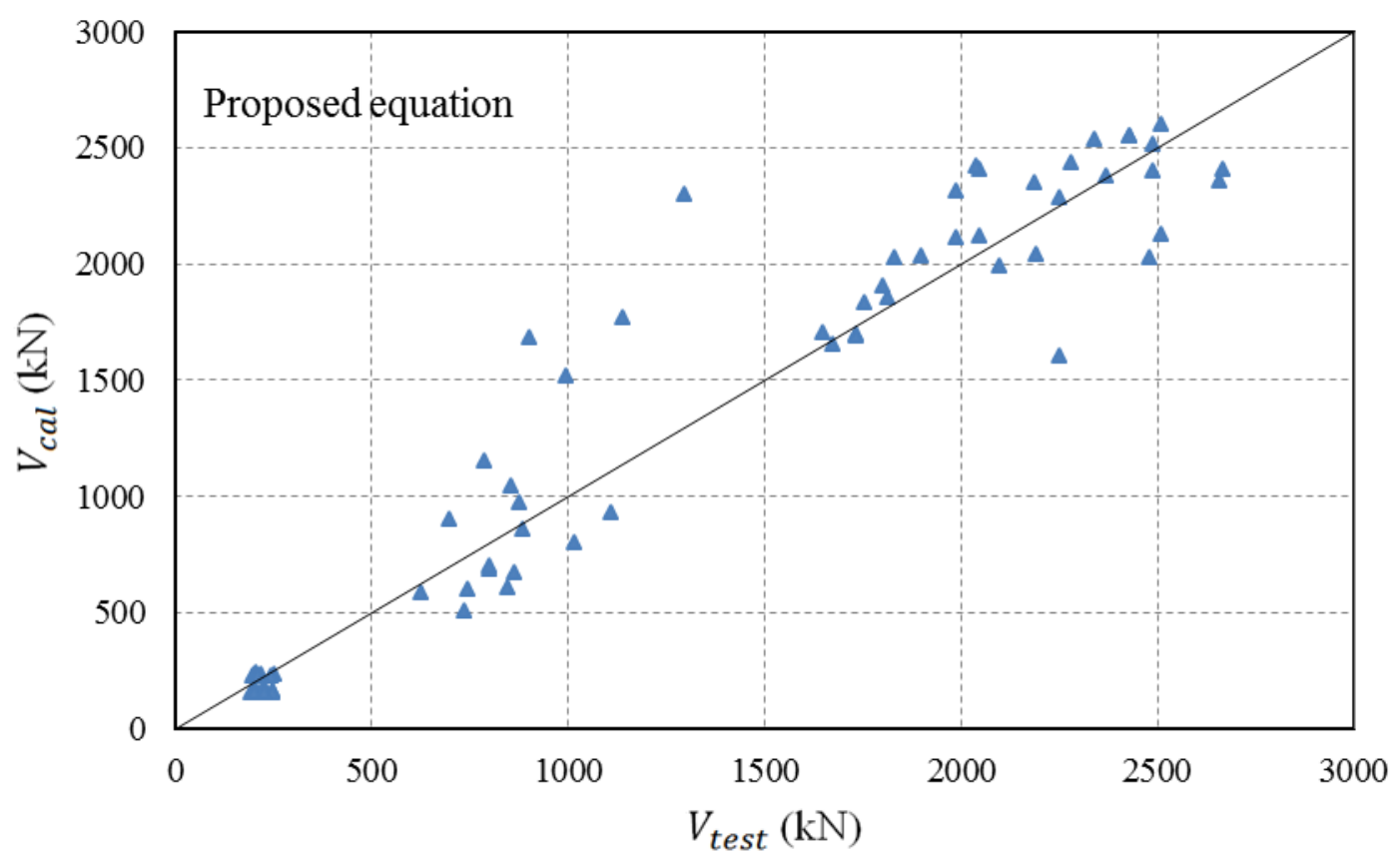

Figure 6.11 Calculated peak shear strength using the proposed equation versus the measured peak shear strength

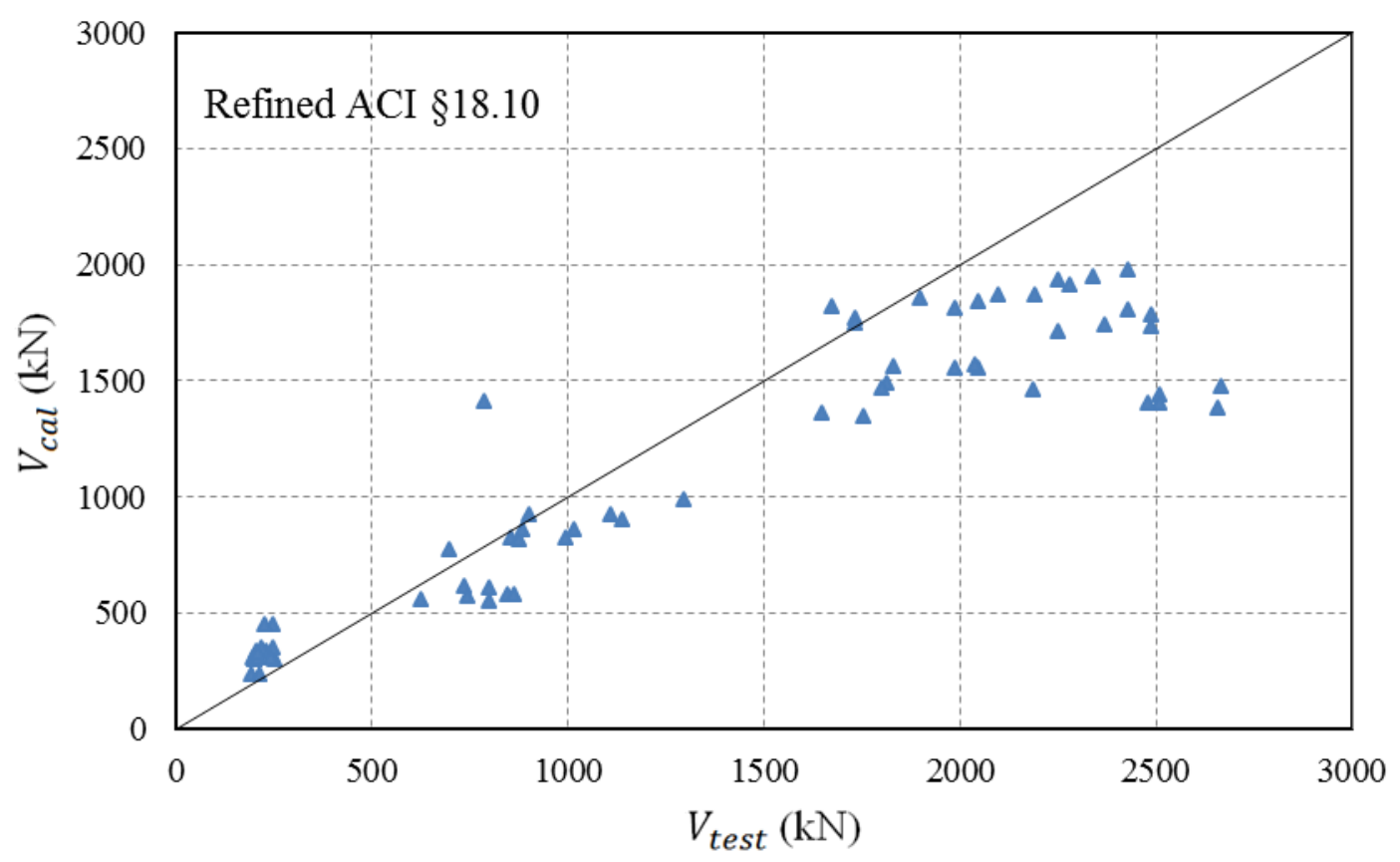

Figure 6.12 Calculated peak shear strength using the refined ACI equation versus the measured peak shear strength 


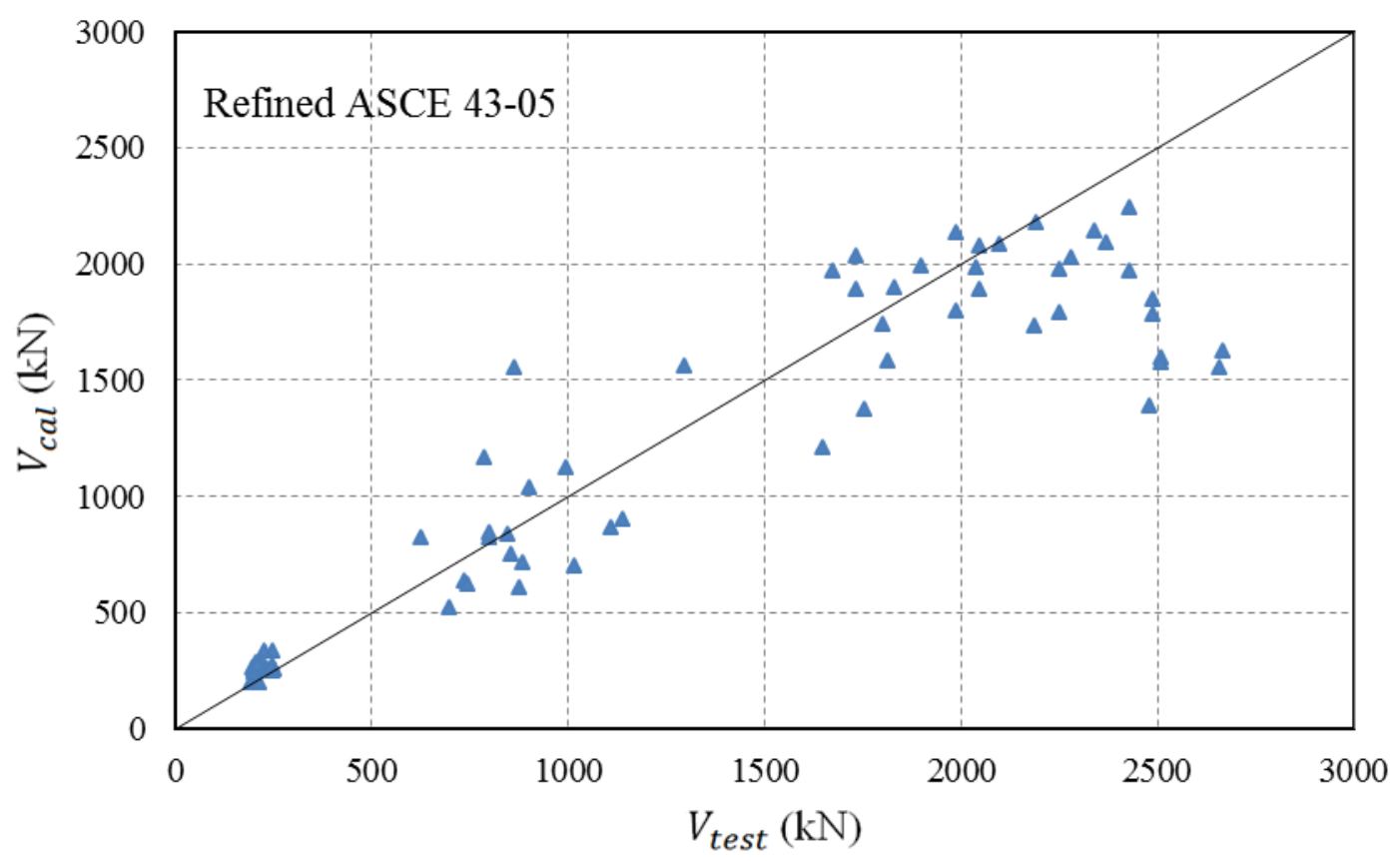

Figure 6.13 Calculated peak shear strength using the refined ASCE equation versus the measured peak shear strength

As seen from the table and figures, equations in Section 11.5 and 18.10 of ACI 31814 [A1], ASCE 43-05 [A3] underestimated the peak shear strength, as the findings of Gulec et al. [G5] also confirmed. The equation proposed by Barda et al. [B1] yielded statistically sound results at first glance. However, deeper examinations revealed the contingency behind these results. Gulec et al. [G5] found this equation overestimated the shear strength of rectangular squat walls by around $30 \%$. Thus, although the contribution from the flange was not included, it might still produce a mean value close to one when used for non-rectangular RC squat walls. Equations developed by Hwang et al. [H4] and Kassem [K1] overestimated the peak shear strength by around $20 \%$, which should be avoided in the design. The equation provided by Gulec and Whittaker [G3], performed best among the equations mentioned above, since it yielded the smallest value of the coefficient of variation (COV) and a mean value close to one. However, several deficiencies were still found. Primarily, the function form of this equation was obtained from a simple free body diagram, which was unable to reasonably account for the force transfer mechanism in non-rectangular RC squat walls. Furthermore, the coefficients in the equations required limits and plausible explanations. It was obvious that the more coefficients one equation included, the smaller value of COV would be produced by 
the nonlinear regression. However, this decrease of $\mathrm{COV}$ did not prove this equation was more applicable and reasonable.

On the other hand, the equations proposed in this study were based on a solid strutand-tie model, and were also able to provide sound statistical results, which were revealed in the mean value equal to one, the small value of the coefficient of variation, and the closest median to one among all the equations. Therefore, the proposed equations were deemed to be best in predicting the peak shear strength of non-rectangular RC squat walls. The refined ACI and ASCE equations performed better than their original counterparts but were less sound compared with the proposed equations. This observation suggested that the shear strength equations from Section 18.10 of ACI 318-14 [A1] and ASCE 43-05 [A3] needed modifications when used for non-rectangular RC squat walls.

As seen in Figure 6.14, generally the normalized shear stress calculated by the proposed equations stayed within the value of $1.2 \sqrt{f_{c}^{\prime}}$. Hence an upper limit was imposed on the shear stress to prevent the diagonal compression failure. The proposed equations for the peak shear strength of non-rectangular RC squat walls with symmetric section shapes are expressed as below, where $A_{t}\left(\mathrm{~mm}^{2}\right)$ is the total section area of non-rectangular RC squat walls.

$$
\begin{gathered}
V_{c a l}=\left(0.26 f_{y f} \rho_{f} t_{f} l_{f}+0.13 f_{y v} \rho_{v} t_{w} z_{w}+\frac{P}{2}\right) \frac{d_{w}}{h_{w}} \\
+f_{y h} \rho_{h} t_{w} h_{w} \leq 1.2 \sqrt{f_{c}^{\prime}} A_{t} \\
d_{w}=l_{w}-t_{f}-\frac{1}{2}\left(\frac{0.26 f_{y f} \rho_{f} t_{f} l_{f}+P}{0.31 f_{c}^{\prime} t_{w}}-\frac{t_{f} l_{f}}{t_{w}}\right)
\end{gathered}
$$




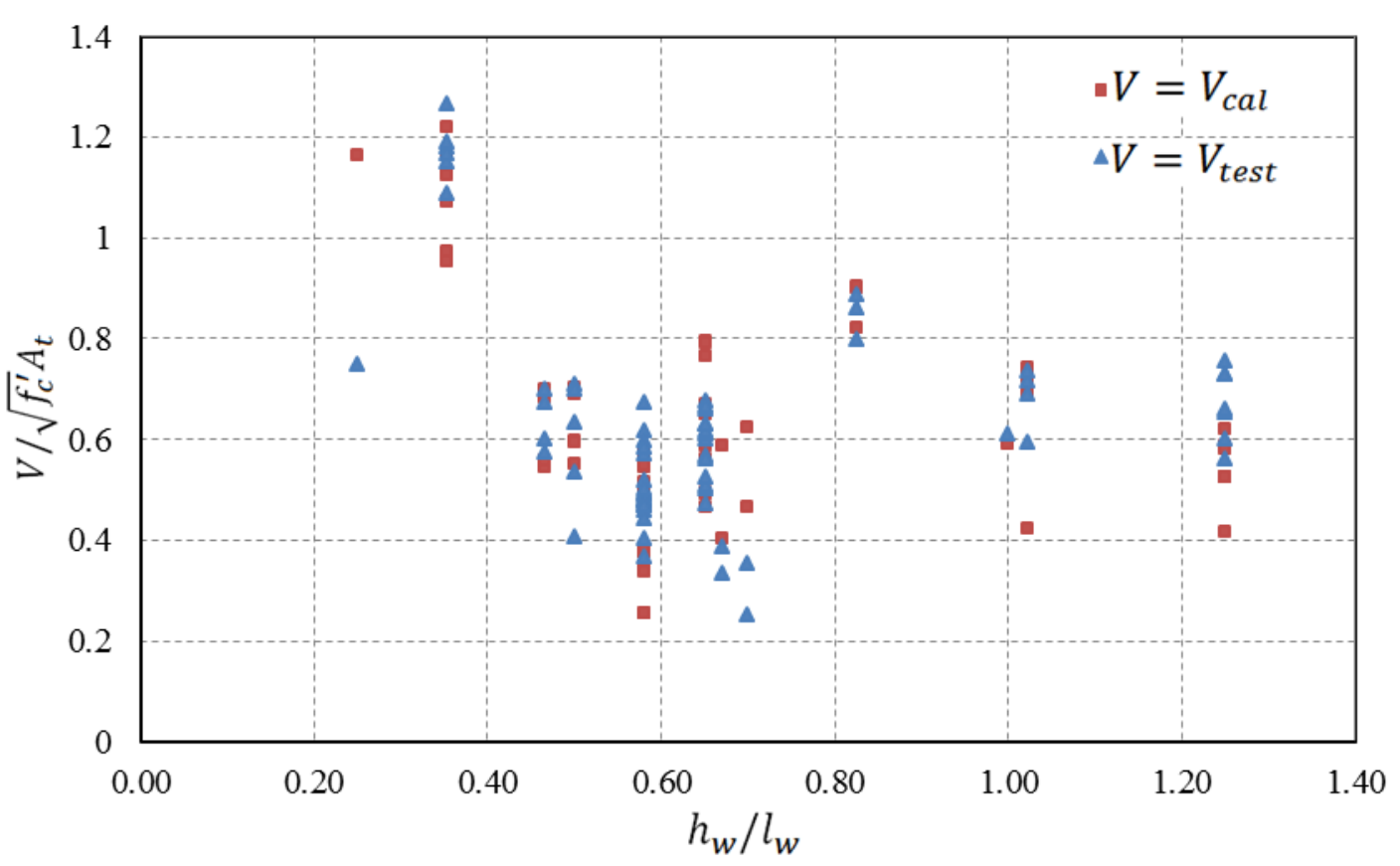

Figure 6.14 Variation of ratios of predicted and measured shear strengths (normalized) with the shear span ratio

\subsection{Probabilistic Approach for the Peak Shear Strength of Non- rectangular RC Squat Walls}

\subsubsection{Introduction}

As reviewed in Chapter 2, different equations [B1, G3, H4, K1, W7] were proposed for the shear strength of squat walls, but none of these was able to demonstrate the influence of the individual parameter on the shear strength. Moreover, contradictory results were found in these studies. For instance, Section 11.5 and Section 18.10 of ACI 318-14 [A1] only recognized the contribution of horizontal reinforcement in resisting shear. However, the research conducted by Barad et al. [B1] and Lefas et al. [L2] indicated that compared with the vertical web reinforcement, the effect of horizontal web reinforcement was negligible on the peak shear strength of squat walls. Thus further research is required to investigate the effect of individual parameter on the shear strength.

More importantly, despite the gradual improvement in understanding the failure mechanism of squat walls, equations currently available are still in a deterministic manner, meaning that the biased estimate could hardly be avoided [G1]. For 
instance, the accuracy of the traditional nonlinear regression was found to be overwhelmingly dependent on the experimental database and the simplified manner to derive the mechanical-based or empirical-based expression. The biased estimate would inevitably exist if bounds of the experimental database changed. Furthermore, as reflected in the statistics in Table 6.4, large scatters existed in the deterministic predictions of peak shear strength of non-rectangular RC squat walls. These large scatters would also occur even if different simplified manners were used to develop deterministic prediction equations. In short, no matter how well a deterministic prediction equation was refined or how extensive the experimental database was, the large scatter and biased estimate in the prediction of peak shear strength would still remain if the deterministic manner was utilized, such as the nonlinear regression approach.

Therefore, the probabilistic approach which has been proven to be effective [N4] is introduced in this study as a reasonable alternative to reconcile the large scatter, avoid the biased estimate and improve the prediction of the peak shear strength of non-rectangular RC squat walls.

\subsubsection{Probabilistic approach}

In mathematics, a capacity model refers to an expression connecting one or more quantities of interest to a set of basic variables $X$. Quantities of interest are predicted by the model at the given deterministic or random values of basic variables [G1]. In this study, only one quantity needs to be predicted, which means the peak shear strength is univariate. Thus, the model has the general form depicting the relationship between basic quantities and peak shear strength of non-rectangular RC squat walls as below:

$$
V=F(X, \Theta)
$$

where $V$ is the predicted quantity, $F$ is the function form of probabilistic shear strength equation, $X$ represents the design parameters in the equation, $\Theta$ is the set of unknown parameters, which is treated as set of random variables to represent the uncertainty. Generally, two kinds of uncertainties are used in the equation, aleatory and epistemic. The former are mainly represented by basic variables and the latter 
are roughly incorporated in the model parameters. Considering the difference of the simplified manner and size of samples in the database, the epistemic uncertainties are investigated in this study.

As for the approaches to developing the function form $F$, two methods are most commonly used, name the empirical regression method and the mechanical derivation method. As mentioned previously, compared with the latter, the empirical regression method usually yields a large scatter in the prediction and the results could hardly be refined with technical advances. For these reasons, the mechanical derivation method is selected in this study. The proposed strut-and-tie model which is composed of the effect of flanges and has been proven to be effective in the last section is continually adopted in the following probabilistic investigation. Each unknown model parameter involved in the function form $F$ is defined mechanically to reasonably limit the corresponding prior distribution ranges.

In the past decades, the uncertainty estimation of unknown model parameters $\Theta$ received considerable attentions [B2, G1, N1]. Numerous approaches have been developed, such as the formal Bayesian method using the Metropolis-Hasting algorithm, the Probability Theory method, the Markov Chain Monte Carlo (MCMC) method and the Generalized Likelihood Uncertainty Estimation (GLUE) method. Among them, the GLUE method, which was proposed by Beven and Binley [B2] is renowned for its reputation for the simplicity and flexible definition on the likelihood function [A2]. Moreover, in the context of multiple uncertainties, the structure of the modeling error using other uncertainty estimation methods is commonly non-stationary and complicated, whereas the GLUE method allows for no strong assumption on the modeling error. As a result, the GLUE method is adopted here for the estimation of unknown model parameters.

To estimate the posterior cumulative distribution of unknown model parameters, an experimental database is needed. Generally, a database including 50 to 150 samples with a minimum size 3 to 20 times' the number of variables is suggested for structural engineering [M6]. An appropriate sample size is actually determined subjectively by researchers based on the database availability. Currently, two major considerations are usually used in determining the size of samples, which are the 
non-statistical consideration and the statistical consideration. The former includes the availability of resources, manpower, and budgets, while the latter contains the preferred accuracy of estimation. In this study, the database containing sixty-eight non-rectangular RC squat walls as selected in the last section was used (excluding the one tested by Synge [S10]), and was rearranged into various groups and demonstrated in Table 6.5. In the database, parameters of specimens such as compressive stress of concrete $f_{c}^{\prime}$, web length $l_{w}$, flange length $l_{f}$ wall height $h_{w}$, thickness of the web $t_{w}$, thickness of the flange $t_{f}$, yield stress of longitudinal reinforcement in web $f_{y v}$, yield stress of longitudinal reinforcement in the flange $f_{y f}$, yield stress of horizontal reinforcement in web $f_{y h}$, longitudinal reinforcement ratio of the web $\rho_{v}$, longitudinal reinforcement ratio of the flange $\rho_{f}$, horizontal reinforcement ratio of the web $\rho_{h}$ and axial load $P$ were summarized and would be further investigated. The measured peak shear strength $V_{\text {exp }}$ of test specimens was calculated as the average maximum strengths in two loading directions. Moreover, statistics for these model parameters were also provided, which included the mean value, the standard deviation (STD) and the coefficient of variance (COV). It was clear that each design parameter demonstrated a large scatter distribution, and the database was able to cover a wide range of design parameters.

Furthermore, to demonstrate the stability of the estimated cumulative distribution, three major aspects in the GLUE method were investigated; these were the selection of the likelihood function, the determination of the threshold values and the assumption of the prior distribution. Since the probabilistically developed equation might be considered inconvenient when applied in engineering practice, it would be further simplified by the estimated mean values and standard deviations of the unknown model parameters as a mean prediction equation and a standard deviation prediction equation. Hence the confidence interval of the shear strength in terms of Mean \pm STD or Mean $\pm 2 \times$ STD could be directly calculated for the comparison with the measured shear strength, which could prove the effectiveness of the proposed probabilistic equation. 
Table 6.5 Experimental database of non-rectangular RC squat walls

\begin{tabular}{|c|c|c|c|c|c|c|c|c|c|c|c|c|c|c|c|c|}
\hline No. & $\begin{array}{c}\text { Group, } \\
\text { Researcher }\end{array}$ & Specimen & $\begin{array}{c}f_{c}^{\prime} \\
(\mathrm{MPa})\end{array}$ & $\begin{array}{c}h_{w} \\
(\mathrm{~mm})\end{array}$ & $\begin{array}{c}l_{w} \\
(\mathrm{~mm})\end{array}$ & $\begin{array}{c}t_{w} \\
(\mathrm{~mm})\end{array}$ & $\begin{array}{c}t_{f} \\
(\mathrm{~mm})\end{array}$ & $\begin{array}{c}l_{f} \\
(\mathrm{~mm})\end{array}$ & $\begin{array}{c}f_{y h} \\
(\mathrm{MPa})\end{array}$ & $\begin{array}{c}f_{y v} \\
(\mathrm{MPa})\end{array}$ & $\begin{array}{c}f_{y f} \\
(\mathrm{MPa})\end{array}$ & $\rho_{h}$ & $\rho_{v}$ & $\rho_{f}$ & $\begin{array}{c}P \\
(\mathrm{kN})\end{array}$ & $\begin{array}{l}V_{\text {exp }} \\
(\mathrm{kN})\end{array}$ \\
\hline 1 & \multirow{6}{*}{$\begin{array}{c}\text { G1, Barda et al. } \\
\text { [B1] }\end{array}$} & B3-2 & 27.0 & 953 & 1905 & 102 & 102 & 610 & 513 & 545 & 414 & 0.50 & 0.50 & 4.10 & 0 & 1108 \\
\hline 2 & & B4-3 & 23.5 & 953 & 1905 & 102 & 102 & 610 & 0 & 535 & 527 & 0.00 & 0.50 & 4.10 & 0 & 1017 \\
\hline 3 & & B5-4 & 19.0 & 953 & 1905 & 102 & 102 & 610 & 495 & 0 & 527 & 0.50 & 0.00 & 4.10 & 0 & 699 \\
\hline 4 & & B6-4 & 21.2 & 953 & 1905 & 102 & 102 & 610 & 496 & 496 & 529 & 0.50 & 0.25 & 4.10 & 0 & 876 \\
\hline 5 & & B7-5 & 25.7 & 476 & 1905 & 102 & 102 & 610 & 501 & 531 & 539 & 0.50 & 0.50 & 4.10 & 0 & 1140 \\
\hline 6 & & B8-5 & 23.5 & 1905 & 1905 & 102 & 102 & 610 & 496 & 527 & 489 & 0.50 & 0.50 & 4.10 & 0 & 886 \\
\hline 7 & \multirow{9}{*}{$\begin{array}{l}\text { G2. Saito et al. } \\
{[\mathrm{S} 2]}\end{array}$} & W12-1 & 35.0 & 750 & 2120 & 120 & 120 & 500 & 369 & 369 & 369 & 1.32 & 1.32 & 4.72 & 706 & 2657 \\
\hline 8 & & W12-2 & 38.0 & 750 & 2120 & 120 & 120 & 500 & 369 & 369 & 369 & 0.90 & 0.90 & 4.72 & 698 & 2511 \\
\hline 9 & & W12-3 & 36.0 & 750 & 2120 & 120 & 120 & 500 & 369 & 369 & 369 & 1.69 & 1.69 & 4.72 & 713 & 2511 \\
\hline 10 & & W12-4 & 36.0 & 750 & 2120 & 120 & 120 & 500 & 369 & 369 & 369 & 1.32 & 1.32 & 4.72 & 350 & 2481 \\
\hline 11 & & W12-5 & 40.0 & 750 & 2120 & 120 & 120 & 500 & 369 & 369 & 369 & 1.32 & 1.32 & 4.72 & 706 & 2668 \\
\hline 12 & & W12-6 & 33.0 & 1750 & 2120 & 120 & 120 & 500 & 369 & 369 & 369 & 1.32 & 1.32 & 4.72 & 701 & 1755 \\
\hline 13 & & W12-7 & 34.0 & 1750 & 2120 & 120 & 120 & 500 & 369 & 369 & 369 & 1.32 & 1.32 & 4.72 & 355 & 1648 \\
\hline 14 & & W15-1 & 25.0 & 750 & 2120 & 150 & 120 & 500 & 369 & 369 & 369 & 1.06 & 1.06 & 4.72 & 830 & 2187 \\
\hline 15 & & W15-2 & 26.0 & 1750 & 2120 & 150 & 120 & 500 & 369 & 369 & 369 & 1.06 & 1.06 & 4.72 & 830 & 1814 \\
\hline
\end{tabular}




\begin{tabular}{|c|c|c|c|c|c|c|c|c|c|c|c|c|c|c|c|c|}
\hline No. & $\begin{array}{c}\text { Group, } \\
\text { Researcher }\end{array}$ & Specimen & $\begin{array}{c}f_{c}^{\prime} \\
(\mathrm{MPa})\end{array}$ & $\begin{array}{c}h_{w} \\
(\mathrm{~mm})\end{array}$ & $\begin{array}{c}l_{w} \\
(\mathrm{~mm})\end{array}$ & $\begin{array}{c}t_{w} \\
(\mathrm{~mm})\end{array}$ & $\begin{array}{c}t_{f} \\
(\mathrm{~mm})\end{array}$ & $\begin{array}{c}l_{f} \\
(\mathrm{~mm})\end{array}$ & $\begin{array}{c}f_{y h} \\
(\mathrm{MPa})\end{array}$ & $\begin{array}{c}f_{y v} \\
(\mathrm{MPa})\end{array}$ & $\begin{array}{c}f_{y f} \\
(\mathrm{MPa})\end{array}$ & $\rho_{h}$ & $\rho_{v}$ & $\rho_{f}$ & $\begin{array}{c}P \\
(\mathrm{kN})\end{array}$ & $\begin{array}{l}V_{\text {exp }} \\
(\mathrm{kN})\end{array}$ \\
\hline 16 & \multirow{17}{*}{$\begin{array}{c}\text { G3. Sato et al. } \\
\text { [S3] }\end{array}$} & $18 \mathrm{M} 12-40$ & 43.1 & 2200 & 2150 & 150 & 150 & 1000 & 422 & 422 & 422 & 0.45 & 0.45 & 2.25 & 1155 & 2250 \\
\hline 17 & & $24 \mathrm{M} 8-30$ & 38.0 & 1400 & 2150 & 150 & 150 & 1000 & 296 & 296 & 296 & 0.80 & 0.80 & 0.80 & 1155 & 1676 \\
\hline 18 & & $24 \mathrm{M} 8-40$ & 35.8 & 1400 & 2150 & 150 & 150 & 1000 & 422 & 422 & 422 & 0.60 & 0.60 & 0.60 & 1155 & 1735 \\
\hline 19 & & $24 \mathrm{M} 8-50$ & 35.0 & 1400 & 2150 & 150 & 150 & 1000 & 528 & 528 & 528 & 0.48 & 0.48 & 0.48 & 1155 & 1735 \\
\hline 20 & & 24M 6-30 & 40.1 & 1000 & 2150 & 150 & 150 & 1000 & 296 & 296 & 296 & 0.80 & 0.80 & 0.80 & 1155 & 2100 \\
\hline 21 & & 24M 6-40 & 40.0 & 1000 & 2150 & 150 & 150 & 1000 & 422 & 422 & 422 & 0.60 & 0.60 & 0.60 & 1155 & 2190 \\
\hline 22 & & $36 \mathrm{M} \mathrm{12-30}$ & 36.3 & 2200 & 2150 & 150 & 150 & 1000 & 296 & 296 & 296 & 1.20 & 1.20 & 5.80 & 1155 & 2490 \\
\hline 23 & & $36 \mathrm{M} 12-40$ & 34.4 & 2200 & 2150 & 150 & 150 & 1000 & 422 & 422 & 422 & 0.90 & 0.90 & 4.50 & 1155 & 2490 \\
\hline 24 & & $36 \mathrm{M} 12-50$ & 37.2 & 2200 & 2150 & 150 & 150 & 1000 & 528 & 528 & 528 & 0.72 & 0.72 & 3.60 & 1155 & 2430 \\
\hline 25 & & 36L 8-30 & 24.5 & 1400 & 2150 & 150 & 150 & 1000 & 296 & 296 & 296 & 1.20 & 1.20 & 1.16 & 1155 & 1800 \\
\hline 26 & & 36L 8-40 & 27.8 & 1400 & 2150 & 150 & 150 & 1000 & 422 & 422 & 422 & 0.90 & 0.90 & 0.90 & 1155 & 1830 \\
\hline 27 & & $36 \mathrm{M} 8-30$ & 39.3 & 1400 & 2150 & 150 & 150 & 1000 & 296 & 296 & 296 & 1.20 & 1.20 & 1.16 & 1155 & 1898 \\
\hline 28 & & $36 \mathrm{M} \mathrm{8-40}$ & 38.8 & 1400 & 2150 & 150 & 150 & 1000 & 422 & 422 & 422 & 0.90 & 0.90 & 0.90 & 1155 & 2046 \\
\hline 29 & & $36 \mathrm{M} 8-50$ & 37.5 & 1400 & 2150 & 150 & 150 & 1000 & 528 & 528 & 528 & 0.72 & 0.72 & 0.72 & 1155 & 1988 \\
\hline 30 & & 36M 6-30 & 33.4 & 1000 & 2150 & 150 & 150 & 1000 & 296 & 296 & 296 & 1.20 & 1.20 & 1.16 & 1155 & 2250 \\
\hline 31 & & 36M 6-40 & 34.6 & 1000 & 2150 & 150 & 150 & 1000 & 422 & 422 & 422 & 0.90 & 0.90 & 0.90 & 1155 & 2370 \\
\hline 32 & & $48 \mathrm{M} 8-30$ & 27.4 & 1400 & 2150 & 150 & 150 & 1000 & 296 & 296 & 296 & 1.60 & 1.60 & 1.60 & 1155 & 1989 \\
\hline
\end{tabular}




\begin{tabular}{|c|c|c|c|c|c|c|c|c|c|c|c|c|c|c|c|c|}
\hline No. & & Specimen & $\begin{array}{c}f_{c}^{\prime} \\
(\mathrm{MPa})\end{array}$ & $\begin{array}{c}h_{w} \\
(\mathrm{~mm})\end{array}$ & $\begin{array}{c}l_{w} \\
(\mathrm{~mm})\end{array}$ & $\begin{array}{c}t_{w} \\
(\mathrm{~mm})\end{array}$ & $\begin{array}{c}t_{f} \\
(\mathrm{~mm})\end{array}$ & $\begin{array}{c}l_{f} \\
(\mathrm{~mm})\end{array}$ & $\begin{array}{c}f_{y h} \\
(\mathrm{MPa})\end{array}$ & $\begin{array}{c}f_{y v} \\
(\mathrm{MPa})\end{array}$ & $\begin{array}{c}f_{y f} \\
(\mathrm{MPa})\end{array}$ & $\rho_{h}$ & $\rho_{v}$ & $\rho_{f}$ & $\begin{array}{c}P \\
(\mathrm{kN})\end{array}$ & $\begin{array}{l}V_{\text {exp }} \\
(\mathrm{kN})\end{array}$ \\
\hline 33 & & $48 \mathrm{M} \mathrm{8-40}$ & 27.5 & 1400 & 2150 & 150 & 150 & 1000 & 422 & 422 & 422 & 1.20 & 1.20 & 1.16 & 1155 & 2048 \\
\hline 34 & & $48 \mathrm{M} \mathrm{8-50}$ & 28.0 & 1400 & 2150 & 150 & 150 & 1000 & 528 & 528 & 528 & 0.96 & 0.96 & 0.96 & 1155 & 2038 \\
\hline 35 & & $48 \mathrm{H} 8-30$ & 41.8 & 1400 & 2150 & 150 & 150 & 1000 & 296 & 296 & 296 & 1.60 & 1.60 & 1.60 & 1155 & 2280 \\
\hline 36 & & $48 \mathrm{H} 8-40$ & 43.1 & 1400 & 2150 & 150 & 150 & 1000 & 422 & 422 & 422 & 1.20 & 1.20 & 1.16 & 1155 & 2340 \\
\hline 37 & & $48 \mathrm{H} 8-50$ & 44.6 & 1400 & 2150 & 150 & 150 & 1000 & 528 & 528 & 528 & 0.96 & 0.96 & 0.96 & 1155 & 2430 \\
\hline 38 & \multirow{12}{*}{$\begin{array}{l}\text { G4. Mo and } \\
\text { Chen [M4] }\end{array}$} & HN4-1 & 32.2 & 500 & 860 & 70 & 80 & 170 & 302 & 302 & 302 & 0.92 & 0.73 & 2.10 & 0 & 205 \\
\hline 39 & & HN4-2 & 32.2 & 500 & 860 & 70 & 80 & 170 & 302 & 302 & 302 & 0.92 & 0.73 & 2.10 & 0 & 247 \\
\hline 40 & & HN4-3 & 32.1 & 500 & 860 & 70 & 80 & 170 & 302 & 302 & 302 & 0.92 & 0.73 & 2.10 & 0 & 202 \\
\hline 41 & & HN6-1 & 29.5 & 500 & 860 & 70 & 80 & 170 & 443 & 443 & 443 & 0.92 & 0.73 & 2.10 & 0 & 255 \\
\hline 42 & & HN6-2 & 29.5 & 500 & 860 & 70 & 80 & 170 & 443 & 443 & 443 & 0.92 & 0.73 & 2.10 & 0 & 204 \\
\hline 43 & & HN6-3 & 31.0 & 500 & 860 & 70 & 80 & 170 & 443 & 443 & 443 & 0.92 & 0.73 & 2.10 & 0 & 205 \\
\hline 44 & & HM4-1 & 37.5 & 500 & 860 & 70 & 80 & 170 & 302 & 302 & 302 & 0.92 & 0.73 & 2.10 & 0 & 223 \\
\hline 45 & & HM4-2 & 37.5 & 500 & 860 & 70 & 80 & 170 & 302 & 302 & 302 & 0.92 & 0.73 & 2.10 & 0 & 231 \\
\hline 46 & & HM4-3 & 39.9 & 500 & 860 & 70 & 80 & 170 & 302 & 302 & 302 & 0.92 & 0.73 & 2.10 & 0 & 250 \\
\hline 47 & & LN4-1 & 18.0 & 500 & 860 & 70 & 80 & 170 & 302 & 302 & 302 & 0.92 & 0.58 & 2.10 & 0 & 193 \\
\hline 48 & & LN4-2 & 18.0 & 500 & 860 & 70 & 80 & 170 & 302 & 302 & 302 & 0.92 & 0.58 & 2.10 & 0 & 217 \\
\hline 49 & & LN4-3 & 29.7 & 500 & 860 & 70 & 80 & 170 & 302 & 302 & 302 & 0.92 & 0.58 & 2.10 & 0 & 203 \\
\hline
\end{tabular}




\begin{tabular}{|c|c|c|c|c|c|c|c|c|c|c|c|c|c|c|c|c|}
\hline No. & & Specimen & $\begin{array}{c}f_{c}^{\prime} \\
(\mathrm{MPa})\end{array}$ & $\begin{array}{c}h_{w} \\
(\mathrm{~mm})\end{array}$ & $\begin{array}{c}l_{w} \\
(\mathrm{~mm})\end{array}$ & $\begin{array}{c}t_{w} \\
(\mathrm{~mm})\end{array}$ & $\begin{array}{c}t_{f} \\
(\mathrm{~mm})\end{array}$ & $\begin{array}{c}l_{f} \\
(\mathrm{~mm})\end{array}$ & $\begin{array}{c}f_{y h} \\
(\mathrm{MPa})\end{array}$ & $\begin{array}{c}f_{y v} \\
(\mathrm{MPa})\end{array}$ & $\begin{array}{c}f_{y f} \\
(\mathrm{MPa})\end{array}$ & $\rho_{h}$ & $\rho_{v}$ & $\rho_{f}$ & $\begin{array}{c}P \\
(\mathrm{kN})\end{array}$ & $\begin{array}{l}V_{\text {exp }} \\
(\mathrm{kN})\end{array}$ \\
\hline 50 & & LN6-1 & 30.7 & 500 & 860 & 70 & 80 & 170 & 443 & 443 & 443 & 0.92 & 0.58 & 2.10 & 0 & 246 \\
\hline 51 & & LN6-2 & 30.2 & 500 & 860 & 70 & 80 & 170 & 443 & 443 & 443 & 0.92 & 0.58 & 2.10 & 0 & 200 \\
\hline 52 & & LN6-3 & 30.2 & 500 & 860 & 70 & 80 & 170 & 443 & 443 & 443 & 0.92 & 0.58 & 2.10 & 0 & 210 \\
\hline 53 & & LM6-1 & 39.3 & 500 & 860 & 70 & 80 & 170 & 443 & 443 & 443 & 0.92 & 0.58 & 2.10 & 0 & 219 \\
\hline 54 & & LM6-2 & 37.0 & 500 & 860 & 70 & 80 & 170 & 443 & 443 & 443 & 0.92 & 0.58 & 2.10 & 0 & 205 \\
\hline 55 & & LM6-3 & 34.5 & 500 & 860 & 70 & 80 & 170 & 443 & 443 & 443 & 0.92 & 0.58 & 2.10 & 0 & 210 \\
\hline 56 & & LM4-2 & 66.0 & 500 & 860 & 70 & 80 & 170 & 302 & 302 & 302 & 0.92 & 0.58 & 2.10 & 0 & 250 \\
\hline 57 & & LM4-3 & 66.0 & 500 & 860 & 70 & 80 & 170 & 302 & 302 & 302 & 0.92 & 0.58 & 2.10 & 0 & 227 \\
\hline 58 & G5, Palermo and & DP1 & 21.7 & 2020 & 3075 & 75 & 95 & 3045 & 605 & 605 & 605 & 0.73 & 0.82 & 0.37 & 940 & 1298 \\
\hline 59 & Vecchio [P2] & DP2 & 18.8 & 2020 & 3075 & 75 & 100 & 3045 & 605 & 605 & 605 & 0.73 & 0.82 & 0.35 & 260 & 904 \\
\hline 60 & \multirow{7}{*}{$\begin{array}{c}\text { G6, Farvashany } \\
\text { et al. }[\mathrm{F} 1]\end{array}$} & HSCW1 & 104.0 & 1100 & 880 & 75 & 90 & 375 & 400 & 400 & 400 & 0.47 & 1.26 & 4.02 & 540 & 735 \\
\hline 61 & & HSCW2 & 93.0 & 1100 & 880 & 75 & 90 & 375 & 400 & 400 & 400 & 0.47 & 1.26 & 4.02 & 954 & 845 \\
\hline 62 & & HSCW3 & 86.0 & 1100 & 880 & 75 & 90 & 375 & 400 & 400 & 400 & 0.47 & 0.75 & 4.02 & 953 & 625 \\
\hline 63 & & HSCW4 & 91.0 & 1100 & 880 & 75 & 90 & 375 & 400 & 400 & 400 & 0.47 & 0.75 & 4.02 & 2365 & 866 \\
\hline 64 & & HSCW5 & 84.0 & 1100 & 880 & 75 & 90 & 375 & 400 & 400 & 400 & 0.75 & 1.26 & 4.02 & 955 & 801 \\
\hline 65 & & HSCW6 & 90.0 & 1100 & 880 & 75 & 90 & 375 & 400 & 400 & 400 & 0.75 & 1.26 & 4.02 & 550 & 745 \\
\hline 66 & & HSCW7 & 102.0 & 1100 & 880 & 75 & 90 & 375 & 400 & 400 & 400 & 0.75 & 0.75 & 4.02 & 952 & 800 \\
\hline
\end{tabular}




\begin{tabular}{|c|c|c|c|c|c|c|c|c|c|c|c|c|c|c|c|c|}
\hline No. & $\begin{array}{c}\text { Group, } \\
\text { Researcher }\end{array}$ & Specimen & $\begin{array}{c}f_{c}^{\prime} \\
(\mathrm{MPa})\end{array}$ & $\begin{array}{c}h_{w} \\
(\mathrm{~mm})\end{array}$ & $\begin{array}{c}l_{w} \\
(\mathrm{~mm})\end{array}$ & $\begin{array}{c}t_{w} \\
(\mathrm{~mm})\end{array}$ & $\begin{array}{c}t_{f} \\
(\mathrm{~mm})\end{array}$ & $\begin{array}{c}l_{f} \\
(\mathrm{~mm})\end{array}$ & $\begin{array}{c}f_{y h} \\
(\mathrm{MPa})\end{array}$ & $\begin{array}{c}f_{y v} \\
(\mathrm{MPa})\end{array}$ & $\begin{array}{c}f_{y f} \\
(\mathrm{MPa})\end{array}$ & $\rho_{h}$ & $\rho_{v}$ & $\rho_{f}$ & $\begin{array}{c}P \\
(\mathrm{kN})\end{array}$ & $\begin{array}{l}V_{\text {exp }} \\
(\mathrm{kN})\end{array}$ \\
\hline 67 & \multirow{2}{*}{ G7, Ma } & HPOD0 & 35.9 & 1000 & 1500 & 100 & 100 & 1500 & 335 & 554 & 554 & 0.71 & 0.97 & 1.26 & 74 & 857 \\
\hline 68 & & HP5D0 & 35.9 & 1000 & 1500 & 100 & 100 & 1500 & 335 & 554 & 554 & 0.71 & 0.97 & 1.26 & 794 & 994 \\
\hline \multicolumn{3}{|c|}{ Mean } & 39.63 & 1060 & 1622 & 108 & 112 & 666 & 391 & 400 & 406 & 0.89 & 0.86 & 2.56 & 598 & 1268 \\
\hline \multicolumn{3}{|c|}{ Standard deviation } & 20.19 & 519 & 653 & 35 & 29 & 559 & 95 & 101 & 87 & 0.31 & 0.33 & 1.50 & 554 & 879 \\
\hline \multicolumn{3}{|c|}{ Coefficient of variance } & 0.509 & 0.490 & 0.402 & 0.325 & 0.262 & 0.840 & 0.242 & 0.253 & 0.215 & 0.345 & 0.389 & 0.585 & 0.928 & 0.693 \\
\hline
\end{tabular}




\subsubsection{Uncertainty estimation of unknown model parameters}

As presented in the previous section, the equations deduced from the proposed strut-and-tie model comprised four unknown parameters, $k_{1}$ to $k_{4}$, ranging from 0 to 1 . Each of them has its physical meaning: $k_{1}$ represents the tensile stage of longitudinal reinforcement in the flange, $k_{2}$ stands for the tensile stage of longitudinal reinforcement in the web, $k_{3}$ reflects the tensile stage of horizontal reinforcement in the web, $k_{4}$ is linked to the compressive force of concrete in flange and web. The database rearranged in the last section was used to estimate the posterior cumulative distribution of these four parameters using the GLUE method and to further obtain the probabilistic equation for the peak shear strength of nonrectangular RC squat walls.

The GLUE method contains four main steps. First, define the prior distribution of unknown model parameters and sample with the Monte-Carlo simulation from a feasible parameter space. Second, select the likelihood function of the model and then define the threshold value of the likelihood function to divide parameter sets into behavior and non-behavior groups. Third, calculate the likelihood function of parameter sets in the behavior group and then rescale the values of the likelihood function to formulate a cumulative distribution. Fourth, identify the posterior probability distribution type, and estimate statistical values of the cumulative distribution before finally obtaining the posterior PDF of each unknown model parameter based on the Kolmogorov-Smirnov (K-S) test method. It is clear from the aforementioned procedures that three major aspects should be separately addressed, which are the selection of likelihood function, the determination of threshold values and the assumption of the prior distribution. However, these factors are usually subjectively determined by researchers. Therefore, the influence of different selections for these three major aspects of the estimated cumulative distribution of $k_{1}$ to $k_{4}$ should be carefully investigated.

In this study, the prior distribution of $k_{1}, k_{2}, k_{3}$ and $k_{4}$ was assumed to be uniform and independent. Moreover, the threshold value of the likelihood function was set equal to 0.8. The Nash-Sutcliffe (NS) efficiency [N1] was adopted as the likelihood function as shown below: 


$$
L\left(\Theta_{i} \mid V_{e x p}\right)=1-\frac{\sum_{k=1}^{N}\left(V_{e x p}-V_{k}\right)^{2}}{\sum_{k=1}^{N}\left(V_{e x p}-V_{a v g}\right)^{2}}
$$

where $V_{k}(\mathrm{~N})$ is the predicted shear strength of $\mathrm{k}^{\text {th }}$ specimen for the $\mathrm{i}^{\text {th }}$ parameter set $\Theta_{i}, V_{\text {exp }}(\mathrm{N})$ is the corresponding experimental shear strength, $V_{a v g}(\mathrm{~N})$ is the average value of experimental shear strength of all specimens, $N$ is the number of the specimen in the database. From the equation, it was clear that a perfect prediction would yield a value of 1 for $L$, which suggested that the shear strength equation of the $\mathrm{i}^{\text {th }}$ parameter set $\Theta_{i}$ had a higher likelihood function value for all the experimental data. On the contrary, $L$ would be 0 if the predicted shear strength equaled the average value of experimental shear strength of all specimens, which indicated that the shear strength equation of the $\mathrm{i}^{\text {th }}$ parameter set $\Theta_{i}$ generated similar results to the equation obtained from the traditional nonlinear regression.

Figure 6.15 illustrates the estimated cumulative distribution of $k_{1}, k_{2}, k_{3}$ and $k_{4}$, in which nearly 10000 independent random samples were generated by the MonteCarlo simulation for each unknown parameter. After that, with the threshold value of the NS efficiency defined by 0.8, 324 parameter sets were selected from the 10000 random samples as the behavior parameter set. Then the posterior cumulative distributions of $k_{1}, k_{2}, k_{3}$ and $k_{4}$ were estimated with the likelihood function value of each behavior parameter set. Based on the estimated cumulative distribution of $k_{1}, k_{2}, k_{3}$ and $k_{4}$, the Kolmogorov-Smirnov (K-S) test method was carried out to obtain the traditional theoretical probability distributions, which were the normal distribution, the lognormal distribution, Weibull distribution, Gamma distribution, Extreme Type I distribution, the exponential distribution, Rayleigh distribution, Poisson distribution and the uniform distribution. Table 6.6 presents tested statistical values of each theoretical probability distribution for each unknown model parameter and critical values with a significant level of 5\%. In this table, if the tested statistical value was smaller than the critical value, the hypothesis that satisfying the particular theoretical distribution was accepted and then denoted by $\mathrm{T}$; otherwise, the hypothesis was rejected and denoted by F. 
Table 6.6 Critical values and tested results of the Kolmogorov-Smirnov test

\begin{tabular}{|c|c|c|c|c|c|c|c|c|c|}
\hline \multirow{2}{*}{$\begin{array}{c}\text { Distribution } \\
\text { type }\end{array}$} & Critical & \multicolumn{4}{|c|}{ Tested statistical value } & \multicolumn{4}{|c|}{ Test results } \\
\hline & \multirow{10}{*}{0.075} & $k_{1}$ & $k_{2}$ & $k_{3}$ & $k_{4}$ & $k_{1}$ & $k_{2}$ & $k_{3}$ & $k_{4}$ \\
\hline Normal & & 0.119 & 0.044 & 0.063 & 0.144 & $\mathrm{~F}$ & $\mathrm{~T}$ & $\mathrm{~T}$ & $\mathrm{~F}$ \\
\hline Lognormal & & 0.100 & 0.132 & 0.098 & 0.086 & $\mathrm{~F}$ & $\mathrm{~F}$ & $\mathrm{~F}$ & $\mathrm{~F}$ \\
\hline Weibull & & 0.039 & 0.052 & 0.061 & 0.105 & $\mathrm{~T}$ & $\mathrm{~T}$ & $\mathrm{~T}$ & $\mathrm{~F}$ \\
\hline Gamma & & 0.039 & 0.086 & 0.085 & 0.104 & $\mathrm{~T}$ & $\mathrm{~F}$ & $\mathrm{~F}$ & $\mathrm{~F}$ \\
\hline Extreme Type I & & 0.214 & 0.096 & 0.075 & 0.177 & $\mathrm{~F}$ & $\mathrm{~F}$ & $\mathrm{~F}$ & $\mathrm{~F}$ \\
\hline Exponential & & 0.091 & 0.224 & 0.313 & 0.254 & $\mathrm{~F}$ & $\mathrm{~F}$ & $\mathrm{~F}$ & $\mathrm{~F}$ \\
\hline Rayleigh & & 0.202 & 0.045 & 0.129 & 0.142 & $\mathrm{~F}$ & $\mathrm{~T}$ & $\mathrm{~F}$ & $\mathrm{~F}$ \\
\hline Poisson & & 0.878 & 0.770 & 0.538 & 0.655 & $\mathrm{~F}$ & $\mathrm{~F}$ & $\mathrm{~F}$ & $\mathrm{~F}$ \\
\hline Uniform & & 0.438 & 0.213 & 0.110 & 0.259 & $\mathrm{~F}$ & $\mathrm{~F}$ & $\mathrm{~F}$ & F \\
\hline
\end{tabular}
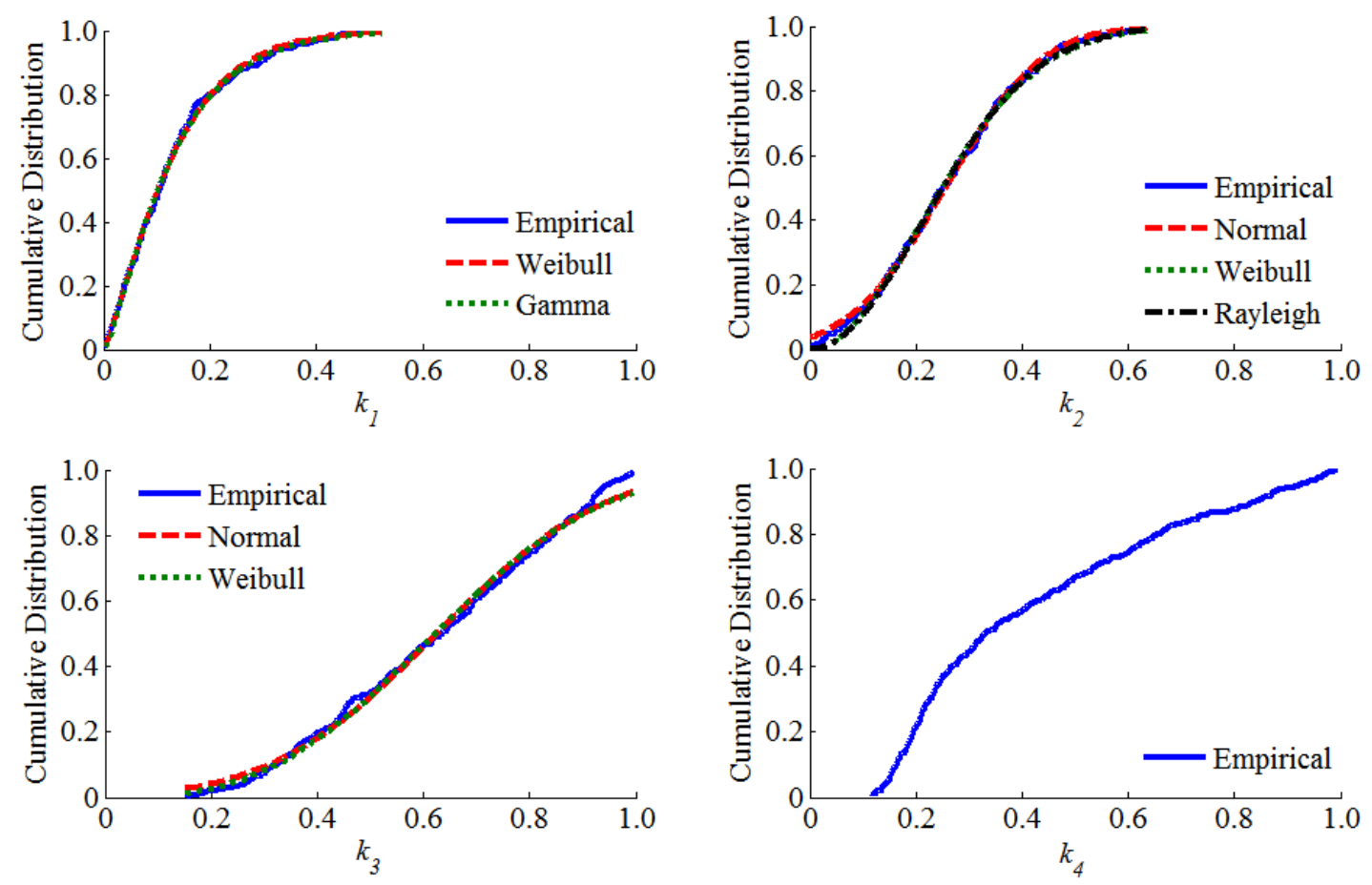

Figure 6.15 Estimated posterior cumulative distributions and theoretical cumulative distributions of $k_{1}, k_{2}, k_{3}$ and $k_{4}$

According to the K-S method, if tested statistical values were smaller than critical values, the hypothesis that satisfied the particular theoretical probability distribution 
would be accepted and denoted as true (T). If not, the hypothesis would be rejected and denoted as false (F). It was clear that the estimated cumulative distribution of $k_{1}$ was able to be accurately represented by the Weibull and Gamma distribution; the estimated cumulative distribution of $k_{2}$ could be well represented by the normal, Weibull and Rayleigh distribution; the estimated cumulative distribution of $k_{3}$ was reasonably fit by the normal and Weibull distribution; the estimated cumulative distribution of $k_{4}$, however, satisfied none of those theoretical probability distributions.

Table 6.7 presents the distributed parameters of the theoretical distribution with the smallest tested statistical values for $k_{1}, k_{2}, k_{3}$ and $k_{4}$, in which the posterior PDF of $k_{1}$ and $k_{3}$ was described by the Weibull distribution; the posterior PDF of $k_{2}$ and $k_{4}$ was described by the Normal distribution and the Lognormal distribution respectively. It should be noted that although the estimated cumulative distribution of $k_{4}$ failed to pass the K-S test, the Lognormal distribution was still selected for the posterior PDF of $k_{4}$, since its tested result was closest to the critical value. As seen in the table, parameters A and B represented the scale and shape parameter for Weibull distribution; mean and standard deviation parameter for Normal distribution; location and scale parameter for Lognormal distribution.

Table 6.7 Distributed parameters of theoretical probability distributions for $k_{1}, k_{2}$, $k_{3}$ and $k_{4}$

\begin{tabular}{ccccc}
\hline Distributed parameter & $k_{1}$ & $k_{2}$ & $k_{3}$ & $k_{4}$ \\
\hline Type & Weibull & Normal & Weibull & Lognormal \\
\hline A & 0.1535 & 0.2390 & 0.7250 & -1.0339 \\
\hline B & 1.3801 & 0.1455 & 3.2545 & 0.7231 \\
\hline
\end{tabular}

Moreover, to examine the posterior distribution range of $k_{1}, k_{2}, k_{3}$ and $k_{4}$ after the GLUE ran, a reduction coefficient $R_{i}$ is given by:

$$
R_{i}=1-\frac{k_{i}^{\text {pos,upp }}-k_{i}^{\text {pos,low }}}{k_{i}^{\text {pro,upp }}-k_{i}^{\text {pro,low }}}
$$


where $i$ stands for $1,2,3$ and $4, k_{i}^{\text {pos,upp }}$ and $k_{i}^{\text {pos,low }}$ are the upper and lower bounds of the posterior distribution range of $k_{i}$ respectively, $k_{i}^{\text {pro,upp }}$ and $k_{i}^{\text {pro,low }}$ are the upper and lower bounds of the prior distribution range of $k_{i}$ respectively. From the results, it could be concluded that $k_{1}$ was reduced by $47.6 \%$ from 0 to 1 to 0 to $0.5245, k_{2}$ was reduced by $36.7 \%$ from 0 to 1 to 0.0014 to $0.6342, k_{3}$ was reduced by $15.6 \%$ from 0 to 1 to 0.1523 to 0.9968 , and $k_{4}$ was reduced by $12.19 \%$ from 0 to 1 to 0.1185 to 0.9966 . According to the GLUE method, a smaller reduction coefficient suggested that the unknown model parameter had a higher level of equifinality, which meant the equifinalities of four unknown model parameters in the proposed probabilistic equation for the peak shear strength of nonrectangular RC squat walls had a descending order from $k_{4}$ to $k_{1}$.

Table 6.8 demonstrates the correlation coefficient of the prior and posterior distribution of the four unknown model parameters. It was clear that after the GLUE ran, the relationship between these four parameters changed from independent to dependent because of the parameter selection by the likelihood function. Nonetheless, all absolute magnitudes of the correlation coefficient between $k_{1}$, $k_{2}, k_{3}$ and $k_{4}$ were smaller than 0.5 . In statistical analyses, two parameters were deemed to have a low correlation when the absolute magnitude of the correlation coefficient was between 0.3 to 0.5 , and little or no correlation when the absolute magnitude of the correlation coefficient was less than 0.3. Thus, it was concluded that those four parameters were still poorly correlated after the GLUE ran. The proposed probabilistic equation for the peak shear strength of non-rectangular RC squat walls would be further simplified as a mean prediction equation and a standard deviation prediction equation. 
Table 6.8 Prior and posterior correlation coefficients of $k_{1}, k_{2}, k_{3}$ and $k_{4}$

\begin{tabular}{|c|c|c|c|c|c|c|c|c|}
\hline \multirow[t]{2}{*}{ Parameter } & \multicolumn{4}{|c|}{$\begin{array}{l}\text { Correlation coefficient of prior } \\
\text { distribution }\end{array}$} & \multicolumn{4}{|c|}{$\begin{array}{c}\text { Correlation coefficient of posterior } \\
\text { distribution }\end{array}$} \\
\hline & $k_{1}$ & $k_{2}$ & $k_{3}$ & $k_{4}$ & $k_{1}$ & $k_{2}$ & $k_{3}$ & $k_{4}$ \\
\hline$k_{1}$ & 1.00 & -0.02 & 0.00 & 0.00 & 1.00 & -0.32 & -0.03 & -0.48 \\
\hline$k_{2}$ & -0.02 & 1.00 & 0.01 & 0.00 & -0.32 & 1.00 & -0.34 & -0.25 \\
\hline$k_{3}$ & 0.00 & 0.01 & 1.00 & -0.01 & -0.03 & -0.34 & 1.00 & 0.03 \\
\hline$k_{4}$ & 0.00 & 0.00 & -0.01 & 1.00 & -0.48 & -0.25 & 0.03 & 1.00 \\
\hline
\end{tabular}

\subsubsection{Sensitivity analysis of the GLUE method}

As mentioned in the introduction to the GLUE method, the assumption of the prior distribution, the selection of the likelihood function and the determination of threshold values play a pivotal role in the estimation of cumulative probability of $k_{1}$, $k_{2}, k_{3}$ and $k_{4}$. In this section, these three important aspects were investigated to demonstrate the stability of the GLUE method.

\section{Assumption of the prior distribution}

Based on the formal Bayesian theory, the prior distribution of $k_{1}, k_{2}, k_{3}$ and $k_{4}$ should be determined by engineering experience. The assumption of the prior distribution should have minimal influence on the posterior probability distribution to avoid the probabilistic inference being affected by information external to the measurement [G1]. In the case of no prior information on unknown model parameters being obtained, the uniform prior distribution could be adopted, whereas the informative prior distribution such as the normal distribution could be adopted if substantial prior information was provided. Therefore, uniform and normal distributions were both adopted to investigate the influence of various prior distributions, where the prior distributions of the four parameters were still assumed to be independent ranging from 0 to 1 . Since the range of the prior distribution was determined, the normal prior distribution became a truncated Gaussian probability distribution with the maximum and minimum limits. As a result, both mean and the 
standard deviation of the Gaussian distribution with a value of 0.5 were employed to generate 10000 random samples within the interval region. Figure 6.16 illustrates estimated cumulative distribution of $k_{1}, k_{2}, k_{3}$ and $k_{4}$ for the uniform and normal prior distributions respectively. It was clear that their estimated cumulative distributions were almost identical, which indicated that a dramatic modification of the prior distribution only led to minor modification on the posterior probability distribution when provided with a sufficient number of test specimens. The observation was in accordance with the findings of Box and Tiao [B4].
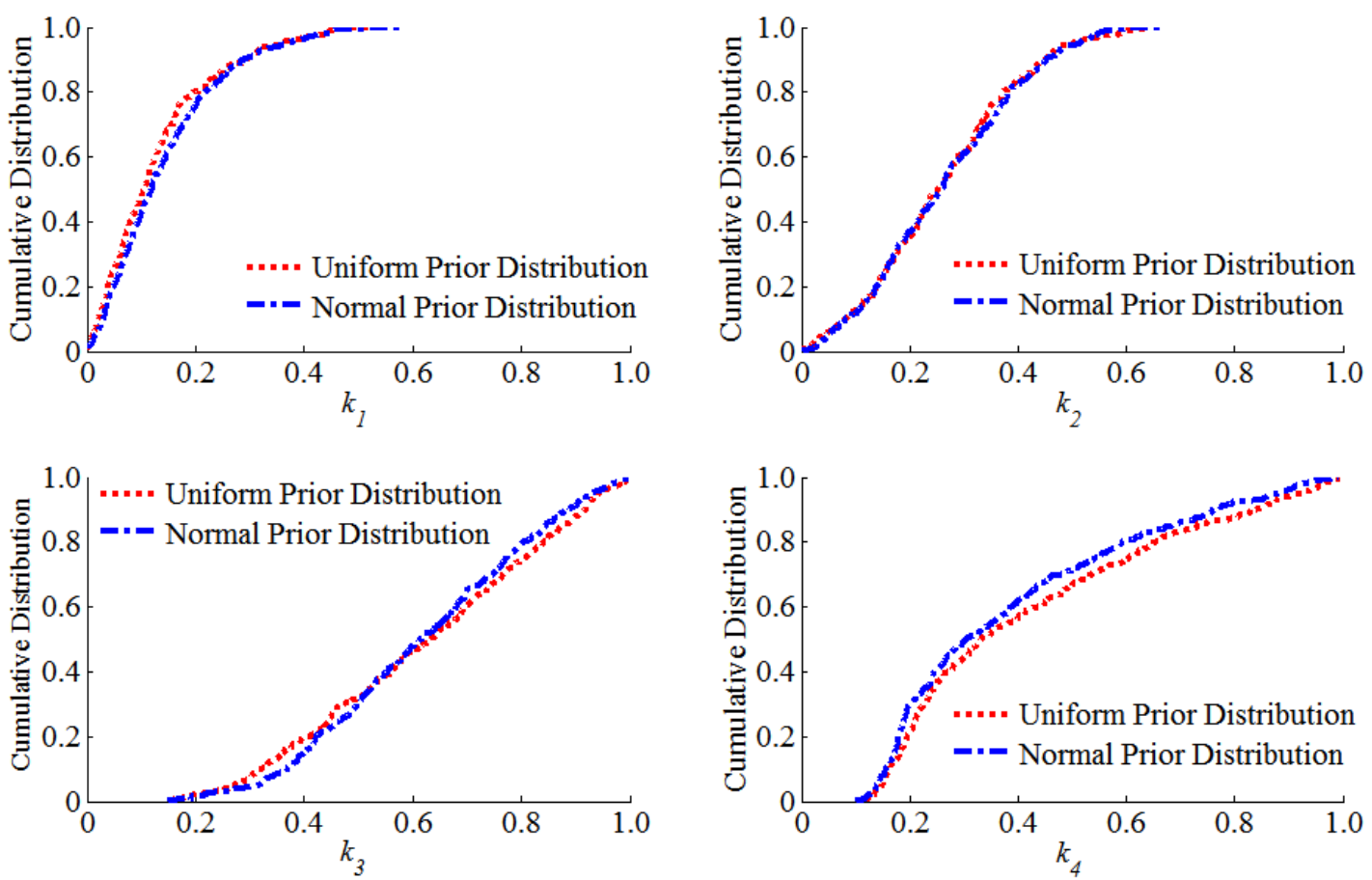

Figure 6.16 Posterior cumulative distributions of $k_{1}, k_{2}, k_{3}$ and $k_{4}$ estimated from the uniform and the normal prior distributions

2. Determination of the threshold value

In the GLUE method, the determination of the threshold value was utilized to divide parameter sets into the behavior and non-behavior groups. This approach has been subjected to criticism for its subjectivity and arbitrariness. Montanari [M5] demonstrated that the determination of the threshold value had a significant impact on the parameter uncertainty and results of simulations. To present the sensitivity of the different threshold value determinations on the estimated cumulative distribution of $k_{1}, k_{2}, k_{3}$ and $k_{4}$, values of $0.6,0.7$ and 0.8 were selected in this 
study. Figure 6.17 shows the estimated cumulative distribution of these four parameters with the aforementioned threshold values. It was witnessed that a smaller threshold value yielded a flatter estimated cumulative distribution curve than a larger threshold value. This was due to the increase of parameter sets, which were further categorized into the behavior group when the threshold value changed from 0.8 to 0.6. Also, more uncertainties occurred for the unknown model parameter and the results of the simulation. However, no more parameter sets were divided into the behavior group once the threshold value of the NS efficiency was too large, such as 0.9. This was because the proposed probabilistic shear strength model could not guarantee a perfect prediction for every specimen due to the inherent nature of epistemic uncertainty. Therefore, the subjectivity of determining the threshold value of the likelihood function should be investigated carefully in the application of the GLUE method. A larger threshold value is generally recommended for the NS efficiency, rather than a larger value and the better model performance. In fact, the determination of the threshold value is strongly dependent on the uncertainty of the unknown model parameters that users want to retain.
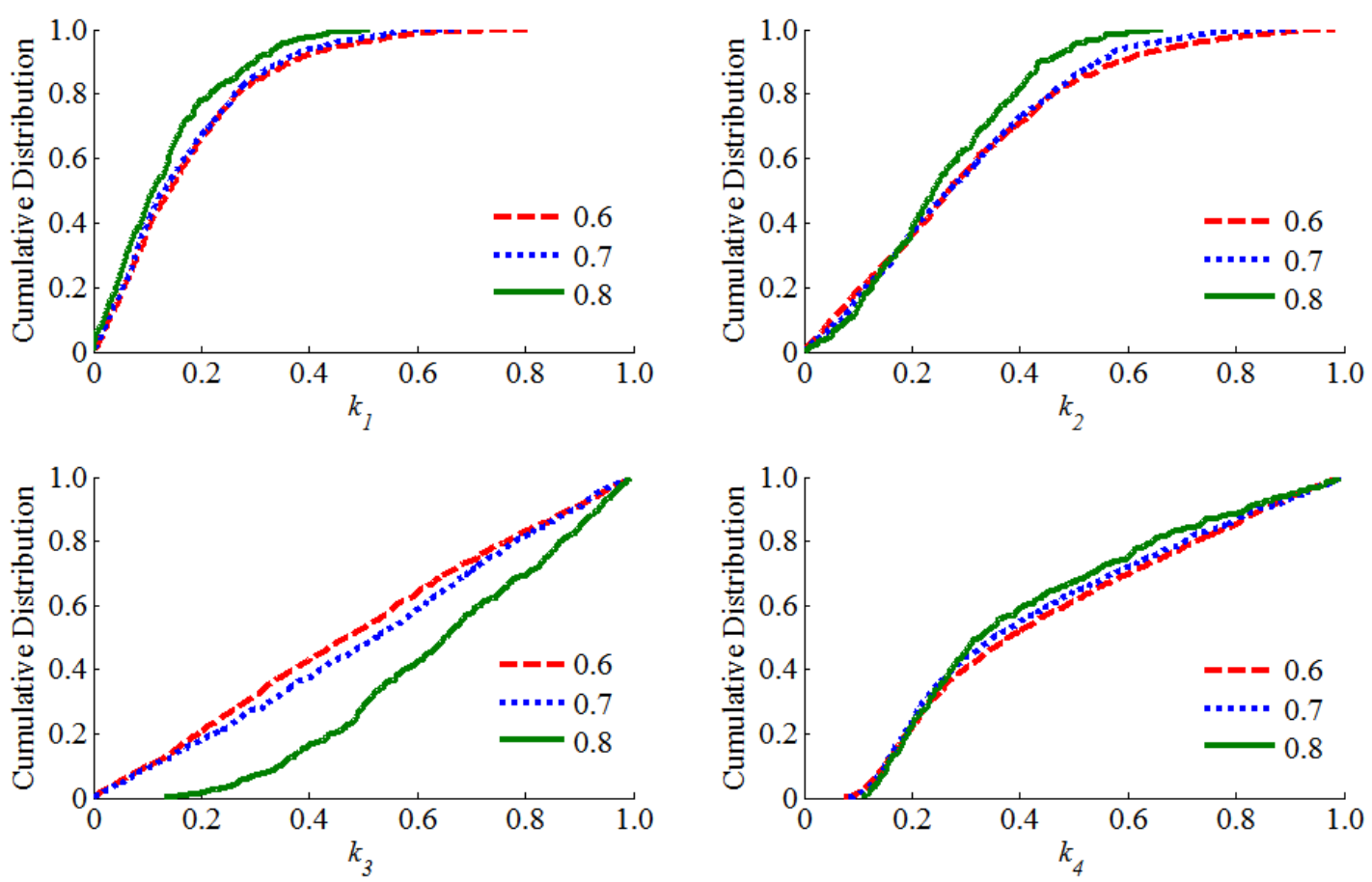

Figure 6.17 Posterior cumulative distributions of $k_{1}, k_{2}, k_{3}$ and $k_{4}$ estimated with threshold values of $0.6,0.7$ and 0.8

3. Selection of the likelihood function 
The selection of the likelihood function was important in estimating the posterior cumulative distribution of the unknown model parameters since it accounted for the performance of the proposed predictive equation. As mentioned previously, various likelihood functions have been proposed and evaluated by researchers over the past decades [B2, N1]. Based on the normal probability distribution assumption, Makowski et al. [M1] proposed the likelihood function as shown below:

$$
L\left(\Theta_{i} \mid V_{\text {peak }}\right)=\prod_{k=1}^{N} \frac{1}{\sqrt{2 \pi \sigma_{0}^{2}}} \exp \left[-\frac{\left(V_{\text {exp }}-V_{k}\right)^{2}}{2 \sigma_{0}^{2}}\right]
$$

where $\sigma_{0}$ is the standard deviation of model error, which is assumed to be the standard deviation of the measurement. Alternatively, based on the minimum mean square error, Wang et al. [W5] defined the likelihood function as:

$$
L\left(\Theta_{i} \mid V_{\text {peak }}\right)=\exp \left[-\frac{\frac{1}{N} \sum_{k=1}^{N}\left(V_{\text {exp }}-V_{k}\right)^{2}}{2 \sigma_{0}^{2}}\right]
$$

In order to investigate the influence of different likelihood functions on estimated cumulative distributions of $k_{1}, k_{2}, k_{3}$ and $k_{4}$, Equation 6.28, Equation 6.30 and Equation 6.31 were selected as the likelihood function in the GLUE method. Figure 6.18 demonstrates the estimated cumulative distribution of the four model parameters, in which the threshold value of each likelihood function was defined by $30 \%$ of the best estimate for comparison. It was observed that different likelihood functions had a moderate influence on the estimated cumulative distributions of the four model parameters. Compared with the determination of threshold value, less sensitivity was found on the selection of likelihood function. Thus, the NS efficiency was recommended as the likelihood function to estimate the posterior cumulative distribution of $k_{1}, k_{2}, k_{3}$ and $k_{4}$ for the proposed probabilistic shear strength equation of non-rectangular RC squat walls. 

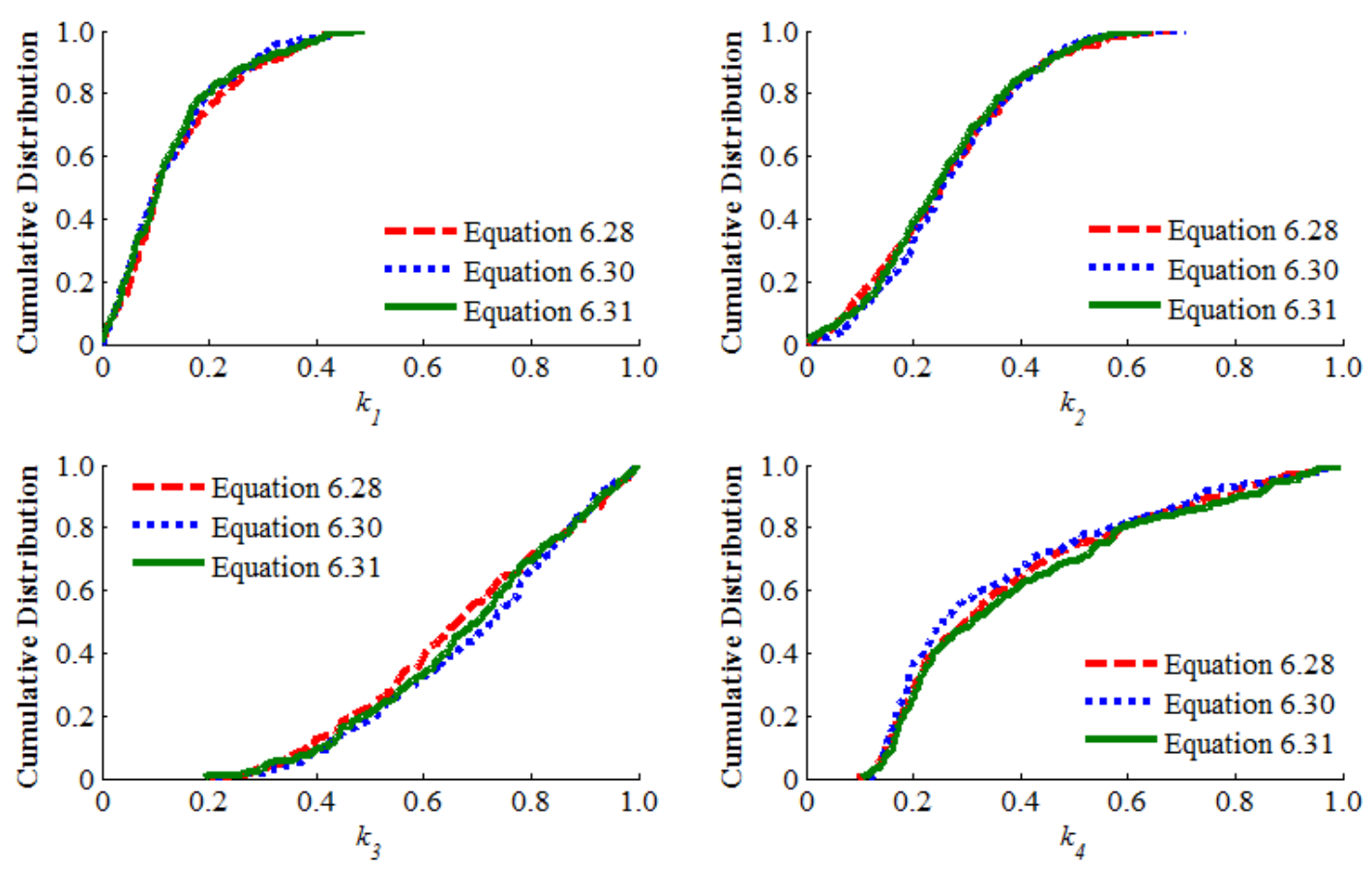

Figure 6.18 Posterior cumulative distributions of $k_{1}, k_{2}, k_{3}$ and $k_{4}$ estimated with different likelihood functions

\subsubsection{Evaluation of the proposed probabilistic equation}

According to the sensitivity analysis of the GLUE method, the estimated cumulative distributions of $k_{1}, k_{2}, k_{3}$ and $k_{4}$ were almost identical under each GLUE ran. This showed the stability of the proposed probabilistic equation. To demonstrate the improved performance of the proposed probabilistic equation in terms of reconciling the large scatter and avoiding the biased estimate, Specimen W12-3 tested by Saito et al. [S2] and Specimen LN4-3 tested by Mo and Chan [M4] were randomly selected from the database for the comparison with the deterministic predictions. The Monte-Carlo simulation was used to generate approximately 10000 parameter sets for $k_{1}, k_{2}, k_{3}$ and $k_{4}$. Then based on Equation 6.14 and Equation 6.18, 10000 values of the shear strength were produced by the corresponding 10000 parameter sets for each specimen. Figure 6.19 and Figure 6.20 show the histogram of the obtained 10000 samples of the shear strength for each specimen, together with the fitted probability density function (PDF) using the K-S test at a significant level of 5\%. It was found that the measured shear strength became a quantile of the predicted PDF and was located near the point with the maximum probability. 


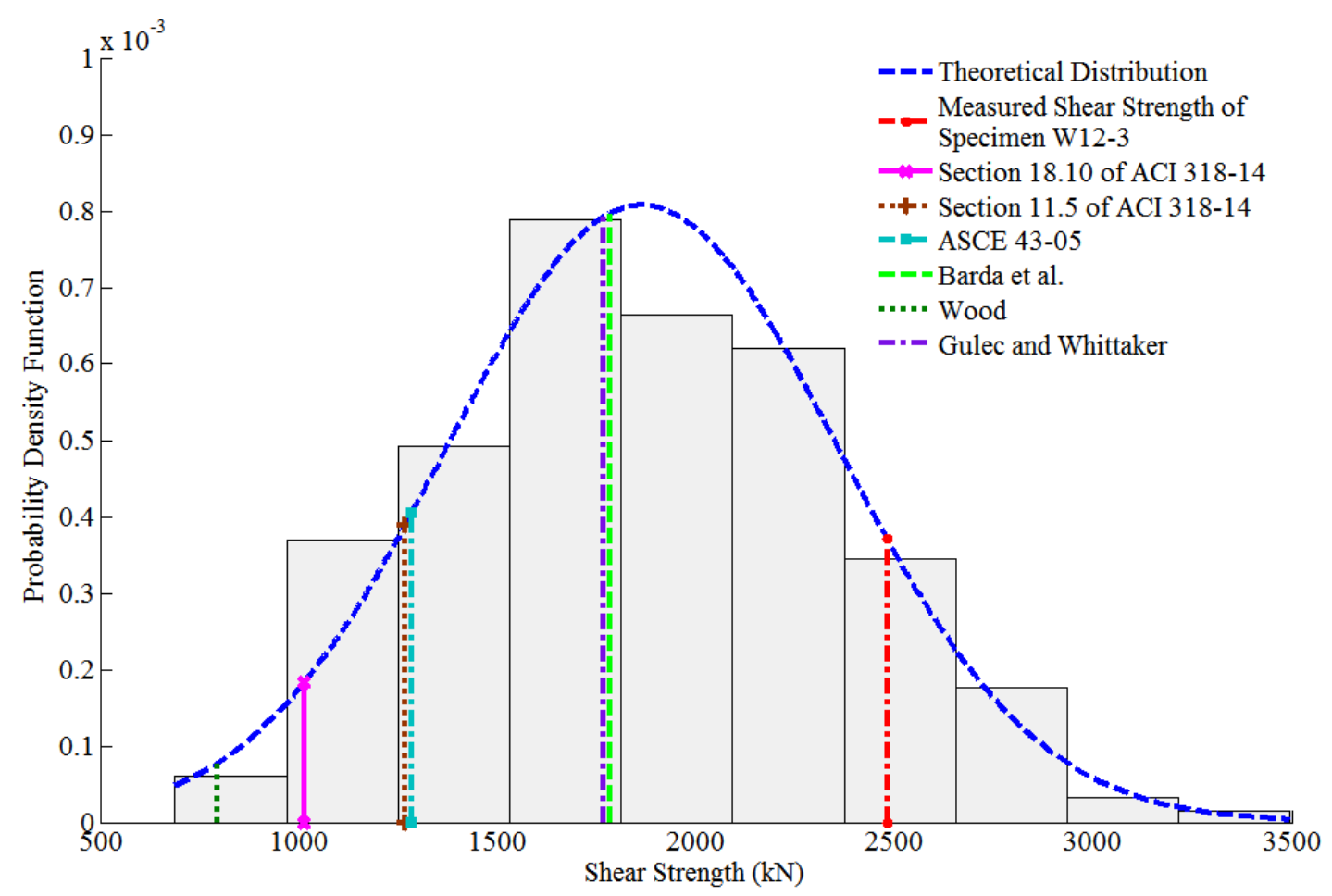

Figure 6.19 Probability density function of the predicted shear strength in comparison with the measured shear strength and deterministic predictions for Specimen W12-3 [S2]

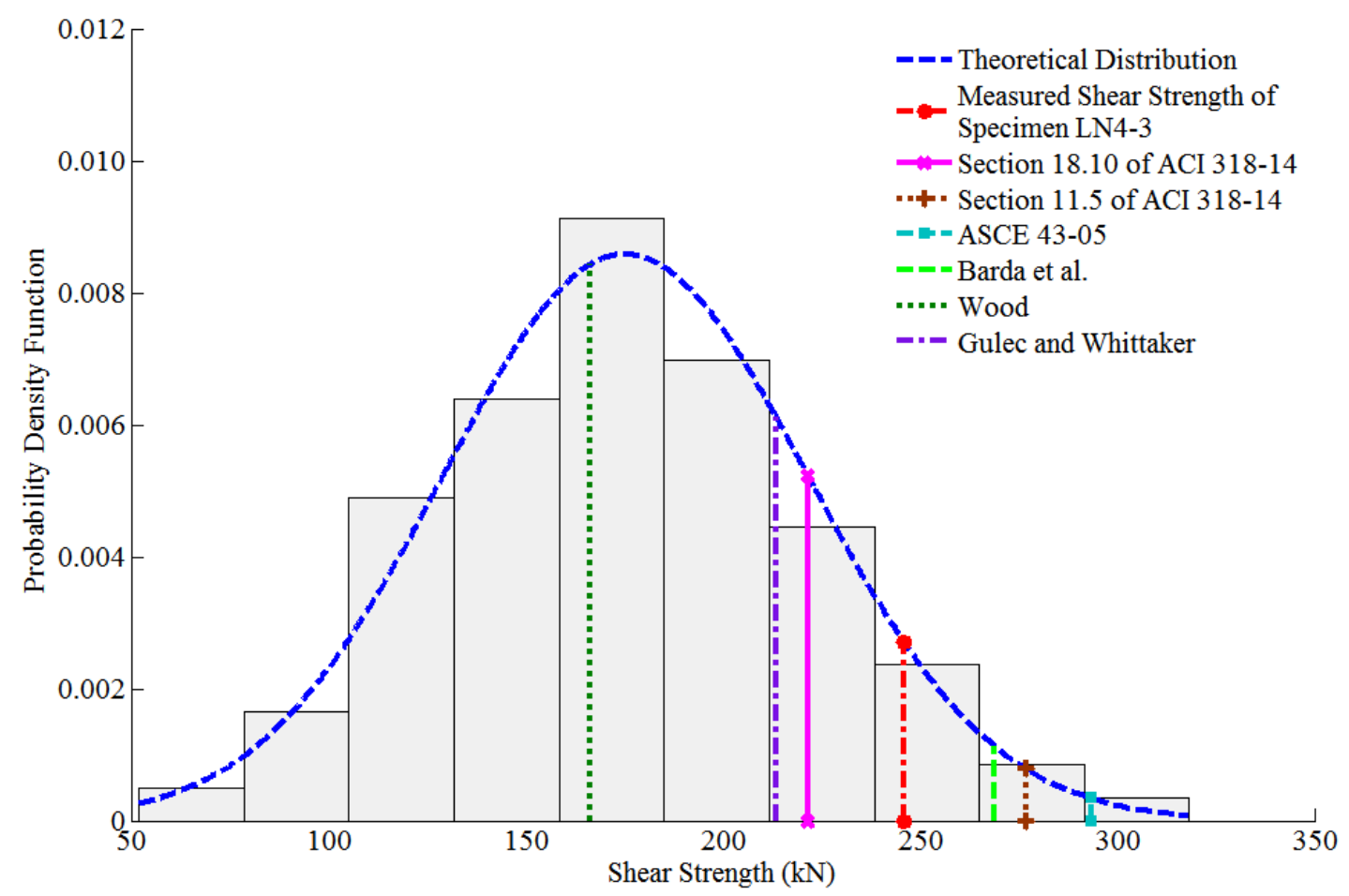

Figure 6.20 Probability density function of the predicted shear strength in comparison with the measured shear strength and deterministic predictions for Specimen LN4-3 [M4] 
This was because the measured data was also deterministic and uncertainties were usually not provided by researchers. Therefore, it was unable to compare the predicted PDF of shear strength with the measured PDF of shear strength. The inherent uncertainties such as those obtained from the measurement and from materials, were barely investigated and discussed in the test. Moreover, as shown by Li et al. [L4], the randomness of materials had a considerable contribution to the structural damage evolution. Therefore, in the experiment, attentions are required for the quantification of uncertainties of macroscopic mechanical properties and the method of obtaining the PDF of the measured data.

Furthermore, as with the experimental data, the peak shear strength predicted by the deterministic equations was also a quantile of the predicted PDF. Different accuracies of prediction were attained by different deterministic equations. Therefore, the accuracies of these deterministic equations could be better evaluated with the probabilistic method by calculating their probabilities of underestimating or overestimating the measured data, rather than by using the traditional assessment based on modeling error. Considering the experimental shear strength as the threshold value of the predicted PDF, the probability of the predicted shear strength exceeding the measured data is written as:

$$
P_{f}=\int_{V_{\text {exp }}}^{V} p(x) d x
$$

where $p(x)$ is the predicted PDF of shear strength. Table 6.9 demonstrates the probabilities of the predicted shear strength underestimating its experimental counterpart using various equations. It was observed that for Specimen W12-3, all the selected deterministic shear strength equations underestimated the measured shear strength, while for Specimen LN4-3, only the equations developed by Wood [W7], Gulec and Whittaker [G3], Section 18.10 of ACI 318-14 [A1] underestimated the peak shear strength. 
Table 6.9 Probabilities of the predicted shear strength underestimating the measured shear strength

\begin{tabular}{|c|c|c|c|c|c|c|}
\hline Specimen & $\begin{array}{c}\text { ACI 318-14 } \\
\S 11.5[\mathrm{~A} 1]\end{array}$ & $\begin{array}{c}\text { ACI 318-14 } \\
\S 18.10 \text { [A1] }\end{array}$ & $\begin{array}{c}\text { ASCE 43- } \\
05[\mathrm{~A} 3]\end{array}$ & $\begin{array}{c}\text { Barda } \text { et } \\
\text { al. }[\mathrm{B} 1]\end{array}$ & $\begin{array}{c}\text { Wood } \\
\text { [W7] }\end{array}$ & $\begin{array}{c}\text { Gulec and } \\
\text { Whittaker [G3] }\end{array}$ \\
\hline W12-3 & 0.84 & 0.78 & 0.77 & 0.47 & 0.87 & 0.49 \\
\hline LN4-3 & 0.09 & -0.05 & -0.06 & -0.04 & 0.50 & 0.14 \\
\hline
\end{tabular}

\subsubsection{Simplification of the proposed probabilistic equation}

The proposed probabilistic equation had proven its ability to provide the predicted PDF of shear strength for each specimen. Nonetheless, the probabilistically proposed equation may be found inconvenient when applied in engineering practice. Thus, it required further simplification as represented by a mean prediction equation and a standard deviation prediction equation. Based on the estimated cumulative distribution of $k_{1}, k_{2}, k_{3}$ and $k_{4}$, the corresponding mean and standard deviation are calculated as:

$$
\begin{gathered}
\mu_{\Theta}=\sum_{i=1}^{N} p\left(\Theta_{i}\right) \cdot \Theta_{i} \\
\sigma_{\Theta}^{2}=\sum_{i=1}^{N} p\left(\Theta_{i}\right) \cdot\left(\Theta_{i}-\mu_{\Theta}\right)^{2}
\end{gathered}
$$

where $N$ is the number of the behavior parameter set, $p\left(\Theta_{i}\right)$ is the probability of the $\mathrm{i}^{\text {th }}$ behavior parameter set. In the behavior parameter set, $p\left(\Theta_{i}\right)$ is determined by rescaling the value of the likelihood function of the behavior parameter set as:

$$
p\left(\Theta_{i}\right)=\frac{L\left(\Theta_{i} \mid V_{\text {exp }}\right)}{\sum_{j=1}^{N} L\left(\Theta_{i} \mid V_{\text {exp }}\right)}
$$

Combining Equation 6.33 to Equation 6.35, the mean of $k_{1}, k_{2}, k_{3}$ and $k_{4}$ was obtained as $0.14,0.24,0.64$ and 0.43 respectively, and the standard deviation of $k_{1}$, $k_{2}, k_{3}$ and $k_{4}$ was obtained as $0.10,0.14,0.21$ and 0.26 respectively. Thus it can be concluded that the longitudinal reinforcement in the flange, the longitudinal reinforcement in the web, the horizontal reinforcement in the web and the 
compressive stress in concrete in flange and web experienced various stress stages when the peak shear strength was achieved. Moreover, it was noted that the horizontal reinforcement in the web $\left(k_{3}\right)$ and the compressive stress in concrete in flange and web $\left(k_{4}\right)$ had higher utilization while the longitudinal reinforcement in the flange $\left(k_{1}\right)$ and the longitudinal reinforcement in the web $\left(k_{2}\right)$ had a lower efficiency to the shear strength.

Furthermore, based on the estimated mean and standard deviation of $k_{1}, k_{2}, k_{3}$ and $k_{4}$, the mean and the variance of shear strength could be obtained by expanding Equation 6.27 into a series of forms consisting of only the linear terms with the stochastic analysis theory, which is given as:

$$
\begin{gathered}
\mu_{V}=F\left(\mu_{\Theta}, X\right) \\
\sigma_{V}^{2}=\sum_{i=1}^{4}\left[\left.\frac{\partial F}{\partial \theta_{i}}\right|_{\theta_{i}=\mu_{\theta_{i}}}\right]^{2} \sigma_{\theta_{i}}^{2}+\left.\left.\sum_{i=1}^{4} \sum_{j=1}^{4} \frac{\partial F}{\partial \theta_{i}}\right|_{\theta_{i}=\mu_{\theta_{i}}} \frac{\partial F}{\partial \theta_{j}}\right|_{\theta_{i}=\mu_{\theta_{j}}} \operatorname{cov}\left[\theta_{i}, \theta_{j}\right]
\end{gathered}
$$

where $\Theta=\left[k_{1}, k_{2}, k_{3}, k_{4}\right], \operatorname{cov}\left[\theta_{i}, \theta_{j}\right]$ is the covariance of random variables $\theta_{i}$ and $\theta_{j}$. As stated in the coefficient analysis, the covariance of four parameters could be assumed to be absolutely uncorrelated after the GLUE ran, so Equation 6.37 could be replaced by:

$$
\sigma_{V}^{2}=\sum_{i=1}^{4}\left[\left.\frac{\partial F}{\partial k_{i}}\right|_{k_{i}=\mu_{k_{i}}}\right] \sigma_{k_{i}}^{2}
$$

After that, the differentiation of Equation 6.27 could be derived with respect to $k_{1}$, $k_{2}, k_{3}$ and $k_{4}$. Therefore, based on the Equation 6.14 obtained from the proposed strut-and-tie mode, the function form $F$ is given with respect to $k_{1}$ as:

$$
\begin{gathered}
\frac{\partial F}{\partial k_{1}}=-\left(\mu_{k_{1}} f_{y f} \rho_{f} t_{f} l_{f}+\mu_{k 2} f_{y v} \rho_{v} t_{w} z_{w}+\frac{P}{2}\right) \frac{1}{h_{w}} \frac{f_{y f} \rho_{f} t_{f} l_{f}}{2 \mu_{k_{4}} f_{c}^{\prime} t_{w}} \\
+\frac{d_{w}}{h_{w}} f_{y f} \rho_{f} t_{f} l_{f}
\end{gathered}
$$

Similarly, the function form $F$ could also be written with respect to $k_{2} k_{3}$ and $k_{4}$ as: 


$$
\begin{gathered}
\frac{\partial F}{\partial k_{2}}=f_{y v} \rho_{v} t_{w} z_{w} \frac{d_{w}}{h_{w}} \\
\frac{\partial F}{\partial k_{2}}=f_{y h} \rho_{h} t_{w} h_{w} \\
\frac{\partial F}{\partial k_{3}}=\left(\mu_{k_{1}} f_{y f} \rho_{f} t_{f} l_{f}+\mu_{k 2} f_{y v} \rho_{v} t_{w} z_{w}+\frac{P}{2}\right) \frac{k_{1} f_{y f} \rho_{f} t_{f} l_{f}+P}{2 f_{c}^{\prime} t_{w} h_{w} \mu_{k_{4}}^{2}}
\end{gathered}
$$

Therefore, substituting Equation 6.39 to Equation 6.42 into Equation 6.38, the variance of the proposed probabilistic equation of the peak shear strength of nonrectangular RC squat walls is calculated as:

$$
\begin{gathered}
\sigma_{V}^{2}=\left[-\left(\mu_{k_{1}} f_{y f} \rho_{f} t_{f} l_{f}+\mu_{k 2} f_{y v} \rho_{v} t_{w} z_{w}+\frac{P}{2}\right) \frac{1}{h_{w}} \frac{f_{y f} \rho_{f} t_{f} l_{f}}{2 \mu_{k_{4}} f_{c}^{\prime} t_{w}}+\frac{d_{w}}{h_{w}} f_{y f} \rho_{f} t_{f} l_{f}\right]^{2} \sigma_{k_{1}}^{2} \\
+\left(f_{y v} \rho_{v} t_{w} z_{w} \frac{d_{w}}{h_{w}}\right)^{2} \sigma_{k_{2}}^{2}+\left(f_{y h} \rho_{h} t_{w} h_{w}\right)^{2} \sigma_{k_{3}}^{2} \\
+\left[\left(\mu_{k_{1}} f_{y f} \rho_{f} t_{f} l_{f}+\mu_{k 2} f_{y v} \rho_{v} t_{w} z_{w}+\frac{P}{2}\right) \frac{k_{1} f_{y f} \rho_{f} t_{f} l_{f}+P}{2 f_{c}^{\prime} t_{w} h_{w} \mu_{k_{4}}^{2}}\right]^{2} \sigma_{k_{4}}^{2}
\end{gathered}
$$

The obtained Equation 6.43 could be rewritten by substituting the mean and standard deviation of $k_{1}, k_{2}, k_{3}$ and $k_{4}$ after the GLUE ran as:

$$
\begin{gathered}
\sigma_{V}^{2}=\left(0.14 f_{y v} \rho_{v} t_{w} z_{w} \frac{d_{w}}{h_{w}}\right)^{2}+\left(0.21 f_{y h} \rho_{h} t_{w} h_{w}\right)^{2} \\
+0.01\left[-\left(0.14 f_{y f} \rho_{f} t_{f} l_{f}+0.24 f_{y v} \rho_{v} t_{w} z_{w}+\frac{P}{2}\right) \frac{1}{h_{w}} \frac{f_{y f} \rho_{f} t_{f} l_{f}}{0.86 f_{c}^{\prime} t_{w}}+\frac{d_{w}}{h_{w}} f_{y f} \rho_{f} t_{f} l_{f}\right]^{2} \\
+\left[0.26\left(0.14 f_{y f} \rho_{f} t_{f} l_{f}+0.24 f_{y v} \rho_{v} t_{w} z_{w}+\frac{P}{2}\right) \frac{k_{1} f_{y f} \rho_{f} t_{f} l_{f}+P}{0.37 f_{c}^{\prime} t_{w} h_{w}}\right]^{2}
\end{gathered}
$$

Similarly, Equation 6.36 could also be rewritten by substituting the mean of $k_{1}$, $k_{2}, k_{3}$ and $k_{4}$ after the GLUE ran as:

$$
\begin{gathered}
\mu_{V}=\left(0.14 f_{y f} \rho_{f} t_{f} l_{f}+0.24 f_{y v} \rho_{v} t_{w} z_{w}+\frac{P}{2}\right) \\
{\left[\frac{l_{w}-t_{f}}{h_{w}}-\frac{1}{2}\left(\frac{0.14 f_{y f} \rho_{f} t_{f} l_{f}+P}{0.43 f_{c}^{\prime} t_{w} h_{w}}-\frac{t_{f}}{t_{w}} \frac{l_{f}}{h_{w}}\right)\right]+0.64 f_{y h} \rho_{h} t_{w} h_{w}}
\end{gathered}
$$


where $d_{w}$ becomes:

$$
d_{w}=l_{w}-t_{f}-\frac{1}{2}\left(\frac{0.14 f_{y f} \rho_{f} t_{f} l_{f}+P}{0.43 f_{c}^{\prime} t_{w}}-\frac{t_{f} l_{f}}{t_{w}}\right)
$$

\subsubsection{Uncertainty bounds of the prediction of shear strength}

Once the model parameters of non-rectangular RC squat walls were determined, the confidence interval of shear strength could be estimated directly by Equation 6.44 and Equation 6.46 to provide upper and lower confidence limits. Figure 6.21 illustrates the comparison of the confidence intervals of predicted shear strength and experimental data. It was observed that the confidence interval in terms of the mean plus or minus one times of standard deviation (Mean \pm STD) was unable to cover all the test data, whereas almost all the measured data were within the confidence interval in terms of the mean plus or minus two times of standard deviation (Mean $\pm 2 \times$ STD). To quantitatively describe the performance of the proposed probabilistic equation, an index is further given as the average relative interval length (ARIL):

$$
\mathrm{ARIL}=\frac{1}{M} \sum_{i=1}^{M} \frac{V_{i}^{U}-V_{i}^{L}}{V_{\text {exp }}}
$$

where $V_{i}^{U}$ and $V_{i}^{L}$ are the calculated upper and lower confidence limits for the $\mathrm{i}^{\text {th }}$ specimen in the database, $M$ is the number of experimental data. It was evident that a smaller ARIL value and a larger percentage of measurement reflected a better performance of the proposed model. Figure 6.22 illustrates the relationship of the ARIL and the percentage of measurement coverage versus the corresponding confidence interval by increasing the factor of standard deviation from 0.5 to 3.0 to determine the confidence interval. It was clear that both the ARIL and the percentage of measurement coverage ascended with the rising of the confidence interval. However, the former was found to grow linearly while the latter increased nonlinearly. For this reason, the confidence interval of shear strength was given as Mean $\pm 2 \times$ STD to cover around $80 \%$ of the measured data, which yielded a corresponding value of the ARIL as 1.06 . 


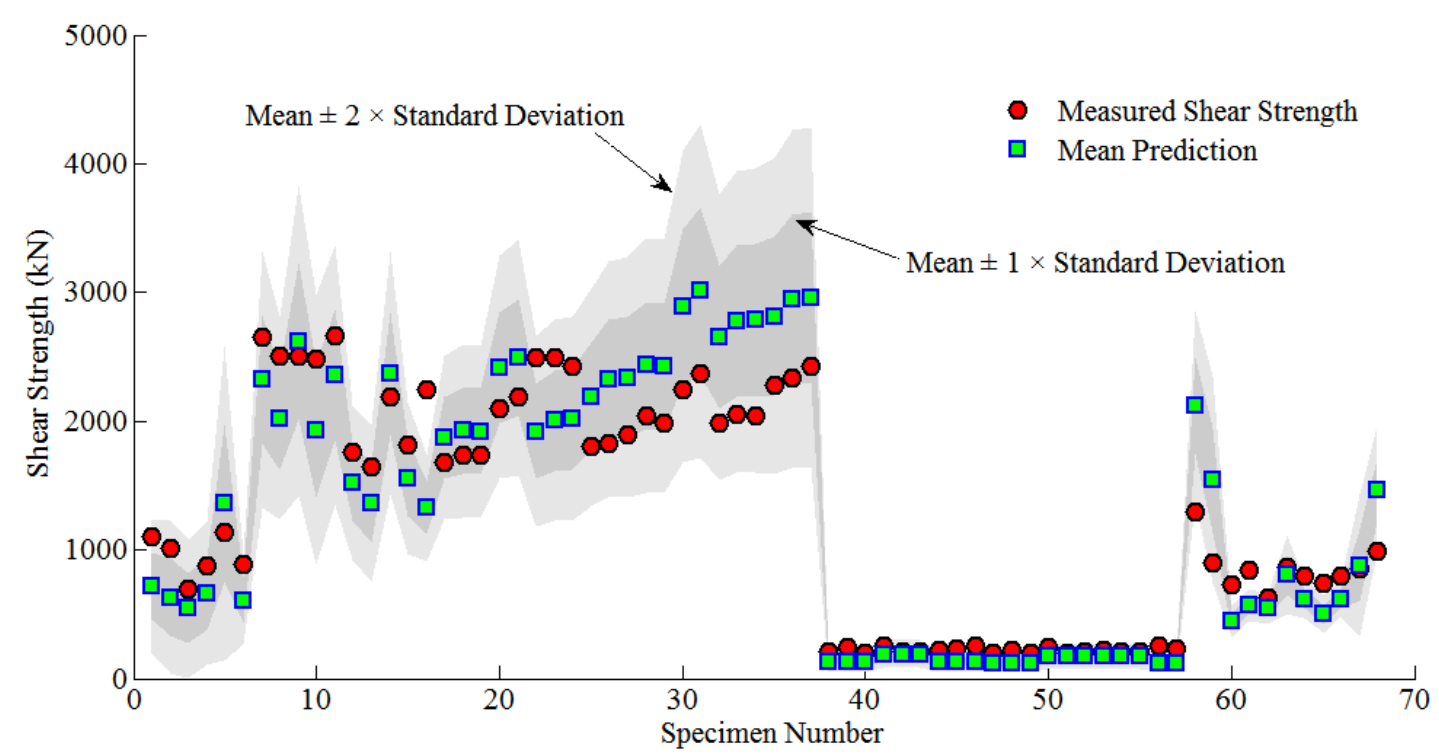

Figure 6.21 Mean prediction, Mean \pm STD and Mean $\pm 2 \times$ STD for the shear strength of non-rectangular RC squat walls
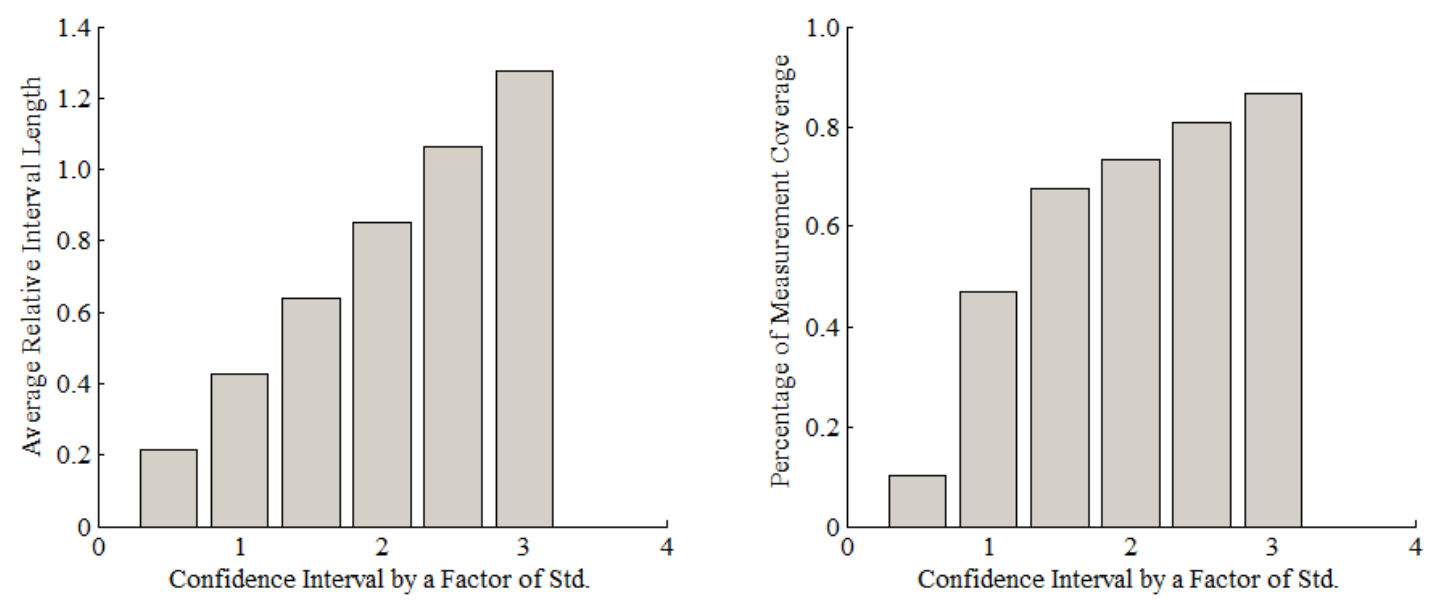

Figure 6.22 Average relative interval length versus the confidence interval and percentage of measurement coverage versus the confidence interval

Similar to the evaluation of the performance of the proposed probabilistic shear strength equation presented previously, the average prediction accuracy of the deterministic equations could also be assessed with the mean prediction model and standard deviation prediction model for all the measured data. Figure 6.23 presents the distribution of shear strength predicted by each deterministic equation with the confidence interval in terms of Mean \pm STD or Mean $\pm 2 \times$ STD. It was clear that the shear strength predicted by Section 18.10 of ACI 318-14 [A1] and Wood [W7] was not even within the area of Mean $\pm 2 \times$ STD for some specimens. As for the other 
deterministic equations, scattering distributions of predictions were witnessed for the same specimen. Hence, in the engineering practice, it was more advisable to evaluate which deterministic shear strength equation had the highest probability to accurately predict the quantity of interest for a particular specimen rather than determine which equation was the most accurate for all the specimens.

Furthermore, the probabilities of the deterministic equations underestimating or overestimating the shear strength of non-rectangular RC squat walls in the database could also be evaluated with Equation 6.32. It was found that average probabilities of the underestimation yielded by the equations from Section 11.5 of ACI 318-14 [A1], Section 18.10 of ACI 318-14 [A1], ASCE 43-05 [A3], Barda et al. [B1] and Wood [W7] were $30.2 \%, 34.1 \% 23.5 \%, 5.41 \%$ and $39.8 \%$ respectively. The equations developed by Gulec and Whittaker [G3], on the other hand, had an average probability of $0.85 \%$ to overestimate the shear strength of walls in the database, which indicated that the equation derived by Gulec and Whittaker [G3] yielded an un-conservative prediction despite its overall accuracy. A similar observation was presented in the last section by examining the statistical results.

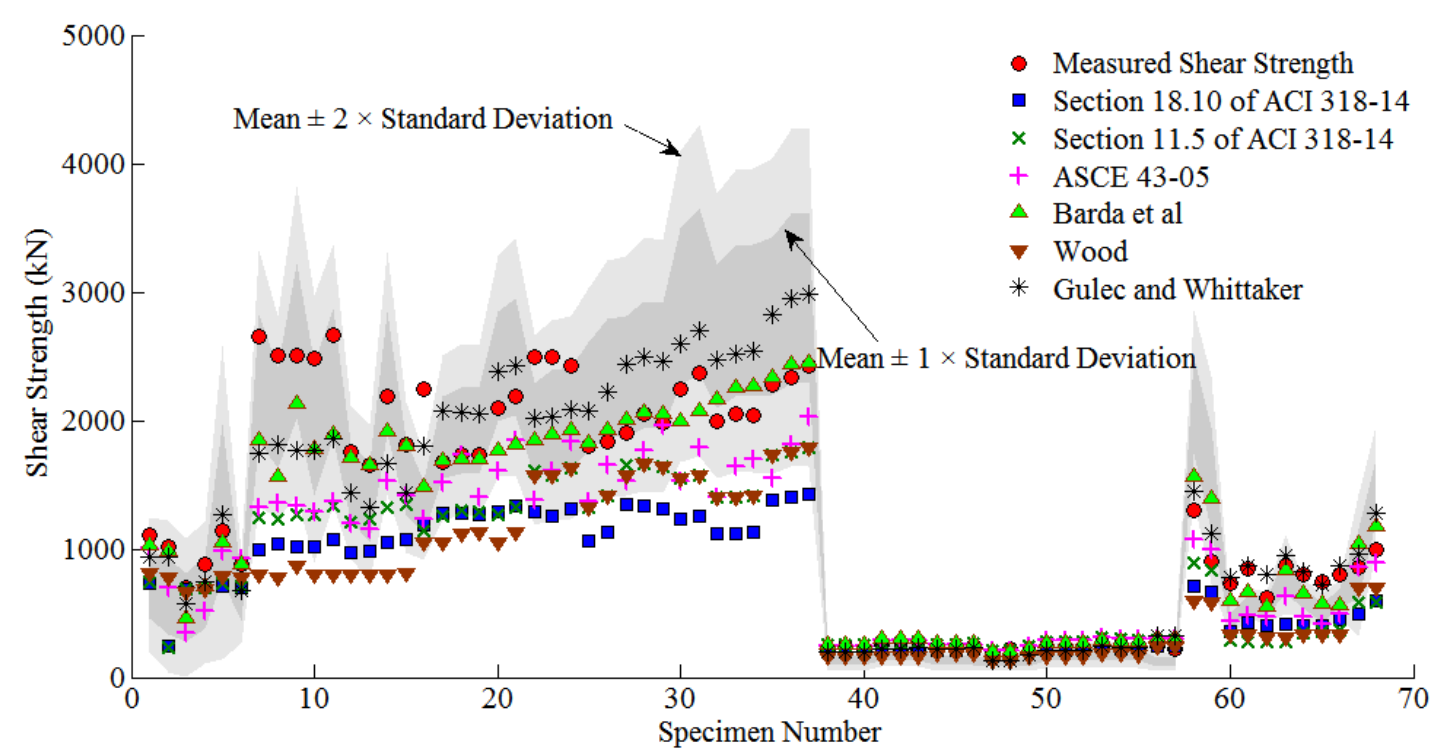

Figure 6.23 Evaluation of the deterministic shear strength equations in a probabilistic manner 


\subsubsection{Conclusion}

A probabilistic equation for the prediction of the peak shear strength of nonrectangular RC squat walls was developed in this section based on the modified strut-and-tie model and the GLUE method. To facilitate the utilization of the proposed probabilistic equation in engineering practice, alternative representations in terms of the mean prediction model and the standard deviation prediction model were also provided. Thus the confidence interval of shear strength in terms of Mean \pm STD or Mean $\pm 2 \times$ STD could be estimated directly once the basic design parameter was determined.

The proposed probabilistic equation showed its superiority since both the experimental results and the shear strength predicted by the deterministic equations were became a quantile of predicted PDF. Therefore, the proposed probabilistic equation was capable of guiding the seismic design of non-rectangular RC squat walls in confidence by specifying an appropriate confidence level. Meanwhile, probabilities of the selected deterministic equations underestimating or overestimating the shear strength of non-rectangular RC squat walls in the database were also evaluated. It was found that the equation derived by Gulec and Whittaker [G3] yielded the most accurate results with an average exceedance probability of $0.85 \%$. The rest of the equations provided by Section 11.5 of ACI 318-14 [A1], Section 18.10 of ACI 318-14 [A1], ASCE 43-05 [A3], Barda et al. [B1] and Wood [W7] underestimated the shear strength of walls in the database with average probabilities of $30.2 \%, 34.1 \%, 23.5 \%, 5.41 \%$ and $39.8 \%$ respectively.

\subsection{Summary}

Numerous analytical models and equations have been proposed in the literature to predict the peak shear strength of RC squat walls. In this study, the performance of equations from Section 11.5 and 18.1 of ACI 318-14 [A1], ASCE 43-05 [A3], Barda et al. [B1], Wood [W7], Hwang et al. [H4], Gulec and Whittaker [G3], Kassem [K1] was first assessed through comparisons with the test results. After that, a modified strut-and-tie model including the effect of large flanges was developed to account for the force-resisting mechanism in non-rectangular RC squat walls, and 
to generate the function form of the peak shear strength equation. Using the fmincon solver in MATLAB [M3], the nonlinear regression was then conducted to obtain the value of unknown coefficients in the function form. The finalized equation was evaluated statistically with the aforementioned equations and two refined equations from the building codes. Finally, the probabilistic approach was adopted to reconcile the large scatter and to avoid the biased estimate found in the deterministic prediction of the peak shear strength of non-rectangular RC squat walls. The main conclusions drawn are as follows:

- Significant scatters existed in the prediction of the peak shear strength of test specimens using equations from Section 11.5 and 18.1 of ACI 318-14 [A1], ASCE 43-05 [A3], Barda et al. [B1], Wood [W7], Hwang et al. [H4], Gulec and Whittaker [G3], Kassem [K1].

- Most equations underestimated the peak shear strength of non-rectangular RC squat walls since the effect of large flanges was not incorporated in these equations. Moreover, contradictory findings were observed in these equations, such as different views to recognize the contribution of horizontal reinforcement to the peak shear strength.

- The equation proposed by Gulec and Whittaker [G3] yielded relatively accurate results in the prediction of the peak shear strength of squat walls with flanged cross section. However, the force mechanism underlying the equation was considered rather simple and the contribution from the longitudinal reinforcement in the flange was overestimated.

- The proposed strut-and-tie model was able to properly account for the forceresisting mechanism in non-rectangular RC squat walls, since it not only comprised the three mechanisms developed for squat walls, but also included the effect of the concrete and reinforcement in the flange.

- The equations derived from the nonlinear regression could precisely predict the peak shear strength of non-rectangular RC squat walls as proven in the sound statistical results. 
- The confidence interval of shear strength in terms of Mean \pm STD or Mean $\pm 2 \times$ STD could be estimated directly by the proposed probabilistic equation, which facilitated its utilization in the engineering practice.

- The proposed probabilistic equation for the peak shear strength of nonrectangular RC squat walls was found to be capable of guiding the seismic design of non-rectangular RC squat walls.

- The equation derived by Gulec and Whittaker [G3] overestimated the shear strength of non-rectangular RC squat walls in the database with an average probability of $0.85 \%$. The equations provided by Section 11.5 of ACI 318-14 [A1], Section 18.10 of ACI 318-14 [A1], ASCE 43-05 [A3], Barda et al. [B1] and Wood [W7] underestimated the peak shear strength of these walls with average probabilities of $30.2 \%, 34.1 \%, 23.5 \%, 5.41 \%$ and $39.8 \%$ respectively. 


\section{CHAPTER 7}

\section{INFLUENCE OF LATERAL LOADING DIRECTION ON THE PEAK SHEAR STRENGTH OF NON-RECTANGULAR RC SQUAT WALLS}

\subsection{Introduction}

The peak shear strength of non-rectangular RC squat walls with symmetric section shape was thoroughly studied in Chapter 6 . The investigation was carried out on the assumption that lateral loading was parallel to the web segment. In reality, however, seismic waves can reach structures from any direction, which necessitates the studies on the behavior of structural walls under various lateral loading directions. Unlike rectangular walls, non-rectangular walls naturally possess the capacity to resist lateral loads in both transverse and longitudinal directions. Thus, during an earthquake, these walls are subjected to biaxial bending actions, and the contribution of different wall segments to the lateral strength could be rather complex. The six specimens presented in Chapter 4, solely provided information regarding loading direction $45^{\circ}$ from the web. To further investigate the peak shear strength of non-rectangular RC squat walls under different lateral loading directions, finite element analyses are employed.

In this study, a widely used commercial nonlinear finite element software Diana 9.4 [D1] is chosen for the modeling of non-rectangular RC squat walls. With an extensive material library, Diana 9.4 is capable of accurately simulating the behavior of RC structures by performing linear and nonlinear analyses in full $2 \mathrm{D}$ and 3D environments. This software has been successfully utilized by researchers and engineers worldwide [H1, L1, X1, Z2].

This chapter first presents a detailed introduction concerning several important aspects involved in the finite element analysis (FEA). After that, appropriate modeling approaches are selected and further validated by comparisons between analytical results and experimental data. Finally, a comprehensive parametric study 
is carried out to investigate the peak shear strength of non-rectangular walls under various lateral loading directions.

\subsection{Finite Element Model}

Diana 9.4 is the flagship product developed by the TNO Building and Construction Research Institute in Delft, which has a comprehensive library of elements and materials. This powerful finite element software has been widely used by civil engineers in the analyses of RC structures. Before using Diana 9.4 to build models for non-rectangular RC squat walls, a review of main factors involved in the modeling RC structures is first presented in this section.

\subsubsection{Element types}

In FEA, different elements act together to model certain structure. Elements generally fall into four major categories: 2D line elements (truss element, beam element, etc.), 2D planar elements (membrane element, plate element, etc.), 3D solid elements (3D tetrahedral element, 3D brick element, etc.), and special elements (spring element, rigid element, etc.). In the 2D nonlinear FEA of RC walls, the most frequently used element is the eight-node quadrilateral isoparametric planar element. In 3D analyses, the twenty-node isoparametric solid brick element is most widely applied.

In this study, a twenty-node isoparametric solid brick element called CHX60 (Figure 7.1) is chosen for the following nonlinear FEA, which is based on the quadratic interpolation and Gauss integration. Compared with the eight-node isoparametric solid brick element, it can more accurately capture the behavior of concrete. 


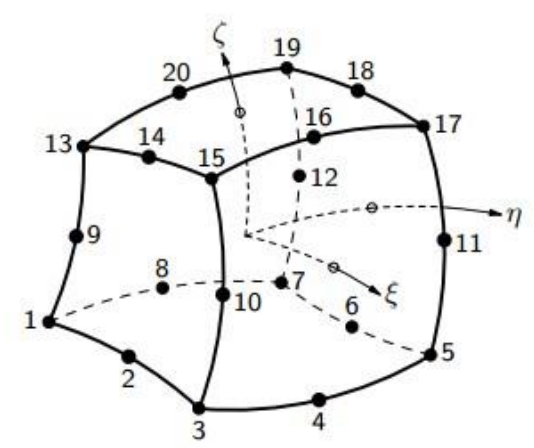

Figure 7.1 CHX60 element [D1]

With regard to the modeling of reinforcement in RC structures, generally, two techniques are widely adopted. The first of these is called the embedded reinforcement or the smeared reinforcement technique (Figure 7.2 and Figure 7.3). This technique adds stiffness to the finite element model. Its main characteristics are as follows:

- Reinforcement is embedded in structural elements, the so-called "mother elements"

- Reinforcement does not have degrees of freedom of its own.

- Reinforcement strains are by default calculated from the displacement field of the mother elements. This implies a perfect bond between the reinforcement and the surrounding material.
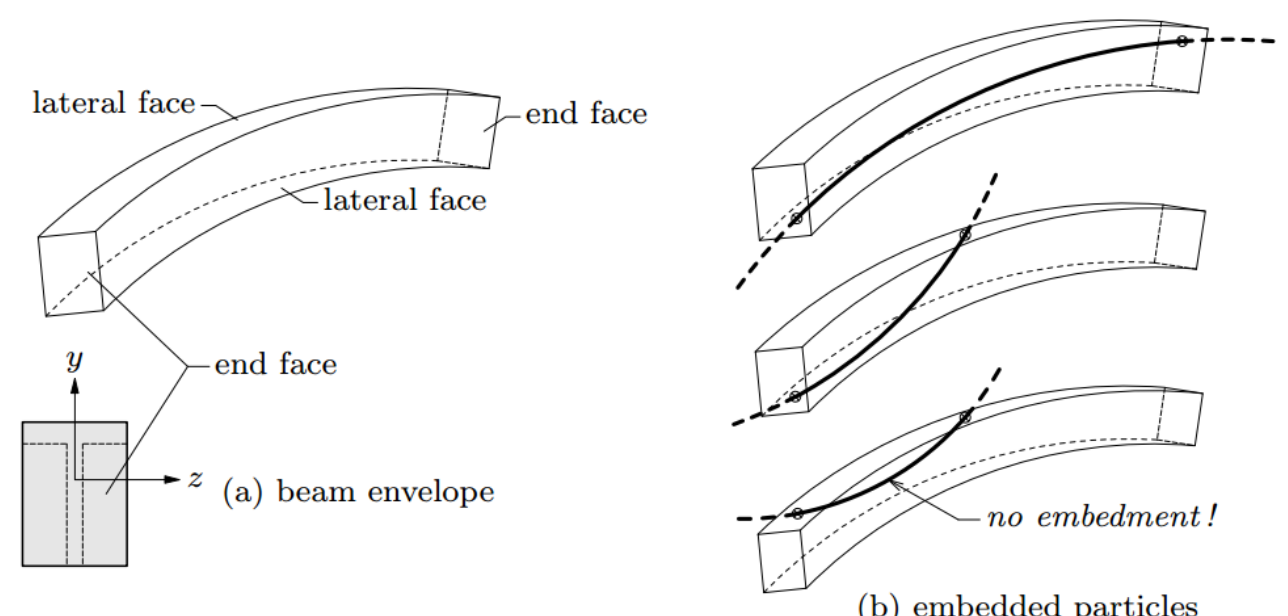

(b) embedded particles

Figure 7.2 Reinforcement in the beam element [D1] 


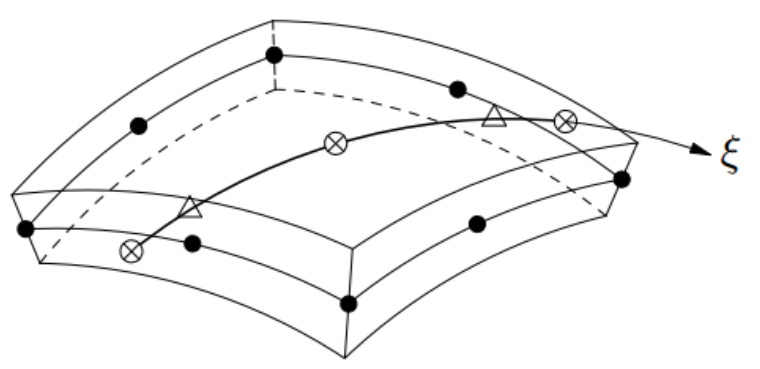

- element node

$\otimes$ location point

$\triangle$ integration point

Figure 7.3 Reinforcement in the curved shell element [D1]

In the second technique, separate elements such as truss and beam elements are used to model reinforcement. The advantage of the latter is that the restricted rotation of beam end nodes can more realistically simulate the behavior of reinforcement if the confinement effect is taken into account [K3]. With this technique, the reinforcement is connected with the surrounding materials at nodes.

Basically, the embedded reinforcement technique requires less computation and allows the geometry of the reinforcement to be different from the geometry of the mesh. However, the bond-slip relationship cannot be explicitly incorporated into the constitutive laws. The second technique, on the other hand, allows the investigation of the bond-slip behavior by adding interface elements between the concrete and the reinforcement. However, significant computational time and cost are foreseeable using this technique as it requires connections of the reinforcement element to the concrete element at nodes. The finite element analysis performed by Palermo [P1], which ignored the bond-slip between concrete and reinforcement, was accurate in simulating the seismic performance of two H-shaped RC squat walls. More importantly, the bond-slip effect was not evident in the experiments presented in Chapter 4 and considered as a less significant factor in affecting the behavior of non-rectangular RC squat walls. Therefore, the embedded reinforcement technique is selected in this study.

\subsubsection{Constitutive laws for concrete}

Nonlinear constitutive laws of materials play an important role in modeling the nonlinear behaviors of RC structures. Hence a reliable FEA should be based on appropriate constitutive models. Concrete is a composite material composed of 
coarse granular material bonded together with cement, water and other additives, which can sustain high compression but low tension. The diversity of components together with different formations makes concrete a material very difficult to model clearly and correctly. The following aspects should be described properly in a valid concrete model, namely (1) crack behavior, (2) compressive behavior, (3) tensile behavior, (4) shear behavior, (5) lateral influence [X1]. In the literature, dozens of constitutive models and modeling techniques have been proposed to describe the nonlinear behaviors of concrete.

The first RC finite element model including the effect of cracking was developed by Ngo and Scordelis [N3] in 1967. The cracks are modeled by separating the nodal points of the finite element mesh. Hence the model is named as the discrete crack model. Through adoption of linkage elements to connect the separated nodes, postcracking behaviors such as tension softening, aggregate interlock, and dowel action, can be incorporated into this model.

In spite of its advantages, especially when modeling lightly reinforced structures whose responses are dictated by a few, wide cracks, the discrete crack model's drawbacks are evident. These are mainly reflected in the following aspects as summarized by Sittipunt and Wood [S6]: (1) Cracking can occur only along element boundaries, which introduce bias into the finite element solution. (2) Unless cracks in the model are pre-defined, cracking will cause continuous updating of the mesh topology as it progresses, destroying the narrow bandwidth in the structural stiffness matrix. (3) Crack closing and reopening has to be defined using a contact algorithm, which significantly complicates the problem.

In order to overcome these defects and improve the simulation of concrete behaviors, Rashid [R1] proposed the smeared crack model (also known as crack band theory) in 1968 by analyzing concrete structures using axisymmetric finite elements. This model represents cracked concrete as an elastic orthotropic material with reduced elastic modulus in the direction normal to the crack plane. Rather than representing a single crack, the smeared crack model represents many finely spaced cracks perpendicular to the principal stress, which facilitates the modeling of RC structures. In this study, the smeared crack model is selected for the FEA since it 
better represents RC structures with homogeneously distributed cracks as shown in the tests.

Two models are widely used to represent the cracking in the smeared crack model, which are the fixed crack model and the rotating crack model. In Diana 9.4, the former comprises the multi-directional fixed crack model (Figure 7.4) and the total strain fixed crack model, while the latter is mainly represented by the total strain rotating crack model. In both models, when the principal tensile stress in the element exceeds a certain limit, cracks are initiated in a direction perpendicular to the principal tensile stress. The material then becomes orthotropic with different material properties normal and parallel to the cracks [G2].

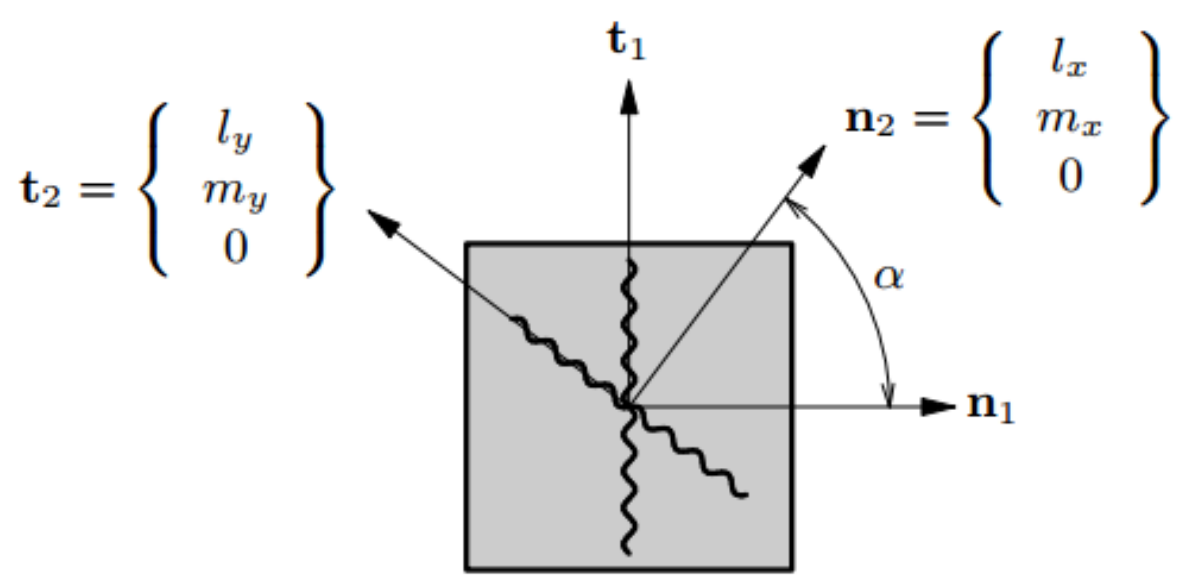

Figure 7.4 Multi-directional fixed crack model [D1]

The most significant difference between these two models is that the fixed crack model assumes that the crack is fixed once it is generated, whereas, in the rotating crack model, the crack direction is continuously aligned orthogonal to the principal tensile stress. Accordingly, in the fixed crack model, when the direction of the principal stress changes, a shear stress parallel to the crack direction should be introduced, while in the rotating crack model, this kind of shear transfer mechanism is not needed. In order to account for the stiffness reduction of elements, which is also known as the shear retention, a constant shear stiffness reduction is defined in the total strain fixed crack model in Diana 9.4 as follows:

$$
G^{c r}=\beta \cdot G
$$


where $G^{r c}\left(\mathrm{~N} / \mathrm{m}^{2}\right)$ is the shear modulus after cracking, $G\left(\mathrm{~N} / \mathrm{m}^{2}\right)$ is shear modulus, $\beta$ is the reduction factor varing from 1 for uncracked concrete to 0 for completely cracked concrete. As mentioned above, for the rotating crack concept, the shear retention factor can be assumed to be equal to one.

Compared with the rotating crack model, the fixed crack model fits the nature of the shear effect, such as the aggregate interlock, which is closer to the real state of cracks in concrete. However, this advantage can complicate the analysis because it is responsible for an uncontrollable rotation of axes of the principal stress, which ceases to coincide with the axes of the principal strain. In fact, it is the simplicity and reasonable accuracy that bring the rotating crack model such popularity among scholars [X1]. Hence in this study, the total strain rotating crack model is adopted, which use one stress-strain relationship to describe the tensile and compressive behavior of concrete.

In Diana 9.4, the compressive behavior of the total strain crack model can be described by seven predefined curves, namely the elastic curve, the constant curve, the brittle curve, the multi-linear curve, the linear hardening and the saturation hardening curves, the Thorenfeldt curve, and the parabolic curve. These curves are presented below in Figure 7.5. The elastic curve only accounts for the elastic part of compression, which cannot be used in nonlinear analyses. Among the remaining six curves, only the Thorenfeldt curve and the parabolic curve incorporate the softening behavior of concrete. The Thorenfeldt curve uses two special parameters $n$ and $k$ together with the compressive strength $f_{c}^{\prime}$ in one equation to define the curve. The parabolic curve is based on the fracture energy $G_{c}$ and the crack bandwidth of the element. In this study, the parabolic curve is adopted for the following parametric study. 


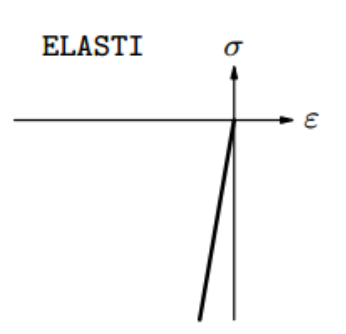

(a) elastic

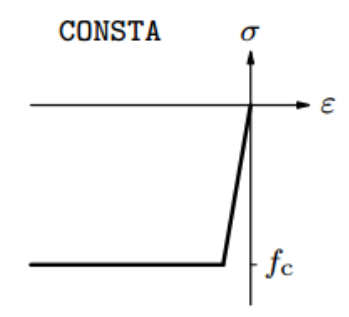

(b) ideal

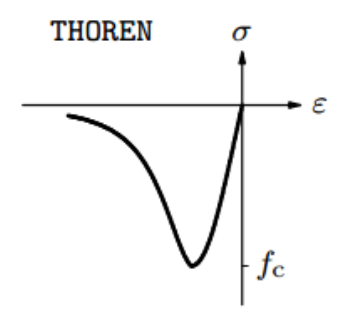

(c) Thorenfeldt

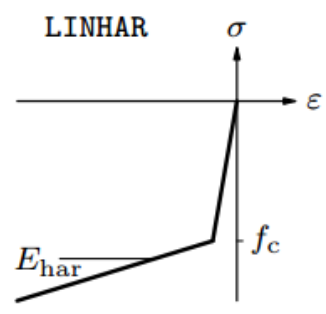

(d) linear

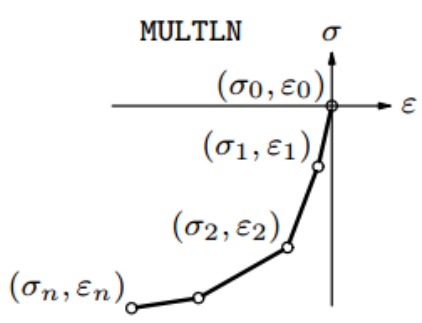

(e) multi-linear

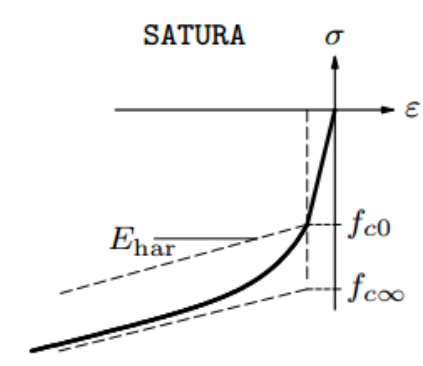

(f) saturation type

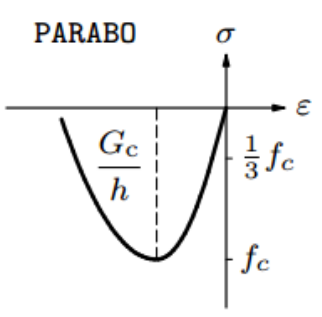

(g) parabolic

Figure 7.5 Predefined stress-strain curves for concrete in compression [D1]

When it comes to the tensile behavior of concrete, Diana 9.4 also predefines seven curves for the total strain crack model, which are the elastic curve, the constant curve, the brittle curve, the multi-linear curve, the linear softening curve, the saturation softening curve and the Hordijk curve (Figure 7.6). The elastic curve does not take the tension softening into consideration, as this is apparently not suitable for the nonlinear modeling approach. In the ideal model, no tension softening behavior is presented. In the brittle model, it is assumed that concrete abruptly loses tensile strength once it cracks, which is close to the tension behavior of plain concrete or concrete with low reinforcement. But in normally or heavily reinforced walls which are also the primary focus in this study, the tension softening behavior of concrete must be considered. The remaining four types of tension curves do satisfy this requirement and they are all based on the fracture energy $G_{f}$, which is nearly $1 / 150$ of the compressive fracture energy $G_{c}$. In this study, the tension softening model proposed by Hordijk et al. is adopted for the following nonlinear FEA. 


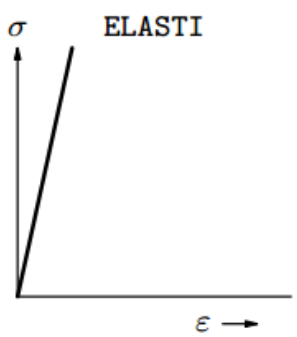

(a) elastic

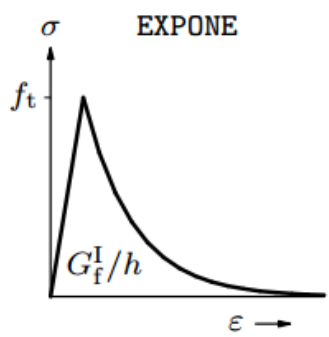

(e) exponential

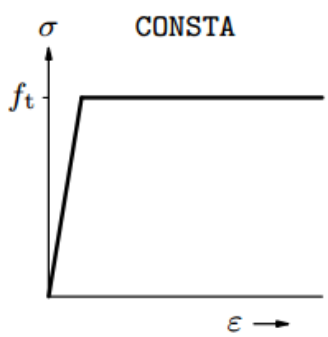

(b) ideal

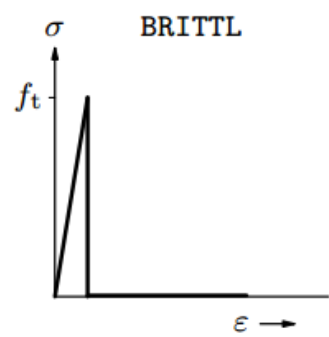

(c) brittle

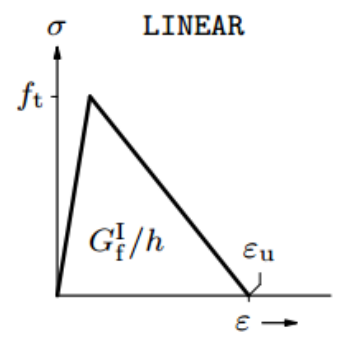

(d) linear

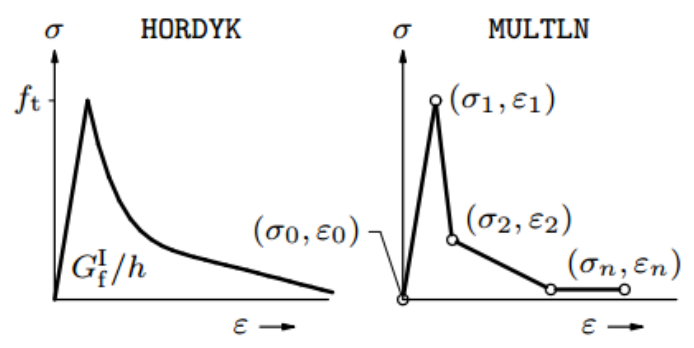

(f) Hordijk

(g) multi-linear

Figure 7.6 Predefined stress-strain curves for concrete in tension [D1]

The lateral influence to concrete mainly comprises two aspects. The first is the drop in the compressive strength of concrete, which happens because the tensile strain perpendicular to the principal compression stress causes cracks and damage in the concrete. The reduction is explained in the model developed by Vecchio and Collins [V1] in Diana 9.4. The other aspect is the lateral confinement. It has long been recognized that the strength, as well as the deformability of concrete, continuously increases when the amount of confinement is increased. Equations have been proposed from numerous experimental studies to predict the improvement of loading capability [D1]. In Diana 9.4, the effects of lateral confinement are incorporated by modifying the compressive stress-strain curves, in which parameters are determined by a failure function defining compressive stress as a function of confining stresses in lateral directions, as explained in the model of Selby et al. [S5].

\subsubsection{Constitutive laws for reinforcement}

As presented in the previous section, two approaches for modeling reinforcing bars in concrete are provided by Diana 9.4. The first one assumes reinforcement to be embedded in surrounding concrete, and the second one uses separate elements to 
model reinforcement. In this study, the embedded reinforcement technique is adopted, for the reasons provided earlier in this section.

With regard to the constitutive relationship of reinforcing bars, the Von Mises yield criteria is applied to describe the uniaxial stress-strain response in Diana 9.4. The stress-strain curves derived from rebar testing are used in the following parametric study.

\subsubsection{Iterative solution methods}

In nonlinear FEA, the relation between a force vector and a displacement vector is no longer linear, which means that the solution vector that equilibrates the internal and external forces cannot be calculated right away as in linear analyses. Therefore, appropriate incremental-iterative solution procedures are indispensable and important in the nonlinear FEA.

Generally, there are four types of iteration methods available in Diana 9.4, namely the Regular Newton-Raphson method, the Modified Newton-Raphson method, the Secant method (the Quasi-Newton method) and the Linear Stiffness iteration method [D1].

As demonstrated in Figure 7.7, in the Regular Newton-Raphson iteration, the stiffness relation is evaluated in every iteration. This means that the prediction of iterative increments is based on the last known or predicted situation, even if this is not an equilibrium state. 


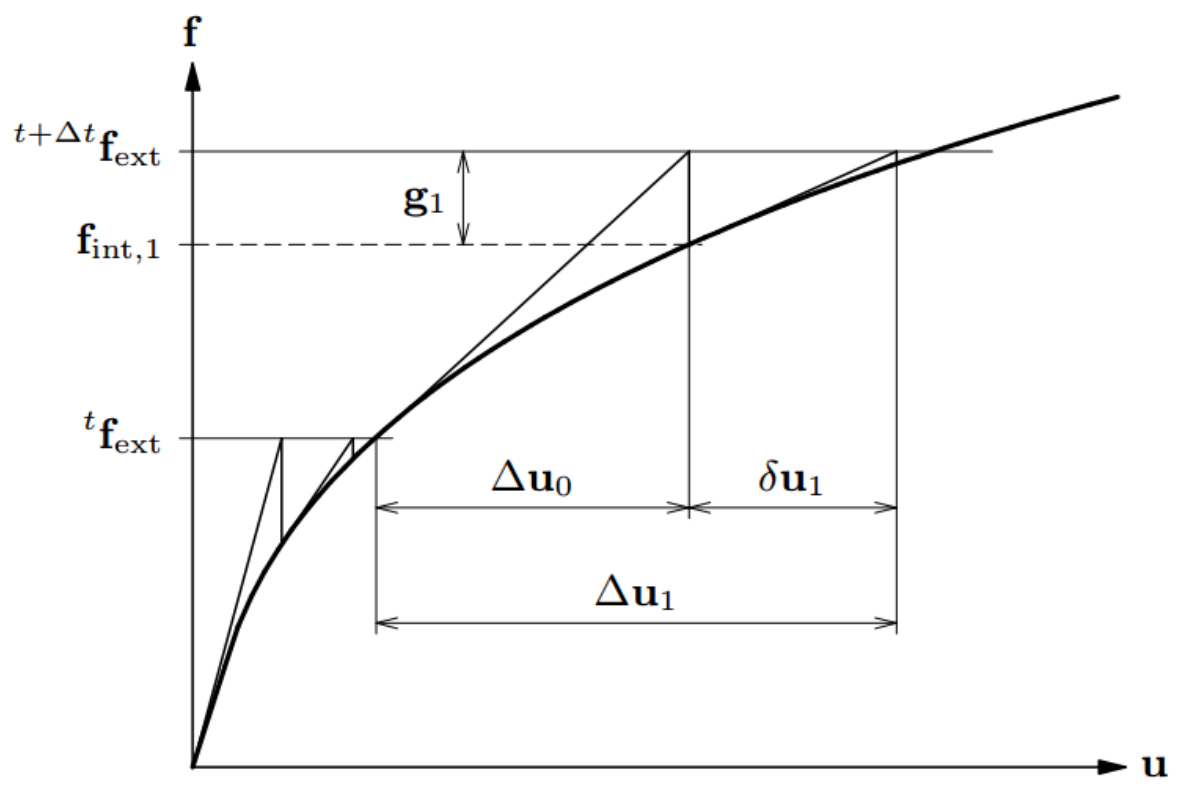

Figure 7.7 Regular Newton-Raphson iteration method [D1]

The Modified Newton-Raphson method only evaluates the tangential stiffness at the start of the increment, as shown in Figure 7.8. This means that the prediction is always based on a converged equilibrium state.

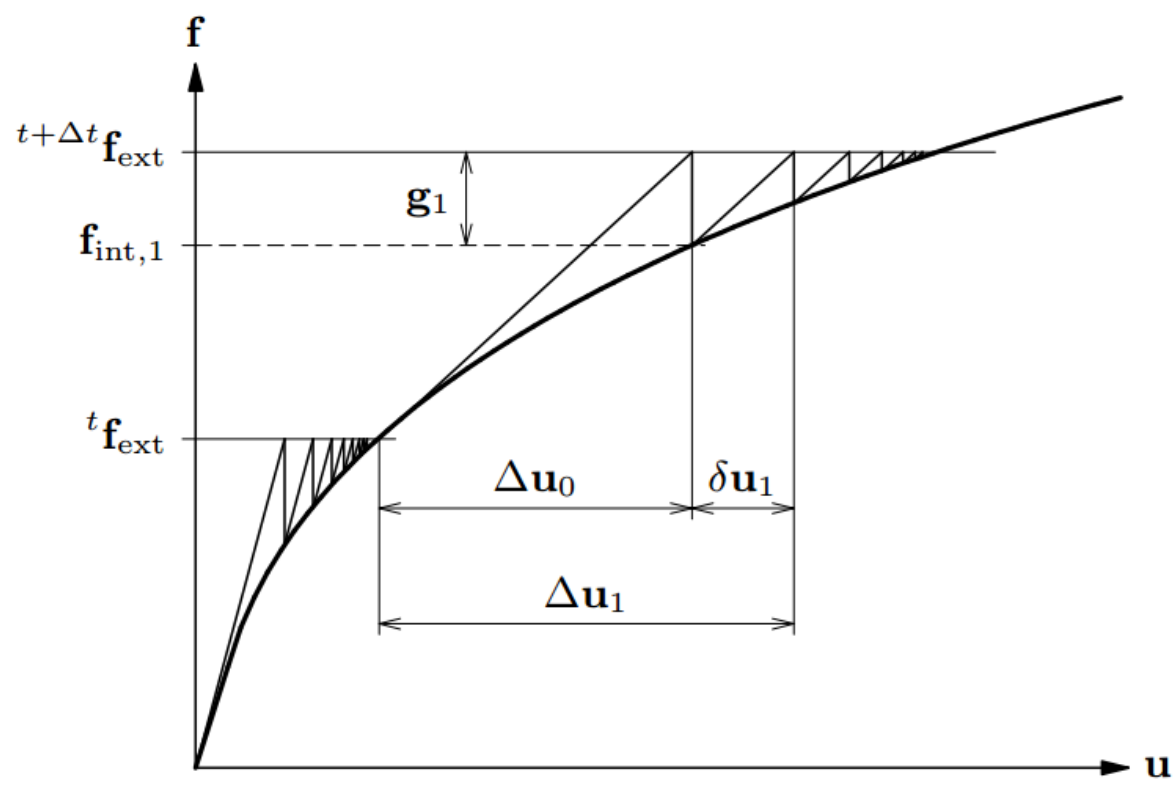

Figure 7.8 Modified Newton-Raphson iteration method [D1]

The Secant method essentially uses the information of previous solution vectors and out-of-balance force vectors during the increment to achieve a better approximation. 


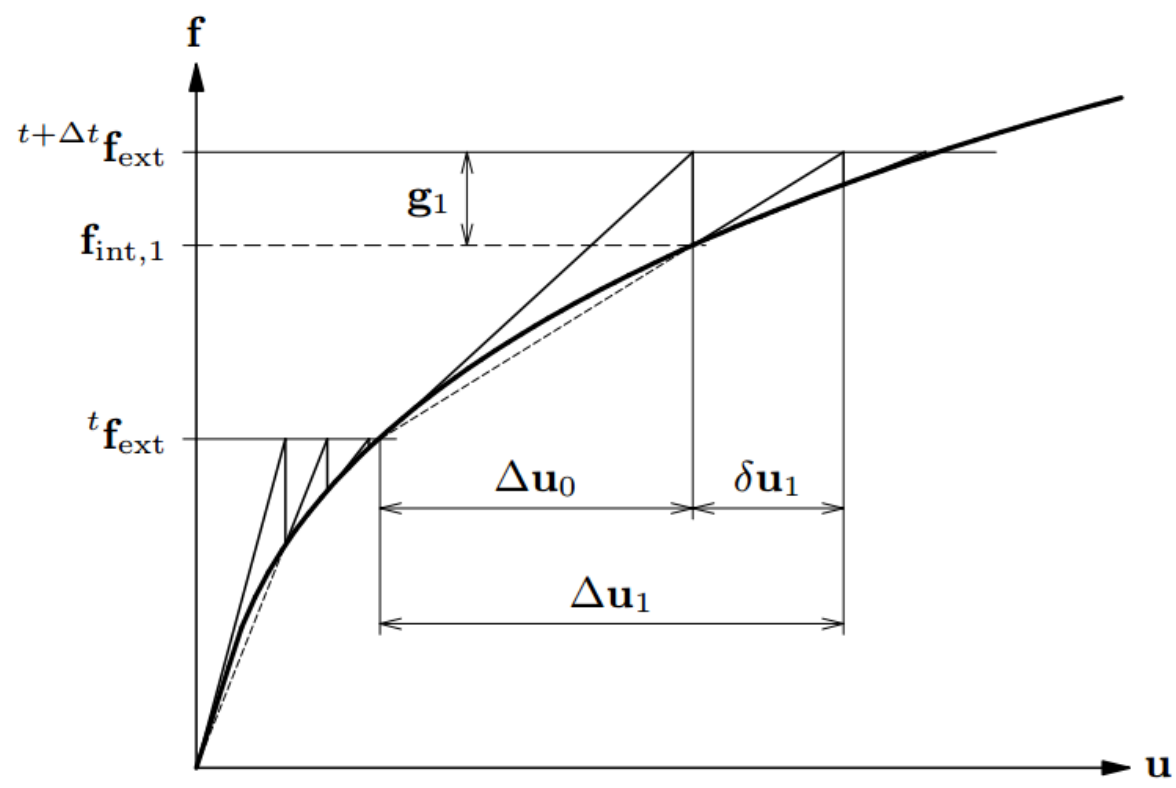

Figure 7.9 Secant method [D1]

The Linear Stiffness iteration method uses the linear stiffness matrix all the time, as seen in the Figure 7.10.

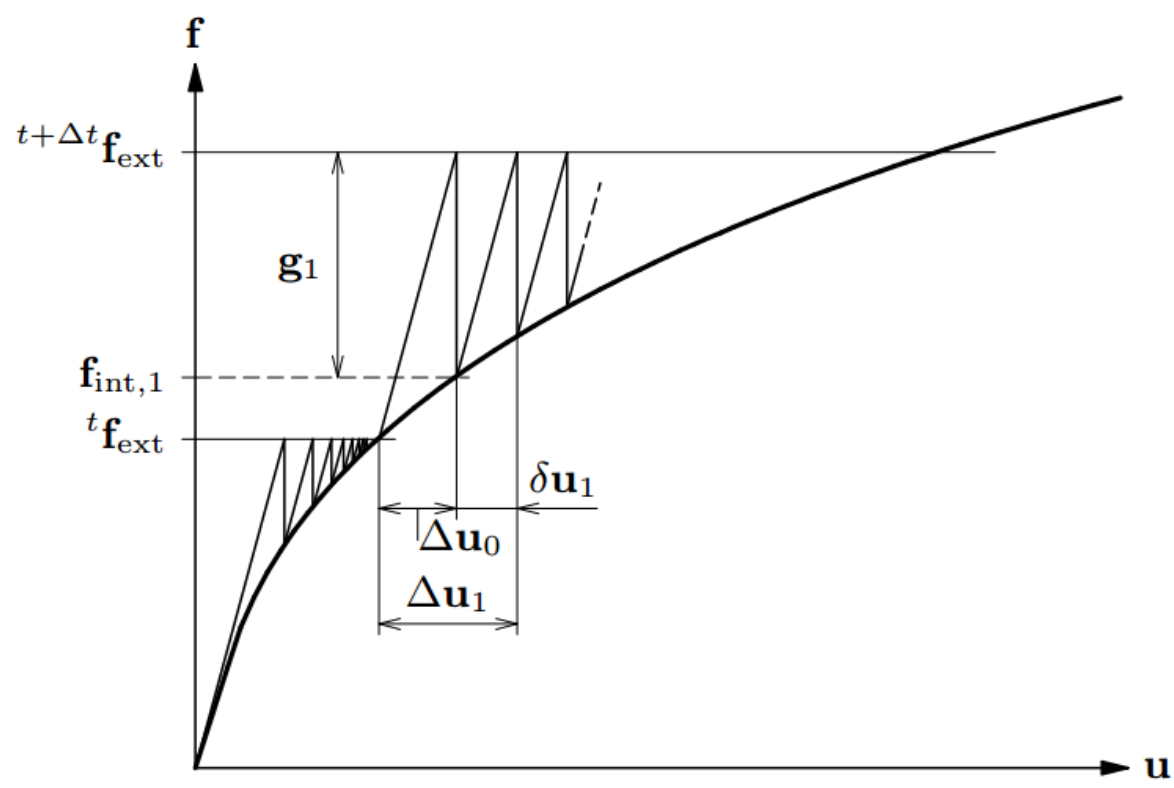

Figure 7.10 Linear stiffness iteration method [D1]

The difference between the Regular and the Modified Newton-Raphson method is the point at which the stiffness matrix is evaluated. In the Regular Newton-Raphson iteration method, the tangential stiffness matrix is recalculated in each step of the 
iteration, yielding a quadratic convergence characteristic, which means that the method converges to the final solution within only a few iterations. On the other hand, it is time-consuming to set up and decompose the stiffness matrix at every step of iteration. Also, the quadratic convergence is only guaranteed if a correct stiffness matrix is used and if the prediction is already in the neighborhood of the final solution. So when the initial prediction is far from the solution, this method can easily fail due to divergence. The Modified Newton-Raphson method, in contrast, iterates faster than the Regular Newton-Raphson iteration method. Because for every iteration, only the iterative incremental displacements and the internal force vector have to be calculated, it is not necessary to set up a new stiffness matrix at every step of iteration. A major drawback of the method is that it converges slower to equilibrium than the Regular Newton-Raphson method.

The Secant method is similar to the Modified Newton-Raphson method since the stiffness matrix is also only derived at the start of every load increment. One evident difference is that secant stiffness matrices are used in this method instead of tangential stiffness matrices.

As for highly nonlinear situations where other methods become unstable, or it is desirable to keep certain characteristics, the Linear Stiffness iteration method can be used. This method potentially has the slowest convergence, but it requires the least time per iteration since the stiffness matrix needs to be set up only once. Moreover, in the case of a direct linear solver, the decomposition has to be performed only once. The Linear Stiffness method can also be advantageous if the stiffness matrix is required to be symmetric. In this study, the Regular Newton-Raphson iteration method is applied to solve the nonlinear equations.

\subsection{Validation of the Finite Element Models}

As mentioned in the previous section, concrete is represented by the twenty-node isoparametric solid brick element $\mathrm{CHX60,}$, and longitudinal, horizontal and transverse reinforcement is modeled as the embedded reinforcement. The total strain rotating crack model with the parabolic compression curve and the tension softening curve proposed by Hordijk et al. is adopted as the constitutive law for 
concrete, with the relevant parameters calculated in accordance to European CEBFIP Model Code 1990 [C1, D1] as shown from Equation 7.2 to Equation 7.5. All reinforcing bars are simulated as the Von Mises plastic material, which follow the strain hardening curves obtained from rebar testing in the lab. The top slab is modelled to be rigid, and the bottom of the base is assumed to be fixed against movements and rotations.

$$
\begin{gathered}
f_{t}=1.4\left(\frac{f_{c}^{\prime}}{10}\right)^{\frac{2}{3}} \\
E_{c}=21500\left(\frac{f_{c}^{\prime}}{10}\right)^{\frac{1}{3}} \\
G_{f}=G_{f 0}\left(\frac{f_{c}^{\prime}}{10}\right)^{0.7} \\
G_{c}=150 G_{f}
\end{gathered}
$$

where $f_{c}^{\prime}(\mathrm{MPa})$ is the concrete compressive strength, $f_{t}(\mathrm{MPa})$ is the concrete tensile strength, $E_{c}(\mathrm{MPa})$ is the elastic modulus of concrete, $G_{f}\left(\mathrm{~N}-\mathrm{mm} / \mathrm{mm}^{2}\right)$ is the fracture energy of concrete, $G_{f 0}$ is the coefficient related to maximum aggregate size, $G_{C}\left(\mathrm{~N}-\mathrm{mm} / \mathrm{mm}^{2}\right)$ is the compressive fracture energy of concrete.

Similar to the test setup illustrated in Chapter 3, the lateral cyclic loading is applied at the center point of the top slab. The top slab is assigned to be rigid enough to uniformly distribute horizontal loads to the entire RC wall. The loading history in the FEA is identical to that in the test. As for the axial load, the uniformly distributed pressure calculated from the experimental data is applied on the top slab, which is demonstrated in Figure 7.11 together with the boundary constraints. The Regular Newton-Raphson iteration method is chosen for the following nonlinear FEA. 


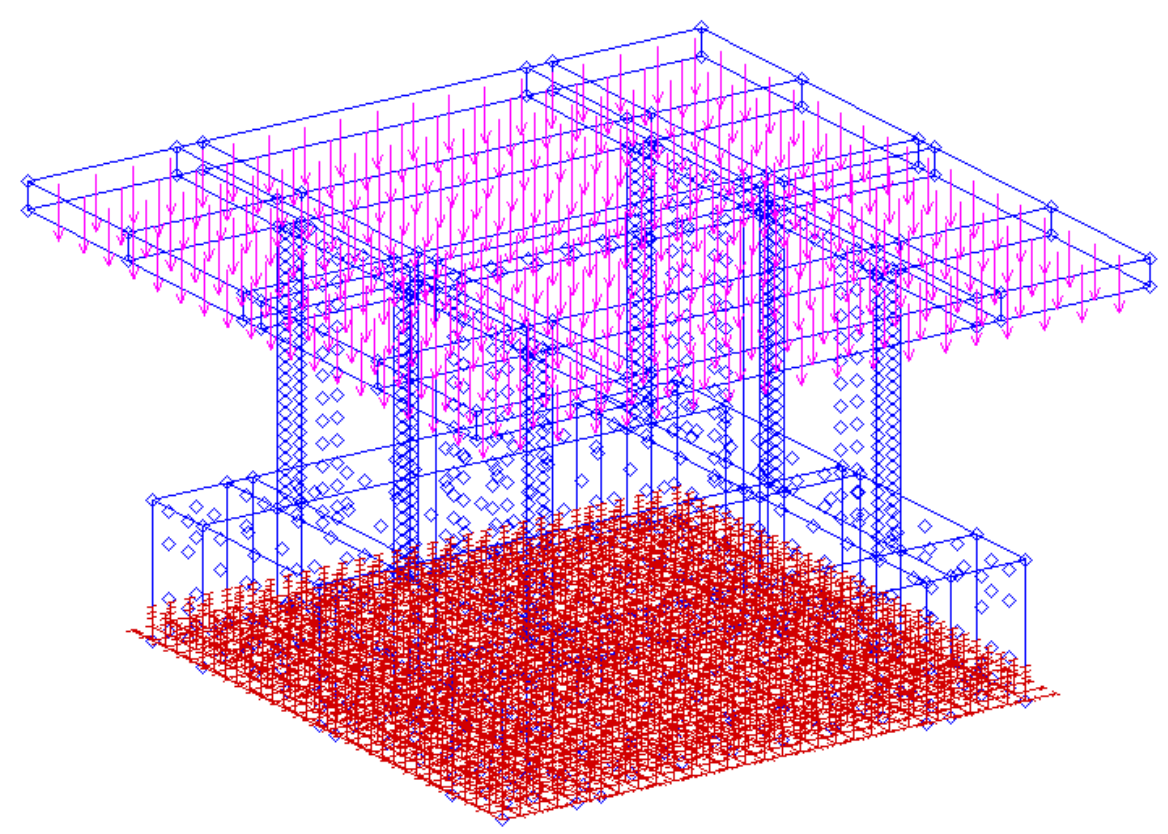

Figure 7.11 Loads and boundary constraints in a representative finite element model

Before the selected modeling approaches are practiced in the parametric study, it is essential to validate these techniques by simulating some past experiments. Six specimens as presented in Chapter 4 are selected for the verification: Specimens HP0D0, HP5D0, LP0D45, LP5D45, TP5D0 and TP5D5. The selection is aimed to show the capability of the modeling approaches in predicting the seismic behavior of non-rectangular RC squat walls under various levels of axial loads and different lateral loading directions. Meanwhile, it also intends to demonstrate the competence of the modeling approaches in accurately simulating the performance of nonrectangular RC squat walls with different section shapes. Table 7.1 presents the material properties of these specimens. Figure 7.12 to Figure 7.14 show the detailed mesh and reinforcement layouts of the finite element models of these specimens. 
Table 7.1 Material properties of test specimens

\begin{tabular}{cccccccc}
\hline \multirow{2}{*}{ Specimen } & $(1)$ & $(2)$ & $(3)$ & $(4)$ & $(5)$ & $(6)$ & $(7)$ \\
& $f_{c}^{\prime}$ & $f_{t}$ & $E_{c}$ & $v_{c}$ & $G_{f}$ & $h$ & $G_{c}$ \\
\cline { 2 - 8 } & $\mathrm{MPa}$ & $\mathrm{MPa}$ & $\mathrm{MPa}$ & & $\mathrm{N}-\mathrm{mm} / \mathrm{mm}^{2}$ & $\mathrm{~mm}$ & $\mathrm{~N}-\mathrm{mm} / \mathrm{mm}^{2}$ \\
\hline LP0D45 & 33.8 & 3.2 & 32266 & 0.15 & 0.070 & 100 & 10.6 \\
\hline LP5D45 & 33.8 & 3.2 & 32266 & 0.15 & 0.070 & 100 & 10.6 \\
\hline HP0D0 & 35.9 & 3.3 & 32920 & 0.15 & 0.073 & 100 & 11.0 \\
\hline HP5D0 & 35.9 & 3.3 & 32920 & 0.15 & 0.073 & 100 & 11.0 \\
\hline TP5D0 & 35.0 & 3.2 & 32643 & 0.15 & 0.072 & 100 & 10.8 \\
\hline TP5D45 & 36.2 & 3.3 & 33012 & 0.15 & 0.074 & 100 & 11.1 \\
\hline
\end{tabular}

Note: (1): Compressive strength of concrete; (2) Tensile strength of concrete; (3) Elastic modulus of concrete; (4) Poisson's ratio of concrete; (5) Fracture energy of concrete; (6) Crack bandwidth; (7) Compressive fracture energy of concrete.
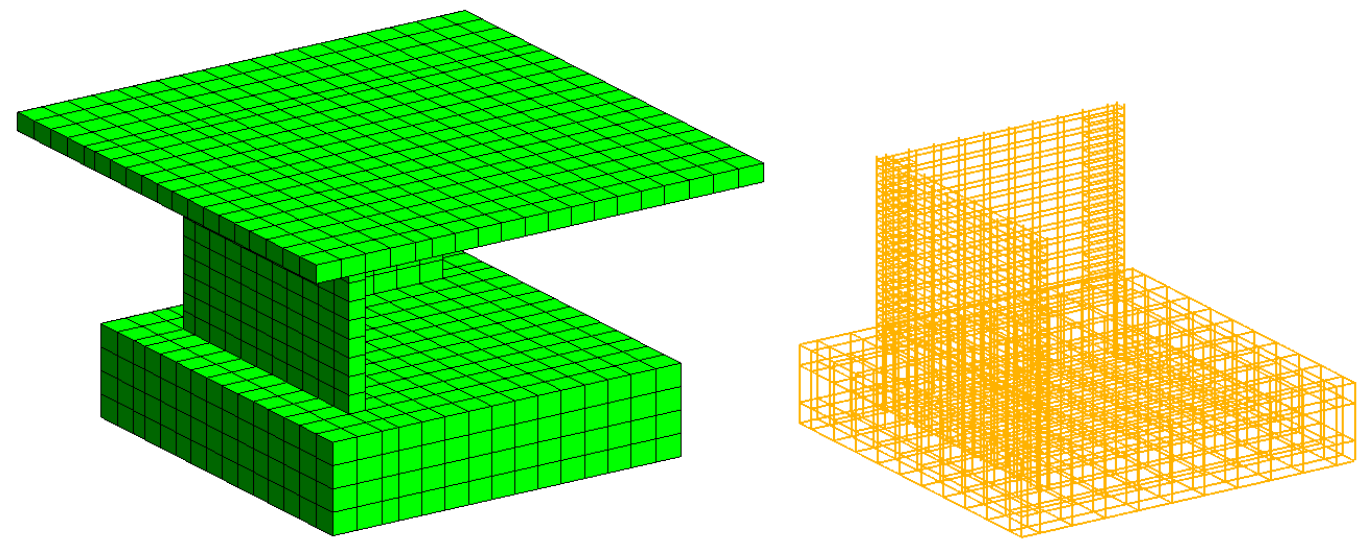

Figure 7.12 Mesh and reinforcement layout of the L-shaped models 

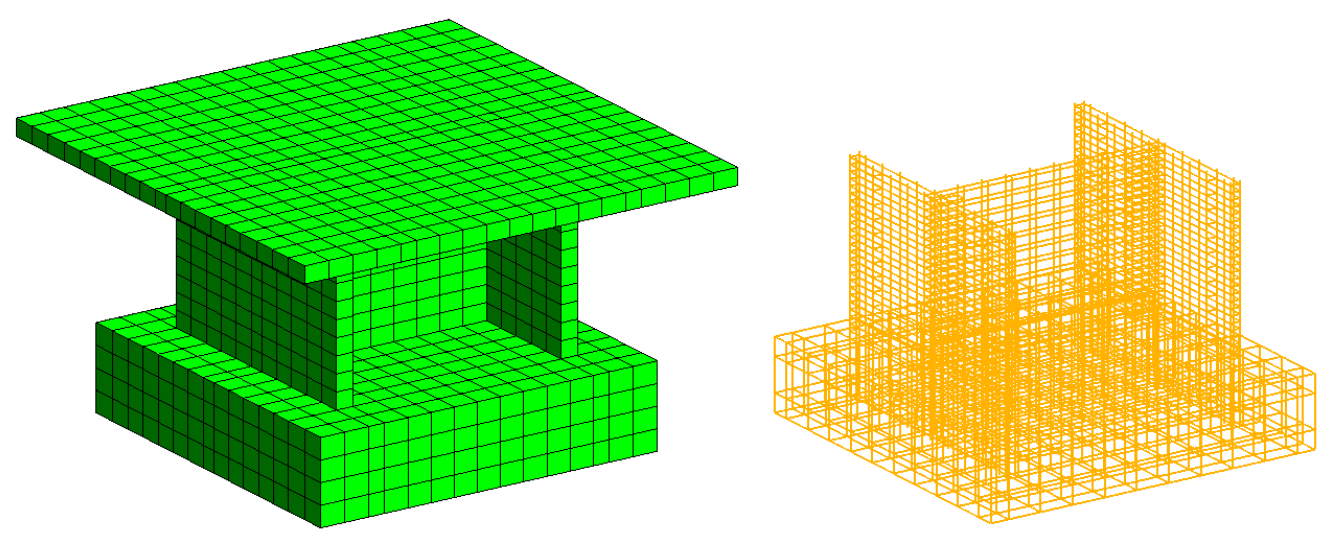

Figure 7.13 Mesh and reinforcement layout of the H-shaped models
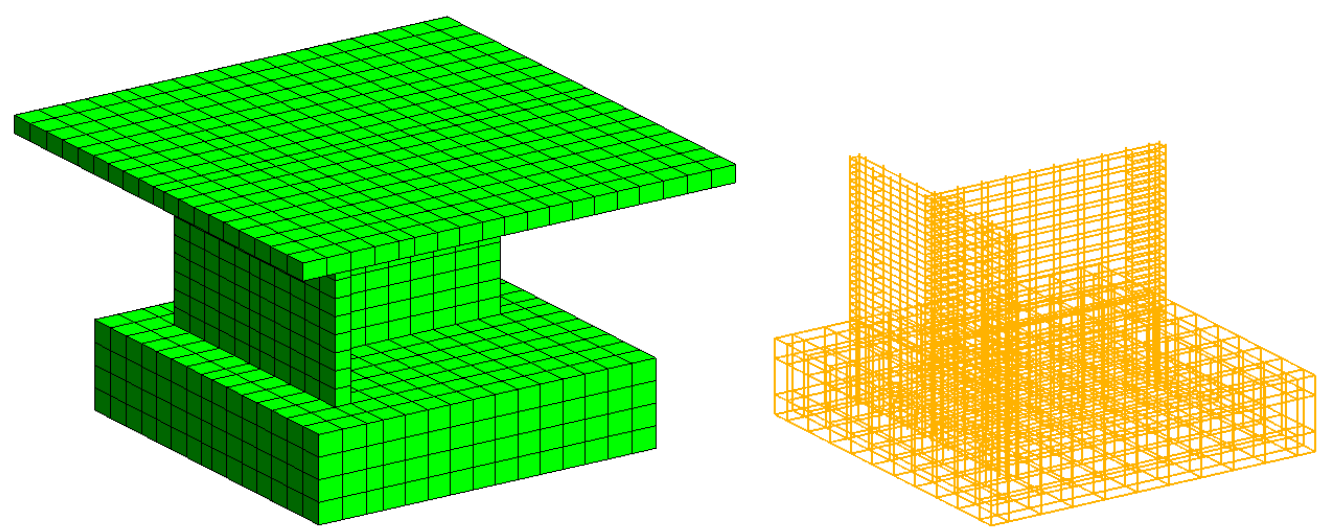

Figure 7.14 Mesh and reinforcement layout of the T-shaped models

Both global and local responses of the selected specimens obtained from finite element analyses were compared with their experimental results. Figure $\mathbf{7 . 1 5}$ to Figure 7.20 show the comparison between experimental and analytical hysteretic loops of each specimen. Figure 7.21 to Figure 7.26 demonstrate the comparison between experimental and analytical strain profiles of longitudinal reinforcement in the web of each specimen. 


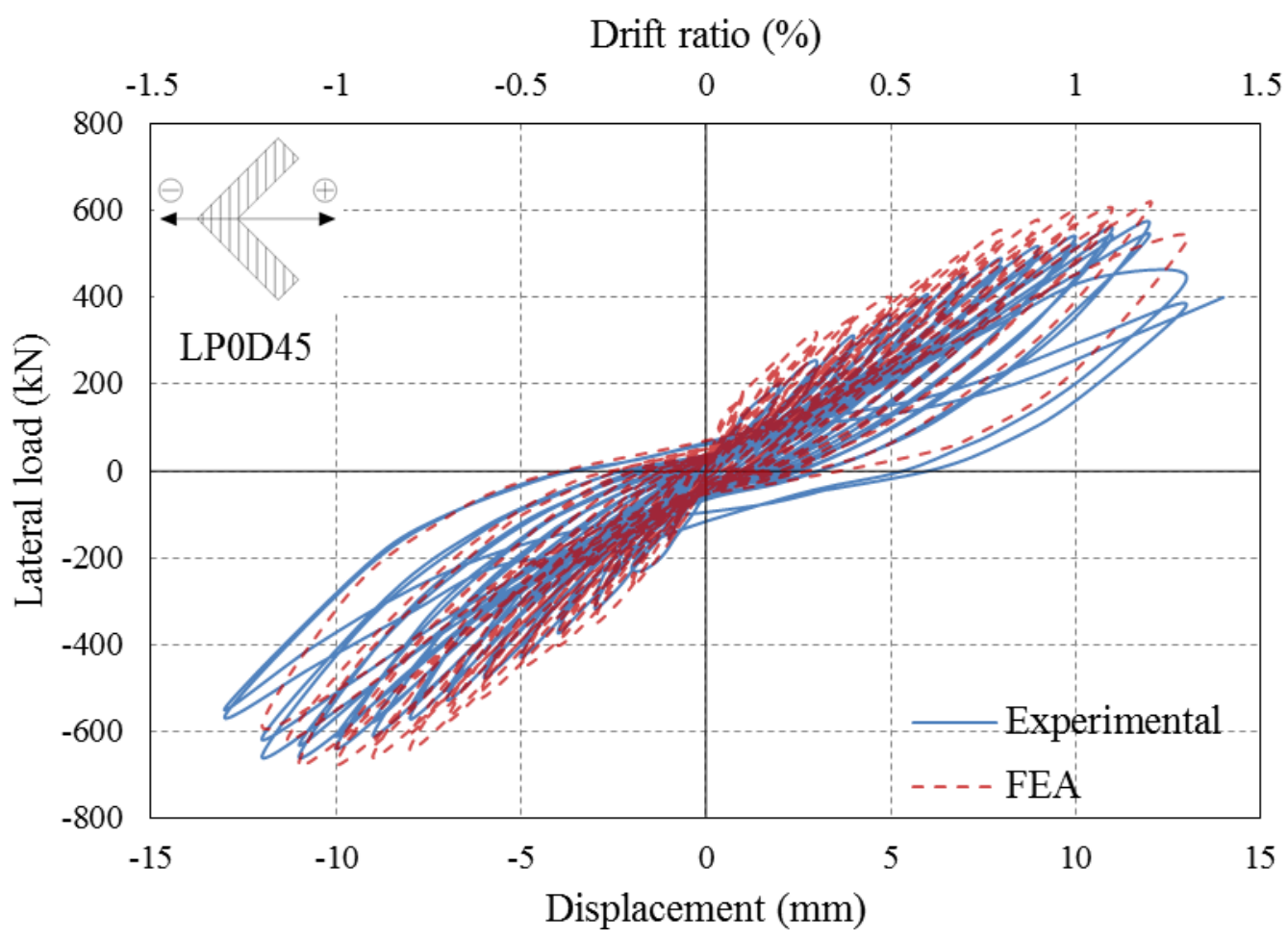

Figure 7.15 Comparison between experimental and analytical lateral loaddisplacement curves of Specimen LP0D45

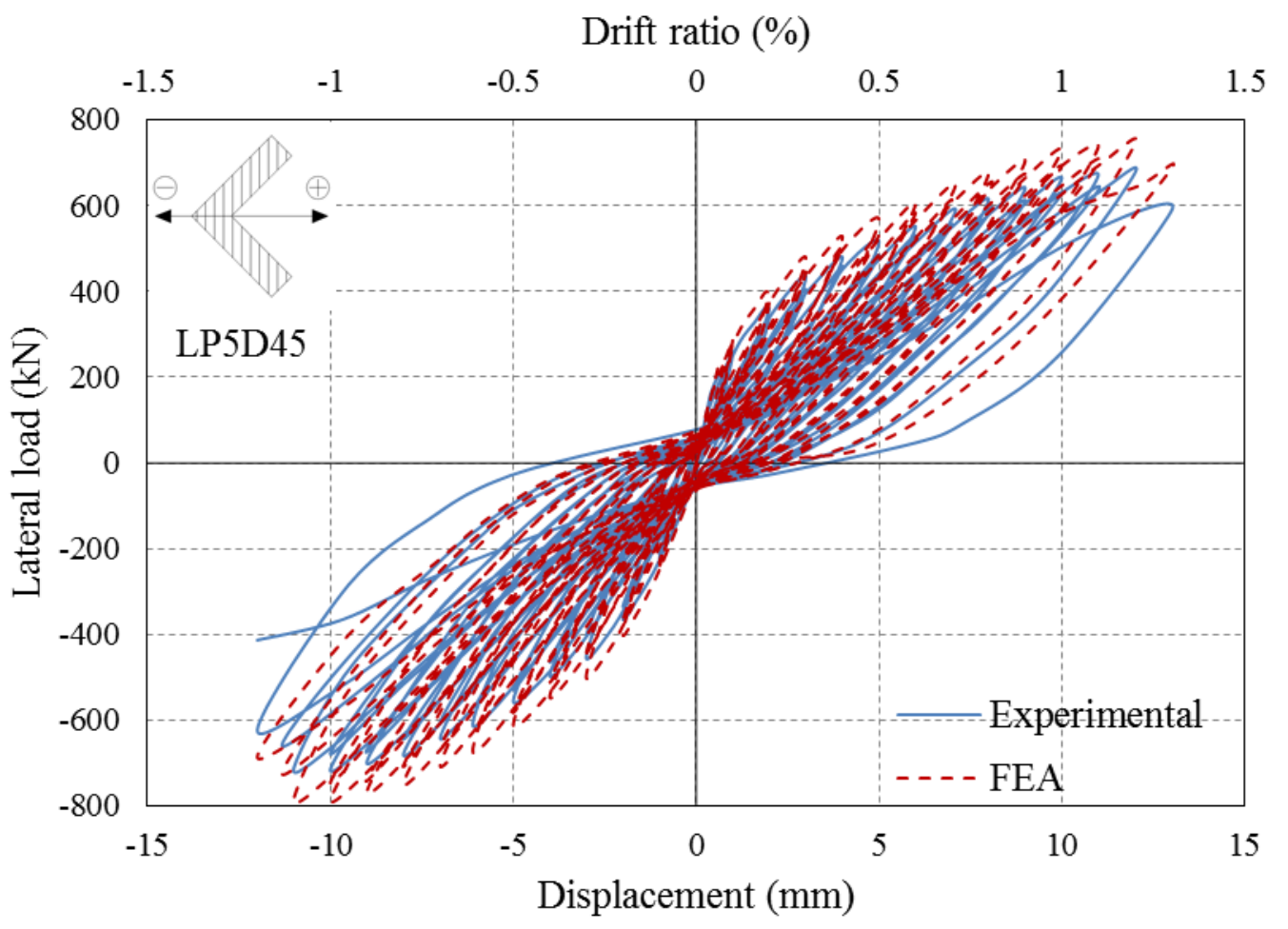

Figure 7.16 Comparison between experimental and analytical lateral loaddisplacement curves of Specimen LP5D45 


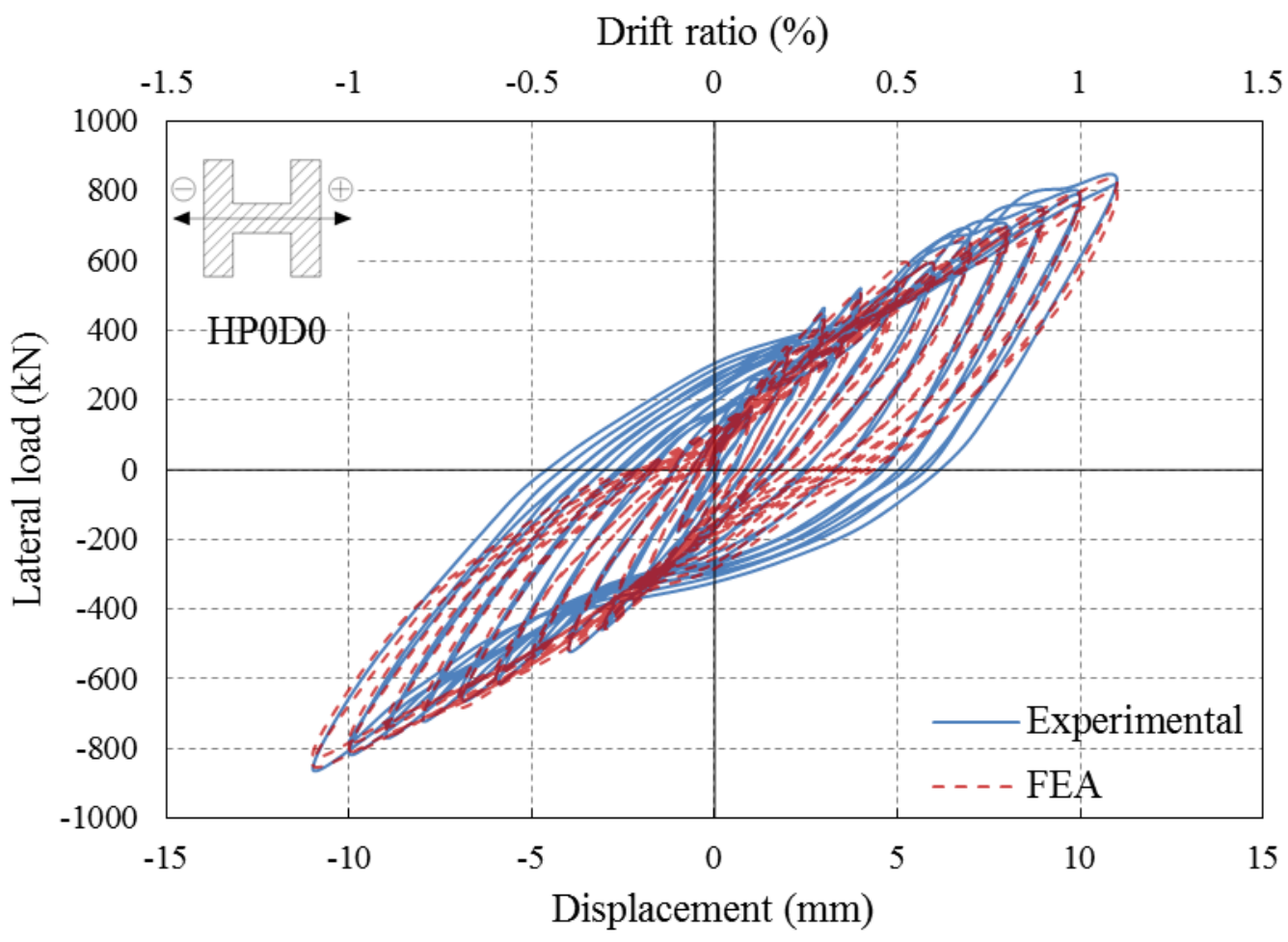

Figure 7.17 Comparison between experimental and analytical lateral loaddisplacement curves of Specimen HP0D0

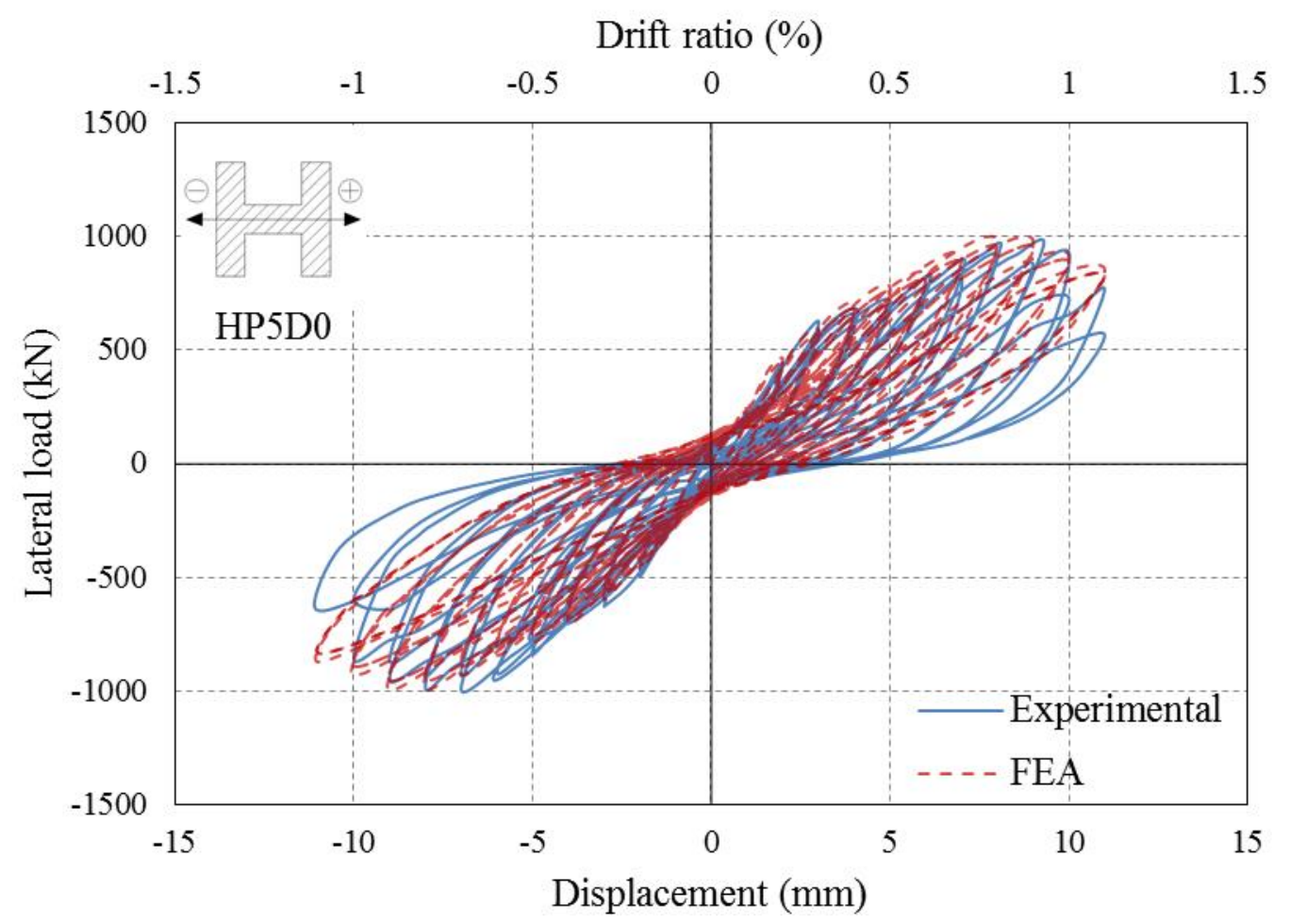

Figure 7.18 Comparison between experimental and analytical lateral loaddisplacement curves of Specimen HP5D0 


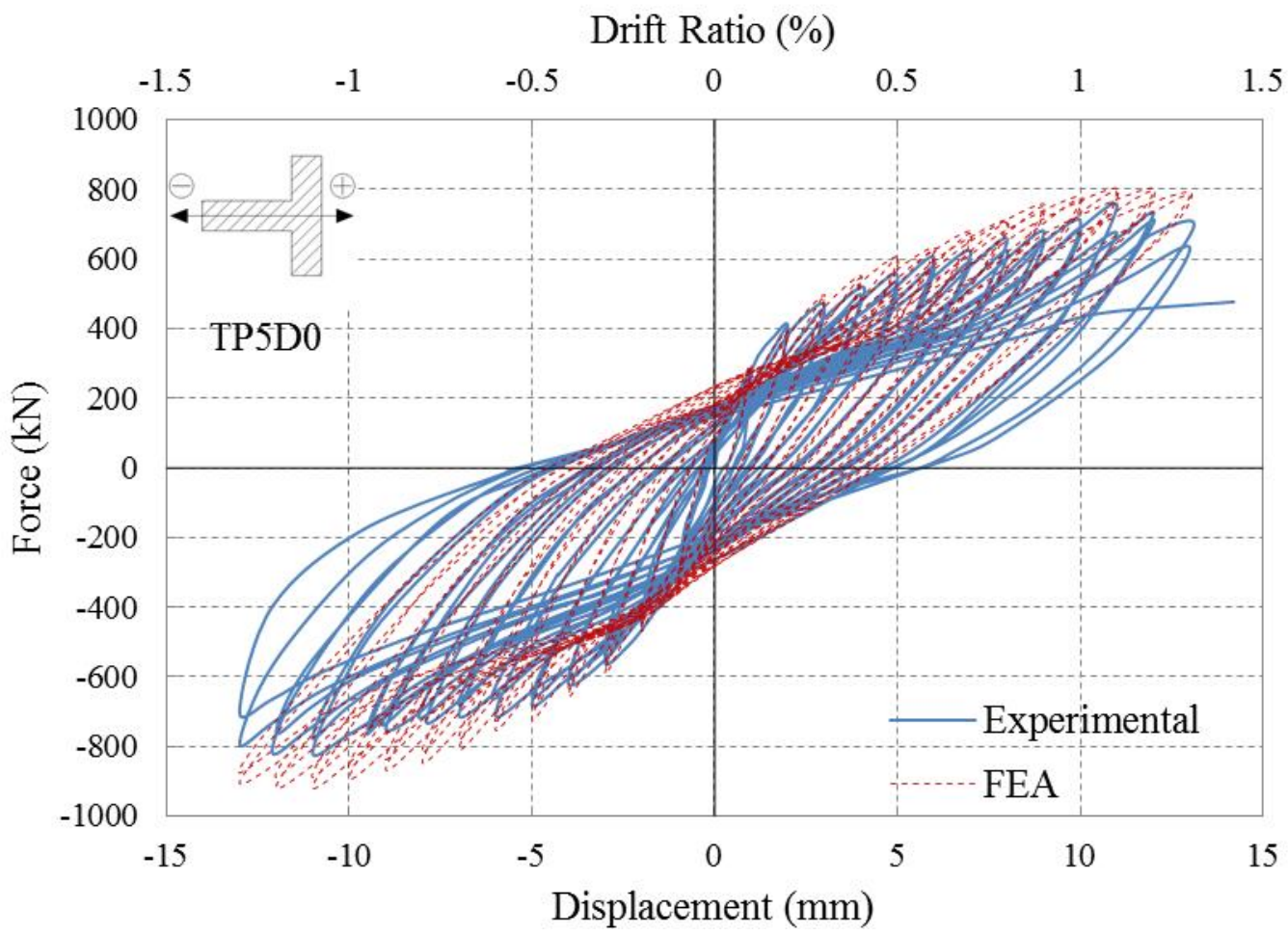

Figure 7.19 Comparison between experimental and analytical lateral loaddisplacement curves of Specimen TP5D0

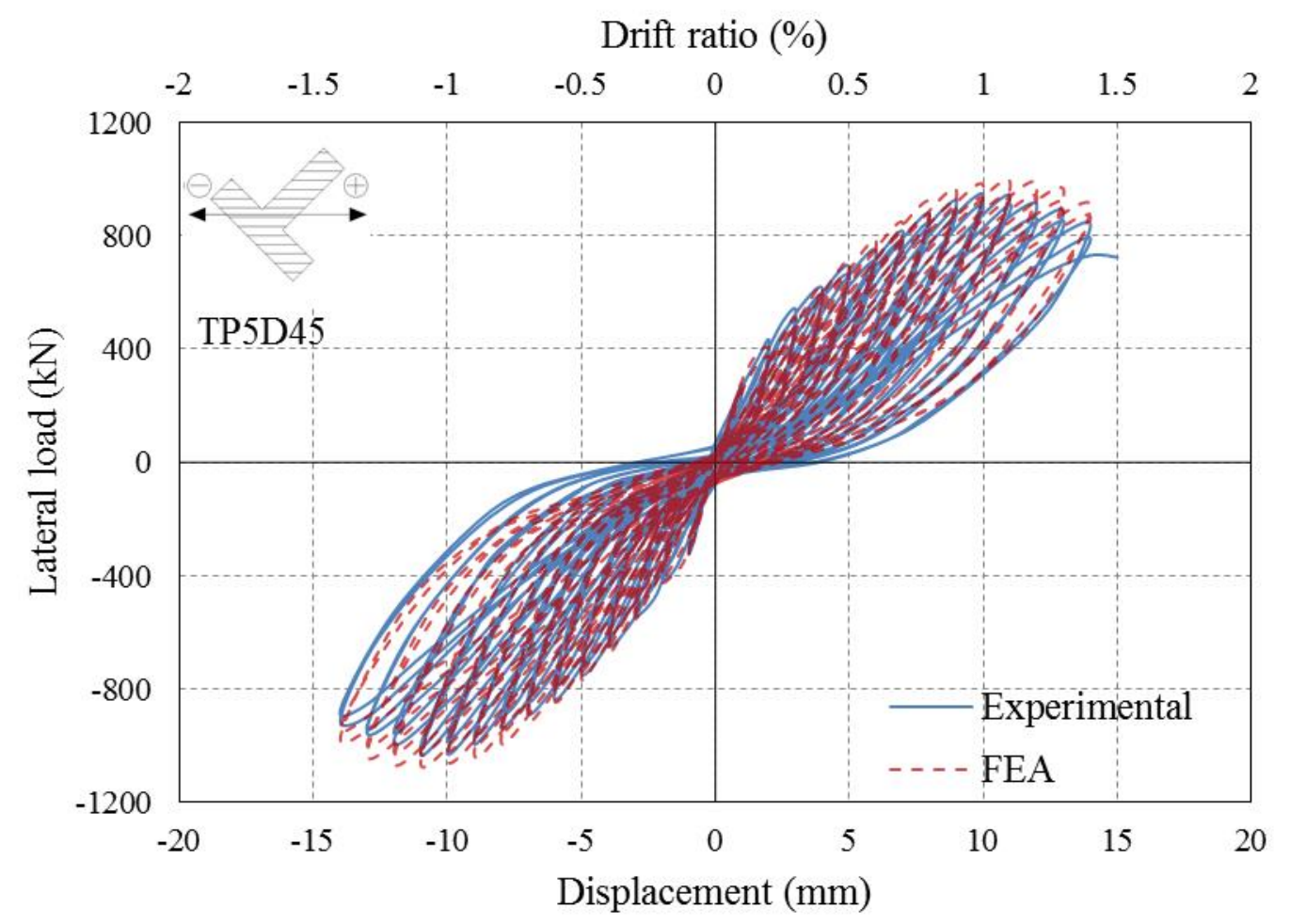

Figure 7.20 Comparison between experimental and analytical lateral loaddisplacement curves of Specimen TP5D45 


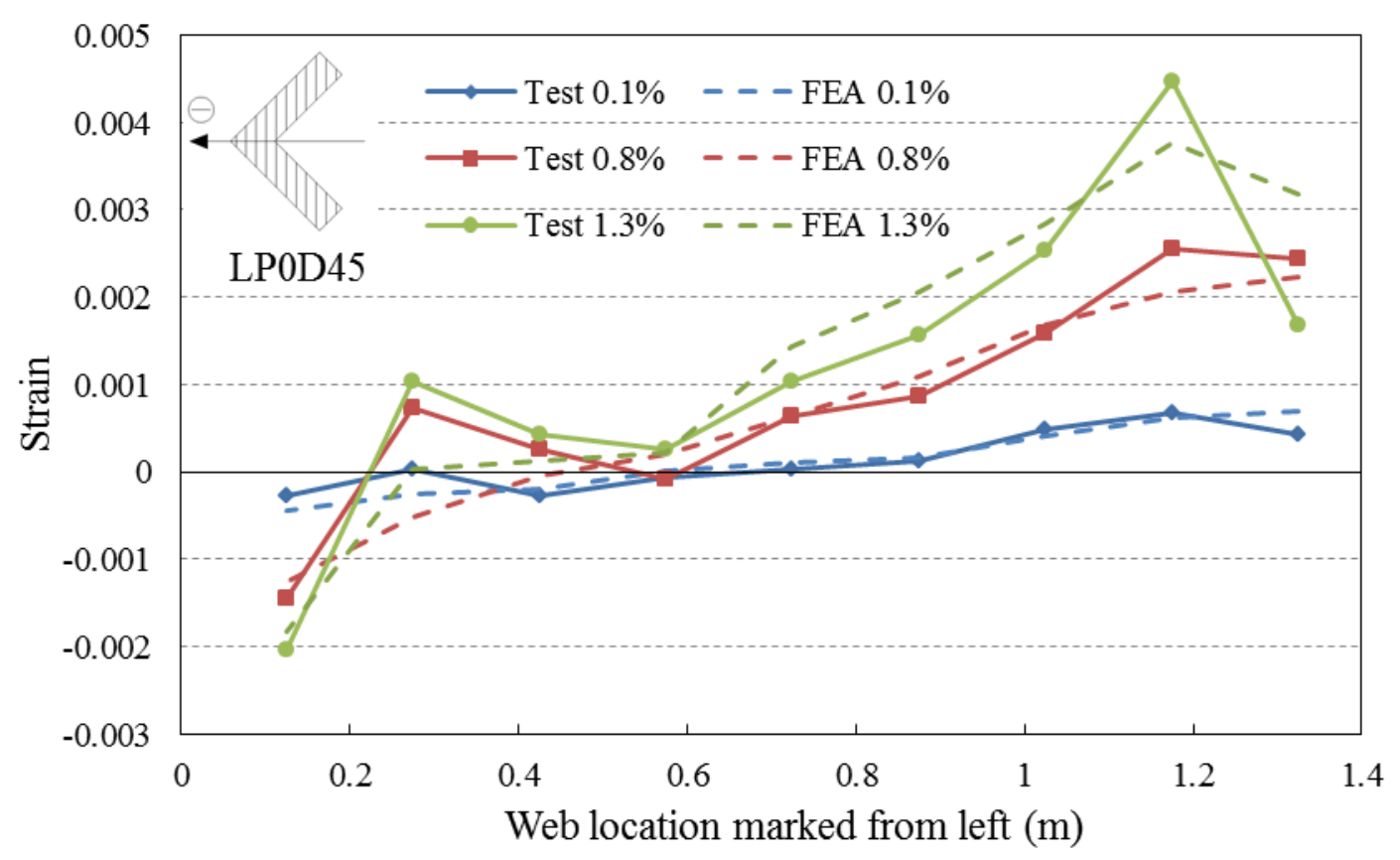

Figure 7.21 Comparison between experimental and analytical strain profiles of longitudinal reinforcement along the web of Specimen LP0D45

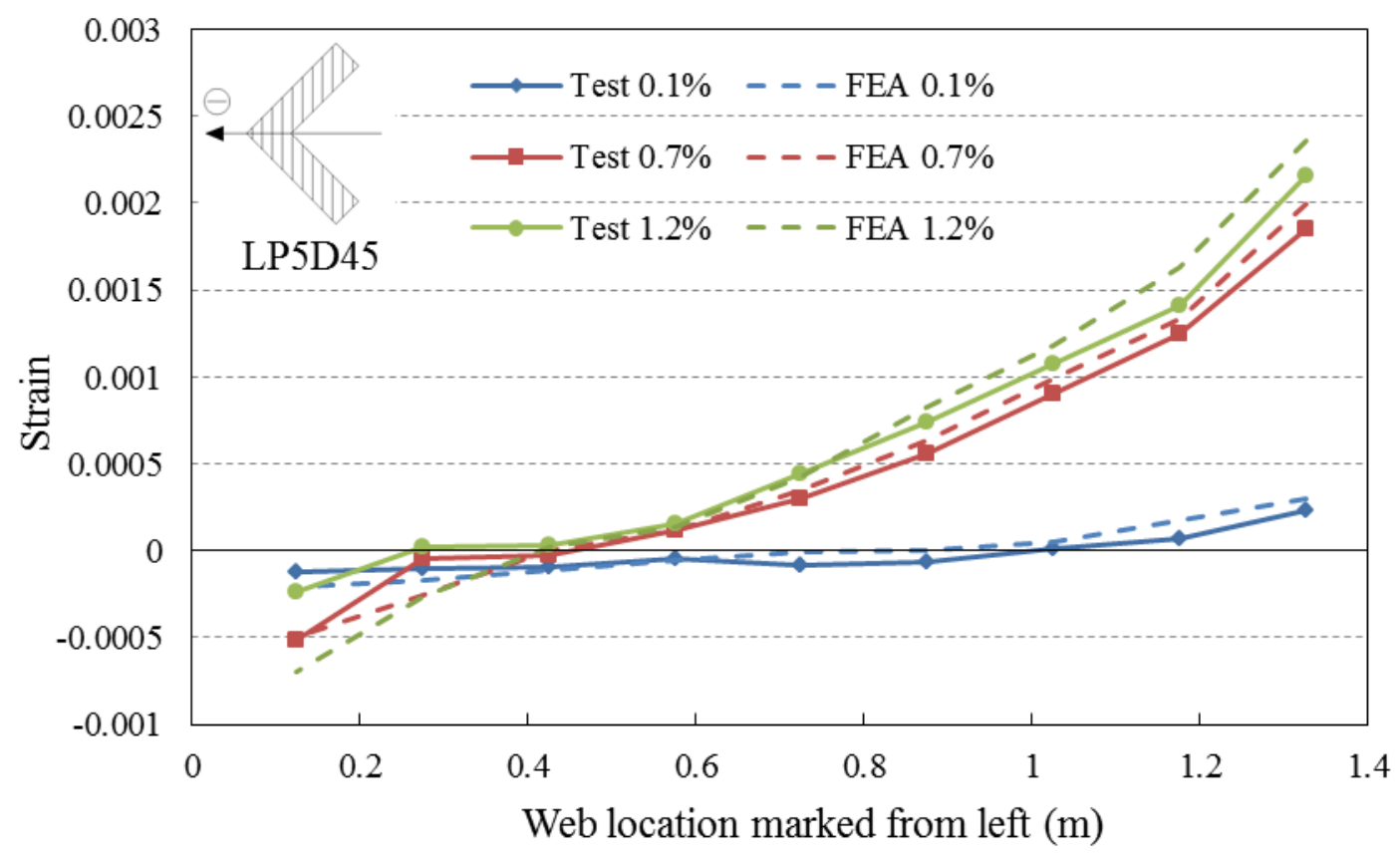

Figure 7.22 Comparison between experimental and analytical strain profiles of longitudinal reinforcement along the web of Specimen LP5D45 


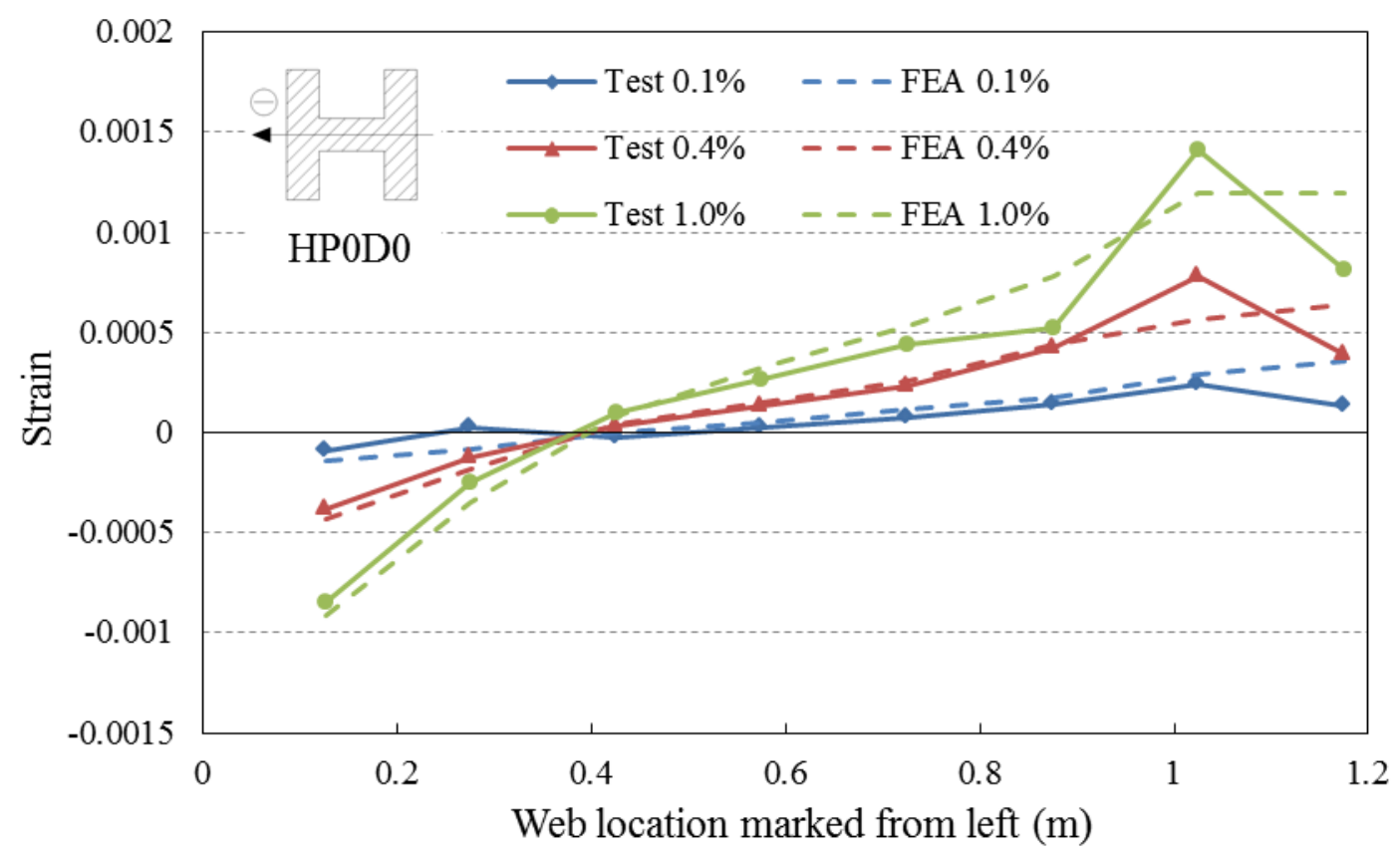

Figure 7.23 Comparison between experimental and analytical strain profiles of longitudinal reinforcement along the web of Specimen HP0D0

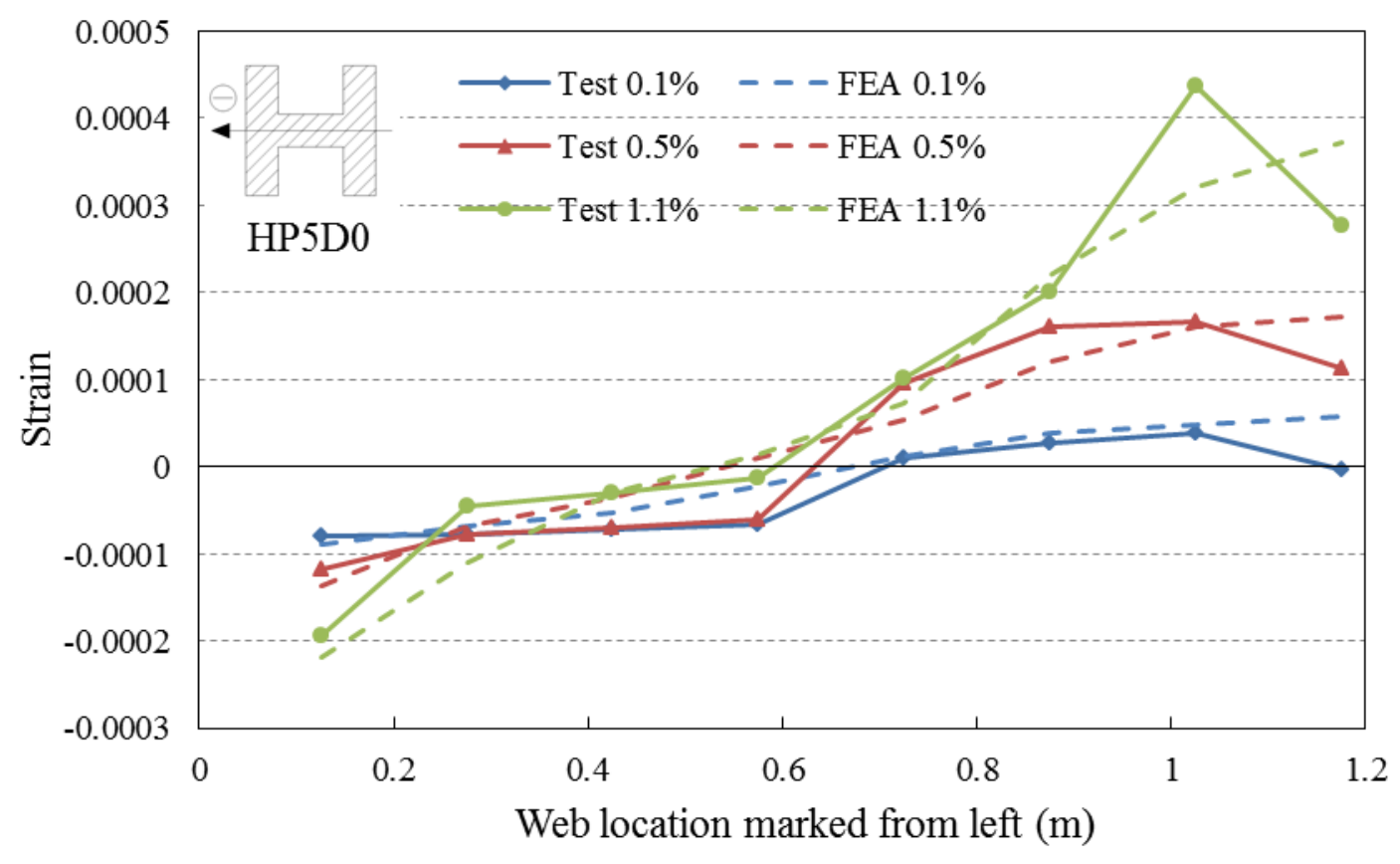

Figure 7.24 Comparison between experimental and analytical strain profiles of longitudinal reinforcement along the web of Specimen HP5D0 


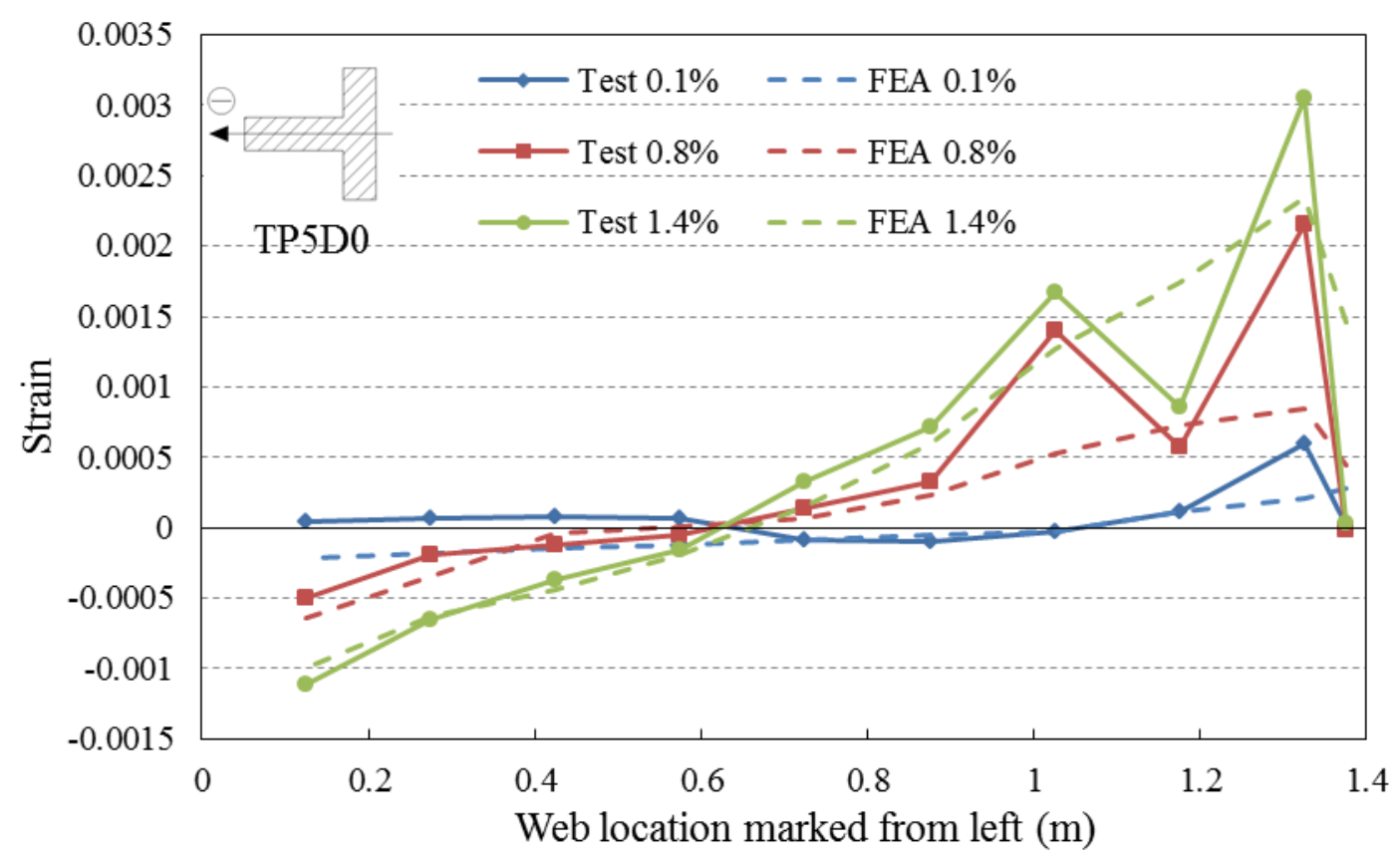

Figure 7.25 Comparison between experimental and analytical strain profiles of longitudinal reinforcement along the web of Specimen TP5D0

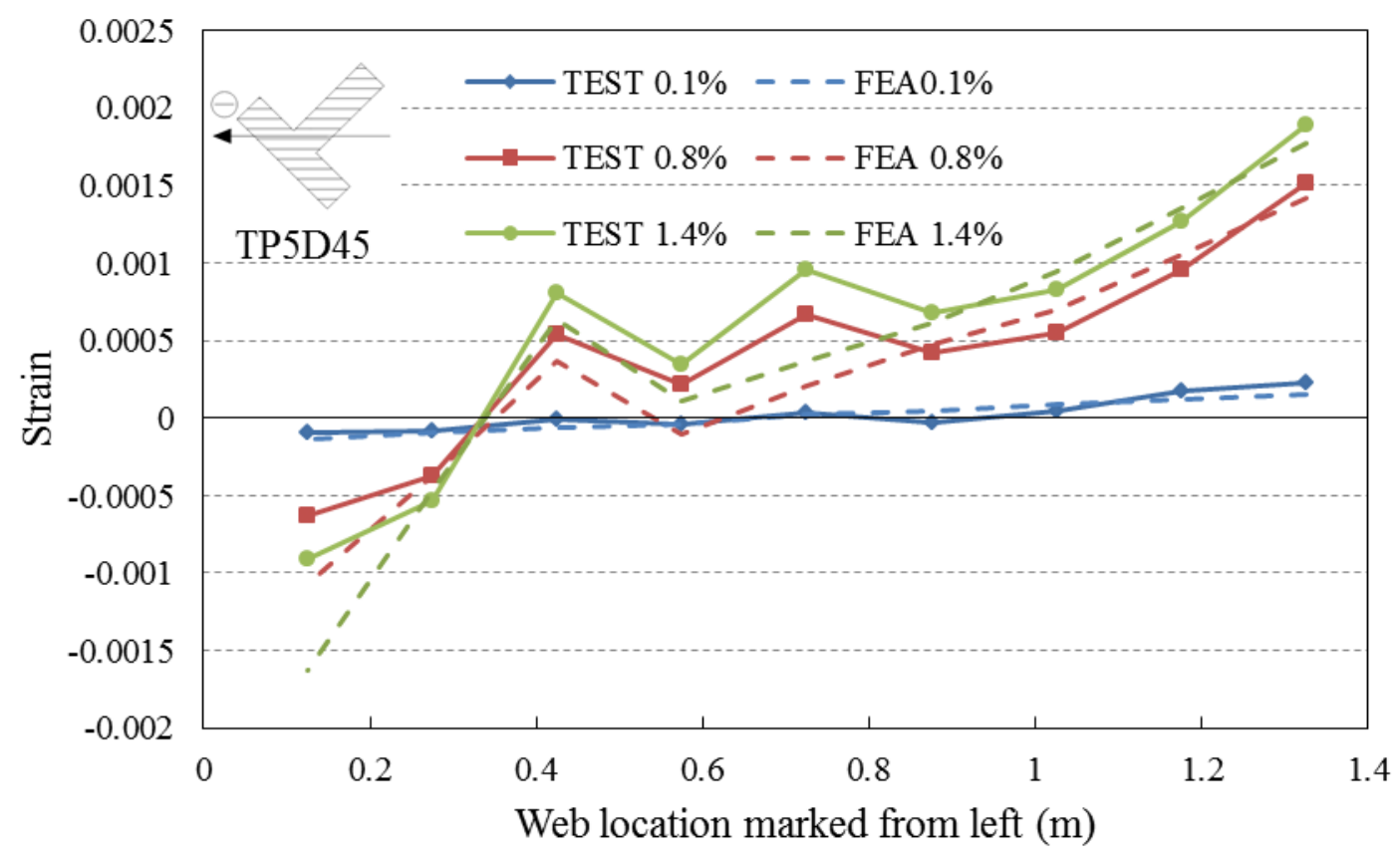

Figure 7.26 Comparison between experimental and analytical strain profiles of longitudinal reinforcement along the web of Specimen TP5D45 
In general, the aforementioned finite element approaches could successfully simulate the global behavior of non-rectangular RC squat walls under lateral cyclic loading. The models were able to capture certain important features of experimental hysteretic loops such as the maximum lateral strength in each loading direction, the drift ratio at which the specimens reached its maximum capacity and the pinching effects. Nevertheless, it was observed that the descending parts diverged slightly from the experimental data in the last few cycles, with the lateral strength being overestimated. The frequent open and close of diagonal cracks could be the primary reason behind this divergence, since it deteriorated the resistance of concrete, and caused the decline of the lateral strength. Overall, differences between these two curves were insignificant.

With regard to the local responses reflected in the strain profiles, the results obtained from the FEA generally matched the experimental results, even though the accuracy was not comparable with that of the hysteretic loop. At relatively low drift ratios, the analytical results agreed well with the experimental counterparts, in which the strain distributed almost linearly along the wall section. However, the simulated results gradually diverged from the test results when the lateral displacement increased, with their maximum difference being obtained at the final stage. But overall, sound correlations were observed between the experimental and analytical strains profiles.

In addition to the six specimens tested in this study, the H-shaped squat wall DP1 tested by Palermo and Vecchio [P2] is also chosen to further verify the competence of the introduced finite element approaches.

Specimen DP1 was designed identical to the NUPEC specimen [K2] in geometry. The wall was constructed with stiff top and bottom slabs. The top slab $(4415 \times 4000 \times 640 \mathrm{~mm})$ was used to distribute the horizontal and axial loads to the wall. The bottom slab $(4415 \times 4000 \times 620 \mathrm{~mm})$ was clamped to the laboratory strong floor, and served as a rigid foundation. The wall consisted of a central web wall (2885 mm in length, $2020 \mathrm{~mm}$ in height, and $75 \mathrm{~mm}$ in thickness) connecting to end flange walls (3045 mm in length, $2020 \mathrm{~mm}$ in height, and in thickness). Figure 7.27 
below demonstrates the front and side views of the finite element model of Specimen DP1.
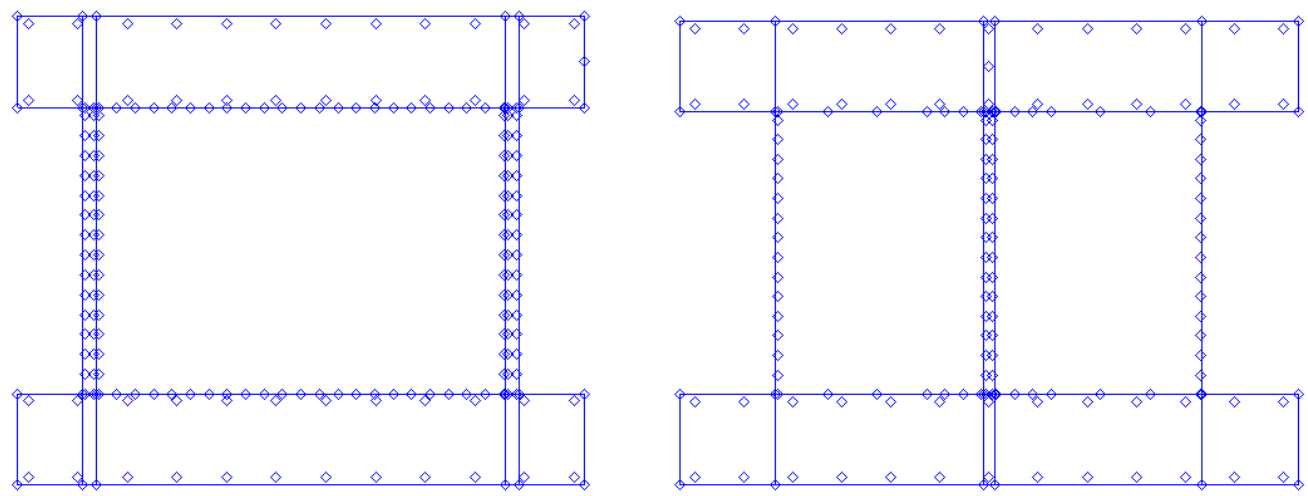

Figure 7.27 Front and side views of the model of Specimen DP1 [P2]

As for the reinforcement, the top and bottom slabs were reinforced with No.30 deformed bars with a spacing of $350 \mathrm{~mm}$ in each direction, with top and bottom layers. The web wall was reinforced with D6 steel bars, the spacing of which was $140 \mathrm{~mm}$ horizontally and $130 \mathrm{~mm}$ vertically in two parallel layers. The flange walls were also reinforced with D6 steel bars, spaced $140 \mathrm{~mm}$ horizontally, and $130 \mathrm{~mm}$ vertically near the web wall and $355 \mathrm{~mm}$ near the tips of flanges. The reinforcement layouts in the finite element model of Specimen DP1 are depicted in Figure 7.28.
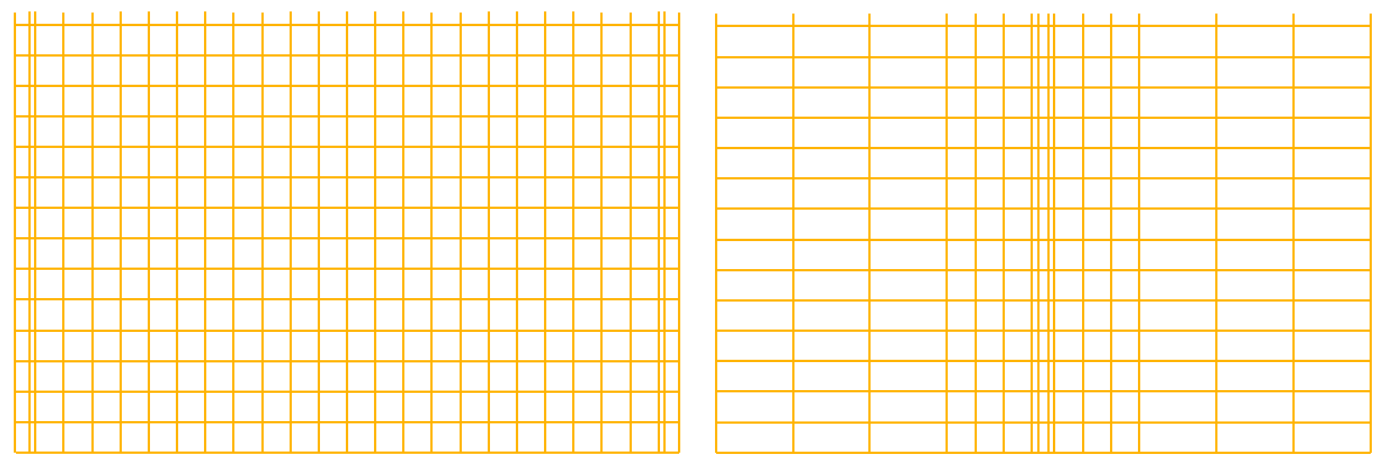

Figure 7.28 Web reinforcement and flange reinforcement in the model of Specimen DP1 [P2]

With regard to the materials, the design compressive strength of concrete were 21.7 $\mathrm{MPa}$ for the web and flange walls, 43.9 $\mathrm{MPa}$ for the top slab and 34.7 $\mathrm{MPa}$ for the bottom slab. Two types of reinforcing bars were used in the construction of 
Specimen DP1: No.30 and D6 steel bars. The former with a yield stress of $605 \mathrm{MPa}$ was used in the top and base slabs, which had a nominal diameter of $29.9 \mathrm{~mm}$ and a cross-sectional area of $700 \mathrm{~mm}^{2}$. The latter with a yield stress of $550 \mathrm{MPa}$ was used in the wall as longitudinal and horizontal reinforcement, which had a nominal diameter of $7.0 \mathrm{~mm}$ and a cross sectional area of $38.71 \mathrm{~mm}^{2}$.

As presented in Chapter 2, four $600 \mathrm{kN}$ actuators were installed at each corner of Specimen DP1 to distribute axial loads. The actuators were mounted to the base slab by bolts and connected to loads spreader beams located on the top slab with threaded rods. The top of the wall was applied with an axial load of approximately $940 \mathrm{kN}$. Cyclic lateral displacements were applied to the wall by two $1000 \mathrm{kN}$ actuators, one end of which was mounted horizontally to the reaction wall and the other was connected to load cells. In the finite element model, a uniformly distributed axial pressure calculated from the experimental data was imposed to the finite element model to simulate the loading condition, which is presented in Figure 7.29. Also, the bottom of the base was fixed against movements and rotations to simulate the boundary constraints in the real test.

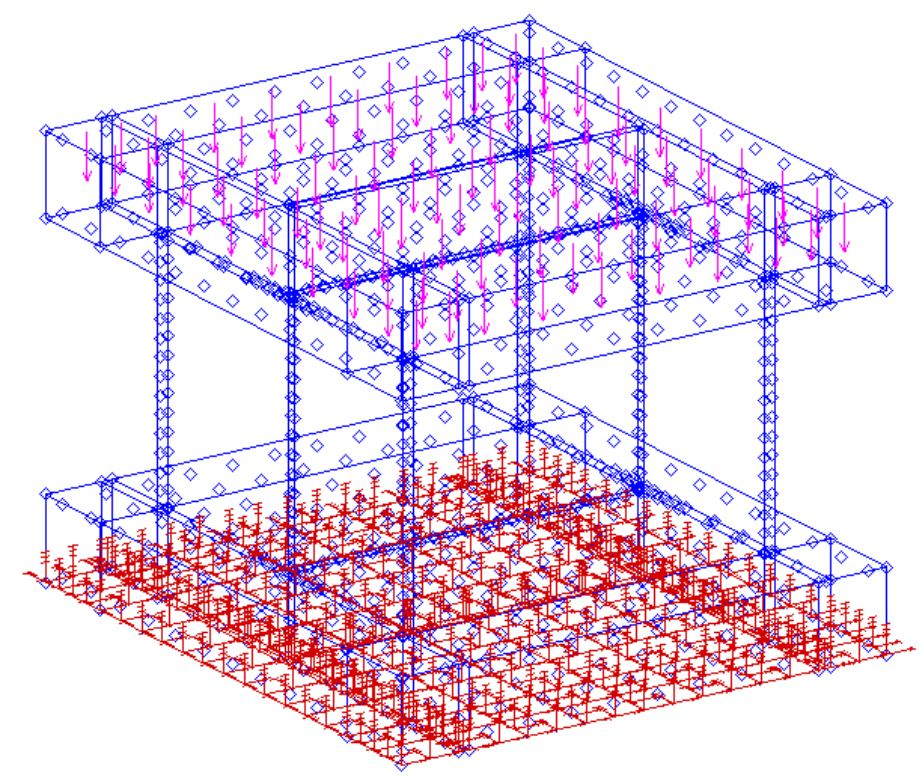

Figure 7.29 Loads and boundary constraints in the model of Specimen DP1 [P2]

The lateral load-displacement curves obtained from the test and the FEA is shown in Figure 7.30. In general, the analytical result showed a good correlation with the 
experimental one in terms of the strength capacity, deformation characteristics and energy dissipations, which demonstrated that the selected finite element approaches could be extended to other non-rectangular RC squat walls with various scales. However, there were still some discrepancies, especially between the first few circles and the last few cycles. At the initial stage of horizontal displacements, the finite element model had slightly larger strength than test specimen in both loading directions. The curve reached its peak at the displacement of $10 \mathrm{~mm}$, which was a little earlier when compared with the experimental results. The reason could be due to the assumed perfect bond between the concrete and reinforcement and the fully fixed base bottom in the finite element model. Furthermore, it was found that the test specimen experienced more severe strength degradation after attaining its maximum lateral strength, which might be caused by the vertical slip planes that did not appear in the finite element model.

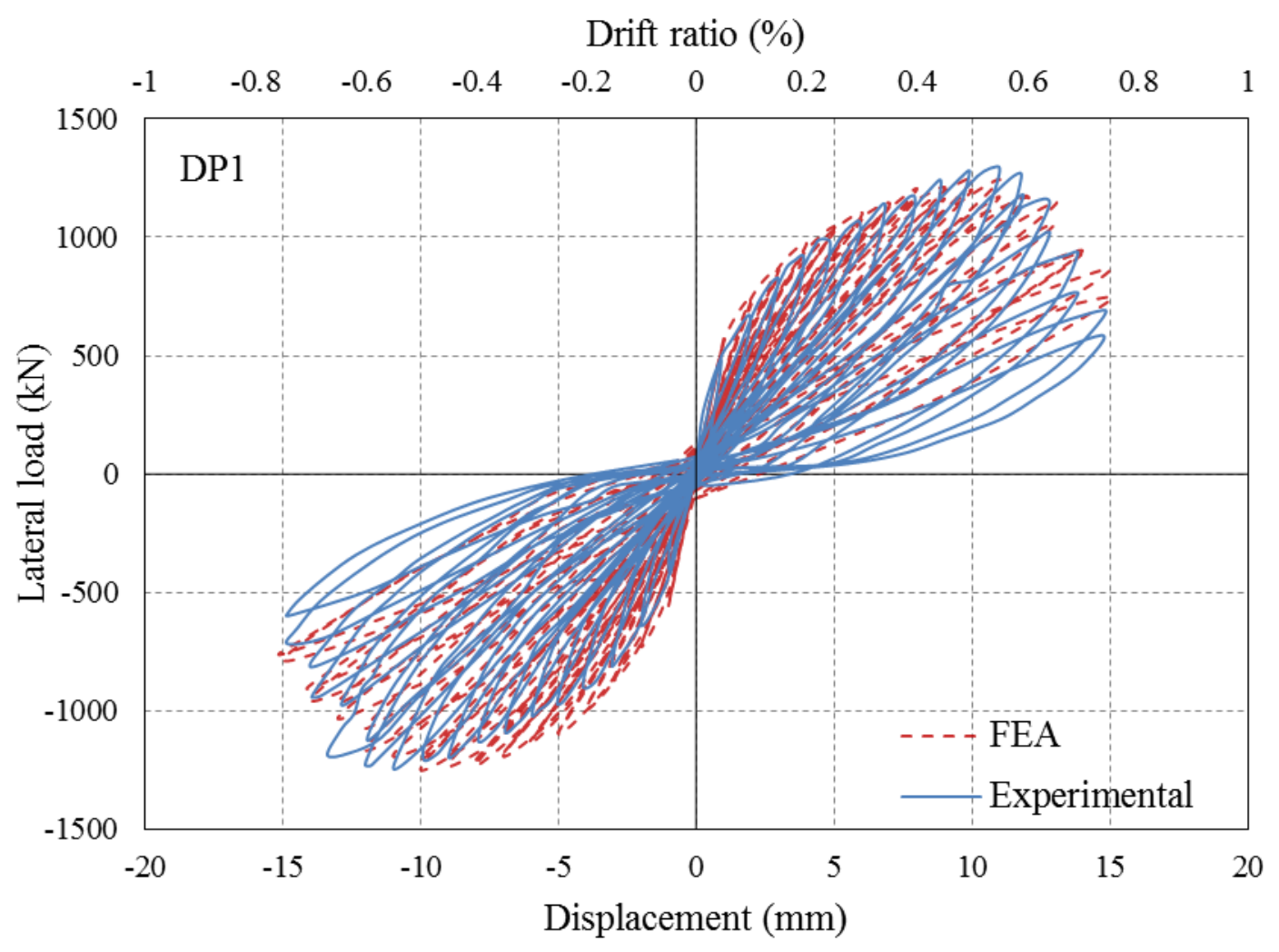

Figure 7.30 Comparison between experimental and analytical lateral loaddisplacement curves of Specimen DP1 [P2]

Therefore, from the comparisons between the prediction yielded by finite element models and results from the experiments, it can be concluded that the adopted finite 
element approaches are capable of accurately predicting the seismic performance of non-rectangular RC squat walls and could be applied to the following parametric study.

\subsection{Parametric Study on the Peak Shear Strength of Non-rectangular RC Squat Walls}

\subsubsection{Model layouts}

The modeling techniques were thoroughly explained in the previous section. The primary parameter under investigation was the lateral loading direction. Besides, important parameters such as the section shape, the flange length, and the axial load which were found to have a prominent impact on the peak shear strength of structural walls [H1, P1, Z2] were also included in this parametric study.

Similar to the test specimens, the finite element models could also be divided into three groups according to their section shapes, which were $\mathrm{H}$-shaped, L-shaped, and T-shaped groups. Among each group, two overhanging flange lengths were assigned to the models: $350 \mathrm{~mm}$ and $700 \mathrm{~mm}$ for the $\mathrm{H}$-shaped and T-shaped walls, and $650 \mathrm{~mm}$ and $1400 \mathrm{~mm}$ for the L-shaped walls. The longer flange length in each group was selected to match the layout of test specimens, as demonstrated in Figure 7.12 to Figure 7.14. The models with shorter a flange length were illustrated in Figure 7.31 to Figure 7.33. As for axial loads, three levels were adopted, namely $0.0072 f_{c}^{\prime} A_{g}, 0.0565 f_{c}^{\prime} A_{g}$ and $0.1 f_{c}^{\prime} A_{g}$ for the T-shaped and L-shaped walls, $0.0048 f_{c}^{\prime} A_{g}, 0.0501 f_{c}^{\prime} A_{g}$ and $0.1 f_{c}^{\prime} A_{g}$ for the H-shaped walls. These axial loads were selected to simulate the loading condition of squat walls in low-rise buildings and nuclear facilities. The first two levels were set in accordance with test specimens. In order to comprehensively explore the effect of lateral loading direction on the shear strength, models were applied with lateral displacements $0^{\circ}$ (orthogonal) to $350^{\circ}$ from the web segment at an interval of $10^{\circ}$. Table $\mathbf{7 . 2}$ below summarized the detail of the parameters. 
Table 7.2 Summary of parameters

\begin{tabular}{cccccccc}
\hline \multicolumn{2}{c}{ Section shape } & \multicolumn{2}{c}{ Flange length } & & Axial load & \multicolumn{2}{c}{ Loading direction } \\
\hline Name & Property & Name & Property & Name & Property & Name & Property \\
\hline L & L-shape & F35 & $350 / 650 \mathrm{~mm}$ & P0 & $0.0048 / 0.0072 f_{c}^{\prime} A_{g}$ & D0 & $0^{\circ}$ \\
\hline H & H-shape & F70 & $700 / 1400 \mathrm{~mm}$ & P5 & $0.0501 / 0.0565 f_{c}^{\prime} A_{g}$ & D10 & $10^{\circ}$ \\
\hline $\mathrm{T}$ & T-shape & & & P10 & $0.1 f_{c}^{\prime} A_{g}$ & $\ldots$ & $\ldots$ \\
\hline & & & & & D350 & $350^{\circ}$ \\
\hline
\end{tabular}

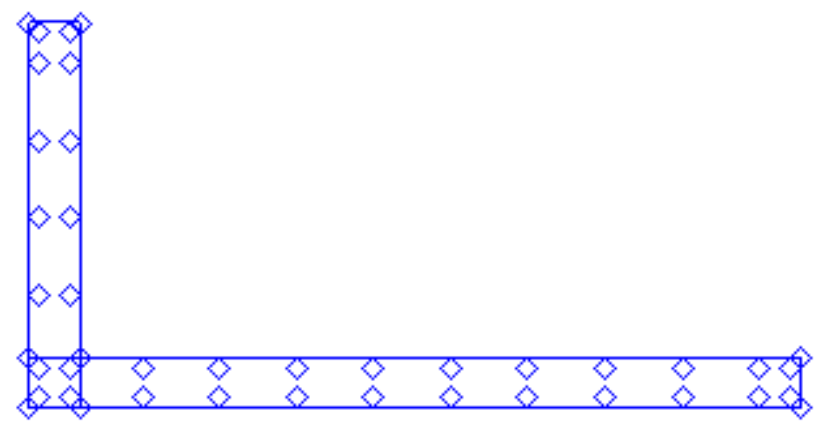

Figure 7.31 L-shaped models with $700 \mathrm{~mm}$ overhanging flange length

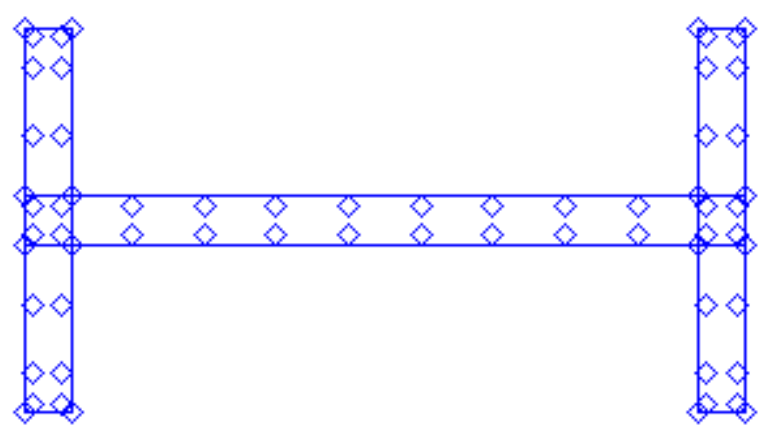

Figure $7.32 \mathrm{H}$-shaped models with $350 \mathrm{~mm}$ overhanging flange length 


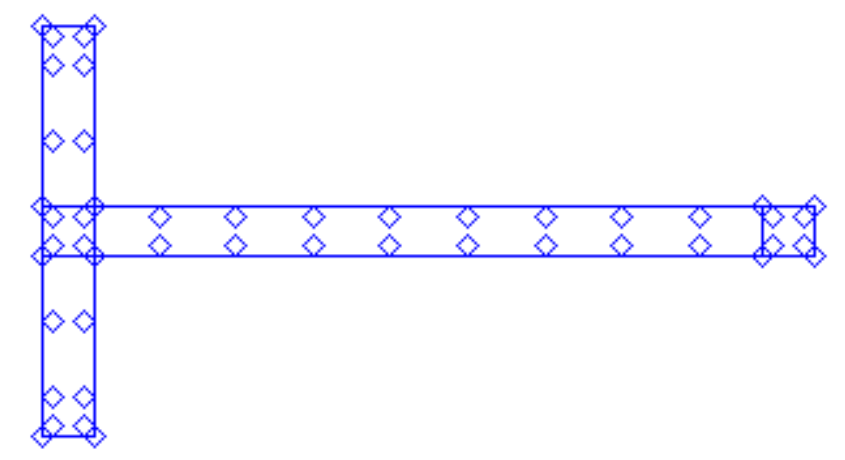

Figure 7.33 T-shaped models with $350 \mathrm{~mm}$ overhanging flange length

As seen from the table, each case was named according to the parameters it contained, which followed the sequence of section shape, flange length, axial load and loading direction. The first letter ( $\mathrm{L}, \mathrm{H}$ and $\mathrm{T}$ ) indicated the section shape of the finite element model, which was straightforward. The next part, F35 and F70, suggested the two flange lengths $(350 / 650 \mathrm{~mm}$ and $700 / 1400 \mathrm{~mm})$ used in the models. After that, P0, P5 and P10 reflected three levels of axial loads. The last part was a combination of a letter and a number. The letter D referred to the direction, and the subsequent number indicated the angle between the lateral loading and the web segment. For instance, the case HF70P10D70 represented an H-shaped squat wall with $700 \mathrm{~mm}$ overhanging flange length, which was imposed with axial loads of $0.1 f_{c}^{\prime} A_{g}$ and lateral loading $70^{\circ}$ from the web.

Except for flange lengths and section shapes, all models shared identical dimensions and reinforcement layouts with test specimens. For the H-shaped and T-shaped walls with an overhanging flange length of $350 \mathrm{~mm}$, longitudinal reinforcement was provided by D10 deformed bars at a spacing of $145 \mathrm{~mm}$, which yielded a reinforcement ratio of $1.57 \%$. For L-shaped walls with an overhanging flange length of $650 \mathrm{~mm}$, longitudinal reinforcement was provided by D10 deformed bars at a spacing of $150 \mathrm{~mm}$, which yielded a reinforcement ratio of $1.47 \%$. The material properties obtained from the test were assigned to the finite element models. Identical modeling techniques as demonstrated in the last section were continually used for the boundary constraints and the external loading. 


\subsubsection{Results of finite element analyses}

Figure 7.34 demonstrates the peak shear strength of L-shaped RC squat walls under lateral loads from different directions. The blue, red and purple curves, labelled as $\mathrm{ALR}=0.72 \%, \mathrm{ALR}=5.65 \%$ and $\mathrm{ALR}=10 \%$, represented the axial loads of $0.0072 f_{c}^{\prime} A_{g}, 0.0565 f_{c}^{\prime} A_{g}$ and $0.1 f_{c}^{\prime} A_{g}$ respectively. The tag 0 indicated the angle between the lateral loading and the web was $0^{\circ}$. That is to say lateral loads were parallel to the web segment. The other tags were designated in the same way. The figure in the middle illustrated the section shape of finite element models. As seen in the figure, the L-shaped model demonstrated identical peak shear strength in two directions with a gap of $180^{\circ}$. This was reasonable since the models were applied with cyclic horizontal displacements. As for walls with two identical segments, the plot of peak shear strength also showed a symmetric distribution about the line connecting 45 and 225 tags. It was evident that the peak shear strength of walls changed with the variation of the lateral loading direction. The model with a flange length of $650 \mathrm{~mm}$ reached its maximum peak shear strength when the horizontal displacement was $120^{\circ}$ from the web, which was around $163 \%$ of the minimum peak shear strength achieved when lateral loads were parallel to the flange. As for the model with $1400 \mathrm{~mm}$ flange length, the maximum peak shear strength was also reached when the lateral loading was $120^{\circ}$ from the web, and the minimum value occurred when horizontal displacements were $20^{\circ}$ from the web. Their difference could be as much as $38 \%$. A reduction of the peak shear strength of $20 \%$ was also witnessed when the direction of horizontal loads changed from parallel to the web to perpendicular to the web for models with $650 \mathrm{~mm}$ flange. Models with a flange length of $1400 \mathrm{~mm}$, on the other hand, had identical peak shear strengths under these two loading directions, due to the symmetric section shape. A similar distribution of peak shear strength was also recorded in the L-shaped slender walls tested by Zhang [Z2]. Moreover, it was found that axial loads played a favorable role in increasing the peak shear strength of models, regardless of the direction of lateral loading, which was consistent with the finding in the test. For models with $650 \mathrm{~mm}$ flange length, the average increases of the peak shear strength were approximately $17 \%$ and $11 \%$ when the axial load rose from 0.0072 to 0.0565 and 0.0565 to $0.1 f_{c}^{\prime} A_{g}$ respectively. The corresponding numbers for models with 1400 
mm flange length were $17 \%$ and $12 \%$. The largest growth of around $35 \%$ was found in walls with a flange length of $650 \mathrm{~mm}$ when horizontal displacements were $100^{\circ}$ from the web. An identical increase of $35 \%$ was observed for walls with $1400 \mathrm{~mm}$ flange length when the lateral loading was $110^{\circ}$ from the web. Furthermore, it was evident that the flange length significantly affected the variation of the peak shear strength of models. As the flange length decreased, the corresponding shear strength along the direction of the flange also declined, which caused the redistribution of the total shear strength. Models with a flange length of $650 \mathrm{~mm}$ apparently processed less strength when the lateral loading was parallel to the flange segment, since the flange segment was much shorter than the web segment. For models with $1400 \mathrm{~mm}$ flange, the peak shear strength showed an ascending trend as the angle between horizontal displacements and the web increased from $0^{\circ}$ to $45^{\circ}$. This was because models with $1400 \mathrm{~mm}$ flange length had identical segments in each principle direction, which ensured more evenly distributed compression and tension zones when the lateral loading was around $45^{\circ}$ from the web.

Figure 7.35 demonstrates the peak shear strength of H-shaped RC squat walls under lateral loads from different directions. The blue, red and purple curves, labelled as $\mathrm{ALR}=0.48 \%, \mathrm{ALR}=5.01 \%$ and $\mathrm{ALR}=10 \%$, represented the axial loads of $0.0048 f_{c}^{\prime} A_{g}, 0.0501 f_{c}^{\prime} A_{g}$ and $0.1 f_{c}^{\prime} A_{g}$ respectively. The tags and middle figure processed identical meanings as stated previously. As can be seen in the figure, the H-shaped model showed identical peak shear strength in two directions with a gap of $180^{\circ}$, due to the imposed cyclic horizontal displacements. The dualaxisymmetrical section shape also contributed to the symmetric distribution of peak shear strength about the horizontal and vertical direction. It was witnessed that the peak shear strength of walls changed with the variation of lateral loading direction. The maximum peak shear strength of models with $350 \mathrm{~mm}$ flange length was reached when horizontal displacements were parallel to the web, which was around $143 \%$ of the minimum peak shear strength achieved when lateral loads were perpendicular to the web. As for the model with a flange length of $700 \mathrm{~mm}$, the opposite was true: the peak shear strength gradually increased as the angle between horizontal loading and web segment rose from $0^{\circ}$ to $90^{\circ}$, with the difference being as much as $40 \%$. Axial loads were found to noticeably increase the peak shear 
strength, regardless of the direction of lateral loading. For models with $350 \mathrm{~mm}$ flange length, the average increases of the peak shear strength were approximately $11 \%$ and $13 \%$ when the axial load rose from 0.0048 to 0.0501 and 0.0501 to $0.1 f_{c}^{\prime} A_{g}$ respectively. The corresponding numbers for models with $700 \mathrm{~mm}$ flange length were $12 \%$ and $13 \%$. The largest growth of approximately $15 \%$ was found in walls with $350 \mathrm{~mm}$ flange length when the lateral loading was $70^{\circ}$ from the web. An increase of $13 \%$ was observed in walls with $700 \mathrm{~mm}$ flange length when the lateral loading was $80^{\circ}$ from the web. Similar to the L-shaped models, the flange length significantly affected the variation of the peak shear strength of models. Models with $700 \mathrm{~mm}$ flange length, as seen in the figure, processed three identical wall segments. Hence the maximum peak shear strength was achieved when two flange segments were parallel to the direction of horizontal displacements. As for walls with a flange length of $350 \mathrm{~mm}$, the contribution of flange segments largely declined when their lengths were reduced by half. Consequently, the maximum peak shear strength was reached when lateral loads were parallel to the web.

Figure 7.36 demonstrates the peak shear strength of T-shaped RC squat walls under lateral loads from different directions. The blue, red and purple curves, labelled as $\mathrm{ALR}=0.72 \%, \mathrm{ALR}=5.65 \%$ and $\mathrm{ALR}=10 \%$, represented the axial loads of $0.0072 f_{c}^{\prime} A_{g}, 0.0565 f_{c}^{\prime} A_{g}$ and $0.1 f_{c}^{\prime} A_{g}$ respectively. The tags and middle figure processed identical meanings as stated previously. As seen in the figure, the Tshaped model demonstrated identical peak shear strength in two directions with a gap of $180^{\circ}$. This was expected since reversed horizontal displacements were applied. Besides, due to the symmetric section shape, the plot also showed symmetric distribution about the line connecting 0 and 180 tags. The peak shear strength of walls changed with the variation of lateral loading direction. The model with $350 \mathrm{~mm}$ flange length reached its maximum peak shear strength when the horizontal displacement was parallel to the web, which doubled the minimum peak shear strength achieved when lateral loads were parallel to the flange. As for the model with $700 \mathrm{~mm}$ flange length, the maximum peak shear strength was reached when the lateral loading was $40^{\circ}$ from the web, and the minimum value occurred when lateral loads were parallel to the flange. Their difference could be as much as $30 \%$. A reduction of the peak shear strength of $20 \%$ was also witnessed when the 
direction of horizontal loads changed from parallel to the web to perpendicular to the web for models with $700 \mathrm{~mm}$ flange length, which was far less than the difference in walls with a shorter flange. A similar decline of around $19 \%$ was also recorded in the T-shaped slender walls tested by Zhang [Z2], which suggested that the influence of lateral loading direction on the peak shear strength of nonrectangular walls was significant. As for the effect of axial loads, evident increases were found when extra axial loads were applied, regardless of the direction of lateral loading, which was also in accordance with Zhang's study [Z2]. For models with $350 \mathrm{~mm}$ flange length, the average increases of the peak shear strength were approximately $17 \%$ and $12 \%$ when the axial load rose from 0.0072 to 0.0565 and 0.0565 to $0.1 f_{c}^{\prime} A_{g}$ respectively. The corresponding numbers for models with 700 mm flange length were $11 \%$ and $9 \%$. The largest growth of around $14 \%$ was found in walls with $350 \mathrm{~mm}$ flange length under orthogonal lateral loading. An increase of $14 \%$ was observed for walls with $700 \mathrm{~mm}$ flange length when the lateral loading was $70^{\circ}$ from the web. Similar to the observation in the L-shaped and H-shaped models, the flange length significantly affected the variation of the peak shear strength of models. This was because the resistance of the model in one principle direction was mainly provided by the flange. As the length of the flange decreased, its contribution declined accordingly, which largely affected the overall shear strength. Models with $700 \mathrm{~mm}$ flange length, as seen in the figure, had similar segments in each principle direction, which enabled the maximum peak shear strength to be achieved when the lateral loading was around $45^{\circ}$ from the web. As for walls with a flange length of $350 \mathrm{~mm}$, the strength in the direction along flange was evidently less than that along the web. Therefore, when the angle between horizontal displacements and web segment changed from $0^{\circ}$ to $90^{\circ}$, the overall resistance was also diminished. 

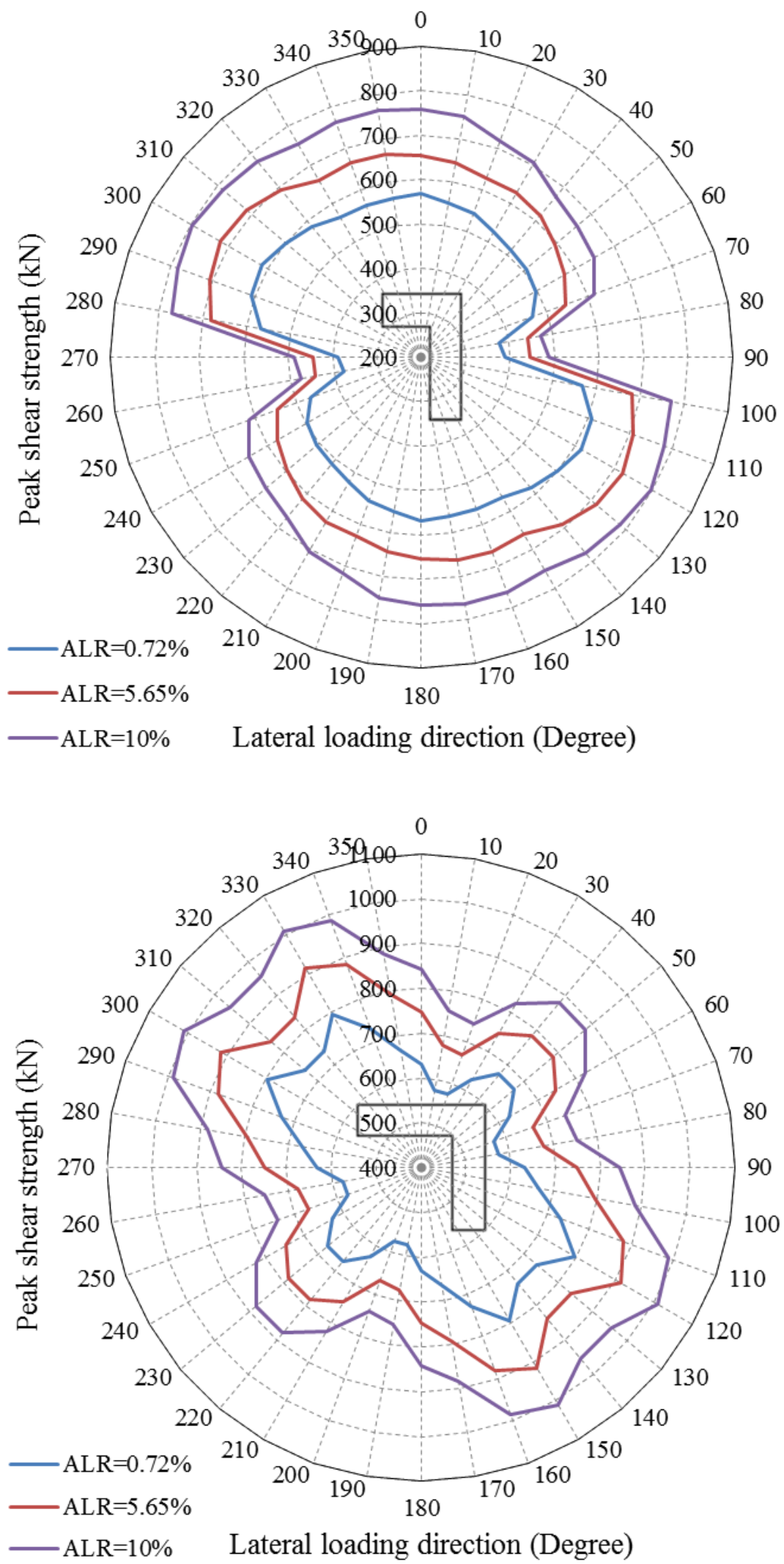

Figure 7.34 The peak shear strength of L-shaped RC squat walls under lateral loading from various directions 

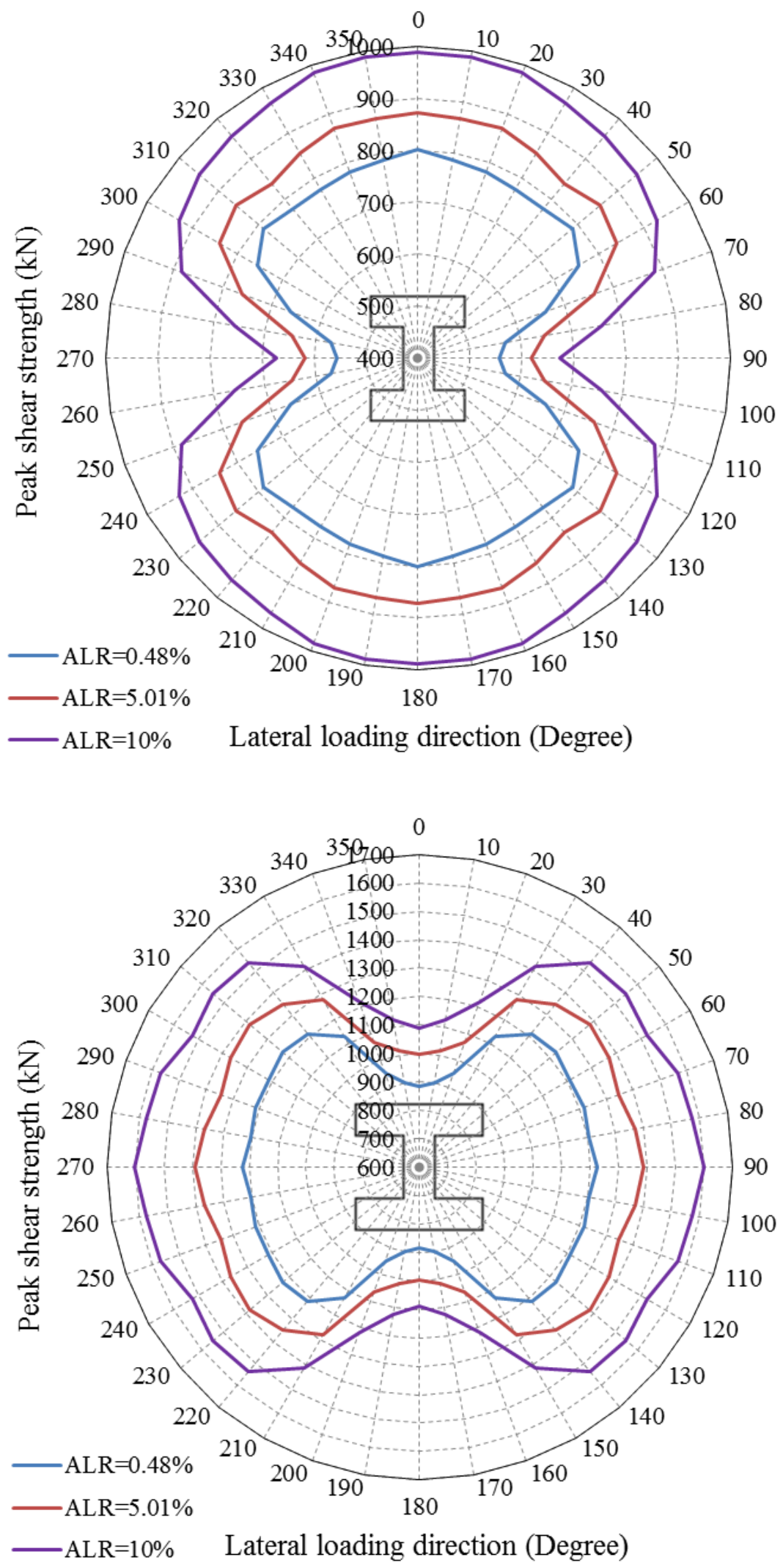

Figure 7.35 The peak shear strength of $\mathrm{H}$-shaped $\mathrm{RC}$ squat walls under lateral loading from various directions 

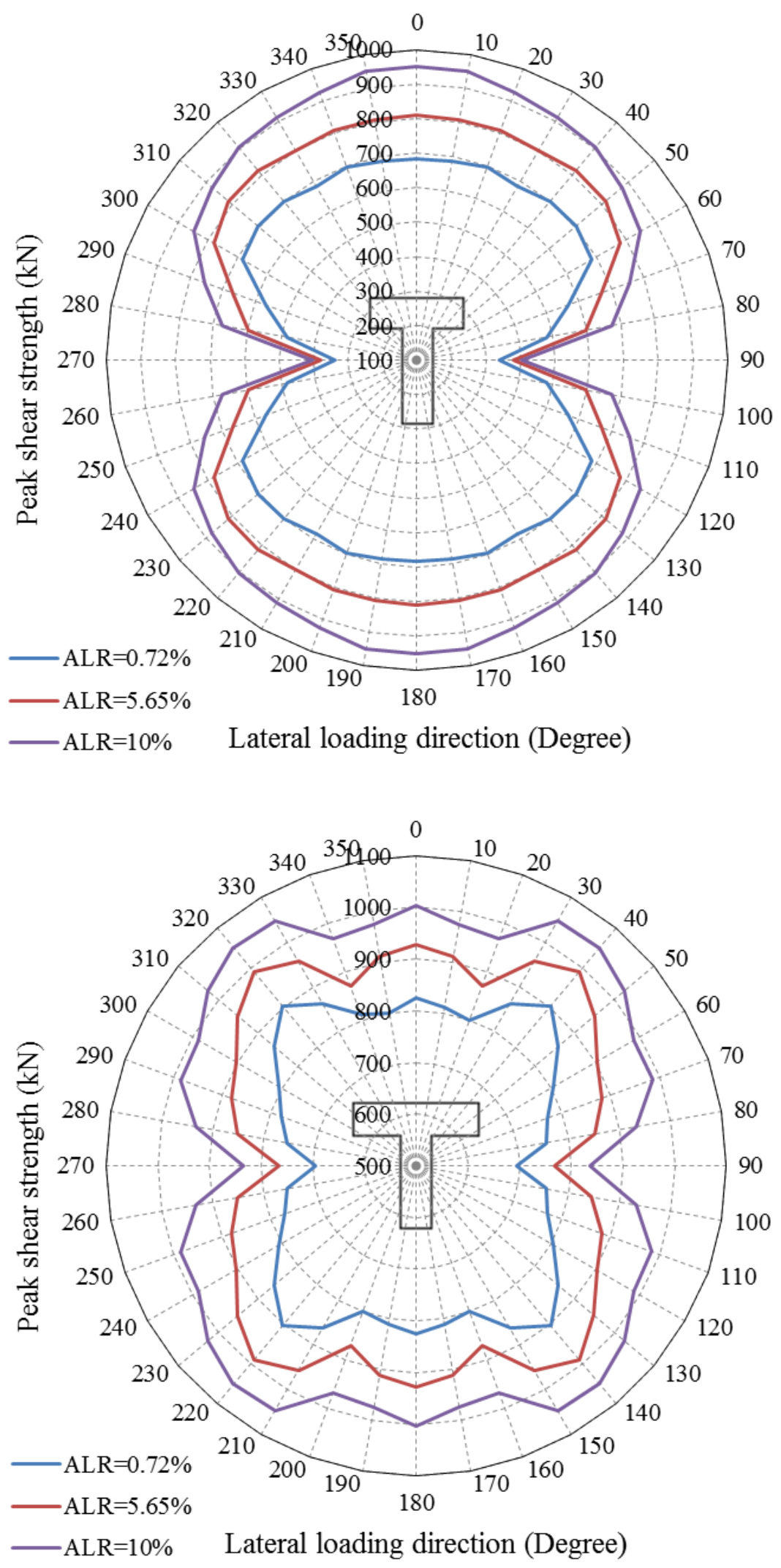

Figure 7.36 The peak shear strength of T-shaped RC squat walls under lateral loading from various directions 


\subsection{Summary}

Since in real earthquakes, seismic waves can reach structures from any direction, it is essential to study the seismic behavior of structural walls under various lateral loading directions. The experimental results provided the information regarding only the lateral loading $0^{\circ}$ and $45^{\circ}$ from the web. Moreover, the equation for peak shear strength proposed in Chapter 6 was developed on the assumption that lateral loads were parallel to the web segment. To investigate the influence of lateral loading direction on the peak shear strength of non-rectangular RC squat walls, a parametric study was carried out by using the nonlinear finite element software Diana 9.4. The effects of section shapes, flange lengths and axial loads on the peak shear strength of walls were also reported. Several conclusions are drawn as follows:

- From the finite element analyses, it was revealed that the peak shear strength of non-rectangular RC squat walls varied at different lateral loading directions.

- As for L-shaped walls, the maximum peak shear strength was reached when lateral loads were $120^{\circ}$ from the web. Among these walls, those with a flange length of $650 \mathrm{~mm}$ attained the minimum peak shear strength when lateral loads were parallel to the flange. Those with $1400 \mathrm{~mm}$ flange length reached the minimum peak shear strength when horizontal displacements were $20^{\circ}$ from the web. The difference could be as much as $63 \%$ and $38 \%$ for these two types of walls respectively.

- The maximum peak shear strength of H-shaped walls with $350 \mathrm{~mm}$ flange length was reached when horizontal displacements were parallel to the web, which was approximately $143 \%$ of the minimum peak shear strength achieved when lateral loads were perpendicular to the web. As for models with a flange length of $700 \mathrm{~mm}$, the opposite was found to be true. The difference between the maximum and the minimum values was $40 \%$.

- As for T-shaped walls, models with $350 \mathrm{~mm}$ flange length reached the maximum peak shear strength when the horizontal displacement was parallel to the web, which doubled the minimum peak shear strength achieved when lateral 
loads were parallel to the flange. Models with a flange length of $700 \mathrm{~mm}$ reached the maximum peak shear strength when the lateral loading was $40^{\circ}$ from the web, and the minimum value when lateral loads were parallel to the flange. Their difference could be as much as $30 \%$.

- Axial loads were found to play a favorable role in increasing the peak shear strength of the finite element models, regardless of the direction of lateral loading, which was consistent with the finding in the test.

- The influence of the flange length on the peak shear strength of non-rectangular RC squat walls was significant. For models with a shorter flange length, the resistance provided by the flange in one principle direction also declined, which affected the overall strength to a large extent. 


\section{CHAPTER 8 SUMMARY AND CONCLUSIONS}

\subsection{Summary and Conclusions}

To investigate the seismic behavior of non-rectangular RC squat walls, an experimental program consisting of twelve specimens with L-shaped, H-shaped and T-shaped sections was conducted at the Protective Engineering Lab in NTU. The specimens resembled structural walls commonly adopted in buildings in Singapore as well as safety-related nuclear facilities around the world. The design of the specimens was based on previous experiments [K2, P2] and provisions of ACI 31814 [A1]. Global and local responses of these walls were reported and discussed in terms of failure mechanisms, cracking patterns, hysteretic responses, displacement components, and strain profiles. Further comparisons and analyses were also conducted on important subjects such as failure modes, load-displacements relationships, shear deformations and shear lag effects.

After that, a modified strut-and-tie model incorporating the effect of flange segments was developed for non-rectangular RC squat walls. The function form of the peak shear strength equation for non-rectangular RC squat walls was derived based on this analytical model. Then the coefficients in the function form were calibrated using the fmincon nonlinear solver in MATLAB [M3]. Comparisons were conducted among various equations proposed in the literature to demonstrate the accuracy and effectiveness of the proposed equation. Besides, improvements were also implemented on the shear strength equations in ACI 318-14 [A1] and ASCE 43-05 [A3] using the identical method. The probabilistic approach was then adopted to reconcile the large scatter and to avoid the biased estimate found in the deterministic prediction of the peak shear strength of non-rectangular RC squat walls.

Finally, to explore the influence of lateral loading direction on the peak shear strength of non-rectangular RC squat walls, a comprehensive parametric study was carried out using the nonlinear finite element software Diana 9.4 [D1]. The effects 
of section shapes, flange lengths and axial loads on the peak shear strength were also reported.

Based on the experimental, numerical and analytical investigations conducted in this study, the main findings are listed below.

\subsubsection{Seismic performance of specimens}

1. Generally, initial shear cracks occur at the middle and upper part of wall segments at a drift ratio ranging from $0.1 \%$ to $0.4 \%$, which is prior to the yielding of longitudinal reinforcing bars. The specimens experience their first yielding at a drift ratio ranging from $0.4 \%$ to $0.7 \%$. As lateral displacements increase, diagonal cracks inclined from $30^{\circ}$ to $65^{\circ}$ quickly spread to the other area of the wall segments. Horizontal flexural cracks are found in the flange of the $\mathrm{H}$-shaped and $\mathrm{T}$-shaped specimens with orthogonal lateral loading, which extend to the full length of the flange. Diagonal shear cracks are observed to have a predominant position in specimens with skew lateral loading.

2. The intersection of diagonal cracks causes severe concrete spalling at the upper part of the web, forms a major horizontal sliding plane, and eventually leads to the failure of Specimens HPOD0 and TPOD0, which is related to the weaker concrete adopted and the stress concentration caused by the stiff top slab.

3. Several vertical slip planes are found on the web of Specimens HP5D0 and TP5D0, which are attributed to the combined effects of axial loads and stiff flanges. The web of these walls is dominated by shear, while the flange is controlled by flexure. Thus a deformation incompatibility exists between the web and flange when the specimen is imposed with cyclic lateral displacements. Further analytical investigations reveal that the deformation incompatibility reaches its maximum at a height of $0.42 h_{w}$, leading to the vertical slip planes forming at the same altitude along the weakened area. 
4. Generally, the axial load contributes to the increase of shear strength and secant stiffness of test specimens, since it can effectively constrain diagonal cracks and limit the development of the sliding. Moreover, specimens with greater axial loads demonstrate better energy dissipation capacities.

5. Shear deformation dominates the response of test specimens since it accounts for more than 50 percent of the total deformation in most specimens. During the test, the shear deformation increases in all T-shaped and $\mathrm{H}$-shaped specimens, with a larger growth found in the latter. In the Lshaped specimens, a slightly lower portion of the shear deformation is witnessed in the group with skew lateral loading, as a result of more evenly distributed shear force in two segments. Sustained and slight increases of sliding shear displacements are observed in all specimens, in which a more rapid growth is found in the specimens with lower axial loads.

6. Data concerning strains of longitudinal reinforcement in the flange shows that a significant shear lag effect exists in some specimens. Due to this effect, some longitudinal reinforcing bars do not yield during the whole test. This effect is more evident in the L-shaped specimens. Besides, the shear lag effect is also observed when the flange is in compression. Axial loads are found to increase the impact of shear lag effect, while less influence of the shear lag effect is witnessed as lateral displacements increase.

7. Surges of strains are detected in the middle of No.7 horizontal rebar and the left and right parts of No.1 horizontal rebar in most specimens, suggesting a diagonal compression strut passing through these places. Hence, the strutand-tie model is considered appropriate for the non-rectangular RC squat walls.

8. The methods proposed Pauley and Priestley [P4] and Li and Xiang [L3] overestimate the effective stiffness of test specimens, as a result of neglecting non-rectangular section shapes and the shear lag effect. Besides, these methods also yield identical results for both loading directions, which is considered improper for the L-shaped and T-shaped specimens since their 
sections are not symmetrical about the bending axis. As corroborated by Zhang [Z2] and Xiang [X1], the increase of axial loads brings growth on the effective stiffness. Also, specimens with skew lateral loading have greater effective stiffness than specimens with orthogonal lateral loading.

\subsubsection{Peak shear strength of non-rectangular RC squat walls}

1. Significant scatters exist in the prediction of the peak shear strength of test specimens using equations from Section 11.5 and 18.1 of ACI 318-14 [A1], ASCE 43-05 [A3], Barda et al. [B1], Wood [W7], Hwang et al. [H4], Gulec and Whittaker [G3], Kassem [K1]. Most equations underestimate the peak shear strength of non-rectangular RC squat walls since the effect of large flanges is not incorporated.

2. The equation proposed by Gulec and Whittaker [G3] yields relatively accurate results in the prediction of the peak shear strength of squat walls with flanged cross section. However, the force mechanism underlying the equation is considered rather simple and the contribution from longitudinal reinforcement in the flange is overestimated.

3. The proposed strut-and-tie model properly accounts for the force-resisting mechanism in non-rectangular RC squat walls, since it not only comprises the three mechanisms developed for squat walls, but also includes the effect of the concrete and reinforcement in the flange. Based on this model, the equations derived from the nonlinear regression are able to precisely predict the peak shear strength of non-rectangular RC squat walls as proven in the sound statistical results.

4. The confidence interval of shear strength in terms of Mean \pm STD or Mean $\pm 2 \times$ STD can be estimated directly by the proposed probabilistic equation, which facilitates its utilization in the engineering practice.

5. The equation proposed by Gulec and Whittaker [G3] overestimates the shear strength of non-rectangular RC squat walls in the database with an average probability of $0.85 \%$. The equations provided by Section 11.5 of ACI 318- 
14 [A1], Section 18.10 of ACI 318-14 [A1], ASCE 43-05 [A3], Barda et al. [B1] and Wood [W7] underestimate the peak shear strength of these walls with average probabilities of $30.2 \%, 34.1 \%, 23.5 \%, 5.41 \%$ and $39.8 \%$ respectively.

\subsubsection{Influence of lateral loading direction on the peak shear strength of non- rectangular $\mathrm{RC}$ squat walls}

1. From the finite element analyses, it is revealed that the peak shear strength of non-rectangular RC squat walls varies at different lateral loading directions.

2. As for L-shaped walls, the maximum peak shear strength is reached when lateral loads are $120^{\circ}$ from the web. Among these walls, those with a flange length of $650 \mathrm{~mm}$ attain the minimum peak shear strength when lateral loads are parallel to the flange. Those with $1400 \mathrm{~mm}$ flange length reach the minimum when horizontal displacements are $20^{\circ}$ from the web. The difference can be as much as $63 \%$ and $38 \%$ for these two types of walls respectively.

3. The maximum peak shear strength of H-shaped walls with $350 \mathrm{~mm}$ flange length is reached when horizontal displacements are parallel to the web, which is approximately $143 \%$ of the minimum peak shear strength achieved when lateral loads are perpendicular to the web. As for models with a flange length of $700 \mathrm{~mm}$, the opposite is true. The difference between the maximum and the minimum values is $40 \%$.

4. As for T-shaped walls, models with $350 \mathrm{~mm}$ flange length reach the maximum peak shear strength when horizontal displacements are parallel to the web, which doubles the minimum peak shear strength achieved when lateral loads are parallel to the flange. Models with a flange length of 700 $\mathrm{mm}$ reach the maximum peak shear strength when the lateral loading is $40^{\circ}$ from the web, and the minimum value when lateral loads are parallel to the flange. Their difference can be as much as $30 \%$. 
5. The influence of the flange length on the peak shear strength of nonrectangular RC squat walls is significant. For models with a shorter flange length, the resistance provided by the flange in one principle direction also declines, which affects the overall strength to a large extent.

\subsection{Suggestions for Future Research}

Based on the literature review and research presented in this study, the following suggestions are summarized for future research:

1. Currently, experiments on RC squat walls with non-rectangular section shapes are still very limited. More tests on non-rectangular squat walls should be carried out to further investigate their seismic behaviors.

2. Experiments should be carried out to study the behavior of non-rectangular $\mathrm{RC}$ walls under various lateral loading directions.

3. Current methods overestimate the effective stiffness of non-rectangular RC squat walls. Further study is required to investigate the effective stiffness of non-rectangular RC squat walls.

4. Methods available in the building codes and the literature are found inappropriate for the estimation of the effective flange width of nonrectangular RC squat walls. Further research is required to improve the evaluation of the effective flange length.

5. In this study, a two-dimensional analytical model incorporating the effects of flange segments is proposed for the non-rectangular RC squat walls. A more sophisticated and realistic 3D model is required to better account for the force-resisting mechanism of these walls.

6. Current codes and standards should be modified to include the equations specifically developed for non-rectangular RC squat walls.

7. The influence of lateral loading direction on the peak shear strength of nonrectangular RC squat walls is significant, and requires corresponding explanations in codes and standards. 


\section{REFERENCES}

[A1] ACI Committee 318 (2014), "Building Code Requirements for Structural Concrete (ACI 318M-14) and Commentary (ACI 318M-14)", American Concrete Institute, Farmington Hills, MI, 503 pp.

[A2] Aghakouchak, A. and Habib, E. (2010), "Application of a Conceptual Hydrologic Model in Teaching Hydrologic Processes", International Journal of Engineering Education, Vol. 26, No. 4, pp. 963-973.

[A3] American Society of Civil Engineers (2005), "Seismic Design Criteria for Structures, Systems, and Components in Nuclear Facilities (ASCE/SEI 4305)", ASCE, Reston, VA, 96 pp.

[B1] Barda, F., Hanson, J. M. and Corley, W. G. (1977), "Shear Strength of LowRise Walls with Boundary Elements", Reinforced Concrete Structures in Seismic Zones, SP 53-8, American Concrete Institute, Farmington Hills, MI, pp. 149-202.

[B2] Beven, K. and Binley, A. (1992), "The Future of Distributed Models: Model Calibration and Uncertainty Prediction", Hydrological Processes, Vol. 6, No. 3, pp. 279-298.

[B3] Beyer, K., Dazio, A. and Priestley, M. J. N. (2011), "Shear Deformations of Slender Reinforced Concrete Walls under Seismic Loading", ACI Structural Journal, Vol. 108, No. 2, pp. 167-177.

[B4] Box, G. E. and Tiao, G. C. (2011), "Bayesian Inference in Statistical Analysis", John Wiley \& Sons, 608 pp.

[B5] Brueggen, B. L. (2009), "Performance of T-Shaped Reinforced Concrete Structural Walls under Multi-Directional Loading", Ph.D Thesis, University of Minnesota, $482 \mathrm{pp}$.

[C1] Comité Euro-International du Béton (1993), "CEB-FIP Model Code 1990", Thomas Telford Services Ltd., London, 437 pp.

[D1] DIANA 9.4 (2012), TNO DIANA BV, Delft, Netherlands.

[F1] Farvashany, F. E., Foster, S. J. and Rangan, B. V. (2008), "Strength and Deformation of High-Strength Concrete Shearwalls", ACI Structural Journal, Vol. 105, No. 1, pp. 21-29. 
[F2] Fenwick, R. and Bull, D. (2000), "What Is the Stiffness of Reinforced Concrete Walls", SESOC Journal, Vol. 13, No. 2, pp. 23-32.

[G1] Gardoni, P., Der Kiureghian, A. and Mosalam, K. M. (2002), "Probabilistic Capacity Models and Fragility Estimates for Reinforced Concrete Columns Based on Experimental Observations", Journal of Engineering Mechanics, Vol. 128, No. 10, pp. 1024-1038.

[G2] Gulec, C. K. (2009), "Performance-Based Assessment and Design of Squat Reinforced Concrete Shear Walls", Ph.D Thesis, State University of New York at Buffalo, 719 pp.

[G3] Gulec, C. K. and Whittaker, A. S. (2011), "Empirical Equations for Peak Shear Strength of Low Aspect Ratio Reinforced Concrete Walls", ACI Structural Journal, Vol. 108, No. 1, pp. 80-89.

[G4] Gulec, C. K., Whittaker, A. S. and Stojadinovic, B. (2008), "Shear Strength of Squat Rectangular Reinforced Concrete Walls", ACI Structural Journal, Vol. 105, No. 4, pp. 488-497.

[G5] Gulec, C. K., Whittaker, A. S. and Stojadinovic, B. (2009), "Peak Shear Strength of Squat Reinforced Concrete Walls with Boundary Barbells or Flanges", ACI Structural Journal, Vol. 106, No. 3, pp. 368-377.

[G6] Gupta, A. and Rangan, B. V. (1998), "High-Strength Concrete (HSC) Structural Walls", ACI Structural Journal, Vol. 95, No. 2, pp. 194-204.

[H1] Hassan, M. and El-Tawil, S. (2003), "Tension Flange Effective Width in Reinforced Concrete Shear Walls", ACI Structural Journal, Vol. 100, No. 3, pp. 349-356.

[H2] Hiraishi, H. (1984), "Evaluation of Shear and Flexural Deformations of Flexural Type Shear Walls", Bulletin of the New Zealand National Society for Earthquake Engineering, Vol. 17, No. 2, pp. 135-144.

[H3] Hsu, T. T. C. and Mo, Y. L. (1985), "Softening of Concrete in Low-Rise Shearwalls", ACI Structural Journal, Vol. 82, No. 6, pp. 883-889.

[H4] Hwang, S. J., Fang, W. H., Lee, H. J., and Yu, H. W. (2001), "Analytical Model for Predicting Shear Strength of Squat Walls", Journal of Structural Engineering, Vol. 127, No. 1, pp. 43-50. 
[K1] Kassem, W. (2014), "Shear Strength of Squat Walls: a Strut-And-Tie Model and Closed-form Design Formula", Engineering Structures, Vol. 84, pp. 430-438.

[K2] Kitada, Y., Akino, K., Terada, K., Aoyama, H., and Miller, A. (1997), "Report on Seismic Shear Wall International Standard Problem Organized by OECD/NEA/CSNI", Fourteenth International Conference on Structural Mechanics in Reactor Technology, Lyon, France.

[K3] Kwak, H. G., and Kim, D. Y. (2004), "Material Nonlinear Analysis of RC Shear Walls Subject to Cyclic Loadings", Engineering Structures, Vol. 26, pp. 1423-1436.

[L1] Lan, W., Ma, J. and Li, B. (2015), "Seismic Performance of Steel-Concrete Composite Structural Walls with Internal Bracings", Journal of Constructional Steel Research, Vol. 110, pp. 76-89.

[L2] Lefas, I. D., Kotsovos, M. D., and Ambraseys, N. N. (1990), "Behavior of Reinforced Concrete Structural Walls: Strength, Deformation Characteristics, and Failure Mechanism", ACI Structural Journal, Vol. 87, No. 1, pp. 23-31.

[L3] Li, B. and Xiang, W. (2011), "Effective Stiffness of Squat Structural Walls", Journal of Structural Engineering, Vol. 137, No. 12, pp. 1470-1479.

[L4] Li, J., Feng, D., Gao, X. and Zhang, Y. (2016), "Stochastic Nonlinear Behavior of Reinforced Concrete Frames. I: Experimental Investigation", Journal of Structural Engineering, Vol. 142, No. 3, pp. 1-15.

[L5] Luna, B. N. (2016), "Seismic Response of Low Aspect Ratio Reinforced Concrete Walls for Buildings and Safety-Related Nuclear Applications", Ph.D Thesis, State University of New York at Buffalo, 435 pp.

[L6] Luna, B. N., Rivera, J. P. and Whittaker, A. S. (2015), "Seismic Behavior of Low-Aspect-Ratio Reinforced Concrete Shear Walls", ACI Structural Journal, Vol. 112, No. 5, pp. 593-603.

[M1] Makowski, D., Wallach, D. and Tremblay, M. (2002), "Using a Bayesian Approach to Parameter Estimation; Comparison of the GLUE and MCMC Methods", Agronomie, Vol. 22, No. 2, pp. 191-203. 
[M2] Markou, J. T. and Durkin, S. A. (2011), "Chilean Earthquake of 2010: Response and Lessons", Nova Science Publishers, Hauppauge, New York, $135 \mathrm{pp}$.

[M3] MatLab R2012b (2012), The MathWorks, Inc., Natick, MA.

[M4] Mo, Y. L. and Chan, J. (1996), "Behavior of Reinforced-Concrete-Framed Shear Walls", Nuclear Engineering and Design, Vol. 166, No. 1, pp. 55-68.

[M5] Montanari, A. (2005), "Large Sample Behaviors of the Generalized Likelihood Uncertainty Estimation (GLUE) in Assessing the Uncertainty of Rainfall-Runoff Simulations", Water Resources Research, Vol. 41, No. 8, pp. $1-13$.

[M6] Mundfrom, D. J., Shaw, D. G. and Ke, T. (2005), "Minimum Sample Size Recommendations for Conducting Factor Analyses", International Journal of Testing, Vol. 5, No. 2, pp. 159-168.

[N1] Nash, J. E. and Sutcliffe, J. V. (1970), "River Flow Forecasting through Conceptual Models: Part I - A Discussion of Principles", Journal of Hydrology, Vol. 10, No. 3, pp. 282-290.

[N2] Naze, P. A. and Sidaner, J. F. (2001), "Presentation and Interpretation of SAFE Tests: Reinforced Concrete Walls Subjected to Shearing", Sixteenth International Conference on Structural Mechanics in Reactor Technology, Washington, DC.

[N3] Ngo, D. and Scordelis, A. C. (1967), "Finite Element Analysis of Reinforced Concrete Beams", American Concrete Institute Journal, Vol. 64, No. 3, pp. 152-163.

[N4] Ning, C. and Li, B. (2016), "Probabilistic Approach for Estimating Plastic Hinge Length of Reinforced Concrete Columns", Journal of Structural Engineering, Vol. 142, No. 3, pp. 1-11.

[P1] Palermo, D. (2002), "Behaviour and Analysis of Reinforced Concrete Walls Subjected to Reversed Cyclic Loading", Ph.D Thesis, University of Toronto, 492 pp.

[P2] Palermo, D. and Vecchio, F. J. (2002), "Behavior of Three-Dimensional Reinforced Concrete Shear Walls", ACI Structural Journal, Vol. 99, No. 1, pp. 81-89. 
[P3] Paulay, T., Priestley, M. J. N. and Synge, A. J. (1982), "Ductility in Earthquake Resisting Squat Shearwalls", ACI Structural Journal, Vol. 79, No. 4, pp. 257-269.

[P4] Paulay, T. and Priestley, M. J. N. (1992), " Seismic Design of Reinforced Concrete and Masonry Buildings", John Wiley \& Sons, New York, 744 pp.

[R1] Rashid, Y. R. (1968), "Ultimate Strength Analysis of Prestressed Concrete Pressure Vessels", Nuclear Engineering and Design, Vol. 7, No. 4, pp. 334344.

[S1] Sørensen, R. K. (2013), "Evaluation of Shear Lag in Standard H-/I-Sections", Master Thesis, Aalborg University Esbjerg, 64 pp.

[S2] Saito, H., Kikuchi, R., Kanechika, M., and Okamoto, K. (1989), "Experimental Study on the Effect of Concrete Strength on Shear Wall Behavior", Tenth International Conference on Structural Mechanics in Reactor Technology, Anaheim, CA.

[S3] Sato, S., Ogata, Y., Yoshizaki, S., Kanata, K., Yamaguchi, T., Nakayama, T., Inada, Y., and Kadoriku, J. (1989), "Behavior of Shear Wall Using Various Yield Strength of Rebar, Part 1: An Experimental Study", Tenth International Conference on Structural Mechanics in Reactor Technology, Anaheim, CA.

[S4] Seki, M., Kobayashi, J., Shibata, A., Kubo, T., Taira, T., and Akino, K. (1995), "Restoring Force Verification Test on RC Shear Wall", Thirteenth International Conference on Structural Mechanics in Reactor Technology, Porto Alegre, Brazil.

[S5] Selby, R. G., Vecchio, F. J. and Collins, M. P. (1996), "Analysis of Reinforced Concrete Members Subject to Shear and Axial Compression", ACI Structural Journal, Vol. 93, No. 3, pp. 306-315.

[S6] Sittipunt, C. and Wood, S. L. (1993), "Finite Element Analysis of Reinforced Concrete Shear Walls", University of Illinois at UrbanaChampaign, Urbana, IL, 384 pp.

[S7] Sittipunt, C. and Wood, S. L. (1995), "Influence of Web Reinforcement on the Cyclic Response of Structural Walls", ACI Structural Journal, Vol. 92, No. 6, pp. 745-756. 
[S8] Stojadinovic, B. and Thewalt, C. R. (1996), "Energy Balanced Hysteresis Models", Eleventh World Conference on Earthquake Engineering, Acapulco, Mexico.

[S9] Syed, S. and Gupta, A. (2015), "Seismic Fragility of RC Shear Walls in Nuclear Power Plant Part 1: Characterization of Uncertainty in Concrete Constitutive Model", Nuclear Engineering and Design, Vol. 295, pp. 576586.

[S10] Synge, A. J. (1980), "Ductility of Squat Shear Walls", Research Report No. 80-8, University of Canterbury, Christchurch, $150 \mathrm{pp}$.

[T1] Thomsen IV, J. H. and Wallace, J. W. (2004), "Displacement-Based Design of Slender Reinforced Concrete Structural Walls-Experimental Verification", Journal of Structural Engineering, Vol. 130, No. 4, pp. 618-630.

[U1] Uniform Building Code (1994), "Uniform Building Code", International Conference of Building Officials, Whittier, Calif, 492 pp.

[V1] Vecchio, F. J. and Collins, M. P. (1993), "Compression Response of Cracked Reinforced Concrete", Journal of Structural Engineering, Vol. 119, No. 12 , pp. $3590-3610$.

[W1] Wallace, J. W. (1994), "A Methodology for Developing New Code Provisions for Seismic Design of RC Shear Walls", Journal of Structural Engineering, Vol.120, No.3, pp. 863-884.

[W2] Wallace, J. W. (1995), "Seismic Design of RC Structural Walls. Part I: New Code Format", Journal of Structural Engineering, Vol. 121, No. 1 pp. 75-87.

[W3] Wallace, J. W. (1996), "Evaluation of UBC-94 Provisions for Seismic Design of RC Structural Walls", Earthquake Spectra, Vol. 12, No. 2 pp. 327-347.

[W4] Wallace, J. W. and Thomsen IV, J. H. (1995), "Seismic Design of RC Structural Walls. Part II: Applications", Journal of Structural Engineering, Vol. 121, No. 1, pp. 88-101.

[W5] Wang, X., He, X., Williams, J. R., Izaurralde, R. C. and Atwood, J. D. (2005), "Sensitivity and Uncertainty Analyses of Crop Yields and Soil Organic Carbon Simulated with EPIC", Transactions of the ASAE, Vol. 48, No. 3, pp. 1041-1054. 
[W6] Whyte, C. and Stojadinovic, B. (2014), "Effect of Ground Motion Sequence on Response of Squat Reinforced Concrete Shear Walls", Journal of Structural Engineering, Vol. 140, No. 8, pp. 1-7.

[W7] Wood, S. L. (1990), "Shear Strength of Low-Rise Reinforced Concrete Walls", ACI Structural Journal, Vol. 87, No. 1, pp. 99-107.

[X1] Xiang, W. (2008), "Seismic Performance of Rc Structural Squat Walls with Limited Transverse Reinforcement", Ph.D Thesis, Nanyang Technological University, $261 \mathrm{pp}$.

[X2] XTRACT version 3.0.8 (2010), TRC Companies, Inc., Lowell, MA.

[Z1] Zhang, Z. and Li, B. (2016), "Seismic Performance Assessment of Slender T-Shaped Reinforced Concrete Walls", Journal of Earthquake Engineering, Vol. 20, No. 8, pp. 1342-1369.

[Z2] Zhang, Z. (2015), "Seismic Behavior of Non-Rectangular RC Walls with Inferior Details Subjected to Loading from Different Direction", Ph.D Thesis, Nanyang Technological University, 223 pp. 